\title{
ROTE-METO COMPARATIVE DICTIONARY
}





\section{ROTE-METO COMPARATIVE DICTIONARY}

OWEN EDWARDS

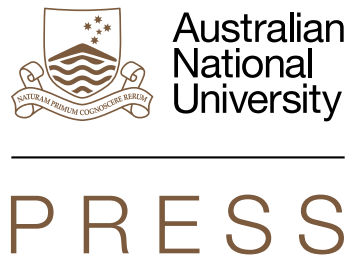




\section{ANU \\ PRESS}

Published by ANU Press

The Australian National University

Acton ACT 2601, Australia

Email: anupress@anu.edu.au

Available to download for free at press.anu.edu.au

A catalogue record for this book is available from the National Library of Australia

ISBN (print): 9781760464561

ISBN (online): 9781760464578

WorldCat (print): 1268571904

WorldCat (online): 1268255637

DOI: 10.22459/RMCD.2021

This title is published under a Creative Commons Attribution-NonCommercial-NoDerivatives 4.0 International (CC BY-NC-ND 4.0).

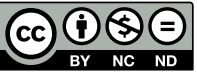

The full licence terms are available at

creativecommons.org/licenses/by-nc-nd/4.0/legalcode

Cover design and layout by ANU Press. Cover photograph by Kirsten Culhane.

This edition $(0) 2021$ ANU Press 


\section{Contents}

Acknowledgements vii

Abbreviations and symbols $\quad$ ix

Speech varieties listed in the dictionary $\quad$ xi

1. Introduction 1

1.1 Purpose 1

1.2 Limitations 1

1.3 Data sources 3

1.3.1 Rote 3

1.3.2 Meto 5

$\begin{array}{ll}\text { 1.3.3 Other languages } & 7\end{array}$

1.4 Transcription $\quad 8$

1.4.1 Jonker's transcription $\quad 8$

1.4.2 Middelkoop's transcription 13

$\begin{array}{ll}1.5 \text { Structure of the dictionary } & 15\end{array}$

$\begin{array}{ll}\text { 1.5.1 Out-comparisons } & 17\end{array}$

$\begin{array}{ll}\text { 1.5.2 Multiple reflexes } & 18\end{array}$

$\begin{array}{ll}\text { 1.5.3 Fields/parts of entries } & 20\end{array}$

$\begin{array}{ll}\text { 1.5.4 Loan distributions } & 27\end{array}$

$\begin{array}{ll}\text { 1.5.5 Finder lists } & 27\end{array}$

2. Language background 29

2.1 Introduction $\quad 29$

2.2 Rote 30

2.3 Meto $\quad 32$

2.4 Segmental phonologies $\quad 34$

2.4.1 Rote 34

2.4.2 Meto 36

2.5 Meto morphophonemic processes 37

$\begin{array}{ll}\text { 2.5.1 Metathesis } & 37\end{array}$

2.5.2 Consonant insertion $\quad 38$

2.5.3 Diphthongisation and vowel assimilation 39 
2.6 Morphology 40

2.6.1 Nominal suffix $-k /-? \quad 41$

2.6.2 Possessive morphology $\quad 42$

2.6.3 Reduplication 43

2.6.4 Nominalisation $\quad 44$

2.6.5 Verb agreement 45

$\begin{array}{ll}\text { 2.6.6 Derivational verbal morphology } & 47\end{array}$

3. Historical background 49

3.1 Introduction 49

$\begin{array}{ll}3.2 \text { Sound correspondences } & 49\end{array}$

3.2.1 Initial and medial consonants $\quad 50$

3.2.2 Final consonants $\quad 55$

3.2.3 Vowels $\quad 55$

$\begin{array}{ll}3.3 \text { Internal sub-grouping } & 57\end{array}$

3.3.1 Nuclear Rote (NRote) $\quad 61$

3.3.2 Central East Rote and Meto (CERM) $\quad 62$

3.3.3 West Rote-Meto (WRM) 64

3.3.4 Meto 64

3.4 Levels of reconstruction $\quad 69$

$\begin{array}{ll}3.5 \text { Rote-Meto within Malayo-Polynesian } & 72\end{array}$

3.5.1 Sound changes between PMP and PRM 72

$\begin{array}{ll}\text { 3.5.2 Sound change and language shift } & 74\end{array}$

3.5.3 Subgrouping within Malayo-Polynesian $\quad 76$

4. Rote-Meto - English 87

5. English - Rote-Meto 405

6. Proto-Malayo-Polynesian - Proto-Rote-Meto 429

References 441 


\section{Acknowledgements}

While this comparative dictionary is collated by only one author, much of the data was collected by many others.

I would like to thank all those who generously shared their unpublished lexical databases, listed in alphabetical order by surname of first author (see $\S 1.3$ for more information): Misriani Balle and Stuart Cameron (Helong); John Christensen (Kisar); João Cristo Rei and Mark Donohue (Galolen); Kirsten Culhane, Laurence Jumetan, and Yedida Ora (Amfo'an); James Fox (Termanu ritual language); Charles E. Grimes, Evelyn Cheng, Enna Adelaide Hayer-Pah, Jonathan Pandie, Neng Mulosing, and Johnny M. Banamtuan (Tii), Yustin Nako, Paulus Nako, Misriani Balle, and Johnny M. Banamtuan (Rikou), Thersia Tamelan (Dela), Catharina Williams-van Klinken (Fehan Tetun); as well as Albert Zacharias, Adika Getroida Balukh, Misriani Balle, and Johnny M. Banamtuan (Lole).

I would also like to thank my many consultants who provided me with data from their languages. It is with great regret that I do not have enough space to list all those who have generously shared knowledge of their languages. Nonetheless, I must mention the following people: Dominggus Atimeta (Timaus), Heronimus Bani (Kotos Amarasi), Toni Buraen and family (Ro'is Amarasi), Yulius Iu (Landu), Melianus Obhetan and family (Ro'is Amarasi), Yedida Ora (Kotos Amarasi), Pieter Sijoen (Oepao), Ferdis Tasae (Funai Helong), and Manuel Un Bria, Emerentiana Uduk, and Aloyisus Nurak (all Kusa-Manea).

I would further like to thank several scholars who have contributed in various ways.

Tom Hoogervorst consulted an earlier version of this dictionary and identified many forms that are ultimately loans. Malcolm Ross also read an earlier version of this dictionary and identified a number of forms that are inherited from a higher node, as well as a few connections between Proto-Rote-Meto reconstructions and Proto-Oceanic.

Antoinette Schapper first invited me to present on Rote-Meto at the international workshop on language contact and substrate in the languages of Wallacea and thus gave me the impetus to launch this project. She also suggested that the work be structured around Proto-Rote-Meto reconstructions with a clearer demarcation between Rote-Meto forms and out-comparisons. This has greatly improved the clarity of the work compared to early drafts. 
Marian Klamer provided me with the job during which much data was added to this dictionary and many revisions were carried out. I am happy to say that this publication was supported by the VICI research project 'Reconstructing the past through languages of the present: the Lesser Sunda Islands', funded by the Netherlands Organisation for Scientific Research, project number 277-70-012.

Mark Donohue has greatly influenced my thinking on the history of Austronesian languages. He also invited me to the workshop in Kupang in July 2012 where I first encountered the languages and people of Timor.

I also need to thank Charles Grimes. Apart from his constant encouragement and support, I'm sure that this is a project that Chuck would have loved to have worked on himself, in some form or another. I am humbled by his generosity in allowing me to do this work.

Finally, the deficiencies that undoubtedly remain in this work are entirely my own responsibility and none of the people mentioned in these acknowledgements are responsible for them. 


\section{Abbreviations and symbols}

\begin{tabular}{|c|c|c|}
\hline$*$ & reconstruction & (asterisk) \\
\hline$* *$ & pseudo-reconstruction & $($ see $\S 1.5 .3 .3)$ \\
\hline$=$ & clitic & (equals sign) \\
\hline \# & cognate set spread by borrowing & (hash) \\
\hline- & productive affix & (hyphen) \\
\hline o & borrowed word & ordinal indicator) \\
\hline / & historic compound; neither member independent & (slash) \\
\hline$\sim$ & reduplication & (tilde) \\
\hline$\frown$ & vowel sequence formed by diphthongisation & (tie; see $§ 2.5 .3$ ) \\
\hline _ & historic compound; one member no longer independent & (underscore) \\
\hline | & historic affix & (vertical bar) \\
\hline CEMP & Central-Eastern Malayo-Polynesian & \\
\hline CER & Central East Rote & cept Tii and Lole) \\
\hline CERM & Central East Rote and Meto & \\
\hline CMP & Central Malayo Polynesian & \\
\hline $\mathrm{dJ}$ & de Josselin de Jong (1947) & \\
\hline $\mathrm{J}$ & Jonker (1908) & \\
\hline M & Middelkoop (1972) & \\
\hline Mo & Morris (1984) & \\
\hline $\mathrm{nRM}$ & Nuclear Rote-Meto & \\
\hline nRote & (Rote except Dela-Oen & (Rote except Dela-Oenale and Dengka) \\
\hline On & Onvlee (1984) & \\
\hline PCEMP & Proto-Central-Eastern Malayo-Polynesian & \\
\hline PCMP & Proto-Central Malayo-Polynesian & \\
\hline PMeto & Proto-Meto & \\
\hline PMP & Proto-Malayo-Polynesian & \\
\hline PnMeto & Proto-Nuclear Meto & pt Ro'is Amarasi) \\
\hline POc & Proto Oceanic & \\
\hline
\end{tabular}




$\begin{array}{ll}\text { ROTE-METO COMPARATIVE DICTIONARY } \\ \text { PnRote } & \text { Proto-Nuclear Rote } \\ \text { PRM } & \text { Proto-Rote-Meto } \\ \text { PWMP } & \text { Proto-Western Malayo-Polynesian } \\ \text { PwRM } & \text { Proto-West Rote-Meto } \\ \text { PwRote } & \text { Proto-West Rote } \\ \text { UBB } & \text { Unit Bahasa dan Budaya } \\ \text { wRM } & \text { West Rote-Meto } \\ \text { wRote } & \text { West Rote }\end{array}$

(Language and Culture Unit)

(Dela-Oenale and Dengka) 


\section{Speech varieties listed in the dictionary}

\section{Rote-Meto speech varieties}

\begin{tabular}{|c|c|c|c|}
\hline Lect & Rote/Meto & ISO & Glottocode \\
\hline Amanatun & Meto & $\mathrm{aoz}$ & $\operatorname{aman} 1264$ \\
\hline Amanuban & Meto & aoz & aman1264 \\
\hline Amanuban, South & Meto & aoz & $\operatorname{aman} 1266$ \\
\hline Amfo'an & Meto & $\mathrm{aoz}$ & amfo1237 \\
\hline Ba'a & Rote & $\operatorname{llg}$ & baaa1237 \\
\hline Baikeno & Meto & bkx & baik1238 \\
\hline Bilbaa & Rote & bpz & bilb1242 \\
\hline Bokai & Rote & twu & boka1251 \\
\hline Dela & Rote & row & dela1252 \\
\hline Dengka & Rote & dnk & deng1253 \\
\hline Fatule'u & Meto & aoz & amfo1237 \\
\hline Keka & Rote & twu & keka1234 \\
\hline Ketun & Meto & - & - \\
\hline Kopas & Meto & - & - \\
\hline Korbafo & Rote & twu & korb1237 \\
\hline Kotos Amarasi & Meto & aaz & koto1251 \\
\hline Kusa-Manea & Meto & aoz & kusa1252 \\
\hline Landu & Rote & rgu & land 1257 \\
\hline Lole & Rote & $11 \mathrm{~g}$ & lole1239 \\
\hline Meto $^{\dagger}$ & Meto & $\mathrm{aoz}$ & uabm1237 \\
\hline Miomafo & Meto & aoz & moll1242 \\
\hline Molo & Meto & $\mathrm{aoz}$ & moll1242 \\
\hline Oenale & Rote & row & oena1237 \\
\hline Oepao & Rote & rgu & oepa1237 \\
\hline Rikou & Rote & rgu & nucl1538 \\
\hline Ro'is Amarasi & Meto & aaz & rois1241 \\
\hline Termanu & Rote & twu & pada1259 \\
\hline Tii & Rote & txq & tiii1241 \\
\hline Timaus & Meto & - & - \\
\hline
\end{tabular}

$\dagger$ 'Meto' is used when a form is taken from Jonker (1908) who does not specify which variety of Meto his data is from. 
ROTE-METO COMPARATIVE DICTIONARY

\section{Speech varieties (with at least four occurrences) in out-comparisons}

\begin{tabular}{|c|c|c|c|c|}
\hline Lect & Region & Sub-region & ISO & Glottocode \\
\hline Alorese & Alor-Pantar & Alor and Pantar & aol & alor1247 \\
\hline Anakalang & Sumba & west Sumba & akg & anak1240 \\
\hline Asilulu & C. Maluku & Ambon Island & asl & asil1242 \\
\hline Bima & Sumbawa & East Sumbawa & bhp & bima1247 \\
\hline Bolok Helong & Timor & west Timor & heg & helo1244 \\
\hline Bugis & Sulawesi & south Sulawesi & bug & bugi1244 \\
\hline Buru & C. Maluku & Buru & mhs & buru1303 \\
\hline Central Lembata & Flores & Solor Islands & lvu & kali1300 \\
\hline Central Nage & Flores & central Flores & nxe & cent 2355 \\
\hline Dadu'a & Timor & Atauro & - & dadu1237 \\
\hline Dhao & Timor & Dhao & nfa & dhao1237 \\
\hline East Tetun & Timor & east Timor & tet & east 2473 \\
\hline Ende & Flores & central Flores & end & ende1246 \\
\hline Fehan Tetun & Timor & central Timor & tet & sout 2898 \\
\hline Funai Helong & Timor & west Timor & heg & funa1237 \\
\hline Galolen & Timor & east Timor & gal & galo1243 \\
\hline Haruku & C. Maluku & Lease Islands & hrk & haru1244 \\
\hline Hawu & Timor & Sabu Island & hvn & sabu1255 \\
\hline Helong ${ }^{\dagger}$ & Timor & west Timor & heg & helo1243 \\
\hline Idate & Timor & east Timor & idt & idat1237 \\
\hline Ili'uun & S.W. Maluku & Wetar Island & ilu & iliu1237 \\
\hline Kaibobo & C. Maluku & west Seram & kzb & kaib1244 \\
\hline Kamarian & C. Maluku & west Seram & kzx & kama1362 \\
\hline Kambera & Sumba & east Sumba & $\mathrm{xbr}$ & nucl1521 \\
\hline Kemak & Timor & east Timor & kem & kema1243 \\
\hline Kisar & S.W. Maluku & Kisar Island & kje & kisa1266 \\
\hline Kodi & Sumba & west Sumba & kod & kodi1247 \\
\hline Komodo & Flores & west Flores & kvh & komo1261 \\
\hline Kupang Malay & Timor & west Timor & mkn & kupa1239 \\
\hline Lewa & Sumba & east Sumba & $\mathrm{xbr}$ & lewa1240 \\
\hline Makassar & Sulawesi & south Sulawesi & mak & maka1311 \\
\hline Mambae, Central & Timor & central Timor & mgm & - \\
\hline Mambae, Northwest & Timor & central Timor & mgm & - \\
\hline Mambae, South & Timor & central Timor & mgm & - \\
\hline Mamboru & Sumba & west Sumba & mvd & mamb1305 \\
\hline Manggarai & Flores & west Flores & mqy & mang1405 \\
\hline Ngadha & Flores & central Flores & nxg & ngad1261 \\
\hline Roma & S.W. Maluku & Roma Island & $\mathrm{rmm}$ & roma1332 \\
\hline Semau Helong & Timor & Semau Island & heg & helo1245 \\
\hline Sika & Flores & east Flores & ski & sika1262 \\
\hline Tokodede & Timor & central Timor & tkd & tuku1254 \\
\hline Tugun & S.W. Maluku & Wetar Island & tzn & tugu1245 \\
\hline Waima'a & Timor & east Timor & wmh & waim 1252 \\
\hline Welaun & Timor & central Timor & wlh & wela1235 \\
\hline Weyewa & Sumba & west Sumba & wew & weje1237 \\
\hline
\end{tabular}

$\dagger$ 'Helong' is used when a form is taken from Jonker (1908) who does not specify which variety of Helong his data is from. 


\section{1}

\section{Introduction}

\subsection{Purpose}

This comparative dictionary provides an initial bottom-up reconstruction of one lowlevel Austronesian subgroup of the linguistic area of Wallacea: the Rote-Meto subgroup. This work forms one part of the larger bottom-up reconstruction of languages in this region, which is needed to fully understand the history of these languages.

This dictionary contains 1,174 reconstructions to Proto-Rote-Meto (PRM) or one of its lower branches (Chapter 3) along with the reflexes in modern languages that support these reconstructions. Proto-Rote-Meto is the hypothesised shared common ancestor of the Rote languages and the Meto language/dialect cluster spoken on the western part of Timor (Chapter 2).

This dictionary is not a reconstruction of any putative node between Proto-MalayoPolynesian (PMP) and Proto-Rote-Meto (PRM). Nodes between these two levels are debated (Blust 1993; Donohue and Grimes 2008; Blust 2009b). Although reconstructions from putative intermediate nodes such as Proto-Central-Eastern Malayo-Polynesian (PCEMP) or Proto-Central Malayo-Polynesian (PCMP) are sometimes included as etyma for PRM forms, this should not be taken as a claim that these nodes exist. I simply follow the labels given by others to their reconstructions without passing judgement on the validity of these labels. For the purposes of this comparative dictionary I am agnostic regarding the validity of Central-Eastern Malayo-Polynesian (CEMP) and Central Malayo-Polynesian (CMP). The most fruitful way of assessing the evidence for such intermediate nodes is to continue and expand the kind of bottom-up work carried out in this dictionary.

\subsection{Limitations}

The reconstruction of Proto-Rote-Meto in this dictionary has a number of limitations. There are a number of minor limitations imposed by the sources on which this dictionary is based, as discussed further in $\S 1.3$ and $\S 1.4$. 
However, probably the most serious limitation on this dictionary is the availability of data. While we have a wealth of data on the Termanu variety of Rote due to the work of the Dutch linguist J. C. G. Jonker in the nineteenth and earlier twentieth centuries, we have comparatively far less data on other varieties of Rote and the Meto cluster. Based on my experience so far, I fully expect that, as more data become available, it will be possible to add more reconstructions to this dictionary, while some of the current reconstructions will need to be modified.

The kind of additional data that will probably be added to this dictionary can be illustrated with two examples of reconstructed bird terms. Firstly, there is PRM *sinara?e 'Todiramphus spp. (kind of kingfisher)'. This is supported by Bilbaa sulae 'Todiramphus chloris', Rikou sirae 'Todiramphus chloris', Lole sinla?e 'kingfisher', Ro'is Amarasi sanae 'Todiramphus spp.', Kotos Amarasi sa?na?e|k 'Todiramphus spp.', and Amfo'an senae-l 'Todiramphus spp.'. None of the data supporting this reconstruction occur in the works of Jonker. Apart from the Kotos Amarasi term, I collected all the other cognates only at the end of 2017.

Secondly, there is PRM *tadengus 'kind of dove, possibly rose-crowned fruit-dove, Ptilinopus regina'. In this case Dengka leygus and Oenale reygus 'pigeon, dove' (Dutch duif) occur in Jonker (1908:772), but none of the other cognates do. These other cognates were also collected at the end of 2017: Ro'is Amarasi kuum treukus 'rose-crowned fruitdove', Kusa-Manea ra rukis 'wild doves', as well as Landu and Rikou rekus 'rosecrowned fruit-dove'.

Such examples indicate that, even though Jonker's works are, perhaps, the most detailed and voluminous works published on any language of eastern Indonesia, more data on the Rote-Meto languages will allow the identification of yet more cognate sets and associated reconstructions.

Some such cognate sets are probably present in Jonker's works and simply await the discovery of a Meto form to allow reconstruction to PRM. One such example is PRM *hida 'how many', which is reconstructed to PRM on the basis of the Rote reflexes combined with Kusa-Manea (Meto) hian 'how many' — a term not otherwise currently known in the Meto cluster.

There are also probably other cognate sets that are not represented in Jonker's works due to his focus on Termanu. One such example is *leu 'now, already', for which the Rote cognates (Bilbaa and Rikou leu) were collected during my own fieldwork.

The relative lack of available data on speech varieties apart from Termanu is partially mitigated by the fact that I am personally most familiar with the Meto cluster (particularly Kotos Amarasi). The language Jonker knew best is thus complemented by the one I know best. Nonetheless, I am quite certain that more detailed and comprehensive data on Rote languages apart from Termanu, as well as more data on the Meto cluster, will allow reconstruction of many more terms than are currently included in this dictionary. 


\subsection{Data sources}

This dictionary is based on a number of different sources of data. This includes published sources, unpublished descriptions and dictionaries, as well as my own field notes. In this section I discuss the sources on which I have drawn, and the limitations associated with these sources. This includes detailed discussions of Jonker (1908), from which most of my Rote data is drawn, as well as Middelkoop (1972), from which most of my Molo (Meto) data is drawn. The transcriptions used by these works and the problems associated with them are discussed in $\S 1.4$.

Data from several languages is frequently cited without any explicit reference to the source from which such data comes. The languages for which this is the case and the sources from which data for them comes are given in Table 1.1 in alphabetical order. All data for these languages come from sources given here, unless otherwise cited. Sources for other languages are always indicated.

Table 1.1: Sources for languages when no source is specified

\begin{tabular}{|c|c|c|}
\hline Language & Source & Notes \\
\hline Amfo'an & Culhane, Jumetan and Ora (2018) & $\begin{array}{l}\text { unpublished Toolbox file, } 1,711 \text { headwords, } \\
\text { revision published as Grimes et al. (2021) }\end{array}$ \\
\hline Dela (Rote) & Tamelan (2017) & unpublished Toolbox file, 1,911 headwords \\
\hline Dhao & Grimes, Ranoh and Aplugi (2008) & \\
\hline Ende & Aoki and Nakagawa (1993) & \\
\hline Galolen & Cristo Rei and Donohue (2012) & unpublished spreadsheet, 1,128 headwords \\
\hline Hawu & Grimes, Lado, Ly and Tari (2008) & \\
\hline Helong, Funai & Edwards (2018b) & \\
\hline Helong, Semau & Balle and Cameron (2014) & unpublished Toolbox file, 3,368 headwords \\
\hline Helong, Bolok & Balle and Cameron (2014) & Semau is the main dialect of this source \\
\hline Kemak & own field notes & archived with PARADISEC $^{1}$ \\
\hline Kisar & Christensen (in progress) & unpublished Toolbox file, 2,518 headwords \\
\hline Meto lects & own field notes & see $\S 1.3 .1 .2$ \\
\hline Rote lects & Jonker (1908) & abbreviated as ' $\mathrm{J}$ ' \\
\hline Sumba lects & Onvlee (1984) & $\begin{array}{l}\text { includes Anakalang, Kodi, Lewa, Mamboru, } \\
\text { and Weyewa }\end{array}$ \\
\hline Tetun Fehan & van Klinken (1995) & unpublished Toolbox file, 3,444 headwords \\
\hline Waima'a & Himmelmann et al. (2006) & archived Toolbox file, 3,890 headwords \\
\hline Welaun & own field notes & on LexiRumah ${ }^{2}$ and PARADISEC \\
\hline
\end{tabular}

\subsubsection{Rote}

In this section I discuss the sources of my data for the Rote languages. Four kinds of Rote data occur in this comparative dictionary: data from Jonker (1908), data from my own fieldwork, data from work by linguists associated with the Language and Culture Unit (UBB) in Kupang, and data from a draft ritual language dictionary by James Fox (Fox 2016b). See the front matter and $\$ 2.2$ for a list of Rote language/dialects and corresponding ISO 693-3 codes and Glottocodes.

catalog.paradisec.org.au/collections/OE8.

2 lexirumah.model-ling.eu/languages/west2547-welaun. 


\subsubsection{Jonker (1908)}

Jonker (1908) is the source for the vast bulk of my data on the Rote languages. Jonker (1908) is an 805-page 'Rote'-Dutch dictionary. Termanu is the primary variety around which this dictionary is organised. The main section of this dictionary is 672 pages long and lists Termanu headwords, often as underlying roots, with derivatives as sub-headings along with definitions and example sentences. Jonker also usually provides cognates/ equivalents of the Termanu headwords in eight other Rote varieties: Korbafo, Bokai, Bilbaa, Rikou, Ba'a, Tii, Dengka and Oenale. He also occasionally gives cognates from Oepao and Keka.

The cognates from these varieties are listed after the Termanu headword and are not given full definitions. Additionally, the final 140 pages or so of Jonker's dictionary are devoted to 'Forms and words from the other dialects'. This section lists non-Termanu words that have already been given in the main body of the dictionary, as well as forms that are not given there. While some of the headwords in this second part have definitions and example sentences, many entries are simply cross-references to the Termanu form in the main body of the dictionary.

In the introduction, Jonker explains that the non-Termanu words were collected by means of the Termanu form (or a derivative) mostly in a sentence and combined with a Malay translation. The non-Termanu equivalents were then given in written form by various schoolteachers from Rote who were Jonker's consultants. He explicitly states that it should not be assumed that the non-Termanu words have the same shades of meaning as their Termanu cognate/equivalent and notes differences in meaning when he is aware of them.

This imposes one limitation on this comparative dictionary. The exact semantics of most words from varieties of Rote other than Termanu is unknown. When I do not give a definition for a particular Rote form, this is because Jonker does not specify any definition. In such cases, while we can assume that the semantics are similar to the Termanu form, we cannot assume that they are identical. A word from another Rote variety may well have additional meanings that are not present in the Termanu form and/ or Termanu may have uses that another variety does not have.

Regarding the form of words from varieties other than Termanu, Jonker states that he has confidence in the accuracy of his data and he does not include words that he suspected were mistakes. Similarly, forms he judged to be dubious are given in brackets and/or with a question mark.

\subsubsection{Other sources}

Three other kinds of Rote data occur in this comparative dictionary: data from my own fieldwork, data from work by linguists associated with the Kupang based Language and Culture Unit (UBB), and data from Fox (2016b). These other sources are summarised in Table 1.2. 
Table 1.2: Sources other than Jonker (1908) for Rote languages

\begin{tabular}{|l|l|l|}
\hline Lect & Source & Notes \\
\hline Dela & $\begin{array}{l}\text { Tamelan (2017) } \\
\text { Tamelan (2021) }\end{array}$ & $\begin{array}{l}\text { Toolbox }{ }^{3} \text { file 1,911 headwords } \\
\text { PhD thesis }\end{array}$ \\
\hline Lole & Zacharias, Balukh Balle and Banamtuan (2014) & Toolbox file 2,688 headwords \\
\hline Rikou & Nako, Nako, Balle and Banamtuan (2014) & Toolbox file 766 headwords \\
\hline Tii & $\begin{array}{l}\text { Grimes, Cheng, Hayer-Pah, Pandie, Mulosing } \\
\text { and Banamtuan (2014) }\end{array}$ & Toolbox file 3,281 headwords \\
\hline Bilbaa & own field notes & archived with PARADISEC \\
\hline Landu & own field notes & archived with PARADISEC \\
\hline Oepao & own field notes & archived with PARADISEC \\
\hline Rikou & own field notes & archived with PARADISEC \\
\hline Termanu & Fox (2016b) & draft ritual language dictionary \\
\hline
\end{tabular}

Note: PARADISEC can be found at catalog.paradisec.org.au/collections/OE9.

I carried out a week's worth of fieldwork at the beginning of November 2017 in eastern Rote on Bilbaa, Landu, Oepao and Rikou. The primary purpose of this trip was to gather preliminary data on Oepao and Landu, neither of which are well represented in Jonker (1908). ${ }^{4}$ During this trip, I also worked through the Rikou forms that were then present in my comparative database, which Jonker gives as orthographically vowel initial to check whether they begin with an underlying glottal stop (see §1.4.1.1).

Another major source of Rote data comes from work carried out by linguists associated with the Kupang-based Language and Culture Unit (UBB). Particularly important is data on Dela collected and provided by Thersia Tamelan. This includes a draft Dela dictionary (Tamelan 2017), as well as her $\mathrm{PhD}$ thesis at The Australian National University (Tamelan 2021), which is a grammar of Dela. In addition to having lexical forms not found in Jonker (1908), Tamelan's work provides a clear, modern linguistic description of Dela, including information that cannot be easily extracted from Jonker's dense grammatical description of the Rote languages, focused on Termanu (Jonker 1915). ${ }^{5}$ One example of the kind of information lacking in Jonker's work, but described in Tamelan's work, is the distinction between vowel initial and glottal stop initial words (§1.4.1.1).

\subsubsection{Meto}

Meto data mainly comes from two sources: Middelkoop (1972) and my own fieldwork. I also have data on Amfo'an compiled by Kirsten Culhane in the form of a toolbox file with 1,711 headwords (Culhane, Jumetan and Ora 2018), as well as Baikeno data provided by Charles E. Grimes. See the front matter and $\$ 2.3$ for a list of varieties of Meto and corresponding ISO 693-3 codes and Glottocodes.

3 software.sil.org/toolbox/. Toolbox is software program for managing lexical and corpus data

4 Particular thanks go to Pieter Sjioen and Yulius Iu who were my main consultants for Oepao and Landu respectively, as well as Paulus Nako who organised my trip and accompanied me on fieldwork in Rote.

5 Fox and Grimes (1995:611f) put it well regarding Jonker's works, stating: 'Neither Jonker's dictionary nor his grammar is, in any conventional sense, the study of a single language. Jonker used both of these works to advance comparative observations on an extensive array of other Austronesian languages. His desire to be comprehensive, exhaustive and at the same time comparative resulted in studies that present formidable obstacles to a simple comprehension of the basic structures of Rotinese [sic]'. 


\subsubsection{Middelkoop (1972)}

One major source of Meto data is Middelkoop (1972), an unpublished draft 673-page Meto-Dutch dictionary, which was still under preparation when Middelkoop passed away. This dictionary is based on the Molo variety, though forms from other varieties of Meto are occasionally included.

Pieter Middelkoop was a missionary linguist posted to Timor in 1922, after which he began to learn Meto. The dictionary was initially compiled in card-file format, which Middelkoop states was mostly ready by about 1947 . The copy that I possess is a photocopy onto (mostly) A4 pages of the typed version of these cards. This photocopy originally belonged to James Fox, who generously gave it to me when he rediscovered it when moving offices. I made a scan of the physical dictionary and had it OCR-ed to make it semi-searchable. References to pages of Middelkoop (1972) are to the pages of the PDF of this scanned copy.

Given that Middelkoop's dictionary is a draft, headwords usually have only a simple Dutch gloss and in many cases only an example sentence with translation occurs, from which the sense of a form can be worked out. There are also many handwritten corrections or emendations on parts of the dictionary. The transcription used in this dictionary is discussed in $\S 1.4 .2$.

\subsubsection{Fieldwork}

My fieldwork on the Meto cluster has been focused on Amarasi, I have spent about eight months working on Kotos Amarasi (based in the desa of Nekmese') and, among other data, have compiled a lexical database of 2,509 headwords of which 2,110 are unique mono-morphemic roots. My work on Kotos Amarasi is the reason that many of the definitions for this language are more extensive than those of other varieties of Meto.

I have also conducted a month's worth of fieldwork on Ro'is Amarasi and 190 of the headwords in my Amarasi lexical database are marked as exclusively Ro'is and a further 234 Kotos Amarasi headwords given with non-identical cognate Ro'is variants. (Many Kotos and Ro'is Amarasi morphemes are also identical.) Ro'is Amarasi data is important from a comparative perspective as, according to the historical phonology, it forms a primary branch within the Meto cluster. All other Meto varieties, including Kotos Amarasi, form a single Nuclear Meto subgroup (\$3.3.4).

In addition to all this Amarasi data, I have also collected data on the following varieties of Meto: Timaus (35 minutes of transcribed and translated texts and 72 minutes untranscribed texts, lexicon of 748 headwords), Kusa-Manea (4 hours of untranscribed texts, lexicon of 489 headwords), Amanuban (22 untranscribed texts, 8 wordlists), Ketun (1 transcribed text, 2 untranscribed texts, 3 wordlists), Kopas (1 transcribed text, 2 untranscribed texts, 5 wordlists), Fatule'u ( 2 wordlists), Amanatun ( 2 wordlists), Molo ( 2 wordlists) and Amfo'an (1 wordlist).

Finally, Jonker (1908) frequently provides forms from an unspecified variety of Meto in his etymological notes. When I include such forms, they are marked as Meto and a reference to Jonker (1908) is given. Jonker usually does not give definitions for these Meto forms. 


\subsubsection{Other languages}

Forms from languages outside the Rote-Meto group are frequently given in the outcomparisons section of this comparative dictionary (§1.5.1). These are usually other languages from the greater Timor region, though data from languages of other regions are also occasionally given.

In most cases, a full citation for such data is given. The exceptions are languages for which data comes from electronic sources or unpublished sources: Dhao, Galolen, Hawu, Helong, Kemak, Kisar, Tetun Fehan, and Waima'a. The sources for these languages were given in Table 1.1 .

Data from other languages are given full references, including page numbers. Languages that commonly occur in the out-comparisons that are drawn from published dictionaries are given in Table 1.3. Some of these sources are abbreviated in the references.

Table 1.3: Sources of other languages frequently cited

\begin{tabular}{|l|l|l|}
\hline Language & Sources & Abbreviation \\
\hline Bima & Ismail, Azis, Yakub, Taufih and Usman (1985), Jonker (1893) & - \\
\hline East Tetun & Morris (1984) & Mo \\
\hline Ili'uun & de Josselin de Jong (1947) & dJ \\
\hline Kambera & Onvlee (1984) & On \\
\hline Mambae & Grimes, Marçal and Fereira (2014), Fogaça (2017) & - \\
\hline Sika & Pareira and Lewis (1998) & - \\
\hline
\end{tabular}

In addition to Kambera, Onvlee (1984) gives cognates/equivalents of his Kambera headwords for other languages of Sumba: Anakalang, Kodi, Lewa, Mamboru, and Weyewa. However, in a very similar way to Jonker (1908), these cognates/equivalents are usually given without any definition. When I cite such forms, I list them after the Kambera word without a definition. In such cases, these Sumba languages have the same reference as Kambera.

In addition to these sources, Jonker (1908) frequently gives putative cognates for his Termanu headwords from many other languages. Among these, the data for Meto, Helong, Ende, Hawu, Kambera and Bima come from his own work. Unfortunately, glosses are only given for languages other than Rote when they diverge significantly from that of the Rote forms.

Whenever possible I have sought independent verification for such cognates from other sources and give the form, definition and citation for such putative cognates according to these other sources. However, many terms from other languages remain known only from Jonker's etymological notes and thus must be given without definition. That Jonker does not include definitions for such forms indicates that their semantics are likely close to that of the Rote forms.

Jonker does not usually specify from which variety of Helong or Meto his data comes. Thus, when I give a form from one of these languages taken from Jonker (1908) I cannot usually specify the variety and each is given respectively as only 'Helong' or 'Meto'. 


\subsection{Transcription}

PRM reconstructions and their reflexes are given in a phonemic transcription according to standard IPA conventions with the exception of the palatal approximant, which is transcribed $\langle y\rangle$ to avoid any confusion with the voiced palatal affricate $<d_{3}>$ [d] $]$.

When the phonemic representation of a form is in doubt, it is enclosed in angled brackets $<>$. This is often the case for Molo forms taken from Middelkoop (1972), as discussed further in $§ 1.4 .2$, as well as out-comparisons from Sumba taken from Onvlee (1984).

As discussed in $§ 1.4 .1 .1$, Jonker does not distinguish between vowel initial and glottal stop initial words in his works. Initial glottal stops in brackets (?) are used to indicate that a word from one of the Rote languages may begin with a glottal stop, but that this has not been confirmed. Initial glottal stops without brackets in Dela, Oenale, Rikou, Oepao and Landu indicate that I have confirmation from other sources or my own fieldwork that this glottal stop is underlying.

A variety of different punctuation marks is used in these phonemic transcriptions to represent different kinds of morphology. These punctuation marks are summarised in the front matter and Table 1.4. Productive affixes are separated from their stem with the hyphen -. Reduplication is indicated by the tilde $\sim$. Fossilised suffixes that are no longer productive are separated from the stem by a vertical bar $\mid$. Historic compounds for which neither member is known to exist as an independent word in the language are separated by a slash /. When one member of a (historic) compound does exist as an independent word, but the other does not, the two are separated by an underscore _.

Table 1.4: Punctuation representing morphological structure

\begin{tabular}{|l|l|l|}
\hline \multicolumn{2}{|l|}{ Punctuation } & Use \\
\hline- & hyphen & productive affix \\
\hline$\sim$ & tilde & reduplication (including frozen reduplication) \\
\hline I & vertical bar & fossilised affix \\
\hline / & slash & historic compound; neither member independent \\
\hline- & underscore & historic compound; one member no longer independent \\
\hline
\end{tabular}

\subsubsection{Jonker's transcription}

Jonker consistently represents the different contrastive sounds of the Rote languages, with the exception of initial glottal stops and some sequences of two identical vowels. Each of these is discussed in further detail in the next sections.

Jonker's representation of consonants (apart from the glottal stop) is mostly straightforward. Most consonant letters correspond to their modern IPA equivalents. The digraph $<n g>$ represents the velar nasal [ $\mathrm{y}]$ and imploded stops are transcribed identically to plain voiced stops. Thus, for instance, Tii $\langle b\rangle=[6]$ and $\langle d\rangle=[\mathrm{d}]$. This is not a problem for comparative purposes as implosion is a non-contrastive feature of voiced stops in the Rote languages. 


\subsubsection{Glottal stop}

Jonker consistently represents the glottal stop word medially and finally. Between two vowels the glottal stop is represented by a diaeresis on the second vowel; thus $<\ddot{\mathrm{V}}>=/ \mathrm{PV} /$ Examples from Termanu are given in Table 1.5.

Table 1.5: Medial glottal stops in Termanu from Jonker (1908)

\begin{tabular}{|c|c|c|c|c|c|}
\hline \multicolumn{3}{|c|}{ Medial glottal stop } & \multicolumn{3}{|c|}{ No medial glottal stop } \\
\hline Jonker & Phonemic & gloss & Jonker & Phonemic & gloss \\
\hline$<$ daï $>$ & $d a ? i$ & 'dirt on body' & $<$ dai $>$ & $d a i$ & 'reach, arrive at' \\
\hline$<s \grave{e}>$ & so?e & 'coconut spoon' & $<$ sòe $>$ & soe & 'disaster' \\
\hline$<l e \ddot{a}>$ & $l e ? a$ & 'fathom' & $<$ leak $>$ & lea-k & 'cave' \\
\hline$<h a \ddot{o}>$ & ha?o & 'mineral lime' & $<$ hao $>$ & hao & 'eat with hand' \\
\hline$<l i \ddot{u}>$ & $l i ? u$ & 'hit with stick' & $<i u>$ & $i u$ & 'shark' \\
\hline
\end{tabular}

Apart from its use to represent a glottal stop, the diaeresis also represents a morpheme break between a reduplicant and its base when partial reduplication applies to a vowel initial word. Thus, for instance, Jonker (1908:10) transcribes Rikou a ana [?a.ana] 'small', which has no underlying glottal stop as $<a \ddot{a} n a>$.

Word finally the glottal stop is represented by a dot under the preceding vowel, thus: $<$ V $>=/$ VP/. Final glottal stops occur most commonly in Korbafo, Bilbaa, Rikou, Dengka and Oenale, in which case they are usually suffixes. Examples from Dengka are given in Table 1.6.

Table 1.6: Final glottal stops in Dengka from Jonker (1908)

\begin{tabular}{|l|l|l|l|l|l|}
\hline \multicolumn{5}{|l|}{ Final glottal stop } & No final glottal stop \\
\hline Jonker & Phonemic & gloss & Jonker & Phonemic & gloss \\
\hline$<$ luị $>$ & lui-? & 'bone' & $<$ lui $>$ & lui & 'take off' \\
\hline$<$ atẹ $>$ & ate-? & 'liver' & $<$ ate $>$ & ate & 'slave' \\
\hline$<$ buna $>$ & buna-? & 'flower' & $<$ bina $>$ & bina & 'k.o. shellfish' \\
\hline$<$ kòlọ $>$ & kolo-? & 'hole' & $<$ lolo $>$ & lolo & 'stretch out' \\
\hline$<$ seụ $>$ & seu-? & 'Alstonia villosa' & $<$ seu $>$ & seu & 'pick fruit' \\
\hline
\end{tabular}

Jonker states in the introduction to his dictionary that a final glottal stop in Bilbaa and Rikou was often not marked. Although regrettable, given that final glottal stops are almost always suffixes in these languages, this does not impact greatly on the application of the comparative method itself or the reconstruction of proto-forms in my comparative dictionary.

More worryingly, Jonker states that for Rikou (and to a lesser extent also Bokai) the difference between a sequence of two vowels without any intervening consonant and a sequence of two vowels with an intervening glottal stop was often not recorded. I have checked the Rikou forms against data from other sources $(\S 1.3 .1 .2)$ wherever possible and the two nearly always agree; though in less than half a dozen cases, Jonker's form has a medial glottal stop where my other sources do not. All such cases are noted. 
Jonker does not write word initial glottal stops. All Rote varieties for which modern phonetic and phonological data is available have a contrast between vowel initial and glottal stop initial roots. The contrast is neutralised phrase initially (including in isolation) due to an automatic process of phrase initial glottal stop insertion. The difference emerges phrase medially.

Thus, for instance, Rikou vowel initial ura-? 'scorpion' and glottal stop initial ?use-? 'navel' are both realised with an initial glottal stop in isolation: ['?ura?] 'scorpion' and ['Pusc?] 'navel'. Phrase medially, however, 'scorpion' is vowel initial, as seen in the phrase au ura $=k a$ [, Pau 'uraka] 'my scorpion', while 'navel' is glottal stop initial, as seen in $a u$ ?use =ka [, Pau' ?uscka] 'my navel'.

Jonker does not distinguish between such words and writes both with an initial vowel. A selection of examples of Rikou vowel initial and glottal stop initial roots (based on my own fieldwork), along with their representation in Jonker, as well as their realisations initially and medially, is given in Table 1.7 to further illustrate.

Table 1.7: Initial glottal stops in Rikou

\begin{tabular}{|c|c|c|c|c|c|}
\hline Jonker & $<$ inde $>$ & $<e a>$ & $<a p a>$ & $<o f a p>$ & $<u s e ̣>$ \\
\hline Phonemic & Pinde & ?ea & ?apa & ?ofa-? & Puse-? \\
\hline Initial & ['Pind $\varepsilon$ ] & ['?ea] & ['Papa] & ['Pofa?] & ['Puse?] \\
\hline Medial & ria Pinde $=n a$ & $a u$ ?ea $=k a$ & ria $? a p a=n a$ & $a u$ ?of $a=k a$ & ria $?$ use $=n a$ \\
\hline Phonetic & [,ria' Pindena] & [, Pau' ?caka] & [,ria' 'apana] & [, Pau' Pofaka] & [,ria'?uscna] \\
\hline Gloss & 'her/his spindle' & 'my turtle' & 'her/his buffalo' & 'my canoe' & 'her/his navel' \\
\hline Jonker & $<i k o>$ & $<e i>$ & $<$ ape $>$ & $<\dot{o}>$ & $<u r a ̣>$ \\
\hline Phonemic & $i k o-?$ & $e i-?$ & ape & oo & ura-? \\
\hline Initial & ['Piko?] & ['Pei?] & ['?ape] & ['?0:] & ['?ura?] \\
\hline Medial & ria $i k o=n a$ & $a u e i=k a$ & ria ape $=n a$ & $a u o o=k a$ & ria ura $=n a$ \\
\hline Phonetic & [,ria'ikona] & [, ?au' eika] & [,ria'apena] & [,Pau's:na] & [,ria'urana] \\
\hline Gloss & 'its tail' & 'my foot' & 'her/his saliva' & 'my bamboo' & 'her/his scorpion' \\
\hline
\end{tabular}

That Jonker does not transcribe initial glottal stops is a problem for comparative purposes, as an initial glottal stop is often a reflex of an earlier consonant. Fortunately, however, I have access to data for both Dela (Tamelan 2017) and Rikou (own field notes), which allows me to distinguish between most vowel initial and glottal stop initial roots for these languages. I have thus transcribed all underlying glottal stops for Rikou, as well as Dela and Oenale as befits their phonemic status. ${ }^{6}$

Initial glottal stops that are suspected to be underlying in other varieties of Rote are transcribed in brackets to indicate their unconfirmed status. Thus, for instance, for 'buffalo' Jonker gives Dengka and Oenale $<a m b a>$ while Tamelan (2017) gives Dela Pamba. I thus transcribe the Dela and Oenale forms as ?amba and the Dengka form (?)amba to indicate that Dengka probably has an initial, but unconfirmed, underlying initial glottal stop.

6 Dela and Oenale are nearly identical and can be treated to some extent as a single lect. 
The status of some initial glottal stops in Dela and Rikou remains ambiguous. This is either because the form in Jonker (1908) is not present in Tamelan (2017) or was not known by my consultants. Additionally, a number of Rikou forms with an ambiguous glottal stop were added to my database after my fieldwork in Rote. Such ambiguous glottal stops are given in brackets (?) in the same way as all unconfirmed initial glottal stops in the Rote languages.

Meto varieties also have a process of glottal stop insertion before vowel initial words. However, unlike the Rote languages this glottal stop insertion affects vowel initial words in all phrase positions - not just phrase initially. The relevance of this process in Meto for comparative purposes is discussed in $\S 2.4 .2$.

\subsubsection{Vowels}

Jonker represents the five phonemic vowels of the Rote languages with and without various accents according to a combination of the placement of stress and phonetic vowel quality. His transcription of vowels is not phonemic, but is consistent. A summary of Jonker's transcription of vowels is given in Table 1.8. Observe in particular that some letters represent both single and double vowels.

Table 1.8: Jonker's transcription of vowels

\begin{tabular}{|l|l|l|l|}
\hline Jonker & Phonetic & Phonemic & Use by Jonker \\
\hline$e$ & {$[\mathrm{e}]$} & $e$ & all environments \\
\hline$o$ & {$[\mathrm{o}]$} & $o$ & all environments \\
\hline$\grave{e}$ & {$[\varepsilon:]$} & $e e$ & final/only vowel of word \\
\hline & {$[\varepsilon]$} & $e$ & elsewhere \\
\hline$\grave{o}$ & {$[\mathrm{0}]$} & $o o$ & final/only vowel of word \\
\hline & {$[\mathrm{o}]$} & $o$ & elsewhere \\
\hline$i$ & {$[\mathrm{i}:]$} & $i i$ & only vowel of word \\
\hline & {$[\mathrm{i}]$} & $i$ & elsewhere \\
\hline$a$ & {$[\mathrm{a}:]$} & $a a$ & only vowel of word \\
\hline & {$[\mathrm{a}]$} & $a$ & elsewhere \\
\hline$u$ & {$[\mathrm{u}:]$} & $u u$ & only vowel of word \\
\hline & {$[\mathrm{u}]$} & $u$ & elsewhere \\
\hline$\dot{y}$ & {$[\mathrm{i}:]$} & $i i$ & final vowel of word \\
\hline$a ́$ & {$[\mathrm{a}:]$} & $a a$ & final vowel of word \\
\hline$\dot{u}$ & {$[\mathrm{u}:]$} & $u u$ & final vowel of word \\
\hline
\end{tabular}

Before this transcription is discussed in detail, several facts concerning the phonology of vowels in Termanu must be summarised. A fuller overview of Rote phonology is given in $\S 2.4$.

- Mid-vowels are lax $[\varepsilon]$ and [o] when stressed and before a syllable containing a midvowel.

- Mid-vowels are tense [e] and [o] before a syllable containing a high vowel.

- Content words contain at least two vowels.

- Nearly all vowel sequences occur, including double vowels /ii/, /ee/, /aa/, /oo/ and /uu/. 
- Double vowels are realised as phonetically long vowels, i.e. $/ \mathrm{V}_{\alpha} \mathrm{V}_{\alpha} / \rightarrow[\mathrm{V}:]$.

- Stress is penultimate. Secondary stress is assigned to every second syllable to the left.

Given this knowledge of Rote phonology, the following generalisations account for Jonker's transcription. Jonker transcribes the lax allophones of the mid vowels $[\varepsilon]$ and [o] with a grave accent $\langle\grave{e}\rangle$ and $\langle\grave{o}\rangle$ whenever these allophones are stressed. Unstressed lax vowels, as well as stressed tense allophones are transcribed as $\langle e\rangle$ and $\left.<_{o}\right\rangle$ without any accent.

Given that stress falls on the penultimate vowel of a word, a double vowel will always contain a vowel that is the locus of stress and the whole double vowel will thus bear stress (either primary or secondary). This - combined with the fact that the first vowel of a sequence of two mid vowels is by nature followed by another mid vowel - means that a double mid-vowel will always be lax. Thus, the double vowels /ee/ and /oo/ are always stressed lax [ع:] and [o:].

Consequently, Jonker's $\langle\dot{e}\rangle$ and $\langle\dot{o}>$ represent double vowels /ee/ and /oo/ when they are the final or only orthographic vowel of a word. Jonker's $\langle\dot{e}\rangle$ and $\langle\dot{o}>$ do not represent double vowels when they are penultimate before another syllable or vowel. Examples of Jonker's transcription of the stressed lax allophones of the mid vowels in Termanu are given in Table 1.9.

Table 1.9: Stressed lax allophones of mid vowels

\begin{tabular}{|c|c|c|c|c|c|c|c|}
\hline Jonker & Phonemic & Phonetic & gloss & Jonker & Phonemic & Phonetic & gloss \\
\hline$<n \grave{e}>$ & nee & ['ne:] & 'six' & $<$ dène $>$ & dene & ['denc] & 'kapok' \\
\hline$<$ na-tanè $>$ & na-tanee & [,nata'n $\varepsilon$ :] & 'contain' & $<$ dèlo $>$ & delo & ['delo] & 'citrus' \\
\hline$<n \dot{o}>$ & noo & ['ns:] & 'coconut' & $<$ bòle $>$ & bole & ['bole] & 'arenga palm' \\
\hline$<$ na-sakò> & na-sa-koo & [,nasa'ko:] & 'sip' & $<b \grave{o}>$ & bo?o & ['bo?o] & 'cough' \\
\hline
\end{tabular}

For the non-mid vowels $/ \mathrm{i} / \mathrm{h} / \mathrm{a} /$, and /u/, Jonker transcribes a final double vowel with an acute accent, to indicate phonetic stress. Examples from Termanu include: $\langle$ nakapi $>=$ na-ka-pii 'tense', <bitiná $>=$ bitinaa 'Kleinhovia hospita', and $<$ na-tafú $>=n a$-tafuu 'inflated'.

In other situations, double /ii/, /aa/, and /uu/ are not distinguished orthographically from single vowels. However, given that Rote content words minimally contain two vowels, any content word that contains only a single orthographic vowel in Jonker (1908) must have a double vowel underlyingly. Termanu examples are given in Table 1.10. That such words do indeed contain a double vowel is confirmed by the fact that when they occur with a prefix or as final members of a compound, Jonker transcribes them with an acute accent, as shown in the right-hand side of Table 1.10 .

Table 1.10: Double vowels in Termanu

\begin{tabular}{|l|l|l|l|l|l|l|l|}
\hline Jonker & Phonemic & Phonetic & gloss & Jonker & Phonemic & Phonetic & gloss \\
\hline$<k i>$ & $k i i$ & {$[$ ['ki:] } & 'left, north' & $<a l u$-kik> & alu kii-k & [,alu'ki:k] & 'left shoulder' \\
\hline$<d a k>$ & $d a a-k$ & {$[$ 'da:k] } & 'blood' & $<n a d a ́>$ & $n a-d a a$ & [na'da:] & 'bleed' \\
\hline$<h u k>$ & $h u u-k$ & {$[$ 'hu:k] } & 'trunk, source' & $<a i-h u ́ k>$ & ai huu-k & [, Rai'hu:k] & 'tree trunk' \\
\hline
\end{tabular}


In summary, Jonker's use of accents to mark stress, combined with the minimal divocalic word requirement, means that there are almost no cases in which it is unclear whether a particular orthographic vowel represents a single vowel or a sequence of two identical vowels.

The only words that remain ambiguous are functors with single $\langle i\rangle,\langle a\rangle$ or $\langle u\rangle$, such as $\langle m a\rangle$ 'and'. This is because functors can be monosyllabic in the Rote languages. In these cases, I have referred to other sources to determine whether such functors contain a single or double vowel. In the case of $m a$ 'and', Tamelan (2017) gives Dela $m a$ 'and' with a single vowel.

\subsubsection{Middelkoop's transcription}

The transcription used in Middelkoop (1972) is not phonemic and under-representation, particularly of the glottal stop and double vowels, is common. Middelkoop's orthography can be used to some extent by those who already know the language, but it cannot be used as a reliable, consistent representation of the phonological structures of Meto. The main issues with Middelkoop's transcription are summarised in the next sections.

- Double vowels /ii/, /ee/, /aa/, /oo/ and /uu/ are written with a single letter.

- The glottal stop is transcribed $<^{\prime}>$ between two vowels.

- Word final $\langle$ ' $>$ indicates either doubling of the previous vowel or doubling of the previous vowel followed by a glottal stop; i.e. $\left\langle\mathrm{V}^{\prime}\right\rangle=/ \mathrm{VV} / \sim / \mathrm{VV}$ /.

- Word finally and before consonants the glottal stop is usually not transcribed.

- The vowel sequences /ao/ and /au/ are both written $<a u>$.

- The vowel sequence /ae/ appears to be written $<a i>$ when non-final.

- Prefixes which are a single consonant are often written with a previous vowel final word.

- The final vowel of the pronouns ina 3sG, sina 3PL and hita 1PL.INCL is usually written with a following inflected verb.

Even with experience of working on Meto and having used Middelkoop (1972) for years, it is still sometimes unclear to me what the exact form of a particular word is. Such forms are indicated in this comparative dictionary with angle brackets $<>$. All words not given in angle brackets from Middelkoop (1972) have been re-transcribed according to their phonemic form.

Thus, Middelkoop's $<$ fule $>$ 'foam' is probably either fule? or ?fule?, but I have no way of knowing whether the Molo form has an initial glottal stop or not; Kotos Amarasi has ?furi-f, indicating that an initial glottal stop might be present in the Molo form, while Kusa-Manea has fa fura-f indicating that a glottal stop might not be present.

Similarly, the word for 'turtle' is given with the forms $\left\langle k e^{\prime}\right\rangle,\left\langle k e^{\prime} a\right\rangle,\langle k e\rangle$ and $\langle k e l\rangle$ with no obvious way of knowing what the orthographic variation means. Indeed, there are probably variant forms of which the final two are certainly kee and kee-l, but whether $<k e^{\prime}>$ is kee or kee? is unclear and whether $<k e^{\prime} a>$ is $k e ? a, k e a, k e a$ ?, $k e e=a a$ and/or $k e e ?=a a$ is also unclear. 


\subsubsection{Glottal stop}

The glottal stop phoneme $/ 2 /$ is usually only written when it occurs between two vowels. Between two vowels $/ \mathrm{R} /$ is written with an apostrophe $\left\langle\right.$ ' $>$. An example is $<m a^{\prime}$ eki $>=$ ma?eki? 'slippery'.

Word finally and before consonants the glottal stop is not usually written. Hence Middelkoop's $<$ mafena $>=$ ma?fena? 'heavy' has two glottal stops in all known varieties of Meto, including my own Molo data collected on the basis of fieldwork.

Initial glottal stops before other consonants can sometimes be detected by the presence of variants with and without initial epenthetic /a/, which often occurs before consonant clusters. Thus, <akalen $>$ and $<$ kale $>$ 'fraenulum' indicate a form with initial / $\mathrm{kk} /$, probably $a$-?kale- $n$ and $? k a l e ?$, respectively. However, such glottal stop detection is a very inexact science. Here my confidence that this word does indeed begin with a glottal stop comes mainly from my own Amarasi data where I have ?kare-f 'palate'.

There are sporadic instances of the glottal stop being written $<^{\prime}>$ when it is stem/ root initial before another consonant, but this is rare and inconsistent. Sometimes it even appears to be written after the initial consonant, such as $\left\langle n a k^{\prime} a i>=n a-? k a i(?)\right.$ 'hook (v.)'.

\subsubsection{Double vowels}

Like Rote, Meto has a minimum word requirement whereby every content word must contain at least two vowels, with double vowels realised phonetically as a single long vowel, e.g. oo $\rightarrow$ [?०:] 'bamboo', $n$-iit $\rightarrow$ [ni:t] 'sees'. The only words that can be exempt from this requirement are functors. But even for functors I found evidence that most also have a minimum of two vowels, at least historically.

Word final sequences of two identical vowels can be marked with the apostrophe $\langle$ ' $\rangle$ in Middelkoop (1972). An example is $\left\langle o^{\prime}\right\rangle=o o$ 'kind of bamboo'. But use of the apostrophe to represent double vowels is not a rule. Thus, for $<n e>=n e e(?)$ 'six' there is no indication of the double vowel. Sometimes a word written with a final $<$ ' $>$ also ends in a glottal stop, such as in $\left\langle n a^{\prime}\right\rangle=n a a$ ? 'blood'. Word final $<^{\prime}>$ indicates either doubling of the previous vowel or doubling of the previous vowel followed by a glottal stop, i.e. $\left\langle\mathrm{V}^{\prime}>=/ \mathrm{VV} / \sim / \mathrm{VV}\right.$ / .

Before a consonant (other than glottal stop), sequences of two identical vowels are normally written with a single letter. Examples include $<$ bifel $>=$ bifee-l 'woman', $<$ hun $>=$ huun 'grass', <lus $>=$ luus 'deer', and $<$ ekfui $>=e e k$ fui 'kind of agave', and so on. ${ }^{7}$

\subsubsection{Other vowel sequences}

The vowel sequence /ao/ is written identically to $/ \mathrm{au} / \mathrm{as}\langle a u\rangle$. As an example, both $a u$ ' $1 \mathrm{sG}$ ' and $a o-f$ 'body' are given under a single $<a u>$ headword. Another example is $<$ nau $>=$ nao 'go'.

7 There are rare exceptions in which a double vowel is written with two letters such as <eem > '2PL come'. 
When it is non-final the vowel sequence /ae/ appears to written as $<a i>$. Thus, Amarasi has na-?aeka? 'soak', which is cognate with the Molo forms <naik>n-(?)aek and $<u$ aikat $>u$-?aeka-t in Middelkoop (1972). Word finally Middelkoop writes /ae/ as $<a \grave{e}>$. Two examples are $<s a \grave{e}>=s a e$ 'go up' and $<$ maè $>=$ mae 'ashamed'.

\subsubsection{Morpheme boundaries}

Verb agreement is usually obligatory in Meto, and one set of agreement prefixes consists of only a consonant: $?-1 \mathrm{SG}, m-2 \mathrm{SG} / 2 \mathrm{PL} / 1 \mathrm{PL}$.EXCL, $n$ - 3SG/3PL, $t$ - 1 PL.INCL $(\$ 2.6 .5)$. When the agreement prefix $m$ - occurs after any of its corresponding pronouns - hoo $2 \mathrm{sG}$, hii 2PL or hai 1PL.EXCL - the prefix is often written as the final consonant of this pronoun. Examples include:

- <hom pau fe> = hoo m-pao fe? 'keep waiting for me'

- $\quad<$ him tok mitloe $>$ = hii m-took mi-tloe 'you sit parallel'

- <haim hek manu> = hai m-heek manu 'we caught a chicken'

Consistent with the fact that Middelkoop (1972) does not usually write initial glottal stops, the 1SG prefix ?- is not usually written. Thus, <au tebi kukis $>=$ au ?-tebi kukis 'I crumble bread'.

The 3SG, 3PL and 1PL.INCL pronouns are in, sin and hit, respectively. These pronouns have vowel final forms with are used before consonant clusters: ina, sina and hita, respectively. When these pronouns occur before an agreeing verb whose root begins with a consonant, the final vowel of these pronouns is written with the verb in Middelkoop (1972). Examples include:

- $\quad$ in anlo'> = ina $n$-loo? 's/he throws up' (p. 280)

- <sin anote hau neki fani> = sina n-?ote hau n-eki fani 'they cut a tree with an axe'

- <hit atlaksaè talali noel> = hita t-laak sae ta-lali noe-l 'we stepped over the river'

In such instances, a case can be made for writing these forms orthographically with the /a/ attached to the verb as this aids morpheme recognition. While this may be helpful for native speakers, it is unhelpful for identifying the underlying structures for the purposes of linguistic analysis and reconstruction.

Occasionally, enclitics are written attached to their host, but given the draft state of Middelkoop's dictionary these could just be typographical errors. One example is $<$ hom nau mankit ho feto $>=$ hoo m-nao $m$-aan =kiit hoo feto? ' $g o$ and get your sister for us'.

\subsection{Structure of the dictionary}

This comparative dictionary is structured around reconstructions. Headwords are reconstructions to Proto-Rote-Meto, or to one of its sub-nodes (see §3.3). Headwords are arranged alphabetically with IPA symbols placed after their nearest equivalent. 
Prenasalised stops are treated as separate letters. The complete order of all letters which occur in PRM reconstructions is as follows: ${ }^{*} \mathrm{a},{ }^{*} \mathrm{~b},{ }^{*} \mathrm{~b},{ }^{*} \mathrm{~d},{ }^{*} \mathrm{~d}, *_{\mathrm{d}},{ }^{*} \mathrm{e},{ }^{*} \mathrm{\partial},{ }_{\mathrm{f}},{ }^{*} \mathrm{~h}, *_{\mathrm{i}},{ }^{*} \mathrm{k}$, *?, *1, *m, *mb, *n, *nd, *n, *ng, *o, *p, *r, *s, *t, *u, *w.

Each reconstruction is defined and a variety of other information is also given. Below each reconstruction are the forms in the Rote-Meto languages, which provide evidence for the reconstruction. A simple example entry is exemplified in Example 1.1.

\section{Example 1.1: Simple PRM reconstruction}

*ika Morph: *ika-k. PRM. fish. Etym: *hikan. Pattern: k-10.

i?a-k Termanu. fish. (J:200)

i?a-? Korbafo.

i?a-k Bokai.

ika-? Bilbaa.

ika-? Landu. fish. (own field notes)

ika-? Rikou.

ika-? Oepao. fish. (own field notes)

i?a-k $B a^{\prime} a$.

i?a-k Tii.

ia-? Dengka.

?u?u_ia-? Dela. all kinds of fish. [Form: $\mathbf{P u} \mathbf{u}$ is the normal word for 'fish' in Dela.]

ika|? Ro'is Amarasi. fish.

ika|? Kotos Amarasi. fish.

ika|? Molo. fish. (M:159)

ika|? Kusa-Manea. fish.

Out-comparisons:

ikan Semau Helong. fish.

ikan East Tetun. fish. (Mo:90)

i?a Dhao. fish.

Each entry is headed by a reconstruction in boldface, in this case *ika. Directly after the root is given any morphology with which this root occurred (§1.5.3.1); in this case, *ika took the nominal suffix *-k (\$2.6.1). The level at which this reconstruction is made $(\$ 3.4)$ is given after this in italics, in this case Proto-Rote-Meto (PRM). This is followed by the reconstructed meaning ('fish'), which in turn is followed by any etymon at a higher level, usually PMP (§1.5.3.3). After the etymology, notes on any issues or problems with this reconstruction are given $(\S 1.5 .3 .7)$.

Below the reconstruction are given its reflexes in the Rote-Meto languages. This begins with the varieties of Rote in the following order: Termanu, (Keka), Korbafo, Bokai, Bilbaa, (Landu), Rikou, (Oepao), Ba'a, (Lole), Tii, Dengka, Oenale and Dela. ${ }^{8}$ Lects in brackets in this list are not always given, while forms from the other lects are given whenever they are known to be cognate.

8 The ordering in which the Rote languages are given is a compromise between geographic position and relatedness. Termanu is given first as this is the variety on which Jonker (1908) is based, followed by Korbafo, which is extremely similar. Bokai is given next with an eastwards progression from there to Bilbaa, Landu, Rikou and Oepao. After this Ba'a is given with a mostly westwards progression to Lole, Tii, Dengka, Oenale and Dela. 
In line with the nature of Jonker (1908) (see §1.3.1), from which most Rote data comes, Termanu is often the only Rote variety defined. The definition is a free translation of Jonker's Dutch definition. When the source of a form does not come from Jonker (1908), it is usually glossed and the source is given. Thus, in the case of *ika 'fish' in Example 1.1 the Landu and Oepao forms $i k a-?$ 'fish' come from my own field notes. When no definition is given for a variety of Rote, the definition must be assumed to be similar to that of Termanu. Thus, the Korbafo, Bokai, Bilbaa, Rikou, Ba'a, Tii and Dengka forms in Example 1.1 can be assumed to also mean 'fish'.

After the definition, any necessary notes are given on issues concerning this reflex. The final part of the entry for a reflex is the source of this form. In Example 1.1, Termanu $i$ i $a-k$ comes from page 200 of Jonker (1908). When no alternate source is given for another Rote variety, it comes from the same page of Jonker (1908) as the Termanu form. The only exception is Dela, data for which always comes from Tamelan (2017). For more discussion on the sources on which this dictionary is based see $\S 1.3$.

After Rote, forms from different varieties of Meto are given. Often only a Kotos Amarasi and Molo form are given, as these are the varieties for which the most data is available. When other lects are given they are ordered roughly from east to west. ${ }^{9}$ These entries follow the same model as the Rote entries except that glosses for all varieties are given.

\subsubsection{Out-comparisons}

The final part of an entry consists of putative cognates in languages outside of the RoteMeto subgroup. These are preceded by Out-comparisons and further indented. These out-comparisons are forms from languages outside of the Rote-Meto group that are formally and semantically similar to the Rote-Meto languages. In most cases, these out-comparisons are from Austronesian languages in the greater Timor region, though occasionally out-comparisons from non-Austronesian or more distant languages are also given.

All languages that occur more than three times in the out-comparisons are listed in the front matter, along with their geographic location, ISO 693-3 codes, and Glottocodes (Hammarström et al. 2020). Equivalent information for languages that occur three or less times in the out-comparisons is included each time a form from that language occurs.

These out-comparisons have not been thoroughly vetted for whether they show regular sound correspondences with the PRM forms and thus cannot be taken unquestioningly as cognate. While it is likely that many of the out-comparisons are indeed cognate with the PRM forms, this cannot be assumed to be the case. A more thorough investigation of the sound correspondences may show that certain forms in certain lects are borrowings and not the result of shared inheritance.

9 The complete ordering of Meto lects is as follows: Ro'is Amarasi, Kotos Amarasi, Amanuban, Amanatun, Ketun, Kopas, Fatule'u, Molo, Amfo'an, Timaus, Baikeno, Kusa-Manea. With the exceptions of Ketun, Kopas and Timaus (which probably originate elsewhere), this is roughly east to west along the south coast, then east to west among remaining varieties, with Timaus after Amfo'an, from which it originated. 
This is particularly the case for Helong out-comparisons. Helong and the Rote-Meto languages appear to have been in contact with one another since the time of PRM and have remained in contact ever since (see \$3.3.4.2). It remains to be determined to what extent it may be possible to detect contact at different time depths between Helong and Rote-Meto. ${ }^{10}$

While I do give preliminary notes on what appear to be irregular sound correspondences between the PRM reconstruction and out-comparisons when I am aware of them, the nature of such irregularities remains to be properly investigated.

In general, I have searched fairly thoroughly for putative cognates in Tetun, Ili'uun, Helong and Kisar based on available sources. I have not made a thorough search for cognates in other regional languages, but have included similarities that I opportunistically stumbled upon.

When the out-comparison field contains forms that can be identified as loans from a Rote-Meto language, or a Rote-Meto entry can be identified as a loan from one of the out-comparisons, the form that is a loan is preceded by the degree sign ${ }^{\circ}$ and the source of the loan is given. An example is *dele 'Job's tears, Coix lachryma-jobi', from PMP *zolay, which is inherited regularly in the Rote languages. Meto, however, has irregular ${ }^{\circ}$ sone, which is probably a loan from Welaun sole (also from *zolay). When all the forms under a single headword can be identified as loans, they are given in the Loan Distribution section $(\S 1.5 .4)$.

\subsubsection{Multiple reflexes}

In many cases, a language has more than one reflex of a single reconstruction; usually morphologically related forms. In such cases each form except the first is numbered sequentially, with these numbers corresponding to the equivalent numbered section of the definition. An example is given in Example 1.2.

\section{Example 1.2: Multiple reflexes}

*lasi2 Morph: *ma-lasi-k, *na-ma-lasi. PRM. old, aged.

lasi-k (2) na-ma-lasi Termanu. 1) old (especially of people and animals). 2) to be or become old, of people and animals. (J:283)
lasi-? Korbafo.
lasi-k Bokai.
lasi-k Bilbaa.
lasi-? Rikou.
lasi-k $B a^{\prime} a$.
lasi-k Tii.
lasi-? Dengka.
lasi-? Oenale.

\footnotetext{
10 As an additional complicating factor, it is not unlikely that Helong and the Rote-Meto languages both had contact with the same non-Austronesian language(s) and/or families that have since been lost due to shift to Helong and/or Rote-Meto. Again, it has not yet been determined to what extent it may be possible to detect which equivalents/cognates shared between Helong and the Rote-Meto languages are the result of mutual borrowing and/or borrowing from a third source.
} 
m|nasi|? Ro'is Amarasi. old, aged.

m|nasi|? (2) na-m|mnasi Kotos Amarasi. 1) old, aged. 2) be or become old/aged. m|nasi|? Molo. old. (M:325)

m|nasi|? (2) m|nasi-k Kusa-Manea. 1) old (e.g. of fruit). 2) old, aged (of people).

In this example, Termanu has two different forms. The definitions of these forms are given after the forms. Form (1) lasi-k means 'old (especially of people and animals)', and form (2) na-ma-lasi means 'be or become old, of people and animals'. When other varieties of Rote taken from Jonker (1908) also have multiple forms, these are given the same numbers as the parts of the Termanu entry to which they correspond.

\section{Example 1.3: Example with multiple reflexes in all Rote varieties}

*beli Rote. price, bride price. Etym: *bəli 'buy, value, price; marriage prestations, bride price; purchase'.

beli (2) belis Termanu. 1) cost, price, value. 2) that which must be paid for a girl when taken for marriage, either paid with goods or money, the purchase price of a woman. (J:41)

beli (2) belis Korbafo.

beli (2) belis Bokai.

beli (2) belis Bilbaa.

beli (2) belis Rikou.

beli (2) belis $B a^{\prime} a$.

beli (2) belis Tii.

feli (2) felis Dengka.

feli (2) felis Oenale.

Out-comparisons:

foli-n East Tetun. price, cost, value; objects for barter. (Mo:35)

heli Ili'uun. property, valuable things. (dJ:117)

weli Kisar. buy.

Example 1.3 shows how the first form in all the Rote lects corresponds to Termanu beli 'cost, price, value' and the second form corresponds to Termanu belis 'bride-price'. Often it is only the Termanu entry that has multiple forms, as in Example 1.2. While equivalent numbered forms across the Rote lects are given the same numerals, such equivalency does not necessarily correspond to any forms among the Meto cluster.

I generally only give multiple reflexes of a single reconstruction when these additional forms contribute to understanding the history or development of the reconstruction. Thus, in Example 1.2, the Termanu and Meto forms attest that *lasiz probably had two forms in PRM: nominal *ma-lasi-k and verbal *na-ma-lasi.

In some cases multiple reflexes occur for a single language with no indication of a semantic difference. In such cases, the two forms are separated by a comma and receive a single gloss. Thus, for instance, two Molo reflexes of *mbeda 'put down' occur with no know semantic difference. They are given as na-pela, na-bela Molo. put down. (M:56, xxxix). Note also that in such cases the ordering of page numbers in the source 
follows the order in which the reflexes are given in this dictionary, rather than their actual ordering in the source — that is, na-pela occurs on page 56 of Middelkoop (1972) and na-bela occurs on page xxxix.

\subsubsection{Fields/parts of entries}

Apart from the forms and definitions, many entries have additional fields that give additional information on the entry. These fields include a morphology field marking any morphology with which the reconstruction occurred (\$1.5.3.2), an etymology field indicating a higher level etymon $(\S 1.5 .3 .3)$, a field for indicating doublets $(\S 1.5 .3 .4)$, fields tracking irregular sound changes $(\S 1.5 .3 .5)$, a field tracking patterns or correspondences among proto-phonemes that undergo unconditioned splits $(\S 1.5 .3 .6)$, and four fields for different kinds of notes $(\S 1.5 .3 .7)$.

\subsubsection{Definitions and glosses}

Glosses of reconstructions are always given, but glosses of the forms in daughter languages are not always given. All glosses of reconstructions are my own proposed semantics for the PRM form, but glosses for the reflexes in daughter languages follow verbatim the sources from which data is drawn (see $§ 1.3$ ). This includes not giving any gloss when no gloss is given in the source, as is usually the case for non-Termanu forms taken from Jonker (1908). ${ }^{11}$ In such cases, we must assume that the meaning of the non-Termanu terms is similar to that of Termanu (see $\$ 1.1 .1 .1$ for more discussion). Thus, most Rote forms are not glossed and the Termanu gloss can be taken as a proxy for the meaning.

The convention is to supply glosses as in the sources verbatim, including a lack of gloss, not to leave out glosses that are identical to the first language listed. Thus, nearly all data not drawn from Jonker (1908) is glossed. This is exemplified in Example 1.1 where the forms in Landu, Oepao, Dela, Meto and the out-comparisons are all glossed according to the sources from which they are drawn, even though theses glosses are usually identical. The forms without glosses are taken from Jonker (1908) where they listed as cognates of the Termanu headword.

In some cases, Jonker (1908) explicitly gives the meaning of one or more Rote forms as differing from their Termanu cognate. Likewise, word sets taken from the final section of his dictionary, which is devoted to 'Forms and words from the other dialects', often have no Termanu cognate. In this case, the gloss for the first language listed is given and the glosses for subsequent varieties of Rote in Jonker (1908) are not glossed and must be assumed to be equivalent to the language for which the gloss is given. When a cognate set is restricted to Dengka and Oenale the gloss is usually repeated for both forms.

The glossing of terms referring to biota follows the practice used elsewhere in the dictionary of citing glosses verbatim from the sources. Many biota terms only receive a vague definition in Jonker (1908), as 'kind of $\mathrm{X}$ ', though they are often accompanied

\footnotetext{
11 The statements here also apply to terms from the Sumba languages taken from Onvlee (1984), in which case we must assume that the unglossed forms are similar to the Kambera forms. These statements also apply to some forms from Maluku, in which case the unglossed forms must be assumed to be similar to the first language listed.
} 
by a description and/or their name in Kupang Malay, which can be used to identify the species referred to. Whenever a biota term is defined precisely in one source (often with a scientific name) and defined only vaguely in other sources, I usually assign the more precise definition to the reconstructed PRM form.

Some discussion of introduced species of biota is needed. There are a number of reconstructed terms in this dictionary that refer to recently introduced species of biota in daughter languages. When the sound correspondences of such terms are regular, this indicates that semantic shift has taken place. In an insightful article on the history of traditional agriculture, Fox (1991) has shown how newly introduced crops were assimilated to pre-existing categories in the Rote languages. Thus, for instance, based on a cursory examination of the present-day semantics, we could assign the meaning 'maize' to PRM *mbela. However, to quote Fox (1991:250):

On Roti [sic], it is clear that maize when it was introduced was culturally assimilated to the category of 'sorghum'. It is also conceivable that at an earlier period when sorghum was introduced, it was assimilated to the category of Job's tears. Thus this category, pela [from PRM *mbela], may subsume three stages of an agricultural progression.

See the discussion in the entry for *mbela for more details on this particular form.

There is evidence that similar patterns of assimilation and subsequent semantic shift have occurred in many other cases with biota terms in the Rote-Meto languages. Thus, for instance, the usual designation of Kotos Amarasi rinah, a reflex of *dilah, is 'pomegranate', which is an introduced species in Timor. However, there is another term riin fui 'wild rinah' that refers to another fruit tree, probably Aegle marmelos, which appears to be native to the region.

In some cases, reflexes of a reconstructed term only refer to introduced species. Thus, for instance, all reflexes of *uas in daughter languages refer to 'jicama Pachyrhizus erosus', which is a tuber native to central America probably introduced into Southeast Asia during the 16th century. If the PRM form is valid, and did not spread by borrowing, this meaning cannot have been the original sense. Instead, *uas must have undergone semantic shift in all daughter languages. However, in the absence of further information as to what other tuber this term may have originally referred to, I have chosen to assign this meaning to the PRM term rather than a vague meaning such as 'kind of tuber'. I have done this on the assumption that any native designation was likely similar to the introduced term. Importantly, however, no conclusions as to the age of PRM should be drawn on the basis of terms that apparently refer to introduced biota in this dictionary.

\subsubsection{Morphology}

The reconstructed headwords are roots. Whenever there is evidence that such a root obligatorily took certain morphology in PRM - that is, the root probably did not occur as a bare stem - the form(s) in which it occurred are given in the morphology field. If the morphology field is filled, this means that I have no evidence that the reconstructed root occurred as a bare stem in PRM. 
Thus, for instance, PRM *lasi 'old, aged' in Example 1.2 is given with the derivatives *ma-lasi-k and *na-ma-lasi. This means that all Rote-Meto reflexes are from either *malasi-k or *na-ma-lasi. No modern-day language attests *lasi alone without morphology. Similarly, the PRM root *ygala 'Sesbania grandiflora' is given with the derivative *yga ngala. This means that all reflexes in the PRM languages are inherited from reduplicated *yga ygala. There is no evidence that *ygala alone occurred at the level of PRM.

When the only reflexes for a reconstruction are morphologically complex, but there is no agreement on a single complex form for PRM, the morphology field is left empty. An example is PRM *bua 'gather', for which no modern language attests a bare root: Termanu has na-ka-bua, bua bua, bu bua-k and be-bua (among other derivatives), while varieties of Meto have na-bua, na-k|bua?, bua? bua? and n-bua. Based on these reflexes we can probably posit that PRM had derivatives *na-ka-6ua and *6ua bua, but we cannot posit that these derivatives were the only forms of this root that occurred in PRM. Thus, the morphology field is left blank for *6ua.

When the reconstructed root itself is a reflex of a morphologically complex PMP term, this is not indicated in the headword. An example is PRM *mea 'red', which is a reflex of PMP *ma-iRaq, without any indication of the earlier *ma- prefix in the PRM headword.

When the root contains frozen morphology, which is not clearly inherited from a reconstructed morphologically complex form, this is indicated in the headword with the vertical bar, which is used for frozen morphology (\$1.4). An example is the PRM root *natu|n 'hundred', which is a reflex of PMP *sa-ya-Ratus (via intermediate **yatus) with a final consonant $*_{n}$ of unclear origin, which is plausibly a suffix.

When a verb is reconstructed as taking agreement prefixes in PRM, the morphology field is filled by the third person form of the prefix, either *na- or *n-. An example is *fada 'say, tell' for which all reflexes take person agreement. Thus, I propose that this verb obligatorily took agreement in PRM and give *na-fada in the morphology field to indicate this.

As discussed further in $§ 2.6 .1$, the Rote languages have a distinction between nouns that take the nominal suffix $-k /-?$ in isolation and as the final member of an attributive phrase and nouns that are not eligible to take this suffix. This distinction is reconstructible to PRM. Thus, when the morphology field is filled by a form with final *-k, this means that I posit that this noun was eligible to take this suffix in the appropriate environments (not that it obligatorily occurred with this suffix in all environments). One example is *ika-k 'fish' in Example 1.1, for which I posit there was an NP final form *ika-k and an NP medial form *ika.

\subsubsection{Etymology}

The etymology field usually contains a reconstruction taken from the online Austronesian Comparative Dictionary (Blust and Trussel ongoing). This is the source of all reconstructions in the etymology field, unless otherwise indicated. 
Most of these reconstructions are to Proto-Malayo-Polynesian (PMP). In a small number of cases no reconstruction at this level exists and I give a reconstruction from one of its putative daughter nodes: Proto-Western Malayo-Polynesian (PWMP), Proto-Central Eastern Malayo-Polynesian (PCEMP) and Proto-Central Malayo-Polynesian (PCMP). As discussed in $§ 1.1$, my use of these labels simply follows the use of others, in this case reflecting the labels given by Blust and Trussel (ongoing). For the purposes of this comparative dictionary, I am agnostic regarding all putative nodes between PMP and PRM, with the exception of Timor-Babar, for which evidence is presented in $\$ 3.5 .3 .1$.

The transcription of PMP reconstructions follows the conventions of Blust and Trussel (ongoing), with the exception of PMP $<\mathrm{e}>$ [ə] which is transcribed $<\mathrm{\vartheta}>$ to avoid confusion with *e [e], which occurs in putative sub-nodes of PMP, such as PCEMP.

Certain symbols within this transcription tradition are not standard IPA or have unexpected phonetic values. The symbols used for $\mathrm{P}(\mathrm{CE}) \mathrm{MP}$ reconstructions in this comparative dictionary, along with the phonetic values ascribed to them by Blust (2009a:547, 623) and Wolff $(2010: 31,241)$ are given in Table $1.11 .{ }^{12}$ Note particularly that the values of $*_{\mathrm{z},} *_{\mathrm{j}},{ }^{\mathrm{r}} \mathrm{r}$ and $* \mathrm{R}$ may be unexpected. ${ }^{13}$

Table 1.11: Proto-Malayo-Polynesian consonants

\begin{tabular}{|c|c|c|c|c|c|c|c|c|c|c|c|c|c|c|}
\hline $\mathrm{P}(\mathrm{CE}) \mathrm{MP}$ & $* p$ & $*_{\mathrm{t}}$ & $*_{\mathrm{k}}$ & $* \mathrm{q}$ & $* b$ & $* d$ & \multicolumn{2}{|l|}{$*_{\mathrm{z}}$} & $*_{\mathrm{j}}$ & $* g$ & $* \mathrm{~m}$ & $*_{\mathrm{n}}$ & $* \tilde{n}$ & $* \eta$ \\
\hline Blust (2009a) & {$[\mathrm{p}]$} & {$[\mathrm{t}]$} & {$[\mathrm{k}]$} & [q] & [b] & [d] & \multicolumn{2}{|l|}{ [d] } & {$\left[\mathrm{g}^{\mathrm{j}}\right]$} & {$[\mathrm{g}]$} & {$[\mathrm{m}]$} & {$[\mathrm{n}]$} & {$[\mathrm{n}]$} & [n] \\
\hline Wolff (2010) & {$[\mathrm{p}]$} & {$[\mathrm{t}]$} & {$[\mathrm{k}]$} & [q] & {$[\mathrm{b}]$} & [d] & \multicolumn{2}{|c|}{$\left[\mathrm{d}_{\bar{n}} \mathrm{j}\right] /[\widehat{\mathrm{d} J}]$} & {$[\mathrm{g}]$} & - & {$[\mathrm{m}]$} & {$[\mathrm{n}]$} & {$[\mathrm{n}]$} & [y] \\
\hline $\mathrm{P}(\mathrm{CE}) \mathrm{MP}$ & $* \mathrm{~h}$ & \multicolumn{2}{|c|}{$* \mathrm{~s}$} & $* 1$ & $*_{\mathrm{r}}$ & $* \mathrm{R}$ & $* \mathrm{w}$ & $* \mathrm{y}$ & $*_{\mathrm{i}}$ & $* \mathrm{e}$ & *a & $* \mathrm{a}$ & $*_{\mathrm{o}}$ & $* \mathrm{u}$ \\
\hline Blust (2009a) & {$[\mathrm{h}]$} & \multicolumn{2}{|c|}{$[\mathrm{s}] /\left[\int\right]$} & [1] & {$[\mathrm{r}]$} & {$[\mathrm{r}]$} & {$[\mathrm{w}]$} & [j] & [i] & {$[\mathrm{e}]$} & [ə] & [a] & [o] & {$[\mathrm{u}]$} \\
\hline Wolff (2010) & {$[\mathrm{h}]$} & \multicolumn{2}{|c|}{$[\widehat{\mathrm{ts}}] /[\widehat{\mathrm{t} \theta} \theta]$} & [1] & - & [в] & {$[\mathrm{w}]$} & [j] & [i] & - & [ə] & [a] & - & {$[\mathrm{u}]$} \\
\hline
\end{tabular}

Several kinds of additional information regarding reconstructions can also occur in entries. Firstly, when the reconstruction does not come from Blust and Trussel (ongoing), the source is given after the reconstruction. Secondly, when the PMP and PRM glosses are substantially different, the gloss ascribed to the PMP form is given in quotation marks after the PMP reconstruction. Often this means that semantic shift has occurred between PMP and PRM.

Thirdly, any comments on the reconstruction are given in brackets. This includes when the reconstruction is not to PMP, but at a putative lower level such as PWMP, PCEMP or PCMP. Other comments relate to problems that the PMP reconstruction presents, or give formally and semantically similar reconstructions that have also been made.

Apart from reconstructions to PMP, I also occasionally give putative forms marked with a double asterisk and assigned to 'pre-Rote-Meto', a level before PRM, which is left deliberately vague. Such pseudo-reconstructions are given when out-comparisons (§1.5.1) indicate that the PRM form is probably inherited from a higher node, but no

\footnotetext{
12 Wolff (2010) uses different symbols for several of his proto-phonemes, and he does not accept all the proto-phonemes posited by Blust. Reconstructions from Wolff (2010) and other sources are (re)transcribed according to the conventions used by Blust and Trussel (ongoing).

13 Blust (2009a) also reconstructs PMP $* c[t]$ and $* D[d]$, but these putative proto-phonemes do not occur in any of the reconstructions in this dictionary.
} 
reconstruction has yet been made by another scholar. These pseudo-reconstructions should not be considered proper reconstructions, as we have only a preliminary understanding of the way in which the Rote-Meto languages relate to other language groups ( 3.5$)$ In the small number of cases in which I am confident of the level and form of my own reconstruction, I give it with a single asterisk and give own reconstruction as the source.

\subsubsection{Doublets}

When a form has an etymologically related counterpart, this counterpart is included preceded by Doublet: . In this comparative dictionary, a 'doublet' is only used for formally distinct reconstructions that are inherited from a single reconstructed etymon without being morphologically related. Thus, for instance, PRM * fai 'day, time' and *hoi 'dry in the sun' are both reflexes of PMP *waRi, but with different sound changes. Similarly, *mane 'man, male' and *mone 'man, male' are both from PMP *maRuqanay via slightly different pathways.

Formally and semantically similar reconstructions that may ultimately be cognate but cannot (yet) be identified as descending from a single etymon are not marked as doublets. Instead, such forms contain cross references to one another. This includes forms that are inherited from those given as doublets by Blust and Trussel (ongoing). An example of the latter is *lea-k 'cave' and *lua|t 'cave', which are inherited from PMP *liay and *luay, respectively.

\subsubsection{Irregular sound changes}

When the forms given do not show the expected correspondences (summarised in §3.2), this is recorded in one of three different fields. Based on our current understanding of the Rote-Meto languages, as well as our reconstruction of PMP, such comparisons require positing irregular sound changes (or irregular retention of certain sounds).

To allow the reader to quickly ascertain the strength of the reconstruction, the notes recording irregular sound changes are usually given in the entry for the reconstruction before all other notes. The only exception is when such notes are given for the outcomparisons, in which case they are given in the entry for the out-comparison.

Firstly, there is a field marked 'irr. from PMP'. This field records irregular sound changes that must be posited to derive the reconstructed PRM form from a putative PMP etymon. Many such irregular sound changes only have one putative attestation.

Secondly, there is a field marked 'minority from $P M P$ '. This field records irregular sound changes between PMP and PRM that have multiple attestations. Thus, for instance, while the usual reflex of PMP *q is $\varnothing$ in PRM, there are seven instances of PMP $* \mathrm{q}>\mathrm{PRM}$ ${ }^{*} \mathrm{~h}$. These latter instances have the note: [minority from PMP: ${ }^{*} \mathrm{q}>* \mathrm{~h}$ ]. See $\$ 3.5 .1$ for discussion of unconditioned splits between PMP and PRM.

The final field for irregular sound changes is marked 'Sporadic'. This field records sporadic sound changes (both between PMP and PRM, and/or PRM and its daughters), such as consonant metathesis, as well as changes that are only partially complete in 
certain lects, such as the raising of final $* \mathrm{a}>e$ in West Rote and Meto (§3.2.3). When a sporadic sound change only affects one language, it is often given in the entry for that language.

Under a strict neo-grammarian view in which sound change is completely regular, all comparisons that involve irregular correspondences would need to be excluded from this comparative dictionary. This is not the approach I take. Instead, I include such comparisons when the form and semantics of two morphemes are so similar that I feel uncomfortable excluding them from the dictionary. Furthermore, the inclusion of such forms means that no potentially valuable information is excluded from the dictionary.

Unfortunately this is an inherently subjective exercise. How similar is similar enough? This is the main reason I explicitly list irregular sound changes. Others may wish to exclude comparisons they judge too dissimilar, or further evidence may show that the identification of a certain cognate set is erroneous in some way. This is also why I give irregular sound changes before most other notes. The problems with such reconstructions should be front and centre in order to allow them to be subject to proper scrutiny. ${ }^{14}$

A second reason for explicitly listing putative irregular sound changes is that they have the potential to advance our understanding of Austronesian comparative linguistics. It may be that a higher level reconstruction is currently in error, or it may be that irregularities similar to those in PRM are found in other languages and can be adduced as evidence for a higher node.

Despite the subjectivity of this criterion, in my experience most cases are actually quite clear-cut. Thus, consider PRM *naa-k 'brother of a woman' (from PMP *ñaRa) with the following reflexes: Rote naa-k/naa-P and Meto na?o/nao-f all 'brother of a woman'. I do not think any comparativist would attribute the formal and semantic similarity between these forms to chance. However, final $o$ in Meto languages is not a regular reflex of *a. The reconstruction *naa-k thus requires irregular *a $>o$ in Meto.

Similarly, consider PRM *ka-nduna-k 'nest' with the following reflexes: Oenale, Dengka nduna-?, Rikou, Oepao runu-?, Bokai, Bilbaa lunu-?, other Rote $n d u n u-k / n d u n u-?$ 'nest', Ro'is Amarasi kuna $\mid$ ?, other Meto ? $\mid$ kuna $\mid ?$ 'nest'. Again, these forms are almost certainly related but final $*^{*}>u$ in Rote languages is not regular and ${ }^{*}$ nd $>k$ in Ro'is Amarasi is also irregular (all other sound correspondences are regular). Thus, these irregular sound changes are flagged.

Whenever the posited irregular sound change can be motivated, such as PRM *ka-nduna-k $>$ Termanu $n d u n u-k$ 'nest' where $* \mathrm{a}>u$ is probably sporadic vowel assimilation, such explanations are given in brackets after the irregular sound change. In this case the sound change is given as: '[irr. from PRM: *a $>u$ in nRote (sporadic assimilation)]'. Particularly speculative or ad-hoc explanations are further followed by a question mark '?'.

14 I welcome correction on any reconstructions in this dictionary, as well as putative connections between my reconstructions and higher levels that can be shown to be false. I only ask that if others debunk reconstructions or connections when I have explicitly flagged them as involving irregular sound changes, that they extend me the generosity of stating that I acknowledge the problems with such reconstructions. 


\subsubsection{Patterns}

The pattern field is used to show patterns of correspondences among the reflexes of $\mathrm{PRM} * \mathrm{k}$ and $* \mathrm{~d}$. Each of these proto-phonemes shows unconditioned splits in which the reflexes are not completely random. Instead, there are patterns of regularity in the correspondences. Four patterns can be identified for initial $* k$, six for medial $* k$, and two for $* \mathrm{~d}$ in all word positions. The correspondences for each pattern are summarised in Table 1.12 for a select number of daughter languages. The full correspondences are given in $\$ 3.2$.

Table 1.12: Patterns for Proto-Rote-Meto *k and *d

\begin{tabular}{|c|c|c|c|c|c|c|c|c|c|c|c|c|c|}
\hline PRM & *k- & & & & & $*-\mathrm{k}-$ & & & & & & $* d$ & \\
\hline env. & \# & \# & & \# & \# & V_V & V_V & V_V & V_V & V_V & V_V & all & all \\
\hline pattern & $\mathrm{k}-1$ & \multicolumn{2}{|l|}{$\mathrm{k}-2$} & $\mathrm{k}-3$ & $\mathrm{k}-4$ & $\mathrm{k}-5$ & $\mathrm{k}-6$ & $\mathrm{k}-7$ & $\mathrm{k}-8$ & k-9 & $\mathrm{k}-10$ & & $\mathrm{~d}-2$ \\
\hline Dela & $k$ & \multicolumn{2}{|l|}{$?$} & $?$ & $h$ & $k$ & $?$ & $k$ & ?/Ø & $?$ & ?/Ø & $r$ & $r$ \\
\hline Dengka & $k$ & \multicolumn{2}{|l|}{$?$} & $?$ & $h$ & $k$ & $?$ & $k$ & $? / \varnothing$ & $?$ & ?/Ø & $l$ & $l$ \\
\hline Tii & $k$ & \multicolumn{2}{|l|}{$k$} & $k$ & $\varnothing$ & $k$ & $k$ & $?$ & $?$ & $?$ & $?$ & $d$ & $r$ \\
\hline Termanu & $k$ & \multicolumn{2}{|l|}{$k$} & $k$ & $\varnothing$ & $k$ & $k$ & $?$ & $?$ & $?$ & $?$ & $d$ & $l$ \\
\hline Bilbaa & $k$ & \multicolumn{2}{|l|}{$k$} & $k$ & $\varnothing / k$ & $k$ & $k$ & $k$ & $k$ & $k$ & $k$ & $d$ & $l$ \\
\hline \multirow[t]{2}{*}{ Rikou } & $k / 2 / \varnothing$ & \multicolumn{2}{|l|}{ ?/Ø } & $?$ & $\varnothing$ & $2 / k$ & $2 / k$ & $k / ?$ & $? / k$ & $? / k$ & $k / 2$ & $d$ & $\mathrm{r}$ \\
\hline & & $\mathrm{k}-2 \mathrm{a}$ & $k-2 b$ & & & & & & & & & & \\
\hline Ro'is & $k$ & $k$ & $k$ & $h$ & $h$ & $k$ & $k / ?$ & $?$ & $\varnothing$ & $?$ & $k$ & $n$ & $r$ \\
\hline Kotos & $k$ & $k$ & $?$ & $h$ & $h$ & $k$ & $k / ?$ & $?$ & $\varnothing$ & $?$ & $k$ & $n$ & $r$ \\
\hline Molo & $k$ & $k$ & $?$ & $h$ & $h$ & $k$ & $k / 2$ & $?$ & $\varnothing$ & $?$ & $k$ & $n$ & $l$ \\
\hline
\end{tabular}

Each of these patterns is tracked throughout this comparative dictionary in the field marked Pattern. Thus, for instance, a reconstruction with *k marked as 'Pattern: $\mathrm{k}-1$ ' indicates that this ${ }^{\mathrm{k}} \mathrm{k}$ has reflexes according the $\mathrm{k}-1$ pattern in Table 1.12, 'Pattern: $\mathrm{k}-2$ ' that it follows the k-2 pattern, and so on. When data from diagnostic languages is lacking, the possible patterns are given. Thus, $\mathrm{k}-2 / 3$ indicates a $* \mathrm{k}$, which could belong to either pattern k-2 or k-3.

Forms that mostly follow one of these patterns, but with one or two deviations in the reflexes, are marked with a prime symbol, with the deviations given afterwards. An example is *koro 'Rainbow Bee-eater' for which the reflexes follow pattern 1 with the exception of Meto which has $*_{\mathrm{k}}>\varnothing$ rather than expected $*_{\mathrm{k}}=k$. Thus, this entry contains 'Pattern: $\mathrm{k}-1$ ' (but $* \mathrm{k}>\varnothing$ in Meto; expect $* \mathrm{k}=k$ )'. Instances of $* \mathrm{k}$ or $* \mathrm{~d}$ that do not follow any of these patterns are marked as k-irr. or d-irr. with the irregularities tracked in the irr. from PRM field, as discussed in $§ 1.5 .3 .5$.

\subsubsection{Notes}

Up to four different kinds of general notes are given for entries. Firstly, there is a field marked Notes:, which is used for all notes that do not clearly fit into any of the categories discussed. After this comes notes on irregular sound changes, discussed in more detail in $\$ 1.5 .3 .5$. 
After this is a note field flagged Form. This is used for notes on the form of an entry. Examples of the kinds of comments given here include giving the possible unmetathesised form(s) of a Meto word, which has only been attested metathesised, giving the gloss of part of a compound, or indicating regular sound changes that might not be immediately apparent. This field is also used for alternate forms from sources apart from the primary source used for a lect.

The note field flagged History is used for notes on the history of a form. This includes discussion of possible higher etyma for a PRM form, or comparisons with reconstructions that cannot straightforwardly be identified as the etymon for my reconstruction. Other miscellaneous notes on the history of a term are also given.

The note field flagged Semantics gives notes on the semantics of an entry. Examples of the kinds of notes given in this field include more detailed discussion of semantic shifts that certain comparisons involve, or indicating when a botanical term has only a vague gloss such as 'kind of tree' in my sources.

\subsubsection{Loan distributions}

In addition to the main part of this comparative dictionary, I have also included a number of loan distributions in the final part of the dictionary. That is, cognate sets that can be identified as being ultimately borrowed from another language. This section has the same organisation and structure as the main part of the dictionary, with the exception that the headwords in this section are preceded by a superscript hash \# indicating that these headwords are not proper reconstructions, but rather generalisations across the forms in daughter languages.

This section does not include every instance of a loan in the Rote-Meto languages, but is focused on sets that might be mistaken for cognates shared by common inheritance. Some of the cognate sets in this section may have been borrowed at the level of PRM or one of its daughter nodes, though this seems unlikely for most sets.

\subsubsection{Finder lists}

Two finder lists are also included in this dictionary. Firstly, there is an English finder list. The glosses by which this finder list is organised include glosses for the reconstruction, and glosses for the reflexes in daughter languages which have undergone semantic shift.

Thus, for instance, PRM *ndelat is reconstructed with the meaning 'lightning', but the Meto cluster has forms that have undergone semantic shift to include 'gun' (e.g. Ro'is Amarasi renet 'gun', other Meto kenat 'gun'). Thus *ndelat is included in the finder list after both 'lightning' and 'gun'. Doing this allows the reader to look up the etymology of a particular word from one of the modern Rote-Meto languages based on its gloss. It also makes it easier for the reader to find potential PRM cognates that may have undergone similar semantic shift in related languages. 
The finder list only gives glosses for PRM reconstructions or reflexes of them. Glosses for PMP forms that are the etymon of a PRM form are not given in the finder list. Thus for instance PMP *kuhkuh 'claw, talon, fingernail' has undergone semantic shift to PRM *kuku 'finger, toe' and no reflexes of this form attest the PMP semantics. As a result, 'fingernail' in the finder list does not point to PRM *kuku.

In addition to the English finder list, a finder list organised by reconstructions to a node higher than PRM is also given. This finder list gives the forms in the etymology field (§1.5.3.3) followed by the PRM reflex(es) and the gloss of the PRM reconstruction. Not all PRM reconstructions occur in this finder list, as not all are known to be inherited from a higher node. 


\section{2}

\section{Language background}

\subsection{Introduction}

In this chapter I give an overview of the synchronic features of the Rote and Meto clusters, necessary for understanding the content of this dictionary. This includes an overview of the phonology (\$2.4), morphophonemic processes (\$2.5) and morphology (\$2.6). The sources of data for this overview are the same as those for the body of the dictionary $(\S 1.3)$.

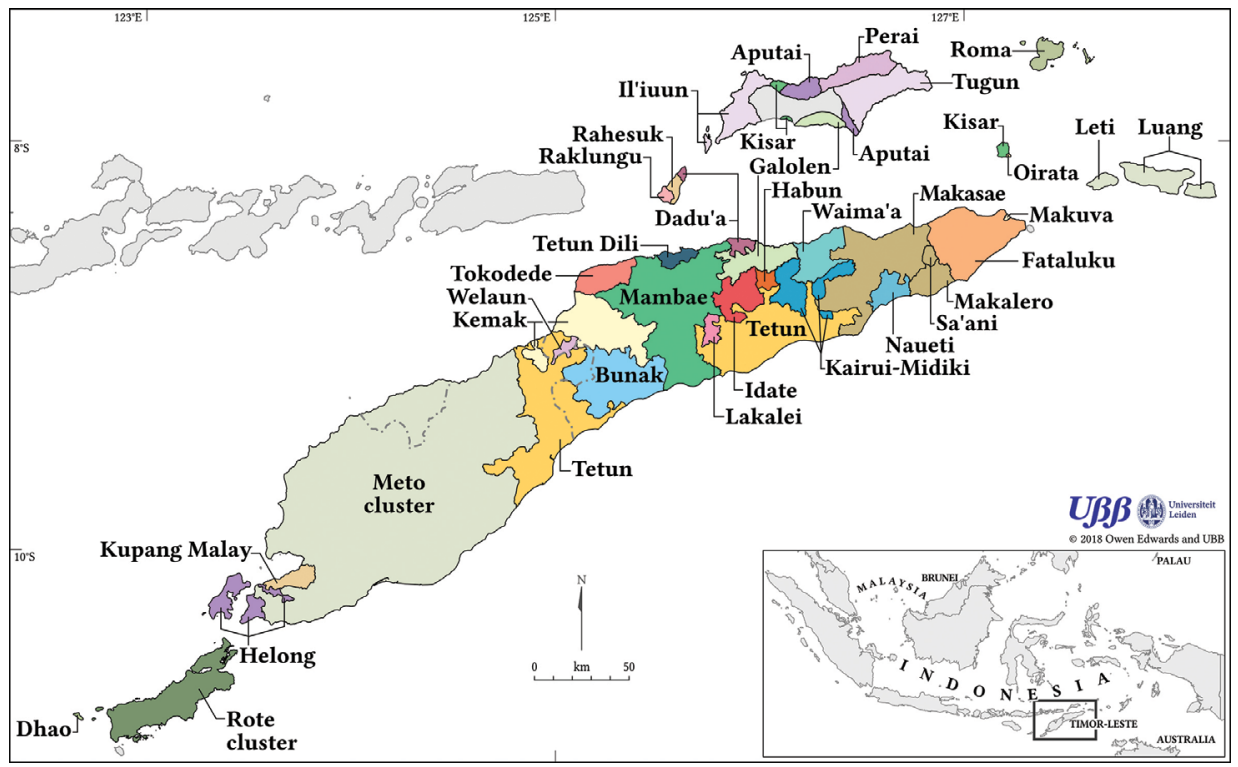

Map 2.1: Language groups of Timor

Source: Owen Edwards and UBB. 
The Rote languages are spoken on the island of the same name immediately to the southwest of the island of Timor. ${ }^{1}$ The Meto cluster is spoken in the western part of Timor including Oecusse, which is politically an enclave of Timor-Leste. The locations of the Rote and Meto clusters along with other languages of Timor are shown in Map 2.1. The location of languages in Timor-Leste is largely based on Williams-van Klinken and Williams (2015), who summarise census data.

\subsection{Rote}

The island of Rote is divided into 19 political units known in the anthropological literature as domains (nusa-k or nusa-? in the languages of Rote), and many speakers claim that each domain has its own language (Fox 2016a:233). A map of the domains of Rote is given in Map 2.2. (The language of Dhao is not part of the Rote cluster.)

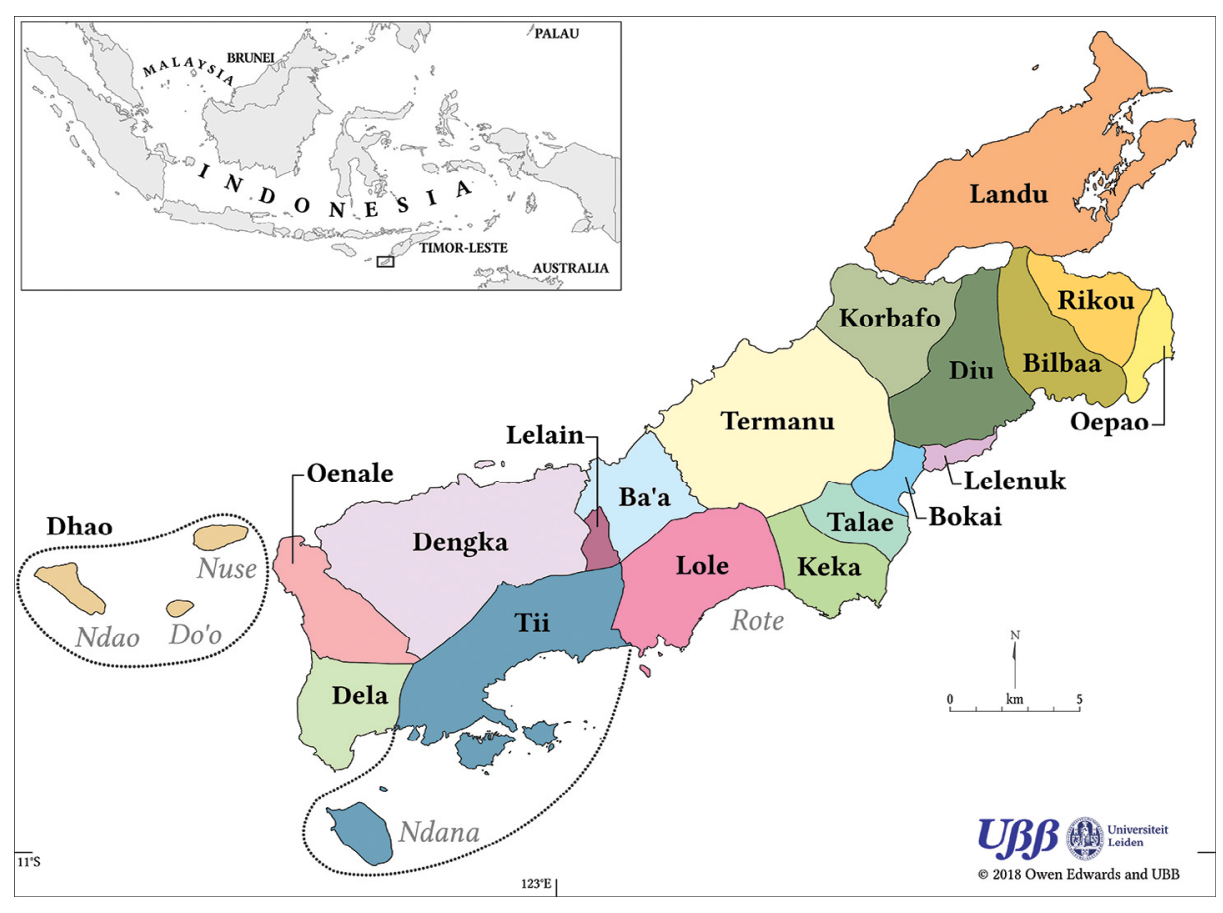

Map 2.2: Rote domains

Source: Owen Edwards and UBB.

The Rote cluster is a language/dialect chain like the Romance or West Germanic chains in Europe. Fox (2016a:233) summarises the complexity stating:

Speakers in neighbouring domains are generally able to understand one another, but for speakers in domains separated from one another intelligibility is reduced. Domains at a distance from one another find mutual intelligibility difficult or impossible. Based on these criteria Rotenese [sic] consists of more than one language.

1 There are also pockets of Rote speakers on the Timor mainland. Such populations are due to migrations in historical times. 
Given that we are dealing with a language/dialect chain, any classification that places an exact figure on the number of languages or dialects on Rote is necessarily somewhat arbitrary, depending on which criteria are privileged. Nonetheless, it is helpful to consider the classifications that have been made by various researchers. The different classifications are summarised in Table 2.1.

The earliest published classification of the speech varieties of Rote is that of Manafe (1889), a Rote speaker. Manafe (1889) identifies nine different Rote lagu (Malay 'song, tune, dialect'): (1) Oepao, Rikou and Landu; (2) Bilbaa, Diu and Lelenuk; (3) Korbafo; (4) Termanu, Keka and Talae; (5) Bokai; (6) Ba'a and Lole; (7) Dengka and Lelain; (8) Tii; (9) Dela and Oenale.

Jonker (1913) reviews the classification of Manafe (1889) and notes differences between varieties that Manafe grouped together as well as similarities between those he separated. One of the main points that arises from Jonker (1913) is that there is a sharper division between Dela-Oenale and Dengka in the west compared with the other Rote lects. This difference is found in morphology and lexicon, as well as the historical phonology, the latter which provides good evidence for a West Rote group separate from all other varieties of Rote (see $\S 3.3$ ).

The most recent classification is that of Fox (2016a) who to some extent follows the earlier classifications, though Fox groups related dialects together rather than differentiating them. Fox identifies six groupings: (1) Eastern dialect area: Rikou, Oepao and some of Landu; (2) East-Central dialect area: Bilbaa, Diu, Lelenuk, Korbafo and some of Landu; (3) Central dialect area: Termanu, Keka, Talae, Ba'a, Lelain and Bokai; (4) South-Western dialect area: Tii and Lole; (5) North-Western dialect area: Dengka; and (6) Western dialect area: Dela and Oenale.

Examining only the historical phonologies of the different speech varieties of Rote (see §3.2), we can identify 12 distinct varieties. (1) Dela and Oenale, (2) Dengka, (3) Tii, (4) Lole, (5) Ba'a, (6) Termanu and Keka, (7) Korbafo, (8) Bokai, (9) Bilbaa, (10) Rikou, (11) Landu ${ }^{2}$ and (12) Oepao. Varieties in each of these 12 groupings currently appear to have undergone the same sound changes. No data is available from Lelain, Talae, Diu or Lelenuk to make a proper assessment of the sound changes in these varieties, though all classifications treat Talae with Termanu, as well as placing Diu and Lelenuk with Bilbaa. ${ }^{3}$

The different classifications of the Rote languages that have been made are summarised in Table 2.1, along with the names, ISO 693-3 codes, and Glottocodes (Hammarström et al. 2020) of each variety.

\footnotetext{
2 The population of Landu was decimated in 1756 by the Dutch. It was later resettled, partly from Rikou (Fox 2016a:236). During my fieldwork, Landu speakers reported that genuine/native Landu is spoken in the western/central villages (desa) of Sotimori, Bolatena and Nifuleu. Rikou is spoken in other areas.

3 Although Manafe (1889) appears to present data from Lelain, Talae, Diu and Lelenuk, such data always consist of a single form, which is labelled 'Dengka dan Lelain', 'Bilba, Diu dan Lělénuk' or 'Termanu, Keka dan Talaě' (where dan is Indonesian for 'and'). Similarly, he presents single forms for 'Baä dan Lålě', including in one instance when other sources show that Ba'a and Lole are different. Thus, Manafe (1889:641) gives Ba'a and Lole $<m$ 'Pui-kah lah $>$ mpui- $k=a$ laa for 'a bird flies' while data from Jonker's works and Zacharias et al. (2014) never attest a voiceless prenasalised plosive in Lole. Instead, Zacharias et al. (2014) have mbui-k 'bird'. This indicates that in other cases the forms in Lelain, Talae, Diu and Lelenuk may differ from those presented in Manafe (1889).
} 
ROTE-METO COMPARATIVE DICTIONARY

Table 2.1: Classifications of Rote speech varieties

\begin{tabular}{|c|c|c|c|c|c|c|}
\hline Lect & ISO & Glottocode & Sound changes & Manafe (1889) & Fox (2016a) & Lect \\
\hline Dela & row & dela1252 & 1 & 1 & 1 & Dela \\
\hline Oenale & row & oena1237 & 1 & 1 & 1 & Oenale \\
\hline Dengka & dnk & deng1253 & 2 & 2 & 2 & Dengka \\
\hline Lelain & dnk & lela1245 & $2 / 5 ?$ & 2 & 4 & Lelain \\
\hline Tii & txq & tiii1241 & 3 & 3 & 3 & Tii \\
\hline Lole & llg & lole1239 & 4 & 4 & 3 & Lole \\
\hline Ba'a & $11 \mathrm{~g}$ & baaa1237 & 5 & 4 & 4 & Ba'a \\
\hline Termanu & twu & pada1259 & 6 & 5 & 4 & Termanu \\
\hline Keka & twu & keka1234 & 6 & 5 & 4 & Keka \\
\hline Talae & twu & tala1297 & $6 ?$ & 5 & 4 & Talae \\
\hline Bokai & twu & boka1251 & 7 & 6 & 4 & Bokai \\
\hline Korbafo & twu & korb1237 & 8 & 7 & 5 & Korbafo \\
\hline Bilbaa & bpz & bilb1242 & 9 & 8 & 5 & Bilbaa \\
\hline Diu & bpz & diuu 1237 & $9 ?$ & 8 & 5 & Diu \\
\hline Lelenuk & $\mathrm{bpz}$ & lele1271 & $9 ?$ & 8 & 5 & Lelenuk \\
\hline Landu & $\mathrm{rgu}$ & land 1257 & 10 & 9 & 6 & Landu \\
\hline Rikou & rgu & nucl1538 & 11 & 9 & 6 & Rikou \\
\hline Oepao & rgu & oepa1237 & 12 & 9 & 6 & Oepao \\
\hline
\end{tabular}

\subsection{Meto}

Meto (a.k.a. Uab Meto, Dawan(ese), Timorese, or Atoni) is a language/dialect chain spoken in the western part of Timor. ${ }^{4}$ Meto speakers usually identify their speech as a single language and call it uab meto?, molok meto?, (uab) Timor, or occasionally, to outsiders, (bahasa) Dawan. Speakers of Meto recognise more than a dozen named varieties. These varieties themselves have named dialects, with further differences being found between different villages and hamlets of a single dialect. A map of self-identified Meto varieties is given in Map 2.3.

While the Meto cluster is numerically and geographically larger than the Rote cluster, ${ }^{5}$ it has less diversity in its segmental phonology. Based only on the historical phonologies of Meto (see §3.2), we can identify four different varieties, each of which is currently known to have undergone different sound changes: (1) Ro'is Amarasi, (2) Kotos Amarasi, Amabi and Kusa-Manea, (3) Amanuban and Amanatun, (4) all other varieties. Among

\footnotetext{
4 In earlier works I referred to this language cluster as Uab Meto. In many varieties uab meto? can be glossed as 'dry/ indigenous speech'. However, not all Meto speaking areas use $u a b$ for 'speech'. Thus, in Amfo'an 'speech' is $a g u a b$, in Timaus it is molok and in Baikeno lasi is used for 'language'. Use of Meto alone thus covers more varieties in an emic manner. It also matches the use of speakers in which meto? alone can refer to the language. Such use is seen in phrases like Kotos Amarasi iin nahiin meto? 'S/he knows (how to speak) Meto'.

5 Ethnologue (Eberhard et al. 2020) estimates 842,000 Meto speakers as opposed to 116,000 speakers for all Rote languages combined. Both these figures are probably underestimated.
} 
other varieties, Amfo'an and Timaus can further be grouped together as, based on current knowledge, these are the only varieties that have developed consonant insertion after all vowel final roots (see $\$ 2.5 .2)$.

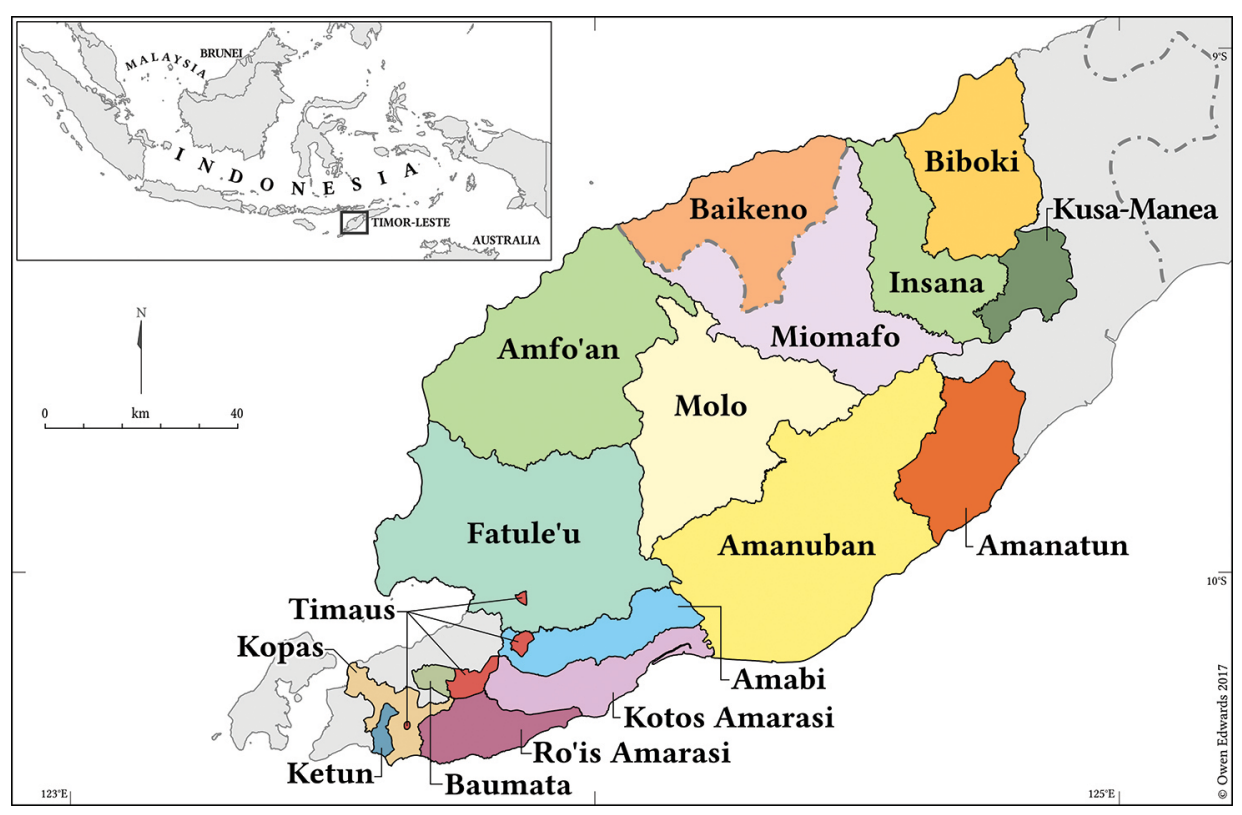

Map 2.3: Self-identified Meto varieties

Source: Owen Edwards.

While the historical sound changes within each of these groupings are mostly the same, there are many lexical and semantic differences between different varieties, as well as significant differences between different varieties in the forms and functions of the morphophonemic processes of metathesis, consonant insertion, vowel assimilation and diphthongisation. Differences in lexicon, semantics and morphophonemic processes are also found between individual hamlets or 'dialects' of the different varieties shown in Map 2.3.

The different varieties of Meto are listed in Table 2.2, along with ISO 693-3 codes, Glottocodes and their classification according to historical phonology. Not all varieties occur in Ethnologue (Eberhard et al. 2020) or Glottolog (Hammarström et al. 2020) and, of those that do, all except Kotos Amarasi, Ro'is Amarasi and Baikeno are listed as dialects/varieties of Meto. ${ }^{6}$

6 Amabi is lumped as part of a single 'Amfo'an-Fatule'u-Amabi' variety of Meto in both Ethnologue (Eberhard et al. 2020) and Glottolog (Hammarström et al. 2020). My consultants report that it is most similar to Kotos Amarasi, with occasional lexical and semantic differences. 
ROTE-METO COMPARATIVE DICTIONARY

Table 2.2: Varieties of Meto

\begin{tabular}{|l|l|l|l|}
\hline Lect & ISO & Glottocode & Sound changes \\
\hline Ro'is Amarasi & aaz & rois1241 & 1 \\
\hline Kotos Amarasi & aaz & koto1251 & 2 \\
\hline Amabi & aoz & amfo1237 & 2 \\
\hline Kusa-Manea & aoz & kusa1252 & 2 \\
\hline Amanuban & aoz & aman1265 & 3 \\
\hline Amanatun & aoz & aman1264 & 3 \\
\hline Molo & aoz & moll1242 & 4 \\
\hline Fatule'u & aoz & amfo1237 & 4 \\
\hline Miomafo & aoz & moll1242 & 4 \\
\hline Baikeno & bkx & baik1238 & 4 \\
\hline Insana & aoz & bibo1238 & 4 \\
\hline Biboki & aoz & bibo1238 & 4 \\
\hline Kopas & - & - & 4 \\
\hline Ketun & - & - & 4 \\
\hline Baumata & - & - & 4 \\
\hline Amfo'an & aoz & amfo1237 & 5 \\
\hline Timaus & - & - & 5 \\
\hline
\end{tabular}

\subsection{Segmental phonologies}

In this section I describe the essential features of the segmental phonologies of the RoteMeto languages. This is necessary to understand the historical sound changes that have occurred in these languages $(\$ 3.2)$.

\subsubsection{Rote}

The Rote languages have different consonant inventories. Consonants occur at four places: labial, coronal, velar and glottal with up to seven manners of articulation: voiceless plosive, prenasalised plosive, voiced plosive (often implosive), voiceless fricative, nasal, trill/tap and lateral.

Four voiceless stops $/ \mathrm{p} \mathrm{t} \mathrm{k} \mathrm{P/,} \mathrm{two} \mathrm{voiced} \mathrm{stops} / \mathrm{b} \mathrm{d} /$ and three fricatives $/ \mathrm{f} \mathrm{s} \mathrm{h/are} \mathrm{present}$ in all varieties. ${ }^{7}$ Among other consonant series there is variation in which segments different varieties attest. Some varieties have two liquids $/ 1 \mathrm{r}$, while others have only a single liquid $/ 1 /$. Some varieties have only two nasals $/ \mathrm{m} \mathrm{n} /$, while others have $/ \mathrm{n} / \mathrm{in}$ addition. Some varieties have a full series of prenasalised stops $/ \mathrm{mb} \mathrm{nd} \mathrm{gg} /$, while others have only a partial series or lack prenasalisation entirely.

7 In all Rote lects for which data is available, the voiceless coronal plosive /t/ is dental [t] while the voiced plosive /d/ or /d/ is alveolar or even slightly retroflex. 
There are also differences in the phonetic qualities of these consonants. In south-western lects including Dela-Oenale, Tii and Lole, voiced plosives are usually lightly imploded in all word positions. For these languages, imploded stops $/ \mathfrak{b} /$ and $/ \mathfrak{d} /$ can be treated as phonemes. Based on two Dengka recordings made available to me by Thersia Tamelan, it appears that Dengka /b/ is usually unimploded [b] while the apical voiced plosive is usually lightly imploded [d].

In Termanu voiced plosives are reported as imploded only medially, thus $/ \mathrm{b} / \rightarrow[\mathrm{b}] / \mathrm{V} \_\mathrm{V}$ and $/ \mathrm{d} / \rightarrow[\mathrm{d}] /$ V_V. During my fieldwork on Bilbaa, Landu and Oepao, I did not record implosion for $/ \mathrm{b} /$, but did occasionally record light implosion for intervocalic $/ \mathrm{d} /$, thus $/ \mathrm{b} /$ $\rightarrow[\mathrm{b}]$ and $/ \mathrm{d} / \rightarrow[\mathrm{d}] \sim[\mathrm{d}] / \mathrm{V}_{-} \mathrm{V}$. The phonetics of voiced plosives in other Rote varieties are currently unknown. Regarding prenasalisation, in Ba'a the bilabial prenasalised stop is voiceless $/ \mathrm{mp} /$ while in other varieties it is voiced $/ \mathrm{mb} /$. The consonant inventories of the Rote lects are summarised in Table 2.3.

Table 2.3: Consonant inventories in Rote Languages

\begin{tabular}{|l|l|l|l|l|l|l|l|l|l|l|l|l|l|l|l|l|l|}
\hline Dela-Oenale, Tii & $p$ & $t$ & $k$ & $?$ & $m b$ & $n d$ & $\eta g$ & $b$ & $d$ & $f$ & $s$ & $h$ & $m$ & $n$ & $(\eta)^{\dagger}$ & $r$ & $l$ \\
\hline Dengka & $p$ & $t$ & $k$ & $?$ & $m b$ & $n d$ & $\eta g$ & $b$ & $d$ & $f$ & $s$ & $h$ & $m$ & $n$ & & & $l$ \\
\hline Lole & $p$ & $t$ & $k$ & $?$ & $m b$ & $n d$ & $\eta g$ & 6 & $d$ & $f$ & $s$ & $h$ & $m$ & $n$ & & & $l$ \\
\hline Ba'a & $p$ & $t$ & $k$ & $?$ & $m p$ & $n d$ & $\eta g$ & $b$ & $d$ & $f$ & $s$ & $h$ & $m$ & $n$ & & & $l$ \\
\hline Termanu, Korbafo & $p$ & $t$ & $k$ & $?$ & & $n d$ & $\eta g$ & $b$ & $d$ & $f$ & $s$ & $h$ & $m$ & $n$ & $\eta$ & & $l$ \\
\hline Bokai, Bilbaa & $p$ & $t$ & $k$ & $?$ & & & & $b$ & $d$ & $f$ & $s$ & $h$ & $m$ & $n$ & $\eta$ & & $l$ \\
\hline Rikou, Landu & $p$ & $t$ & $k$ & $?$ & & $n d$ & & $b$ & $d$ & $f$ & $s$ & $h$ & $m$ & $n$ & & $r$ & $l$ \\
\hline Oepao & $p$ & $t$ & $k$ & $?$ & & $(n d)^{\ddagger}$ & & $b$ & $d$ & $f$ & $s$ & $h$ & $m$ & $n$ & & $r$ & $l$ \\
\hline
\end{tabular}

$\dagger$ In Dela the velar nasal $/ \mathrm{y} /$ is a marginal phoneme only occurring in onomatopoeic words.

† In my Oepao data /nd/ occurs in Rikou borrowings and one possible native word.

All (known) varieties of Rote have five vowels /i e a o u/. For Termanu the mid-vowels are described as lax $[\varepsilon]$ and $[0]$ in unstressed syllables, and when they bear stress followed by a syllable containing another mid vowel. Tense mid-high allophones [e] and [o] occur in stressed syllables followed by a syllable containing a high vowel. When the following syllable has /a/ as its nucleus there is apparently variation between tense [e] [o] and lax [ع] [o] in Termanu (Jonker 1915:2f, Fox and Grimes 1995:614).

In Dela the low vowel /a/ is centralised to [ $\mathrm{e}$ ] after stressed syllables and often further reduced towards schwa [ə] in antepenultimate syllables. The mid-vowels are mid-high [e] and [o] before a syllable containing a high vowel and mid-low $[\varepsilon]$ and [o] elsewhere. The high front vowel $/ \mathrm{i} /$ is [I] in unstressed closed syllables while $/ \mathrm{u} /$ is slightly centralised to [ซ] after stressed syllables (Tamelan 2021:22).

Content words contain at least two vowels. Some functors, such as the conjunction $m a$ 'and', are monosyllabic. Stress falls on the penultimate vowel of a word with secondary stress assigned to every second syllable to the left. All vowel sequences occur with the exception of $/ \mathrm{uo} / .^{8}$ This includes double vowels (sequences of two identical vowels)

8 The vowel sequence /ie/ does occur in the Rote languages, but is rare. Based on current data it appears to only be found in Dela-Oenale, Dengka, Tii and Lole. 
/ii/, /ee/, /aa/, /oo/ and /uu/. Such double vowels are realised phonetically as a single long vowel, i.e. [i:], [ع:], [a:], [o:] and [u:]. Given the placement of stress, one member of a vowel sequence will always bear stress.

\subsubsection{Meto}

All (known) Meto varieties have 11 core consonants $/ \mathrm{p} \mathrm{t} \mathrm{k}$ ? b f s h m n/ and either / $/$ or /1/. A few varieties have both $/ \mathrm{r} /$ and $/ 1 /$ and thus have 12 core consonants. To these core consonants most varieties of Meto add voiced obstruents /dz/ and /gw/, though some lack $/ \mathrm{gw} /$.

The obstruents /dz/ and /gw/ are restricted in distribution. They only occur under processes of consonant insertion $(\S 2.5 .2)$ that operate at clitic boundaries and/or word finally in different varieties of Meto, as well as between certain historic sequences of vowels. Both these obstruents are a result of glide fortition. These glides are still present in Amanuban which lacks these obstruents but has glides $/ \mathrm{y} /$ and $/ \mathrm{w} /$ in comparable environments.

All voiced obstruents are realised as plosives [b d $\mathrm{gw}]$ or fricatives $[\beta 3 \mathrm{\gamma w}]$. Fricative realisations are most common in unaffected speech, except after nasals, when the plosive realisations usually occur. The consonant inventories of several different varieties of Meto are summarised in Table 2.4 for comparison.

Table 2.4: Consonant inventories in Meto

\begin{tabular}{|l|l|l|l|l|l|l|l|l|l|l|l|l|l|l|}
\hline Amarasi & $p$ & $t$ & $k$ & $?$ & $b$ & $d s$ & $g w$ & $f$ & $s$ & $h$ & $m$ & $n$ & $r$ & \\
\hline Amanuban & $p$ & $t$ & $k$ & $?$ & $b$ & $y$ & $w$ & $f$ & $s$ & $h$ & $m$ & $n$ & & $l$ \\
\hline Amfo'an, Kopas & $p$ & $t$ & $k$ & $?$ & $b$ & $d s$ & $g w$ & $f$ & $s$ & $h$ & $m$ & $n$ & & $l$ \\
\hline Timaus & $p$ & $t$ & $k$ & $?$ & $b$ & $d s$ & $g w$ & $f$ & $s$ & $h$ & $m$ & $n$ & $r$ & $l$ \\
\hline Baikeno, Molo & $p$ & $t$ & $k$ & $?$ & $b$ & $d s$ & & $f$ & $s$ & $h$ & $m$ & $n$ & & $l$ \\
\hline Kusa-Manea & $p$ & $t$ & $k$ & $?$ & $b$ & & & $f$ & $s$ & $h$ & $m$ & $n$ & $r$ & $l$ \\
\hline
\end{tabular}

Known Meto varieties have five vowels /i e a o u/. The mid vowels are usually phonetically mid-low $[\varepsilon$ o ] but are raised to mid high [e o] in certain environments, particularly before high vowels. In some varieties of Meto this difference is becoming phonemic due to vowel assimilation after metathesis (\$2.5.1). Thus, for instance, in Naitbelak Amfo'an na-leko $\rightarrow$ na-leek [na'le:k] 'good' contrasts with na-henu $\rightarrow$ naheen [na'he:n] at the surface level. As in the Rote languages, content words are minimally disyllabic, stress is penultimate, and double vowels /ii/, /ee/, /aa/, /oo/ and /uu/ are realised phonetically as a single long vowel.

Varieties of Meto have a process of glottal stop insertion whereby an automatic glottal stop is inserted before vowel initial words in all phrase positions. While there is a contrast between vowel initial roots and glottal stop initial roots, this contrast only surfaces after the addition of certain prefixes, e.g. Kotos Amarasi $n$-ani 'before' contrasts with $n$-?ani 'head towards'. Because many Meto roots have never been attested with any prefixes, the status of many initial glottal stops is thus ambiguous. The issues surrounding initial glottal stops in one variety of Meto, Kotos Amarasi are discussed at length in Edwards (2017). 
For the purposes of this dictionary, all words with an initial ambiguous glottal stop are transcribed as vowel initial, with the exception of words that have $k$ in Ro'is Amarasi but initial glottal stop in other varieties of Meto (see Table 3.4). ${ }^{9}$ One such example is the reflexes of *katefuan 'wasp' > Ro'is Amarasi katfua|?, Kotos Amarasi, Molo Patfuan, Kusa-Manea ?aetfuan, all 'kind of wasp'. Wherever evidence from prefixes is available for such words, it shows that their initial glottal stop is underlying and not an automatic insertion.

\subsection{Meto morphophonemic processes}

Meto has many complex morphophonemic processes including metathesis, multiple processes of vowel assimilation, consonant insertion, diphthongisation and epenthesis. All of these processes can co-occur to different extents depending on a number of semantic, phonological and syntactic factors. This results in a single word having a diversity of surface forms in a single variety and across different varieties of Meto. In this section I discuss only those processes that affect the presentation of words in this dictionary: metathesis, consonant insertion, vowel assimilation and diphthongisation.

\subsubsection{Metathesis}

All varieties of Meto have productive final $\mathrm{CV} \rightarrow \mathrm{VC}$ metathesis producing alternates such as Kotos Amarasi fafi $\rightarrow$ faif 'pig' or neno $\rightarrow$ neon 'sky, day'. Metathesis is a morphological process in Meto. In Kotos Amarasi it marks attributive modification in the noun phrase and resolution for verbs. Edwards (2020) provides a detailed description of metathesis in Kotos Amarasi.

When the final vowel of such words is /a/, it assimilates to the quality of the previous vowel after metathesis in most known varieties of Meto, except for Kusa-Manea. Thus, Kotos Amarasi $n$-sena $\rightarrow n$-seen 'plant' and nima $\rightarrow$ niim 'five'. This results in surface minimal pairs such as Kotos n-nene $\rightarrow$ n-neen 'push' and n-nena $\rightarrow$ n-neen 'hear'. In Kusa-Manea such vowel assimilation does not occur, thus Kusa-Manea $n$-sena $\rightarrow$ $n$-sean 'plant' and nima $\rightarrow$ niam 'five'. Similarly, in Ro'is Amarasi assimilation of /a/ does not occur after metathesis when the medial consonant is $/ 2 /$, but does occur in all other situations. Thus, Kotos Amarasi $n$-ro? $a \rightarrow n$-roo? 'vomit' but Ro'is Amarasi $n$-ro? $a$ $\rightarrow n$-roa? 'vomits'.

Many other processes of vowel assimilation also occur after metathesis to different extents in different varieties of Meto, but none are present in forms included in this dictionary. An overview of known instances of these processes is included in Edwards (2020:163-167).

Meto words are given in the unmetathesised form throughout this dictionary whenever these forms are known. When such data is lacking, I give the metathesised form with the putative unmetathesised form(s) given in the notes when it may be ambiguous.

9 All words with initial $k$ in Ro'is Amarasi and $?$ in other varieties of Meto belong to the $\mathrm{k}-2 \mathrm{~b}$ pattern for initial PRM *k. See $§ 1.5 .3 .6$ for discussion of patterns. 


\subsubsection{Consonant insertion}

Most varieties of Meto have a process of consonant insertion that operates after vowel final words, before vowel initial enclitics, as well as phrase finally in some varieties. Consonant insertion has been described most fully for Amfo'an by Culhane (2018). Throughout this dictionary inserted consonants are separated from the stem by a hyphen. This means they are represented in the same way as suffixes.

Amfo'an, Timaus, Kopas, Fatule'u and Baikeno all have consonant insertion for certain nouns when phrase final, including in citation form. Consonant insertion also occurs with transitive verbs to mark a known third person object. Examples of consonant insertion in six different varieties of Meto are given in Table 2.5 to illustrate the following discussion.

Table 2.5: Phrase final consonant insertion

\begin{tabular}{|l|l|l|l|l|l|l|l|l|}
\hline & & Amfo'an & Timaus & Kopas T.' & Kopas U. & Fatule'u & Baikeno & Gloss \\
\hline$a i$ & $\rightarrow$ & aids & aar & aads & aads & aads & aids & 'fire' \\
\hline tei & $\rightarrow$ & teids & teer & teeds & teeds & teeds & teids & 'faeces' \\
\hline oe & $\rightarrow$ & oel & oel & oel & oel & oel & oel & 'water' \\
\hline fee & $\rightarrow$ & feel & feel & feel & feel & feel & feel & 'wife' \\
\hline ao & $\rightarrow$ & aog & aagw & aag & aagw & aob & aob & 'mineral lime' \\
\hline meo & $\rightarrow$ & meog & meegw & meeg & meegw & & meob & 'cat' \\
\hline hau & $\rightarrow$ & haug & haads & haag & haagw & haub & haub & 'tree, wood' \\
\hline kiu & $\rightarrow$ & kiug & kiids & kiig & kiigw & kiub & kiub & 'tamarind' \\
\hline uki & $\rightarrow$ & ukids & ukar & - & - & - & - & 'banana' \\
\hline tani & $\rightarrow$ & tanids & tanar & - & - & - & - & 'rope' \\
\hline ume & $\rightarrow$ & umel & umal & - & - & - & - & 'house' \\
\hline ane & $\rightarrow$ & anel & anal & - & - & - & - & 'field rice' \\
\hline neno & $\rightarrow$ & nenog & nenugw & - & - & - & - & 'day, sky' \\
\hline kolo & $\rightarrow$ & kolog & kolugw & - & - & - & - & 'bird' \\
\hline fatu & $\rightarrow$ & fatug & fatids & - & - & - & - & 'stone, rock' \\
\hline feru & $\rightarrow$ & ferug & ferids & - & - & - & - & 'new' \\
\hline
\end{tabular}

$\dagger$ Kopas T. is from Tuale'u hamlet and Kopas U. is from Usapisonba'i hamlet. The difference between final [g] and final [gw] is distinctive and noticeable to native speakers.

In Amfo'an and Timaus final consonant insertion affects all vowel final nouns while in other varieties in which it is attested it only affects words that end in a vowel sequence VV\#. Which consonant is inserted is conditioned by the quality of the final vowel. All varieties have insertion of $/ \mathrm{l} / \mathrm{after} / \mathrm{e} /$ and most have insertion of $/ \mathrm{d} / \mathrm{after} / \mathrm{i} /$, with the exception of Timaus, which has insertion of $/ \mathrm{r} /$ after $/ \mathrm{i} /$. After $/ \mathrm{o} /$ and $/ \mathrm{u} /$ Amfo'an and Kopas have insertion of $/ \mathrm{gw} /$ (with $/ \mathrm{gw} / \rightarrow[\mathrm{g}] / \#$ in some varieties), Fatule'u and Baikeno have insertion of $/ \mathrm{b} /$, while Timaus has insertion of $/ \mathrm{d} z /$ after $/ \mathrm{u} /$ and $/ \mathrm{gw} /$ after $/ \mathrm{o} /$.

Furthermore, consonant insertion is often accompanied by assimilation or shift of the final vowel. When the root ends in a vowel sequence the final vowel undergoes complete assimilation in Kopas and Timaus with the exception of /e/, which does not assimilate. 
In Fatule'u only /i/ assimilates after consonant insertion. In Timaus if the root ends in a consonant followed by a vowel (that is, $\mathrm{CV} \#$ ), the following vowel shifts occur: $/ \mathrm{i} / \rightarrow$ $/ \mathrm{a} /, / \mathrm{o} / \rightarrow / \mathrm{u} /$, and $/ \mathrm{u} / \rightarrow / \mathrm{i} / .^{10}$

Phrase final consonant insertion similar to that in Baikeno occurs to a lesser extent in Molo, though for the varieties of Molo on which I have collected data it is not as consistent or regular and does not seem to have become phonological to the same extent as other Meto varieties with consonant insertion. In the variety of Molo represented by Middelkoop (1972), insertion of $l$ occurs after $e$ for some, but not all, words with a final vowel sequence.

In Middelkoop (1972) insertion of $l$ also occurs for some words with final $a$. The three examples that are included in this comparative dictionary are *sikəh $>* *$ sia $>$ sia-l 'lath', *fia $>$ fia-l 'kind of wild tuber' and *hunga $>u k a-l$ 'chop big branches'. Insertion of $l$ after $a$ is also attested in Fatule'u and Amfo'an, but in both varieties (and probably also in Molo) it does not appear to have exactly the same distributions and functions as consonant insertion in other varieties of Meto.

\subsubsection{Diphthongisation and vowel assimilation}

Ro'is Amarasi has two productive phonological processes that affect the citation forms of consonant final words: diphthongisation and assimilation of /a/. Both these processes affect words of the shape (C)VCVC\# so long as the final consonant is not the glottal stop $/ \mathrm{R}$. Both processes are productive and affect vowel final words when their final syllable is closed by a following mono-consonantal enclitic or a word with an initial consonant cluster. See Edwards (2020:137f, 181) for more examples and discussion.

Firstly, the penultimate vowel of such words is diphthongised by addition of an off-glide of the same quality as the final vowel; that is, $\mathrm{V}_{\alpha} \mathrm{CV}_{\beta} \mathrm{CH} \rightarrow \mathrm{V}_{\alpha} \mathrm{V}_{\beta} \mathrm{CV}_{\beta} \mathrm{C}$. This process does not affect words that have /a/ as final vowel in varieties of Ro'is for which I have collected most data. Examples are given in Table 2.6 alongside their Kotos Amarasi cognates for comparison. Throughout this dictionary Ro'is Amarasi diphthongs that are a result of this process are transcribed with a tie-bar $<->$ to distinguish them that underlying vowel sequences.

Table 2.6: Ro'is Amarasi diphthongisation

\begin{tabular}{|c|c|c|c|c|c|}
\hline Kotos & Ro'is & Gloss & Kotos & Ro'is & Gloss \\
\hline tefis & teifik & 'roof' & niis eno- $f$ & niis êno- $f$ & 'incisors' \\
\hline masik & mâisik & 'salt' & n-Pator & $n$-?âator & 'arrange' \\
\hline to?is & tôi?is & 'trumpet' & si?u-f & sิü? $u-f$ & ‘elbow’ \\
\hline hunik & huinik & 'turmeric' & esuk & êusuk & 'mortar' \\
\hline anet & aenet & 'needle' & manus & mânus & 'betel pepper' \\
\hline rone- $f$ & roene-f & 'brain' & ponu- $f$ & poิ̄nu-f & 'body hair’’ \\
\hline
\end{tabular}

10 To further complicate matters, the variety of Timaus spoken in Sanenu hamlet where most of my Timaus data was gathered has * $\mathrm{e}>\mathrm{a} / \mathrm{C}_{-}$\#. Thus, Proto-Meto *ume $>$uma 'house' phrase medially and uma-l phrase finally. This change occurred after the development of consonant insertion in Timaus. 
$\dagger$ Ro'is Amarasi pounu- $f$ is 'body hair' and Kotos ponu- $f$ is moustache.

Secondly, when the final vowel of (C)VCVC\# words is /a/ (and the final consonant is not $/ \mathrm{R} /$ ), this vowel usually assimilates to the quality of the penultimate vowel. Examples are given in Table 2.7. There is some variation in the application of this process and some words have been attested with and without variants with assimilation of final $/ \mathrm{a} /$. Assimilation of /a/ in final closed syllables also occurs in Timaus.

Table 2.7: Ro'is Amarasi assimilation of final /a//_C\#

\begin{tabular}{|l|l|l|}
\hline Kotos & Ro'is & Gloss \\
\hline ?nima- $f$ & nimi- $f$ & 'arm, hand' \\
\hline sbeta- $f$ & sbete- $f$ & 'upper arm' \\
\hline ekam & erem, eram & 'wild pandanus' \\
\hline na-tenab & na-teneb & 'think' \\
\hline okam & okom, okam & 'gourd, melon' \\
\hline oras & oros & 'time' ' \\
\hline ruman & rumun & 'empty' \\
\hline utan & utuk, utak & 'vegetables; pumpkin, squash'‘ \\
\hline surat & surut & 'paper, book'\# \\
\hline
\end{tabular}

$\dagger$ From Portuguese horas [oras] 'hours'.

\$ Kotos Amarasi utan means only 'vegetables'.

\# From Malay surat 'letter'.

Finally, Kotos Amarasi has historic assimilation of final /a/ in syllables preceded by a glottal stop and closed by a consonant other than a glottal stop; that is, $\mathrm{V}_{\alpha} \mathrm{PaCH} \rightarrow$ $\mathrm{V}_{\alpha}$ PV ${ }_{\alpha} \mathrm{CH}$. Examples include Kotos Amarasi ke?en, other Meto ke?an 'room' and Kotos Amarasi po?on, other Meto po?an 'orchard'.

\subsection{Morphology}

In this section I provide an overview of the affixal morphology of the Rote-Meto languages. The purpose here is to present a succinct summary of the forms and usual semantic functions of morphology that occurs in this dictionary. This summary is based on Jonker (1915) and Tamelan (2021) for the Rote languages, as well as my own fieldwork for Meto. A more comprehensive description of Meto affixal morphology is Edwards (2020:439-458).

A summary of morphology is also needed as many forms included in this dictionary are only attested in morphologically complex forms with no putative mono-morphemic root attested. In most cases all such morphology is marked in the same way as productive morphology. Strings that can be confidently identified as an instance of frozen morphology are separated from the (historic) base by the vertical bar |, as discussed at the beginning of $\S 1.4$. 


\subsubsection{Nominal suffix $-k /-$ ?}

Many nouns in the Rote languages occur with the suffix $-k /-2$. Tii, Lole, Ba'a, Keka, Termanu and Bokai have $-k$, while Dela-Oenale, Dengka, Korbafo, Bilbaa, Landu, Rikou and Oepao have -?. The presence or absence of this suffix is partially lexically dependent. Nouns can be divided into two classes: those that are eligible to take this suffix, and those that never take this suffix (except after derivation with other nominalising morphology, see §2.6.4). Thus, for instance, Termanu bafi 'pig' and manu 'chicken' never occur with this suffix while $n e$ ? $e-k$ 'ant' and bau- $k$ 'bat' do take this suffix in appropriate environments. Nouns that are eligible to take this suffix in the Rote languages are given in this dictionary with this suffix.

One function of this suffix is to mark the end of the noun phrase. Thus, nouns modified by another noun do not take this suffix, with the final noun of an attributive phrase taking this suffix as long as it is a member of the class of eligible nouns. Examples of attributive phrases from Termanu extracted from Jonker (1908) are given in Table 2.8. Note also that there does not appear to be a grammatical basis for a noun/adjective distinction in the Rote languages.

Table 2.8: Termanu nominal $-k$

\begin{tabular}{|c|c|c|c|c|c|c|c|}
\hline \multicolumn{3}{|l|}{ Noun } & \multicolumn{3}{|c|}{ Modifier } & \multicolumn{2}{|l|}{ Phrase } \\
\hline lima-k & 'arm/hand' & + & $k u ? u-k$ & 'finger/toe' & $\rightarrow$ & lima $k u ? u-k$ & 'finger' \\
\hline lima-k & 'arm/hand' & + & dale-k & 'inside’ & $\rightarrow$ & lima dale- $k$ & 'palm' \\
\hline$e i-k$ & 'foot/leg' & + & $k u$ ? u-k & 'finger/toe' & $\rightarrow$ & ei ku?u-k & 'toe' \\
\hline$e i-k$ & 'foot/leg' & + & bu?u-k & 'joint' & $\rightarrow$ & ei bu?u-k & 'ankle' \\
\hline$n e ? e-k$ & 'ant' & + & ngeo-k & 'black' & $\rightarrow$ & ne?e ngeo-k & 'black ant' \\
\hline timi-k & 'jaw’ & + & $d u i-k$ & 'bone' & $\rightarrow$ & timi dui-k & 'jawbone’ \\
\hline beba-k & 'leaf stalk' & + & tula & 'gebang' & $\rightarrow$ & beba tula & 'gebang palm leaf stalk' \\
\hline$i ? a-k$ & 'fish' & + & tasi & 'sea' & $\rightarrow$ & i?a tasi & 'ocean fish' \\
\hline
\end{tabular}

There also appear to be certain Termanu nouns that only take the suffix $-k$ in isolation and do not take it when they are the final member of a phrase. Two examples are mene 'snake' + maa-k 'tongue' $\rightarrow$ meye maa 'snake tongue', and kaa-k 'fig tree' + daa- $k$ 'blood' $\rightarrow$ kaa daa 'fig tree sap'. The extent to which there are similar nouns in other varieties of Rote is currently unknown.

When a noun that is eligible to take the suffix $-k$ is modified by a non-nominal modifier, such as a quantifier, numeral or demonstrative, the suffix $-k$ occurs on the head noun. One example is Termanu bula-k 'moon, month' + telu 'three' $\rightarrow$ bula- $k$ telu 'three months'.

Tamelan (2021) identifies two main (non-derivational) functions for Dela nominal -? The first is as an attributive suffix with functions similar to Termanu $-k$. Like in Termanu, there are certain words that are eligible to occur with this suffix and certain words that are not. Those nouns that are eligible take this suffix in isolation and when they are the final member of an attributive phrase. 
The second function is as a generic genitive in non-referential genitive constructions for nouns that are in a part-whole relationship with the possessor. In this second function the suffix $-?$ is not restricted to a subset of eligible nouns (as in the attributive use), but occurs on all nouns. Two examples are noo oe-? 'water of a coconut' and sapi tei-P 'faeces of a cow'. Neither $o e$ 'water' nor tei 'faeces' occurs with -? in isolation or as the final member of an attributive phrase.

The non-derivational uses of this suffix do not occur in Meto. However, Meto cognates of words that take the nominal suffix $-k /-?$ in Rote languages often have a final $k$ or $?$. These final consonants are probably not best analysed as suffixes in Meto, but as part of the root. When all Rote members of a cognate set are eligible to take $-k /-?$ and at least one variety of Meto has a final $k$ or ?, I reconstruct the PRM noun as eligible to take *-k. This is indicated by the presence of this suffix in the morphology field ( 1 1.5.3.1).

In some instances when PRM *-k has become a glottal stop in Meto, and Meto has a root final vowel sequence, perceptual metathesis (Blevins and Garrett 1998:510-522) of the glottal stop (from *-k) with the root final vowel has occurred; thus $* \mathrm{~V}_{1} \mathrm{~V}_{2}$ ? $>$ $\mathrm{V}_{1} \mathrm{PV}_{2}$. Examples include PRM *beu-k 'new' $>$ Amanuban $f e u \mid ?>$ Amarasi $f e ? u$, and *doo-k 'leaf' $>$ Amanuban noo $\mid$ ? $>$ Amarasi no?o 'leaf'. Consistent with the origin of these glottal stops as affixes, they do not occur when such nouns take a productive suffix. Thus, Amarasi has moen feu-f 'son-in-law' and noo-n 'leaf-3sG.GEN'.

Finally, Kusa-Manea has a nominal suffix $-k$, the function and productivity of which is unclear. While it is possible that this suffix is a direct reflex of PRM *-k, this is not certain. Instead, this suffix may be borrowed from/influenced by Tetun $-k$, which occurs with a number of different nominal functions (van Klinken 1999:58ff). Examples include Kusa-Manea fatu $\sim$ fatu- $k$ 'stone, rock' (Tetun fatu $\sim$ fatuk) and no?o- $k$ 'leaf' (but noo- $n$ 'leaf-3GEN').

\subsubsection{Possessive morphology}

The Rote-Meto languages have productive possessive morphology that is attached to the possessed noun and agrees with the possessor in person and number. The Rote languages have enclitics and Meto has suffixes. The possessive paradigms of the Rote-Meto languages are given in Table 2.9.

Table 2.9: Rote-Meto possessive morphology

\begin{tabular}{|l|l|l|l|l|l|l|l|}
\hline & $1 \mathrm{SG}$ & 2SG & 3SG & 1PL.INCL & 1PL.EXCL/2PL & 3PL & 0 \\
\hline PRM & $*$ gga & $*$ ma & $*$ na & *nda & *ma & *nda & \\
\hline Dela & $=\eta g a$ & $=m a$ & $=n a$ & $=n a /=t a$ & $=m a$ & $=n a$ & \\
\hline Oenale, Dengka, Tii & $=\eta g a$ & $=m a$ & $=n a$ & $=n a$ & $=m a$ & $=n a$ & \\
\hline Termanu, Bilbaa, Bokai, Korbafo, Ba'a & $=\eta a$ & $=m a$ & $=n a$ & $=n a$ & $=m a$ & $=n a$ & \\
\hline Rikou & $=k a$ & $=m a$ & $=n a$ & $=n a$ & $=m a$ & $=n a$ & \\
\hline Ro'is & $-k$ & $-m$ & $-n$ & $-r$ & $-m$ & $-r$ & $-f$ \\
\hline Kotos Amarasi & $-k$ & $-m$ & $-n$ & $-k$ & $-m$ & $-k$ & $-f$ \\
\hline Amfo'an & $-k$ & $-m$ & $-n$ & $-k$ & $-m$ & $-k$ & $-f$ \\
\hline Kusa-Manea & $-k$ & $-m$ & $-n$ & $-k$ & $-m$ & $-n$ & $-f$ \\
\hline
\end{tabular}


In Rote all possessed nouns take a genitive enclitic. These enclitics have four forms. In addition to the $=C a$ forms given in Table 2.9, these enclitics also have a reduced form with no final vowel, thus $=\mathrm{C}$. When they attach to a consonant final stem the vowel $/ \mathrm{a} /$ is inserted between the stem and the enclitic. Thus, for example, the 3sg genitive enclitic has four forms: $=n a$ or $=n$ with vowel final stems and $=a n a$ or $=a n$ with consonant final stems. The only Rote genitive enclitic that occurs in this dictionary is the $3 \mathrm{sG}$ enclitic, which occurs on a small number of terms given by Jonker (1908), as well as some Landu words collected during my fieldwork $(\S 1.3 .1 .2)$.

The Meto languages have a paradigm of genitive suffixes. Unlike the Rote languages, only some nouns take genitive suffixes in Meto. Most such nouns are in a part-whole relationship with the possessor and typically include body parts or properties of the possessor. Furthermore, many nouns have only been attested with a genitive suffix. Such nouns are given in this dictionary with the suffix $-f$, which is used for unpossessed parts. Exceptions are a number of Ro'is Amarasi nouns that have only been attested with the $3 \mathrm{SG}$ suffix $-n$, as well as a number of Molo nouns that are only given by Middelkoop (1972) with either the 1sG suffix $-k$ or 3sG suffix $-n$.

Some kin terms also occur with genitive suffixes in Meto. In Ro'is Amarasi the suffixes taken by kin terms are the same as those taken by other nouns, while in most other known varieties of Meto kin terms with 3sG possessors take the suffix $-f$ while other possessors usually trigger no suffix. ${ }^{11}$ In Kusa-Manea, kin terms do not appear to take any genitive suffixes, though this observation is based only on words elicited in isolation.

\subsubsection{Reduplication}

The Rote-Meto languages have two kinds of reduplication: partial reduplication and full reduplication. In the Rote languages partial reduplication copies the first (C)V syllable of a base and places it to the left of that base. ${ }^{12}$ Termanu examples include $d e$ ? $a$ 'say, speak' $\rightarrow$ de de?a-k 'that which is said', bali 'mix' $\rightarrow$ ba bali- $k$ 'mixture' and $n$-inu 'drink' $\rightarrow$ ni ninu- $k$ 'a drink'.

In all known varieties of Meto except Kusa-Manea, partial reduplication copies the first CVC syllable of a disyllabic $\mathrm{CV}(\mathrm{C}) \mathrm{V}(\mathrm{C})$ foot and places it to the left of this foot. If a root contains consonant clusters or has more than two syllables, the reduplicant is an infix. One productive use of syllable reduplication in Meto is as an intensive. Examples from Kotos Amarasi include: $k o$ ? $u$ 'big' $\rightarrow \boldsymbol{k o}$ ? ko? $u$ 'very big', ana? $\rightarrow \boldsymbol{a n} \sim a n a ?$ 'very small', ?roo 'far' $\rightarrow$ ?ro $\sim$ roo 'very far', ma?fena? $\rightarrow$ ma?fen $\sim$ fena? 'very heavy' and paumaka? 'near' $\rightarrow$ paumak maka? 'very near'.

\footnotetext{
11 The situation regarding kin terms and possessive suffixes in Meto is complex. Thus, in Kotos Amarasi third person plural possessors also occasionally trigger use of the suffix $-f$ while in Koro'oto hamlet (where most of my Amarasi data was collected) all possessors optionally trigger use of the suffix $-f$ with this suffix only not occurring for unpossessed kin terms, such as vocatives.

12 The base for reduplication in the Rote is identical to an underlying root in nearly all cases. The only exception is vowel initial verbs, which obligatorily occur with a consonantal prefix $(\S 12.6 .5)$ for which the third person form is the base for reduplication; e.g. the reduplicative base for $\sqrt{ }$-inu 'drink' is $n$-inu.
} 
The reduplicant in Kusa-Manea consists of the first consonant of the final foot and the default vowel /a/. Like other varieties of Meto, this reduplicant is placed to the immediate left of the foot; thus, $\mathrm{C}_{1} \mathrm{~V}(\mathrm{C}) \mathrm{V}(\mathrm{C}) \rightarrow \mathrm{C}_{1} \mathrm{a} \sim \mathrm{C}_{1} \mathrm{~V}(\mathrm{C}) \mathrm{V}(\mathrm{C})$. This structure is the same as partial reduplication in Tetun (van Klinken 1999:44). Examples include the following: neno 'sun, day' $\rightarrow$ na neno-? 'bright, midday', mii 'urine' $\rightarrow$ ma $\sim$ mii- $f$ 'bladder', *mukə $>* * \mathrm{k} \mid$ mu?a $\rightarrow k \mid m a \sim m u a$ ? $-r=a a$ 'wild pigeon', and na-Pboni? 'hang' $\rightarrow$ ?ba boni? 'hanging'.

Full reduplication in Meto copies the entire root. Examples include batuur 'true' $\rightarrow$ batuur batuur 'truly' and neno 'day' $\rightarrow$ neno neno 'every day'. Full reduplication has the same structure in the Rote languages. Examples from Dela include ume 'house' $\rightarrow$ ume $\sim$ ume 'various houses', nduar 'sleepy' $\rightarrow$ nduar nduar 'very sleepy', and na-mbeta '3SG-painful' $\rightarrow$ nambeta nambeta 'intensely painful' (Tamelan 2021:41).

Many forms in the Rote-Meto languages are only attested in a reduplicated form without any known base. Such frozen reduplication is not differentiated from productive reduplication in this dictionary. In both cases the reduplicant is separated from the base by a tilde ' $\sim$ '. Likely cases of frozen reduplication can be identified by the lack of any base without reduplication in their entry.

\subsubsection{Nominalisation}

The Rote languages have a handful of nominalising processes, which are attested in this dictionary. Firstly, partial reduplication combined with the nominal suffix $-k /-?$, as seen in Termanu $d e ? a$ 'say, speak' $\rightarrow$ de de?a-k 'that which is said', bali 'mix' $\rightarrow$ $b a \sim$ bali-k 'mixture', and $n$-inu 'drink' $\rightarrow$ ni ninu- $k$ 'a drink'. In Dengka and DelaOenale such reduplication combines with one of three suffixes: $-?$, $-s$ or $-t$. Examples from Dela include $6 a e^{\prime}$ 'pay' $\rightarrow 6 a \sim 6 a e-P$ 'payment', lemba 'carry with a shoulder pole' $\rightarrow$ le lemba-t 'shoulder pole', and fiti? 'fire slingshot' $\rightarrow f i \sim f i t i-s$ 'fire with a slingshot'. In Dela, stems with a / $t$ / only take $-s$ while other stems take any of the three suffixes.

Another Rote nominalising morpheme in this dictionary is the property prefix ma-. Examples from Termanu include tane- $k$ 'sharp, pointy, sharpness' $\rightarrow$ ma-tane 'sharp', $d a l u-k$ 'long' $\rightarrow$ ma-dalu 'long, length', and tobi 'burn, scorch' $\rightarrow$ ma-tobi- $k$ 'hot'. The prefix $m a$ - is also a verbal prefix, in which case it forms stative verbs (\$2.6.6).

The Meto cluster has half a dozen affixes that serve a nominalising function, given in Table 2.10. All except one of these affixes are circumfixes. A more complete discussion of the different functions of these affixes in Kotos Amarasi is given in Edwards (2020:439-458).

Table 2.10: Meto nominalisation morphology

\begin{tabular}{|l|l|l|l|l|}
\hline \multicolumn{2}{|l|}{ Form } & \multicolumn{2}{l|}{ Gloss } & Notes \\
\hline & & $-t$ & result NMLZ & $-s$ when root contains $/ \mathrm{t} /$ \\
\hline$? a-$ & $\ldots$ & $-t$ & actor NMLZ & $k a-\ldots-t$ in Ro'is Amarasi, suffix $-s$ when root contains $/ \mathrm{t} /$ \\
\hline$?-$ & $\ldots$ & $-?$ & tool NMLZ & final $-?$ is an intervocalic infix for VV\# final roots \\
\hline$m a-$ & $\ldots$ & $-?$ & property NMLZ & final $-?$ is an intervocalic infix for VV\# final roots \\
\hline$m-$ & $\ldots$ & $-?$ & stative NMLZ & not fully productive, final $-?$ intervocalic infix for VV\# final roots \\
\hline$C a \sim$ & $\ldots$ & $-?$ & NMLZ & only known in Kusa-Manea, initial element is a reduplicant \\
\hline
\end{tabular}




\subsubsection{Verb agreement}

The Rote-Meto languages have productive verbal subject agreement prefixes. In all RoteMeto languages there are two paradigms: a vocalic paradigm with prefixes of the shape $\mathrm{CV}$-, and a consonantal paradigm in which the prefixes consist of a single consonant, which is the first consonant of the corresponding prefix in the vocalic paradigm. The vocalic and consonantal agreement prefixes are given in Table 2.11 and Table 2.12, respectively. ${ }^{13}$

Table 2.11: Vocalic agreement prefixes

\begin{tabular}{|c|c|c|c|c|c|c|}
\hline & $1 \mathrm{SG}$ & $2 \mathrm{sG}$ & $3 \mathrm{sg}$ & 1PL.INCL & 1PL.EXCL/2PL & $3 \mathrm{PL}$ \\
\hline PRM & *ku- & *mu- & $*_{\mathrm{i}-} / *_{\mathrm{n}}$ - & $*$ ta- & *mi- & *ra- \\
\hline Dela, Oenale & $? u-$ & $m u-$ & $n a-$ & ta- & $m i-$ & $r a-$ \\
\hline Dengka & $P u-$ & $m u-$ & $n a-$ & $t a-$ & $m i-$ & la- \\
\hline Tii & $? a-$ & $m a-$ & $i-/ n a-\dagger$ & $t a-$ & $m a-$ & $r a-$ \\
\hline Lole & $? a-$ & $m a-$ & $n i-/ i-/ n a_{-}^{\dagger}$ & $t a-$ & $m a-$ & $l a-$ \\
\hline Termanu, Ba'a & $P a-$ & $m a-$ & $n a-$ & $t a-$ & $m a-$ & la- \\
\hline Bilbaa, Bokai, Korbafo & $k a-$ & $m a-$ & $n a-$ & $t a-$ & $m a-$ & $l a-$ \\
\hline Rikou, Oepao & $? a-$ & $m a-$ & $n a-$ & ta- & $m a-$ & $r a-$ \\
\hline Ro'is Amarasi & $k u$ - & $m u-$ & $n a-$ & $t a-$ & $m i-$ & $n a-$ \\
\hline Nuclear Meto & $? u-$ & $m u-$ & $n a-$ & ta- & $m i-$ & $n a-$ \\
\hline
\end{tabular}

$\dagger$ Jonker (1915:411) has Tii 3sg $i$ - and Lole 3sg $n i$-. Unit Bahasa and Budaya (2016:vi) and Zacharias et al. (2014) have Tii and Lole $n a$-. For Lole, Adika Balukh (pers. comm. September 2020) reports that $i$ - is still used regularly and is particularly associated with northeast regions. She reports that she may have heard $n i$ - in the past, but is not confident. For Tii, Yanti TunliuMooy (pers. comm. September 2020) recalls hearing $i$ - more often in the past and associates this form with certain regions.

The $n a$ - form is dominant.

Table 2.12: Consonantal Agreement prefixes

\begin{tabular}{|c|c|c|c|c|c|c|}
\hline & $1 \mathrm{SG}$ & $2 \mathrm{SG}$ & $3 \mathrm{SG}$ & 1PL.INCL & 1PL.EXCL/2PL & 3PL \\
\hline PRM & *k- & $*_{\mathrm{m}}$ & $* n-$ & $*_{\mathrm{t}-}$ & $*_{\mathrm{m}}-$ & $*_{\mathrm{r}-}$ \\
\hline Bilbaa, Bokai, Korbafo & $k-$ & $m-$ & $n-$ & $t-$ & $m-$ & $l-$ \\
\hline Dela, Oenale, Rikou, Tii & ?- & $m-$ & $n-$ & $t-$ & $m-$ & $r-$ \\
\hline Dengka, Lole, Termanu, Ba'a & $?-$ & $m-$ & $n-$ & $t-$ & $m-$ & l- \\
\hline Ro'is Amarasi & $\begin{array}{l}k-\# \mathrm{~V} \\
\text { ?-\#C }\end{array}$ & $m-$ & $n-$ & $t-$ & $m-$ & $n-$ \\
\hline Nuclear Meto (all other Meto) & P- & $m-$ & $n-$ & $t-$ & $m-$ & $n$ - \\
\hline
\end{tabular}

Verbs that take agreement prefixes are usually cited throughout this comparative dictionary in the third person form. Tii and Lole verbs that take vocalic prefixes are usually cited with the third person prefix $n a$-, except where Jonker (1908) gives only a form with $i$ - or $n i$ -

In Meto nearly all verbs take agreement in most circumstances. One exception is KusaManea, for which consonant initial verbs that take consonantal prefixes are often cited without any prefix. When this is the only form of a Kusa-Manea verb I have so far collected, I give this form as it is attested in my data without any prefix.

13 In addition to the prefixes given in Table 2.11, Tamelan (2021:129) also gives 0 person (a kind of obviative used when the subject is irrelevant or not in focus) $n e$ - for Dela. 
The 1sG consonantal prefix displays allomorphy in Meto. In all known varieties it is realised as $\varnothing$ before another glottal stop; that is, ?-? $\rightarrow$ ?. In Ro'is Amarasi it has the form $k$ - before vowels and $?$ - before consonants other than glottal stop (where it is realised as Ø).

Which paradigm is used in Meto is partially determined by the phonotactic shape of the verb, partially determined by the semantics of the verb and partially lexically determined. The use of each paradigm is summarised in Table 2.13, with examples from Kotos Amarasi to illustrate. Roots that have more than two syllables, as well as vowel initial roots, take consonantal prefixes. ${ }^{14}$ Roots that begin with a consonant cluster take the vocalic set. Consonant initial roots that consist of a single foot take either set with the choice lexically specified. Verbs that have both transitive and intransitive forms are an exception. Such verbs take the vocalic set when transitive and the consonantal set when intransitive.

Table 2.13: Meto agreement according to stem shapes

\begin{tabular}{|l|l|l|l|l|}
\hline Stem Shape & & Paradigm & Kotos Example & Gloss \\
\hline$(\sigma) \sigma \sigma \sigma$ & three or more syllables & consonantal & $n$-Peus/fani & 'sneeze' \\
\hline$\# \mathrm{CC}$ & cluster initial disyllable & vocalic & $n a-m \mid$ naha & 'hungry' \\
\hline$\# \mathrm{~V}$ & vowel initial disyllable & consonantal & $n$-inu & 'drink' \\
\hline$\# \mathrm{C}$ & consonant initial disyllable & $75 \%$ consonantal & $n$-sae & 'go up' \\
\hline & & $25 \%$ vocalic & $n a$-sai & 'flow' \\
\hline$\# \mathrm{C}$ & transitive & vocalic & $n a$-tama & 'make enter' \\
\hline & intransitive & consonantal & $n$-tama & 'enter' \\
\hline
\end{tabular}

$\uparrow$ Percentages for Kotos Amarasi based on 836 verbs, of which 557 are consonant initial disyllables.

Like in Meto, there are a range of phonological, lexical and morphological factors that determine verb agreement in the Rote languages. However, unlike in Meto, there are many verbs that do not take any agreement prefix. My discussion in this section is based mainly on data from Dela, which is most clearly described (Tamelan 2021:129-138). The main conditioning factors determining verb agreement in the Rote languages are summarised in Table 2.14, illustrated with examples from Dela.

Table 2.14: Rote agreement according to stem shapes

\begin{tabular}{|l|l|l|l|l|}
\hline Stem Shape & & Paradigm & Dela Example & Gloss \\
\hline \#V & vowel initial & consonantal & n-ala & 'get' \\
\hline & & vocalic & na-iru & 'pregnant' \\
\hline \#C & & none & ale & 'chew' \\
\hline & consonant initial & vocalic & na-riu & 'bathe' \\
\hline $\mathrm{N} / \mathrm{V} \rightarrow \mathrm{V}$ & derived verb & none & mae & 'ashamed' \\
\hline & & vocalic & oe $\rightarrow$ na-oe & 'watery' \\
\hline$m a-$ & with stative $m a-$ & mae $\rightarrow$ na-mae-? & 'make ashamed' \\
\hline $\mathrm{CV}-$ & with other prefix $(\S 2.6 .6)$ & vocalic & na-sa-roi & 'good' 'lean' \\
\hline
\end{tabular}

14 Loan words in Meto also take prefixes drawn from the consonantal paradigm. 
Derived verbs nearly always take vocalic prefixes. This includes verbs derived from a nominal base and verbs derived from another verb. For verbs derived from other verbs, agreement prefixes are used with causatives, such as in Dela mae 'ashamed' $\rightarrow$ na-mae-? 'make ashamed' or reciprocals, such as Dela Pidu 'kiss' $\rightarrow$ na-Pidu 'kiss one another'. ${ }^{15}$ Similarly, verbs that take any derivational CV-prefix (see \$2.6.6) take vocalic prefixes, with the exception of verbs derived with the stative/inchoative prefix $\mathrm{ma}^{-}$, which do not take prefixes. All other verbs are divided into lexical classes. Vowel initial verbs are divided between verbs that take consonantal agreement prefixes, those that take vocalic agreement prefixes, and those that take no agreement prefixes. Consonant initial verbs are divided between those that take vocalic agreement prefixes, and those that do not take agreement prefixes.

Apart from the verbs that take agreement prefixes, there are also two verbs that have partially suppletive paradigms in some of the Rote-Meto languages. These are the reflexes of *emə 'come' and *eu 'go, to'. See the respective entries for each of these verbs for their declensions.

\subsubsection{Derivational verbal morphology}

There are two fully productive verbal derivational prefixes in the Rote languages and a number of unproductive or semi-productive prefixes. The most productive prefix in the Rote languages is $m a-$, which derives non-active verbs with a stative or process sense. In addition to productive prefixes, the Rote languages have a number of nonproductive, or semi-productive, CV-prefixes that occur with verbs. Verbs that occur with these prefixes also take vocalic agreement prefixes. The semi-productive verb prefixes that can be identified in the Rote languages are given in Table 2.15, listed roughly in order of their productivity in Dela.

Table 2.15: Rote verb prefixes ${ }^{\dagger}$

\begin{tabular}{|l|l|l|l|l|l|l|l|l|l|l|l|}
\hline Dela, Oenale & $2 V-$ & $m V-$ & $k V-$ & $s V-$ & $m b V-$ & $l V-$ & $t V-$ & $f V-$ & $p V-$ & $\eta g V-$ & $r V-$ \\
\hline Dengka & $2 V-$ & $m V-$ & $k V-$ & $s V-$ & $m b V-$ & $l V-$ & $t V-$ & $f V-$ & $p V-$ & $\eta g V-$ & $l V-$ \\
\hline Tii & $k V-$ & $m V-$ & $k V-$ & $s V-$ & $m b V-$ & $l V-$ & $t V-$ & $f V-$ & $p V-$ & $\eta g V-$ & $r V-$ \\
\hline Ba'a & $k a-$ & $m a-$ & $k a-$ & $s a-$ & $m p a-$ & $l a-$ & $t a-$ & $f a-$ & $p a-$ & $\eta g a-$ & $l a-$ \\
\hline $\begin{array}{l}\text { Termanu, Bilbaa, } \\
\text { Bokai, Korbafo }\end{array}$ & $k a-$ & $m a-$ & $k a-$ & $s a-$ & $p a-$ & $l a-$ & $t a-$ & $f a-$ & $p a-$ & $\eta a-$ & $l a-$ \\
\hline Rikou & $\varnothing$ & $m a-$ & $\varnothing$ & $s a-$ & $p a-$ & $l a-$ & $t a-$ & $f a-$ & $p a-$ & $k a-$ & $r a-$ \\
\hline
\end{tabular}

$\dagger$ The unspecified vowel in Dela, Oenale, Dengka and Tii assimilates to the quality of the vowel of the previous agreement prefix.

There are many verb roots in the Rote languages that always occur with one of these prefixes and do not occur without any verbal prefix. Thus, for instance, in Dela the verbs na-Pa-mina? 'play' and na-sa-pedo? 'fold legs' always occur with these prefixes and the hypothetical roots $\sqrt{\text { mina? }}$ and $\sqrt{ }$ pedo? do not occur independently.

15 The use of vocalic prefixes to derive verbs from a verbal base also occurs with vowel initial verbs that take consonantal prefixes, such as in $n$-inu 'drink' $\rightarrow n a-n$-inu-? 'make drink'. 
Apart from this, some of these prefixes have some use in deriving verbs from nouns or deriving alternate meanings from verbal stems. Tamelan (2021:139) describes the selection of these prefixes in Dela as partly semantically determined and partly lexically determined. The prefixes $m a$ - and $m b a$ - tend to form stative or inchoative verbs, while $2 a$ - often derives process verbs from property nominals. ${ }^{16}$ However, even among the more productive prefixes, any semantic properties are only tendencies and none are fully semantically determined.

All verbs that occur with a prefix usually also take an agreement prefix (\$2.6.5). The vowel of the derivational prefix assimilates to the quality of the vowel of the agreement prefix. Examples from Dela are given in Table 2.16. Whether this assimilation occurs in the Nuclear Rote languages is mostly a moot point as the vowel of nearly all agreement prefixes in these languages is /a/. However, this assimilation does occur with Tii 3sG $i$ - and Lole 3sG $n i$-. Examples include Tii $i$-mi-nene, and Lole $n i$-mi-nene 'hear' (see *nene), as well as lole $n i$-fi-lende 'remember' (see *farəndən).

Table 2.16: Assimilation of prefix vowel in Dela

\begin{tabular}{|l|l|l|l|l|l|}
\hline & 'play' & 'become smart' & 'remember' & 'reach' & 'scatter' \\
\hline & -?V-mina? & -mV-hine & -sV-neda & -mbV-nai & -lV-ono \\
\hline 1SG & ?u-?u-mina? & ?u-mu-hine & ?u-su-neda & ?u-mbu-nai & ?u-lu-ono \\
\hline 2SG & mu-?u-mina? & mu-mu-hine & mu-su-neda & mu-mbu-nai & mu-lu-ono \\
\hline 3SG & na-?a-mina? & na-ma-hine & na-sa-neda & na-mba-nai & na-la-ono \\
\hline 1PL.INCL & ta-?a-mina? & ta-ma-hine & ta-sa-neda & ta-mba-nai & ta-la-ono \\
\hline 1PL.EXCL/2PL & mi-?i-mina? & mi-mi-hine & mi-si-neda & mi-mbi-nai & mi-li-ono \\
\hline 3PL & ra-?a-mina? & ra-ma-hine & ra-sa-neda & ra-mba-nai & ra-la-ono \\
\hline 0 & ne-?e-mina? & ne-me-hine & ne-se-neda & ne-mbe-nai & ne-le-ono \\
\hline
\end{tabular}

In several instances, the presence of one of these prefixes on a verb in the Rote languages is due to reanalysis of a historic antepenultimate syllable that was lost in one form but retained in another, leading to alternations with and without the prefix. Once example is PRM * salili 'armpit' for which Meto has snini-f 'armpit' and na-snini 'carry slung under the armpit' while Termanu has the noun lili_bolo- $k$ 'armpit' (bolo- $k=$ 'hole') and the verb na-sa-lili 'carry slung under the armpit'.

Apart from these prefixes, the only other Rote derivational verbal morphology that features in this dictionary is the applicative/transitive suffix $-k /-2$. This suffix has the same form as the nominal $-k /-2$ suffix. That is, Tii, Lole, Ba'a, Keka, Termanu and Bokai have $-k$, while Dela-Oenale, Dengka, Korbafo, Bilbaa, Landu, Rikou and Oepao have -?. Because verbs that take this suffix are derived, they also usually take agreement prefixes, as discussed in $\S 2.6 .5$.

\footnotetext{
16 There are two formally similar prefixes in Dela: $m a$-, which productively derives non-active state or process verbs from nouns; and $m V$-, which is semi-productive. They can be distinguished, as verbs with fully productive $m a$ - do not take agreement prefixes while those with semi-productive $m V$ - always take vocalic agreement prefixes.
} 


\section{3}

\section{Historical background}

\subsection{Introduction}

In this chapter I discuss the historical background of the Rote-Meto languages. This includes the sound correspondences ( $\$ 3.2)$, the internal subgrouping of the Rote-Meto group (§3.3), and the way Rote-Meto fits into the Austronesian language family as a whole (§3.5).

Slightly earlier versions of the sound correspondences and internal subgrouping, along with detailed discussion, can be found in Edwards (2016, 2018a, 2018c). In this chapter I only discuss those parts of my analysis that have been revised since the appearance of these publications.

\subsection{Sound correspondences}

The reconstructed PRM phoneme inventory is given in Table 3.1. This is the same as the inventory posited in Edwards (2018a), with the addition of PRM *ds and ${ }^{*} w$ for both of which evidence has since been adduced. Both these proto-phonemes are marginal, with only two attestations each.

Table 3.1: Proto-Rote-Meto phoneme inventory

\begin{tabular}{|c|c|c|c|c|c|c|c|c|c|}
\hline & \multicolumn{5}{|c|}{ Consonants } & & \multicolumn{3}{|c|}{ Vowels } \\
\hline & Labial & Coronal & Palatal & Velar & Glottal & & Front & Central & Back \\
\hline Voiceless plosive & $* p$ & $* \mathrm{t}$ & & $*_{\mathrm{k}}$ & $* ?$ & High & $*_{\mathrm{i}}$ & & $* \mathrm{u}$ \\
\hline Voiced plosive & $* b$ & $* \mathrm{~d}$ & $(* d \mathrm{~d})$ & & & Mid & $* \mathrm{e}$ & $*_{0}$ & $*_{\mathrm{o}}$ \\
\hline Implosive & $* 6$ & $* d$ & & & & Low & & $* \mathrm{a}$ & \\
\hline Prenasalised plosive & $*_{\mathrm{mb}}$ & *nd & & *yg & & & & & \\
\hline Fricative & $*_{\mathrm{f}}$ & $*_{\mathrm{s}}$ & & & $* \mathrm{~h}$ & & & & \\
\hline Nasal & $*_{\mathrm{m}}$ & $*_{\mathrm{n}}$ & & $* \eta$ & & & & & \\
\hline Rhotic & & $* \mathrm{r}$ & & & & & & & \\
\hline Lateral & & $* 1$ & & & & & & & \\
\hline Glide & $(* w)$ & & & & & & & & \\
\hline
\end{tabular}


The sound correspondences between PRM and its daughter languages are summarised in $\$ 3.2 .1-\$ 3.2 .3$, in a number of tables. Where there are multiple reflexes in a single language due to unconditioned splits, the more common reflex is given first. The number of attestations of each correspondence set is given in the second last row (attestations), while the final row (irr. attestations) shows the number of irregular correspondence sets that attest the reconstructed proto-phoneme. Most of these irregular correspondences have only one or two unexpected reflexes, which are noted in the irr. from PRM field (see $§ 1.5 .3 .5)$ in the relevant entries.

Most of these correspondences have been discussed in full detail in Edwards (2018a), and I do not repeat that discussion in the following sections. Since the publication of that paper, however, my understanding of some correspondence sets is better developed, which has led to a number of minor adjustments. I discuss these adjustments in the following sections.

\subsubsection{Initial and medial consonants}

Table 3.2, Table 3.3 and Table 3.4 give the correspondences for consonants in intervocalic and foot initial position. ${ }^{1}$ Discussion of word initial consonants in trisyllables can be found in Edwards (2018a:395f). PRM *k shows several unconditioned splits, with a number of different patterns identifiable. These splits are discussed in detail in Edwards (2018a:383387 ), though that discussion is based on a slightly earlier understanding of the data. ${ }^{2}$ Similarly, PRM *d shows a two-way unconditioned split, discussed further in $§ 3.2 .1 .1$.

Table 3.2: Proto-Rote-Meto voiceless plosive correspondences

\begin{tabular}{|c|c|c|c|c|c|c|c|c|c|c|c|c|c|}
\hline Pattern & & & k-1 & $\mathbf{k}-\mathbf{2}^{\dagger}$ & k-3 & $k-4$ & k-5 & k-6 & k-7 & k-8 & k-9 & k-10 & \\
\hline PRM & *p & $* t$ & $* \mathbf{k}-+$ & & & & *-k-\# & & & & & & $* ?$ \\
\hline Dela-Oenale & $p$ & $t$ & $k$ & $?$ & $?$ & $h$ & $k$ & $?$ & $k$ & ?/Ø & $?$ & ?/Ø & $? / \varnothing$ \\
\hline Dengka & $p$ & $t$ & $k$ & $?$ & $?$ & $h$ & $k$ & $?$ & $k$ & ?/Ø & $?$ & ?/Ø & ?/Ø \\
\hline Tii & $p$ & $t$ & $k$ & $k$ & $k$ & $\varnothing$ & $k$ & $k$ & $?$ & $?$ & $?$ & $?$ & $?$ \\
\hline Lole & $p$ & $t$ & $k$ & $k$ & $k$ & $\varnothing$ & $k$ & $k$ & $?$ & $?$ & $?$ & $?$ & $?$ \\
\hline Ba'a & $\mathrm{p} / \mathrm{mp}$ & $t$ & $k$ & $k$ & $k$ & $\varnothing$ & $k$ & $k$ & $?$ & $?$ & $?$ & $?$ & $?$ \\
\hline Termanu & $p$ & $t$ & $k$ & $k$ & $k$ & $\varnothing$ & $k$ & $k$ & $?$ & $?$ & $?$ & $?$ & $?$ \\
\hline Korbafo & $p$ & $t$ & $k$ & $k$ & $k$ & $\varnothing / k$ & $k$ & $k$ & $?$ & $?$ & $?$ & $?$ & $?$ \\
\hline Bokai & $p$ & $t$ & $k$ & $k$ & $k$ & $\varnothing / k$ & $k$ & $k$ & $?$ & $?$ & $?$ & $?$ & $?$ \\
\hline Bilbaa & $p$ & $t$ & $k$ & $k$ & $k$ & $\varnothing / k$ & $k$ & $k$ & $k$ & $k$ & $k$ & $k$ & $\varnothing$ \\
\hline Landu & $p$ & $t$ & & $k / ?$ & $?$ & $\varnothing$ & $k$ & & $\mathrm{k}$ & & $k$ & $k$ & ?/Ø \\
\hline Rikou & $p$ & $t$ & $k / 2 / \varnothing$ & ?/Ø & $?$ & $\varnothing$ & $? / k$ & $? / k$ & $k / ?$ & $? / k$ & $? / k$ & $k / ?$ & ?/Ø \\
\hline Oepao & $p$ & $t$ & $k / 2 / \varnothing$ & ?/Ø & $?$ & $\varnothing$ & P/k & $\mathrm{P} / \mathrm{k}$ & & $\mathrm{P} / \mathrm{k}$ & & $k / ?$ & ?/Ø \\
\hline
\end{tabular}

\footnotetext{
1 'Foot initial' is before the stressed syllable of the disyllabic foot. A quadrisyllabic reconstruction such as *banakudu 'Morinda citrifolia' has two foot initial consonants: * $\mathrm{b}$ and $* \mathrm{k}$, respectively. Similarly, the foot initial consonant in a trisyllabic reconstruction such as *6alafo 'Mallotus philippensis' is the second consonant, in this case *1.

2 The patterns of PRM *k in Table 3.2 broadly match the discussion in Edwards (2018a:383ff), though with different labels. The cross-references between the labels in each work for initial *k- are as follows (label in this dictionary $=1$ label in Table 22 of Edwards 2018a:383): k-1 = set 2, k-2 = set 1, k-3 = set 3, k-4 = set 4. Set 5 in Edwards (2018a) is now considered irregular (marked k-irr). The patterns identified for medial *-k- in this dictionary are more fine-grained than those identified in Edwards (2018a). As a result, the two sets of patterns and their members do not exactly match. Cross-references that mostly hold for medial *-k- are as follows (label in this dictionary $=$ label in Table 27 and Table 28 of Edwards 2018a:387): k-5= set 1, k-8= set $5, \mathrm{k}-9=$ set $3, \mathrm{k}-10=$ set 4 .
} 


\begin{tabular}{|c|c|c|c|c|c|c|c|c|c|c|c|c|c|}
\hline Pattern & & & k-1 & $\mathbf{k}-2^{\dagger}$ & k-3 & k-4 & k-5 & k-6 & k-7 & k-8 & k-9 & k-10 & \\
\hline PRM & *p & $* t$ & $* k-\ddagger$ & & & & *-k-\# & & & & & & *? \\
\hline Proto-Meto & $* p$ & $*_{\mathrm{t}}$ & $* \mathrm{k}$ & $*_{\mathrm{k}}$ & $* h$ & $* \mathrm{~h}$ & $* \mathrm{k}$ & $* \mathrm{k} / * ?$ & *? & $\varnothing$ & *? & $*_{\mathrm{k}}$ & *? \\
\hline Ro'is Amarasi & $p$ & $t$ & $k$ & $k$ & $h$ & $h$ & $k$ & $k / ?$ & $?$ & $\varnothing$ & $?$ & $k$ & $?$ \\
\hline Kotos Amar. & $p$ & $t$ & $k$ & $k / ?$ & $h$ & $h$ & $k$ & $k / ?$ & $?$ & $\varnothing$ & $?$ & $k$ & $?$ \\
\hline Amanuban & $p$ & $t$ & $k$ & $k / ?$ & $h$ & $h$ & $k$ & $k / ?$ & $?$ & $\varnothing$ & $?$ & $k$ & $?$ \\
\hline Molo & $p$ & $t$ & $k$ & $k / ?$ & $h$ & $h$ & $k$ & $k / ?$ & $?$ & $\varnothing$ & $?$ & $k$ & $?$ \\
\hline Kusa-Manea & $p$ & $t$ & $k$ & $k / ?$ & $h$ & $h$ & $k$ & $k / ?$ & $?$ & $\varnothing$ & $?$ & $k$ & $?$ \\
\hline attestations & 14 & 222 & 28 & 27 & 9 & 11 & 24 & 13 & 8 & 10 & 10 & 7 & 19 \\
\hline irr. attest. & 5 & 17 & & & & & & & & & & & 7 \\
\hline
\end{tabular}

$\dagger$ Pattern k-2 has two sub-patterns: 2a, in which Nuclear Meto (all Meto varieties except Ro'is Amarasi) has *k $=k$, and $2 \mathrm{~b}$ in which Nuclear Meto has ${ }^{*} \mathrm{k}>$ ? .

$\ddagger$ There are 36 reconstructions with initial $*_{k}$ - that are ambiguous between more than one pattern due to missing reflexes in diagnostic languages: eight are ambiguous between patterns 1 and $2 \mathrm{a}(\mathrm{k}-1 / 2 \mathrm{a})$, eight are ambiguous between patterns 1,2 and 3 (k-1/2/3), and 20 are ambiguous between patterns 2 and $3(\mathrm{k}-2 / 3)$. There are also eight instances of initial *k- that are irregular and fit into no pattern.

\# There are 22 reconstructions with medial *-k- that are ambiguous between multiple patterns: seven are ambiguous between patterns 5 and $6(\mathrm{k}-5 / 6)$, nine are ambiguous between 8 and $9(\mathrm{k}-8 / 9)$, two are ambiguous between 7, 8, 9 and $10(\mathrm{k}-7 / 8 / 9 / 10)$, three are ambiguous between 6 and $9(\mathrm{k}-6 / 9)$, and one is ambiguous between 7 and 8 (k-7/8) There are also eight cases of medial *k which fit into no pattern.

\section{Table 3.3: Proto-Rote-Meto voiced plosive correspondences}

\begin{tabular}{|c|c|c|c|c|c|c|c|c|c|c|c|c|c|}
\hline Pattern & & d-1 & d-2 & & & & & & & & & & \\
\hline PRM & ${ }^{*} \mathbf{b}$ & $* d$ & & $* d s$ & $* 6$ & ${ }^{*} \mathbf{d}$ & & $* \mathbf{m b}$ & *nd & & & *ng & \\
\hline environment & & & & & & & $/ * 1, * \mathrm{rV}$ & & \#_ & $\mathrm{V} \_\mathrm{V}$ & $\partial_{-}$ & \# & V_V \\
\hline Dela-Oenale & $f$ & $r$ & $r$ & $r$ & 6 & $d$ & $d$ & $m b$ & $n d$ & $n d$ & $n d / n$ & $\eta g$ & $\eta g$ \\
\hline Dengka & $f$ & $l$ & $l$ & $l$ & $b$ & $d$ & $d$ & $m b$ & $n d$ & $n d$ & $n d / n$ & $\eta g$ & $\eta g$ \\
\hline Tii & 6 & $d$ & $r$ & $d$ & 6 & $d$ & $d$ & $m b$ & $n d$ & $n d$ & $n / n d$ & $\eta g$ & $\eta g$ \\
\hline Lole & 6 & $d$ & $l$ & $d$ & 6 & $d$ & $d$ & $m b$ & $n d$ & $n d$ & $n / n d$ & $\eta g$ & $\eta g$ \\
\hline Ba'a & $b$ & $d$ & $l$ & $d$ & $b$ & $d$ & $d$ & $m p$ & $n d$ & $n$ & $n$ & $\eta g$ & $\eta g$ \\
\hline Termanu & $b$ & $d$ & $l$ & $d$ & $b$ & $d$ & $d$ & $p$ & $n d$ & $n$ & $n$ & $\eta g$ & $\eta$ \\
\hline Korbafo & $b$ & $d$ & $l$ & $d$ & $b$ & $d$ & $d$ & $p$ & $n d$ & $n$ & $n$ & $\eta g$ & $\eta$ \\
\hline Bokai & $b$ & $d$ & $l$ & $d$ & $b$ & $d$ & $d$ & $p$ & $l$ & $n$ & $n$ & $\eta$ & $\eta$ \\
\hline Bilbaa & $b$ & $d$ & $l$ & $d$ & $b$ & $d$ & $d$ & $p$ & $l$ & $n$ & $n$ & $\eta$ & $\eta$ \\
\hline Landu & $b$ & $d$ & $r$ & $d$ & $b$ & $d$ & $d$ & $p$ & $n d$ & $n d$ & $n$ & $k$ & $k$ \\
\hline Rikou & $b$ & $d$ & $r$ & $d$ & $b$ & $d$ & $d$ & $p$ & $r$ & $n d$ & $n$ & $k$ & $k$ \\
\hline Oepao & $b$ & $d$ & $r$ & $d$ & $b$ & $d$ & $d$ & $p$ & $r$ & $r$ & $n$ & $k$ & $k$ \\
\hline Proto-Meto & $*_{\mathrm{f}}$ & $*_{\mathrm{n}}$ & $* d$ & $* d$ & $* b$ & $* d$ & $*_{\mathrm{n}}$ & $* \mathrm{p}$ & $*_{\mathrm{r}}$ & $*_{\mathrm{r}}$ & $*_{\mathrm{r}}$ & $* \eta$ & $* y$ \\
\hline Ro'is Amarasi & $f$ & $n$ & $r$ & $r$ & $b$ & $r$ & $n$ & $p$ & $r$ & $r$ & $r$ & $k$ & $k$ \\
\hline Kotos Amarasi & $f$ & $n$ & $r$ & $r$ & $b$ & $r$ & $n$ & $p$ & $k$ & $k$ & $k$ & $k$ & $k$ \\
\hline Amanuban & $f$ & $n$ & $l$ & $l$ & $b$ & $l$ & $n$ & $p$ & $k$ & $k$ & $k$ & $k$ & $k$ \\
\hline Molo & $f$ & $n^{\dagger}$ & $l$ & $l$ & $b$ & $l$ & $n$ & $p$ & $k$ & $k$ & $k$ & $k$ & $k$ \\
\hline Kusa-Manea & $f$ & $n$ & $r$ & $r$ & $b$ & $r$ & $n$ & $p$ & $k$ & $k$ & $k$ & $k$ & $k$ \\
\hline attestations & 19 & 20 & 17 & 2 & 77 & 90 & 4 & 76 & 16 & 17 & 4 & 33 & 32 \\
\hline irr. attestations & 18 & 2 & & & 5 & 5 & 1 & 4 & 7 & 3 & & 5 & 2 \\
\hline
\end{tabular}

$\dagger$ Molo and other varieties of North Meto have ${ }^{*} \mathrm{n}>l / l V_{-}$. See $\$ 3.3 .4$ for details. 
ROTE-METO COMPARATIVE DICTIONARY

Table 3.4: Proto-Rote-Meto fricative and sonorant correspondences

\begin{tabular}{|c|c|c|c|c|c|c|c|c|c|c|c|}
\hline PRM & $* f$ & $*_{\mathbf{S}}$ & $* h$ & & & $* m$ & $* n$ & $* \boldsymbol{n}$ & $* 1$ & $* \mathbf{r}$ & $* \mathbf{k l}$ \\
\hline environment & & & \# & V_V & a_a & & & & & & \\
\hline Dela-Oenale & $f$ & $s$ & $h$ & $\varnothing$ & $\varnothing$ & $m$ & $n$ & $n$ & $l$ & $r$ & $k$ \\
\hline Dengka & $f$ & $s$ & $h$ & $\varnothing$ & $\varnothing$ & $m$ & $n$ & $n$ & $l$ & $l$ & $k$ \\
\hline Tii & $f$ & $s$ & $h$ & $?$ & $\varnothing$ & $m$ & $n$ & $n$ & $l$ & $r$ & $k$ \\
\hline Lole & $f$ & $s$ & $h$ & $?$ & $\varnothing$ & $m$ & $n$ & $n$ & $l$ & $l$ & $k$ \\
\hline Ba'a & $f$ & $s$ & $h$ & $?$ & $\varnothing$ & $m$ & $n$ & $n$ & $l$ & $l$ & $k$ \\
\hline Termanu & $f$ & $s$ & $h$ & $?$ & $\varnothing$ & $m$ & $n$ & $n$ & $l$ & $l$ & $k$ \\
\hline Korbafo & $f$ & $s$ & $h$ & $?$ & $\varnothing$ & $m$ & $n$ & $n$ & $l$ & $l$ & $k$ \\
\hline Bokai & $f$ & $s$ & $h$ & $?$ & $\varnothing$ & $m$ & $n$ & $n$ & $l$ & $l$ & $k$ \\
\hline Bilbaa & $f$ & $s$ & $h$ & $\varnothing$ & $\varnothing$ & $m$ & $n$ & $n$ & $l$ & $l$ & $k$ \\
\hline Landu & $f$ & $s$ & $h$ & $\varnothing$ & $\varnothing$ & $m$ & $n$ & $n$ & $l$ & $r$ & \\
\hline Rikou & $f$ & $s$ & $h$ & $\varnothing$ & $\varnothing$ & $m$ & $n$ & $n$ & $l$ & $r$ & $?$ \\
\hline Oepao & $f$ & $s$ & $h$ & $\varnothing$ & $\varnothing$ & $m$ & $n$ & $n$ & $l$ & $r$ & \\
\hline Proto-Meto & $* f$ & $*_{\mathrm{S}}$ & $* \mathrm{~h} / \varnothing$ & $* \mathrm{~h} / \varnothing$ & $* \mathrm{~h}$ & $*_{\mathrm{m}}$ & $* \mathrm{n}$ & $* \mathrm{y} / *_{\mathrm{n}}$ & $* \mathrm{n}$ & $* \mathrm{n}$ & $*_{\mathrm{k} l}$ \\
\hline Ro'is Amarasi & $f$ & $s$ & $h / \varnothing$ & $h / \varnothing$ & $h$ & $m$ & $n$ & $k / n$ & $n$ & $n$ & $k r$ \\
\hline Kotos Amarasi & $f$ & $s$ & $h / \varnothing$ & $h / \varnothing$ & $h$ & $m$ & $n$ & $k / n$ & $n$ & $n$ & $k r$ \\
\hline Amanuban & $f$ & $s$ & $h / \varnothing$ & $h / \varnothing$ & $h$ & $m$ & $n$ & $k / n$ & $n$ & $n$ & $k l$ \\
\hline Molo & $f$ & $s$ & $h / \varnothing$ & $h / \varnothing$ & $h$ & $m$ & $n^{*}$ & $k / n$ & $n$ & $n$ & $k l$ \\
\hline Kusa-Manea & $f$ & $s$ & $h / \varnothing$ & $h / \varnothing$ & $h$ & $m$ & $n$ & $k$ & $n$ & $n$ & \\
\hline attestations & 110 & 183 & 49 & 23 & 6 & 124 & 155 & 10 & 214 & 99 & 3 \\
\hline irr. attestations & 8 & 5 & 13 & 3 & 1 & 3 & 9 & 6 & 15 & 11 & \\
\hline
\end{tabular}

$\uparrow \mathrm{PRM} * \mathrm{n}$ underwent a split in Proto-Meto between ${ }^{*} \mathrm{y}$ and ${ }^{*} \mathrm{n}$ with subsequent Proto-Meto ${ }^{*} \mathrm{y}>k$ and ${ }^{*} \mathrm{n}>n$. See $\S 3.3 .4$ for more details.

$\$$ Molo and other varieties of North Meto have $*_{n}>l / l V_{-}$. See $\$ 3.3 .4$ for details.

\subsubsection{PRM *d}

In Edwards (2018a) I identified two correspondence sets for PRM *r. In this dictionary I now reconstruct $* \mathrm{~d}$ for the second of these sets. Thus, I identify two correspondence sets that attest PRM *d. On the basis of current evidence, I propose that these two correspondence sets are due to an unconditioned split of PRM *d. However, it may be that these correspondence sets attest different proto-phonemes. Throughout this dictionary the first set is not indicated, while the second set is marked as d-2 in the pattern field.

The first $* \mathrm{~d}$ correspondence set has 19 unambiguous attestations at the level of PRM. It is retained as $d$ or $d$ in the Nuclear Rote languages and has changed in West-RoteMeto. The first step was $* \mathrm{~d}>r$, which is still the reflex in Dela-Oenale, followed by $*_{\mathrm{r}}>l$ as retained in Dengka, with subsequent $* 1>n$ in Meto.

Of the 19 unambiguous PRM attestations of this set, 13 are word initial, and all except one are retentions of PMP * $\mathrm{d}^{3}$ There are only six word medial instances of this correspondence set, of which four trace back to PMP $* \mathrm{j}$ and one to PMP ${ }^{*} \mathrm{z}^{4}$

3 The only instance of initial PRM *d-1 that is not a reflex of PMP *d is PRM *dene 'kapok tree', which is clearly connected with Helong deyen 'kapok tree'. This may be a borrowing from pre-Helong into PRM, though borrowing in the opposite direction cannot currently be ruled out.

4 The only instance of medial PRM *d that does not come from PMP is that in *soda-k 'space'. This reconstruction has irregular medial $* \mathrm{~d}>d$ in West Rote, and an alternate reconstruction would be $*$ soda-k with irregular $* \mathrm{~d}>(* \mathrm{~d}>* \mathrm{r}>* 1)>n$ in Meto. 
The second $* \mathrm{~d}$ correspondence set has 15 unambiguous PRM attestations. For this set $* \mathrm{~d}$ changes in all languages. It is reflected as $r$ in those languages that have this consonant (Dela-Oenale, Landu, Rikou, Oepao, Amarasi, and Kusa-Manea) with subsequent ${ }^{*} r>$ $l$ in other languages. This set is the one I conflated with PRM *r in Edwards (2018a), but the need to reconstruct Proto-Meto *d to account for the reflexes of this set in Meto (see Table 3.3 and $\$ 3.3 .4$ ) means that $*$ d should also be reconstructed to PRM to avoid positing $\mathrm{PRM} * \mathrm{r}>$ Proto-Meto $* \mathrm{~d}$ - an unlikely sound change.

There are only four instances of this set that are known to be inherited from PMP: *dayday $>$ PRM *dada 'warm near a fire', *pandak > PRM *mbada-k 'short in height', *yadas > PRM *ygadas 'palate, gills', and *duyun $>$ PRM *dui 'dugong'. Of these, the first two attest an earlier nasal-stop cluster. This suggests that this second $* \mathrm{~d}$ correspondence set may have been a nasal-obstruent combination/cluster in PRM. Given that a prenasalised plosive *nd is well supported by different correspondence sets, the best candidate if this analysis were taken would be PRM *nr. ${ }^{5}$

However, three of the four instances of the second set of PRM *d that can be traced back to PMP show additional irregularities: *dandan $>*$ dada has irregular initial $* \mathrm{~d}>$ $*_{\mathrm{d}} \mathrm{f}($ expect $* \mathrm{~d}=* \mathrm{~d}),{ }^{*}$ pandak $>*_{\text {mbada-k has }}^{*} \mathrm{p}>*^{*} \mathrm{mb}($ expect $* \mathrm{p}>* \mathrm{~h})$, and $*_{\text {yadas }}$ $>*_{\text {ygadas has minority } * \eta}>*^{*} \mathrm{gg}$ (against usual $*_{\mathrm{y}}>*_{\mathrm{n}}$ ). Given these irregularities, I prefer to propose that these instances of $\mathrm{PRM} * \mathrm{~d}$ are also irregular rather than positing that they descend from another PRM sound. As suggested in Edwards (2018a:379), these irregularities may be attributable to these words not being normal inheritances in Meto and/or the Rote languages.

An alternate approach to positing a nasal-stop cluster or an unconditioned split would be to posit an extra PRM phoneme. ${ }^{6}$ If this approach were taken, the most likely candidate is probably retroflex $* \mathrm{~d}$ as retroflex consonants are regionally attested. Kisar, to the east of Timor, has retroflex / $t$ / (Christensen and Christensen 1992), while Dhao, spoken on the island of the same name just to the west of Rote, has /d/. In Dhao /d/ is a 'slightly retroflexed, lightly articulated alveolar affricate' (Grimes 2010:256), thus [dz] or [ḍ].

While further evidence, perhaps from other languages of the region, may lead me to reconstruct a nasal-stop cluster and/or an additional PRM proto-phoneme for the second PRM *d set, based on current evidence I attribute the two *d correspondence sets to an unconditioned split.

\subsubsection{PRM *ds}

One difference between the system proposed here and that in Edwards (2018a) is that I now reconstruct PRM *dy, though there are only two reconstructions I am confident attest this proto-phoneme: *fudzo 'foam' (from PMP *bujəq) and *nadza-k 'name'

\footnotetext{
5 If it is accepted that the second set attesting PRM *d was *nr, it may have even been the case that this was a separate prenasalised trill phoneme, as is attested in several languages of Oceania and indeed has been reconstructed for Proto-Oceanic (Lynch et al. 2002:64).

6 It is, of course, also possible that some instances of the second PRM *d correspondence set are from an earlier nasal-stop cluster and some are from another PRM phoneme and/or PRM *d.
} 
(from PMP * yajan). The evidence for reconstructing medial *ds in these forms comes both from the distinct reflexes of the medial consonant and raising, or palatalisation, of the following vowel in some reflexes.

The first piece of evidence in favour of reconstructing $* d z$ is its reflexes. The reflexes of $* d z$ overlap with those of $* d, * d$ and $*$ r, but are not identical to any. The reflexes of $* d$, $* \mathrm{~d},{ }^{*} \mathrm{~d}$ and ${ }^{*} \mathrm{r}$ in indicative languages are summarised in Table 3.3.

Table 3.5: Proto-Rote-Meto voiced coronal obstruent correspondences

\begin{tabular}{|l|l|l|l|l|l|l|}
\hline \multirow{5}{*}{ West Rote } & PRM & *ds & *d & *d & *d & ${ }^{*} \mathbf{r}$ \\
\cline { 2 - 8 } & Dela-Oenale & $r$ & $r$ & $r$ & $d$ & $r$ \\
\cline { 2 - 7 } & Dengka & $l$ & $l$ & $l$ & $d$ & $l$ \\
\hline \multirow{5}{*}{ Nuclear Rote } & Tii & $d$ & $d$ & $r$ & $d$ & $r$ \\
\cline { 2 - 8 } & Lole & $d$ & $d$ & $l$ & $d$ & $l$ \\
\cline { 2 - 7 } & Termanu & $d$ & $d$ & $l$ & $d$ & $l$ \\
\cline { 2 - 7 } & Rikou & $d$ & $d$ & $r$ & $d$ & $r$ \\
\hline \multirow{3}{*}{ Meto } & Amarasi & $r$ & $n$ & $r$ & $r$ & $n$ \\
\cline { 2 - 7 } & Molo & $l$ & $n$ & $l$ & $l$ & $n$ \\
\hline
\end{tabular}

While the reflexes of $* \mathrm{~d} 3$ and $* \mathrm{~d}$ are the same in the Rote languages, they are different in Meto. Similarly, while the reflexes of $* d z$ and $* d$ are the same in Nuclear Rote and Meto, they are different in West Rote. In no case can the reflexes of *ds be conflated with those of another proto-phoneme. This indicates that $* d s$ was distinct from other protophonemes in PRM.

The second piece of evidence in favour of PRM *ds is that it often triggers raising of a following vowel. Regarding *fudzo 'foam', the normal reflex of final *ə in DelaOenale, Dengka and Meto for other words is $a$. However, reflexes of *fudzo 'foam' in all these languages except the Kusa-Manea variety of Meto have final $e$ : Dela-Oenale na-fu fure, Dengka na-fu fule, Kotos Amarasi P|furi-f (from intermediate **?|fure), Molo $<$ fule $>$, but Kusa-Manea fa fura-f 'foam'?

A similar, though not identical, situation holds regarding reflexes of *nadza-k 'name'. This reconstruction only has direct reflexes in the Rote languages: ${ }^{8}$ Dengka nala-?, DelaOenale nara-?, Tii, Lole nade- $k$, other Rote nade- $k$ or nade-? 'name'. In this case final $*_{\mathrm{a}}>a$ is expected in all languages, but Rote languages apart from Dela-Oenale and Dengka have unexpected $* \mathrm{a}>e$.

The unexpected vowel changes in each of PRM * fudzo 'foam' and *nadza-k 'name' can be explained as cases of sporadic raising of final $* a>e$ after a palatal consonant - a case of assimilation. This change is also attested in several PRM forms inherited from PMP: *añam $>$ *ane 'braid', *suja $>$ *sure-k 'caltrop, sword', and *utaña $>$ *tane $\sim$ *tana 'ask'.

\footnotetext{
7 The normal reflex of PMP *ə before *q in PRM is *e. Thus, *bujəq $>*$ fudzə also requires irregular *ə $* *$ *. Kusa-Manea with final /a/ provides the evidence for PRM *ə, as final Kusa-Manea $a$ is a regular reflex of either final *ə or *a.

8 Meto kana- $f$ 'name, clan' is a borrowing from Helong yala 'name, clan' (also ultimately from PMP * yajan). The evidence for the Meto form being a borrowing from Helong comes both from irregular initial $* \eta>k$ and the identical semantic expansion from 'name' to include 'clan'.
} 
The main difference between palatalisation in these forms and that in *fudzo 'foam' and *nadza-k 'name' is that for these two latter reconstructions palatalisation of the vowel and subsequent de-palatalisation of the consonant occurred after the break-up of PRM.

Reconstruction of medial *d ${ }^{*}$ in *fudzo 'foam' and *nadza-k 'name' thus accounts for both the medial consonants and provides an explanation for otherwise unexpected final mid-high vowels in some languages. More complete discussion of these forms can be found in the relevant entries for these reconstructions.

\subsubsection{PRM *W}

Another difference between the proto-phonemes posited here and in Edwards (2018a) is the addition of the glide ${ }^{*} \mathrm{w}$. Only two forms have been identified with this protophoneme: *wani 'honey bee' and *wadi 'younger sibling'.

\subsubsection{Final consonants}

Eight consonants can be reconstructed in word final position in PRM: ${ }^{*},{ }^{*} \mathrm{k},{ }^{*} \mathrm{~m},{ }^{*} \mathrm{n}$, $*_{\mathrm{r}}, *_{\mathrm{h}}, *_{\mathrm{r}}$ and $*_{\mathrm{s}}$. Of these consonants, *t and $*_{\mathrm{s}}$ in particular may be analysable as nominalising suffixes for some forms in the daughter languages, and thus also in PRM. In general, Dela-Oenale, Dengka and Meto preserve word final consonants in the most forms, while they are usually lost in other languages. The correspondence sets attesting word final consonants in PRM are given in Table 3.6.

Table 3.6: Proto-Rote-Meto word final consonant correspondences

\begin{tabular}{|c|c|c|c|c|c|c|c|c|}
\hline PRM & $* t$ & ${ }^{*} \mathbf{k}$ & $* \mathbf{m}$ & $* n$ & $* \mathbf{r}$ & $* h$ & $* ?$ & $*_{\mathbf{S}}$ \\
\hline Dela-Oenale & $t / \varnothing$ & $\varnothing / \mathrm{k}$ & $\varnothing$ & $\varnothing / n$ & $r$ & $\varnothing$ & $\varnothing / 2$ & $s / \varnothing$ \\
\hline Dengka & $t / \varnothing$ & $\varnothing$ & $\varnothing$ & $\varnothing / n$ & $\varnothing / l$ & $\varnothing$ & $\varnothing$ & $s / \varnothing$ \\
\hline Ba'a, Tii, Lole & $\varnothing$ & $\varnothing$ & $\varnothing$ & $\varnothing / n$ & $\varnothing$ & $\varnothing$ & $\varnothing$ & $s / \varnothing$ \\
\hline Termanu & $\varnothing$ & $\varnothing$ & $\varnothing$ & $\varnothing / n$ & $\varnothing$ & $\varnothing$ & $\varnothing$ & $s / \varnothing$ \\
\hline Korbafo, Bokai, Bilbaa & $\varnothing$ & $\varnothing$ & $\varnothing$ & $\varnothing$ & $\varnothing$ & $\varnothing$ & $\varnothing$ & $\varnothing$ \\
\hline Rikou, Oepao & $\varnothing$ & $\varnothing$ & $\varnothing$ & $\varnothing$ & $\varnothing$ & $\varnothing$ & $\varnothing$ & $s / \varnothing$ \\
\hline Proto-Meto & $* t / \varnothing$ & $* \mathrm{k} / * ?$ & $* \mathrm{~m}$ & $* \mathrm{n}$ & $\varnothing / * \mathrm{n}$ & $* \mathrm{~h}$ & *? & $* \mathrm{~s}$ \\
\hline Meto & $t / \varnothing$ & $k / ?$ & $m$ & $n$ & $\varnothing / n$ & $h$ & $?$ & $s$ \\
\hline regular attestations & 26 & 6 & 12 & 19 & 9 & 6 & 3 & 40 \\
\hline irregular attestations & & 3 & 1 & 5 & 1 & & & 7 \\
\hline
\end{tabular}

\subsubsection{Vowels}

Table 3.7 gives the sound correspondences for vowels and glides in the PRM languages in penultimate and final syllables. Vowels in antepenultimate and pre-antepenultimate position are often deleted in daughter languages due to the preference among the RoteMeto languages for disyllabic words. 
ROTE-METO COMPARATIVE DICTIONARY

Table 3.7: Proto-Rote-Meto vowel correspondences (penultimate and final syllables)

\begin{tabular}{|c|c|c|c|c|c|c|c|c|c|c|}
\hline PRM & $* \mathbf{i}$ & $* \mathbf{e}$ & $* \mathbf{a}$ & $* \mathbf{a}$ & $* a$ & *o & *o & $*_{0}$ & $* \mathbf{u}$ & *wa \\
\hline environment & & & & /_\# & 1_\# & 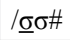 & $/ \sigma \underline{\sigma} \#$ & & & \\
\hline Dela-Oenale & $i$ & $e$ & $a$ & $a$ & a/e & e/o & a/e & $o$ & $u$ & $o$ \\
\hline Dengka & $i$ & $e$ & $a$ & $a$ & $a / e$ & e/o & $a / e$ & $o$ & $u$ & $o$ \\
\hline Tii & $i$ & $e$ & $a$ & $a$ & $a$ & e/o & $e$ & $o$ & $u$ & $f a$ \\
\hline Lole & $i$ & $e$ & $a$ & $a$ & $a$ & e/o & $e$ & $o$ & $u$ & $f a$ \\
\hline Ba'a & $i$ & $e$ & $a$ & $a$ & $a$ & e/o & $e$ & $o$ & $u$ & $f a$ \\
\hline Termanu & $i$ & $e$ & $a$ & $a$ & $a$ & e/o & $e$ & $o$ & $u$ & $f a$ \\
\hline Korbafo & $i$ & $e$ & $a$ & $a$ & $a$ & elo & $e$ & $o$ & $u$ & $f a$ \\
\hline Bokai & $i$ & $e$ & $a$ & $a$ & $a$ & e/o & $e$ & $o$ & $u$ & $f a$ \\
\hline Bilbaa & $i$ & $e$ & $a$ & $a$ & $a$ & e/o & $e$ & $o$ & $u$ & $f a$ \\
\hline Landu & $i$ & $e$ & $a$ & $a$ & $a$ & e/o & $e$ & $o$ & $u$ & $f a$ \\
\hline Rikou & $i$ & $e$ & $a$ & $a$ & $a$ & e/o & $e$ & $o$ & $u$ & $f a$ \\
\hline Oepao & $i$ & $e$ & $a$ & $a$ & $a$ & e/o & $e$ & $o$ & $u$ & $f a$ \\
\hline Proto-Meto & $*_{\mathrm{i}}$ & $*_{\mathrm{e}}$ & $* \mathrm{a}$ & $* \mathrm{a}$ & $*_{\mathrm{e}}$ & $* \mathrm{e}$ & $* \mathrm{a}$ & $*_{0}$ & $* u$ & $*_{0}$ \\
\hline Ro'is Amarasi & $i$ & $e$ & $a$ & $a$ & $e$ & $e$ & $a$ & $o$ & $u$ & $o$ \\
\hline Kotos Amarasi & $i$ & $e$ & $a$ & $a$ & $e$ & $e$ & $a$ & $o$ & $u$ & $o$ \\
\hline Amanuban & $i$ & $e$ & $a$ & $a$ & $e$ & $e$ & $a$ & $o$ & $u$ & $o$ \\
\hline Molo & $i$ & $e$ & $a$ & $a$ & $e$ & $e$ & $a$ & $o$ & $u$ & $o$ \\
\hline Kusa-Manea & $i$ & $e$ & $a$ & $a$ & $e$ & $e$ & $a$ & $o$ & $u$ & $o$ \\
\hline attestations & 372 & 371 & 384 & 229 & 28 & 10 & 33 & 295 & 494 & 2 \\
\hline irr. attestations & 20 & 20 & 14 & 21 & 4 & 2 & 10 & 8 & 19 & \\
\hline
\end{tabular}

While PRM * o is well-supported on the basis of the bottom-up evidence in final syllables, there is much less support for it in penultimate syllables, with only 12 putative cases identified. Firstly, there are five words in which PRM *o undergoes a split between $e$ and $o$ in daughter languages: *dəma 'drown', *səru 'meet', *səu 'Alstonia villosa', *təlo 'egg' and *dəmbə 'dip'.

Secondly, there are five words that have $*_{\partial}>e$ in all daughter languages. Nonetheless, penultimate schwa can be detected as several Rote languages undergo otherwise unexpected *nd $>n$ for the consonant following schwa (see Table 3.3). These five words are: *əndi 'bring', *farəndən 'thoughts', *həndi 'finish', *kənda 'close' and *londe 'still (water)'.

Apart from these 10 words, I have also reconstructed initial schwa in the root forms of *omə 'come' and *ou 'go'. The initial vowel of these verbs shows irregularities in the 1sG, $2 \mathrm{SG}$ and 1 PL/1PL.EXCL verb forms, which appear to be a result of loss and/or coalescence of the initial vowel with the historic vowel of the agreement prefixes ( $\$ 2.6 .5)$. The $3 \mathrm{sG}$, 3 PL and 1 PL.INCL forms of these verbs have regular *ə $>e$ in most daughter languages.

As discussed in $\$ 3.5 .1 .2$, PMP * $ə$ usually became *e in PRM, and we can propose that the change of $*_{\partial}>e$ in penultimate syllables was a change in progress that was not completed until after the break-up of the proto-language. It is likely that there were many more instances of penultimate schwa in PRM than can be reconstructed on the basis of bottom-up evidence from the modern-day languages. 


\subsection{Internal sub-grouping}

The internal sub-grouping of the Rote-Meto languages is complex, with several overlapping and conflicting sets of sound changes. On the basis of shared sound changes, three subgroups can be identified within Rote-Meto: West Rote-Meto (Dela-Oenale, Dengka, and Meto), Nuclear Rote (all other Rote languages) and Central East Rote and Meto (Meto and all Rote languages except Dela-Oenale, Dengka, Tii and Lole). The sound changes that would define each subgroup are summarised in Table 3.8.

Table 3.8: Subgrouping within Rote-Meto

\begin{tabular}{|c|c|c|}
\hline West Rote-Meto & Nuclear Rote & Central East Rote and Meto \\
\hline$* \mathrm{~b}>f$ & $*_{\partial}>e / \sigma \underline{\sigma} \#$ & $* m b>m p>p$ \\
\hline$* \mathrm{~d}>r$ & $*_{\mathrm{wa}}>f a$ & $* \mathrm{nd}>r$ \\
\hline$*_{\partial}>a / \sigma \underline{\sigma} \#$ & $* \mathrm{~d} / \mathrm{d} / \mathrm{d} \mathrm{s}>d \sim d$ & $* \eta \mathrm{g}>\eta>k$ \\
\hline$* \mathrm{a}>a, e / \#$ & & \\
\hline${ }^{*} \mathrm{k}>k, h / \#$ & & \\
\hline${ }^{*}$ wa $>o$ & & \\
\hline
\end{tabular}

Meto shares a number of sound changes with both West Rote and Central East Rote. As discussed in Edwards (2018a), I propose that this is because it has shared a period of common development with each. However, it is likely that the period of common development Meto shared with each group was not of the same kind. While Meto and West-Rote probably descend from a single ancestral language, Meto and East-Rote appear to descend from a common ancestral speech community comprised of two languages: pre-Central East Rote and pre-Meto. Thus, while West Rote-Meto is a 'classic' subgroup in which the defining sound changes arose once in a language ancestral to all daughters, the Central East Rote and Meto grouping is a result of contact and convergence between its members.

My current hypothesis is that the first split was between West Rote-Meto and Nuclear Rote. After this initial split, pre-Meto split from West Rote and entered into a period of intense contact with Central East Rote, which resulted in common development. During the initial period of this contact, pre-Meto and pre-Central East Rote were probably mutually intelligible.

Two different visual representations of the relationships of the Rote-Meto languages are given as a simplified tree diagram in Figure 3.1 and a wave diagram in Figure 3.2. As discussed in Edwards (2018a), I do not think these models are incompatible. Changes can diffuse between proto-languages and can thus be used to define the descendants of such a proto-language. Instead, each model captures and emphasises different aspects of the social history of the Rote-Meto languages. A complete tree diagram of the RoteMeto languages is shown in Figure 3.3. 


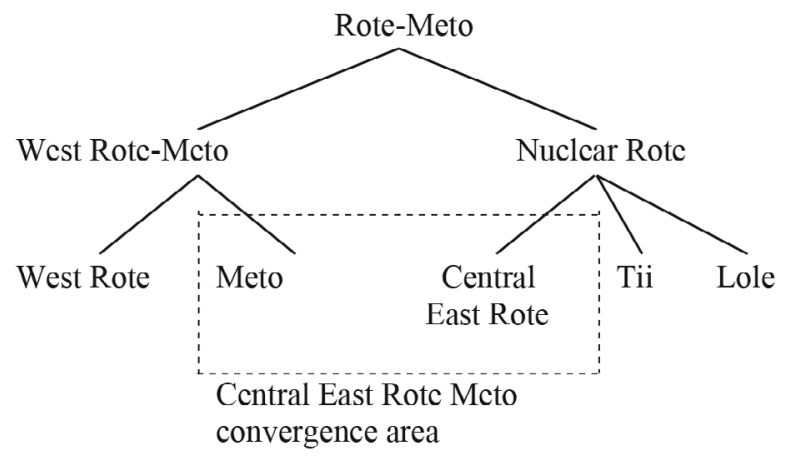

Figure 3.1: Simplified Rote-Meto family tree

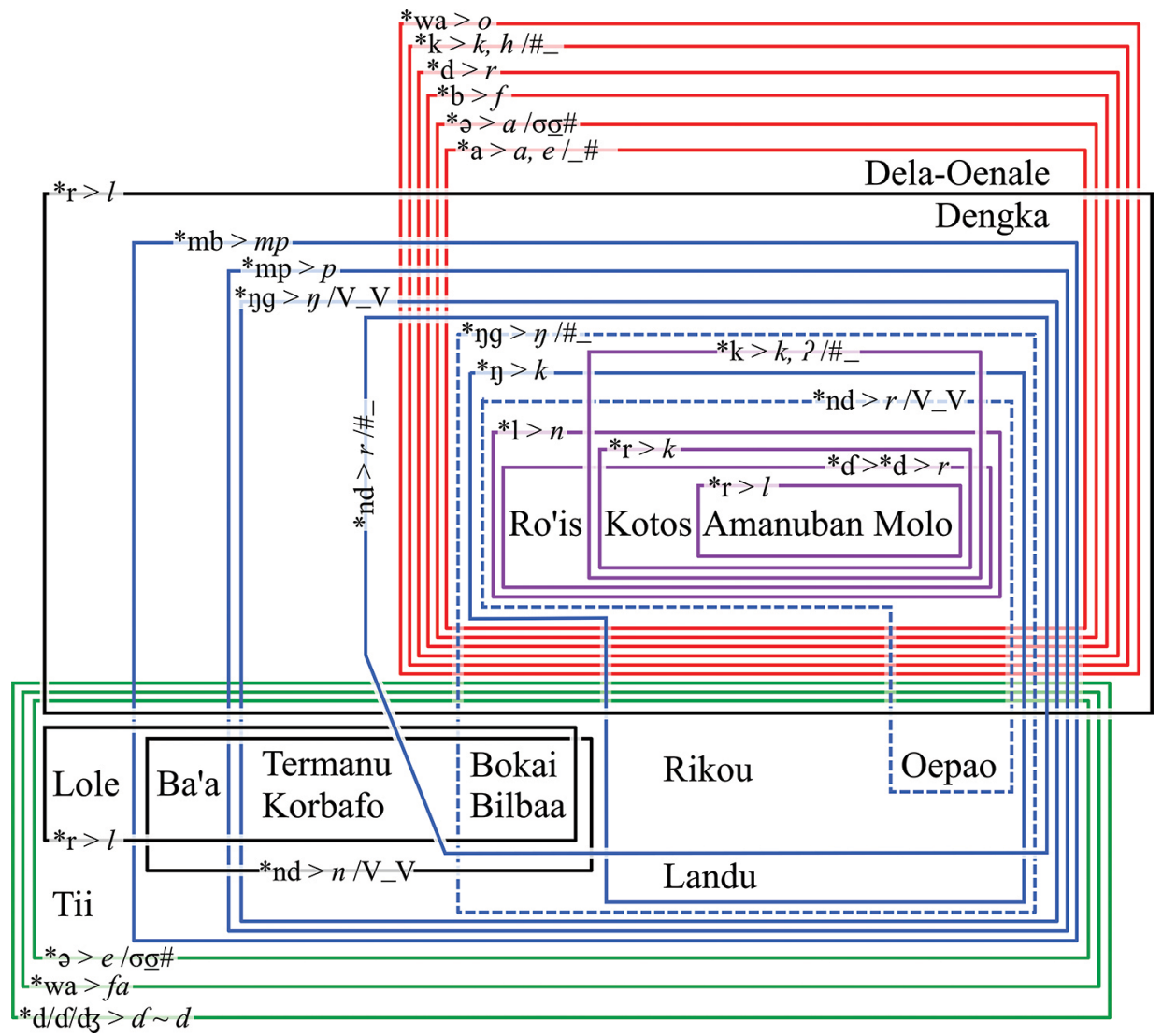

Figure 3.2: Rote-Meto wave diagram 
Figure 3.2 is a wave diagram showing the main sound changes that affect individual languages. Red lines are for changes that define West Rote-Meto, blue lines are those shared between Central East Rote and Meto (dashed lines are used only to indicate more clearly which isoglosses affect which languages), green lines indicate Nuclear Rote, and purple lines are for the changes that affect only Meto. Black lines are used for other changes.

When one change occurred before another, it is overlayed before the secondary change. Thus, for instance, ${ }^{*} \mathrm{mb}>m p$ in Central East Rote and Meto occurred before ${ }^{*} \mathrm{mp}>p$ in these languages. Likewise, ${ }^{*} \mathrm{r}>l$ happened after $*_{\mathrm{nd}}>r / \#_{\text {in }}$ in Bokai and Bilbaa. ${ }^{9}$ In other cases the order in which the lines are placed is essentially arbitrary, with visual clarity being the main consideration.

The wave diagram in Figure 3.2 shows visually that the sound changes that are shared between Meto and central East Rote languages (represented by blue lines) fully encircle the Meto languages but only encircle some of the Rote languages, and do so in a more unordered fashion. This indicates that the direction of influence was mostly from Meto. Additional evidence for this probably comes from the fact that the change of $* y>k$ must have occurred after the change of $* \mathrm{k}>k$, ? /\#, which only affects some varieties of Meto. Thus. $*_{y}>k$ must have spread between these languages after the break-up of Proto-Meto. This is discussed in more detail in $§ 3.3 .4$.

A complete tree diagram showing the proposed internal structure of the Rote-Meto languages is given in Figure 3.3, along with sound changes that define each node. Due to the complexity involved, changes that likely spread by diffusion are only represented for the Rote languages. Of these changes, it is likely that diffusion happened between proto-languages in some cases. Thus, for instance $*_{n}>n / \mathrm{V} \_\mathrm{V}$ and ${ }^{*} \mathrm{r}>l$ may have arisen once in Proto-Central Rote, from which they then spread into Proto-Bokai-Bilbaa, or vice versa.

9 The change $*_{r}>l$ is shown twice in Figure 3.5 as it occurred at different times in relation to $*_{\text {nd }}>r / \#$ in different languages. In Bokai and Bilbaa *nd $>r / \#$ _occurred before $* \mathrm{r}>l$, thus resulting in a merger of $* \mathrm{nd} / *_{\mathrm{r}}>l$ word initially. In Meto, on the other hand, ${ }^{*} \mathrm{r}>l$ occurred before ${ }^{*}$ nd $>r$ and these proto-phonemes did not merge. 


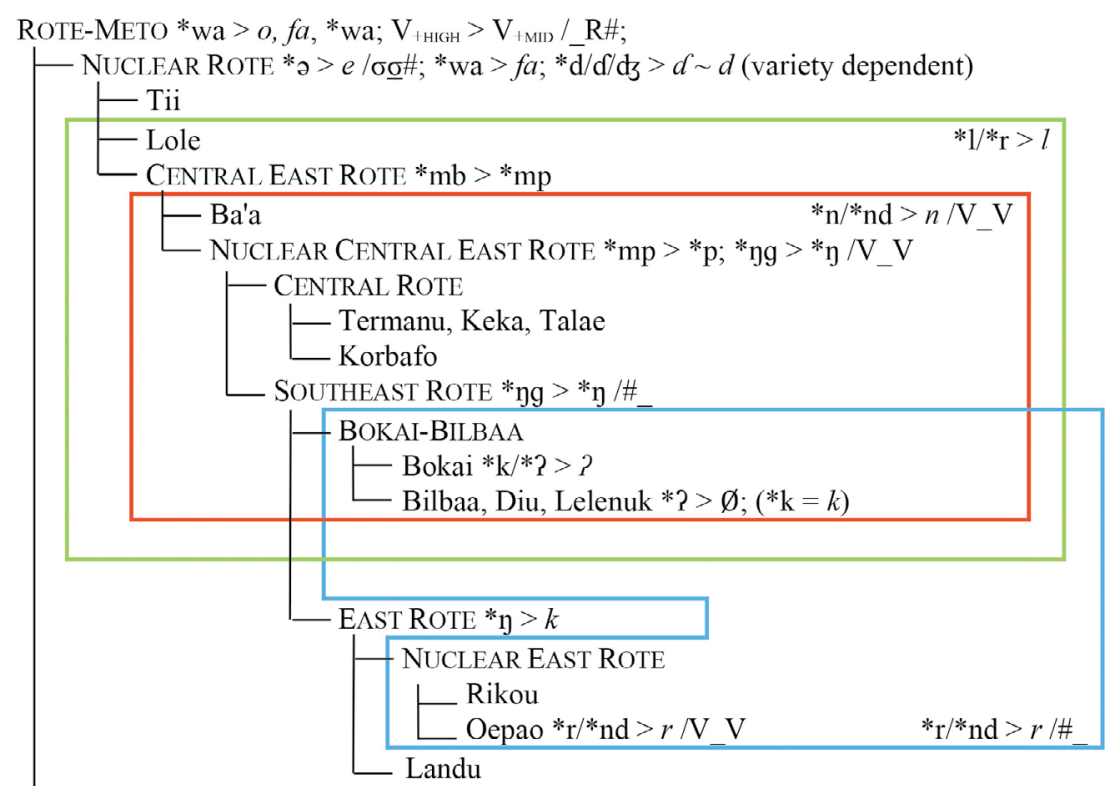

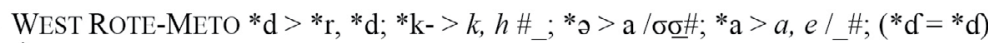

- Dela-Oenale

— DENGKA-METO *1/*r $>* 1$

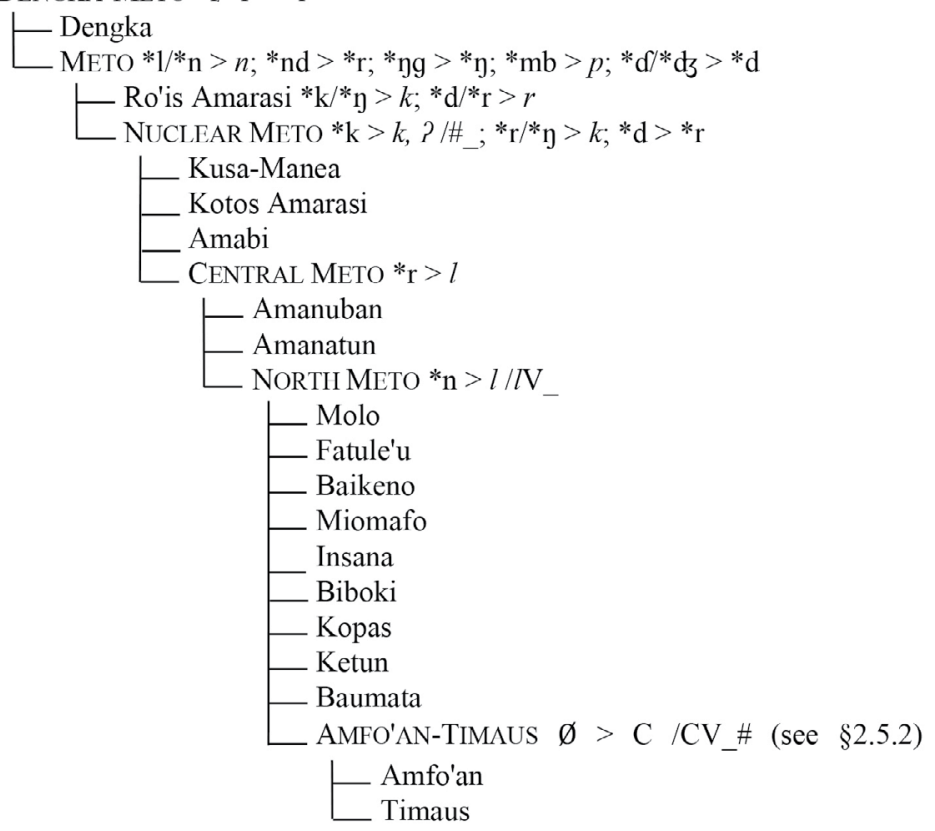

Figure 3.3: Rote-Meto family tree 
There are no sound changes that affect all of the Rote languages to the exclusion of Meto. That is, there is no 'Rote' subgroup. The only changes shared between all the languages of Rote that are not found in Meto are shared phonological irregularities in specific lexical items. There are nine lexically specific shared irregularities that unambiguously affect only the Rote languages: ${ }^{10}$

\begin{tabular}{|c|c|c|}
\hline - $\quad * \mathrm{u}>o$ & in & *bua-k $>$ boa- $k /-2$, boa- $k /-2$ 'fruit' \\
\hline - $\quad * \mathrm{u}>o$ & in & *lua $>$ loa- $k /-$ ? 'open, wide' \\
\hline$*_{\mathrm{i}}>e$ & in & *hiri > here, hele 'choose' \\
\hline$* \mathrm{u}>a$ & in & *tufu $>$ tufa 'punch' \\
\hline$*_{\partial}>o$ & in & *səru > na-so $\sim$ soru, na-so $\sim$ solu 'meet' \\
\hline - $\quad * \mathrm{~m}>* \mathrm{mb}$ & in & *simo > simbo, simpo, sipo 'receive' \\
\hline$* \mathrm{t}>d / d$ & in & *tui > dui-k/-P, dui-k, dui 'stripe' \\
\hline$* \mathrm{~h}>\varnothing$ & in & *halu-k $>$ alu- $k /-$ ? 'pestle' \\
\hline$* \mathrm{~h}>\varnothing$ & & *haru-k $>$ aru- $k /-2$, alu- $k /-$ ? 'shoulder' \\
\hline
\end{tabular}

While these shared irregularities do show that the Rote languages have undergone some common development apart from Meto, the evidence for splitting the Rote languages into a West Rote and Central East Rote subgroup (each of which has shared a period of common development with Meto) is much stronger as both these branches are supported both by shared sound changes and shared irregularities in specific lexical items.

\subsubsection{Nuclear Rote (NRote)}

There are three sound changes shared between the Nuclear Rote languages; ${ }^{*} \mathrm{~W}>f$ (attested in only two lexical items), ${ }^{*} \mathrm{\partial}>e$ in final syllables, and merger of $* \mathrm{~d} / *^{*} \mathrm{~d} / *_{\mathrm{d}} \mathrm{d}>d$ $\sim d$ (see $\$ 2.4 .1$ for the realisation of the voiced alveolar plosive in the Rote languages). There are also at least 10 lexically specific innovations which support Nuclear Rote. These are the changes:

$$
\begin{aligned}
& \text { - } *_{\mathrm{ii}}>*_{\mathrm{oe}} \text { in } \\
& \text { *ka-mii }>\text { *moe 'urine' } \\
& \text { - *a }>*_{0} \text { in } \quad *^{\text {sou }}>*^{*} \text { sou 'Alstonia villosa' } \\
& \text { - } *>*_{0} \text { in } \quad * \text { təlo-k }>* \text { tolo-k 'egg' } \\
& \text { - } * \mathrm{a}>*_{\mathrm{e}} \text { in } \quad *_{\text {nadga-k }}>*^{*} \text { nade-k 'name' } \\
& \text { - } *_{\mathrm{a}}>*_{\mathrm{u}} \text { in } \quad * \text { ka-nduna-k }>* \text { ndunu-k } \\
& \text { - } * \text { au }>* \text { eu in } * \text { taun }>* \text { teu-k 'year' }
\end{aligned}
$$

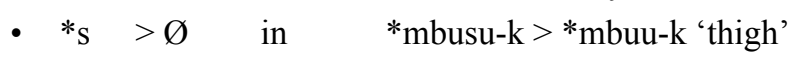

$$
\begin{aligned}
& \text { - } * \mathrm{k}>*_{\mathrm{yg}} \text { in } \quad * \text { ka-kelas }>*^{*} \text { ygelas 'winter melon' } \\
& \text { - } *_{\mathrm{t}}>*_{\mathrm{d}} \text { in } * \text { tahi }>* \text { dahi 'winnow' }
\end{aligned}
$$

- vowel metathesis in *midu $>*$ mudi 'spit'

\footnotetext{
10 The lexically specific irregularities in these sections include only those that can confidently be assigned to a specific subgroup, often on the basis of external evidence. For instance, I posit irregular $*_{r}>l$ in Nuclear Rote for *saraa 'shine, sunbeam'. An alternate hypothesis would be to reconstruct *salaa with irregular *1 $>r$ in Oenale. Ambiguous cases such as this are excluded from my discussion.
} 
In addition to the phonological evidence and these lexically specifically phonological irregularities, evidence for Nuclear Rote also comes from the forms of the vocalic agreement prefixes (see Table 2.15) as all Nuclear Rote languages have changed the vowels of these prefix to /a/. Specifically, PRM $1 \mathrm{sG} * \mathrm{ku}->$ Proto-Nuclear Rote *ka- $>$ Bilbaa $k a-$, other Nuclear Rote $2 a-$, and PRM 1 PL.EXCL/2PL *mi- and 2sG *mu- $>$ ProtoNuclear Rote $*$ ma- $=$ all Nuclear Rote 1 PL.EXCL/2PL/2SG $m a-$ Within Nuclear Rote, we can identify a number of subgroups on the basis of changes affecting prenasalised plosives, as discussed further in the next section.

\subsubsection{Central East Rote and Meto (CERM)}

The Central East Rote and Meto grouping contains Meto and all members of Nuclear Rote except Tii and Lole. While this group reflects a period of shared development between members, it probably does not reflect descent from a single common ancestor, but rather descent from a common ancestral speech community of closely related languages. The languages that belong to this group share the changes affecting the prenasalised plosives, with these changes diffusing between these languages after Meto had split from Proto West Rote-Meto.

The changes affecting the bilabial and velar prenasalised plosives each follow a single pathway, which is carried out to different extents in different languages. The bilabial prenasalised plosive undergoes $* \mathrm{mb}>m p>p$, while the velar prenasalised plosive undergoes *yg $>\eta>k$. All members of Central East Rote-Meto share *mb $>m p$, with voiceless prenasalised $m p$ retained in Ba'a. All other Central East Rote-Meto languages share $* m p>p$ and medial $*$-ng- $>\eta$. All these languages except Termanu and Korbafo further share initial *yg- $>\eta$. A tree showing these changes is given in Figure 3.4.

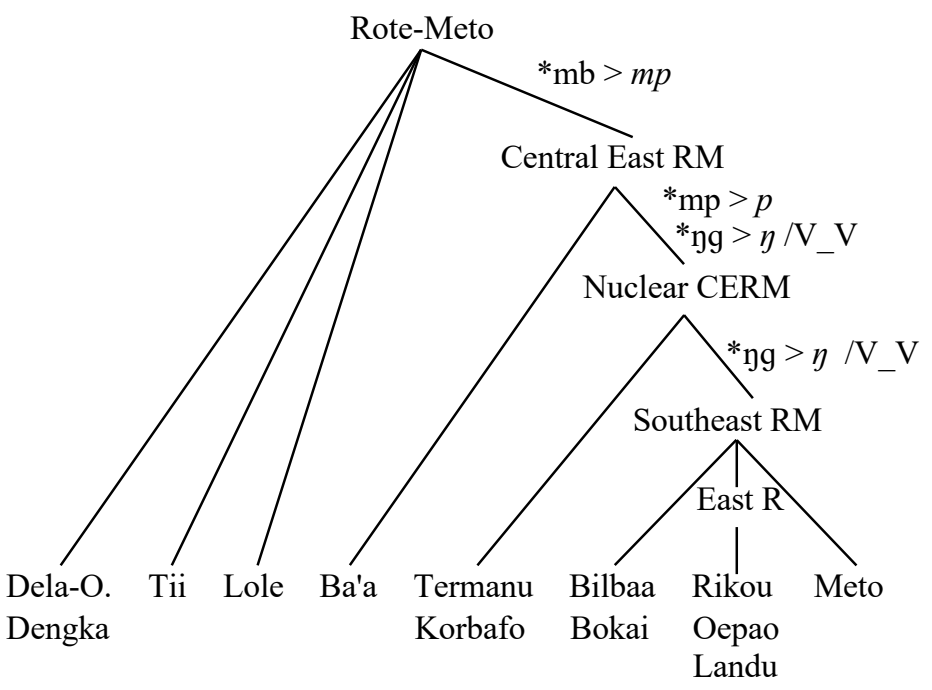

Figure 3.4: Rote-Meto bilabial and velar prenasalised plosives 
The velar nasal $*_{\eta}$ (from earlier *yg) further changes into $k$ in Rikou, Oepao, Landu and all Meto lects. However, as discussed further in $\S 3.3 .4$, this change must have been completed after Meto had split from this group and after the break-up of Proto-Meto.

The changes affecting *nd are more complex, with more than one pathway of change. *nd is retained unchanged in Landu and is unchanged word initially in Ba'a, Termanu and Korbafo. Ba'a, Termanu, Korbafo, Bokai and Bilbaa have medial *nd $>n$. Medial *nd is unchanged in Rikou.

The other reflexes of *nd in Central East Rote-Meto can be derived from intermediate *r. Rikou has initial *nd $>r$, Bokai and Bilbaa have initial *nd $>*_{r}>l$, and Oepao and Meto have *nd $>*_{r}$ in all word positions with all varieties of Meto apart from Ro'is Amarasi subsequently having $* r>k$. The reflexes of PRM *nd are summarised:

\begin{tabular}{|c|c|c|}
\hline - $\quad * \mathrm{nd}=n d$ & $\#_{-}$ & Ba'a, Termanu, Korbafo, Bokai, Bilbaa, Landu \\
\hline - $\quad *_{\mathrm{nd}}=n d$ & V_V & Rikou, Landu \\
\hline - $\quad * \mathrm{nd}>n$ & V_V & Ba'a, Termanu, Korbafo, Bokai, Bilbaa \\
\hline$* \mathrm{nd}>r>l$ & \# & Bokai, Bilbaa \\
\hline - $\quad * \mathrm{nd}>r$ & \#_ & Rikou, Oepao, Ro'is Amarasi \\
\hline$*_{\mathrm{nd}}>r$ & V_V & Oepao, Ro'is Amarasi \\
\hline - $\quad * \mathrm{nd}>r>k$ & $\#, V_{-} \mathrm{V}$ & Nuclear Meto (Meto apart from Ro'is Am \\
\hline
\end{tabular}

The main difficulty these changes provide with regards to subgrouping concerns the position of Bokai and Bilbaa. The change of medial *nd $>n$ groups these languages with those to their west (Termanu and Korbafo), while the change of initial *nd $>r>l$ groups them with the languages to the east (Rikou and Oepao).

On the basis of current evidence there is no principled reason to privilege either of these changes in defining which languages Bokai and Bilbaa subgroup with. For this reason, I have privileged the evidence from other sound changes in representing the relationship of Bokai and Bilbaa to other languages in the tree diagrams in Figure 3.3 and Figure 3.4. This has resulted in a Southeast Rote subgroup in which Bokai and Bilbaa are placed with East Rote (Rikou, Oepao and Landu) on the basis of *yg $>*^{*} \mathrm{y} / \#$.

Nonetheless, while the changes of $*$ nd $>n / \mathrm{V}_{-} \mathrm{V}$ and initial $*$ nd $>r>l$ are contradictory in determining with which languages Bokai and Bilbaa subgroup, the fact that they are both shared by Bokai and Bilbaa is evidence for placing these languages in a single subgroup. Similarly, shared $*^{n}>n$ in Termanu and Korbafo provides evidence for placing these languages in a single subgroup. The historical phonologies of Termanu and Korbafo are almost identical, with the exception of some of the reflexes of *k (Table 3.2) and word final consonants (Table 3.6). There are also morphological differences between Termanu and Korbafo (see §2.6). The changes affecting *k, word final consonants, and the morphology of Korbafo are the same as in Bokai and Bilbaa.

While the Central East Rote and Meto grouping, along with several internal subgroups, is well defined on the basis of the changes affecting the prenasalised plosives, there are no phonological irregularities in specific lexical items which unambiguously only occur in these languages. 


\subsubsection{West Rote-Meto (WRM)}

The West Rote-Meto subgroup is well supported by six shared sound changes: ${ }^{*} \mathrm{~b}>f$, $*_{\mathrm{d} / * \mathrm{~d} \mathrm{~s}}>r, *_{\mathrm{\partial}}>\mathrm{a} / \sigma \underline{\sigma} \#, *_{\mathrm{a}}>a, e / \ldots, * \mathrm{k}>k, h / \#$, and $*_{\mathrm{wa}}>o$. However, with the exception of $* \mathrm{~d} /{ }^{*} \mathrm{~d} J>r$ and ${ }^{*} \mathrm{wa}>\bar{o}$, none of these sound changes is complete in DelaOenale and Dengka. That is, there are forms that have undergone these sound changes in Meto while cognates in West Rote have not.

This probably indicates that (the speech community of what was to become) Meto was the centre of diffusion with changes originating there and spreading into West Rote. Additionally, it probably indicates that Proto-West Rote-Meto was not a single language for long enough for pre-Meto to exert the pressure needed for these sound changes to be completed in Dela-Oenale and Dengka. In concrete terms, this probably indicates that speakers of what was to become Meto were more socially influential during the development of Proto-West Rote-Meto and that they were exerting influence for a relatively short period of time before leaving the speech community.

In addition to the sound changes that are shared between West Rote-Meto, there are also at least seven irregularities in specific lexical items which are exclusively shared by West Rote-Meto:

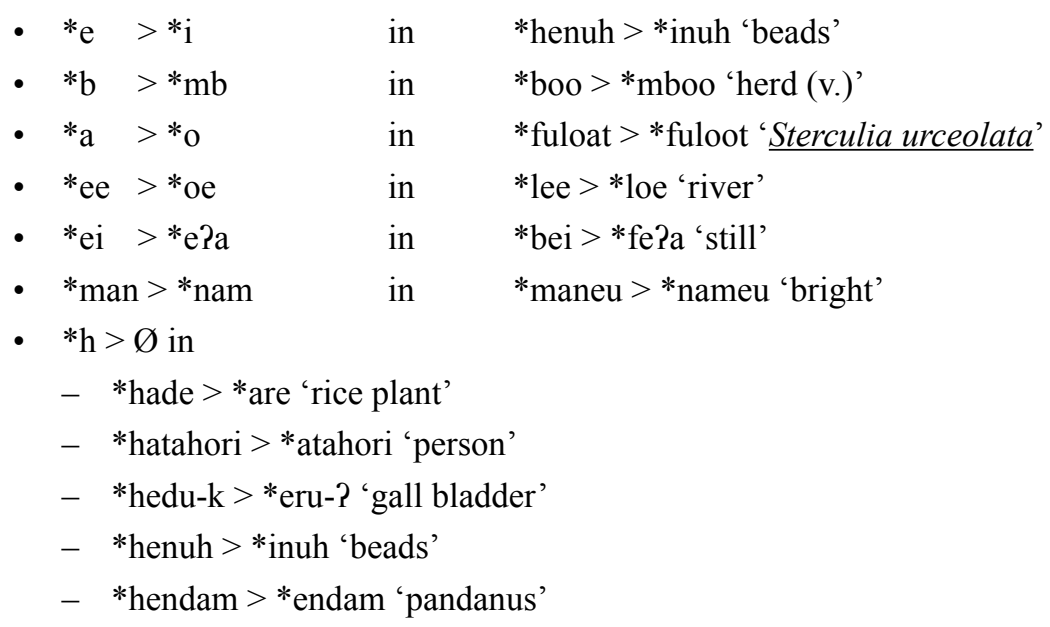

\subsubsection{Meto}

The main defining sound change of Meto as a distinct subgroup is $* 1>n$ in nearly all forms. This change also affected PRM *d and $* r$ as these had already merged with $* 1$ in the pre-Meto period. Despite this change, there are half a dozen or so forms in which PRM *1 appears to have been retained as *1 in Proto-Meto. Thus, we must posit that this consonant was present, at least marginally, in Proto-Meto. Forms that retain *1 unchanged may represent borrowings into Proto-Meto after $* 1>n$ and/or borrowings after the break- 
up of Proto-Meto. ${ }^{11}$ The Proto-Meto consonant inventory is given in Table 3.9. The velar nasal ${ }^{*} \mathrm{y}$ may have become ${ }^{*} \mathrm{~g}$ by the time of Proto-Meto. This is discussed in $§ 3.3 .4 .1$ below. Proto-Meto also had five vowels: $*_{\mathrm{i}},{ }^{*} \mathrm{e}, *_{\mathrm{a}}, *_{\mathrm{o}}$ and $*_{\mathrm{u}}$.

Table 3.9: Proto-Meto consonant inventory

\begin{tabular}{|l|c|c|c|c|}
\hline & Labial & Coronal & Velar & Glottal \\
\hline Voiceless stops & $*_{\mathrm{p}}$ & $*_{\mathrm{t}}$ & $*_{\mathrm{k}}$ & $*^{\mathrm{p}}$ \\
\hline Voiced stops & $*_{\mathrm{b}}$ & $*_{\mathrm{d}}$ & & \\
\hline Fricatives & $*_{\mathrm{f}}$ & $*_{\mathrm{s}}$ & & $*_{\mathrm{h}}$ \\
\hline Nasals & $*_{\mathrm{m}}$ & $*_{\mathrm{n}}$ & $*_{\mathrm{g}}$ & \\
\hline Rhotic & & $*_{\mathrm{r}}$ & & \\
\hline Lateral & & $\left(*_{1}\right)$ & & \\
\hline
\end{tabular}

Within Meto there is a primary split between Ro'is Amarasi and all other Meto varieties (Nuclear Meto). Nuclear Meto is defined by initial $*_{\mathrm{k}}>k$, ? followed by Proto-Meto $*_{\mathrm{r}}($ from PRM *nd $)>k$. While Meto shares PRM *ng $>*_{\eta}>k$ with Landu, Rikou and Oepao (East Rote), no instances of PRM *yg become? in Nuclear Meto. Thus, the change of $*_{\eta}>k$ probably occurred in Meto after the change of Nuclear Meto initial $* \mathrm{k}>k$, $?$ and thus after the split between Ro'is Amarasi and Nuclear Meto.

PRM * $y$ split into Proto-Meto * $y$ and *n. For some cognate sets we can only reconstruct Proto-Meto ${ }^{*} \mathrm{y}$, while for others there is variation. Some Meto varieties have $k\left(<*^{*}\right)$ and some varieties have $n$. There is also occasional variation within a single variety. This indicates that such words themselves had variants in Proto-Meto. An example of such a word is PRM *rinin 'cold', which is reflected as mainikin or $<$ maniki $>$ in most varieties of Meto, but with the variant $<$ mainini $>$ in Amanuban. This points to ProtoMeto *ma|ninin *ma|ninin. Similarly, PRM *nuna 'Cordia species' is reflected in Molo as nun/ba?i, nuk/ba?i, kuk/ba?i, which probably attests Proto Meto *nun/ba?i *nuy/ba?i * *uy/ba?i.

While Kusa-Manea shares $*_{\mathrm{k}}>k, ?$ /\#_ and $*_{\mathrm{r}}>k$ with Nuclear Meto, it also has a number of conservative features where most other known varieties of Nuclear Meto have innovated. On the basis of these innovations, we can place these other varieties of Meto together, leaving Kusa-Manea as a primary branch of Nuclear Meto. The retentions and innovations which separate Kusa-Manea from most other known varieties of Meto are given as follows:

- retention of final /a/ after metathesis

- other known Nuclear Meto varieties have assimilation of /a/

- optional retention of initial $* \mathrm{n}=n$ in *nudu $>$ nuru-f, ruru- $f$ 'lips'

- other known Nuclear Meto varieties have assimilation; ruru-f, lulu-f 'lips'

11 Proto-Meto $* 1>r$ in Amarasi and $* 1=l$ in all other varieties of Meto for which data is available. (No clear unambiguous reflexes of Proto-Meto *1 have so far been identified in Kusa-Manea.) The forms for which I posit Proto-Meto *1 are: *6olo1 'hole', *hela 'pull', *laa2 'hut', *laba 'wind (v.)', *lasa 'underlay', *lole1 'kind of tuber', as well as three forms with initial *kl: *klaha 'coals', *klou 'bow (and arrow)' and *kleet 'mock'. Finally, there is Proto-Meto *kalusa 'fingernail', which has no known cognates in the Rote languages. 
- retention of PRM *hida > hian 'how many'

- other known Meto varieties have fauk 'how many'

(see *6a?u)

- retention of final *m in PMP *ma-qitəm > metom 'black'

- other known Meto varieties have *m $>n$; metan 'black'

- retention of initial *e in PwRM *ela $>n$-Pean 'run, flee'

- other known Meto varieties have *e $>a e$; $n$-aena 'run, flee'

- retention of semantics for *hanas $>$ manas 'hot'

- other known Meto varieties have manas 'sun'

- retention of semantics 'sun' for *ledo > neno 'sun, day, sky'

- other known Meto varieties have neno 'day, sky' 12

Ro'is Amarasi groups with known varieties of Meto, and groups apart from Kusa-Manea, for most of these features, except retention of * $n=n$ in *nudu $>$ nuru- $f$ 'lips' and retention of /a/ after metathesis in forms with a medial glottal stop. Nuclear Meto shares sound changes with Kusa-Manea, but other innovations with Ro'is Amarasi. The relationship between Ro'is Amarasi, Kusa-Manea and other varieties of Meto is represented with a simplified wave diagram in Figure 3.5.

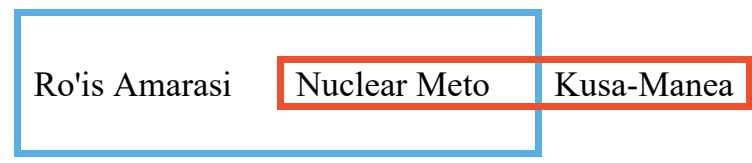

Figure 3.5. Simplified Meto wave diagram

Proto-Meto $* \mathrm{~d}$ (usually from PRM $* \mathrm{~d}$, though some instances are from PRM $* \mathrm{~d}$ or $*_{\mathrm{d}}$ — see $\$ 3.2 .1 .1$ and $\left.\$ 3.2 .1 .2\right)$ undergoes changes in all varieties of Meto. Ro'is Amarasi, Kotos Amarasi, Amabi and Kusa-Manea all have $*^{*}>r$ while all other known Meto varieties have $* \mathrm{~d}>*_{\mathrm{r}}>l$. However, we must reconstruct Proto-Meto $*_{\mathrm{d}}$ for this correspondence set rather than *r, as reconstruction of *r would falsely predict that it merges with Proto-Meto *r (from PRM *nd) as $k$ in Nuclear Meto. Thus, we must posit that $* \mathrm{~d}>*^{*}$ spread between Nuclear Meto and Ro'is Amarasi after the breakup of ProtoMeto; Proto-Meto $* \mathrm{~d}>$ Proto-Nuclear Meto $*$ r.

Within Nuclear Meto the change of $(* d>) *_{r}>l$ defines a Central Meto subgroup that excludes Kotos Amarasi, Amabi and Kusa-Manea. Within Central Meto we can further identify a North Meto subgroup on the basis of $*_{\mathrm{n}}>l / l \mathrm{~V}$ _V. North Meto contains all members of Central Meto except Amanuban and Amanatun. Examples of this $*_{n}>l$ sound change include PRM *dalan 'path, way' > Proto-Meto *danan $>$ Kotos Amarasi ranan $>$ Amanuban $>$ lanan $>$ Molo lalan, and PRM *dilah 'Aegle marmelos' $>$ ProtoMeto *dinah $>$ Kotos Amarasi rinah $>$ Amanuban linah $>$ Molo lilah.

12 The meaning 'sun' is retained in certain set phrases for neno in Nuclear Meto, such as the terms for 'east' and 'west'. See the notes on *ledo. 


\subsubsection{Contact with languages of central Timor}

There is evidence for a period of pre-historic contact between Meto and languages of the Central Timor subgroup: Welaun, Kemak, Tokodede and Mambae (see Edwards (2019) for the evidence for this subgroup). This contact almost certainly occurred before the northwards spread of Tetun, which now separates these groups.

Firstly, there are a number of words that are shared between Meto and Central Timor languages that have no (regular) cognates in the Rote languages and are almost certainly due to borrowing, though it is not always possible to determine whether these words were borrowed from Central Timor into Meto or vice versa. Examples of likely loans between Meto and Central Timor languages.

- Meto sone 'Job's tears' from Welaun sole 'corn, maize'. Weluan sole is regular from PMP *zolay 'Job's tears'. Semantic shift to 'maize' probably occurred in Welaun after it was borrowed into Meto.

- Meto mneas 'hulled rice', Kemak mreas 'hulled rice'. Both forms are irregular from $\mathrm{PMP} *$ bəRas. While irregular retention of $* \mathrm{R}$ and metathesis of *əR $>* \mathrm{R} ə$ is common throughout Timor — pointing to widespread borrowing — Meto and Kemak are the only two languages known to also have irregular $* \mathrm{~b}>m$ thus indicating a specific case of borrowing between these languages.

- Amarasi, Kusa-Manea baba? 'paternal aunt, maternal uncle', Kemak, Welaun baba 'maternal uncle' (Rote languages have to?o 'maternal uncle').

- Meto kase 'foreigner', Kemak kase 'foreigner' (probably borrowed from Meto).

- Ro'is Amarasi aka?ta?e, Kotos Amarasi ata?ra?e, Molo ata?la?e 'praying mantis', Welaun astatae 'praying mantis'.

- Ro'is Amarasi kupu 'fog', Kotos Amarasi kupu 'water vapour', Tokodede api kupu 'smoke' (Tokodede api = 'fire').

- Amarasi naiPbesi, Amfo'an bePi-db, Kusa-Manea be?i 'crocodile', Welaun (Oele'u hamlet) na?ibein, Welaun (Mahein hamlet) bei liurai 'crocodile'.

- Amarasi na-Pba?e 'play', Welaun ba?an 'play'.

In addition to such likely borrowings, denasalisation of $* y>*_{\mathrm{g}}$ is one of the defining sound changes of Central Timor as a subgroup. Welaun and some varieties of Mambae further have $*_{\mathrm{y}}>*_{\mathrm{g}}>k$. Given the lexical evidence for contact between Meto and Central Timor, it is likely that denasalisation of $* \eta$ in both groups also spread by contact between these languages, though parallel development also could have taken place.

There are three language groups that have denasalisation of $*_{y}$ in Timor: East Rote (Landu, Rikou and Oepao), Meto and Central Timor. Furthermore, we have evidence of a genetic relationship between East Rote and Meto and a contact relationship between Meto and Central Timor, but no evidence for any kind of relationship between East Rote and Central Timor.

Thus, if $* \eta>k$ spread by contact in this region, positing East Rote as the ultimate source of $* \eta>k$ would require positing that Central Timor acquired this change via influence from Meto. On the other hand, positing that $*_{y}>k$ spread from Meto into East Rote 
leaves open the possibility that the ultimate origin of this change was either with Meto or with Central Timor. On the balance of probabilities then, it seems more likely that East Rote acquired $* \eta>k$ from Meto rather than vice versa. Additional support for the idea that $* \eta>k$ had a westwards spread from Meto into East Rote comes from the fact that the most eastern variety of Meto, Kusa-Manea, only has Proto-Meto ${ }^{*} y>k$, while the most western variety, Ro'is Amarasi, has the most instances of Proto-Meto * $y>n$. This is expected if $* \eta>k$ spread westwards from the east. ${ }^{13}$

Given that Central Timor has $*^{*}>*_{g}$ (with subsequent $*_{g}>k$ in some languages), this provides circumstantial evidence that Meto ${ }^{*} y>k$ also went through an intermediate $*_{\mathrm{g}}$ stage. If this was the case, we could propose $*_{\mathrm{g}}>*_{\mathrm{g}}$ had occurred by Proto-Meto and that $* \mathrm{~g}>k$ occurred after the split between Ro'is Amarasi and Nuclear Meto. Similarly, $*_{r}>k$ (which occurred in Nuclear Meto) is cross-linguistically extremely rare, while $*_{r}>$ $*^{*} \mathrm{~g}$ is more common. Thus, it could be that the merger of $*_{\mathrm{r}} / *_{\mathrm{y}}>k$ in Nuclear Meto went through an intermediate $* \mathrm{~g}$ stage at some point.

In addition to contact with languages of the Central Timor subgroup, there is also evidence for prehistoric contact between Meto and Tetun. Five terms that are probably shared between Tetun and Meto due to borrowing are given below. Each of these terms would have undergone irregular sound changes in Tetun, but regular sound changes in Meto. Thus, they were probably borrowed from Meto into Tetun. Note that these five terms are not only found in varieties of Tetun spoken in central Timor (Foho Tetun and Fehan Tetun), but also in East Tetun that is currently separated from Meto by the speakers of Bunak (non-Austronesian) and Mambae (Central Timor).

- Tetun feto 'woman' from Meto feto 'man's sister, woman'

- Tetun na?i(n) 'lord; male honorific' from Meto na?i-f 'grandfather', nai? 'Mr'

- Tetun koto 'variety of bean' from Meto koto?, koto 'hyacinth bean, Lablab purpureus'

- Tetun kunus 'capsicum', from Meto; Ro'is Amarasi, Kusa-Manea kunus 'chilli', other Meto ?unus 'chilli'

- Tetun na?i bei 'respectful name for the crocodile' from Meto; Amarasi nai?besi, Amfo'an be?i-ds, Kusa-Manea be?i 'crocodile'

In addition to terms that were borrowed from Meto into Tetun, there are also a number of terms that are probably shared by borrowing but for which the direction of borrowing cannot be determined as neither language has undergone distinctive sound changes. One example is Tetun bukae 'provisions for a trip, food taken on a journey', Molo bukae-l 'provisions', n-bukae 'eat', Amarasi n-bukae 'eat, drink, dine'. There are also several forms that are probably loans into Meto from Tetun. One example is Tetun fukar 'season, spice', Kotos Amarasi fukar 'herbs, spices, seasonings', Molo fukal 'seasonings'.

Finally, Kusa-Manea has been in more intense contact with Tetun than other varieties of Meto. Kusa-Manea is spoken in territory that was historically part of the Tetun kingdom of Wehali and Kusa-Manea speakers are nearly universally multilingual and also speak

13 Two examples of Ro'is Amarasi $*^{*}>n$ where other Meto varieties have ${ }^{*} \mathrm{y}>\mathrm{k}$ are PRM * $\mathrm{yilu}>$ niu 'tamarind' and PMeto *yinu > na-ninu 'spit'. 
Tetun. Tetun is also the language used by them in traditional ceremonies. Unsurprisingly, there are many loans from Tetun into Kusa-Manea across all semantic spheres, including functors such as mos 'to, also' or tan 'because, therefore'.

\subsubsection{Contact with Helong}

Meto has been in extensive contact with Helong since prehistoric times and this contact is still ongoing. At present, Meto is dominant and the nature of contact is overwhelmingly from Meto into Helong. However, in prehistoric times the relationship appears to have been more equal and many borrowings can be identified that are from Helong into Meto.

Examples of likely borrowings from Helong into Meto include Helong yala 'name, tribe, clan, people group' (regular from PMP * yajan) $\rightarrow$ Meto kana- $f$ 'name, clan', Helong blapas 'side' $\rightarrow$ Meto bnapa- $f$ 'side' (initial $b l$ is common in Helong, but $b n$ is rare in Meto), Helong nale- $n$ 'daughter-in-law' $\rightarrow$ Meto nane- $f$ 'daughter-in-law, opposite sex sibling's daughter' (Rote languages have compounds of feto 'female' and feu- $k$ 'new'), Helong el 'like, similar; to, towards' $\rightarrow$ Ro'is Amarasi en 'like, similar; to, towards', Nuclear Meto on 'like, similar' (Rote languages have leo 'like, similar').

However, this is not to say that the direction of prehistoric borrowing was exclusively from Helong into Meto. Many loans in the other direction can also be identified, including PRM *hatahori 'person' > Pre-Meto **atoli $\rightarrow$ Helong atuli 'person' > Proto-Meto *atoni 'man, person', and PRM *sarakaen 'sand' $>$ Pre-Meto **slaen $\rightarrow$ Funai Helong slaen, Semau Helong hlaen 'sand'> Proto-Meto *snaen 'sand'. The fact that many of these borrowings involve items of basic vocabulary points to long-term intense contact between Helong and Meto involving extensive bilingualism. This contact continues today, with the difference that Meto is the dominant language.

Apart from contact between Helong and Meto, there is also evidence that Proto-RoteMeto was in contact with Proto-Helong. There are a little over 100 vocabulary items in this comparative dictionary, for which cognates are only known in Rote-Meto and Helong. Many of these cognates sets show regular sound changes in both groups, thus indicating borrowing at an early stage. In addition to this vocabulary, the change of ${ }^{*} \mathrm{~W}$ $>{ }^{*} \mathrm{f}$ is partly shared between Rote-Meto and Helong. (See $\$ 3.5 .3 .2$ for more discussion of this change.)

\subsection{Levels of reconstruction}

Reconstructions in this dictionary are assigned to one of 10 levels according to the language varieties in which their reflexes occur. These levels are summarised in Table 3.10, along with the number of reconstructions made to each level. Of these levels, the first six are all proto-languages, which can be identified on the basis of exclusively shared innovations, while the final four are groupings that have a shared common history but do not necessarily descend from a single unitary proto language below the level of PRM or, in the case of Central East Rote, below the level of PnRote (§3.3). 
ROTE-METO COMPARATIVE DICTIONARY

Table 3.10: Reconstructive levels ${ }^{\dagger}$

\begin{tabular}{|l|l|l|l|}
\hline Abbreviation & Level & Must include at least one of & No. \\
\hline PRM & Proto-Rote-Meto & West Rote, Nuclear Rote, and Meto & 664 \\
\hline PwRM & Proto-West Rote-Meto & West Rote, and Meto & 37 \\
\hline PMeto & Proto-Meto & Meto & 39 \\
\hline PnMeto & Proto-Nuclear Meto & Nuclear Meto & 22 \\
\hline PnRote & Proto-Nuclear Rote & Nuclear Rote & 23 \\
\hline PwRote & Proto-West Rote & West Rote & 3 \\
\hline Rote & Rote & West Rote, and Nuclear Rote & 264 \\
\hline nRM & Nuclear Rote-Meto & Nuclear Rote, and Meto & 50 \\
\hline CERM & Central East Rote-Meto & Central East Rote, Meto & 45 \\
\hline CER & Central East Rote & Central East Rote & 27 \\
\hline
\end{tabular}

$\dagger$ West Rote includes Dela, Oenale and Dengka. Nuclear Rote includes all other Rote lects. Nuclear Meto includes all varieties of Meto except Ro'is Amarasi. Central East Rote includes all Nuclear Rote except Tii and Lole.

There are two factors which determine to which level a reconstruction is assigned. Firstly, when cognate forms with (mostly) regular sound correspondences are identified, a reconstruction is made to the appropriate level according to the lects that attest the cognate forms. Thus, for instance, *6ole 'arenga palm, Arenga pinnata' is reconstructed to PRM on the basis of cognates in West Rote languages, Nuclear Rote and Meto.

Secondly, when a Rote-Meto cognate set has likely cognates in other Austronesian languages, a reconstruction is made to the highest level justified by the Rote-Meto evidence. This is accompanied by any $\mathrm{P}(\mathrm{CE}) \mathrm{MP}$ reconstruction in the etymology field for terms that have been reconstructed to $\mathrm{P}(\mathrm{CE}) \mathrm{MP})$ and/or the external cognates in the out-comparison section ( $\$ 1.5 .1)$. Thus, for instance, PRM *langa 'head' has cognates in Helong, Kisar, Tokodede and Kemak, which are all included in the out-comparisons section (§1.5.1). Similarly, PRM *ate 'liver' has numerous cognates in Austronesian languages and its PMP etymon * qatay is given in the etymology field (§1.5.3.3).

A reconstruction to any of the levels lower than PRM has three logical explanations:

- the reconstruction is an innovation at that level

- the reconstruction is inherited from PRM, but cognates are unknown (perhaps due to loss) in all daughter languages of a particular branch

- the cognate set is ultimately a loan.

While we can never, in principle, definitively decide which of these three explanations explains any particular lower level reconstruction (or any reconstruction at any level for that matter), there are certain situations in which one explanation is more likely than another.

When a reconstruction is made on the basis of internal Rote-Meto evidence and is assigned to Rote, Nuclear Rote-Meto or Central East Rote-Meto, the second explanation is most likely: the terms are probably inherited from PRM, but cognates are not known in the languages that do not belong to these groups. Innovation is unlikely as none of 
these groups are descended from unitary proto-languages (see §3.3), though we cannot rule out innovation in one branch with borrowing into the other branch (a combination of the first and third explanations).

Similarly, when a reconstruction is made to a level below PRM on the basis of evidence external to Rote-Meto, and these cognates are widely distributed, the second explanation is probably most likely: the form was present in PRM, but is unknown or lost in daughter languages from a particular branch. Thus, for instance, Proto-Meto *metam 'black' is a reflex of PMP *ma-qitom. While it cannot technically be reconstructed to PRM on the basis of the internal evidence, it was almost certainly present in PRM but has been lost without known trace in all the modern Rote languages. Likewise, Proto-Meto *k|teom 'sea-urchin' has cognates in Flores and other languages of Timor. There is no reason to suppose that borrowing accounts for all these cognate forms (though it might account for some), thus unless the Proto-Meto term itself is a borrowing after the breakup of ProtoMeto, it is likely that Proto-Meto ${ }^{*} \mathrm{k} \mid$ teom is a reflex of an earlier PRM etymon, which has been lost in the Rote languages.

On the other hand, when a lower level reconstruction is made for which external cognates have only a limited range, we cannot yet decide between any of the explanations, though the second explanation (loss in all daughter languages of a particular branch) usually seems unlikely. Thus, for instance, Proto-Meto *domi 'love, like' has cognates in Tetun and languages of central Timor. It could be a Proto-Meto innovation that was then borrowed by these other languages, or it could have been borrowed by (Proto)-Meto.

When I am fairly confident that a particular cognate set is due to borrowing into the Rote-Meto languages, the cognate set is included in the loan distribution section of the dictionary (see $\$ 1$ 1.5.3.6 for more discussion of this section) usually with discussion of the evidence as to why this set is identified as a loan. When the evidence is ambiguous, it is included in the main section, again often with discussion as to the possibility that this term is a borrowing.

The cognate sets that are most ambiguous between explanation one (innovation at a lower level) and explanation two (PRM innovation with loss in daughter languages) are those that are made to a lower level only on the basis of Rote-Meto internal evidence. Thus, for instance, Proto-West Rote-Meto * batus 'sea-snail' is reconstructed on the basis of Dengka $6 a \sim 6 a t u-P$ 'sea-snail', Ro'is Amarasi k|bautus, and Kotos Amarasi k|batus 'sea-snail, oyster'. At present, this term has no other known cognates. It may be a West Rote-Meto innovation, it may have been present in PRM but lost in the other Rote languages, or further work may reveal cognates in other languages of Rote or other regional languages.

To summarise, I assign reconstructions to one of 10 levels as is appropriate according to the internal Rote-Meto evidence. Reconstructions assigned to Rote, Nuclear Rote-Meto or Central East Rote-Meto were probably present in PRM, but cognates are unknown (perhaps due to complete loss) in West Rote or Meto. Similarly, when external cognates of lower level reconstructions are widely distributed, borrowing into Rote-Meto is unlikely. 


\subsection{Rote-Meto within Malayo-Polynesian}

In this section I discuss the way in which Rote-Meto fits within Malayo-Polynesian. This includes discussion of the sound correspondences between PMP and PRM (§3.5.1), the probable role of language shift from non-Austronesian languages (§3.5.2) and the position of Rote-Meto within Malayo-Polynesian (\$3.5.3).

\subsubsection{Sound changes between PMP and PRM}

Edwards (2018c) provides a detailed discussion of most aspects of the top-down history of the Rote-Meto languages, though that paper is based on an earlier and smaller database, as well as an earlier understanding of the sound changes. In this section I summarise the latest understanding of the sound changes that have occurred between PMP and PRM. This includes the changes affecting consonants (§3.5.1.1), vowels and glides (§3.5.1.2) and word final consonants (\$3.5.1.3). Two different kinds of sound correspondences between PMP and PRM can be identified: those that are mostly regular and those which involve unconditioned splits.

\subsubsection{Initial and medial consonants}

The PMP consonants that have regular reflexes in PRM are summarised in Table 3.11, along with the number of attestations of each correspondence set. PMP $*_{\mathrm{t}}, *_{\mathrm{k}}, *_{\mathrm{m}}$, $*_{\mathrm{n}}$, $*_{\mathrm{s}}$ and $*_{1}$ are usually retained unchanged in PRM. PMP $*_{\mathrm{h}}>\varnothing$ and $*_{\tilde{n}}>*_{\mathrm{n}}$. The correspondences shown in Table 3.11 represent more than 95 per cent of cases for each PMP consonant, with the exception of $* \mathrm{k}=*_{\mathrm{k}}$ which is retained unchanged in 82 per cent of cases $(68 / 83){ }^{14}$

Table 3.11: Regular P(CE)MP to PRM consonant correspondences

\begin{tabular}{|l|l|l|l|l|l|l|l|l|}
\hline PMP & $*_{\mathrm{t}}$ & $*_{\mathrm{k}}$ & $*_{\mathrm{m}}$ & $*_{\mathrm{n}}$ & $*_{\tilde{\mathrm{n}}}$ & $*_{\mathrm{h}}$ & $*_{\mathrm{s}}$ & $*_{1}$ \\
\hline PRM & $*_{\mathrm{t}}$ & $*_{\mathrm{k}}$ & $*_{\mathrm{m}}$ & $*_{\mathrm{n}}$ & $*_{\mathrm{n}}$ & $\varnothing$ & $*_{\mathrm{s}}$ & $*_{1}$ \\
\hline No. & 117 & 72 & 59 & 55 & 8 & 36 & 60 & 97 \\
\hline
\end{tabular}

Other PMP consonants undergo unconditioned splits in PRM. The main reflexes are summarised in Table 3.12 and Table 3.13, along with the number of times each reflex is attested. Correspondences with only a single attestation, as well as those that constitute less than 10 per cent of all cases, are not included.

Table 3.12: P(CE)MP to PRM consonant correspondences with unconditioned splits: Part 1

\begin{tabular}{|c|c|c|c|c|c|c|c|c|c|c|c|c|c|c|c|c|}
\hline env. & & & & & \multicolumn{4}{|l|}{ /\#_ } & \multicolumn{3}{|c|}{$/ \mathrm{V}_{-} \mathrm{V}$} & \multicolumn{3}{|l|}{ /\#_ } & \multicolumn{2}{|c|}{$/ \mathrm{V}_{-} \mathrm{V}$} \\
\hline PMP & $* \mathrm{p}$ & & $* \mathrm{q}$ & & *b- & & & & *-b- & & & $* \mathrm{~d}-$ & & & $*$-d & \\
\hline PRM & $* h$ & $* \mathrm{p}$ & $\varnothing$ & $* \mathrm{~h}$ & $*_{\mathrm{f}}$ & $* \mathrm{~b}$ & $* 6$ & $*_{\mathrm{mb}}$ & $*_{\mathrm{f}}$ & $* 6$ & $* \mathrm{mb}$ & $* \mathrm{~d}$ & $* d$ & $* \mathrm{r}$ & $*_{\mathrm{r}}$ & $* d$ \\
\hline No. & 39 & 6 & 45 & 8 & 36 & 29 & 25 & 14 & 24 & 5 & 3 & 16 & 7 & 4 & 16 & 3 \\
\hline
\end{tabular}

14 The other reflexes of PMP *k are as follows: six instances of *k $>\varnothing$, four of *k> $*^{*}$, three of *k $>*_{h}$ and two of *k $>$ *yg. None of these constitute more than 10 per cent of the reflexes of PMP *k. 
Table 3.13: P(CE)MP to PRM consonant correspondences with unconditioned splits: Part 2

\begin{tabular}{|c|c|c|c|c|c|c|c|c|c|c|c|c|c|c|c|c|}
\hline PMP & $*_{\mathrm{Z}}$ & & & $*_{j}$ & & & & $* g$ & & $* \eta$ & & & ${ }^{*} \mathrm{R}$ & & ${ }^{*} \mathrm{r}$ & \\
\hline PRM & $*_{d}$ & $*^{n}$ nd & $* \mathrm{~d}$ & $*_{d}$ & $* \mathrm{~d}$ & $*_{r}$ & $* d s$ & *ng & ${ }^{*} \mathrm{k}$ & $*_{\mathrm{n}}$ & $*_{\eta}$ & $*^{*} \mathrm{gg}$ & $\varnothing$ & $r$ & $\varnothing$ & $\mathrm{r}$ \\
\hline No. & 7 & 2 & 2 & 7 & 4 & 3 & 2 & 6 & 6 & 21 & 8 & 4 & 42 & 9 & 3 & 9 \\
\hline
\end{tabular}

The reflexes of PMP nasal-plosive clusters in PRM are given in Table 3.14. Of these, bilabial nasal-plosive clusters merge as PRM *mb, while others have a split between a PRM prenasalised plosive or plain plosive.

Table 3.14: P(CE)MP nasal-plosive clusters

\begin{tabular}{|c|c|c|c|c|c|c|c|}
\hline PMP & ${ }^{*} \mathrm{mb} / *^{*} \mathrm{mp}$ & \multicolumn{2}{|c|}{$*_{\mathrm{nd}} / *_{\mathrm{nd}}$} & \multicolumn{2}{|c|}{$* \mathrm{nt}$} & \multicolumn{2}{|l|}{ *nk } \\
\hline PRM & $* m b$ & *nd & $* \mathrm{~d}$ & $* \mathrm{t}$ & *nd & *ng & $*_{\mathrm{k}}$ \\
\hline No. & 8 & 3 & 2 & 4 & 3 & 3 & 2 \\
\hline
\end{tabular}

\subsubsection{Vowels and glides}

The reflexes of PMP vowels are given in Table 3.15 and the reflexes of vowel-glide sequences are given in Table 3.16. Of these, PMP *o undergoes an unconditioned split word finally, ${ }^{*} \mathrm{u}$ undergoes a split before $\mathrm{PMP} * \mathrm{R}$ and the vowel-glide sequences ${ }^{*}$ wa and *ya each undergo unconditioned splits (see $§ 3.5 .3 .2$ for more discussion of the reflexes of *wa and vowels before final $* \mathrm{R}$ ). Other reflexes are mostly regular. As in previous tables, correspondences with only a single attestation and those which constitute less than 10 per cent of instances are not included.

Table 3.15: P(CE)MP to PRM vowel correspondences

\begin{tabular}{|c|c|c|c|c|c|c|c|c|c|c|c|}
\hline env. & & I_R\# & /\#西 $\sigma$ & $/ \# \sigma \underline{\sigma}$ & & & /_q\# & & & /_R & \\
\hline PMP & $*_{\mathrm{i}}$ & $*_{\mathrm{i}}$ & *ə & *a & & & $*_{\partial}$ & $* \mathrm{a}$ & $* u$ & $*_{u}$ & \\
\hline PRM & $*_{\mathrm{i}}$ & $*_{\mathrm{e}}$ & $* \mathrm{e}$ & $*_{\partial}$ & $*_{\mathrm{e}}$ & $* \mathrm{a}$ & $* \mathrm{e}$ & $* \mathrm{a}$ & $* u$ & $* u$ & $*_{0}$ \\
\hline No. & 180 & 3 & 64 & 22 & 14 & 7 & 5 & 328 & 247 & 7 & 7 \\
\hline
\end{tabular}

Table 3.16: $P(C E) M P$ to $P R M$ vowel-vowel and vowel-glide correspondences

\begin{tabular}{|c|c|c|c|c|c|c|c|c|c|}
\hline PMP & $* \mathrm{w}$ & & & $*_{\mathrm{wi}} / *_{\mathrm{iw}}$ & *au/*aw & *ya & & *ay/*ai & $*_{\mathrm{uy}} /$ *yu \\
\hline PRM & $*_{0}$ & $*_{f a}$ & *wa & $*_{\mathrm{i}}$ & $*_{\mathrm{o}}$ & $* \mathrm{e}$ & $* a$ & $* \mathrm{e}$ & $*_{\mathrm{i}}$ \\
\hline No. & 9 & 3 & 2 & 7 & 25 & 4 & 2 & 22 & 7 \\
\hline
\end{tabular}

\subsubsection{Final consonants}

Most final PMP consonant are lost in PRM, though in some cases *p, *t, ${ }^{*} \mathrm{~s}, *_{\mathrm{m}}, *_{\mathrm{n}}$, $*_{\eta}$ and $*_{k}$ have been retained. Among all the Rote-Meto languages, Meto is the most conservative in retaining final consonants. Table 3.17 summarises the extent to which final consonants have been lost or retained in reconstructions in PRM. In addition to these retentions, there is one example each of final $* \mathrm{q}>*^{\mathrm{h}}\left({ }^{*}\right.$ buaq $>*$ mbuah 'betel nut') and $* \mathrm{R}>* \mathrm{~h}(*$ niuR $>*$ noh 'coconut'). 
ROTE-METO COMPARATIVE DICTIONARY

Table 3.17: Retention and loss of final consonants

\begin{tabular}{|c|c|c|c|c|c|c|c|c|c|c|c|c|c|c|c|}
\hline PMP & $* p$ & $* \mathrm{t}$ & $* \mathrm{k}$ & & & $*_{\mathrm{s}}$ & & $*_{\mathrm{m}}$ & & & $* \mathrm{n}$ & & & $* \eta$ & \\
\hline PRM & $*_{s} \quad \varnothing$ & $*_{\mathrm{t}} \quad \varnothing$ & $* \mathrm{k}$ & $* h$ & $\varnothing$ & $*_{\mathrm{s}}$ & $\varnothing$ & $* \mathrm{~m}$ & $* \mathrm{n}$ & $\varnothing$ & $* n$ & $* \mathrm{~m}$ & $\varnothing$ & $* \mathrm{n}$ & $\varnothing$ \\
\hline No. & 5 & $\begin{array}{ll}7 & 27\end{array}$ & 2 & 1 & 36 & 11 & 14 & 4 & 1 & 11 & 10 & 1 & 38 & 1 & 22 \\
\hline $\begin{array}{l}\text { Retention } \\
\text { rate }\end{array}$ & $40 \%$ & $21 \%$ & $7 \%$ & & & $48 \%$ & & $31 \%$ & & & $22 \%$ & & & $4 \%$ & \\
\hline
\end{tabular}

The change of $* p>*^{*}$ is unusual and requires further discussion. The four forms with this change are *qudip $>*$ horis 'alive, living', *ma-qudip $>*$ moris 'live, be alive', *malip $>$ *malis 'laugh, smile' and *qatip $>*$ atis 'breast beam of a loom'. All these forms have $*_{i}$ as the final vowel, and this appears to be a conditioning environment. The usual reflex of PMP *p in PRM is $* h$, and final $* p>*_{s}$ in these forms is probably assimilation of earlier $* * h$ to the place of the previous palatal vowel. The complete pathway may have been $* \mathrm{p}>* * \mathrm{~h}>* * \mathrm{c}>*_{\mathrm{s}}$.

For most of these forms the Rote-Meto languages have multiple reflexes, some of which have loss of the final consonant and some of which have retention of $* s=s$. Thus, for instance, Meto has *malis $>n$-manis 'laugh at (transitive)' and *malis $>n$-mani 'laugh, smile (intransitive)', similarly Termanu has *moris > moli 'live, be alive', molis 'life! health! (said to a child who sneezes)'. Because of such variation, it would be plausible to analyse the final /s/ in forms in which it is retained as a reflex of a suffix. While there is a suffix $-s$ in the Rote-Meto languages, this is a nominalising suffix and thus probably cannot account for final *s in forms such as Meto $n$-manis 'laugh at (transitive)'.

Further support for identifying $* \mathrm{p}>(* * \mathrm{~h})>*^{*}$ as a sound change in these forms rather than positing that the final $/ \mathrm{s} /$ is a historic suffix comes from external witnesses. Putative cases of $* \mathrm{p}>s /$ i_ \# have been identified in several other languages of the region, none of which are known to have a suffix $-s$. Additionally, all languages with this change are members of the Timor-Babar subgroup to which Rote-Meto belongs (see §3.5.3), which is defined by $* \mathrm{p}>* \mathrm{~h}$. Thus, the proposed assimilation of $* * \mathrm{~h}>*_{\mathrm{s}} / \mathrm{i}$ \# may have occurred at the level of Proto-Timor-Babar. Forms for which the change of final $* \mathrm{p}>s$ /i_\# have been identified in other languages are as follows:

- *qudip > Semau Helong nulis 'life' (nuli 'live', kuli? 'alive'), Fehan Tetun horis 'living', East Tetun houris 'alive, with life'

- *ma-qudip > Fehan Tetun moris 'live, be alive', East Tetun moris $\sim$ mouris 'live, be alive, exist, be born'

- *qatip > Helong, Fehan Tetun atis 'breast beam of a loom', East Tetun atis 'fine cloth, or cloth still on the loom; a part of the loom', Galolen atis 'weaving loom'

\subsubsection{Sound change and language shift}

The correspondences between PMP and PRM raise two problems that require explanation. Firstly, there are a large number of unconditioned splits between PMP and PRM. PMP $* \mathrm{~b},{ }^{*} \mathrm{~d}, *_{\mathrm{j}},{ }^{*} \mathrm{~g}$ and $*_{\mathrm{y}}$ have all undergone unconditioned splits with each of the PRM reflexes relatively well attested, even if one reflex is a majority. Similarly, ${ }^{*} \mathrm{p},{ }^{*} \mathrm{q}$ and $*_{z}$ also show unconditioned splits, though for these correspondences one reflex clearly 
predominates. Among the vowels and glides there are fewer splits. Nonetheless, word final PMP *o has undergone a split between PRM *e and *ə, while *wa has undergone a three-way split.

Secondly, several PRM proto-phonemes are not particularly well represented in inheritances from PMP. The prenasalised plosives do not have a robust and consistent source in PMP. In particular, *nd has no robust PMP source and initial *ng is mostly unexpected based on inheritance from PMP. Only about a third of prenasalised plosives have a PMP source, and furthermore — as discussed in Edwards (2018a:398f) — PMP forms that develop a prenasalised plosive in PRM often show additional irregularities. Similarly, the mid-vowel $*_{0}$ is mostly unexpected based on inheritance from PMP.

The two main issues that are not accounted for by inheritance from PMP are the large number of splits between PMP and PRM, as well as the fact that the PRM phoneme inventory has been restructured when compared with its PMP source. In this section I propose a tentative scenario that may go some way towards partly explaining these two issues. I do not think that this scenario provides a complete explanation and it is almost certainly an over-simplification of a much more complex process. With those disclaimers in mind, I present it as a starting point for understanding the history of PRM and other languages in this region.

PRM is partly a result of large-scale language shift from at least one non-Austronesian language to the incoming Austronesian language, which was to become PRM. The large number of unconditioned splits between PMP and PRM is due to adaptation of the new Austronesian phonological system to the pre-Austronesian phonological system. The 'Austronesian' system probably contrasted plain voiced plosives *b $*^{*} *^{\mathrm{g}}$ with voiceless plosives $*_{\mathrm{p}} *_{\mathrm{t}} *_{\mathrm{k}}$, as is reconstructed for PMP, while the 'non-Austronesian' system probably contrasted three series of plosives: voiceless $*_{\mathrm{p}} *_{\mathrm{t}} *_{\mathrm{k}}$, prenasalised $*_{\mathrm{mb}}{ }^{*} \mathrm{nd}$ $*^{*} \mathrm{~g}$, and imploded $*^{*} *^{*}$, as is areally common in this region.

The way the incoming Austronesian phonological system may have been transformed by speakers of the pre-Austronesian languages can be illustrated with the labial obstruents. The Austronesian language probably had a single bilabial obstruent $/ \mathrm{b} /$ with both plosive $[\mathrm{b}]$ and fricative $[\beta]$ realisations. The fricative realisation was probably most common intervocalically while there was some variation word initially. That is, the Austronesian language $\mathrm{had} / \mathrm{b} / \rightarrow[\beta] / \mathrm{V}_{-} \mathrm{V}, / \mathrm{b} / \rightarrow[\mathrm{b}] \sim[\beta] / \#+{ }^{15}$

The pre-Austronesian language(s), on the other hand, contrasted three labial obstruents: $/ 6 /, / \mathrm{mb} /$ and $/ \mathrm{f} /$. As speakers of this language began to acquire the new Austronesian language, the fricative realisation $[\beta]$ of $/ \mathrm{b} /$ may have been interpreted as $/ \mathrm{f} /$, while the plosive realisation was interpreted as either $/ \mathfrak{b} /$ or $/ \mathrm{mb} /$. As language contact intensified and the non-Austronesian speakers became more fluent in the new Austronesian language, they may have acquired /b/ 'correctly' as /b/, thus leading to the PRM system with $* b, * 6,{ }^{*} \mathrm{mb}$ and $* \mathrm{f}$.

15 Modern-day varieties of Meto similarly have plosive and fricative realisations of voiced stops $(\$ 2.4 .2)$. 
As previously stated, the scenario sketched in the previous three paragraphs is highly tentative and is almost certainly a simplification. As currently presented, language shift is treated as a unitary event that affected an entire population at a single time. This is a gross over-simplification. Instead, different speakers would have acquired the new Austronesian language with different degrees of proficiency and at different times.

Thus, for instance, as discussed in Edwards (2018a:398f), forms with PMP *b > PRM ${ }^{*} \mathrm{mb}$ often show additional irregularities. If we envisage a scenario in which the new Austronesian language was learnt at first by only a minority of the non-Austronesian speaking population, forms with PMP *b $>$ PRM *mb may represent the first layer of vocabulary acquired by the Austronesian learners before they were fully proficient. These forms may have then spread to non-Austronesian speakers who were not otherwise learning the Austronesian language. Forms with PMP *b $>$ PRM *6 do not usually show additional irregularities and these may represent forms that were acquired later when more of the non-Austronesian-speaking population was learning the Austronesian language, while forms with $\mathrm{PMP} * \mathrm{~b}=\mathrm{PRM} * \mathrm{~b}$ may have been the most recent acquisitions perhaps being brought into PRM by bilingual children.

Furthermore, this scenario treats every sound change that has occurred between PMP and PRM as due to contact. This was almost certainly not the case. It is likely that there was a mix of changes due to contact and changes due to 'natural' causes. Thus, for instance, the shift of $* \beta>*$ f could also quite easily have come about without contact as intervocalic devoicing of fricatives is not uncommon.

While this represents one step towards a more realistic picture, I must emphasise that this scenario remains too simplistic. Many different variables remain unaccounted for. What about the possibility that different Austronesian learners had different accents (e.g. some with $* b>* m b$, other with $* b>* 6)$ ? What effect would the children of the Austronesian learners have had on the formation of PRM? What could have been the effects of borrowing between the Austronesian and non-Austronesian languages? What about the effects of acquisition of the non-Austronesian language by Austronesian speakers? What about the possibility of multiple language shift events, such as one at the level of ProtoTimor-Babar and another at the level of PRM?

There is a significant mismatch between the data revealed by a bottom-up reconstruction and that expected by simple 'normal' top-down inheritance from PMP. This mismatch demands explanation. Language shift with substrate transference of non-Austronesian features is almost certainly part of the explanation.

\subsubsection{Subgrouping within Malayo-Polynesian}

In this section I discuss the position of the Rote-Meto family within the Austronesian language family and its relationships with other nearby languages. Rote-Meto can be placed in a larger Timor-Babar family, which includes most, but not all, the Austronesian languages of Timor and Southwest Maluku. Rote-Meto is justified as a separate subgroup within Timor-Babar on the basis of two shared phonological innovations. 


\subsubsection{Timor-Babar}

Within the greater Timor region, languages belonging to two families are spoken: Austronesian languages; and Timor-Alor-Pantar languages, which are non-Austronesian, or 'Papuan'. In addition, there are also Austronesian-based contact creoles in the region that do not fit normal genetic classification.

Among the Austronesian languages of this region, four primary branches of (CE)MP can be identified on the basis of shared sound changes from PMP, one of which is TimorBabar, within which Rote-Meto is located. The other branches of (CE)MP within the greater Timor region are Bima-Lembata, Central Timor and Tanimbar-Bomberai. Other branches of (CE)MP that are coordinate with these branches are found in other parts of eastern Indonesian.

The Bimba-Lembata subgroup contains the languages of Bima, Sumba, Flores and the Solor archipelago, as well as Hawu and Dhao, the last of which is spoken just to the west of Rote. The Central Timor subgroup contains four languages of central Timor: Welaun, Kemak, Tokodede and Mambae. The Tanimbar-Bomberai subgroup contains Yamdena and Fordata of the Tanimbar Islands, as well as Kei and several languages of the Bomberai Peninsula of New Guinea. The Timor-Babar subgroup contains all other Austronesian languages of this region extending from Rote to Wetar and across to Selaru in the east.

The distribution of the different language families in the greater Timor, along with their member languages, is shown in Map 3.1. A tree showing the higher order subgroups in the region along with primary subgroups of Timor-Babar is given in Figure 3.6. As discussed in $\S 1.1$ and $\S 1.5 .3 .3$, for the purposes of this dictionary I am agnostic regarding the putative intermediate nodes of CEMP and CMP. 


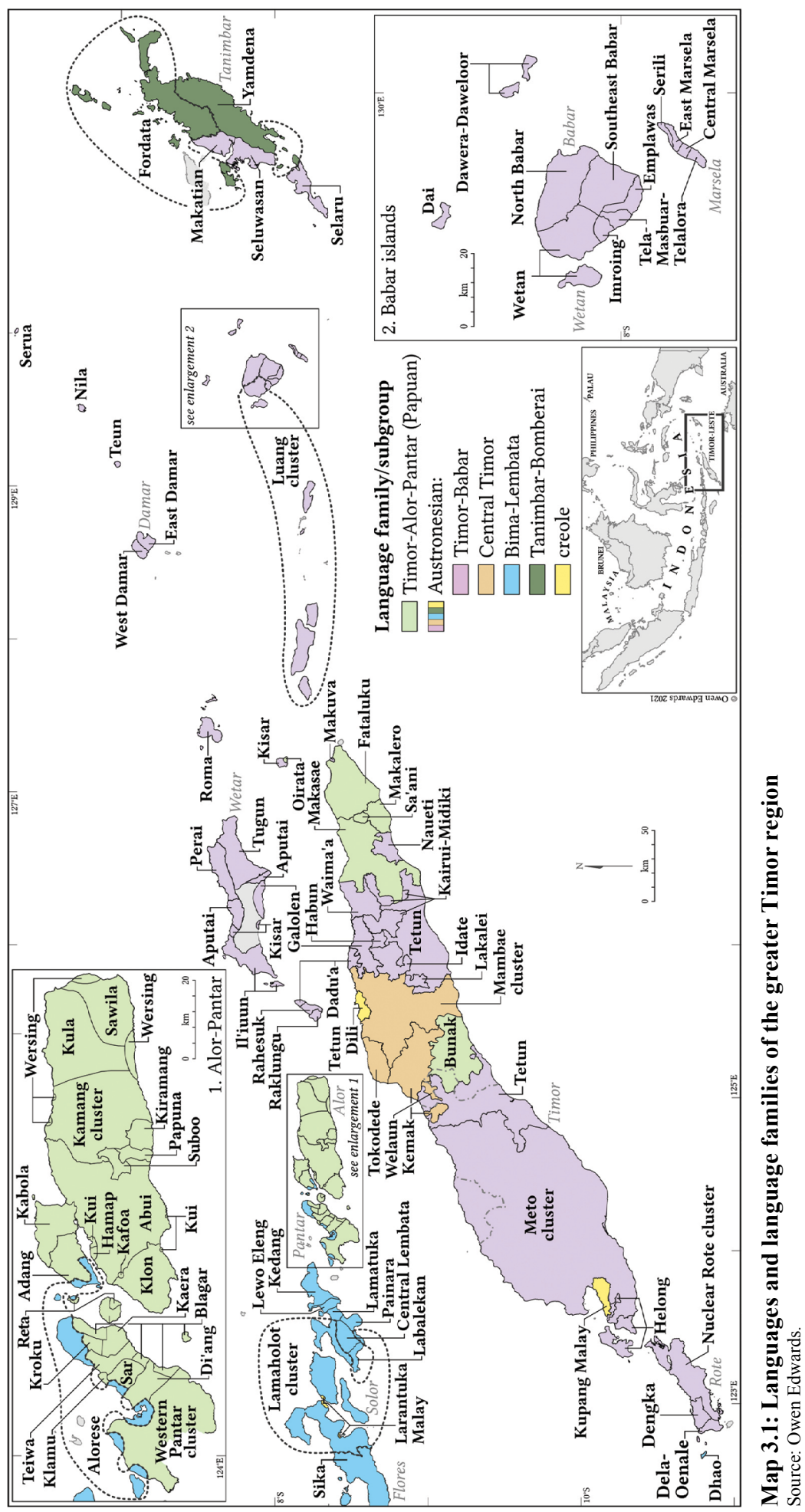




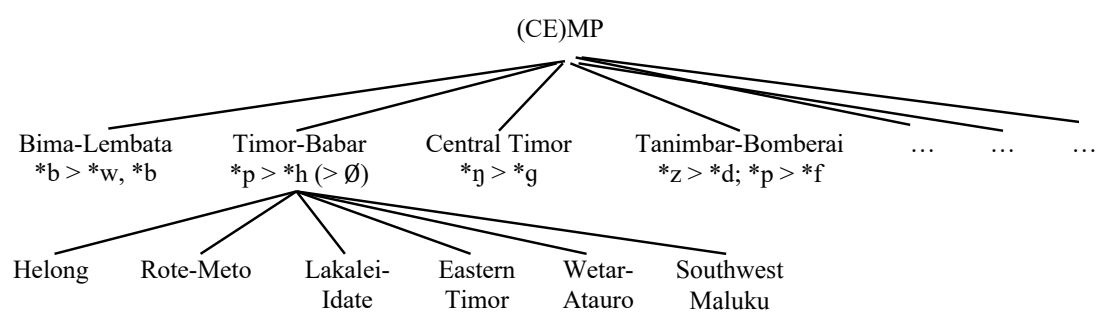

Figure 3.6: Timor-Babar and Rote-Meto within Malayo-Polynesian

It is beyond the scope of the present work to justify the proposed classification shown in Figure 3.6. In this section I only discuss the evidence for Timor-Babar as a valid subgroup of (CE)MP, and the evidence for Rote-Meto as a valid subgroup within TimorBabar. Evidence for Bima-Lembata has been presented by Fricke (2019:229ff), Central Timor by Edwards (2019:42ff) and evidence for Tanimbar-Bomberai (under different names) is presented in Mills (1991) and Blust (1993:276f).

The Timor-Babar subgroup is supported by reflexes of PMP *p as $h$ or $\varnothing$, with several languages showing a split of $* \mathrm{p}>h, \varnothing$. To account for this, we can posit $* \mathrm{p}>*_{\mathrm{h}}$ in Proto-Timor-Babar with subsequent $* \mathrm{~h}>\varnothing$ where relevant. The change $* \mathrm{p}>h$ is crosslinguistically well attested. However, within the geographical context of the greater Timor region, this sound change does set Timor-Babar apart. The Bima-Lembata and Central Timor subgroups retain *p $* * p$ (Fricke 2019:176f; Edwards 2019:43f) and the Tanimbar-Bomberai subgroup has $* \mathrm{p}>*_{\mathrm{f}}$ (Mills 1991:243). Given that $*_{\mathrm{p}}>*_{\mathrm{f}}>*_{\mathrm{h}}$ is a natural sequence of changes, it may be possible at a higher level to link Timor-Babar with Tanimbar-Bomberai on the basis of shared $* p>{ }^{*}$. However, even if this is done, Timor-Babar would still be a valid subgroup on the basis of shared *f $>* h$. Reflexes of word initial *ma-p must be treated separately to those of *p as most Timor-Babar languages have *ma-p $>p$ or *ma-p $>b$, probably as a result of an intermediate nasalstop cluster stage, either **mp and/or **mb.

Examples of $* \mathrm{p}>h, \varnothing$ in most languages of the Timor-Babar subgroup are given in Table 3.18 to illustrate this defining sound change. Languages are grouped according to their likely subgroup within Timor-Babar. Non-cognate forms with the same gloss as the PMP reconstructions are given in brackets, to indicate potential lexical replacement.

Table 3.18: Reflexes of *p in Timor-Babar

\begin{tabular}{|c|c|c|c|c|c|c|}
\hline Gloss & 'four' & 'seven' & $\begin{array}{l}\text { 'how } \\
\text { much' }\end{array}$ & 'banana' & 'fire' & 'thin' \\
\hline PMP & *əpat & *pitu & *pija & *punti & *hapuy & *ma-nipis \\
\hline Proto Timor-Babar & *haat & *hitu & *hija & *hundi & *ahi & *manihis \\
\hline \multicolumn{7}{|l|}{ Rote-Mето } \\
\hline PRM & *haa & *hitu & *hida & *hundi & *ahi & *nihis \\
\hline \multicolumn{7}{|l|}{ HELONG } \\
\hline Funai Helong & aat & $i t u$ & ila & (bua) & $a i$ & mnihis \\
\hline Semau Helong & aat & $i t u$ & ila & (bua) & $a i$ & nihis \\
\hline
\end{tabular}


ROTE-METO COMPARATIVE DICTIONARY

\begin{tabular}{|c|c|c|c|c|c|c|}
\hline Gloss & 'four' & 'seven' & $\begin{array}{l}\text { 'how } \\
\text { much' }\end{array}$ & 'banana' & 'fire' & 'thin' \\
\hline \multicolumn{7}{|l|}{ EASTERN TIMOR } \\
\hline East Tetun & haat & hitu & hira & hudi & ahi & mihis \\
\hline Fehan Tetun & haat & hitu & hira & hudi & $h a i^{\dagger}$ & ni?is \\
\hline Habun & ha?a & hitu & hira & (muku) & $a h i$ & \\
\hline Waima'a & kai-haa & kai-hitu & kai-hire & hudi & (daha) & nihi \\
\hline Midiki & kai-haa & kai-hitu & kai-hira & hudi & (yaha) & \\
\hline \multicolumn{7}{|l|}{ LAKALEI-IDATE } \\
\hline Lakalei & aat & hitu & ila & & wai & \\
\hline Idate & aat & hitu & yila & $(m u ? u)$ & wai & mihis \\
\hline \multicolumn{7}{|l|}{ Wetar-Atauro } \\
\hline Galolen & ih-aat & i-hitu & i-hila & (mu?u) & i-morin & miis \\
\hline Tugun & f-aat & fa?-itu & far-ira & (тии) & ai-moriy & miis \\
\hline Perai & $a a k$ & itu & far-ira & (muu) & ai-moriy & miis \\
\hline Aputai & $a a k$ & itu & fa?-ira & (muи) & ai-moriy & miis \\
\hline Dadu'a & war-itu & $w$-aak & wa?-ira & (тuи) & ai-mori & miih \\
\hline Raklungu & ha-itu & $h-a a ?$ & ha-ira? & $(m u ? u)$ & $a-m o r i$ & \\
\hline Rahesuk & he?-itu & h-aat & he?-ira & (mu?u) & $i$-mori & ne-mniis \\
\hline \multicolumn{7}{|c|}{ SOUTHWESt MALUKU } \\
\hline Makuva $^{\ddagger}$ & & & & utu & $a-k e$ & \\
\hline Selaru & ena-at & $i t w$ & (enai) & (kwe) & ay & mani $\sim$ nias \\
\hline East Damar & wo-atu & wo-itu & $w i$ & $(w u)$ & $a i$ & na-mnihu \\
\hline Roma & wor-atta & wo?-itu & wo?-ira & (wui) & $a i$ & mnihana \\
\hline Kisar & wor-akka & wor-iku & wo-ira & (тu?u) & $a i$ & na-mnisa \\
\hline Luang & wog-ata & wo-itu & wo-iro & $u d i$ & $a i$ & mnihə \\
\hline Serua & natu & itu & ira & (wia) & $a i$ & ni-mnisu \\
\hline Teun & natu & yitu & yiru & (fiwa) & wai & ni-mnis $\sim$ nisu \\
\hline Nila & watu & itu & ira & (hia) & wai & n-nisu \\
\hline West Damar & wi-oto & $w i-i t i$ & wo-ire & $(u s-o)^{\#}$ & $O S-O^{\#}$ & minidoi \\
\hline Dawera & aty & ity & irei & (urus) & (am) & me-lid lid \\
\hline Dai & at & ity & ire & (diamny-on) & (amp-on) & me-mnit \\
\hline North Babar & ato & $i t i$ & ire & ud wia & (ami-ai) & ne-mnido \\
\hline Central Marsela & $f i-a k$ & wo-ie & fi-era & $u t$ & ei-ei & ne-mni nit \\
\hline East Marsela & $w u-a$ & $w o-i k$ & fi-era & $u t$ & $u i$ & le-mni nit \\
\hline Serili & bor? $-a$ & bo?-ot & (nomia) & $u t$ & ui-e & me-li lit \\
\hline Southeast Babar & $w o-a x$ & wo-ixy & (nomya) & uty & $u i$ & (pilpil) \\
\hline Emplawas & wi-ek & wo-ik & vi-era & uti-e & $u i-e$ & (piliti) \\
\hline Tela-Masbuar & wi-eky & wo-iky & wo-iri & uti-e & ui-e & ne-minita \\
\hline
\end{tabular}

${ }^{\dagger}$ Initial $/ \mathrm{h} /$ in East Tetun $h a ? i$ 'fire' is an insertion before a glottal stop.

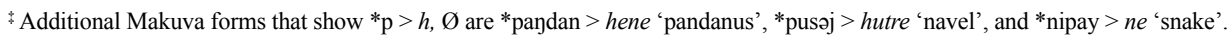
\#West Damar os-o 'fire' has regular $* \mathrm{i}>s /-\mathrm{V}$ and comes thus from *hapuy $>*$ ahi $>* *$ oi. Despite the superficial similarity between PMP * punti and West Damar us-o 'banana', this latter form is from earlier **ui, which is cognate with Roma wui, and East Damar $w u$. These forms are not inherited from *punti. 
Examples of the reflexes of PMP *p in illustrative languages of other subgroups of (CE)MP neighbouring Timor-Babar are given in Table 3.19 to show that they do not share $* \mathrm{p}>h, \varnothing$. PMP $* \mathrm{p}$ is retained as $p$ in most forms in Bima-Lembata, and Central Timor. While some languages have subsequent changes, *p must be reconstructed for each of these subgroups. The only exception is Central Timor *pitu > hitu 'seven', which may represent later borrowing in daughter languages from Tetun. (Note also that all Welaun forms except hoat 'four' are identical to Fehan Tetun forms from which they may be borrowed.) Tanimbar-Bomberai languages usually have $* \mathrm{p}>f$, with subsequent sporadic $* \mathrm{f}>\varnothing$ in Yamdena.

Table 3.19: Reflexes of *p in subgroups neighbouring Rote-Meto

\begin{tabular}{|c|c|c|c|c|c|c|}
\hline *Gloss & 'four' & 'seven' & $\begin{array}{l}\text { 'how } \\
\text { much' }\end{array}$ & 'banana' & 'fire' & 'thin' \\
\hline PMP & *opat & *pitu & *pija & *punti & *hapuy & *ma-nipis \\
\hline BIMA-LEMBATA & *opat & *pitu & *pida & *punti & *api & *manipis \\
\hline Bima & upa & pidu & pila & (kalo) & afi & nipi \\
\hline Manggarai & paat & pitu & pisa & punti $^{\dagger}$ & api & mipis \\
\hline Sika & (hutu) & pitu & pira & (mu?u) & api & (blelar) \\
\hline Lewotobi Lamaholot & paa & pito & pira & (muko) & ape & mənipi \\
\hline Kedang & apa? & pitu & pie & (тиРи) & api & mipi \\
\hline Central Lembata & paat & pito & pira & (muku) & ape & mipiv \\
\hline Kambera & pat-u & pihu & piray & (kaluu) & epi & manipa \\
\hline Hawu & әра & pidu & pori & $(m u ? u)$ & $a i$ & menii \\
\hline Dhao & opa & pidzzu & pari & (muи) & $a i$ & manii \\
\hline Central Timor & *pəat & *hitu & *pija & & *api & \\
\hline Welaun & hoat & hitu & hira & hudi & ha?i & ni?is \\
\hline Kemak & paat & hitu & pila & (тии) & api & (diren) \\
\hline Tokodede & paat & (hoho ruu) & piil & & api & (lihire) \\
\hline Northwest Mambae & paat & (hoho rua) & ar-piil & (muuk-a) & $a e p-a$ & (hilire) \\
\hline Central Mambae & faat & hitu & ar-fiil & $($ (тии- $a)$ & aif-a & $(\text { mihis })^{\ddagger}$ \\
\hline South Mambae & paat & (liim nai rua) & piil & (muu) & aap & (hilire) \\
\hline TANIMBAR-BOMBERAI & *faat & $*$ fitu & $*$ fija & $*$ fundi & *afuy & *manifis \\
\hline Yamdena & fat & $i t w$ & fir & fundi & $a u$ & manisik \\
\hline Fordata & (i)fa?at & (i)fitu & ifira & (mu?u) & yafu & masnifit \\
\hline Kei & faak & fit & $n$-fir & $(m u k)$ & yaf & manifin \\
\hline Sekar & fat & (buteras) & firas & fudi & yafi & (bair) \\
\hline
\end{tabular}

$\dagger$ The Pacar dialect of Manggarai has punti 'banana'. Other Manggarai varieties have muku 'banana'.

* Central Mambae mihis 'thin' is probably a borrowing from East Tetun mihis 'thin'.

In Edwards (2018c) I adduced the change $* \mathrm{n} / *^{*} \mathrm{n}>n$ as evidence for Timor-Babar. However, since publication of that paper a closer examination of a wider range of data has shown that this cannot be cited in support of Timor-Babar. While PMP *y usually undergoes change in most Timor-Babar languages, it is retained unchanged in Helong (e.g. *yajan $>$ yala 'name', *hayin > ayin 'wind') and languages of South Atauro (e.g. Raklungu 
*yajan > yai 'name' and *hanin > a $i$ 'wind'). Some languages of Southwest Maluku also have different outcomes of $* \eta$ compared with *n (Mills 2010:284ff). Similarly,

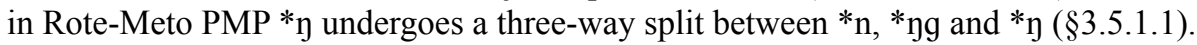

Apart from the change of $* \mathrm{p}>h, \varnothing$, the Timor-Babar languages usually share $* \tilde{\mathrm{n}}>* \mathrm{n}$, $* \mathrm{~h}>\varnothing$ and $* \mathrm{q}>\varnothing$, though there are reflexes of $* \mathrm{q}$ in some languages in some forms that indicate that a reflex of it probably needs to be reconstructed in some forms for Proto-Timor-Babar. Most Timor-Babar languages also have medial $* \mathrm{~d}>r$. All of these changes are extremely common among all Austronesian languages and have negligible subgrouping value. Apart from these four changes, the proto-phonology of Timor-Babar appears to be the same as that reconstructed for $\mathrm{P}(\mathrm{CE}) \mathrm{MP}$.

\subsubsection{Rote-Meto within Timor-Babar}

Within Timor-Babar, there is phonological evidence that Rote-Meto forms a distinct subgroup. The main defining change of Rote-Meto is *wa $>o$. There are 15 examples of $\mathrm{PMP}^{*}$ wa in the current database, of which nine show *wa $>o$. Six of these examples are given in Table 3.20 alongside cognates in representative languages from other subgroups of Timor-Babar. Discussion follows.

Table 3.20: Reflexes of *wa in Timor-Babar

\begin{tabular}{|l|l|l|l|l|l|l|}
\hline Gloss & 'water' & 'root' & 'dry in sun' & 'right' & 'nine' & 'spouse' \\
\hline PMP & *wahiR & *wakaR & *(pa)-waRi & *kawanan & *siwa & *qasawa \\
\hline PRM & *oe & *oka-k & *hoi & *kona & *sio & *sao \\
\hline Funai Helong & ui & (kbakat) & hui? & kanan & sipa & safa \\
\hline Semau Helong & ui & (klaput) & hui? & kanan & sipa & sapa \\
\hline Fehan Tetun & wee, ue & (abut) & ha-wai & kwana & siwi & fetosawa \\
\hline East Tetun & bee, uee & $($ abut) & ha-bai, ha-uai & kuana & sia & \\
\hline Waima'a & wai & (?kaka) & ?wai & wali wana & siwe & \\
\hline Lakalei & weer & (rabut) & & (kloon) & sia & \\
\hline Idate & weer & (raput) & a-wari & (he kaer) & sia & \\
\hline Galolen & wee & (amut) & (re?a) & mawana & i-sia & \\
\hline Ili'uun & eer & akar & (rese) & wana & ha-sia & \\
\hline Raklungu & (irai) & a?a & (krari) & & ha-sia & saa \\
\hline Kisar & oir & a?arn & (loilere) & malanna & wo-hii & hoo \\
\hline Luang & gero & ga?ra & gari & malgana & wo-siewa & \\
\hline S.E. Babar & wey & (wia) & (mo-yare) & & wu-si & \\
\hline Dawera & wee & (woi-el-ol) & mewael & & siuw & daw-ei \\
\hline
\end{tabular}

†Fehan Tetun fetosawa is glossed as 'sister (of a man)'.

*Dawera siuw 'nine' has irregular * $\mathrm{s}=s$ (expect * $\mathrm{s}>d$, as in *sawa $>d a w-e i$ 'husband').

The three forms not shown in Table 3.20 that also have *wa $>o$ are: *sawa 'python' $>$ *kai/sao 'green viper' (see under *sao2), PWMP *qambaway $>$ *mbao 'mango', and *hawak 'waist, back of waist' $>$ *ao 'body'. Of the seven forms that do not have *wa $>o$ three have ${ }^{*} \mathrm{w}>f$, two have ${ }^{*} \mathrm{w}=w$, one has ${ }^{*} \mathrm{w}>\varnothing$, and one has ${ }^{*}$ wa $>u$. These seven forms are: 
- $*_{\mathrm{wa}}>*_{\mathrm{f}}$

_ *walu $>*$ falu 'eight'

_ *waRi $>$ *fai 'day, time'

_ *bayawak $>$ *baiafa 'monitor lizard'

- $*_{\mathrm{W}}=*_{\mathrm{W}}$

_ *huaji $>* *$ waji $>$ *wadi 'same-sex younger sibling'

_ *wani $={ }^{*}$ wani 'bee'

- other

_ *kali-wati $>$ *kalati 'earthworm'

_ *lawaq $>* 6 o /$ lau 'spider'

Although some other Timor-Babar languages also have *wa $>o$ in some forms, there is no reason to suppose that this is due to a period of common development shared between Rote-Meto and these languages. Thus, Kisar also has *wa $>o$ in two forms in which this change occurs in Rote-Meto: *wahiR > oir 'water' and *qasawa > hoo 'marry'. However, cognates in other languages of Southwest Maluku show that this sound change occurred in Kisar after the break-up of Proto-Southwest Maluku. ${ }^{16}$ It thus cannot be assigned to a single common ancestor of Rote-Meto and Southwest Maluku.

Likewise, Wetar-Atauro languages have *wa $>o$ in reflexes of *sawa 'python', which is also seen in PRM *kai/sao 'green viper'. The available reflexes are Galolen, Ili'uun, Tugun, Aputai sao and Perai soo 'snake'. However, this is the only change among reflexes of ${ }^{*} \mathrm{~W}$ which is the same in Rote-Meto and Wetar-Atauro. It is best viewed as a case of parallel development.

Helong has *wa $>u$ in *wahiR $>u i$ 'water' and *pa-waRi $>$ hui? 'dry in sun'. Helong does not allow sequences of a mid vowel followed by a high vowel, and thus both these forms could be from intermediate **oi and **hoi? respectively, though they could also be from intermediate ${ }^{* *}$ ue or $* *$ hue?. Thus, in these two forms Helong may share ${ }^{*}$ wa $>*^{*}$ o with Rote-Meto. However, *pa-waRi $>$ hui? 'dry in sun' has irregular *R $>\varnothing$ instead of regular $* \mathrm{R}>l$ and is thus probably a borrowing from a Rote-Meto language. Thus, at best, there is one form in which Rote-Meto and Helong may share ${ }^{*}$ wa $>{ }^{*} \mathrm{o}$; reflexes of *wahiR 'water', and in this case Helong $u i$ may be from earlier **ue, in which case it would not show $*$ wa $>0 .{ }^{17}$

The usual reflex of ${ }^{*} \mathrm{w}$ in other Helong forms is ${ }_{\mathrm{w}}>f$ in Funai Helong and ${ }^{*} \mathrm{w}>*_{\mathrm{f}}>p$ in Semau Helong. A form in addition to those shown in Table 3.20 in which this sound change occurs is *hawak 'waist, back of waist' > Funai Helong afa? 'body, self', Semau Helong apa 'body, self', both of which are in contrast to Rote-Meto *ao 'body' with $*_{\mathrm{wa}}>o$.

16 Roma also has *wa $>o$ in *wahiR > ori 'water'.

17 The only other reflex of *ahi currently known in Helong is *bahi 'female, woman, wife; female of animals', which is reflected in the first syllable of Semau Helong bihata behata, Funai Helong bihata 'woman, female'. There are also two reflexes of *aqi: *taqi > Semau and Funai Helong tai 'faeces', and *baqi 'grandmother' > Semau Helong bee 'mother-in-law'. 


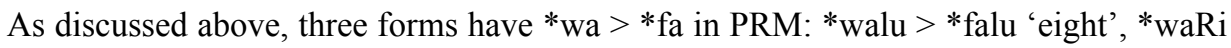
$>$ *fai 'time, day' and *bayawak $>*$ baiafa 'monitor lizard'. In addition, both forms in which *wa is retained unchanged in PRM have *wa $>*^{*}$ fa in Nuclear Rote: PRM *wani $>$ Nuclear Rote *fani 'bee' (West Rote-Meto *oni) and *huaji $>* *$ waji $>$ PRM *wadi $>$ Nuclear Rote *fadi 'same-sex younger sibling' (West Rote-Meto *odi). Of the five forms that show ${ }^{*}$ wa $>*^{*}$ fa in at least some Rote-Meto languages, three are known to occur in Helong in which they have regular ${ }^{*} \mathrm{w}>*_{\mathrm{f}}$ : *walu $>$ Funai Helong falu 'eight', Semau Helong palu 'eight', *huaji $>* *$ waji $>$ Funai Helong falin, Semau Helong palin 'samesex younger sibling' and *wani $>$ Semau Helong pani 'bee'.

The best explanation of most of this data is that *wa $>o$ was a change in progress in Proto-Rote-Meto. At the same time, ${ }^{*} \mathrm{w}>{ }^{*}$ f was probably taking place in Proto-Helong. The two proto-languages then came into contact and the change of $*_{\mathrm{w}}>f$ spread by contact from Proto-Helong into Proto-Rote-Meto and/or Proto Nuclear Rote and affected instances of $*$ wa, which had not yet undergone $*$ wa $>o$.

The change *wa $>o$ can be posited as a sound change that defines Rote-Meto. While we cannot posit *wa $>o$ in all forms, ${ }^{*}$ wa $>o$ took place in $9 / 15$ forms for which *wa has been reconstructed to PMP. Of all changes of *wa $>o$ in PRM, only the change of *wa $>o$ in *wahiR $>*^{*}$ oe 'water' is potentially shared with Helong and in this case Helong $u i$ 'water' may be from earlier **ue, in which case it would not show *wa $>o$. In addition to the nine instances of PMP *wa $>o$ there is at least one other likely instance of $* * w a$ $o$ in a form that has not yet been reconstructed to PMP. This is *osi 'garden in a village', which has cognates in Maluku with initial /wa/. (See the entry *osi for details.)

An additional sound change that provides evidence that Rote-Meto is a valid subgroup within Timor-Babar is sporadic lowering of high vowels to mid before *R. Six examples of this change are shown Table 3.21 along with examples from representative languages other languages of Timor. (No cognates of *qənuR 'animal path, trail' are yet known in other languages of Timor.)

Table 3.21: Reflexes of * $u R$ and *iR /_\# in Timor-Babar

\begin{tabular}{|c|c|c|c|c|c|c|}
\hline Gloss & 'tail' & 'egg' & 'calcium' & 'grain head' & 'water' & 'trail' \\
\hline PMP & *ikuR & *qatəluR & *qapuR & *buliR & *wahiR & *qənuR \\
\hline PRM & $*_{\mathrm{ik} o-\mathrm{k}}$ & $*$ təlo-k & *aho & *mbule-k & $*_{\mathrm{oe}}$ & *eno \\
\hline \multicolumn{7}{|l|}{ TIMOR-BABAR } \\
\hline Helong & $i k u-n$ & tilu-n & $a o$ & bulin & $u i$ & \\
\hline East Tetun & $i k u-n$ & tolu- $n$ & $a h u$ & fulin & uee, bee & \\
\hline Waimaha & $i k u$ & thelu & $a u$ & wulin & wai & \\
\hline Lakalei & hi?on & manu telor & aur & & weer & \\
\hline Idate & (y)i?an & manu telor & wa?ur & & weer & \\
\hline Galolen & i?un & manu telun & aur & hulin & wee & \\
\hline Ili’uun & $i k u$ & telu & a?ur & hulin & eer & \\
\hline Raklungu & $i ? u ?$ & manu? telu? & $a u ?$ & & (irai) & \\
\hline Kisar & $i ? u r-n$ & kerru-n & aur & wurna & oir & \\
\hline Luang & li?iru & ternu & auru & wurani & gera & \\
\hline Southeast Babar & iy & kely & uir & & wey & \\
\hline
\end{tabular}




\begin{tabular}{|l|l|l|l|l|l|l|l|}
\hline Gloss & 'tail' & 'egg' & 'calcium' & 'grain head' & 'water' & 'trail' \\
\hline CENTRAL TImor & hi?u- $n$ & man tolu- $n$ & $(\text { haul })^{\dagger}$ & fulin & wee & \\
\hline Welaun & hi?o- $n$ & telo- $n$ & (rapo) & hulen & bia, bea & \\
\hline Kemak & iko & manu telo & (rapo) & & ee & \\
\hline Tokodede & io & maun telo & (gau) & & eer & \\
\hline South Mambae &
\end{tabular}

$\dagger$ While both Welaun haul and South Mambae gau 'calcium, mineral lime' are ultimately from *qapuR, neither are regular and both are probably borrowed from an unidentified intermediate source. Kemak and Tokodede rapo 'calcium, mineral lime' are either from *dapuR 'hearth' with semantic shift via intermediate 'ash in hearth' > 'ash' (also attested in Meto, see PRM *raho) or are irregular from *dabuk reconstructed to PMP by Wolff (2010:778).

The two forms not shown in Table 3.21 with this lowering are *bibiR $>$ *6ife 'lip' and *dapuR $>$ *raho 'three stone hearth'. Thus, there are eight forms that have lowering of mid vowels before *R. Apart from these eight forms there are six forms in which a high vowel is retained as high before $* \mathrm{R}$. These six forms are listed: ${ }^{18}$

- *muRmuR > *mumu 'gargle, rinse the mouth'

- *tuquR $>*$ tuu 'dry'

- *busuR $>*$ busu 'bow for cleaning cotton'

- *kuluR > Proto-Meto *kunu 'breadfruit'

- *qizuR $>$ *midu 'saliva, spit out', Proto-Meto *ninu 'spit out'

- *qusiR > *usi 'pursue'

The penultimate vowel of all these forms is high. While this provides a conditioning environment for why the final vowel was not lowered in these forms, there are also three forms with a penultimate high vowel that $d o$ have lowering of final mid vowels (*bibiR > *6ife 'lip', *buliR > *mbule-k 'grainhead', and *ikuR > *iko-k 'tail').

Within Timor-Babar, Lakalei and Idate also have vowel lowering before $* \mathrm{R}$ in reflexes of *qatəluR 'egg' and *ikuR 'tail' but do not have it in reflexes of *qapuR. No other Timor-Babar languages have lowering of final high vowels, with the possible exception of Helong *qapuR > ao 'calcium, mineral lime', which is identical to Meto ao 'calcium, mineral lime' and may be a loan. While we could subgroup Lakalei-Idate and Rote-Meto together on the basis of lowering of high vowels to mid, this would currently rest on only two forms.

All languages of the Central Timor subgroup also have lowering of high vowels to mid before final $* \mathrm{R}$, with the exception of Welaun. Indeed, Lakalei and Idate may have acquired such vowel lowering due to influence from Mambae, with which they are in contact. Vowel lowering before $* \mathrm{R}$ is a regional feature of west and central Timor. Nonetheless, even if vowel lowering before $* \mathrm{R}$ in Rote-Meto is due to contact with Central Timor, the fact that it had occurred in a lexically specific subset of words by the time of Proto-Rote-Meto can thus be taken as supporting evidence for Rote-Meto as a subgroup. 
Other sound changes shared between all members of Rote-Meto are common throughout the region. The change of $* \mathrm{R}>\varnothing$ is found in most languages of the Timor mainland. ${ }^{19}$ The change of $*_{\partial}>e$ in penultimate syllables is found in all other Timor-Babar languages except Tetun and Habun, which have $*_{\partial}>o$. The other changes that occur in RoteMeto are $*_{\mathrm{q}}>\varnothing, h ; * \mathrm{~h}>\varnothing$, and medial $*$-d- $>*$ r. Some of these changes had probably occurred by the level of Proto-Timor-Babar and in any case are extremely widespread among Austronesian languages and are of negligible subgrouping value.

In summary, within the greater Timor region, there is phonological evidence for a Timor-Babar subgroup on the basis of shared $* p>h, \varnothing$. This subgroup contains all the Austronesian languages of Timor and Southwest Maluku, except for the languages that belong to the Central Timor subgroup (Welaun, Kemak, Tokodede and Mambae). RoteMeto can in turn be identified as a valid member of Timor-Babar on the basis of shared ${ }^{*}$ wa $>*_{0}$ and lowering of high vowels before $* \mathrm{R}$, though neither of these changes take place in all Proto-Roto-Meto forms.

19 The only languages of the Timor mainland that do not have $* \mathrm{R}>\varnothing$ are Helong $(* \mathrm{R}>l)$, Lakelei-Idate $(* \mathrm{R}=r)$, Mambae $(* \mathrm{R}>\varnothing, r)$ and Makuva $(* \mathrm{R}=r)$. 
4

Rote-Meto - English 
*aa PRM. the; marker of definiteness.

$=\mathbf{a}$ Termanu. singular form of the definite article. (J:1)

$=\mathbf{a}$ Landu . nominal article. Frequently occurs on nouns in the citation form. [Form: Stress remains on the penultimate syllable of the host. In citation form the enclitic is often non-syllabic, e.g. ani=a ['?anip] 'the wind'.] (own field notes)

$=\mathbf{a}$ Dela . the, a (known referent).

$=\mathbf{a a}$ Ro'is Amarasi. zero person nominal determiner. Usage: Frequently occurs with nouns in the citation form during wordliststyle elicitation. [Form: This enclitic surfaces as $=\mathbf{i a},=\mathbf{e a}$ or $=\mathbf{0 a}$ after nouns after stems of a certain phonological shape, thus confirming that this enclitic has two underlying vowels. An example is atoni? 'man, person' + $=\mathbf{a a} \rightarrow$ atoon $\mathbf{P}=\mathbf{i a}$.]

$=\mathbf{a a}$ Kotos Amarasi. zero person nominal determiner which occurs on nouns that are both given/ accessible (in the sense of Chafe 1994) and definite. Cannot occur on referents which have the pragmatic function of focus (= 'The semantic component of a pragmatically structured proposition whereby the assertion differs from the presupposition' (Lambrecht 1994:213)). Usage: In Kotos Amarasi from Nekmese' village (where the bulk of my data comes from) $=\mathbf{a a}$ is used less frequently than in other varieties of Meto. Instead, the first person nominal determiner $=\mathbf{i i}$ is most frequent, followed by third person $=\mathbf{e e}$.

=aa Kusa-Manea. zero person nominal determiner. Usage: Only post-nominal determiner currently known in Kusa-Manea. Nouns are almost always cited with this determiner.

*aem PRM. tame, domesticated. Etym: *qayam 'domesticated animal' (PWMP).

ae-k Termanu. native, own; the opposite of foreign, belonging to the house, the opposite of running around in the wild; cultivated, the opposite of growing in the wild (of a plant). (J:3)

ae-? Korbafo.

ae-k Bokai.

ae-? Bilbaa.

ae-? Rikou.

ae-k $B a^{\prime} a$.

ae-k Tii.

ae-? Dengka.

ae-? Oenale.

aem Kotos Amarasi. tame.

aem Molo. tame. (M:4)

Out-comparisons:

baen Helong. [irr. from PMP: Ø $>$ b] (J:3)

*afa CER. insipid. [History: Blust and Trussel (ongoing) connect the Rote form with PMP *qambaR (a doublet of $*$ tabaR) but this requires irr. ${ }^{*} \mathrm{mb}>f$. Similarly any connection with $*$ tabaR would require irr. $* \mathrm{t}>\varnothing$.]

afa afa Termanu. without odour or taste; insipid. ( $\mathrm{J}: 3$ )

\section{Out-comparisons:}

<kàba> Kambera. insipid, tasteless. (On:73)

<kòba> Kodi. [Note: also in Mangili, Lewa, Anakalang, Mamboru, and Weyewa]

*afi PRM. yesterday. See: *beni, *esak. Etym: *Rabiqi 'late afternoon, evening; evening meal; yesterday'. $\mathbf{a f i - k}=\mathbf{a} B a^{\prime} a$. yesterday. (J:676) afi-k Tii. afis Dengka. 
afis Oenale.

afis Dela. yesterday.

afi naa $R o^{\prime}$ is Amarasi. yesterday, the other day.

afi naa Kotos Amarasi. yesterday.

afi, aif neno Molo. lately, recently. (M:5)

Out-comparisons:

slahin Funai Helong. yesterday.

lahin Semau Helong. yesterday.

klahin Bolok Helong. yesterday.

*afu PRM. ash, dust. Etym: *qabu 'ash, hearth, cinder, powder, dust; grey'.

afu Termanu. ash, dust. (J:4)

afu Korbafo.

afu Bokai.

afu Bilbaa.

afu Rikou.

afu $B a^{\prime} a$.

afu Tii.

afu Dengka.

afu Oenale.

afu Dela. dust.

afu Ro'is Amarasi. ground, earth.

afu Kotos Amarasi. soil, ground, floor.

[Note: $\mathbf{s k u k u ? ~}=$ 'dust']

afu Molo. dust, ground. (M:6)

afu, auf Kusa-Manea. ash, dust.

Out-comparisons:

ahu Semau Helong. ash, dust.

apu uban Kemak. ashes. [irr. from PMP: *b $>p$ ]

apu Kisar. ashes. [irr. from PMP: $* \mathrm{~b}>p]$

*ahi1 PRM. tread out. [Sporadic: $\varnothing>h$

/\#_V? in Ba'a]

a a?i Termanu. tread on, trample, tread out. (J:6)

a a?i Bokai.

a ai Bilbaa.

a ai Rikou. trample on, tread on. (J:6; own field notes)

ha ha?i $B a^{\prime} a$.

a $\mathbf{a}$ ?i Tii.

ai Dengka.

ai Oenale. <ume ahi> Molo. the house where the field rice is trodden out of its stalk. [Note: Jonker (1908:6) gives 'Amarasi' ahi.] (M:7)

*ahi2 PRM. fire. Etym: *hapuy. [Sporadic: $\varnothing>h$ /\#_VP in Termanu, Korbafo, Bokai, Ba'a and Lole]

ha?i Termanu. fire. (J:155)

ha?i Korbafo.

ha?i Bokai.

ai Bilbaa.

ai Rikou.

ha?i $B a^{\prime} a$.

ha?i Lole. (Zacharias et al. 2014)

a?i Tii.

ai Dengka.

ai Oenale.

ai Dela. fire.

ai Ro'is Amarasi. fire.

ai Kotos Amarasi. fire.

ai Molo. fire. (M:7)

ai Kusa-Manea. fire.

Out-comparisons:

ai Semau Helong. fire.

haPi Fehan Tetun. flame, fire. (Mo:48)

ahi East Tetun. fire. (Mo:1)

ai Kisar. fire.

ai Ili'uun. fire. (dJ:111)

*aho PRM. mineral lime, calcium. Etym:

*qapuR. [Sporadic: *u $>*_{\mathrm{o}} /{ }^{*} \mathrm{R} \#, \varnothing$

$>h$ /\#_V? in Termanu, Korbafo, Bokai,

Ba'a and Lole]

ha?o Termanu. mineral lime. (J:162)

ha?o Korbafo.

ha?o Bokai.

ao Bilbaa.

ao Rikou.

ha?o $B a^{\prime} a$.

ha?o Lole. (Zacharias et al. 2014)

a?o Tii.

ao Dengka.

ao Oenale.

ao Dela. lime paste.

ao Ro'is Amarasi. mineral lime.

ao Kotos Amarasi. mineral lime.

ao Molo. mineral lime, chalk. (M:34) 
ao Kusa-Manea. mineral lime.

Out-comparisons:

${ }^{\circ}$ o Semau Helong. chalk, lime. Borrowed from: either Rote or Meto, as shown by irr. final ${ }^{*} \mathrm{u}$ $>o$.

ahu Fehan Tetun. lime. [irr. from $P M P: * \mathrm{p}>h / \mathrm{V} \_\mathrm{V}$ (expect?, perhaps actually a reflex of *qabu 'ash, dust')]

ahu East Tetun. lime, calcium. (Mo:2)

aPur Ili'uun. lime. (dJ:113)

haul Welaun. mineral lime. [irr.from PMP: * $\mathrm{q}>h ; * \mathrm{R}>l$ ] [History:

The irregular correspondences indicate that this is a loan from an intermediate source, but no likely donor language has been identified. The closest match is Bunak hau, which also has initial $h$ but this Bunak form too is ultimately a loan from an unidentified Austronesian source.]

aur Lakalei. mineral lime. [Note: language of east Timor ISO 639-3 [lka]] (Klamer 2002)

*ahu CER. dew. See: *aygum. Etym: *hapun.

aPu(s) Termanu. dew. Usage: poetic. [Note: The non-poetic word for 'dew' is dinis.] (J:17)

*ala Morph: *n-ala. PRM. get, fetch, take; converts verbs into an accomplishment carried out with purposeful intent (Jacob and Grimes 2011). Etym: *ala[q/p].

n-ala Termanu. he (she) gets, takes. (J:374)

n-ala Korbafo.

n-ala Bokai.

n-ala Bilbaa.

n-ala Rikou.

n-ala $B a^{\prime} a$.

n-ala Tii.

n-ala Dengka. n-ala Oenale.

n-ala (2) n-ala-? Dela. 1) take, get; accomplishment, purposeful action, toward speaker. 2) can, able.

n-ana Ro'is Amarasi. get; converts verbs into an accomplishment carried out with purposeful intent.

n-ana Kotos Amarasi. get; converts verbs into an accomplishment carried out with purposeful intent.

n-ana Molo. get, buy. (M:21)

*ama Morph: *ama-k. PRM. father. Etym: *ama.

ama-k Termanu. father. (J:9)

ama-? Korbafo.

ama-k Bokai.

ama-k Bilbaa.

ama-? Rikou.

ama-k $B a^{\prime} a$.

ama-k Tii.

ama-? Dengka.

ama-? Oenale.

ama-? Dela. father, father's brother (paternal uncle).

ama? Ro'is Amarasi. father.

ama?, ama-f Kotos Amarasi. father. ama-f Molo. father. (M:20) ama? Kusa-Manea. father.

*anak PRM. child, son/daughter, small. Etym: *anak.

ana-k (2) ana-k (3) na-ana (4) na-ana-k Termanu. 1) child in respect to its parents, the young of an animal. [Form: usually without -k as the second member of an expression.] 2) be small, small, as a specific word both predicative and attributive. [Form: usually used in the form with a final -k.] 3) have a child, adopt or consider someone as a child; have sprouts (of a plant). 4) call someone a child, address someone as 'child'. (J:10)

ana-? (2) ana(-2) Korbafo.

ana-k (2) ana(-k) Bokai.

ana-? (2) ana(-?) Bilbaa. 
ana=na $L a n d u$. son, daughter. (own field notes)

ana-? (2) a ana Rikou.

ana-k (2) ana(-k) $B a^{\prime} a$.

ana-k (2) ana(-k) Tii.

ana-? (2) ana(-?) Dengka.

ana-? (2) ana(-?) Oenale.

anak (2) ana-? Dela. 1) child.

2) small.

ana|? Ro'is Amarasi. son/daughter; small, baby (animal). [Form: Unlike other varieties of Meto, Ro'is is only known to have a single form.]

anah (2) ana|? (3) n-Pana Kotos Amarasi. 1) son/daughter, nephew/ niece from a same-sex sibling. 2) small, baby (animal). 3) address as 'child'; produce a sapling (said of plants).

anah (2) ana|? Molo. 1) child.

2) diminutive, young. (M:21f)

anah (2) ana|? Kusa-Manea.

1) child. 2) small.

*ane $n R M$. braid. Etym: *añam. [irr. from PRM: ${ }^{*} \mathrm{e}>i$ in Kusa-Manea and Molo verbal form; * $\varnothing>n / \#$ in Ba'a and Tii] [Sporadic: $* \mathrm{a}>*_{\mathrm{e}} /{ }^{*} \mathrm{C}+$ palatal] ane Termanu. braid something. (J:12) ane Korbafo.

ane Bilbaa.

ane Rikou.

nane $B a^{\prime} a$. (J:736)

nane Tii. (J:736)

aene|t Ro'is Amarasi. needle. [Form:

Final $-t$ is a productive nominaliser.

The hypothetical independent root

*ane is not attested in Meto.]

ane|t Kotos Amarasi. needle.

n-ani (2) ane|t Molo. 1) braid.

2) needle. (M:23)

ani|t Kusa-Manea. needle.

*anin PRM. wind. Etym: *hanin. ani(n) Termanu. wind. (J:13)

ani Korbafo.

anin Bokai.

ani Bilbaa. ani Rikou.

anin $B a^{\prime} a$.

anin Tii.

anin Dengka.

anin Oenale.

anin (2) na-la-ani Dela. 1) wind.

2) windy.

ainn Ro'is Amarasi. wind.

anin Kotos Amarasi. wind.

anin Molo. wind. (M:23)

anin Kusa-Manea. wind.

Out-comparisons:

ayin Semau Helong. wind, breeze.

anin East Tetun. wind, current of air. (Mo:4)

*angum CERM. dew.

a ayu-k Bokai. mist, fog. (J:677f)

a ayu-? Bilbaa.

a aku-? Rikou.

akum Fatule'u. dew.

akum Molo. evening dew. (M:17)

*ao Morph: *ao-k. PRM. body. Etym:

*hawak 'waist, back of the waist'.

ao-k Termanu. body. (J:13)

ao-? Korbafo.

ao-k Bokai.

ao-? Bilbaa.

ao-? Rikou.

ao-k $B a^{\prime} a$.

ao-k Tii.

ao-? Dengka.

ao-? Oenale.

ao-? Dela. body, self, reflexive pronoun.

ao-n Ro'is Amarasi. body.

ao-f Kotos Amarasi. body.

ao-f Molo. body. (M:34)

Out-comparisons:

afa Funai Helong. body, self. $(\mathrm{J}: 13)$

apa Semau Helong. body, self.

*arum PMeto. cuscus. See: *?|mauka|?. [Note: Schapper (2011:264) gives Meto urem without indicating which variety it is from. If this form is not an error, it attests additional complications in the cognate sets I have included 
here.] [irr. from PRM: *a $>u$ in all Meto except Kusa-Manea] [Form: Penultimate *a has been reconstructed instead of $* u$ as $* a>u$ can be motivated as a case of sporadic assimilation to the quality of the following vowel. The alternate hypothesis, ${ }^{*} \mathrm{u}>a$ in Kusa-Manea, would be phonetically unmotivated.] [History: Although PMeto $*^{*}$ is regular from PRM *nd, connecting this reconstruction with PCEMP *mansər requires positing far too many irregularities for me to make this connection.]

urum Ro'is Amarasi. cuscus.

ukum Kotos Amarasi. cuscus.

ukum Amanuban, South. cuscus.

ukum $A m f o$ 'an. cuscus.

akum Kusa-Manea. cuscus.

*aso CER. fetch water, scoop up water. Etym: *qasu. [irr. from PMP: ${ }^{*} \mathrm{u}>{ }^{*} \mathrm{o}$ ]

aso Termanu. scoop water out of a river or water source with one's hand. (J:15)

*asu CERM. dog. Etym: *asu.

asu Termanu. dog. Usage: poetic. [Note: The non-poetic word for 'dog' is busa.] ( $\mathrm{J}: 16)$

asu Ro'is Amarasi. dog.

asu Kotos Amarasi. dog.

asu Molo. dog. (M:30)

asu Kusa-Manea. dog.

Out-comparisons:

asu Semau Helong. cow. [Note: noot $\left.={ }^{\prime} \operatorname{dog}{ }^{\prime} \cdot\right]$

aus Mambae, South. dog. (Fogaça 2017:233)

asu East Tetun. dog. (Mo:23)

asu Galolen. dog.

ahu Kisar. dog.

*ata1 PRM. above, up, on top. Etym:

*atas 'high, tall'.

ata Termanu. the heavens, the top (of); above, on top of. Usage: possibly poetic. (Fox 2016b:2)

ata Dengka. above, up. (J:678)

ata Oenale. above, up. (J:678) ata-? Dela. above, upper, on, on top. ata Kotos Amarasi. up, top. Usage: Most often occurs in the phrase ata nee 'up there' and rarely in other constructions with the meaning 'top'.

*ata2 Rote. first person plural possessive pronoun; our. Etym: *ata.

ata Termanu. non-emphatic form of the first person plural inclusive pronoun ita. (J:16)

ata $B a^{\prime} a$.

ata Dengka. ata Oenale.

*atas PRM. slave. Doublet: *hatahori. Etym: *qaRta 'negrito, black person' (The semantics across a wide range of MP languages point to the original meaning being 'black/Negrito person', which, depending on the race of the speakers, was applied either to themselves or a subjugated population (Mahdi 1994:464ff).). [Sporadic: *a > e/_\# in wRM]

ata Termanu. slave. (J:16)

ata Korbafo.

ata Bokai.

ata Bilbaa.

ata Rikou.

ata $B a^{\prime} a$.

ata Tii.

ate Dengka.

ate Oenale.

ate Dela. servant, slave.

ate Kotos Amarasi. servant, slave.

ate Molo. slave. (M:32)

Out-comparisons:

ata (2) at (3) hutu ata Semau Helong. 1) underling, slave. 2) counter (classifier) for people. 3) citizen. [Note: hutun $=$ 'society, populace, citizens, common people'.]

ata East Tetun. slave, servant; shepherd, herdsman. (Mo:5)

aka, ake Kisar. slave. 
*ate Morph: *ate-k. PRM. liver. Etym: *qatay 'liver; seat of the emotions, inner self'.

ate-k Termanu. liver, also heart of person or animal in the physical (non-metaphorical) sense. (J:16)

ate-? Korbafo.

ate-k Bokai.

ate-? Bilbaa.

ate $=$ na $L a n d u$. liver. (own field notes)

ate-? Rikou.

ate-k $B a^{\prime} a$.

ate-k Tii.

ate-? Dengka.

ate-? Oenale.

ate-? Dela. liver.

aete-f $R o$ 'is Amarasi. liver.

ate-f Kotos Amarasi. liver. [Semantics:

Only refers to the physical organ, the seat of emotions is neka-f.]

ate-f Molo. liver. (M:32)

ate-f Kusa-Manea. liver.

Out-comparisons:

faten Funai Helong. liver. [irr. from PMP: $\varnothing>\left({ }^{*} \mathrm{w}\right)>f$ in Helong (also seen in Sika vaten)]

paten Semau Helong. liver.

ate-n East Tetun. liver. (Mo:5)

akin Kisar. liver.

*atis CERM. breast beam of a loom consisting of two wooden bars. Etym: *qatip 'breast beam of a back loom'. [Form: expected *p $>$ /_\#(§3.5.1.3).] atis Termanu. the part of the loom which the weaver holds in front of themself. (J:16)

atis Molo. piece of weaving equipment. (M:32)

\section{Out-comparisons:}

atis Helong. ( $\mathrm{J}: 16)$

atis East Tetun. fine cloth, or cloth still on the loom; a part of the loom. (Mo:5)

*atu PRM. charcoal, soot. atu-k (2) hade atu-k Termanu.

1) soot, the black part of a lamp or candle, what remains of burnt grass.

2) the fine dust that flies up at the stamping out of the field rice. (J:16)

(2) hade atu-? Korbafo.

ai_atu-? (2) hade_atu-? Rikou. 1) that which is left over after burning something. 2) dust from rice. (own field notes)

(2) hade_atu-? $B a^{\prime} a$.

(2) hade_atu-? Dengka.

ai atu $R o^{\prime} i$ is Amarasi. charcoal.

atu Kotos Amarasi. charcoal.

atu Molo. charcoal. (M:33)

atu Kusa-Manea. charcoal.

*au PRM. first person singular pronoun;

I, me. Etym: *aku. [irr. from PMP:

$\left.*_{\mathrm{k}}>\varnothing\right]$

au Termanu. first person singular pronoun. (J:16)

au Korbafo.

au Bokai.

au Bilbaa.

au Landu. first person singular pronoun. (own field notes)

au Rikou.

au $B a^{\prime} a$.

au Tii.

au Dengka.

au Oenale.

au Dela. first person singular pronoun.

au Ro'is Amarasi. first person singular nominative pronoun.

au (2) kau Kotos Amarasi. 1) first person singular nominative pronoun. 2) first person singular accusative pronoun.

au Molo. first person singular nominative pronoun. (M:34)

au Kusa-Manea. first person singular nominative pronoun.

*auee $C E R$. exclamation of joy or sorrow. Etym: *qaué (PCEMP).

au-é, awé (2) au au Termanu. 1) cry of joy, also of pain, e.g. while mourning for the dead. (J:17)

2) shout, shout constantly. (J:16) 


\section{B - b}

*baa Morph: *baa-k. Rote. lung. Etym: *baRaq.

baa-k (2) ba/de?e-k Теrтапи.

1) lungs of people and animals. $(\mathrm{J}: 18)$ 2) heart of people and animals. (J:19)

baa-? Korbafo.

baa-k Bokai.

baa-? Bilbaa.

baa-? Rikou.

baa-k $B a^{\prime} a$.

baa-k Tii.

faa-? Dengka.

faa-? Oenale.

Out-comparisons:

afaak Fehan Tetun. lungs.

*baba|? PMeto. parent's opposite sex sibling.

baba-f Ro'is Amarasi. parent's opposite sex sibling, spouse's parent.

baba-f Kotos Amarasi. parent's opposite sex sibling, spouse's parent.

aam baba? Molo. father-in-law. (M:39)

baba? Kusa-Manea. parent's opposite sex sibling, spouse's parent.

\section{Out-comparisons:}

baba Kemak. maternal uncle. Usage: Kutubaba and Leosibe dialects.

baba Welaun. maternal uncle.

baba Ende. father, father's brother, mother's sister's husband, male third cousin on the father and grandfather's side (FFBSS).

*badoe Morph: *na-badoe. PRM. grope, touch. Pattern: d-2. [irr. from PRM: vowel metathesis in Meto *oe $>e o ; * b$ $=b$ in Meto]

na-fa-loe Termanu. grope, touch. (J:321)

na-fa-loe Korbafo. na-fa-loe Bokai. na-fa-loe Bilbaa.

na-fa-roe Rikou.

na-fa-loe $B a^{\prime} a$.

na-fa-roe $T i i$.

na-fa-loe Dengka.

na-fa-roe Oenale.

na-breo Kotos Amarasi. grope around.

na-bleo Molo. gropes. [Form: Middelkoop (1972:265) also gives lefo 'grope' and Amfo'an anleok 'grope' (p.359) which may be connected.] (M:73)

*bafi PRM. pig. Etym: *babuy.

bafi Termanu. pig. (J:22)

bafi Korbafo.

bafi Bokai.

bafi Bilbaa.

bafi Rikou.

bafi $B a^{\prime} a$.

bafi Tii.

fafi Dengka.

fafi Oenale.

fafi Ro'is Amarasi. pig.

fafi Kotos Amarasi. pig.

fafi Molo. pig. (M:107)

fafi Kusa-Manea. pig.

Out-comparisons:

bahi Semau Helong. pig.

fahi East Tetun. pig, swine. (Mo:31)

haeh Mambae, South pig, swine. (Grimes et al. 2014b:20)

wawi Kisar. pig.

hahi Dhao. pig.

vavi Hawu. pig.

*bafo PRM. upper surface, top, above. Etym: *ba(w)baw. [irr. from PRM: $*_{\mathrm{o}}>a$ in Termanu, Bokai and KusaManea]

bafa-k Termanu. the top layer of a liquid substance. (J:21)

bafo-? Korbafo.

bafa-k Bokai.

bafo-? Bilbaa. 
bafo-? Rikou.

bafo-k $B a^{\prime} a$.

bafo-k Tii.

fafo-? Dengka.

fafo-? Oenale.

fafo Ro'is Amarasi. above.

fafo Kotos Amarasi. above, on top of.

fafo-n Molo. upper, or top part of something. (M:108)

faaf Kusa-Manea. above, on top.

Out-comparisons: wawan(ne) Kisar. top.

*bali1 PRM. again, go back, return. Etym: *baliw2 'return'. [irr. from PRM: *1 $>\varnothing$ in Termanu, Bilbaa, Rikou, Dengka, and Dela] [Semantics: Collapse/interference from reflexes of *baliz $<*$ balik. It is not always clear which forms are reflexes of which reconstruction. I have grouped them by likely semantics.]

bai Termanu. again, once more. ana mai bai he comes back (J:22)

bai Bilbaa.

bai Rikou.

Bali $B a^{\prime} a$.

bali Tii.

Fai Dengka.

fali Oenale.

fai Dela. again.

n-fani Ro'is Amarasi. again, go back, return.

n-fani Kotos Amarasi. again, go back, return.

n-fani Molo. go back. (M:110)

fain Kusa-Manea. go back, return.

Out-comparisons:

palit (2) pait Semau Helong.

1) return, go home; again, once more. 2) again, once more. [irr. from PMP: *b $>p$ (perhaps through intermediate *f); *1 $>\varnothing$ in second sense. Both irregular changes possibly point to borrowing from a Rote language] fali East Tetun. again, another time. (Mo:31)

fali Fehan Tetun. in turn.

vari (2) vəri Hawu. 1) return, go home; again, repeatedly; times. 2) anymore.

*baliz PRM. turn back, turn around. Etym: *balik 'reverse, turn around'. [irr. from PRM: ${ }^{*} \mathrm{~b}=b$ in Meto] [Semantics: Collapse/interference from reflexes of *bali1 < *baliw2. It is not always clear which forms are reflexes of which reconstruction. I have grouped them by likely semantics.]

fali (2) na-sa-fali Termanu. 1) turn back; turning back, back. (J:122f) 2) turn something around, reverse. (J:123)

fali (2) na-sa-fali Korbafo.

fali (2) na-sa-fali Bokai.

fali (2) na-sa-fali Bilbaa.

fali (2) na-sa-fali Rikou.

fali (2) na-sa-fali $B a^{\prime} a$.

fali (2) na-sa-fali Tii.

fali (2) na-sa-bali, na-sa-fali Dengka. 1) turn back; turning back, back. (J:122f) 2) reverse, translate. (J:680,123)

fali (2) na-sa-bali, na-sa-fali Oenale. 1) turn back; turning back, back. (J:122f) 2) reverse, translate. (J:680,123)

bali? Dela. return, again.

n-bani Ro'is Amarasi. let it be.

n-bani (2) na-?-bani-? (3) nabanit Kotos Amarasi. 1) let, turn, work. 2) turn something/someone around. 3) turn something/someone around, translate. [Note: Amarasi also has n-bani 'work' that may be related.]

na-bani $A m f o$ 'an. turn.

Out-comparisons:

bali Semau Helong. turn, back. Contrary to the momentum of what has been happening or assumptions. 
balik Fehan Tetun. soon, at (future time).

bale Hawu. return, go home.

*balu1 PRM. mourn the dead. Etym: *baluq (Blust and Trussel (ongoing) state: 'Jonker (1908) points out that the Korbaffo [sic] dialect of Rotinese has fali "mourn the dead", falu "orphan, widow", and he gives this as evidence that ... Termanu falu "mourn the dead" and falu "orphan, widow" are distinct words.' I agree that this is evidence they are distinct words.). [irr. from PRM: ${ }^{*} \mathrm{u}>i$ in Korbafo]

falu Termanu. mourn for the deceased. (J:124)

fali Korbafo. [History: Jonker (1908:124) considers this to be a different (non-cognate) word.] (J:124,694)

\section{falu Bokai.}

falu $B a^{\prime} a$.

(falu ?) Tii.

falu Dengka.

(falu ?) Oenale.

$<$ banu> Molo. mourning atmosphere, mourning taboo, widow. (M:49)

*balu2 PRM. widow(er). Etym: *balu. [irr. from PRM: ${ }^{*} \mathrm{~b}=b$ in Meto]

falu Termanu. widow, widower. (J:124)

falu Korbafo.

falu Bokai.

falu Bilbaa.

falu Rikou.

falu $B a^{\prime} a$.

falu Tii.

falu Dengka.

falu Oenale.

banu? Kotos Amarasi. widow, widower.

$<$ banu> Molo. mourning atmosphere, mourning taboo, widow. (M:49)

Out-comparisons:

bebalu Semau Helong. widow, widower.

faluk East Tetun. widowed, deprived. (Mo:31) balu $H a w u$. widow, widower.

wal walum Kisar. widow. [irr. from $P M P: * \varnothing>m]$

*balus CER. cover, envelop. Doublet: *mbalu. Etym: *balun 'bind, bundle, wrap in cloth; death shroud; cloth(ing)' balu Termanu. cover, envelop. (J:28)

balu Korbafo.

balu Bilbaa.

balu Rikou.

balu $B a^{\prime} a$.

Out-comparisons:

falun East Tetun. packet, parcel, bundle; $v$. wrap up, bag up. (Mo:31)

*banakudu PRM. Indian mulberry. Morinda citrifolia. Etym: *baykudu 'tree with white fruit and roots that yields a useful dye: Morinda citrifolia' (PWMP). Pattern: k-2b. [minority from PMP: *d $>*_{\mathrm{d}}($ expect $* \mathrm{r}) ; *_{\mathrm{y}}=$ ${ }^{*} \mathrm{y}$ ] [irr. from PRM: $* \mathrm{~b}>m$ in Rote; $* \mathrm{~b}=b$ in Meto; $*_{\mathrm{y}}>r$ in KusaManea] [Form: The addition of the antepenultimate vowel occurred to bring this form into line with the disyllabic CVCV foot structure of PRM. Landu, Rikou and Dengka have added $a$. (This vowel is also possibly attested by Kotos Amarasi bak?uru? and Molo <bakulu> with $\mathrm{CV} \rightarrow \mathrm{VC}$ metathesis of earlier initial $* *$ baka with subsequent reduction of the double vowel.) Other languages have added $u$ which can be derived from $*$ a via sporadic assimilation to the following vowel. Initial irregular $* \mathrm{~b}>m$ in Rote is also paralleled in Malay mengkudu and could be sporadic assimilation to the following nasal and/or due to borrowing from a source different to the source of the Meto forms. The first $r$ in the Kusa-Manea form is currently unexplained.] [History: A likely loan spread after the break-up of PRM connected with the spread of weaving and textile dyeing.] 
manukudu Termanu. kind of tree, the bengkudu $[\mathrm{OE}=\underline{\text { Morinda }}$ citrifolia], also the red dye which can be extracted from it. (J:347)

manukudu Korbafo.

manukudu Bokai.

manukudu Bilbaa.

mana?udu-? Landu. Indian Mulberry. Morinda citrifolia. (own field notes)

mana?udu Rikou.

manukudu $B a^{\prime} a$.

manukudu Tii.

mana?udu Dengka.

manu?udu Oenale.

kuru? Ro'is Amarasi.

Pbak?uru? Kotos Amarasi. Indian Mulberry. Morinda citrifolia.

$<$ bakulu>, <baukulu> Molo. kind of tree, dye is made from the bark of the roots. Morinda tinctora. (M:46)

baur?uru? Kusa-Manea. kind of plant mixed with nohob (kind of plant) to make a red dye.

\section{Out-comparisons:}

kudu, pkudu Helong. (J:347)

*batu PRM. stone, rock. Etym: *batu.

[irr. from PRM: ${ }^{*} \mathrm{~b}>6$ in second Dela form]

batu Termanu. stone or rock. (J:33)

batu Korbafo.

batu Bokai.

batu Bilbaa.

batu Rikou.

batu $B a^{\prime} a$.

batu Tii.

fatu Dengka.

fatu Oenale.

fatu (2) tua batu-? Dela. 1) stone, rock. 2) solid sugar. [Form: tua-? = 'lontar palm'.]

fatu Ro'is Amarasi. rock, stone.

fatu Kotos Amarasi. rock, stone.

fatu Molo. stone. (M:112)

fatu, fatu-k Kusa-Manea. rock, stone.

\section{Out-comparisons:}

batu Semau Helong. rock, stone.

fatu(k) East Tetun. stone, rock. (Mo:32)

waku Kisar. rock; classifier for animals.

*bau PRM. small shore tree, sea hibiscus.

Hibiscus tiliaceus. Etym: *baRu. [irr.

from PRM: $* \mathrm{~b}=b / 6$ in wRote]

bau Termanu. Sea Hibiscus. (J:33)

bau Korbafo.

bau Bokai.

bau Bilbaa.

bau Rikou.

bau $B a^{\prime} a$.

bau Tii.

bau Dengka.

bau Oenale.

fau Molo. kind of tree, Sea Hibiscus. Hibiscus tiliaceus. (M:112)

Out-comparisons:

bau Helong. Sea Hibiscus. [irr. from $P M P: * \mathrm{R}>\varnothing($ expect $l)]$ $(\mathrm{J}: 33)$

kfau Fehan Tetun. type of tree, whose new shoots are used to bathe a newborn baby.

(k)fau(k) East Tetun. tree with stringy bark for making strong rope. Hibiscus tiliaceus. (Mo:32,106)

hau Ili'uun. hibiscus tree. (dJ:117)

*bei PRM. still, yet. [irr. from PRM: glottal stop insertion and final vowel in wRM attesting PwRM *fe?a]

bei Termanu. still, yet. (J:38)

bei Korbafo.

bei Bokai.

bei Bilbaa.

bei Rikou.

bei $B a^{\prime} a$.

bei Tii.

fe?a Dengka.

fePe Oenale.

fe? Ro'is Amarasi. still, yet.

fe?e, fe? Kotos Amarasi. still, yet.

fe?a Molo. still, yet. (M:113)

Out-comparisons:

bii Semau Helong. still, yet. 
be, bei Fehan Tetun. also.

be East Tetun. particle (not translatable). (Mo:12)

*belas PRM. machete. Etym: *belas

(Blust and Trussel (ongoing) reconstruct this form to PCMP. While reconstruction to a lower level, such as Proto-Timor-Babar, may be justified, this form is not (currently) known to occur outside greater Timor and reconstruction to PCMP is unlikely to be supported unless more cognates are forthcoming.). [irr. from PRM: ${ }^{*} \mathrm{~b}=b$ in most Meto but not Ro'is and KusaManea]

felas Termanu. machete. (J:130)

fela-? Korbafo.

fela? Bokai.

fela-? Bilbaa.

felas Rikou.

felas $B a^{\prime} a$.

felas Tii.

felas Dengka.

felas Oenale.

fenas, fenes Ro'is Amarasi. machete.

benas Kotos Amarasi.

benas Molo. machete. (M:57)

fenas Kusa-Manea. machete.

Out-comparisons:

hela? Semau Helong. machete. [Form: Jonker (1908:130) gives Helong $<\mathbf{k h e l a}>$.]

vela $H a w u$. machete.

hela Dhao. machete.

$<$ kabela $>$ Kambera. machete. (On:123)

wela Komodo. machete. (Verheijen 1982:134)

wehla Luang. machete. [Note: language of southwest Maluku ISO 639-3 [lex].] (Taber 1993:428)

kawela, ngawela Pamona. machete, iron. [Note: language of central Sulawesi ISO 639-3 [pmf].] (Adriani 1928:268) *beli Rote. price, bride price. Etym: *bəli 'buy, value, price; marriage prestations, bride price; purchase'.

beli (2) belis Termanu. 1) cost, price, value. 2) that which must be paid for a girl when taken for marriage, either paid with goods or money, the purchase price of a woman. (J:41)

beli (2) belis Korbafo.

beli (2) belis Bokai.

beli (2) belis Bilbaa.

beli (2) belis Rikou.

beli (2) belis $B a^{\prime} a$.

beli (2) belis Tii.

feli (2) felis Dengka.

feli (2) felis Oenale.

Out-comparisons:

foli-n East Tetun. price, cost, value; objects for barter. (Mo:35)

heli Ili'uun. property, valuable things. (dJ:117)

weli Kisar. buy.

*beni PRM. last night. See: *afi, *esak. Etym: *bəRyi 'night'. [irr. from PRM: $* \mathrm{e}>i$ in $\mathrm{nRM}$ (sporadic assimilation to $* \mathrm{i}$ ?); ${ }^{*} \mathrm{i}>a / \varnothing$ in nRote (antepenultimate vowel reduction)] [Form: Obligatorily compounded with reflexes of *esak in Rote.]

bina_esa-k (2) (ndee) bina_ esa-k=a Termanu. 1) day after tomorrow. 2) day before yesterday. (J:50)

bina_esa-? (2) bina_esak=a Korbafo.

bina_esa-k (2) bina_esa-k=a Bokai. bin/esa-? (2) bin/esak=a Bilbaa. bina esa-? (2) bina esa-?=a Rikou. bina_esa-k (2) bina_esa-k=a $B a^{\prime} a$. bin/esa-k Tii.

feni_esa-? Dengka. feni_esa-? Oenale. 
afiin, afiin=ii Ro'is Amarasi. yesterday, a few days ago. [Form: Initial $a$ is probably due to influence from afi naa 'yesterday' (see *afi).]

fini (2) afina (3) nmeu_n-fini (4) n-fini Kotos Amarasi. 1) last night. 2) yesterday. 3) early in the morning. 4) go past, pass. Usage: fourth sense poetic.

fini (2) fini fai (3) n-fini Molo.

1) recently. 2) yesterday evening.

3) goes past. (M:119)

Out-comparisons:

binin (2) binin tai Semau Helong. 1) day. [Semantics: Used with numbers to indicate a future day; e.g. binin dua 'in two days', binin aat 'in four days'.] 2) midnight.

*berat PRM. heavy, hard work, weight. Etym: *bəRəqat 'weight, heaviness, weightiness; difficult; pregnant'. [minority from PMP: * $\mathrm{R}=*_{\mathrm{r}}$ (fairly widespread in the region for this term)] [irr. from PRM: ${ }^{*} \mathrm{~b}=b / 6$ in wRote]

bela-k (2) bela-k (3) ma-bela Termanu. 1) heavy, heaviness, weight. 2) hard work (in general), corvée (in particular). (J:40) 3) heavy, (usually used attributively, except in a few expressions mabela does not tend to be used predicatively in equative clauses or before hiik 'very'). (J:41)

bela-k (2) bela-k (3) ma-bela Bokai.

bela-? (2) bela-? (3) ma-bela Bilbaa.

bera-? (2) bera-? (3) ma-bera Rikou.

bela-k (2) bela-k (3) ma-bela $B a^{\prime} a$. bera-k (2) bera-k (3) ma-bera Tii. bela-? (2) belat (3) ma-bela, ma ma-bela-? Dengka.

bera-? (2) berat (3) ma-bela, ma ma-bera-? Oenale.

ma?|fena|? Ro'is Amarasi. heavy. ma?|fena|? Kotos Amarasi. heavy, weight.

ma?|fena|? Molo. heavy. (M:296)

Out-comparisons:

werek Kisar. heavy.

*betə Morph: *betə-k. Rote. foxtail millet. Etym: *bətəy 'millet species, probably foxtail millet Setaria italica'. bete-k Termanu. millet, both the grain and the fruit. (J:47)

bete-? Korbafo.

bete-k Bokai.

bete-? Bilbaa.

bete-? Rikou.

bete-k $B a^{\prime} a$.

bete-k Tii.

feta-? Dengka.

feta-? Oenale.

Out-comparisons:

boto? Semau Helong. foxtail millet. [irr. from PMP: *ə $>o$ (expect $e$ )]

heten Ili'uun. millet. (dJ:117)

*beu1 Morph: *beu-k. PRM. new. Etym:

*baqəRu 'new, fresh; recent(ly)'.

[Sporadic: *VV-k $>* \mathrm{VV}$ ? $>\mathrm{V}$ ?V in most Meto (perceptual metathesis).]

beu-k Termanu. new. (J:47)

beu-? Korbafo.

beu-k Bokai.

beu-? Bilbaa.

beu-? Rikou.

beu-k $B a^{\prime} a$.

beu-k Tii.

feu-? Dengka.

feu-? Oenale.

fe?u Ro'is Amarasi. new.

fe?u Kotos Amarasi. new.

feu|? Amanuban/Amanatun. new.

fe?u Molo. new. (M:117)

fe?u-k Kusa-Manea. new.

Out-comparisons:

balu Semau Helong. new.

foun East Tetun. new, recent. (Mo:36)

woru Kisar. new. 
*beur PRM. bogo tree. Garuga Floribuna. Etym: *bəRus 'tree Acalypha amentacea' (Blust and Trussel (ongoing) reconstruct *bəRus to proto-Philippine.). [Semantics: vague Rote semantics.]

beu Termanu. kind of tree. (J:47)

beu Korbafo.

beu Bokai.

beu Bilbaa.

beu Rikou. designates three trees: the kedondong, a tree similar to the kedondong, and the same tree which Amarasi feu designates. Spondias dulcis; Garuga floribuna. (J:47; own field notes)

beu $B a^{\prime} a$.

beu Tii.

feu Dengka.

feu Oenale.

feu Kotos Amarasi. Bogo tree, a kind of tree the leaves of which are fed to cows. Garuga floribuna.

feu Molo. kind of tree. Garuga floribuna. (M:117)

Out-comparisons:

bilu Helong. (J:47)

kfeu Fehan Tetun. type of tree. Goats like to eat the flowers.

kfeu (2) feu East Tetun. 1) tree with good dark timber. Garuga floribunda. (Mo:106) 2) tree with medicinal bark. (Mo:33)

*biae PMeto. buffalo. [Form: Amarasi, Amfo'an, and Molo have consonant insertion to break up *VVV. Ro'is Amarasi has antepenultimate vowel deletion after consonant insertion. Other varieties have vowel deletion to avoid a sequence of three vowels.] [Semantics: The original meaning was 'buffalo' with later semantic shift to 'cow' in many varieties as cows supplanted buffalo as the dominant kind of cattle.] bdzae (2) bdzae meetn Ro'is Amarasi. 1) cow. [Form: antepenultimate vowel deletion from earlier *bidzae.] 2) buffalo. Lit: 'black cow'.

bidzae (2) bidzae metan Kotos Amarasi. 1) cow. 2) buffalo. Lit: 'black cow'.

bie, bia Amanuban, South. cow.

bidzae-l (2) bidzae metan Amfo'an. 1) cow. 2) buffalo. Lit: 'black cow'. bidzae-l Kopas. cow.

bia, bidzae-l Molo. buffalo. (M:64, 66)

bea (2) bea ma meto? (3) bea Pbaka? Kusa-Manea. 1) cow, cattle.

2) buffalo. Lit: 'dry/indigenous cow'. 3) cow (specifically not buffalo). Borrowed from: Pbaka? from Portuguese vaca 'cow'.

*bibi CER. pinch. Doublet: *biti, *fitis. Etym: *bitbit 'pull at body part; hold something dangling from the fingers'. [irr. from PRM: ${ }^{*} \mathrm{~b}>m p$ in $\left.\mathrm{Ba}^{\prime} \mathrm{a}\right]$

fifi? pou=na Bilbaa . holds her sarong tightly with her fingertips as she walks. [Note: Under the Termanu sub-entry fiPi-k.] (J:135)

bifi? Rikou. pinch with the finger and thumb. (J:49,682)

mpimpik mpou=na $B a^{\prime} a$. holds her sarong tightly with her fingertips as she walks. [Note: Under the Termanu sub-entry fi?i-k.] (J:135)

bifik Lole. pinch. (J:682)

*bini PRM. seed for replanting. Etym:

*binəhiq 'seed rice, rice set aside for the next planting'.

bini Termanu. seed, seed for sowing. (J:50)

bini Korbafo.

bini Bokai.

bini Bilbaa.

bini Rikou.

bini $B a^{\prime} a$.

bini Tii.

fini Dengka.

fini Oenale. 
fini Ro'is Amarasi. seed.

fini Kotos Amarasi. seed for replanting.

fini Molo. seed. (M:119)

Out-comparisons:

bini Semau Helong. seed, grain seed.

fini East Tetun. seed (grain for sowing). (Mo:34)

wini Kisar. seed.

*biti1 PRM. calf of leg. Etym: *bitios

'lower leg (below the knee); calf of the

leg'. [irr. from PRM: $*_{\mathrm{t}}>k$ in Kusa-

Manea]

biti_boa-k Termanu. calf of leg. $\overline{(J}: 51)$

biti_boa-? Korbafo.

biti_boa-k Bokai.

biti_boa-k Bilbaa.

biti_boa-? Rikou.

biti boa-k $B a^{\prime} a$.

biti_boa-k Tii.

fiti_isi Dengka.

fiti_boa-? Oenale.

fiti-n Ro'is Amarasi. calf (of leg).

fiti-f Kotos Amarasi. calf (of leg).

fiti-k Molo. calf. (M:121)

fiki-f Kusa-Manea. calf of leg.

Out-comparisons:

pitis_boa Helong. calf (of leg). (J:51)

*biti2 Rote. jerk, jump up. Doublet: *fiti1.

Etym: *bitik 'snare, noose trap; spring up suddenly, jerk up (as a fishing line or noose trap)'. [irr. from PRM: *b > $p$ in Meto]

na-ka-biti Termanu. suddenly jump up, like a shrimp. (J:51)

pitil, pitir Meto. fish with a fishing rod. (J:137)

Out-comparisons:

pitin Semau Helong. leap. [irr. from PMP: *b $>p$ ]

*bitiz PRM. slingshot. [irr. from PRM: $* \mathrm{i}>e$ in Meto]

fi fiti-k Termanu. kind of weapon made from bamboo with pebbles for ammunition. ( $\mathrm{J}: 137)$ fi fiti-? Bilbaa.

fi fiti-? Rikou.

fi fiti-? Dengka.

bi -bitis Oenale.

fi fitis (2) fiti? Dela. 1) slingshot.

2) slingshot, pulled.

k|feiti-s Ro'is Amarasi. slingshot.

na-k|feti (2) k|feti-s Kotos Amarasi.

1) fire a slingshot. 2) slingshot.

fa feti? Kusa-Manea. slingshot.

Out-comparisons:

fiti Alorese. shoot with a slingshot. Usage: Pandai village. (Moro 2016)

*boa? PMeto. ten. [Note: While Helong has a cognate, positing this as a simple case of borrowing between Helong and Meto is problematic. If Helong was the donor, this would not explain the form boa? used with twenty and above in Ro'is. Similarly, if Meto was the donor it would not explain the $k$ in the Helong form. One possible explanation is that the final $?$ in Meto is from an earlier * $\mathrm{k}$ and that this is an early borrowing from pre-Meto into Helong before ${ }^{*} \mathrm{k}>$ ? occurred in Meto.] [History: Middelkoop (1950:48) connects the Meto forms with Meto bua 'gather' (see *bua) and this is possible.]

bo?=ees (2) boa? nua Ro'is Amarasi.

1) ten. 2) twenty. [Semantics: Multiples of ten from twenty upwards use boa?.]

bo? Kotos Amarasi. ten, multiples of ten (e.g. bo?=es 'ten', bo?=nua 'twenty').

bo?=ees Molo. ten. (M:76)

bo?=eas Kusa-Manea. ten.

Out-comparisons:

buk Semau Helong. multiples of ten; e.g. buk dua 'twenty', buk tilu 'thirty', etc. [Note: hyulu = 'ten'; e.g. hyul esa 'eleven', hyul dua 'twelve', etc.] 
*boo1 PRM. drive forward, herd. [irr. from PRM: *b $>*$ mb in $w R M ; * b>(* \mathrm{f})>$ $h$ in Ba'a] [Form: Jonker (1908:138) suggests this is an onomatopoeic word from a sound made by herders.]

foo Termanu. drive, drive forward. (J:138)

foo Korbafo.

foo Bokai.

foo Bilbaa.

foo Rikou.

hoo $B a^{\prime} a$.

foo Tii.

mboo Oenale.

na-po?o Kotos Amarasi. herd.

<npo'> Molo. herds. (M:442)

Out-comparisons:

poa Semau Helong. chase out, shoo, hunt, herd.

*boo2 Morph: *boo-k, *na-boo. PRM. smell (good or bad), odour. Etym: *bahuq 'odour, stench'.

boo-k (2) na-boo (3) ma-boo-k Termanu. 1) the air of something, smell, odour, stink. 2) smell, have an aroma. 3) have a smell, odour, as an individual word especially: stinking, smell bad. (J:51)

boo-? Korbafo.

boo-k Bokai.

boo-? Bilbaa.

boo-? Rikou.

boo-k $B a^{\prime} a$.

boo-k Tii.

foo-? Dengka.

foo-? Oenale.

na-foo Ro'is Amarasi. stink.

na-foo (2) foo meni (3) foo punu?

Kotos Amarasi. 1) stink. 2) fragrant

smell. 3) rotten smell.

na-foo Molo. stink. (M:124)

na-foo Kusa-Manea. stink.

*bua Morph: *bua-k. PRM. fruit.

Doublet: *mbuah. Etym: *buaq 'fruit; areca palm and nut ...'. [irr. from PRM: ${ }^{*} \mathrm{u}>o$ in Rote] [Sporadic: $* \mathrm{~b}=$ $b / 6$ in wRote] boa-k Termanu. fruit; mainly used as a separate word to refer to the fruit of a tree already mentioned. (J:52)

boa-? Korbafo.

boa-k Bokai.

boa-? Bilbaa.

boa=na $L a n d u$. fruit. (own field notes)

boa-? Rikou.

boa-k $B a^{\prime} a$.

boa-k Lole. fruit. (Zacharias et al. 2014)

boa-k Tii.

boa-? Dengka.

boa-? Oenale.

boa-? Dela. fruit.

fua|? Ro'is Amarasi. fruit.

fua|? Kotos Amarasi. fruit.

$<$ fua $>$ Molo. fruit. (M:129)

fua|? Kusa-Manea. fruit.

Out-comparisons:

bua Semau Helong. banana.

fuan East Tetun. fruit (plants and trees ai fuan); heart (of persons or living things). (Mo:36)

hua Mambae, South. a) fruit. b) counter (e.g. classifier). (Grimes et al. 2014b:22)

*bufu PRM. fish trap. Etym: *bubu 'conical bamboo basket trap for fish'. [irr. from PRM: $* \mathrm{~b}=b$ in Meto; ${ }^{*} \mathrm{u}>$ $o$ in Meto]

bufu (2) te?ek bufu Termanu. 1) fish trap. 2) set a fish trap. (J:62)

bufu Korbafo.

bufu Bokai.

bufu Bilbaa.

bufu Rikou.

bufu $B a^{\prime} a$.

bufu Tii.

fufu Dengka.

fufu Oenale.

bofu Meto. (J:62)

Out-comparisons:

buhu Helong. (J:62)

*bulan PRM. moon, month. Etym: *bulan. bula-k Termanu. moon, month. (J:63) bula-? Korbafo. 
bula-k Bokai.

bula-? Bilbaa.

bula-? Rikou.

bula-k $B a^{\prime} a$.

bula-k Tii.

fula-? Dengka.

fulan Oenale.

funun, fuunn Ro'is Amarasi. moon, month.

funan Kotos Amarasi. moon, month.

funan Molo. moon. (M:132)

funan Kusa-Manea. moon.

Out-comparisons:

bulan Semau Helong. moon.

fulan East Tetun. moon; lunar month, the period between the new moons. (Mo:37)

wollo Kisar. moon, month.

*bulu Morph: *bulu-k. PRM. body hair;

fur; feather. Etym: *bulu.

bulu-k Termanu. hair, feather(s). [Semantics: Both laya_bulu-k and laya_doo-k (literally 'head leaf') are given for 'head hair'.] (J:63)

bulu-? Korbafo.

bulu-k Bokai.

bulu-? Bilbaa.

bulu-? Landu. body hair. (own field notes)

bulu-? Rikou.

bulu-k $B a^{\prime} a$.

bulu-k Tii.

fulu-? Dengka.

fulu-? Oenale.

funu-f Ro'is Amarasi. body hair. [Note: naak_bu?u = 'head hair'.]

funu|? Kotos Amarasi. hair (including head hair, though 'head hair' is usually specified in a compound as ?naak funu-f).

$<$ funu> Molo. body hair. (M:133)

Out-comparisons:

bulu Semau Helong. hair.

wulla Kisar. fur, feathers, hair.

*buni PRM. ringworm. Tinea imbricata.

Etym: *buqəni. [irr. from PRM: *b > $h$ in Meto; $*_{\mathrm{i}}>e$ in Meto; ${ }^{*} \mathrm{~b}=b / 6$ in wRote] bu buni Termanu. ringworm, kind of skin disease. (J:65)

bu buni Korbafo.

bu buni Bokai.

buni Bilbaa.

bu buni Rikou.

bu buni $B a^{\prime} a$.

buni Lole. ringworm. (Zacharias et al. 2014)

bu buni Tii.

buni Dengka.

buni Oenale.

hune Kotos Amarasi. ringworm.

hune Phonis Molo. kind of ringworm. (M:156)

Out-comparisons:

buna Helong. ( $\mathrm{J}: 65)$

wuni Kisar. ringworm.

vuni Hawu. (J:65)

*buu PRM. blow, blowpipe. Doublet:

*fuu. Etym: *buu (PCEMP). [irr. from

PRM: ${ }^{*} \mathrm{~b}>p$ in wRote; ${ }^{*} \mathrm{~b}=b / \mathrm{V}_{-} \mathrm{V}$ in some Meto]

na-ta-fuu Termanu. inflated, blown up. (J:143)

na-ta-fuu Korbafo.

na-ta-fuu Bokai.

na-ta-fuu Rikou.

na-ta-fuu $B a^{\prime} a$.

na-ta-fuu $T i i$.

na-sa-puu Dengka.

na-sa-puu Oenale.

sfuut Kotos Amarasi. blowpipe, darts.

sbuut Amanuban. blowpipe made from a kind of bamboo that is also used to make flutes. (M:457)

na-sbuu, na-sfuu Molo. someone blows a dart from a blowpipe. [Note: The form <tfo' $>$ 'kind of reed, cat's-tail, Thypha latifolia' may be related.] (M:479)

\section{Out-comparisons:}

hahuuk East Tetun. blowpipe, a long length of bamboo used for blowing darts (to hunt birds and sometimes small animals). (Mo:48) 


\section{$\mathbf{B}-\mathbf{b}$}

*baat PRM. crossbeam. Etym: *baRat. [minority from PMP: $* \mathrm{~b}>* 6$ ]

na-ta-baa Termanu. across (lying, etc.). (J:17)

na-ta-ba baa Dengka. lying down, of objects. (J:678)

opola?a na-ta-ba baa Oenale. speech is obstructed or blocked. (J:678)

t|baa-t Ro'is Amarasi. something which lies across or in between two other things.

t|baa-t Kotos Amarasi. large thick beam that is supported by the nii ainaf ('mother post' = main supporting post) in a house. 'Each of the four 'mother posts' has a curved fork at the top, and they support two large beams termed atbaat which must run parallel to the centre-line of the house. Lying above and across them are beams called kranit that are each the same length and that number $8,12,16$, or 24 , depending upon house size. The rafters, nesa?, lie above and across these, parallel to the atbaat, and usually number the same as the kranit'. (Cunningham 1964:37, 43)

<na-baat> (2) <na-kbata> Molo. 1) closes with planks. 2) lays across. a-moe lele na-kbata lalan neki hau uu? ees someone who makes a garden lays a tree across the path (as a barrier) [Form: Medial $t$ in na-kbata may be a retention of the final consonant with reanalysis of $* *$ baat as the metathesised form of **bata.] (M:51)

*bafa1 Morph: *bafa-k. Rote. valley. Etym: *babaq 'lower surface, bottom; short, low; below, beneath, under'. [minority from PMP: *b $>* 6$ ] bafa-k Termanu. valley, space between the mountains. ( $\mathrm{J}: 21)$

bafa-? Korbafo.

bafa-k Bokai.

ba -bafa-? Bilbaa.

bafa-? Rikou.

bafa-k $B a^{\prime} a$.

bafa-k Tii.

bafa-? Dengka.

bafa-? Oenale.

*bafa2 Morph: *bafa-k. Rote. mouth, opening. See: *fefa. Etym: *baqbaq 'mouth, opening; speak, say' (Reconstructed with the doublet *bəqbəq. Both forms are attested in the Timor region.). [minority from PMP: $* \mathrm{~b}>* 6$ ]

bafa-k Termanu. mouth of a person or animal, beak of a bird, mouth of a river, opening of a water course.

bafa-? Korbafo.

bafa-k Bokai.

bafa-? Bilbaa.

bafa-? Landu. mouth. (own field notes)

bafa-? Rikou.

bafa-k $B a^{\prime} a$.

bafa-k Tii.

bafa-? Dengka.

bafa-? Oenale.

bafa-? Dela. mouth.

Out-comparisons:

baha Semau Helong. mouth.

waban Habun. mouth. [Note: language of east Timor ISO 639-3 [hbu]] (Dawson 2014)

kahan Ili'uun. mouth (of man or animal). [irr. from PMP: $* \mathrm{~b}>$ $\left({ }^{*} \varnothing\right)>k$ (compare ${ }^{*}$ pandan $>$ ketfan, *pusəj $>$ kusan, and *hutək > gutan $)](\mathrm{dJ}: 119)$

*baiafa PRM. monitor lizard. Varanus species. Etym: *bayawak (PWMP). [minority from PMP: $* \mathrm{~b}>* 6$ ] 
baPiafa Termanu. monitor lizard. [History: Jonker attributes the irregular glottal stop to the word becoming associated with that for 'grandfather'; ba?i.] (J:23)

ba?iafa Korbafo.

baPiafa Bokai.

baiafa Bilbaa.

baiafa-? Landu. monitor lizard. (own field notes)

baiafa Rikou.

baiafa Dengka.

baiafa Oenale.

bairafa Timaus. lizard. [Form: regular $\left.(* \mathrm{y})>*_{\mathrm{d}}>r.\right]$

baiafa, baidzafa Meto. (J:23)

*Gakos PRM. barn owl. Pattern: k-8' (*k

$=k$ in Korbafo, Tii, and Oenale; expect ?).

kolo ba?o-k Termanu. kind of bird called koro ba?ok in Kupang. (J:248)

kolo_bako-? Korbafo.

kolo_ba?o-k Bokai.

kolo_bako-? Bilbaa.

koro bako-? Rikou.

kolo_ba?o-k $B a^{\prime} a$.

koro bako-k Tii.

kolo_ba?o-? Dengka.

koro_bako-? Oenale.

baos Ro'is Amarasi. barn owl.

baos Kotos Amarasi. barn owl.

Out-comparisons:

baos Semau Helong. evil spirit, ghost, apparition.

*ba?i Rote. grandfather. Etym: *baki

(Reconstructed to PAN based only on

Formosan reflexes.). [minority from $P M P: * \mathrm{~b}>* 6$ ]

ba?i Termanu. grandfather, also used in conjunction with proper names for all old men. (J:22)

ba?i Korbafo.

ba?i Bokai.

bai Bilbaa.

ba?i=na $L a n d u$. grandfather. (own field notes)
baPi Rikou.

ba?i $B a^{\prime} a$.

ba?i Tii.

ba?i Dengka.

ba?i Oenale.

ba?i Dela. grandfather.

Out-comparisons:

baki Semau Helong. mother's brother.

* ba?u CERM. several, many.

ba?u Termanu. many, great in number. (J:33)

ba?u Korbafo.

ba?u Bokai.

bau Bilbaa.

bau bea Landu. how many? (own field notes)

bau bea Oepao. how many? (own field notes)

ba?u|k Kotos Amarasi. several, many. [Form: Two forms with initial $f$ are probably related: mfaun = 'many', and fauk = 'how many?, several'.] $<$ ba?u> Molo. great, many. (M:52)

Out-comparisons:

bakun Semau Helong. how many, several.

wa?u Waima'a. many.

*balafo Morph: *balafo-k. PRM. kamala tree. Mallotus philippensis. [Semantics: vague Rote semantics]

kai lafo-k Termanu. kind of tree. $\overline{(\mathrm{J}}: 216)$

kai lafo-? Korbafo.

kai_lafo-k Bokai.

kai_lafo-? Bilbaa.

ai_lafo-? Rikou. (J:33; own field notes)

ai lafo-k $B a^{\prime} a$.

hau_lafo-? Dengka.

bnafu|?, bnafo|? Kotos Amarasi. Kamala tree. Mallotus philippensis.

$<$ nafu $>$, <banafo $>$, <benafo $>$ Molo. kind of tree. Mallotus philippensis. (M:341) 
*bali Rote. mix, add something to something else. Etym: *balik (PCMP. Blust and Trussel (ongoing) only give Yamdena and Fordata valik 'mixture of liquid and dry substances' and the Rote forms as evidence for this reconstruction.). [minority from PMP: $* b>* 6]$

bali (2) ba bali-k Termanu. 1) mix, add something to something else. $(\mathrm{J}: 27)$ 2) mixture, mixing, all mixed up. (J:28)

bali Korbafo.

bali Bokai.

bali Bilbaa.

bali Rikou.

bali $B a^{\prime} a$.

bali Tii.

bali Dengka.

bali Oenale.

* banafi $P R M$. sea cucumber. [irr. from

PRM: $* \mathrm{n}>r$ in Meto]

nafi Termanu. sea cucumber. (J:373)

nafi Korbafo.

nafi Bokai.

nafi Bilbaa.

nafi $L a n d u$. sea cucumber. (own field notes)

nafi Rikou.

nafi $B a^{\prime} a$.

nafi $T i i$.

nafi Dengka.

nafi Oenale.

brafi Ro'is Amarasi. sea cucumber.

brafi Kotos Amarasi. sea cucumber.

Out-comparisons:

nahe Semau Helong. sea cucumber. [Note: Jonker (1908:373) gives Helong hnahe.]

banahi East Tetun. round edible shellfish. (Mo:10)

$<$ kanewi> Kambera. sea cucumber. (On:164)

$<$ kanawi $>$ Kodi. edible sea cucumber. (On:543)

<nawi> Sika. kind of blue fat fish without a shell. (Calon 1891:334) *bandae PRM. hover, hang over. [irr. from PRM: *nd $>d$ in Bilbaa; *nd $>$ $* \mathrm{~d}>r / l$ in Meto]

ndae Termanu. hang something over somewhere. ( $\mathrm{J}: 412)$

ndae Korbafo.

lae Bokai.

dae Bilbaa.

rae Rikou.

ndae $B a^{\prime} a$.

ndae Tii.

ndae Dengka.

ndae Oenale.

na-brae Kotos Amarasi. hover.

na-blae Molo. it stays hanging from the hair (in a tree); it floats away. (M:72)

*baraka Rote. box. Pattern: k-6. [Form: This could be a loan but no likely donor language has yet been identified.]

balaka Termanu. box or suitcase made from wood or iron. (J:26)

balaka Korbafo.

balaka Bokai.

balaka Bilbaa.

bara?a Rikou.

balaka $B a^{\prime} a$.

baraka Tii.

bala?a Dengka.

bara?a Oenale.

Out-comparisons:

baraka Hawu. box. (J:26)

barakan Sika. wooden box for betel-vine. (Pareira and Lewis 1998:12)

*basoko PRM. slant, sway, dance. Pattern: $\mathrm{k}-7^{\prime}\left({ }^{*} \mathrm{k}=k\right.$ in Tii; expect $* \mathrm{k}$ $>$ ?) for sense $1,{ }^{*} \mathrm{k}-6$ for sense $2,{ }^{*} \mathrm{k}$-irr for senses 3 and 4. [irr. from PRM: *k $>\varnothing / \mathrm{V} \mathrm{V}$ in Rote for senses 3 and 4.$]$ na-so?o (2) soko $\sim$ soko, so?o $\sim$ so?o

(3) soo (4) so $\sim$ soo bapa $=a$ Termanu. 1) slant, sloping. (J:563)

2) very slanted. (J:557, 563) 3) swinging, swaying, wobbling, like a drunken man, etc. (J:550) 4) dancing to the sound of the 
drum, including both men and women, each gender according to their way. (J:550)

na-so?o (2) - (3) soo (4) so soo Korbafo.

na-so?o (2) soko soko (3) soo (4) so soo Bokai.

so?o Rikou.

na-so?o (2) - (3) soo (4) so soo $B a^{\prime} a$.

na-soko (2) soko soko Tii.

soko (2) soko soko (3) — (4) ili_ so $\sim$ soo Dengka.

soko (2) soko soko (3) (4) so $\sim$ soo Oenale.

na-bso?o Kotos Amarasi. dance, sway (jocular).

na-sbo?o Amanuban. dance.

na-bso?o Molo. one dances. (M:85)

*bate Morph: *ka-bate. PRM. edible grub. Etym: *qabatəd 'sago grub'. [irr. from PMP: *o $>*_{\mathrm{e}}$ (expect $*_{\partial}>a$ in wRM, possibly *ə $>*_{\mathrm{a}}>e$ in wRM)] [minority from PMP: * $\mathrm{b}>* 6$ ]

ba bate Termanu. kind of fat grub or worm, called ular babate in Kupang Malay, it occurs in the bubuni tree. These grubs are used as medicine to heal thrush in children. (J:32)

ba bate Korbafo.

ba bate Bokai.

ba bate Bilbaa.

ba bate Rikou.

ba bate Tii.

buni_bate Oenale.

buni_bate-? Dela. kind of maggot usually found at the $\mathbf{b u} \sim \mathbf{b u n i}$ tree.

k|bate|? Kotos Amarasi. kind of edible grub that eats rotten wood, reported to turn into a cicada.

k|bate|? Molo. grub that lives in a certain tree (Indonesian tengguli, Meto nikis $=\underline{\text { Cassia fistula), }}$, it is as long as a thumb, yellowish and very fat. When cooked in a pan its fat drips out and is used as medicine against thrush. This grub makes itself a chrysalis and turns into a moth/butterfly. (M:189)

ka|bata|? Timaus. kind of edible grub which eats rotten wood. [Form: regular final $* \mathrm{e}>a$.]

k|ba bate Kusa-Manea. kind of edible grub.

* bati CERM. distribute.

ba?e_ba bati Termanu. distribute. $(\mathrm{J}: 32 \mathrm{f})$

bati Bokai.

bati Bilbaa.

bati Rikou.

n-bati Ro'is Amarasi. distribute, share, divide.

n-bati (2) n-batis Kotos Amarasi.

1) distribute, share, divide.

2) separate.

n-bati Molo. distribute. (M:51)

bati Kusa-Manea. share, divide.

Out-comparisons:

batin Semau Helong. divide, give.

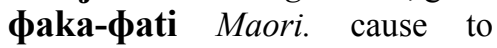
disperse. [Note: language of New Zealand ISO 639-3 [mri].] (Tregear 1891:615)

*batus Morph: *ka-batus. PwRM. sea snail.

ba batu-? Dengka. shellfish. (J:681) k|bautus Ro'is Amarasi. sea-snails; oysters.

k|batus Kotos Amarasi. sea-snails; oysters.

*bau Morph: *ka-bau-k. PRM. bat, flying fox. [Sporadic: *VV-k > *VV? > V?V in Meto (perceptual metathesis); consonant metathesis *kb $>b k$ in Amarasi.]

bau-k Termanu. bat. (J:33)

bau-? Korbafo.

bau-k Bokai.

bau-? Bilbaa.

bau-? Landu. bat. (own field notes)

bau-? Rikou.

bau-k $B a^{\prime} a$.

bau-k Tii. 
bau-? Dengka.

bau-? Oenale.

bau-? Dela. bat.

bka?u Ro'is Amarasi. bat, flying fox.

bka?u Kotos Amarasi. bat, flying fox.

$\mathbf{k} \mid \mathbf{b a} \mathbf{u}$ Kopas. bat, flying fox.

?|ba?u Molo. bat. (M:52)

?|ba?u Kusa-Manea. bat, flying fox.

Out-comparisons:

khau Funai Helong. bat, flying fox.

fau Semau Helong. bat, flying fox. [Note: Jonker (1908:33) gives Helong kfau.]

hau Bolok Helong. bat, flying fox. kabau Kupang Malay. bat.

*beba Morph: *ka-beba-k. PRM. palm leaf stems or stalks (the middle bit without the leaves). Etym: *papaq 'frond of a palm'. [irr. from PMP: * $\mathrm{p}$ $>* 6 ; * \mathrm{a}>* \mathrm{e}]$

beba-k Termanu. palm leaf stalks. [Note: Jonker lists beba as the headword with the note that beba-k occurs in compounds. The examples have the bare form as the first member of the compound and the suffixed form phrase finally.] (J:36)

beba Korbafo.

beba Bokai.

beba Bilbaa.

beba Rikou.

beba $B a^{\prime} a$.

beba Tii.

beba Dengka.

beba Oenale.

?|beba|? Kotos Amarasi. palm leaf stems, typically used to make walls for houses or fences.

$<$ beba> Molo. gebang palm. (M:53)

Out-comparisons:

hepan Semau Helong. gebang stem.

beba|k East Tetun. stalk or stem of a palm frond, used for building walls of houses and panels in fences. (Mo:12) әра Dhao. stem (of leaf).

әра Hawu. stem (of leaf). [Note: Jonker (1908:36) gives Hawu pipa.]

*bee PRM. where. [Note: Possibly combines two cognate sets: *mee and *bee, though the formal and semantic similarity leads me to present them together.] [irr. from PRM: $* 6>m$ in Meto (also Ili'uun); $* \mathrm{e}>a$ in Bilbaa, Landu, Rikou, and Oepao]

bee Termanu. where? wherever (with a preposition); how, what, whatever. (J:35)

bee Korbafo.

bee Bokai.

bea Bilbaa.

bea Landu. who, where, how. (own field notes)

(u)bea Rikou.

bea Oepao. who, where, how. (own field notes)

bee $B a^{\prime} a$.

bee Tii.

bee Dengka.

bee Oenale.

bee Dela. where.

mee Ro'is Amarasi. where.

mee Kotos Amarasi. where.

mee Molo. where. (M:317)

mee Kusa-Manea. where.

Out-comparisons:

la mee Ili'uun. which, where; also used as a general interrogative particle. (dJ:128)

bee Bima. where. (Ismail et al. 1985:14)

*bei PRM. grandmother. Etym: *baqi. [minority from PMP: *b $>*^{*}$ ] [Sporadic: $* \mathrm{VV}-\mathrm{k}>* \mathrm{VV}$ ? $>\mathrm{V}$ ?V in Meto (perceptual metathesis).]

bei Termanu. grandmother, also used to address older women or before the name of older women. (J:37)

bei Korbafo.

bei Bokai.

bei Bilbaa.

bei Rikou. 
bei $B a^{\prime} a$.

bei Tii.

bei Dengka.

bei Oenale.

bei Dela. grandmother.

be?i Ro'is Amarasi. grandmother.

bei-f, be?i Kotos Amarasi. grandmother.

be2i (2) bai-f Molo. 1) mother-in-law. (M:54) 2) mother-in-law. (M:41)

be?i Kusa-Manea. grandmother.

Out-comparisons:

bee (2) been Semau Helong.

1) mother's brother's wife.

2) mother-in-law.

bei-n East Tetun. grandparent, ancestor. (Mo:12)

*beis PRM. crocodile. See: *foes. [irr. from PRM: *VVs $>\mathrm{VsV}$ in many varieties of Meto] [Form: As noted by Jonker (1908:38) some of the irregular correspondences (especially forms with $a$ and ?) probably arose because the crocodile is addressed as ba?i 'grandfather'.] [History: Possibly connected with PMP *buqaya, though the vowel correspondences and final consonant are difficult to account for. *buqaya is regularly inherited in Nuclear Rote as *foe.]

beis Termanu. crocodile. ( $\mathrm{J}: 38)$

bei Korbafo.

bei Bokai.

bai Bilbaa.

bais Rikou.

ba?is Oepao. crocodile. (own field notes)

ba?is $B a^{\prime} a$.

bei Tii.

bei Dengka.

ba?i Oenale.

ba?i Dela. crocodile.

nai? besi Ro'is Amarasi. crocodile. [Form: Initial nai? from na?i 'grandfather'.]

nai? besi Kotos Amarasi. crocodile.

besi_mnasi? Amanuban. crocodile. [Form: mnasi? = 'old'.] besi_mnasi? Molo. crocodile. (M:61)

be?i-d's Amfo'an. crocodile.

nai? bais Amfo'an. name of an Amfo'an clan

na/basa-r, na/besi_nasi? Timaus. crocodile.

be?i Kusa-Manea. crocodile.

Out-comparisons:

'na?i_bei East Tetun. respectful name for the crocodile. (Mo:145)

${ }^{\circ}$ naPibein Welaun. crocodile. Usage: Oele'u village.

bei liurai Welaun. crocodile. Usage: Mahein village.

*Geki Morph: *na-beki. PRM. strong, capable. Pattern: k-9' $\left({ }^{*} \mathrm{k}>\varnothing\right.$ in Rikou; expect ? or $k$ ).

na-be?i Termanu. strong, can, is able to. (J:38)

na-be?i Korbafo.

na-beki Bilbaa.

na-bei Rikou.

na-be?i $B a^{\prime} a$.

na-be?i Tii.

na-be?i Dengka.

na-be?i Oenale.

na-be?i Ro'is Amarasi. strong, capable, able, can.

na-be?i Kotos Amarasi. strong, capable, able, can.

na-be?i Molo. strong, able, can. (M:54)

na-bei? Kusa-Manea. able, possible.

Out-comparisons:

biki Bolok Helong. power, exceptional strength.

biit East Tetun. force, strength. [irr. from PRM: ${ }^{*} \mathrm{k}=\varnothing$ correspondence] (Mo:14)

bik Wersing. strong. [Note: nonAustronesian language of Alor ISO 639-3 [kvw].] (Schapper 2017:263)

bi'ki Sawila. strong. [Note: nonAustronesian language of Alor ISO 639-3 [swt].] (Schapper 2017:263) 
*beko CERM. sway, shake. Pattern: k-5/6.

beko Termanu. shake from behind. (J:39)

na-beko Kotos Amarasi. sway, shake.

na-beko Molo. shake. (M:55)

Out-comparisons:

beko (2) heko Semau Helong.

1) shake, move, sway. 2) shake.

*beku Rote. bent over. Etym: *bənkuq 'bend, curve'. Pattern: k-6' $\left({ }^{*} \mathrm{k}>\varnothing\right.$ in Rikou, expect $?$ or $k$ ). [minority from PMP: *b $>* 6$; * $\left.\mathrm{jk}>*_{\mathrm{k}}\right]$

beku_tee Termanu. hanging bent over like an ear of rice or a broken branch. (J:39)

beku_tee Korbafo.

beku_tee Bokai.

(beu_tee ?) Rikou.

beku tee $B a^{\prime} a$.

beku tee Tii.

be?u tee Dengka.

be?u_tee Oenale.

be?u_tee Dela. bow (in respect).

*be?e $P \bar{R} M$. stay awake.

be?e Termanu. watch, remain awake.

be?e $B a^{\prime} a$.

be?e Tii.

be?e Dengka.

bePe Oenale.

bePe Dela. stay up late.

n-be?e Kotos Amarasi. stay awake. Normally implies someone has died, and one is staying awake overnight with the family of the deceased in the presence of the corpse.

n-be?e Amfo'an. be aware.

n-be?a Molo. watch. (M:53)

bePan Kusa-Manea. stay up overnight.

Out-comparisons:

beke Hawu. (J:37)

*bela PRM. spread out, flat. Doublet:

*fela. Etym: *bəkəlaj 'spread out, unroll (mats, etc.), open out, unfold (as the hand); wide' (Blust and Trussel (ongoing) reconstruct PEMP *bolaj and the Rote-Meto forms provide evidence for PCEMP *bəlaj.). [minority from PMP: *b $>* 6$ ]

bela (2) be bela-k Termanu. 1) spread out as a mat, cover something with something that is spread out. (J:39) 2) flat. (J:40)

bela (2) be bela-? Korbafo.

bela (2) be bela-k Bokai.

bela (2) be bela-? Bilbaa.

bela (2) be -bela-? Rikou.

bela (2) be bela-k $B a^{\prime} a$.

bela (2) be bela-k Tii.

bela (2) be bela-? Dengka.

bela (2) be bela-? Oenale.

bela (2) be bela-? Dela. 1) unfold or place on the ground. 2) flat, smooth.

na-?bena Kotos Amarasi. spread out (e.g. mat, blanket).

na-Pbena Molo. spreads something out. (M:57)

\section{Out-comparisons:}

bela Semau Helong. open eyes.

belar East Tetun. level, flat, even, broad; $v$. to spread, to strew, multiply. [irr. from PMP: *ə > $e$ (expect $o$ )] (Mo:13)

hele Ili'uun. unfold, open, spread out. (dJ:117)

bəla Hawu. spread out, expanse, open by separating.

*besa Morph: *besa-k. PRM. reonja tree.

Vachellia leucophloea.

kai/besa-k Termanu. kind of tree the bark of which is used to colour sails and nets brown, deer like to eat the leaves of this tree. Vachellia leucophloea. $\quad(\mathrm{J}: 215, \quad$ Heyne 1950:713, lxxii)

kai/besa-k Bokai.

kai besa-? Bilbaa.

ai_besa-? Rikou.

kaì/besa-k $B a^{\prime} a$.

ai_besa-k Tii.

hau_besa-? Dengka. 
besa-? Oenale.

besa|k Kotos Amarasi. kind of tree with branches like the white leadtree (= Leucaena leucocephala).

besa|k Molo. name of a screen tree. Acacia leucophloea. (M:60)

Out-comparisons:

besa|? Semau Helong. kind of thorn tree.

* beta Rote. cut vegetation. Etym: *bontas 'hack a passage through vegetation, blaze a trail'. [minority from PMP: *b $>* 6]$

beta ai Termanu. cut a tree, cut off branches, prune. (J:46)

beta Korbafo.

beta Bokai.

beta Bilbaa.

beta Rikou.

beta $B a^{\prime} a$.

beta Tii.

beta Dengka.

beta Oenale.

*beto Morph: *na-ka-beto. PRM. tense, tight. Etym: *bəntən 'extended, stretched taut, put under tension'. [minority from PMP: *b $>* 6$ ] [Form: The unmetathesised form has not yet been attested in Meto. It could be *kbeta or *kbete.]

na-ka-bete Termanu. tense, tight. (J:46)

na-ka-bete Korbafo.

na-ka-bete Bokai.

na-ka-bete Bilbaa.

na-bete Rikou.

na-ka-bete $B a^{\prime} a$.

na-ka-bete $T i i$.

na-Pa-beta Dengka.

na-Pa-beta Oenale.

na-k|beet Kotos Amarasi. stiff, tight.

na-k|beet Molo. tight, braced.

(M:189)

*betu Morph: *na-ka-betu. PRM. bent, folded. Doublet: *fedu. Etym: *bəntuk 'curve'. [minority from PMP: *b $>* 6$ ] na-ka-betu Termanu. flexible (mainly said of fingers); bent in, bent through; crooked; stick up crookedly. (J:47)

betu Dengka.

na-k|betu Molo. folded. [History: Middelkoop (1972:63) also gives na-kfetu, and na-kfeti as variants meaning 'jumps or bounces back' which may be connected.] (M:63)

\section{Out-comparisons:}

(s)beton Helong. (J:128)

*bia PRM. split. Etym: *biqak. [Note:

The irregularities in the Meto forms indicate that they may not be cognate with the Rote forms.] [minority from PMP: *b $>* 6$ ] [irr. from PRM: *6 > $p$ in Meto; $* \mathrm{i}>e$ in Meto] [Sporadic: glottal stop insertion in Meto]

bia Termanu. split. (J:48)

bia Korbafo.

bia Bokai.

bia Bilbaa.

bia Rikou.

bia $B a^{\prime} a$.

bia Tii.

bia Dengka.

bia Oenale.

n-pe?a Ro'is Amarasi. break.

n-pe?e Kotos Amarasi. break, crack.

n-pee? (2) pe?as Molo. 1) broken through. 2) fissure or split in the ground. (M:428)

\section{Out-comparisons:}

mbi?a (2) bi?a Bima. 1) piece, broken off from glass and earthenware, etc. in the meaning of Malay pecah. (Jonker 1893:55) 2) break something. (Jonker 1893:9, Ismail et al. 1985:15)

mbiPa (2) pi?a Ende. 1) broken. 2) blow, hit, break.

*bibi Morph: *ka-bibi. PRM. goat. [irr. from PRM: *6 $>$ ? in Termanu, Korbafo, Bokai, and Ba'a] [Semantics: onomatopoeia] 
bi?i_hii-k, bi?i_ae-k (2) bi?i_lopo Termanu. 1) goat, buck. 2) ram, sheep. (J:49)

bi?i Korbafo.

bi?i Bokai.

bibi Bilbaa.

bibi Rikou.

bi?i $B a^{\prime} a$.

bibi Tii.

bibi Dengka.

bibi Oenale.

bibi Dela. sheep, goat.

P|bibi Kotos Amarasi. goat.

P|bibi Molo. goat. (M:65)

?|bibi Kusa-Manea. goat.

Out-comparisons:

bibi East Tetun. goat. (Mo:14)

pipi Ili'uun. goat. (dJ:133)

biub Mambae, South. goat.

(Grimes et al. 2014b:14)

pipi Kisar. goat.

kahibi Dhao. goat.

*bife Rote. lip. Etym: *bibiR 'lower

lip'. [minority from PMP: *b $>* 6$ ]

[Sporadic: $* \mathrm{i}>*_{\mathrm{e}} /{ }_{-} \mathrm{R} \#$ ] [Form:

Assimilations of $* 6>d$ in the first sense are due to this term being compounded with reflexes of *doo-k 'leaf', compare *ndiki > di?i_doo-k and *ndake_doo-k $>$ da?e_doo-k.]

difa_doo-k (2) bifa-k (3) na-bifa ule-k Termanu. 1) lips (of people and animals). (J:86) 2) the cut off edge of a leaf. 3) shape the earth to follow the edge of a pot. (J:49)

bife_doo-? (2) bifa-? Korbafo.

difa_doo-k Bokai.

bife doo-? Bilbaa.

bifi_doo-? Landu. lips. (own field notes)

bifi_doo-? Rikou.

bifi_doo-? Oepao. (own field notes)

difi_doo-k (2) bifa-k $B a^{\prime} a$.

bifi_doo-k (2) bifa-k Tii.

bife Dela. side of, edge.

*bina Rote. kind of volute shell. Etym:

*binaq. [minority from PMP: *b $>$ *6] bina Termanu. kind of shellfish; the shell or a part of the shell of this shellfish is used to rest the ine (spindle) on. (J:49)

bina Korbafo.

bina Bokai.

bina Bilbaa.

bina Rikou.

bina $B a^{\prime} a$.

bina Tii.

bina Dengka.

Out-comparisons:

bijan Helong. (J:49)

*bisu PRM. ulcer, pimple. Etym: *bisul

'boil, abscess'. [minority from PMP: $* \mathrm{~b}>* 6$ ]

bisu Termanu. ulcer, pimple; have an ulcer or wound. (J:50)

bisu Korbafo.

bisu Bokai.

bisu Bilbaa.

bisu Rikou.

bisu $B a^{\prime} a$.

bisu Tii.

bisu Dengka.

bisu Oenale.

bisu Dela. wound.

bisu Kotos Amarasi. kind of wound, pustule.

na-bisu Molo. pimply. (M:71)

Out-comparisons:

fisur, fisul East Tetun. abscess, boil. (Mo:34)

vihu Hawu. (J:50)

*biti PRM. pinch. Doublet: *bifi, *fitis. Etym: *bitbit 'pull at body part; hold something dangling from the fingers'. [minority from PMP: *b $>* 6$ ] [irr. from PRM: *t $>$ ? /V_V in Rote] [Form: The Meto forms meaning 'scorpion' are mostly from *ka-biti with the nominal *ka- prefix.]

biPi Termanu. pinch with the finger and thumb. (J:49)

bi?i Tii.

bi?i Dengka.

k|biti Ro'is Amarasi. scorpion. 
k|biti Kotos Amarasi. scorpion.

<biti> (2) ka|biti Molo. 1) give a little pinch. (M:71) 2) scorpion. (M:166)

biti Kusa-Manea. scorpion. Usage: Upper Manulea village.

ka|biti Kusa-Manea. scorpion. Usage: Uabau' village.

\section{Out-comparisons:}

bitin Semau Helong. flick something (especially rock or marble).

*bitinaa PRM. guest tree. Kleinhovia hospita.

bitinaa Termanu. kind of tree. Kleinhovia hospita. (J:51, Heyne 1950:1064, lxxv)

bitinaa Korbafo.

bitinaa Bilbaa.

bitinaa $B a^{\prime} a$.

bitinaa Tii.

betinaa Dengka.

betinaa Oenale.

$<$ bitna $>$ Molo. kind of tree which becomes green quickly. Kleinhovia hospita. (M:72)

*boho PRM. cough.

bo?o Termanu. cough. (J:58)

bo?o Korbafo.

bo?o Bokai.

boo Bilbaa.

boo Landu. cough. (own field notes)

boo Rikou. [Note: Jonker (1908) gives the Rikou form as bo?o and marks it as dubious. If this form is accurate, it would be only one of two forms with medial $* \mathrm{~h}>$ ? in Rikou, the other being *kahu > ka?u).] (own field notes)

bo boo-s Oepao. cough. [Form: nominalisation]

bo?o $B a^{\prime} a$.

bo?o Tii.

boo Dengka.

boo Oenale.

boo Dela. cough.

n-boho Ro'is Amarasi. cough. n-boho Kotos Amarasi. cough.

<boho> Molo. cough. (M:77)

ba booh Kusa-Manea. cough.

*boki Morph: *ka-boki-k. PRM.

hollowed out coconut shell used as a container. Pattern: k-9.

bo bo?i-k Termanu. whole hollowed out coconut shell with an opening used to contain sugar sap. (J:54)

bo bo?i Korbafo.

bo bo?i-k Bokai.

bo boki Bilbaa.

bo boki Rikou.

bo bo?i-k $B a^{\prime} a$.

bo bo?i-k Tii.

bo bo?i Dengka.

bo bo?i Oenale.

a-?|bo?i Meto. (J:54)

Out-comparisons:

buki Helong. (J:54)

*bo/lau Rote. spider. Doublet: *k|naba|?.

Etym: *lawaq 'spider; spider web'

(Possibly a chance similarity.). [irr. from PMP: *wa $>*_{\mathrm{u}}$ in Rote] [Form:

source of initial *bo unknown]

bolau Termanu. spider. (J:55)

bolau Korbafo.

bolau Bokai.

bolau Bilbaa.

bolau Landu. spider. (own field notes)

bolau Rikou.

bolau $B a^{\prime} a$.

bolau Tii.

bolau Dengka.

bolau Oenale.

Out-comparisons:

lailaon Helong. [irr. from PMP:

*wa $>o$ (expect $p a)](\mathrm{J}: 55)$

*bole PRM. areng palm. Arenga pinnata.

bole Termanu. areng palm, called gemuti in Kupang. (J:56)

bole Korbafo.

bole Bokai.

bole Bilbaa.

bole Rikou.

bole $B a^{\prime} a$.

bole Tii. 
bole Dengka.

bole Oenale.

bone Kotos Amarasi. areng palm. Arenga pinnata.

bone Molo. areng palm. Arenga pinnata. (M:82)

bone Kusa-Manea. areng palm. Arenga pinnata.

\section{Out-comparisons:}

'bone Fehan Tetun. type of tree which is as tall as a sago palm. Borrowed from: Meto, as shown by irr. $* 1>n$.

*bolo1 Morph: *bolo-k. PRM. hole. [irr. from PRM: *1 $>r / l$ in Meto]

bolo-k Termanu. hole, cavity, hollow, pit. (J:56f)

bolo-? Korbafo.

bolo-k Bokai.

bolo-? Bilbaa.

bolo-? Rikou.

bolo-k $B a^{\prime} a$.

bolo-k Tii.

na-boor, n-boor Kotos Amarasi. make a hole.

bola|? Meto. (J:57)

Out-comparisons:

bolo? Funai Helong. hole.

bolo Semau Helong. hole.

*boloz PwRM. scabies, smallpox.

bo bolo Oenale. have scabies. (J:683)

bono Kotos Amarasi. smallpox.

bono Molo. smallpox. (M:83)

Out-comparisons:

bobolo Helong. smallpox. (J:683)

*bona Morph: *bona-k. CERM. fragrant pandanus.

bona-k Termanu. fragrant pandanus. (J:57)

bona-k Bokai.

bona-k $B a^{\prime} a$.

bona|k, bono|k Ro'is Amarasi. fragrant pandanus.

bona|k Kotos Amarasi. fragrant pandanus.

bona|? Amfo'an. fragrant pandanus. bona|k Molo. fragrant pandanus. (M:82)

boan|k=aa Kusa-Manea. fragrant pandanus.

Out-comparisons: bonak Kupang Malay. pandanus.

*bone CERM. circle dance.

bo bone Termanu. kind of circle dance, only performed by men. (J:57)

n-bone (2) bone-t Molo. 1) sings in a choir dance. 2) choir dance in a large circle, singing in a choir dance. (M:82)

*boni $n R M$. hang, depend on.

tai_boni Termanu. hang out for something $=$ desire something, demand something. (J:588)

tai_boni Korbafo.

tai boni Bokai.

tai_boni Bilbaa.

tai_boni Rikou.

tai_boni $B a^{\prime} a$.

tai_boni Tii.

na-?boni? Kotos Amarasi. depending on.

<na-boni> Molo. it depends on something. (M:83)

ta-Pboni? Kusa-Manea. hang.

*boygo1 PRM. round, bulbous.

bo boyo-k Termanu. round. (J:58)

bo boyo-? Korbafo.

bo bono-k Bokai.

bo boyo-? Bilbaa.

bo boko-? Rikou.

bo boygo-k $B a^{\prime} a$.

bo boygo-k Tii.

bo boygo-? Dengka.

bo bongo-? Oenale.

bo boygo-? Dela. round.

siim tai_boko Kotos Amarasi. kind of large katydid with a large belly. [Form: simah $=$ 'katydid', tai-f $=$ 'belly'.]

Out-comparisons:

bukas Semau Helong. round. [irr. from PRM: ${ }^{*} \mathrm{u}=o$; final ${ }^{*} \mathrm{o}=a$; $\left.*_{\mathrm{yg}}=k\right]$ 
bokur East Tetun. fat, fatty, well fed; $n$. fat, tallow, grease. (Mo:16)

boku Waima'a. fat, round.

$<$ kabunggulu> Kambera. squat of stature, short and round. [Note: also in Mangili and Lewa.] (On:126)

$<$ kaḅugulu> Anakalang.

$<$ kawonggila $>$ Mamboru.

$<$ kamunggila $>$ Weyewa.

*boygor Morph: *ka-bongo. PRM. gourd, pumpkin, squash.

boyo Termanu. calabash, called bongko in Kupang, which is hollowed out and used for the storage of liquids. (J:57f)

bojo Korbafo.

boyo Bokai.

boyo Bilbaa.

boko Rikou.

boygo $B a^{\prime} a$.

boygo Tii.

boygo_melu? Dengka.

boygo Oenale.

boko Ro'is Amarasi. kind of vegetable the skin of which is used as a water container. [Note: uut bo?o $=$ 'pumpkin blossom' may be related with irr. *yg $>(* \mathrm{k})>$ ?.]

P|boko Kotos Amarasi. pumpkin, squash.

P|boko (2) atoni na-k|boko Molo.

1) pumpkin. 2) bald-headed man (used for a Christian, who has had his hair cut off). (M:79)

Out-comparisons:

boyo Helong. (J:58)

boko Fehan Tetun. type of chilli, so named as the fruits are fat.

boygo Ende. vessel made of gourd.

*boto1 Morph: *na-ka-boto. CERM. whisper, mutter.

na-ka-bo boto-? Bilbaa. whisper. (J:684)

na-bo boto-? Rikou.
na-Pboto Kotos Amarasi. remind.

<bot -boto> Meto. mutter. (J:684)

Out-comparisons:

hakbotuk East Tetun. whisper, speak in a secret and low voice, to mutter to oneself. (Mo:51)

*botor PRM. Indian beech tree. Millettia pinnata. [Semantics: Scientific identification given on the basis of the Molo reflex and the Termanu example sentence. 'While the oil and residue of the plant [Millettia pinnata] are toxic and will induce nausea and vomiting if ingested, the fruits and sprouts, along with the seeds, are used in many traditional remedies.' ('Millettia pinnata', Wikipedia. en.wikipedia. org/wiki/Millettia_pinnata. Accessed 17 September 2020).]

bo boto Termanu. plant kind. see na?a boboto $=a$ boa-na naa see mate whoever eats the fruit of the bo -boto will die (J:59)

bo boto Dela. kind of tree.

boto? Kotos Amarasi. cluster fig tree; Indian fig tree; goolar fig. Ficus racemosa. [Semantics: Scientific identification from Meijer-Drees (1950:4).]

<boto'is> Molo. kind of tree. Millettia pinnata. (M:85)

*botos PRM. fontanelle. [Form: The second element of the Rote forms is a reflex of *lii $<*$ liqəR.]

boto_lii-k Termanu. neck. (J:59)

boto_lii-? Korbafo.

boto_lii-k Bokai.

boto lii-? Bilbaa.

bo/lii-? Landu. neck. (own field notes)

bo/lii-? Rikou.

bo/lii-? Oераo. neck. (own field notes)

boto_lii-k $B a^{\prime} a$.

boto lii-k Tii.

boto_lii-? Oenale.

boto-n Ro'is Amarasi. fontanelle. 
boto-f Kotos Amarasi. fontanelle (the pulsating part of a baby's head).

boto-f Kusa-Manea. head.

Out-comparisons:

ba boto-n East Tetun. fontanel [sic], the gap between the bones in the skull of young children. (Mo:7)

*bua PRM. gather. [Sporadic: *a > e/\# in wRote]

na-ka-bua (2) bua bua (3) bu bua-k (4) bebua Termanu. 1) gathering, coming together, accumulating. 2) together, gathered, in piles. 3) being together, that which is together, heap, herd, swarm, clod, clump. 4) together in piles. (J:60)

na-ka-bua Korbafo.

na-ka-bua Bokai.

na-ka-bua Bilbaa.

na-bua Rikou.

na-ka-bua Tii.

na-Pa-bue Dengka.

na-Pa-bue Oenale.

na-bua $R o$ 'is Amarasi. gather.

na-bua (2) na-k|bua?, na-?|bua?

(3) bua? bua? Kotos Amarasi.

1) gather together (intransitive).

2) gather (transitive). 3) together.

[Note: kbo?es 'clump' may be related with final $* \mathrm{a}>e$, vowel height harmony, lowering $* \mathrm{u}>o$, and addition of various affixes.]

na-bua (2) <atoni> na-k|bua?

(3) bua? bua? (4) <anbua nakan> Molo. 1) coming together.

2) man with his hair tied together in as opposed to a Christian who cuts his hair off. 3) close together.

4) makes a hair roll. (M:85)

ta-bua Kusa-Manea. gather.

Out-comparisons:

${ }^{\circ}$ nakbua (2) 'obuan Semau Helong. 1) gather. 2) gather, collect, group, cluster. Borrowed from: Rote or Meto, as indicated by the irregular lack of any reflex for pre-RM * $\mathrm{k}$ which is evidenced by the Tetun form below $\left({ }^{*} \mathrm{k}>\right.$ ? is attested in Tetun).

fuPa|k East Tetun. group of things or animals very close together. (Mo:36)

*buas Rote. thing, equipment.

buas Termanu. equipment. (J:61)

bua-? Korbafo.

bua-? Bokai.

bua-? Bilbaa.

buas Rikou.

buas $B a^{\prime} a$.

buas Tii.

buas Dengka.

buas Oenale.

buas Dela. generic clothes.

Out-comparisons:

buat East Tetun. thing, object. (Mo:18)

*bubu PRM. bubbling, boiling. Etym:

*bukbuk1. [minority from PMP: *b > *6 (both initially and medially)]

na-sa-bubu Termanu. bubbling up, boiling up of water. (J:61f)

bubu Bokai.

bubu Bilbaa.

bubu Dengka.

na-sa-bubu Oenale.

n-bubu Molo. boils, trembles. (M:87)

Out-comparisons:

bubu East Tetun. swell. (Mo:18)

bubu Hawu. boil over, overflow; anger.

*buə Morph: *buə-k. nRM. head hair, frizzy haired. Etym: *buhək 'head hair'. [minority from PMP: *b > *6] [irr. from PRM: *o $>u$ in Ro'is Amarasi] [Sporadic: *VV-k $>* \mathrm{VV}$ ? $>$ VPV in Ro'is Amarasi (perceptual metathesis)]

laya_bue-k Bokai. frizzy haired. (J:684)

laka doo bue-? Rikou. frizzy haired. (own field notes) 
(layga) bue-k Tii. frizzy haired. (J:684)

naak_bu?u Ro'is Amarasi. head hair. bu/ratu Kotos Amarasi. frizzy (of hair). [Form: Neither element of this (probable) historic compound is known to be attested independently.]

\section{Out-comparisons:}

fuhuk Fehan Tetun. hair of the head.

fuuk East Tetun. hair of the head. (Mo:38)

*buit PRM. rear, backside. Etym: *buRit 'hind part, rear, back'. [minority from PMP: *b $>* 6$ ] [irr. from PRM: * $\mathrm{i}>$ $a$ in Molo; $*_{\mathrm{i}}>u$ in Kotos Amarasi (sense 1)] [Sporadic: *VV-k $>* V V$ ? $>\mathrm{VPV}$ in some Meto (perceptual metathesis)]

bui-k (2) tane_bui Termanu. 1) rear. (J:62) 2) kind of black ant with an acutely painful sting. Lit: 'sharp rear'. (J:817)

bui-? (2) tane_bui Korbafo.

bui-k (2) tane bui Bokai.

bui-? (2) tene_bui Bilbaa.

bui-? (2) tande_bui Rikou.

(2) tane_bui $B \bar{a}^{\prime} a$.

bui-k Tii.

na-Pa-buit Dela. go last, from behind.

(2) kas/bu?i Ro'is Amarasi. 2) ant. P|bu?u-f (2) sa/buit Kotos Amarasi.

1) buttocks, anus. 2) ant. [Form: I have an (unconfirmed) variant bu?i for the first sense in my dictionary. This would be a regular reflex.]

$<$ bu'an> Molo. posterior. (M:86)

(2) as/bui|k Kusa-Manea. 2) ant.

\section{Out-comparisons:}

hui Dhao. stern of boat.

vui Hawu. stern, rear.

*buku Morph: *buku-k. PRM. node, joint. Etym: *buku 'node (as in bamboo or sugarcane); joint; knuckle; knot in wood; knot in string or rope'. Pattern: $\mathrm{k}-7$. [minority from PMP: *b $>* 6$ ] bu?u-k Termanu. protuberance, joint. (J:66)

bu?u-? Korbafo.

bu?u-k Bokai.

buku-? Bilbaa.

buku-? Rikou.

bu?u-k $B a^{\prime} a$.

bu?u-k Tii.

buku-? Dengka.

buku-? Oenale.

bu?u-f Ro'is Amarasi. joints.

bu?u-f Kotos Amarasi. joints; knuckles, wrist, ankle, etc.

\section{Out-comparisons:}

buku-n Semau Helong. joint, knot, knee.

fuku-n East Tetun. knot (of trees, ropes, etc.); knuckle, joint (of limbs). (Mo:37)

*bula Rote. open one's eyes wide. Etym: *bulat 'open the eyes wide, stare with round eyes'. [minority from PMP: $* \mathrm{~b}>* 6]$

na-ka-bu bula (2) bula bula (mata de?e-n-) Termanu. 1) he opens his eyes wide. 2) his eyes are wide open. (J:63)

na-ka-bu bula Korbafo.

na-ka-bu bula Bokai.

na-ka-bu bula Bilbaa.

na-bu bula Rikou.

na-ka-bu bula-k $B a^{\prime} a$.

na-ka-bu bula-k Tii.

na-ta-bu bula-? Dengka.

na-?a-bu bula-? Oenale.

Out-comparisons:

$<$ kabula $>$ Kambera. with the eyes wide open. (On:125)

*buna Morph: *buna-k. PRM. flower, blossom. Doublet: "buyga. Etym: *buna. [minority from PMP: *b $>$ *6] [irr. from PRM: $* \mathrm{u}>o$ in Meto]

buna-k Termanu. flower, blossom. (J:64)

buna-? Korbafo.

buna-k Bokai.

buna-? Bilbaa. 
buna=na Landu. flower. (own field notes)

buna-? Rikou.

buna-k $B a^{\prime} a$.

buna-k Tii.

buna-? Dengka.

buna-? Oenale.

fu/bona|? Ro'is Amarasi. ornamental flower.

fu/bona|?, fua_bona|? Kotos Amarasi. ornamental flower. [Form: first element from fua? $=$ 'fruit'.]

Out-comparisons:

buya? Semau Helong. flower.

funan East Tetun. flower, bloom. (Mo:37)

*buni PRM. Cassia tree, kinds of legume trees.

bu buni hedu (2) bu buni doo lutu Termanu. 1) kind of tree with hard wood the leaves of which can be used as medicine for ringworm. 2) kind of tree with small leaves the bark of which can be used as a substitute for betel nut. Lit: 'slender leaved bubuni'. [Semantics: Heyne (1950:741, lxxvi) gives <boeboeni sèla $>$ as Cassia fistula.] (J:65)

bu buni_hedu Korbafo.

bu buni hedu Bilbaa.

bu buni lama (2) bu buni lutu

(3) bu buni sela-? Rikou. 1) kind of tree the bark of which can be used as a substitute for betel nut.

2) kind of tree with white flowers.

3 ) kind of tree with yellow flowers. (own field notes)

bu buni hedu $B a^{\prime} a$.

buni, bu $\sim$ buni mali Tii.

bu buni_melū? Dengka.

buni meru? Oenale.

bu buni Dela. kind of tree.

buni Kotos Amarasi. kind of tree the bark of which can be used as a substitute for betel nut. buni Molo. kind of tree. Cassia javanica; Cassia siamea. (M:90)

\section{Out-comparisons:} vuni Hawu. (J:65)

*bunda Rote. fat bellied. Etym: *buntor 'distended, inflated (of the belly)' (Blust and Trussel (ongoing) give a host of semantically and formally similar reconstructions some of which they posit as doublets and some of which they mark as 'disjuncts': *buntəR 'round', *bə(n)tur 'glutted, sated; swollen (of the belly)', *bo(n)tuR 'glutted, sated; swollen (of the belly)', *buntu? 'bloated, swollen (of the belly)', *buntuD 'swollen, distended, of the belly', *buntuR 'bloated, swollen', and *bu(n)tu 'bloated'. It seems extremely likely that several of these forms are spurious and we are dealing with cases of irregular sound change. Manggarai and Rembong forms are given as evidence for multiple reconstructions and are the only witnesses from Wallacea.). [irr. from PMP: *a $>*_{a}$ (expect $*_{\partial}>e$ in nRote)] [minority from PMP: $* \mathrm{~b}>* 6$; $*_{n t}>*_{\text {nd }}($ expect $\left.* \mathrm{t})\right]$

buna tei-k (2) iPa buna-k Termanu. 1) have a fat belly, get a fat belly (only said of children). 2) a kind of fish which inflates itself like a ball at the slightest touch. (J:64)

buna_tei-? Korbafo.

buna tei-? Bilbaa.

bunda_tei-? Rikou.

bunda_tei-k Tii.

bunda - bunda Dengka. stuffed. [Semantics: Jonker gives a cross reference to Termanu lamu lamu which is glossed as "very stuffed, of the belly'. Thus, it seems likely that the usual reference of the Dengka form was also to the belly.] (J:684)

*busa Rote. dog. [History: possibly connected with PWMP *musay 'civet' or Dutch poes [pu:s] 'cat'.]

busa Termanu. dog. (J:65) 
busa Korbafo.

busa Bokai.

busa Bilbaa.

busa Landu. dog. (own field notes)

busa Rikou.

busa $B a^{\prime} a$.

busa Tii.

busa Dengka.

busa Oenale.

busa Dela. dog.

Out-comparisons:

busa East Tetun. cat. (Mo:19)

busa Kemak. cat.

busan Lamaholot, Ile Mandiri. cat. Usage: Lewoingu dialect. [Note: language of east Flores ISO 639-3 [slp].] (Nishiyama and Kelen 2007:90) *busu Rote. bow for cleaning cotton. Etym: *busuR 'hunting bow'. [minority from PMP: *b $>* 6$ ]

busu (2) bu busu-k Termanu.

1) clean cotton with a bow.

2) cleaning; the bow used to clean cotton. (J:65)

busu Korbafo.

busu Bokai.

busu Bilbaa.

busu Rikou.

busu $B a^{\prime} a$.

busu Tii.

busu (2) bu busu-t Dengka.

(2) bu busu-t Oenale.

Out-comparisons:

fusu East Tetun. an arched comb used for carding cotton. (Mo:38)

\section{D - d}

*daa Morph: *daa-k. PRM. blood. Etym: *daRaq.

daa-k Termanu. blood, sap. (J:66)

daa-? Korbafo.

daa-k Bokai.

daa-? Bilbaa.

daa-? Landu. blood. (own field notes)

daa-? Rikou.

daa-k $B a^{\prime} a$.

daa-k Tii.

laa-? Dengka.

raa-? Oenale.

naa|? Ro'is Amarasi. blood.

naa|? Kotos Amarasi. blood.

naa|? Molo. blood. (M:338)

naa|? Kusa-Manea. blood.

Out-comparisons:

dala Semau Helong. blood.

raa-n East Tetun. blood. (Mo:159)

laa-t Welaun. blood.

rara Kisar. blood.

rara(n) Ili'uun. blood. (dJ:135) *dae PRM. soil, land, earth. Etym: *daRəq 'soil, probably clay'. [irr. from PRM: ${ }^{*} \mathrm{e}>i$ in Meto with additional subsequent irregularities in the Meto vowels] [Form: regular *a $>e$ /_q\#.] dae Termanu. soil, land, earth. (J:68) dae Korbafo.

dae Bokai.

dae Bilbaa.

dae Rikou.

dae $B a^{\prime} a$.

dae Tii.

lae Dengka.

rae Oenale.

nain (2) nai ra?e Kotos Amarasi. 1) ground. 2) clay. Usage: nain occurs in place names and phrases, such as nai muti? 'white ground'. (The normal word for ground in Amarasi is afu.). [History: The second element of nai ra?e 'clay' could also be an irregular reflex of *dae.] 
nain Amanuban/Amanatun. ground.

naidzan $A m f o$ 'an. earth.

naidzan Molo. earth. [Form: Insertion of medial $d_{3}$ is unexpected. It probably came about via a pathway such as $* *$ nain $>* *$ naiyin $>* *$ naiyan.] (M:342)

naidzaan Baikeno. earth. (Charles E. Grimes pers. comm.)

nian Kusa-Manea. earth. [Form: This form is derivable from intermediate **naian with subsequent reduction of the initial vowel sequence.]

\section{Out-comparisons:}

dale Semau Helong. ground.

rai East Tetun. earth, soil, ground; land estate, kingdom; the world. (Mo:158)

${ }^{\circ}$ rai Kisar. king. Borrowed from: perhaps Tetun, as shown by irr. $* \mathrm{R}>\varnothing$ instead of expected $* \mathrm{R}>r$ combined with the semantic shift.

ra(ra), rare Ili'uun. land, ground, especially in contrast to sea, water. (dJ:134)

*daem $n R M$. termites.

(?)ana/dae Termanu. termites. (J:12)

hana/dae Korbafo.

(?)ana/dae Bokai.

(?)ana/dae Bilbaa.

Pana/dae Rikou. [Form: My consultants gave ?andae.]

(?)ana/dae $B a^{\prime} a$.

(?)ana/dae Tii.

naem Ro'is Amarasi. termites.

naem Kotos Amarasi. termites.

<name> Molo. flying white ants. [Form: Molo may have unmetathesised an original CVVC root.] (M:347)

na $\sim \mathbf{n a e m}=\mathbf{a a}$ Kusa-Manea . termites.

Out-comparisons:

'naen Semau Helong. termite. Borrowed from: probably Amarasi (shown by irr. $* d=n$ correspondence). *dai PMeto. rooster comb or wattle. [irr. from PRM: ${ }^{*} \mathrm{i}>e$ in Ro'is]

took rae-n (2) iuk rae-n $R o$ 'is Amarasi. 1) rooster wattle (the bit which hangs down). 2) the long tail feathers of a rooster.

fo/lai-n Amanuban. rooster wattle. [Form: nai-n $=$ 'chicken/rooster comb'.]

Out-comparisons:

manu lain East Tetun. the long tail feathers of a rooster. (Mo:124)

*daki PRM. body dirt. Etym: *daki 'dirt on skin; dandruff; tartar on teeth'. Pattern: k-irr. [irr. from PRM: ${ }^{*} \mathrm{~d}>r$ in Meto (expect $n) ; * \mathrm{k}>h / \mathrm{V}$ V in Meto] da?i Termanu. dirt on the body. (J:71) da?i Korbafo.

da?i Bokai. da daki Bilbaa. da?i Rikou. da?i $B a^{\prime} a$. da?i Tii.

rai Oenale. rahi Kotos Amarasi. body filth. lahi Molo. skin filth. (M:256)

Out-comparisons:

dakin Semau Helong. ra?i Hawu. filth, dirt, body filth.

*dalo Morph: *dalə-k. PRM. in, inside, feelings. Etym: *daləm 'in, area within, inner part of something; between; below, under; deep; mind, feelings, liver (fig.)'.

dale-k Termanu. a) inside. b) the interior of a person, the heart, the mind. (J:72)

dale-? Korbafo.

dale-k Bokai.

dale-? Bilbaa.

dale $=$ na $L a n d u$. seat of emotions. (own field notes)

dale-? Rikou.

dale-k $B a^{\prime} a$.

dale-k Tii.

lala-? Dengka.

rala-? Oenale. 
nana|? Ro'is Amarasi. inside. [Note: nere-f $=$ 'seat of emotions'.]

nana-f Kotos Amarasi. inside, in. [Note: neka-f $=$ 'seat of emotions'.] $<$ nana> Molo. inner, inside. (M:425) naan Kusa-Manea. inside.

Out-comparisons:

dale Semau Helong. inside; seat of emotions, lower, ground.

laran East Tetun. the interior, the inside part. [Sporadic: consonant metathesis * $\mathrm{IVr}>$ rVl] (Mo:127)

raram (2) rorom Kisar. 1) inside. 2) down into earth or into seas, depth.

dara Hawu. 1) inside. 2) character, seat of emotions, heart, thoughts.

*damei PRM. lick. Pattern: d-2. [irr. from PRM: *ei $>i$ in Meto; *ei $>o i$ in Rikou (possibly sporadic assimilation to previous $m$ )] [Form: The Galolen and Dadu'a forms are similar to the RM forms, but it is unclear what vowels or medial consonant a higher level reconstruction might have.] [History: Ross and Osmond (2016a:268) reconstruct $\mathrm{POc} * \mathrm{~d}(\mathrm{r}) \mathrm{am}^{\mathrm{w}}{ }^{\mathrm{i}}(\mathrm{s})$ 'lick (intr.)'.]

na-la-mei (2) mei mei Termanu.

1) lick. 2) the tongue of the snake constantly goes in and out, as in licking motion. (J:354)

na-la-mei Korbafo.

na-la-mei Bokai.

na-la-moi Bilbaa.

na-ra-moi, na-la-moi Rikou.

na-la-mei $B a^{\prime} a$.

na-ra-mei $T i i$.

na-la-mei Dengka.

na-ra-mei Oenale.

n-rami Kotos Amarasi. lick.

lami Molo. lick. [Note: Jonker

(1908:354) gives Meto lame.]

(M:261)

\section{Out-comparisons:}

rema Galolen. lick, flatter.

rabi Dadu'a. lick. (Penn 2006:103)

*daru Morph: *daru-k, *ma-daru.

CER. long, length. Doublet: *naru.

Etym: *adaduq.

dalu-k (2) ma-dalu Termanu.

1) long. 2) long, length. (J:74)

dalu-? Korbafo.

dalu-k Bokai.

dalu-? Bilbaa.

daru-? Rikou.

dalu-k $B a^{\prime} a$.

*dei Morph: *dei-k. Rote. forehead. Etym: *daqih.

de $\sim$ dei-k Termanu. forehead. (J:81)

de dei-? Korbafo.

de dei-k Bokai.

de dei-? Bilbaa.

de dei-? Rikou.

lee-? Dengka.

ree-? Oenale.

Out-comparisons:

ree-n East Tetun. forehead, brow. (Mo:160)

*deki CER. kiss. Pattern: d-1/2, k-7/8/9/10. [Note: Jonker (1908:81) gives Loura (ISO 693-3 [lur]) diki as possibly cognate, but I have been unable to locate this form anywhere.] [irr. from PRM: $* \mathrm{i}>a$ in Meto (possibly influence from neka-f 'feelings')] [Form: The lack of reflexes in wRote and Meto means that the initial consonant is ambiguous between $* \mathrm{~d}$ and $*^{*}$. I have tentatively reconstructed $* \mathrm{~d}$ on the basis of the Helong reflex as this may attest borrowing from Meto or wRote in which $* d>*$ r had taken place. The other out-comparisons provide conflicting evidence for PRM $* d$ and $* d$. Fehan Tetun would be consistent with PRM *d but East Tetun would be consistent with PRM *d.]

de?i Termanu. kiss. (J:81)

de?i Korbafo.

de?i Bokai. 
deki Bilbaa.

de?i Rikou.

Out-comparisons:

liki Semau Helong. kiss, sniff. [irr. from PRM: *d $=l / \#$ correspondence]

de?i Fehan Tetun. kiss. [irr. from PRM: *d $=d$ correspondence (expect $r$ )]

rei East Tetun. kiss. (Mo:160)

ri?i Sika. kiss. (Pareira and Lewis 1998:174)

*deku PnMeto. strike, knock. Pattern: $\mathrm{k}-5 / 6$.

n-reku Kotos Amarasi. strike (e.g. clock).

n-leku Molo. knocks. (M:266)

reuk Kusa-Manea. hit, knock.

Out-comparisons:

diku Semau Helong. hit, pound, punish, flail, whip, beat.

deku East Tetun. give light blows, tap, knock. (Mo:23)

*dene PRM. kapok tree.

dene Termanu. kapok, kapok tree. (J:84)

dene Korbafo.

dene Bokai.

dene Bilbaa.

dene Rikou.

dene $B a^{\prime} a$.

dene Tii.

lene Dengka.

rene Oenale.

neke Ro'is Amarasi. kapok tree.

neke Kotos Amarasi. kapok tree.

neke Molo. kapok tree. (M:361)

Out-comparisons:

deyen Semau Helong. kapok. [Note: Jonker (1908:84) gives Helong kdejen/denen.]

${ }^{\circ}$ neke Fehan Tetun. kapok. Borrowed from: Meto neke (shown by irr. $* \mathrm{~d}=n$ correspondence).

rini Bima. kapok. (Jonker 1893:86) *deras Morph: *ka-deras. PRM. tree. Erythrina species. Etym: *dəpdəp (Blust and Trussel (ongoing) reconstruct doublets *dəpdəp and *dapdap. The Termanu form appears as evidence for both, though the vowels are not regular from either. They would be regular from (unreconstructed) *dədap (final $*_{\partial}>e$ in Termanu, but $* \mathrm{a}>a$ ).). [irr. from PMP: $\varnothing>*_{\mathrm{s}} ; *_{\mathrm{o}}>*_{\mathrm{a}} /(\mathrm{C}) \#$ ] [irr. from PRM: ${ }^{*} \mathrm{a}>e$ in Amarasi; ${ }^{*} \mathrm{~d}>l$ in Oenale (possibly sporadic dissimilation from earlier *rVr)]

delas Termanu. the dedap, or shadowtree, varieties thereof. (J:82)

dela-? Korbafo.

dela-k Bokai.

dela-? Bilbaa.

deras Rikou.

delas $B a^{\prime} a$.

deras Tii.

lelas Dengka.

relas Oenale.

?|nenes Kotos Amarasi. kind of large tree.

$<$ nenas $>$ Molo. kind of tree. Erythrina species. (M:362)

Out-comparisons:

kdela Helong. ( $\mathrm{J}: 82$ )

*dete PMeto. Blackboard tree. Alstonia scholaris. Etym: *ditaq. [irr. from PMP: $*_{\mathrm{i}}>*_{\mathrm{e}}$ ] [minority from PMP: $* \mathrm{~d}=* \mathrm{~d}($ expect $* \mathrm{~d}>* \mathrm{r}>* 1>* \mathrm{n})]$ [Sporadic: *a $>e / \#$ in Meto] [Form: The PMeto forms are probably cognate with the Tetun form, and both could be derived from earlier **dətəq which would require irregular PMP $* \mathrm{~d}>$ $*_{\mathrm{d}}$ and $*_{\mathrm{i}}>*_{\partial}$. However, unless the Waima'a form is a borrowing from Tetun, it indicates that Tetun $o$ is from earlier $*_{0}\left(*_{\partial}>e\right.$ in Waima'a). Despite the phonological problems in explaining the Meto, Tetun and Waima'a forms, they are too similar (and furthermore too similar to PMP *ditaq) to be ignored.] 
rete Ro'is Amarasi. Blackboard tree; a kind of evergreen tree. Alstonia scholaris.

rete Kotos Amarasi. Blackboard tree; a kind of evergreen tree. Alstonia scholaris.

lete Molo. kind of tree. Alstonia scholaris. (M:271)

\section{Out-comparisons:}

(k)doti East Tetun. tree whose bark is used as an antifebrile; the bark has two varieties, one white and the other black. Alstonia scholaris. [Note: ai henak is given as having the same meaning.] [History: Morris (1984:119) gives kroti 'a tree with a very straight trunk' with the same scientific name. Van Klinken (1995) identifies kroti as 'a tall thin palm with feathery leaves. Is used to make balok, papan, ai riin (house posts)'.] (Mo:27, 104)

doti-buto, doti Waima'a. devil tree. Alstonia scholaris.

*dii1 Morph: *na-mba-dii. Rote. stand. Doublet: *dii2. Etym: *diRi. [irr. from $P R M: * \mathrm{i}>e$ in nRote (sporadic medial lowering before $* \mathrm{R}$ ?)]

na-pa-dei-? (2) na-pa-de-

dei Termanu. 1) stand, stand still, remain standing. 2) stand something up. (J:80f)

na-pa-dei-? (2) na-pa-de dei-? Korbafo.

na-pa-dei-k (2) na-pa-de dei Bokai.

na-pa-dei-? (2) na-pa-de $\sim$ dei Bilbaa.

na-pa-dei-? (2) na-pa-de dei Rikou.

na-pa-dei-? Oераo.

na-mpa-dei-k (2) na-mpa-de dei $B a^{\prime} a$.

na-mba-dei-k (2) na-mba-de dei Tii. na-mba-lii-? Dengka. na-mba-rii-? Oenale.

Out-comparisons:

dili Semau Helong. stand.

harii East Tetun. stand upright, straight, erect, or raised. (Mo:78)

*dii2 PRM. house post. Doublet: *diii. Etym: *hadiRi.

dii Termanu. pole, stile pillar. (J:86)

dii Korbafo.

dii Bokai.

dii Bilbaa.

dii Rikou.

dii $B a^{\prime} a$.

dii Tii.

lii Dengka.

rii Oenale.

nii Kotos Amarasi. pole, post, pillar.

nii Molo. pole, mast. (M:367)

Out-comparisons:

hdiin Semau Helong. [irr. from $P M P: * \mathrm{R}>\varnothing($ expect $l)]$

rii(n) East Tetun. column, pillar, post, pier, or stake. (Mo:161)

gerii Hawu. pole, post.

agarii Dhao. pole, post.

*di?u PMeto. chase, chase away. [History: Given that Kemak has both $/ \mathrm{r} /$ and $/ 1 /$, the Kemak form is most likely a borrowing from a variety of Meto in which PMeto $* \mathrm{~d}>* \mathrm{r}>$ l.]

n-ri?u Ro'is Amarasi. chase out, expel.

n-ri?u Kotos Amarasi. chase out, expel.

n-li?u Molo. chase. (M:279)

Out-comparisons:

li?u Kemak. chase.

*dindi PRM. wall made of dried palm leaf stems. Etym: *dindin 'wall of a house; partition off'. [irr. from PRM: $* \mathrm{~d}>l$ in Lole (expect $d$ )]

dini Termanu. wall, side (of a house) made of bebak ['palm leaf stems'], verb dini uma, provide a house with a wall. (J:88) 
dindi Rikou. wall. (own field notes)

diri Oepao. wall. (own field notes)

lindi Lole. wall. (Zacharias et al. 2014)

li lindi Dengka. (J:88)

ri rindi Dela. palm wall.

P|niki-t (2) < na-niki> Molo. 1) wall of a house. 2) protects. (M:369)

na niki Kusa-Manea. edge.

Out-comparisons:

didin East Tetun. wall of house, both internal and external. (Mo:25)

*diu Morph: *na-diu. PRM. bathe. Etym: *diRus.

na-diu Termanu. bathe. (J:90)

na-diu Korbafo.

na-diu Bokai.

na-diu Bilbaa.

na-diu Rikou.

na-diu $B a^{\prime} a$.

na-diu Tii.

na-liu Dengka.

na-riu Oenale.

na-niu $R o$ 'is Amarasi. bathe.

na-niu Kotos Amarasi. bathe.

na-niu Kusa-Manea. wash, bathe.

Out-comparisons:

diu Semau Helong. bathe, wash. [irr. from PMP: $* \mathrm{R}>\varnothing$ (expect $l$ )]

hariis East Tetun. bathe, take a bath. (Mo:78)

*domi PMeto. love, like. [History: Ross and Osmond (2016b:545) reconstruct POc *drom-i (from PMP *dəmdəm) 'think, worry; love, be sorry for, long for', but the similarity between this form and the PMeto form may be chance.]

n-romi Ro'is Amarasi. like, want to.

n-romi Kotos Amarasi. like, want to.

n-lomi Molo. like. (M:285)

Out-comparisons:

hadomi East Tetun. love, give love, have an affection for. (Mo:43) domi Waima'a. love.

domi Mambae, South. love. (Fogaça 2017:267)

adomi Welaun. love, like. (da Silva 2012:113)

*doo Morph: *doo-k. PRM. leaf. Etym: *dahun. [Sporadic: *VV-k > *VV? $>$ V?V in Amarasi (perceptual metathesis)]

doo-k Termanu. leaf. (J:92)

doo-? Korbafo.

doo-k Bokai.

doo-? Bilbaa.

doo=na Landu. leaf. (own field notes)

doo-? Rikou.

doo-k $B a^{\prime} a$.

doo-k Tii.

loo-? Dengka.

roo-? Oenale.

no?o Ro'is Amarasi. leaf.

no?o, noo-f Kotos Amarasi. leaf.

noo? Molo. leaves of trees. (M:375)

no?o-k, noo-n Kusa-Manea. leaf.

Out-comparisons:

roon Ili'uun. leaf. (dJ:136)

*dua PRM. two. Etym: *duha.

dua Termanu. two, both. (J:103)

dua Korbafo.

dua Bokai.

dua Bilbaa.

dua Rikou.

dua $B a^{\prime} a$.

dua Tii.

lua Dengka.

rua Oenale.

nua $R o$ 'is Amarasi.

nua Kotos Amarasi.

nuga Amfo'an. two. [Form: Medial consonants in Amfo'an and Baikeno are a result of fortition of a medial phonetic glide.]

nua Molo. two. (M:388)

nuban Baikeno. two. (Charles E. Grimes pers. comm.)

nua Kusa-Manea. two. 


\section{Out-comparisons:}

dua Semau Helong. two. rua East Tetun. two. (Mo:162) woro?o Kisar. two.

*dui1 Morph: *dui-k. PRM. bone. Etym: *duRi 'thorn, splinter, fish bone' (Helong retains the meaning 'thorn'.). dui-k Termanu. bone. (J:106)

dui-? Korbafo.

dui-k Bokai.

dui-? Bilbaa.

dui-? Landu. bone. (own field notes)

dui-? Rikou.

dui-k $B a^{\prime} a$.

dui-k Tii.

lui-? Dengka.

rui-? Oenale.

nui-f Ro'is Amarasi. bone.

nui-f Kotos Amarasi. bone.

nui-f Amanuban. bone.

nui-f Molo. bone. (M:390)

nui-f Kusa-Manea. bone.

Out-comparisons:

duli? Semau Helong. thorn.

rui-n East Tetun. bone, bones of the skeleton. (Mo:162)

lui-t Welaun. bone.

ruri (2) rurna Kisar. 1) thorn.

2) bones.

rurin Ili'uun. bone. (dJ:136)

*duir 2 Rote. dugong. Etym: *duyun (Blust and Trussel (ongoing) posit PCEMP *ruyuy, but initial *r cannot regularly account for the PRM form or Helong duin.). Pattern: d-2.

lui-k Termanu. sea cow. (J:332)

lui-? Korbafo.

lui-k Bokai.

lui-? Bilbaa.

rui-? Rikou.

lui-k $B a^{\prime} a$.

rui-k Tii.

lui-? Dengka.

rui-? Oenale.

lui Meto. (J:332)
Out-comparisons:

duin Helong. ( $\mathrm{J}: 332)$

*duman PRM. some. Pattern: d-2. [Note: Jonker (1908) gives ruma as being found in Seram and Ambon, but I have been unable to track down his source.]

luma Termanu. portion, some, a few. (J:333)

luma Korbafo.

luma Bokai.

luma Bilbaa.

ruma Rikou.

luma $B a^{\prime} a$.

ruma Tii.

luma Dengka.

ruma Oenale.

rumun Ro'is Amarasi. empty.

ruman (2) ruum Kotos Amarasi.

1) empty. 2) plain, 'emptily', without anything extra. [Note: Jonker (1908:333) glosses Meto luum, ruum as 'just, plainly'.]

luman Molo. empty. (M:292)

ruman Kusa-Manea. empty, blank.

Out-comparisons:

ruman Fehan Tetun. some.

ruma East Tetun. some, any, several, few. (Mo:162)

*dupi PnMeto. wall. Etym: **dumbi (pre-Meto).

krupit, rupit Kotos Amarasi. wall.

klupit, lupit, klipi, klipit Molo. wall (of a house). (M:218, 369)

\section{Out-comparisons:}

rupi Kisar. wall, make walls of the house, plaster walls(?). 


\section{D - d}

*dada PRM. warm near a fire. Etym: *danday 'warm oneself or something near a fire; heat or dry near a fire'. Pattern: d-2. [minority from PMP: $* \mathrm{~d}>*_{\mathrm{d}}($ expect $\left.* \mathrm{~d})\right]$

dala (2) dala ha?i Termanu. 1) roast.

2) warm oneself by a fire, warm up, like a woman after childbirth. (J:72)

dala Korbafo.

dala Bokai.

dala Bilbaa.

dara Rikou.

dala $B a^{\prime} a$.

dara Tii.

dala Dengka.

dara Oenale.

n-rara Ro'is Amarasi. warm oneself by a fire.

n-rara Kotos Amarasi. warm oneself by a fire.

malala? Amfo'an. hot.

n-lala Molo. roast, warm oneself. (M:259)

Out-comparisons:

e?-rara (2) e?-rara-n Buru.

1) warm over fire. 2) heat, fever. (Grimes and Grimes 2020:245)

*dade PRM. basil. Pattern: d-2. [irr. from PRM: *a $>e$ in Meto; $* \mathrm{~d}>r$ in Amarasi] [Sporadic: antepenultimate vowel reduction in most Rote.]

dala_dae, dale_dae Termanu. basil. (J:72)

dali_dai Bokai.

dara_dae Rikou.

dala_dai $B a^{\prime} a$.

dara dae Tii.

dale_mbake Dengka.

dare_dae Oenale.

dare Dela. basil.

to/rere Ro'is Amarasi. basil. [Form: The source of initial to is currently unknown.] to/rere Kotos Amarasi. basil.

to/lene, to/nene Molo. kind of mint. (M:569)

to/nena-l Timaus. basil. [Form: regular *a $>e$ word finally.]

*dafu1 Morph: *ka-dafu. PRM. useless, waste. [Form: Helong indicates earlier medial *b.]

ka|dafu-k (2) na-ka-da dafu Termanu. 1) garbage, dry garbage.

2) make dirty. (J:70)

ka|dafu-? Korbafo.

ka|dafu-k Bokai.

ka|dafu-? Bilbaa.

ka|dafu-? Rikou.

ka|dafu-k $B a^{\prime} a$.

ka|dafu-k Tii.

ka|dafu-? Dengka.

ka|dafu-? Oenale.

k|rafun Kotos Amarasi. useless.

<ma(k)lafu> Molo. dust, riddle. (M:255)

Out-comparisons:

dabun Semau Helong. not enough.

(k)rahuk East Tetun. brittle, fragile. (Mo:158)

*dafu2 Morph: *dafu dafu. PRM. chaotic, random.

dafu dafu Termanu. indifferent, wrong. (J:69)

dafu dafu Korbafo.

dafu dafu Bokai.

dafu dafu Bilbaa.

dafu dafu Rikou.

dafu dafu $B a^{\prime} a$.

dafu dafu Tii.

dafu dafu Dengka.

dafu dafu Oenale.

rafu? rafu? Kotos Amarasi. without cause, reason, or purpose, or thought as to the consequences, usually resulting in chaos for someone.

lafu? lafu? Molo. in a wild manner. (M:255) 
Out-comparisons: da dahut, dahut $\sim$ dahut Semau Helong. messy, chaotic, commotion, uproar, tumult. rahuk

rahun rahu rahun East Tetun. 1) rain in large scattered drops. 2) powder, small fragments, small pieces. 3) small things, little objects. (Mo:158)

*dai1 Morph: *na-dai. PRM. store, put inside.

na-dai Termanu. put something in somewhere, store. (J:71)

na-dai Korbafo.

na-dai Bokai.

na-dai Bilbaa.

na-dai Rikou.

na-dai $B a^{\prime} a$.

na-dai $T i i$.

na-dai Dengka.

na-dai Oenale.

na-dai Dela. fill (bottle).

na-rai Kotos Amarasi. put inside, fill.

na-lai Meto. (J:71)

\section{Out-comparisons:}

rai East Tetun. put down, retain, remain, keep, to guard. (Mo:158)

rai Waima'a. put, lay.

*dai2 Rote. reach, arrive at.

dai Termanu. reach, arrive at. (J:70)

dai Korbafo.

dai Bokai.

dai Bilbaa.

dai Rikou.

dai $B a^{\prime} a$.

dai Tii.

dai Dengka.

dai Oenale.

Out-comparisons:

dai $H a w u$. arrive, until, the point that. [Form: $\mathbf{d a e}=$ singular.]

*dalan PRM. path. Etym: *zalan. dala-k Termanu. course. [Semantics: In Termanu the meaning is restricted to 'course'; in other Rote varieties the meaning is broader.] (J:72)

dala-? Korbafo. course, way.

dala-k Bokai.

dala-? Bilbaa.

dala-? Rikou.

dala-k $B a^{\prime} a$.

dala-k Tii.

dala-? Dengka.

dala-? Oenale.

dala-? Dela. way, path, road.

ranan Ro'is Amarasi. way, path, road.

ranan Kotos Amarasi. way, path, road.

lanan Amanuban. way. (M:259)

lalan Molo. way. [Form: regular *n > $\left.l / 1 \mathrm{C}_{-}.\right](\mathrm{M}: 259)$

ranan Kusa-Manea. way, path, road.

Out-comparisons:

lalan Semau Helong. road, trail, path.

dalan East Tetun. road, track, path. (Mo:22)

saal Mambae, South. road, path, way. (Grimes et al. 2014b:39)

salan Galolen. road, path, way.

fara Dhao. path, trail, road, way.

kalla Kisar. road, path, street.

sala(n) Ili'uun. road, path, way. (dJ:136)

rufara $H a w u$. way, path, road.

*dama PRM. resin, gum, plaster. Etym: *damaR 'resin or gum exuded by certain trees, notably of the genera Shorea and Hopea; resinous torch; resinous tree'. [minority from PMP: $* \mathrm{~d}>*_{\mathrm{d}}($ expect $* \mathrm{~d})$ ] [Sporadic: $* \mathrm{a}>$ $e$ /_\# in Meto.] [History: If this is a borrowing (from Malay damar or similar), then it is an early borrowing as final $*_{\mathrm{a}}>e$ in Meto does not otherwise occur in loans.] 
dama Termanu. a) gum, resin. b) dama ofa-k tar a boat. From that, more generally: cover with; also: work with mortar, or concrete masonry: dama uma, plaster a house; dama lates, make a grave with mortar; late na-na-dama-k, a plastered grave or a grave made with mortar. (J:74)

dama Korbafo.

dama Bokai.

dama Bilbaa.

dama Rikou.

dama $B a^{\prime} a$.

dama Tii.

dama Dengka.

dama Oenale.

dama Dela. build concrete wall.

rame? (2) n-rame Kotos Amarasi.

1) concrete wall, safety, security.

2) plaster, build concrete wall. [Semantics: The semantic expansion to include 'concrete' is obviously recent.]

\section{Out-comparisons:}

${ }^{\circ}$ dame Helong. tar, plaster, cover. Borrowed from: Meto before $*_{\mathrm{d}}>r$ (shown by final $* \mathrm{a}=e$ correspondence). (J:74)

*dano Rote. lake. Etym: *danaw. [minority from PMP: $* \mathrm{~d}>*_{\mathrm{d}}$ (expect $* \mathrm{~d})$ ] [History: possibly a borrowing from Malay danau or similar.]

dano Termanu. lake, pool. (J:75)

dano Korbafo.

dano Bokai.

dano Bilbaa.

dano Rikou.

dano $B a^{\prime} a$.

dano Tii.

dano Dengka.

dano Oenale.

*danga1 PRM. step over. [History: possibly connected with PMP *lankaq.] daya Termanu. softly tiptoe, step over. <ana danga seli-n $>$ he steps over him (J:75) daya Korbafo.

dana Bokai.

daya Bilbaa.

daka Rikou.

dayga $B a^{\prime} a$.

danga Tii.

dayga Dengka.

dayga Oenale.

n-raka, n-rakan Kotos Amarasi. step over, cross, stride.

n-laka Molo. steps over. (M:256)

*daygar PwRM. hand span. Etym:

*zankal (PWMP. Blust and Trussel

(ongoing) also reconstruct PMP

*zanan 'hand span'.).

dayga Dengka. span. (J:621,686)

n-raka (2) raka-t Kotos Amarasi.

1) measure with hand span. 2) hand span.

hae-n laka-n (2) < nima-n> laka-n Molo. 1) step of foot. 2) span of hand. (M:256)

Out-comparisons:

daya Semau Helong. span of hand, inch. [Note: Jonker (1908:621) gives Helong sdana.]

*dasi $n R M$. discussion, speech.

dasi (2) dasi-k Termanu. 1) singing of birds; used in combination with soda $[\mathrm{OE}=$ 'sing']. Also (though less common in Termanu): say, tell (frequently with this meaning in poems). 2) song (also of people), speech, word. Usage: mainly poetic. (J:76)

dasi Tii. discuss, speak. (Grimes et al. 2014a)

rasi Ro'is Amarasi. matter, affair, issue.

rasi Kotos Amarasi. matter (nonphysical), affair, issue.

lasi Molo. case, ritual, story, lawsuit. (M:263)

rasi Kusa-Manea. language, speech.

\section{Out-comparisons:}

dasi Semau Helong. language, matter, thing, dispute, litigation, affairs. 
*dee PRM. subordinating particle.

dee Termanu. and, and then, and therefore. (J:76)

dee Korbafo.

dee Bokai.

dee Bilbaa.

dee Rikou.

dee $B a^{\prime} a$.

dee Tii.

dee Dengka.

dee Oenale.

de Dela. then, thus, so.

he? Ro'is Amarasi. RELATIVISER. [irr. from PRM: * $\mathrm{r}>h$ ]

re? Kotos Amarasi. a) general purpose relativiser, including time ('when') and location ('where'). b) marker of an NP that is already a known referent.

le? Molo. which. (M:264)

on ro? Kusa-Manea. like, similar to. Usage: In Amarasi on and on re? (when the following NP is a topic) are used to mean 'like'. Kusa-Manea ro? has not yet been attested without preceding on. [irr. from PRM: *e > o]

\section{Out-comparisons:}

fe Hawu. then, while. Realis, indicating actual action or result.

*deha Rote. speak.

de?a (2) de $\sim$ de?a-k Termanu. 1) say, speak. 2) that which is said, saying, word, language, report; therefore: matter. (J:79)

de?a (2) de $\sim$ de?a-? Korbafo.

de?a (2) de de?a-k Bokai.

dea (2) de $\sim$ dea-? Bilbaa.

de dea Landu. speak. (own field notes)

dea (2) de dea-? Rikou.

de?a (2) de de?a-k $B a^{\prime} a$.

de?a (2) de de?a-k Tii.

(2) de dea-t Dengka.

(2) de $\sim$ dea-t Oenale.

\section{Out-comparisons:}

dehet (2) nahdehe Semau Helong.

1) story, news, language. 2) talk, tell a story. [irr. from PRM: *a $=$ $e$ correspondence]

dePan Fehan Tetun. speak angrily; speak abusively (e.g. 'you're a dog').

dehan East Tetun. speak. (Mo:23)

*deke Morph: *deke-k. Rote. kernel, pip of fruit. Pattern: k-7.

dePe-k Termanu. kernel, pip of fruit. ( $\mathrm{J}: 80)$

de?e-? Korbafo.

de?e-k Bokai.

deke-? Bilbaa.

deke-? Rikou.

de?e-k $B a^{\prime} a$.

de?e-k Tii.

deke-? Dengka.

deke-? Oenale.

Out-comparisons:

ai tahan dikin East Tetun. bud, shoot, sprout. [Form: Jonker (1908:80) gives Tetun Dili dikin $=$ 'eye, knot of a plant'.] (Mo:2)

*de?u PwRM. sacred, awe inspiring, awful, bad.

de?u Dengka. cattle plague. (J:688)

re?u (2) re?uf (3) n-re?u Kotos Amarasi. 1) sacred, holy, aweinspiring. 2) bad, evil. 3) broken.

le?u Molo. magic. (M:272)

na-mreu? Kusa-Manea. broken.

*dele PRM. Job's tears. Coix lachrymajobi. Etym: *zolay.

dele Termanu. kind of millet, which is a staple food in eastern Rote. (J:82)

dele Korbafo.

dele Bokai.

dele Bilbaa.

dele Rikou.

dele $B a^{\prime} a$.

dele Tii.

dele Dengka.

dele Oenale. 
'sone Ro'is Amarasi. Job's tears. Borrowed from: Welaun (or extinct related language) sole before $* 1>n$ in Meto. For Welaun $*_{\mathrm{Z}}>s$ and $*^{2}$ $>o$ are both regular.

${ }^{\circ}$ sone Kotos Amarasi. Job's tears.

${ }^{\circ}$ sone Molo. kind of grain. (M:513)

Out-comparisons:

sele Kemak. corn, maize.

sole (2) sole fatun Welaun.

1) corn, maize. 2) Job's tears. [Note: fatun = 'stone'.] [Semantics: It is likely that the original designation was 'Job's tears' and that maize was assimilated as a kind of Job's tears. With increased importance of corn at the expense of Job's tears, the core semantic meaning also shifted. See the discussion under *mbela.]

sela Mambae, Northwest. corn, maize. (Fogaça 2017:236)

otsele Fataluku. corn, maize. Borrowed from: an Austronesian language. [Note: non-Austronesian language of east Timor ISO 639-3 [ddg].] (Heston 2015:84)

*deli CERM. tendril, vine.

deli Termanu. kind of climbing plant with bean shaped fruit, small oval leaves, and blue flowers with white calyx. (J:83)

uut k|reni|? Ro'is Amarasi. pumpkin tendril.

k|reni|? Kotos Amarasi. tendril (particularly of a pumpkin).

*dene $n R M$. field, garden. [irr. from PRM: ${ }^{*} \mathrm{~d}>r \sim l$ in Rikou and Bilbaa for the second sense]

na-dene Termanu. give a field (etc.) to others in return for the cost of wages. ( $\mathrm{J}: 84)$

na-dene Korbafo.

na-dene Bokai. na-dene (2) lene Bilbaa. 2) wet rice field. (J:721)

na-dene (2) rene Rikou. 2) wet rice field. (J:721)

na-dene $B a^{\prime} a$.

na-dene Tii.

rene $R o$ 'is Amarasi. field.

rene Kotos Amarasi. field.

lene Amanuban. field. (M:267)

lele-l Amfo'an. field. [Form: regular $*_{\mathrm{n}}>l / 1 \mathrm{C}$. $]$

lele Molo. garden. [Form: regular *n $>l / 1 \mathrm{C}_{\text {_.j }}(\mathrm{M}: 267)$

rene Kusa-Manea. field.

*denu Morph: *na-denu. PRM. order, command.

na-denu Termanu. send someone; command. (J:84)

na-denu Korbafo.

na-denu Bokai.

na-denu Bilbaa.

na-denu Rikou.

na-denu $B a^{\prime} a$.

na-denu Tii.

denu Dengka.

denu Oenale.

n-renu Kotos Amarasi. order, command.

n-lenu Amanuban. commands. (M:268)

n-lelu Molo. commands. [Form: regular *n $>l / 1 \mathrm{C}].(\mathrm{M}: 268)$

reun Kusa-Manea. order, command.

Out-comparisons:

donu Welaun. order, command.

*dere Rote. beat a drum.

dele labu Termanu. beat the drum quickly, beat the ruffle, ruffle up. (J:82)

dele Korbafo.

dele Bilbaa. beat.

dere Rikou.

de dele $B a^{\prime} a$.

dere Tii.

dele Dengka. beat.

dere Oenale. 


\section{Out-comparisons:}

dele Helong. beat. ( $\mathrm{J}: 82$ )

dere East Tetun. give repeated blows with a hitting implement.

(Mo:24)

dere Hawu. (J:82)

*dero Morph: *ka-dero. PRM. citrus. [History: Ultimately connected with Malay jeruk [dzeru?] or a related form, but $* 1>n$ in Meto indicates this was an early borrowing.]

delo Termanu. all kinds of citrus trees and fruit. (J:83)

delo Korbafo.

delo Bokai.

dero Bilbaa.

dero munde $L a n d u$. (own field notes)

delo $B a^{\prime} a$.

delo Tii.

delo-? Dengka.

dero Oenale.

reno|? Ro'is Amarasi. sweet orange.

?|reno|? Kotos Amarasi. sweet orange.

<lelo> Molo. orange (fruit). Citrus species. (M:268)

P|lelo|? Timaus. citrus.

reno|? Kusa-Manea. citrus.

Out-comparisons:

derok East Tetun. orange fruit. (Mo:24)

*deta Rote. dip, dunk.

deta Termanu. dip. (J:85)

deta Korbafo.

deta Bokai.

deta Bilbaa.

deta Rikou.

deta $B a^{\prime} a$.

deta Tii.

deta? Dengka.

deta Oenale.

Out-comparisons:

ketəda Hawu. [irr. from PRM: consonant metathesis] (J:85)

<dàtalu> Kambera. momentarily touch. [Note: also in Mangili, Lewa, and Anakalang.] (On:33)

<dàtala> Mamboru.

$<$ dètala $>$ Weyewa. *deu Rote. make a fire, strike up a fire with a fire lighter.

deu Termanu. make a fire, strike up a fire with a fire lighter. $(\mathrm{J}: 85)$

deu Korbafo.

deu Bokai.

deu Bilbaa.

deu Rikou.

deu $B a^{\prime} a$.

deu Tii.

deu Dengka.

deu Oenale.

Out-comparisons:

deu Hawu. (J:85)

*dəma PRM. deep. [irr. from PRM: * $\mathrm{d}>$ $r$ in Dela (expect $d) ; *^{*}>o$ in Dela]

ma-dema (2) na-ma-dema (3) dema-k (4) na-dema Termanu. 1) high, deep. 2) be or become high or deep. 3) high, height, deep, depth. 4) make high or deep. ( $\mathrm{J}: 83$ )

ma-dema Korbafo.

ma-dema Bokai.

ma-dema Bilbaa.

ma-dema Rikou.

ma-dema $B a^{\prime} a$.

ma-dema Lole. tall, high, deep. (Zacharias et al. 2014)

ma-dema Tii.

ma-dema Dengka.

roma-? Dela. deep.

n-rema Kotos Amarasi. drown.

n-lema Molo. drown. (M:268)

Out-comparisons:

merəma Hawu. deep.

.əma Ende. deep.

ləman Sika. deep or far-away place. [irr. from PRM: ${ }^{*} \mathrm{~d}=l$ correspondence] (Pareira and Lewis 1998:12)

ləma Ngadha. deep. (Djawanai 1995:Part 4, 1)

*dəmbə PRM. dip into. [irr. from PRM:

$*_{\partial}>o$ in Rote]

dope Termanu. dip. (J:101)

dope Korbafo. 
dope Bokai.

dope Rikou.

dompe $B a^{\prime} a$.

dombe Tii.

đombo Dela. dip, swim.

na-repa? Kotos Amarasi. dip.

<n-lepe> Molo. dip, dunk. (M:270)

Out-comparisons:

dopon Semau Helong. dip.

*dii1 Morph: *ka-dii. PwRM. left

(side). Doublet: *kii. Etym: *diwiRi (combination of *di 'locative case marker' and *wiRi 'left side or direction'.).

dii Dengka. left, north. (J:688)

dii-? Oenale. left, north. (J:688)

?|rii Kotos Amarasi. left.

?|lii Molo. left. (M:273)

Out-comparisons:

kliu Semau Helong. [irr. from PRM: ${ }^{*} \mathrm{~d}=l$ correspondence]

mariri Kisar. left.

*diiz PRM. whinny. [Semantics:

onomatopoeia.]

na-ka-dii Termanu. make the sound dii, whinny of a horse. (J:86)

na-ka-dii Korbafo.

na-ka-dii Bokai.

na-ka-dii Bilbaa.

na-dii Rikou.

na-ka-dii $B a^{\prime} a$.

na-ka-dii $T i i$.

na-?a-dii Dengka.

na-Pa-dii Oenale.

eku_lii, euk_rii Meto. whinny. (J:86)

*diki $\bar{P} R M$. small. Etym: *dikit 'little,

few, small in amount'. Pattern: k-7.

[minority from PMP: $* \mathrm{~d}>*_{\mathrm{d}}$ (expect

$\left.{ }^{*} \mathrm{~d}\right)$ ] [irr. from PRM: ${ }^{*} d>h$ in Dengka]

ka-di?i Termanu. be(come) small. (J:86)

ka-di?i Korbafo.

ka-di?i $B a^{\prime} a$.

ka-di?i Tii.

ana_hiki-? Dengka. small. (J:677)

ana_diki-? Oenale. small. (J:677) ana_diki-? (2) ana_hiki-? Dela.

1) small child. 2) small.

ri?i|t (2) ri?/ana? (3) ri?/feto

(4) ri?/mone Kotos Amarasi.

1) younger sibling. 2) child. 3) girl.

4) boy.

liPa|t (2) li?/mone (3) li?/feto Molo.

1) young man. 2) boy. 3) girl.

(M:273)

Out-comparisons:

kiPik East Tetun. small or little in size. [irr. from PMP: *d $>k$ ] (Mo:107)

iki Hawu. tiny, small.

Siki Dhao. tiny, small.

*dila1 Morph: *dila-k. CER. wing. See: *lida.

dila-? Landu. (own field notes)

dila-? Rikou.

dila-? Oepao. (own field notes)

Out-comparisons:

kdila?, klila? Funai Helong. wing.

dila? Semau Helong. wing.

dila/paan Kemak. wing. Usage: Leosibe dialect.

*dilaz Rote. shine, glitter. Etym: *dilap 'sparkle, shine'. [minority from PMP: $* \mathrm{~d}>* \mathrm{~d} 7$

na-na-dila (2) dila dila Termanu.

1) shine, glitter. 2) shine, glitter. (J:87)

na-na-dila Korbafo.

na-na-dila Bokai.

na-na-dila Bilbaa.

na-ka-dila Rikou.

na-nga-dila $B a^{\prime} a$.

na-nga-dilak Tii.

na-Pa-dila? Dengka.

*dilah PRM. bael, wood apple. Aegle marmelos. [Semantics: The designation was almost certainly a native tree, probably the bael/wood apple (Aegle marmelos $)$, which has fruit similar to the pomegranate. The pomegranate was then classified as a sub-type of this tree and eventually took over the meaning entirely.] 
dila-k (2) dila dae loo-k Termanu.

1) kind of wild pomegranate tree.

2) the normal pomegranate. (J:87)

dila-? Korbafo.

dila-k Bokai.

dila(-?) Bilbaa. [Form: final -1 given with a question mark.]

dila(-?) Rikou. [Form: final -? given with a question mark.]

dila boi-k $B a^{\prime} a$.

dila-k Tii.

dila Dengka.

dila Oenale.

rinah Ro'is Amarasi. kind of tree with a fruit like a pomegranate.

rinah (2) riin fui Kotos Amarasi. 1) pomegranate. 2) kind of tree with a fruit like a pomegranate which grows in the wild.

liin hau (2) liin kase Amanuban.

1) kind of tree the fruit of which has a gelatinous fluid. Aegle marmelos.

2) kind of pomegranate. (M:275)

lilah (2) liil kase, lila kase Molo.

1) kind of tree whose fruit has gelatinous fluid. Aegle marmelos.

2) kind of pomegranate. Punica granatum. [Form: regular $*_{\mathrm{n}}>l /$ 1C_.] (M:275)

rinah Kusa-Manea. pomegranate.

Out-comparisons:

dila Fehan Tetun. pomegranate, pawpaw.

ai dila (2) ai dila tukun, ai dila

fatuk East Tetun. 1) pawpaw tree and fruit. (Mo:2) 2) tree known as the quince tree of Timor, bearing hard orange coloured fruit. (Mo:25)

kai dile wai Waima'a bael fruit. Aegle marmelos.

<diliméné> Kisar. pomegranate. Punica granatum. (Heyne 1950:1158, lxxxv)

dila Kemak. papaya, paw-paw.

dila Welaun. papaya, paw-paw. dila Mambae, Northwest. papaya. (Fogaça 2017:235)

*dio Morph: *dio-t. PRM. crushed grain. di dio-k (2) dio-k Termanu. 1) grit of grains. 2) kind of rash on the face with (the appearance of) the grit of granules. (J:89)

di dio-? (2) dio-? Korbafo.

kadio-k (2) dio-k Bokai.

kadio-? (2) dio-? Bilbaa.

(2) dio-? Rikou.

di dio-k (2) dio-k $B a^{\prime} a$.

di dio-k (2) dio-k Tii.

di $\sim$ dio-? (2) dio-t Dengka.

di dio-? (2) dio-t Oenale.

reo|t Kotos Amarasi. crushed grains (typically corn or rice). [Sporadic: vowel height harmony $* \mathrm{i}>e$ /_o.]

lio|t Amanatun. crushed rice grains. (M:278)

leo|t Molo. fine grains of crushed rice or maize. (M:270)

*dito PRM. gum, resin, glue, sticky, stick to. [irr. from PRM: *ə $>a$ in Ba'a] [Sporadic: $*_{\partial}>e / \sigma \#$ in wRote (perhaps $*_{\partial}>*_{\mathrm{a}}>\mathrm{e} /$ \#).] [History: Blust and Trussel (ongoing) reconstruct PAN *ditəq 'sticky substance' on the basis of the Termanu reflex and Amis dita? 'a lump of clay for making pottery; clay; a type of soil'. Unless more evidence is forthcoming, I do not find this reconstruction convincing. They also reconstruct PMP *litəq 'sap of a tree or plant' on the basis of much better evidence, though this reconstruction cannot account regularly for the forms given here.]

di dite (2) na-ka-dite Termanu. 1) plant gum, bird lime. 2) be sticky, stick to. (J:89)

di dite Korbafo.

di dite Bokai.

di dite Bilbaa.

di $\sim$ dita $B a^{\prime} a$.

di dite Tii.

dite Dengka. 
dite Oenale.

n-rita (2) a-rita-s Kotos Amarasi.

1) frozen, coagulated (can be said of blood or liquid). 2) thick (e.g. of milk).

n-liit (2) < lit> Molo. 1) sticks to, cures (milk). 2) glue, adhesive. (M:278)

Out-comparisons:

'litas Semau Helong. thick. Eta unit ta litas son nam nakin tia. When the sugar is thick, take it off the heat. Borrowed from: Meto rita-s (shown by irr. ${ }^{*} \mathrm{~d}=l$ correspondence and final $s$ ).

ritan (2) ha-ritan East Tetun. 1) resin, gum. (Mo:161) 2) glue, stick together with glue, gum or resin. [irr. from PRM: $* d=r$ correspondence] (Mo:78)

krite Waima'a. sticky.

*doa PRM. burp, belch. [Sporadic: glottal stop insertion in Meto.]

doa Termanu. have an eructation, burp. (J:92)

doa Korbafo.

doa Bokai.

doa Bilbaa.

doa Rikou.

doa $B a^{\prime} a$.

doa Tii.

doa Dengka.

doa Oenale.

doa Dela. burp.

n-ro?a Ro'is Amarasi. vomit.

n-ro?a Kotos Amarasi. vomit.

n-loo? Molo. throws up. (M:280)

roa? Kusa-Manea. vomit.

Out-comparisons:

deaך Semau Helong. burp, belch. [irr. from PRM: $*_{\mathrm{o}}=e$ correspondence]

*dodo PRM. kill by stabbing. Etym:

*dodok 'pierce, stab' (PCMP).

[minority from PMP: $* \mathrm{~d}>*_{\mathrm{d}} / \# ; * \mathrm{~d}$

$\left.>*_{\mathrm{d}} / \mathrm{V}_{-} \mathrm{V}\right]$ dodo Termanu. slit the throat, slaughter. (J:94)

dodo Korbafo.

dodo Bokai.

dodo $B a^{\prime} a$.

dodo Tii.

dodo Dengka.

n-roro Ro'is Amarasi. kill.

n-roro Kotos Amarasi. kill by stabbing. [Semantics: Does not refer exclusively to slitting the throat.]

n-lolo Molo. slaughters. (M:284)

roor Kusa-Manea. kill.

Out-comparisons:

dodo Semau Helong. technique of multiple stabbing of carotid artery (for goat).

* doi PRM. carry on shoulder with pole.

na-la-doi Termanu. carry something on a pole so that the load hangs from one end of the pole above one's back. (J:95)

na-la-doi Korbafo.

na-na-doi Bokai.

na-ka-doi Rikou.

doi $B a^{\prime} a$.

na-la-doi $T i i$.

na-nga-doi Dengka.

doi Oenale.

n-roi Kotos Amarasi. carry on shoulder with pole.

n-loi Molo. carries a pole on his shoulder. (M:282)

roi Kusa-Manea. carry.

Out-comparisons:

dui, dui Dhao. carry on shoulder, carry with a pole.

dui Hawu. carry on shoulder.

doi Keo. carry something with stick on shoulder. [Note: language of central Flores ISO 639-3 [xxk].] (Baird 2002:547) 
*doka Morph: *doka_dalə-k. Rote. knee cavity. Pattern: k-5. [History: Perhaps related to PMP *dəkuk 'bow, bend downward', though this requires irr. ${ }^{*} \mathrm{~d}$ $>*_{d}$ and $*_{\partial}>*_{0}$.]

doka_dale(-k) Termanu. knee cavity. (J:96)

loka_dale-? Korbafo.

doka_dale-k Bokai.

doka_dale-k $B a^{\prime} a$.

doka_dale-k Tii.

doka_rala-? Oenale. [Form: This form is given as 'resp. doka dale-?' but the word for 'inside' in Oenale is rala-?.]

Out-comparisons:

kerəki Hawu. (J:96)

$<$ karoka $>$ Kambera . cavity, bend. [Note: also in Mangili, Lewa, and Anakalang.] (On:188)

$<$ kaleka $>$ Mamboru.

*doki Morph: *do doki. Rote. desire. Pattern: k-5' ${ }^{*} \mathrm{k}>\varnothing$ in Rikou; expect $?$ or $k$, and $* \mathrm{k}>$ ? in Dengka; expect $\left.*_{\mathrm{k}}=k\right)$.

do doki Termanu. desire or crave something. (J:97)

do doki Korbafo.

do doki Bokai.

do doi, (do do?i ?) Rikou.

do doki $B a^{\prime} a$.

do $\sim$ doki Tii.

do $\mathbf{d o}$ ?i Dengka.

do doki Oenale.

Out-comparisons:

duki Hawu. (J:97)

*do?i PRM. prise out, lever out.

do?i Termanu. picks out, e.g. to lever something up with a stick like a lever. (J:96)

do?i Korbafo.

do?i Rikou.

do?i $B a^{\prime} a$.

do?i Tii.

do?i Dengka.

do?i Oenale. n-ro?e Kotos Amarasi. pick out, pick seeds from a piece of fruit. [Sporadic: vowel height harmony $*_{\mathrm{i}}>$ e /oC_.]

lo?i Molo. churns the ground up. (M:282)

ro?i Kusa-Manea. dig out.

Out-comparisons:

tuki Semau Helong. dig out, peck, adze. [irr. from PRM: $* \mathfrak{d}=t$ correspondence]

<ruki> Kambera. take out. (On:449)

$<$ rauku> Lewa.

$<$ rauki $>$ Anakalang.

dokit Sika. dig out. (Pareira and Lewis 1998:40)

*dole Morph: *dole-k. $n R M$. brains, marrow.

do dole-k (2) laya do dole-k (3) mata do dole-k (4) nado dole Termanu. 1) anything like grease, therefore brains. 2) marrow from bones. 3) eye grease, dirt from the eyes. 4) have brains or marrow. (J:98)

do dole-? Korbafo.

do dole-k Bokai.

do dole-? Bilbaa.

do dole-? Rikou.

do dole-k $B a^{\prime} a$.

do dole-k Tii.

roene-f $R o$ 'is Amarasi. brain.

rone-f (2) maat rone? Kotos Amarasi. 1) brain. 2) rheum, eye gunk.

lone-f Amanuban/Amanatun. brains. (M:283)

lole-f Molo. brains. [Form: regular *n $>l / 1 \mathrm{C}$.] (M:283)

rone-f Kusa-Manea. brain.

Out-comparisons:

dole-n East Tetun. marrow (of bones). (Mo:26)

dole Hawu. marrow. (J:98)

*dolu PRM. draw water, lower, fish with a rod. 
dolu Termanu. a) draw water by tying the dipper onto a rope and lower it down into the well by means of a rope; also: lower on a rope. c) well (n.). b) fish, fish with a rod. (J:99)

dolu Korbafo.

dolu Bokai.

dolu Bilbaa.

dolu Rikou.

dolu $B a^{\prime} a$.

dolu Tii.

dolu Dengka. fish, fish with a rod. [Semantics: Only the final Termanu sense is recorded as being present in Dengka and Oenale.] (J:99)

dolu Oenale. fish, fish with a rod. (J:99)

<lolu> (2) an-lolu Molo. 1) hook, anchor of ship. 2) taken with a harvesting hook. (M:284)

\section{Out-comparisons:}

dulu Hawu. enter deeply, pierce downward.

<dúlungu> Kambera. hang down, dangle. [Note: also in Mangili.] (On:41)

$<$ dolungu $>$ Lewa.

$<$ daulungu> Anakalang.

<padulungu> Mamboru.

$<$ dòluna $>$ Weyewa.

$<$ dalungo $>$ Kodi.

*dombe Rote. knife. See: ${ }^{\#}$ kopi.

dope Termanu. knife. (J:101)

dope Korbafo.

dope Bokai.

dope Bilbaa.

dope Rikou.

dompe $B a^{\prime} a$.

dombe Tii.

dombe Dengka.

dombe Oenale.

Out-comparisons:

supi Sika. knife. (Pareira and Lewis 1998:187)

fobe Hawu. knife. (J:101)

*doo Morph: *ka-doo. PRM. far, distant.

Etym: *zauq. doo-k Termanu. long of distance, far, long of time. (J:90)

doo-? Korbafo.

doo-k Bokai.

doo-? Bilbaa.

doo-? Rikou.

doo-k $B a^{\prime} a$.

doo-k Tii.

doo-? Dengka.

doo-? Oenale.

na-?|roo Ro'is Amarasi. far.

na-?|roo Kotos Amarasi. far.

na-k|loo-g Amfo'an. far. (own field notes)

2|loo Molo. far. (M:280)

roo Kusa-Manea. far.

Out-comparisons:

(k)dook (2) dodook East Tetun.

1) far, distant, remote. (Mo:27,

104) 2) go further away. (Mo:26)

soo Galolen. far.

ko?u Kisar. far.

soo Ili'uun. far. (dJ:137)

kafəu Dhao. far.

fəu Hawu. far.

*dosa Rote. vinegar.

dosa (2) na-ka-do dosa Termanu.

1) vinegar. 2) make sour. (J:102)

dosa Korbafo.

dosa Bokai.

dosa $B a^{\prime} a$.

dosa Tii.

dosa Dengka.

dosa Oenale.

Out-comparisons:

ei do kedoha Hawu. [Form: ei $=$ 'water', do $=$ 'relativiser'.] (J:102)

*doto Morph: *na-ka-doto. PRM. thunder, make a loud noise.

na-ka-doto (2) doto doto Termanu.

1) make noise, cause a din. 2) make a din. (J:102)

na-ka-doto Korbafo.

na-ka-doto Bokai.

na-ka-doto Bilbaa.

na-doto Rikou. 
na-ka-doto $B a^{\prime} a$.

na-ka-doto $T i i$.

na-?a-doto Dengka.

na-Pa-doto Oenale.

na-?|roto (2) P|roto-s Kotos Amarasi. 1) rumbling, thundering.

2) thunder.

na-P|loto Molo. it thunders. (M:287)

na-Proot Kusa-Manea. noisy.

Out-comparisons:

loto Semau Helong. rumble, grumble. [irr. from PRM: $* \mathrm{~d}=l$ correspondence]

doro Hawu. thunder. [irr. from PRM: * $\mathrm{t}=r$ correspondence (expect $d)]$

*dudi PRM. stoop, bow.

dudi Termanu. crouch down to go through or into somewhere, creep out or into somewhere, like a chick under or out of their mother's wing, or as a thief through the trees. (J:105)

dudi Korbafo.

dudi Bokai.

dudi Bilbaa.

dudi Rikou.

dudi $B a^{\prime} a$.

dudi Tii.

dudi Dengka.

dudi Oenale.

na-Pruri? Ro'is Amarasi. bend down, bow.

na-Pruri? Kotos Amarasi. bow (in respect).

<na-luli> Molo. bows. (M:291)

*dudu Rote. tinder, oakum.

dudu Termanu. tinder; also oakum used to plug up a vessel, and from that everything which is used to plug things up, e.g. putty. (J:105)

dudu Korbafo.

dudu Bokai.

dudu Bilbaa.

dudu Rikou.

dudu $B a^{\prime} a$.

dudu Tii. dudu Dengka.

dudu Oenale.

Out-comparisons:

dudu Semau Helong.

duduk East Tetun. tinder, made from the velvety exterior of tua naa, a palm tree. (Mo:28)

dudu Sika. tinder from an areng palm used to make a fire. (Pareira and Lewis 1998:42)

dudu Ende. stuff for catching a fire, made of sugar palm.

$<$ kadudu $>$ Kambera . tinder (from the areng palm). (On:130)

nduru Bima. tinder. (Jonker 1893:65)

dzu?dzu? (2) andzu?dzu?

Makassar. 1) coconut husk with rags twisted into a wick.

2) set on fire. (Cense 1979:190)

*dula PRM. engrave, tattoo, pattern.

dula (2) dula-k Termanu. 1) making drawings, figures or patterns on sarongs and sheets, etc. 2) drawing, pattern, design on a scarf, sarong, etc. $(\mathrm{J}: 106)$

dula Korbafo.

dula Bokai.

dula Bilbaa.

dula Rikou.

dula $B a^{\prime} a$.

dula Tii.

dula Dengka.

dula Oenale.

dula-? Dela. pattern.

runu-t Ro'is Amarasi. plan, scheme, event.

n-runa (2) runa-t Kotos Amarasi. 1) carve, chisel, inscribe. 2a) cloth pattern, tattoo, branding. 2b) plan, scheme, event.

luna (2) luna-t Amanuban/ Amanatun. 1) engrave. (M:291) 2) tattooed figure, also pattern in cloth and carved figures in bamboo or bone. (M:291, 293) 
lula (2) lula-t Molo. 1) engrave. (M:291) 2) tattooed figure, also pattern in cloth and carved figures in bamboo or bone. [Form: regular $\left.*_{\mathrm{n}}>l / 1 \mathrm{C} \_.\right](\mathrm{M}: 291,293)$

ruan Kusa-Manea. tattoo, writing.

Out-comparisons:

dula (2) hdulat Semau Helong. 1) write. 2) picture, illustration, portrait, image, sculpture, carving; colour, characteristic.

*dungu Rote. insert. duyu Termanu. insert somewhere, prod with something. (J:107)

dunu Korbafo.

dunu Bokai.

dunu Bilbaa.

duku Rikou.

dungu $B a^{\prime} a$.

dungu Tii.

dungu Dengka.

dungu Oenale.

Out-comparisons:

duyun Helong. (J:107)

\section{$\mathbf{E}-\mathbf{e}$}

* eda PRM. ladder, stairs. Etym: *haRəzan 'notched log ladder'. [Sporadic: $\varnothing>$ ? /\# in Dela-Oenale.]

eda-k (2) heda_huu-k Termanu. 1) stairs, ladder. (J:108) 2) ladder. [irr. from PRM: $\varnothing>h$ in sense 2 probably influenced by following huu-k]. (J:167)

eda (2) heda_huu-? Korbafo. eda-k Bokai.

(2) heda_huu-k Bilbaa.

eda Rikou.

eda-k (2) heda_huu-k $B a^{\prime} a$.

eda-k (2) heda huu-? Tii.

(?)e (?)eda-? (2) (?)e (?)eda huu-? Dengka.

Pe Peda-? Oenale.

Pe Peda-? Dela. ladder.

era|?, era|k Kotos Amarasi. steps, ladder.

ela|k Molo. ladder. (M:98)

Out-comparisons:

elan Semau Helong. ladder.

$\operatorname{oda}(\mathbf{n})$ East Tetun. stairs, staircase. (Mo:156)

rokon Kisar. ladder.

*ee PwRM. 3SG.ACC. [History: Possibly connected with PRM *ia 'this, here' (from PMP $* \mathrm{ia}$ ), though this is retained with a demonstrative meaning in all branches of Rote-Meto.] ee, nee Dengka. shortened form of Dengka and Oenale eni $=$ Termanu n, ana (3SG) as direct object or subject suffix ... sometimes also nee. (J:691)

ee, nee Oenale.

ee Dela. 3SG.ACC.

=ee (2) =nee Kotos Amarasi. 1) 3SG. OBJ, 3DET. 2) 3SG.OBJ. Usage: Occurs with the verb n-ok 'with, accompany' and occasionally with n-fee 'give'.

*ei Morph: *ei-k. Rote. foot, leg. Doublet: *hae. Etym: *qaqay. [irr. from PMP: *ay $>* \mathrm{i} ; *_{\mathrm{a}}>*_{\mathrm{e}}$ (sporadic assimilation)]

ei-k Termanu. foot, leg of a person, paw of an animal. (J:109)

ei-? Korbafo.

ei-k Bokai.

ei-? Bilbaa.

ei-? Landu. leg. (own field notes)

ei-? Rikou.

ei-k $B a^{\prime} a$.

ei-k Tii.

ei-? Dengka.

ei-? Oenale.

ei-? Dela. foot/leg.

Out-comparisons:

ii-n Funai Helong. foot/leg.

ii-n Semau Helong. foot.

ai-n East Tetun. leg, foot. (Mo:3) 
ai-n Habun. leg/foot. [Note: language of east Timor ISO 639-3 [hbu].] (Dawson 2014)

wei-r Idate. leg/foot. [History: This form may be from *waqay, but initial [w] also occurs on at least two other words where it is unexpected: *qahəlu > walu 'pestle' and *hapuy > wai 'fire.] (Dawson 2014)

ee-n Galolen. foot/leg. Usage: Talur (Wetar Island) dialect. (Hinton 2000:104)

ee Dadu'a. foot/leg. (Penn 2006:93)

ei-n Kisar. foot/leg.

ai-t Welaun. leg, foot.

*eki Rote. shout, cry out. Etym: *akit 'squeak, shriek' (Blust and Trussel (ongoing) give the Termanu form as evidence for both *akit and the 'disjunct' *ə(y)kik. The Termanu form is their only eastern evidence for $* \partial(\mathrm{y})$ kik.). Pattern: k-5. [Sporadic: $\varnothing>h$ /\#_V? in Rikou.]

eki Termanu. shout, cry out loudly. $(\mathrm{J}: 110)$

eki Korbafo.

eki Bokai.

eki Bilbaa.

he?i Rikou.

eki Tii. shout, make a racket. (J:700)

eki Oenale.

*eko Rote. shake, sift. Pattern: k-5/6'

(Dengka ? Dela $k$ correspondence, expect either $k$ in both for pattern 5, or $?$ in both for pattern 6).

(?)e (?)eko Termanu. sift the rice through the horizontal sift by rotating and shaking. (J:110f)

(?)eko Korbafo.

(?)eko Bokai.

(?)eko Bilbaa.

PePo Rikou. (J:110f; own field notes)

(?)eko $B a^{\prime} a$.

(?)eko Tii.

e?o Dengka. eko Dela.

Out-comparisons:

keko Semau Helong. sift.

keku East Tetun. shake, wag. (Mo:105)

kerifi-kerəgu Hawu. tremble, shake. [Note: Jonker(1908:111) gives herego, kereko, keko.]

ekok Sika. sift rice round and round. (Pareira and Lewis 1998:45)

*ekut PRM. palm fibres, woven ring from palm fibres. Pattern: k-6. [irr. from PRM: *u $>e$ in Rote] [Form: I have reconstructed final $* u$ rather than $* \mathrm{e}$ as irr. ${ }^{*} \mathrm{u}>e$ in Rote can be motivated as an instance of sporadic vowel assimilation, while alternate $*_{\mathrm{e}}$ $>u$ in Meto would be unmotivated.]

eke-k (2) eke_naa-k Termanu.

1) kind of ring braided from rattan or palm stems to put a hot pot or pan on, also something similar that is placed on the head to carry a load, a kind of braided ring around the opening of a popper. (J:110) 2) the outermost hard part of a young gebang palm (called tula pato), which is processed (called dusi) and separated from the useless inner part (called tula tei-k) and used to make a kind of rope or string called tali_eke/ naa-k (in Kupang called tali heknaak), which is used to make various things (lapik). (J:110)

eke-? (2) eke_naa-? Korbafo.

eke-k (2) eke_naa-k Bokai.

eke-? (2) heke/na-? Bilbaa.

eke-? (2) ? henaa-? Rikou.

eke-k (2) heke/naa-k $B a^{\prime} a$.

eke-k (2) aki/naa-k Tii. [irr. from PRM: * $>a$; $* \mathrm{e}>i$ for second form]

e?e-t Dengka.

ePe-t Oenale.

eku|t Kotos Amarasi. base of a cooking pot on the ground. 
eku|t Molo. braided ring of twigs used a support for a pot carried on the head. (M:98)

\section{Out-comparisons:}

eket Helong. ( $\mathrm{J}: 110)$

ekat East Tetun. fibres of the palm Piassava. (Mo:29)

*ela1 PRM. already, marker of perfective aspect. [irr. from PRM: *1 $>n$ in wRote; $* \varnothing>h$ in Ro'is Amarasi]

la Termanu. an enclitic which attaches to the end of verbs to exclude something else; it can sometimes be represented with 'but, only'. (J:260)

ela Rikou. so that. (Nako et al. 2014)

ena, ela, la Tii. already, PRF. (Grimes et al. 2014a)

ena, en, na Dengka. already. (J:692)

ena Oenale. already. (J:692)

ena Dela. already. With active non-punctual verbs can either indicate that the situation (seen as a whole) is completed or that a particular stage of the situation, such as the beginning of the situation, is completed. With punctual verbs it indicates that the situation is completed.

=hena Ro'is Amarasi. perfective aspect.

=ena Kotos Amarasi. perfective aspect.

=een Molo. perfective suffix. [Form: metathesised form of =ena.] (M:101)

\section{Out-comparisons:}

ela Semau Helong. like that.

ele, le Dhao. already, PRF.

ola Hawu. already, PRF, has, completed.

*elar PwRM. run, flee. [Sporadic: diphthongisation $* \mathrm{e}>a e$ in most Meto.]

n-ela Dengka. he flees. (J:736) n-ela Oenale. he flees. (J:736)

n-ela-? Dela. run.

n-aena Ro'is Amarasi. run.

n-aena Kotos Amarasi. run, evade, flee.

n-aen Molo. run away, flee. (M:10)

n-?ean Kusa-Manea. run. [irr. from PRM: $\varnothing>$ ?] [Form: metathesised form of (currently unattested) *n-?ena.]

*ele $n R M$. yonder, there. [irr. from PRM: $* \mathrm{e}>\varnothing$ in Amarasi]

ele Termanu. yonder, points at something that is further off than naa. (J:113)

ele Korbafo.

ele Bokai.

ele Bilbaa.

ele Rikou.

ele $B a^{\prime} a$.

ele Tii.

nee Kotos Amarasi. 3DEM, 3rd person demonstrative.

\section{Out-comparisons:}

nee, one Hawu. that, there, then. Usage: Seba dialect. [irr. from PRM: $* 1=n$ correspondence]

*elus $P R M$. rainbow.

elus Termanu. rainbow. (J:113)

elu-? Korbafo.

elu-? Bokai.

elu-? Bilbaa.

elus Rikou.

elus $B a^{\prime} a$.

elus Tii.

elus Dengka.

elus Oenale.

elus Dela. rainbow.

eunus Ro'is Amarasi. rainbow.

enus Kotos Amarasi. rainbow.

enus Molo. rainbow. (M:102)

euns=aa Kusa-Manea . rainbow.

*ena Rote. property, possession. [Sporadic: $\varnothing>$ ? /\# in Dela-Oenale.] ena-na (2) na-ena $B a^{\prime} a$. 1) his property. 2) possess.

ena Tii. 
(?)ena Dengka.

Pena Oenale.

Pena (2) ma-Pena-? Dela. 1) have, own. 2) possess, have, rich.

Out-comparisons: nena Semau Helong. share, portion, own.

*eni $P w R M$. third person.

eni Dengka. $=$ ndia (3SG) as personal pronoun. (J:693)

eni Oenale.

eni Dela. 3SG.ACC.

=eni Kotos Amarasi. PL, DEF.PL.

*eno Morph: *eno-k. $n R M$. way, path.

Etym: *qənuR 'animal path, trail'.

[Sporadic: $* \mathrm{u}>*_{\mathrm{o}} /{ }_{-} \mathrm{R} \#$.]

eno-k (2) na-eno Termanu. 1) way, path, road. Usage: in Termanu, eno is the more common word and dala-k the more unusual (Fox 2016b:11). 2) serve as a path. (J:114)

eno-? Korbafo.

eno-k Bokai.

eno-? Bilbaa.

eno-? Rikou.

eno-k Tii.

eno|? (2) eno_sneer Kotos Amarasi. 1) door. 2) window.

eno|? Molo. door. [Form: Middelkoop gives a parallelism with lalan 'way'; eno? ma lalan. This probably attests older semantics.] (M:101)

\section{Out-comparisons:}

enon Helong. path. (J:114)

*endən PRM. soak. See: *lende. Etym: *(R)ədəm (Blust and Trussel (ongoing) reconstruct *ədəm with 'disjunct' *Rədəm. Blust and Trussel (ongoing) give Termanu ene and Mongondow onop as evidence for *əñəp 'sunken, submerged'. This connection is spurious as the other Rote reflexes all point to medial *nd.). [irr. from PMP: * $\mathrm{d}>* \mathrm{nd}$ ] [minority from $P M P: *^{*}>*_{\mathrm{e}} / \_$\# in second Oenale form (expect $*_{\partial}>a$, possibly $*_{\partial}>$ $*_{\mathrm{a}}>$ e)] [Sporadic: diphthongisation $*_{\partial}>\left(*_{\mathrm{e}}\right)>a e$ in Meto; $\varnothing>$ ? /\#_ in Dela-Oenale.] [Form: The nasal-stop cluster reconstructed for PRM is also supported by Malay rondam.]

ene (2) oe ma-na|ene-k Termanu.

1) soak something, have something soak, make soak. 2) still-standing water. [Form: This form probably has consonant metathesis from earlier *na-ma-ene-k.] (J:114)

ene Korbafo.

ene Bokai.

ene (2) oe $=\mathbf{a}$ ene Bilbaa. 2) standing still, said of water. (J:692)

enden Landu. soak. (own field notes)

ende Rikou.

ere Oepao. (own field notes)

ene $B a^{\prime} a$.

ende Lole. submerge. (Zacharias et al. 2014)

ende Tii.

(?)enda Dengka.

Penda (2) ende $\sim$ ende Oenale.

2) standing still, said of water. (J:692)

Penda Dela. soak.

na-Paera? Ro'is Amarasi. soak. [Form: automatic glottal stop insertion between CV- prefix and \#V-initial stem.]

na-Paeka? Kotos Amarasi. soak.

$<$ n-aika> Molo. soak. (M:9)

Out-comparisons:

neney Semau Helong. soak.

*esa PRM. one. Etym: *əsa.

esa Termanu. one. (J:115)

esa Korbafo.

esa Bokai.

esa Bilbaa.

esa Rikou.

esa $B a^{\prime} a$.

esa Tii.

esa Dengka.

esa Oenale. 
esa, es, sa (2) ka-esan Dela. 1) one.

2) first.

=esa Ro'is Amarasi. one.

=esa Kotos Amarasi. one.

=ees Molo . one. [Form: metathesised form of $=$ esa.] (M:103)

eas (2) =ees Kusa-Manea. 1) one. 2) one (enclitic). [Form: The second form apparently shows assimilation of final $a$ after metathesis, which is not normally expected in Kusa-Manea.]

Out-comparisons:

esa Semau Helong. one.

*esak PRM. day after tomorrow. See: *afi, *beni. [Form: Final *k has been reanalysed in most Rote forms as the nominal suffix *-k.]

bina_esa-k (2) (ndee) bina esa-k=a Termanu. 1) day after tomorrow. 2) day before yesterday. (J:50)
bina esa-? Korbafo.
(2) bina_esak=a
bina_esa-k
(2) bina_esa-k=a Bokai.

bin/esa-? (2) bin/esak=a Bilbaa. bina esa-? (2) bina esa- $?=a$ Rikou.

bina_esa-k (2) bina_esa-k=a $B a^{\prime} a$. bin/esa-k Tii.

feni esa-? Dengka.

feni esa-? Oenale.

esah Kotos Amarasi. day after the day after tomorrow, two days from now.

<anesa > Molo. day after tomorrow. (M:103)

*eti PRM. come by, go by. [irr. from PRM: * $\mathrm{t}>? / \mathrm{V}$ V in Termanu]

n-e?i Termanu. go, (often translated with 'come'). (J:387)

n-eti $B a^{\prime} a$. he goes. (J:737)

n-eti Tii. go. (Grimes et al. 2014a)

eti Dengka. go. (J:737) n-eti Dela. indicates motion away from the speaker and motion towards the addressee.

n-eiti Kotos Amarasi. go, come.

eti Molo. come round. au P-eti I'll come to you (M:105)

*eto Morph: *eto-?. PwRote. bran. See: *taa2. [Sporadic: $\varnothing>$ ? /\# in DelaOenale.] [History: Possibly related to PMP *qata, though this is retained as taa-? in wRote.]

(?)eto-? Dengka. bran. (J:694)

Peto-? Oenale. bran. (J:694)

Peto-? Dela.

Out-comparisons:

əto Hawu. (J:694)

*etu1 PRM. catfish. [irr. from PRM: *t >

$?$ in Keka]

etu Termanu. kind of small poisonous fish. (J:116)

e?u Keka. [Note: The form $\mathbf{e} \mathbf{u}$ is noted as also occurring in other (unspecified) varieties.] (J:694)

etu Korbafo.

etu Bokai.

etu Bilbaa.

etu Rikou.

etu $B a^{\prime} a$.

etu Tii.

etu Dengka.

etu Oenale.

etu Dela. catfish.

iik etu Ro'is Amarasi. catfish.

Out-comparisons:

<naétu> Hawu. (J:116)

*etu2 PMeto. therefore, that is why. [History: The Meto and Tetun forms are likely borrowings, but the direction of borrowing is unclear.]

etu naa $R o$ 'is Amarasi. that is why, because of that.

etun Kotos Amarasi. that is why, because of that.

etun Amanuban. therefore. (M:105)

Out-comparisons:

etuk East Tetun. therefore, for that reason. (Mo:30) 


\section{$\boldsymbol{\partial}-\boldsymbol{\partial}$}

*əmə Morph: $1 \mathrm{SG}$ *kumə, 2SG *mumə, 3SG *nemə, 2PL/1PL.EXCL *mimə, 1PL.INCL *temə, 3PL * remə. PRM. come. See: *mai. [irr. from PRM: *ə $>a / \#$ in Lole and Tii; initial $*_{\mathrm{m}}>\varnothing$ in $2 \overline{S G}$ form in Lole, Tii, and wRM; initial $* \mathrm{~m}>\varnothing$ in 2PL/1PL.EXCL form in wRM] [Form: With the exception of Tii and Lole, all the Nuclear Rote languages have a vowel initial root -eme which takes the expected consonantal agreement prefixes (see $\S 2.6 .5)$. The other languages all have irregular inflections none of which I can explain adequately at this stage. While I propose a possible account for these irregular forms here, I am not completely satisfied with it, as it requires positing several ad-hoc changes. Firstly, I have reconstructed irregular inflections to PRM as it seems simpler to posit regularisation of the paradigm between PRM and some daughter languages than to posit creation of irregular forms from an originally regular paradigm. Secondly, I propose that this root took vocalic prefixes for all forms at a stage prior to PRM and that the root was originally disyllabic **əmə. Thus, I propose pre-RM $1 \mathrm{SG} * *$ ku-əmə, $2 \mathrm{SG} * * \mathrm{mu}-$ әmə, 3SG **na-əmə, 2PL/1PL.EXCL **mi-əmə, 1PL.INCL **ta-əmə, 3PL **ra-əmə. The 3SG, 3PL, and 1PL. INCL forms all show **aə $>*$ e which is supported by four other forms (*maəsa $>$ *mesa 'alone', *ma-qəti $>$ *meti 'low tide', *baqəRu $>* *$ baəRu $>$ *beu 'new', and *haRəzan $>*$ aəzan $>$ *eda 'ladder'), though there are also three forms that attest $*$ a $>*_{\mathrm{a}}$ (*ma-həmis $>* *$ maəmis $>*^{*}$ mamis 'insipid', *mahəyaq $>$ *mae 2 'shy', *qahəlu $>* *$ qaəlu $>$ *halu 'pestle').
Thus, I propose, as illustrated with the 3SG form, pre-RM **na-əmə > PRM *nemə. The Nuclear Rote languages (apart from Tii and Lole) then reanalysed 3SG *nemə, 3PL remə, and 1PL.INCL *tem root *-emə and a consonantal prefix. They then regularised the paradigm by using this root and a consonantal prefix for all persons. The PRM 1SG, 2SG, and 2PL/1PL.EXCL forms are a result of $* * \partial>\varnothing$ in the inflected forms. This follows the normal pattern for trisyllables with medial schwa and initial $* \mathrm{i}$ or $* \mathrm{u}$, though there are only three putative examples: *binəhiq $>$ *bini 'seed', *buqəni > *buni 'ringworm', and perhaps *quhənap $>$ *unə 'scale'. Thus, the complete pathway for the 2PL/1PL.EXCL form in wRM, Tii, and Lole was probably: pre-RM **mi-əmə > PRM *mimə > mima. wRM further has loss of initial $*_{m}$ in both this form and the $2 \mathrm{SG}$ form, while Tii and Lole only have loss of $* \mathrm{~m}$ in the $2 \mathrm{SG}$ form. Thus, **mu-əmə > PRM *mumə > (?)uma. The only explanation I can offer for initial $u$ in Tii and Lole 3SG numa and $3 \mathrm{PL}$ ruma/luma is that this is due to the initial vowel of the $1 \mathrm{SG} / 2 \mathrm{SG}$ form (?)uma exerting paradigmatic pressure. While this goes against crosslinguistic norms where we expect the more common 3SG form to be the one to exert paradigmatic pressure, it finds some support from the fact that in the inflection of *ou ' $g o$ ' in Ro'is Amarasi it is also the $1 \mathrm{SG} / 2 \mathrm{SG}$ stem that has spread to other parts of the paradigm. Regarding the vowels of the Meto forms, apart from the Amarasi and Amfo'an forms, other known varieties of Meto show irregular vowel 
developments in the metathesised 1SG/2SG and 2PL/1PL.EXCL forms. These changes have three sequenced stages: (1) vowel breaking, as seen in Fatule'u 1SG/2SG **uum > aum and 2PL/1PL.EXCL **iim > aim, (2) partial assimilation, as seen in Amanatun $1 \mathrm{SG} / 2 \mathrm{SG} * *$ aum $>$ oum, and (3) complete assimilation, as seen in Baikeno 2PL/1PL.EXCL **aim $>* *$ eim $>$ eem. The unmetathesised forms of the 1SG/2SG and 2PL/1PL. EXCL forms in these varieties of Meto are unknown, and I cannot confidently predict what they might be.] [History: The final syllable of *omo is possibly related to *ma(R)i with irregular *ai $>$ ${ }^{*}$, , but note that ${ }^{*} \operatorname{ma}(\mathrm{R}) \mathrm{i}$ is regularly reflected as mai in the Nuclear Rote languages.]

-eme Termanu. can sometimes mean 'come from or come out' but usually 'from' or 'out of'. Morph: 1SG (?)-eme, 2SG m-eme, 3SG n-eme, 2PL/1PL.EXCL m-eme, 1PL.INCL t-eme, 3PL l-eme. (J:387)

-eme Korbafo. Morph: 1SG k-eme, 2SG m-eme, 3SG n-eme, 2PL/1PL. EXCL m-eme, 1PL.INCL t-eme, 3PL l-eme.

-eme Bokai. Morph: 1SG k-eme, 2SG m-eme, 3SG n-eme, 2PL/1PL. EXCL m-eme, 1PL.INCL t-eme, 3PL l-eme.

-eme Bilbaa. Morph: 1SG k-eme, 2SG m-eme, 3SG n-eme, 2PL/1PL. EXCL m-eme, 1PL.INCL t-eme, 3PL l-eme.

-eme Rikou. Morph: 1SG (?)-eme, 2SG m-eme, 3SG n-eme, 2PL/1PL. EXCL m-eme, 1PL.INCL m-eme, 3PL r-eme.

-eme $B a^{\prime} a$. Morph: 1SG (?)-eme, 2SG m-eme, 3SG n-eme, 2PL/1PL. EXCL m-eme, 1PL.INCL t-eme, 3PL l-eme.
-Vma Lole. Morph: 1SG/2SG (?)uma, 3SG numa, 2PL/1PL.EXCL mima, 1PL.INCL (no form given), 3PL luma. [Form: Zacharias et al. (2014) gives only Lole neme 'from, of'.] (J:739)

-Vma Tii. Morph: 1SG/2SG (?)uma, 3SG numa, 2PL/1PL.EXCL mima, 1PL.INCL (no form given), 3PL ruma. [Form: Grimes et al. (2014a) gives singular numa and plural ruma. A search of the Tii Bible indicates that ruma is used with third person plural subjects and numa with all other persons, including other plural persons.] (J:739)

-Vma Dengka. come. Morph: 1SG/2SG (?)uma, 3SG nema, 2PL/1PL.EXCL, (?)ima, 1PL. INCL tema, 3PL lema. ( $\mathrm{J}: 736)$

-Vma Oenale. come. Morph: 1SG/2SG ?uma, 3SG nema, 2PL/1PL.EXCL 2ima, 1PL.INCL tema, 3PL rema. (J:736)

-Vma Dela. come. Morph: 1SG/2SG ?uma, 3SG nema, 2PL/1PL.EXCL Pima, 1PL.INCL tema, 3PL rema.

-Vma Ro'is Amarasi. come. Morph: 1SG kuma, 2SG uma, 3SG nema, 2PL/1PL.EXCL ima, 1PL.INCL tema, 3PL nema-n.

-Vma Kotos Amarasi. come. Morph: 1SG/2SG uma, 3SG nema, 2PL/1PL.EXCL ima, 1PL.INCL tema, 3PL nema-n.

-Vma Amanatun. come. Morph: $1 \mathrm{SG} / 2 \mathrm{SG}$ oum, $3 \mathrm{SG}$ neem.

-Vma Amfo'an. come. Morph: $1 \mathrm{SG} / 2 \mathrm{SG}$ uma, 3SG nema, 2PL/1PL.EXCL ima, 1PL.INCL tema, 3PL nema-n.

-Vma Fatule'u. come. Morph: 1SG/2SG aum, 3SG neem, 2PL/1PL.EXCL aim, 1PL.INCL teem, 3PL nema-n. 
-Vma Baikeno. come. Morph: $1 \mathrm{SG} / 2 \mathrm{SG}$ oum, 3SG neem, 2PL/1PL.EXCL eem or aim, 1PL. INCL teem, 3PL nema-n. (Charles E. Grimes pers. comm.)

-Vma Kusa-Manea. come. Morph: $1 \mathrm{SG} / 2 \mathrm{SG}$ oom, 3SG neam, 2PL/1PL.EXCL eem, 1PL. INCL team, 3PL nema-n. [Form: Although at first sight the 1SG/2SG form oom and 1PL/2PL. EXCL form eem seem to show the third stage of development (full assimilation of earlier diphthong) as discussed above, this is not straightforward as in Kusa-Manea /a/ does not undergo assimilation after metathesis. Thus, I would predict that these forms are reflexes of earlier $* *$ uam and $* *$ iam rather than **uum and **iim.]

*ondi Morph: *n-əndi. PRM. bring, take. [Form: *nd $>n / \partial_{-}$in Tii and Rikou.] [History: Blust and Trussel (ongoing) ACD give a Minangkabau and Ngadha cognate as 'noise'.]

n-eni Termanu. brings. (J:388)

n-eni Korbafo.

n-eni Bokai.

n-eni Bilbaa.

n-eni Rikou.

n-eni Oepao. (own field notes)

n-eni $B a^{\prime} a$.

n-eni Tii.

n-endi Dengka.

n-endi Oenale.

n-endi Dela. bring; use, with.

n-eri Ro'is Amarasi. bring, take.

n-eki Kotos Amarasi. bring, take, use.

n-eki Molo. brings. (M:99)

Out-comparisons:

nini Semau Helong. use, with, using.

hodi East Tetun. bring; prep. with (an instrument). (Mo:87) odi Galolen. bring, carry, take. [irr. from PMP: *o >o (expect e)]

oid Mambae, South. take, bring, use. [irr. from PMP: *o > $o$ (expect e)] (Grimes et al. 2014b:36)

n-oti Kisar. bring. [irr. from PMP: *a $>o$ (expect $e)]$

n-əti Dhao. carry, bring.

məndi Ende. bring, carry. n-əti Sika. bring, carry. [Form: n-əti 3SG-bring, m-əti 2SG/1PL. EXCL-bring.] (Pareira and Lewis 1998:136)

<ngàndi> Kambera. bring. [Note: also in Kodi.] (On:342)

<ngidi> Anakalang.

<ngindi> Weyewa. [Note: also in Mamboru.]

nenti Bima. hold, hold in the hand, e.g. a staff. (Jonker 1893:60)

onti Sumbawa. [Note: language of Sumbawa ISO 639-3 [smw].] (J:389)

*əu Morph: 1SG *kuu, 2SG *muu, 3SG *neu, 1PL.INCL *teu, 2PL/1PL. EXCL *miu, 3PL *reu. PRM. go; dative marker: to, for. [irr. from PRM: $* \mathrm{u}>i$ in wRM (for 2PL/1PL.EXCL mii)] [Form: The inflection shows several irregularities which are not dissimilar to those seen in the inflection of *omə 'come, from'. As with that stem, I propose that these irregularities are due to this stem taking vocalic prefixes at a stage prior to PRM with subsequent loss of the root initial vowel in some cases. Similarly, for the $3 \mathrm{SG}$, 3PL, and 1PL.INCL inclusive forms I propose ${ }^{* *}$ aə $>e($ e.g. $* *$ na-əu $>$ n-eu). I have reconstructed the $1 \mathrm{SG}$ and $2 \mathrm{SG}$ forms with irregular double *uu as this is attested in all branches. Similarly, I have reconstructed the 2PL/1PL.EXCL form as *miu as this best explains 
the Tii, Lole, and West Rote forms. West Rote further shows irregular assimilation of the final vowel of the 2PL/1PL.EXCL form. Other varieties of Rote have regularised the 2PL/1PL. EXCL slot of the paradigm with the stem -eu. Similarly, known varieties of Nuclear Meto have regularised the entire paradigm by using the stem -eu for all persons. In Ro'is Amarasi, on the other hand, the original $1 \mathrm{SG} / 2 \mathrm{SG}$ stem -uu has spread at the expense of -eu, which is only retained in the third person in my data.]

-eu Termanu. go. Morph: 1SG (?)uu, 2SG muu, 3SG n-eu, 1PL.INCL t-eu, 2PL/1PL.EXCL m-eu, 3PL l-eu. (J:392f)

-eu Korbafo. Morph: 1SG kuu, 2SG muu, 3SG n-eu, 1PL.INCL t-eu, 2PL/1PL.EXCL m-eu, 3PL l-eu.

-eu Bokai. Morph: 1SG kuu, 2SG muu, 3SG n-eu, 1PL.INCL t-eu, 2PL/1PL.EXCL m-eu, 3PL l-eu.

-eu Bilbaa. Morph: 1SG kuu, 2SG muu, 3SG n-eu, 1PL.INCL t-eu, 2PL/1PL.EXCL m-eu, 3PL l-eu.

-eu Rikou. Morph: 1SG (?)uu, 2SG muu, 3SG n-eu, 1PL.INCL t-eu, 2PL/1PL.EXCL m-eu, 3PL r-eu.

-eu Ba'a. Morph: 1SG (?)uu, 2SG muu, 3SG n-eu, 1PL.INCL t-eu, 2PL/1PL.EXCL m-eu, 3PL l-eu.

-Vu Lole. Morph: 1SG (?)uu, 2SG muu, 3SG n-eu, 2PL/1PL.EXCL miu. [Note: miu is given by Jonker (1908:733). The other forms are from Zacharias et al. (2014). The 1PL.INCL and 3PL forms are missing from both sources.] (Zacharias et al. 2014; Jonker 1908:733)

-Vu Tii. Morph: 1SG (?)uu, 2SG muu, 3SG neu, 1PL.INCL teu, 2PL/1PL.EXCL miu, 3PL reu. [Form: Jonker (1908:393) implies that the Tii 2PL/1PL.EXCL form is the same as the Termanu form, but does not explicitly list it. Grimes et al. (2014a) only has miu for the 2PL/1PL.EXCL form. This is also the form used throughout the Tii Bible translation.]

-eu/-uu/-ii Dengka. Morph: 1SG (?) uu, 2SG muu, 3SG neu, 1PL. INCL teu, 2PL/1PL.EXCL mii, 3PL leu.

-eu/-uu/-ii Oenale. Morph: 1SG ?uu, 2SG muu, 3SG neu, 1PL.INCL teu, 2PL/1PL.EXCL mii, 3PL reu.

-eu/-uu/-ii Dela. go, to. Morph: $1 \mathrm{SG}$ Puu, 2SG muu, 3SG neu, 1PL. INCL teu, 2PL/1PL.EXCL mii, 3PL reu.

-uu/-eu Ro'is Amarasi. 3SG-DATIVE. Morph: 1SG kuu, 2SG muu, 3SG/3PL nuu/n-eu, 1PL.INCL tuu. [Form: The 2PL/1PL.INCL form is not currently known. Both nuu and neu are attested for the third person, though neu is about twice as common in my current database.]

n-eu Kotos Amarasi. DATIVE. [Form: All persons have the root -eu.]

n-eu Amanuban. DATIVE, ALLATIVE. 
*fa PRM. little, just, NEG.

fa Termanu. a little. In phrases that contain a negative fa also often means 'a little', but is mostly meaningless (like Meto fa). (J:117)

fa Korbafo.

fa Bokai.

fa Bilbaa.

fa $B a^{\prime} a$.

fa Tii.

fa Dengka.

faa Dela. some.

$\mathbf{k a}=. .=\mathbf{f a}$ Kotos Amarasi. negator.

[Semantics: Kotos Amarasi has a two part negator with the element $=$ fa occurring after the negated predicate. Prescriptive norms dictate that $=\mathbf{f a}$ is always present, but in natural data it is occasionally omitted.]

fa Molo. still, not. (M:106)

\section{Out-comparisons:}

ve Hawu. just, only, mitigative; must, command. Particle used with manipulative verbs to indicate direct command or imperative.

<-wa> Kambera. modal particle after an imperative, request or proposal. (On:519)

*faa Rote. current; flows, streams, overflows. Etym: *bahaq 'flood; to overflow, be in flood'.

faa Termanu. current; flowing, streaming, overflowing. (J:116)

faa Korbafo.

faa Bokai.

faa Bilbaa.

faa Rikou.

faa $B a^{\prime} a$.

faa $T i i$.

faa Dengka.

fa faa Oenale.

Out-comparisons:

baa Semau Helong. flow, spread. əi-vaa Hawu. overflowing. (J:116)

*faat PRM. rainy season, southwest monsoon. Etym: *habaRat 'southwest monsoon'.

oe faa-k, fai oe faa-k (2) ani faa-k

Termanu. 1) the rainy season, the west monsoon. (J:454) 2) in Termanu mostly sea wind, in the other varieties also west wind. (J:118)

oe faa-? (2) ani faa-? Korbafo. [Form: Jonker gives Korbafo, Bilbaa, Rikou <òe-fá $>$ without a final glottal stop for the first form, but this probably a typographical error given that it is included for the second form and Termanu is clearly given as $<$ Òe-fák $>$ oe faa-k with the nominal suffix -k.]

oe faa-k (2) ani faa-k Bokai.

oe faa-? (2) ani faa-? Bilbaa.

oe faa-? (2) ani faa-? Rikou.

oe faa-k (2) ani faa-k $B a^{\prime} a$.

oe faa-k (2) ani faa-k Tii.

oe faat (2) ani faat Dengka.

oe faat (2) ani faat Oenale.

oe_faat Meto. (J:454)

Out-comparisons:

oe_haat (2) haat Semau Helong.

1) rainy season. 2) west monsoon. [Note: Jonker (1908:454) gives ui haat which has the normal Helong word for 'water', ui.] [irr. from $P M P: * \mathrm{R}>\varnothing($ expect $l)]$

*fado PnMeto. pierce.

faro Kotos Amarasi. ear-ring, nose ring.

falo, faol noni? (2) n-falo (3) n-falo bia (4) n-falon Molo. 1) silver earring. 2) wear or put on earrings. 3) put a rope through the nostrils of a buffalo. [Form: bia $=$ cow, buffalo.] 4) stabs. (M:109) 


\section{Out-comparisons:}

fadu Fehan Tetun. introduce, insert (key, etc.). fadu karau inur put a piece of wood through a buffalo's nose (to stop it suckling) (Mo:30)

*fada Morph: *na-fada. PRM. say, speak, tell, inform. Etym: *bajaq 'know, understand; ask, inquire'. [Note: The putative Meto reflexes may be chance resemblances. The semantic match is not great and they have an unexplained final consonant.] [Sporadic: $* \mathrm{a}>e /$ \# in wRM.]

na-fada Termanu. speak, say, inform, enumerate. (J:118)

na-fada Korbafo.

na-fada Bokai.

na-fada Bilbaa.

na-fada $R i k o u$.

na-fada $B a^{\prime} a$.

na-fada, i-fada Tii. (J:118,705)

na-fade Dengka.

na-fade Oenale.

na-fade Dela. tells.

n-fare? (2) uab fare? Kotos Amarasi. 1) ridicule, scoff at. Usage: collocates with n-mani 'laugh'. 2) round about talk, oblique speech.

n-falek Molo. care about, care for. au ka ?-falek fa in uab I don't care about what s/he said (M:109)

*fae Morph: *fae-k. CER. kind of large lizard.

ngolo/fae-k Termanu. kind of lizard. (J:446)

ygolo/fae-? Korbafo.

Out-comparisons:

lafaek East Tetun. crocodile, and some large lizards. (Mo:123)

*fai PRM. day, time. Doublet: *hoi. Etym: *waRi 'day, sun'.

fai Termanu. day, in the sense of time in general. (J:120f)

fai Korbafo.

fai Bokai.

fai Bilbaa. fai Rikou.

fai $B a^{\prime} a$.

fai Tii.

fai Dengka.

fai Oenale.

fai Ro'is Amarasi. night.

fai Kotos Amarasi. night.

fai Molo. night. (M:108)

fai Kusa-Manea. night.

Out-comparisons:

wain Foho Tetun. day. Usage: archaic. [Note: variety of Tetun spoken in the northern part of the Tetun-speaking area of central Timor ISO 693-3 [tet].] (van Klinken 1995)

bai(n) (2) bai-bain, uai-uain (3) bai(n)_hira, uai_hira(k) East Tetun. 1) day. 2) often, continually. (Mo:8) 3) when, how long, since when. (Mo:8, 191)

*faka1 Rote. split in half. Doublet: *faka2, *paha. Etym: *bakaq 'spread apart, split'. Pattern: k-8/9.

fa?a Termanu. split something cut through. (J:118)

fa?a Korbafo.

faka Bilbaa.

fa?a Rikou.

fa?a $B a^{\prime} a$.

fa?a Tii.

faPa Dengka.

faPa Oenale.

Out-comparisons:

bəka Hawu. divide, split.

*faka2 Morph: *faka-k. Rote. split in ground, crack in ground. Doublet: *faka1, *paha. Etym: *bakaq 'spread apart, split'. Pattern: k-5.

faka-k Termanu. split, crack in the ground. (J:122)

faka-? Korbafo.

faka-k Bokai.

faka-? Bilbaa.

faka-? Rikou.

faka-k $B a^{\prime} a$.

faka-k Tii. 
faka-? Dengka.

fa faka-? Oenale.

*fake $P R M$. petai tree; kind of tree with pods. Parkia speciosa. Pattern: k- 8 ' $\left({ }^{*} \mathrm{k}>\varnothing\right.$ in Bilbaa; expect $\left.{ }^{*} \mathrm{k}=k\right)$.

fa fa?e Termanu. kind of hard flat round fruit, which is called fafake in Kupang Malay, children play with it. (J:120)

fa fa?e Korbafo.

fa fa?e Bokai.

fa fae Bilbaa.

fa fake Rikou.

fa fa?e $B a^{\prime} a$.

fa fa?e Tii.

fa faPe Dengka.

fa fape Oenale.

fae Kotos Amarasi. kind of wild legume tree.

fae Molo. kind of liana, the fruits of which are cooked as bush-pods, the sap of which foams up. The sap or decoction is used as an anthelminthic $[\mathrm{OE}=$ kills parasitic worms] agent and is lain on bruises and cramps, a paste of the leaves heals unclean wounds. Curanga amara. (M:106)

Out-comparisons:

kbaki Helong. (J:120)

fae matan metan East Tetun. tree whose berries are used as glue by goldsmiths. (Mo:30)

$<$ kawaka $>$ Kambera. kind of creeper, or climbing plant on the riverbank; the fruit has the shape of long beans, the sap serves as an adhesive to seal a crack in a pot. (On:199)

*fakur PRM. pull out. Pattern: k-10. [irr. from PRM: ${ }^{*} \mathrm{u}>i$ in Meto]

fa?u Termanu. pull out (e.g. grass). (J:126)

fa?u Korbafo.

faku Bilbaa. (J:122)

fa?u Rikou.

fa?u Tii.
na-Pa-fa fa?u Dengka.

na-Pa-fa faPur Oenale.

n-faki Kotos Amarasi. draw

(a weapon).

n-faki Molo. pull, draw (sword).

(M:109)

*fali Rote. help, stand with. Etym: *baliw1 'dual division, moiety = cluster (a) answer, oppose, opposite side or part; partner, friend, enemy'.

fali Termanu. help, stand with. (J:122)

fali Korbafo.

fali Bokai.

fali Bilbaa.

fali Rikou.

fali $B a^{\prime} a$.

fali $T i i$.

fali Dengka.

fali Oenale.

*falu PRM. eight. Etym: *walu. [irr. from PRM: * $\mathrm{u}>a$ in Ro'is Amarasi]

falu Termanu. eight. (J:124)

falu Korbafo.

falu Bokai.

falu Bilbaa.

falu Rikou.

falu $B a^{\prime} a$.

falu Tii.

falu Dengka.

falu Oenale.

fana $R o$ 'is Amarasi. eight.

fanu Kotos Amarasi. eight.

fanu Molo. eight. (M:111)

Out-comparisons:

falu Funai Helong. eight.

palu Semau Helong. eight.

walu Fehan Tetun. eight.

ualu East Tetun. eight. (Mo:192)

balu Kemak. eight.

*fandi PRM. cut. Etym: **panti (preRM). [Note: Jonker (1908:125) also gives 'Bul.' wanti 'chop down', pati 'chop'. I have been unable to figure out which language/dialect 'Bul.' refers to.] [irr. from PRM: *nd $>n$ in Meto]

fani Termanu. incising, cutting. (J:125) 
fani Korbafo.

fani Bokai.

fani $B a^{\prime} a$.

fandi Dengka.

fani Kotos Amarasi. axe.

fani Molo. axe. (M:110)

Out-comparisons:

fani Waima'a. hook.

fati (2) manti Bima. 1) chop, chop down, e.g. a tree. (Jonker 1893:21) 2) chop, e.g. wood. (Jonker 1893:50)

*fandu Morph: *fandu-k. PRM. dry season.

fanu-k Termanu. the dry season. (J:125)

fanu-? Korbafo.

fanu-k Bokai.

fanu-? Bilbaa.

fai fandu-? Landu. drought. (own field notes)

fandu-? Rikou.

faru-? Oepao. (J:x)

fanu-k $B a^{\prime} a$.

fandu-k Tii.

fandu-? Dengka.

fandu-? Oenale.

faur/nais Ro'is Amarasi. dry season.

fauk/nais Kotos Amarasi. dry season, drought.

fauk/Pais Ketun. dry season, drought. Usage: Bone village.

fauk/nais Molo. dry season. (M:288)

fak/nais Kusa-Manea. dry season, drought.

Out-comparisons:

vəru vadu $H a w u$. dry season, hot season. [Form: vəru = 'month, moon'.]

$<$ wandu> Kambera. dry season. (On:525)

$<$ wadu> Anakalang.

*fanduun PRM. star. Etym: *bituqən. [irr. from PMP: *ə $>*_{\mathrm{u}}$ (sporadic assimilation); $*_{\mathrm{t}}>*_{\mathrm{nd}}$ (Several other languages show a medial $n t$ cluster, such as Sikule bintun 'star'
(Kähler 1959:17, Barrier Islands ISO 693-3 [skh]), or Pazeh bintun (Blust 1999:361, Taiwan, ISO 6933 uun), and it is likely that PRM *nd developed from earlier *nt.)] [Sporadic: consonant metathesis $* \mathrm{fk}>$ $k f$ in some Meto.] [Form: The change $* \mathrm{i}>*_{\mathrm{a}}$ is due to the general reduction of ante-penultimate syllables seen also, for instance in reflexes of *sumayəd > *sumanə. *nd follows the pattern for word initial *nd in Rote.]

nduu-k Termanu. star. (J:425)

nduu-? Korbafo.

luu-k Bokai.

luu-? Bilbaa.

fanduu-? Landu. (own field notes)

ruu-? Rikou.

ruu-? Oepao. (own field notes)

nduu-k $B a^{\prime} a$.

nduu-k Lole. star. (Zacharias et al. 2014)

nduu-k Tii.

nduu-? Dengka.

nduu-? Oenale.

fruun Ro'is Amarasi. stars.

kfuun Kotos Amarasi. stars. [Form: The final $n$ has been reanalysed by at least some speakers as the plural enclitic/suffix.]

kfuun, fkuun Molo. star. (M:204)

fkuun Kusa-Manea. stars.

\section{Out-comparisons:}

bduun Funai Helong. star.

duun Semau Helong. star.

fitu(n) East Tetun. star. (Mo:15)

hiut Mambae, South. star. (Grimes et al. 2014b:22)

tuu Ili'uun. star. (dJ:140)

*fayga Morph: *fayga-k. Rote. nail.

faja-k Termanu. finger or toe nail.

( $\mathrm{J}: 124)$

faya-? Korbafo.

fana-k Bokai.

faya-? Bilbaa.

faka-? Rikou.

fayga-k $B a^{\prime} a$. 
fayga-k Lole. nail. (Zacharias et al. 2014)

fanga-k Tii.

fayga-? Dengka.

fayga-? Oenale.

Out-comparisons:

waya-n Buru. digit, limb, strip, finger, slat; counter for cylindrical shaped things. (Grimes and Grimes 2020:994)

*farəndən $n R M$. thoughts, mind, feelings, hope. [irr. from PRM: * $\mathrm{r}>(* 1)$ $>n$ in Korbafo] [Sporadic: consonant metathesis *rVnd $>*$ ndVr in Termanu, Ba'a, and Korbafo.] [Form: *nd $>n$ /o_ in Rikou and Oepao.] [Semantics: It is questionable whether the required semantic shift from 'think, be mindful' in Rote to 'hope' in Meto is a likely one.]

na-fa-ndele Termanu. think about something, be mindful, remember. (J:417)

na-fa-ndene Korbafo.

na-fa-lene Bokai.

na-fa-lene Bilbaa.

na-fa-rene Rikou.

na-fa-re $\sim$ rene Oераo. remember. (own field notes)

na-fa-ndele $B a^{\prime} a$.

ni-fi-lende Lole. remember. (J:721)

na-fa-rende Tii. remember. (Grimes et al. 2014a)

(2) nere-f (4) ma-nere-t Ro'is Amarasi. 2) seat of emotions. 4) love (n.). [Form: ma- = productive reciprocal prefix.]

na-fneka, na-fnekan (2) neka-f (3) n-neka (4) ma-neka-t Kotos Amarasi. 1) hope, trust, have assurance. 2) seat of emotions; feelings, mind, thoughts. cannot be equated with any physical organ in the body. 3) love, nose kiss. 4) love (n.). na-fnekan (2) neka-n (3) n-maneka (4) ma-neka-t Molo. 1) hopes. (M:122) 2) character, nature. 3) give one another a nose kiss, love one another. 4) beloved, dear. (M:360)

*fase PRM. wash (clothes). Etym: *basəq 'wet; wash clothes'. [Sporadic: consonant metathesis $* \mathrm{fVs}>s V f$ in all instances except Amfo'an and Timaus.] [Form: regular *ə> $>$ /_q\#.]

safe Termanu. wash, clean with water. (J:511)

safe Korbafo.

safe Bokai.

safe Bilbaa.

safe Rikou.

safe $B a^{\prime} a$.

safe Tii.

safe Dengka.

safe Oenale.

n-safe Ro'is Amarasi. wash (clothes).

n-safe Kotos Amarasi. wash (clothes).

safe Molo. undergo cleaning. leaves of the safe plant would be used with water to clean clothes. (M:111, 465)

n-fase Amfo'an. wash. [Form: Middelkoop (1972:111) gives Amfo'an fasel.]

n-fasa Timaus. wash. [Form: regular final $* \mathrm{e}>a$.]

Out-comparisons: base Semau Helong. wash clean. fasi, fase East Tetun. wash. (Mo:32)

bahe Hawu. wash. wase Ili'uun. wash (objects). (dJ:141)

*fata Rote. female. Etym: **bata (preRM. Blust (1993:277) reconstructs *bat-bata 'woman' as evidence for a Yamdena-North Bomberai subgroup and also notes cognates in Ili'uun and Wetan (Luang cluster).).

tua_fata Tii. female lontar palm. (J:695) 
Out-comparisons:

bata? (2) bi/hata Funai Helong.

1) sister of man. 2) woman, female. [Form: initial syllable of bihata from PMP *bahi (see *fee 1$)$.]

bata (2) bi/hata, be/hata Semau Helong. 1) sister, opposite sex sibling (male speaking). 2) female, woman.

babata Galolen. woman.

wawata Dadu'a. woman, wife, female. (Penn 2006:111)

hahata Ili'uun. female (of human beings), woman. (dJ:116)

fafata Tugun. woman. (Hinton 2000:125)

awatwata Wetan. woman. [Note: language of southwest Maluku, member of Luang language/ dialect cluster ISO 639-3 [lex].] (de Josselin de Jong 1987)

pata Roma. woman. [irr. from PRM: $* \mathrm{~b}=p$ correspondence (expect $* \mathrm{~b}=w)] \quad($ Taber 1993:426)

*fati Morph: *na-fa fati. Rote. feed, give a drink to.

na-fa fati Termanu. give a drink to a child. (J:126)

na-fa fati Korbafo.

na-fa fati Bokai.

na-fa fati Bilbaa.

na-fa fati Rikou.

na-fa fati $B a^{\prime} a$.

na-fa fati $T i i$.

na-Pa-fa fati Dengka.

na-Pa-fa fati Oenale.

Out-comparisons:

vati $H a w u$. feed, maintain animals.

*faun Morph: *ma-faun. PwRM. thick.

fau-? Dengka. thick. (J:695)

fau-? Oenale. thick. (J:695)

fau-? (2) na-ma-fau Dela. 1) thick.

2) be thick (e.g. skin).

ma|faun Ro'is Amarasi. thick.

ma|faun Kotos Amarasi. thick. n-ma|fau-b Molo. it has many layers, thick. (M:113)

Out-comparisons:

ba bakun Semau Helong. thick, dense.

*fedu Rote. bent through. Doublet:

*betu. Etym: *bəntuk. [irr. from PMP:

$*$ nt $>*^{*}$ d 7

fedu (2) fe fedu Termanu. 1) bent through. 2) be flexible. (J:128)

fedu Korbafo.

fedu Bokai.

fedu Bilbaa.

fedu Rikou.

fedu $B a^{\prime} a$.

fedu Tii.

fedu Dengka.

fedu Oenale.

*fee1 PMeto. wife, woman. Etym: *bahi 'female, woman, wife; female of animals'. [History: The forms fenai 'wife of an important person' and Kusa-Manea fa/nai 'woman' are historically compounds of *fee 'wife' + *la?i 'male, grandfather, king'. Despite the superficial similarity, they are not from PMP $* \mathrm{~b}<$ in $>$ ahi.]

fee (2) bi/fee Ro'is Amarasi. 1) wife. 2) woman. [Form: bi is used before female names in Meto.]

fee (2) fe/nai (3) bi/fee Kotos Amarasi. 1) wife. 2) wife of an important person. [Form: nai in fenai is probably connected with na?i-f 'grandfather'.] 3) woman.

fee (2) fe/nai Amanuban. 1) wife. 2) woman. Usage: Kualiin village.

fee (2) fe/nai (3) bi/fee-l Molo. 1) wife. (M:113) 2) wife of a chief. (M:113) 3) woman. (M:66)

fee (2) fa/nai Kusa-Manea. 1) wife. 2) woman.

Out-comparisons:

bi/hata Funai Helong. woman. [Form: second element hata from **bata (see *fata).] 
bi/hata, be/hata Semau Helong. female, woman.

fee-n East Tetun. wife. (Mo:33)

*fee2 PRM. give. Etym: *bəRay.

fee Termanu. give, give gift. (J:127)

fee Korbafo.

fee Bokai.

fee Bilbaa.

fee Rikou.

fee $B a^{\prime} a$.

fee Tii.

fee Dengka.

fee Oenale.

n-fee Ro'is Amarasi. give.

n-fee Kotos Amarasi. give.

fee Molo. give. (M:113)

fee Kusa-Manea. give.

Out-comparisons:

bele Semau Helong. give.

foo East Tetun. give, grant, deliver. (Mo:35)

vie $H a w u$. give, present.

*fefa Morph: *fefa-f. PMeto. mouth (internal). See: *bafa2. Etym: *bəqbəq 'mouth, opening; speak, say' (Reconstructed with the doublet *baqbaq. Both forms are attested in the Timor region.). [irr. from PMP: *ə > *a /_q\# (expect *e)]

fefe-f Ro'is Amarasi. mouth.

fefa-f Kotos Amarasi. mouth (internal).

fefa-f Molo. mouth. (M:113)

Out-comparisons:

foha-t Welaun. voice.

*fei PRM. open. Etym: *boriq 'split, tear open'. [irr. from PMP: * $\mathrm{r}>\varnothing$ (expect *r)]

fei Termanu. open. (J:130)

fei Korbafo.

fei Bokai.

fei Bilbaa.

fei Rikou.

fei $B a^{\prime} a$.

fei Tii.

fei Dengka.

fei Oenale. n-fei Kotos Amarasi. open.

fai, fei Amanuban/Amanatun. (M:108)

*fekə Rote. separate, other. Doublet:

*feka. Etym: *bəkaq 'split, crack

open'. Pattern: k-8. [irr. from PMP:

$\left.* \mathrm{aq}>*^{*}(\operatorname{expect} * \mathrm{a})\right]$

fe?e (2) fe?e-k Termanu. 1) separate oneself out from, individuate oneself, be alone. 2) other. (J:128)

fe?e (2) fe?e-? Korbafo.

fe?e Bokai.

feke Bilbaa.

feke (2) feke-? Rikou.

fe?e (2) fe?e-k $B a^{\prime} a$.

fe?e (2) fe?e-k Tii.

fea (2) fea-? Dengka.

fea (2) fea-? Oenale.

Out-comparisons:

weke Kisar. split.

bəka (2) bəke Hawu. 1) divide, split (pl.). 2) divide, split (sg.).

*feku PRM. flute. Pattern: k-5.

feku Termanu. kind of flute from wood or bamboo with two holes at each end, used to whistle at animals. (J:130)

feku Korbafo.

feku Bokai.

feku Bilbaa.

feku-k $B a^{\prime} a$.

feku Tii.

feku Oenale.

feku Kotos Amarasi. flute.

n-feku Amfo'an. blow.

feku Molo. flute. (M:114)

feku Kusa-Manea. trumpet.

Out-comparisons:

heko Ende. flute. [Form: Jonker (1908:130) gives Ende feko.]

*fela1 $P w R M$. get up, rise.

fela Dengka. get up. (J:695)

fela Oenale. get up. (J:695)

n-fena Ro'is Amarasi. get up.

n-fena Kotos Amarasi. get up.

n-fena Molo. gets up. (M:115) 
*fela2 Morph: *na-fe fela. CER. spread out, flat. Doublet: *bela. Etym: *bəkəlaj 'spread out, unroll (mats, etc.), open out, unfold (as the hand); wide' (Blust and Trussel (ongoing) reconstruct PEMP *bolaj and there is also evidence for PCEMP *bəlaj.).

na-fe $\sim$ fela Termanu. spread out, said of plants; also to cover a large expanse, said of a landslide. (J:130)

na-fe fela Korbafo.

na-fe fela Bokai.

na-fe fela Bilbaa.

na-fe fela Rikou.

la-sa-fe fela $B a^{\prime} a$.

Out-comparisons:

felar East Tetun. unfold, open. [irr. from PMP: *ə $>e$ (expect o)] (Mo:33)

*felu Morph: *fe felu-k. CER. bend.

Doublet: *helu, *fenu. Etym: *boluk.

fe felu-k Termanu. trap used to catch birds, the snare is attached to a bent piece of wood. (J:131)

* femba Rote. hit in general, especially with a stick. [irr. from PRM: $*_{\mathrm{f}}>h$ in wRote] [History: Blust and Trussel (ongoing) reconstruct a number of similar terms the first or second syllable of which could be reconciled with this PRM reconstruction, but in no case can both syllables be regularly reconciled with *femba. Similar reconstructed terms include PAN *pəkpək, PMP *tambak, *tambək, and *sambak.]

fepa Termanu. hit in general, especially with a stick or something like that. (J:133)

fepa Korbafo.

fepa Bokai.

fepa Bilbaa.

fepa Rikou.

fempa $B a^{\prime} a$.

femba Lole. beat, hit. (Zacharias et al. 2014)

femba Tii.

hemba Dengka. hemba Oenale.

hemba Dela. hit with a wooden stick.

Out-comparisons:

vəbe (2) dede vabe Hawu.

1) beat, smash; as in waves against a boat. 2) hit, pound; Repeatedly. By hand, or with something else, most commonly with a stick. Usage: Raijua dialect.

*fenu PRM. bend. Doublet: *helu, *felu. Etym: *boluk. [irr. from PMP: *1>*n] [irr. from PRM: $* \mathrm{f}>p$ in Meto]

fenu (2) fe felu-k Termanu. 1) bend. (J:131) 2) trap used to catch birds, the snare is attached to a bent piece of wood.

fenu Korbafo.

fenu Bokai.

fenu Bilbaa.

fenu Rikou.

fenu $B a^{\prime} a$.

fenu Tii.

fenu Dengka.

fenu Oenale.

penu Kotos Amarasi. crooked, twisted.

n-penu Molo. crooked, bent. [Note: Jonker (1908:131) gives fenu as a Meto variant.] (M:433)

*fera1 Morph: *na-fera. PMeto. decide, break. [Form: Probably from earlier **fenda.]

na-fera (2) na-t|fer fera $R o^{\prime} i s$ Amarasi. 1) decide. 2) broken up, stop-start.

na-feka Kotos Amarasi. decide. n-feek (2) na-t|feek Molo. 1) breaks. 2) broken off. (M:114)

*ferar Rote. landslide, ravine. See: *ndefa. Etym: **foran (pre-RM). [irr. from PRM: $* \mathrm{f}>h$ in Oepao, Bilbaa and Korbafo (also Tetun)] [Form: Interference/collapse with *ndefa, which is likely related with consonant metathesis.] 
fela (2) (fe $)$ fela-k Tеrmanu. 1) landslide, but only said about the ground (not stones). dae $=\mathbf{a}$ fela the ground has collapsed/there has been a landslide [Semantics: Sense one is a verbal form.] 2) ravine. $(\mathrm{J}: 130)$

hela (2) hela-? Korbafo.

fela (2) fela-k Bokai.

hela Bilbaa.

hera Oepao. landslide into a space. (own field notes)

fela (2) fela-k $B a^{\prime} a$.

fera (2) fera-k, re refa-k Tii. [Form: Jonker actually gives Tii fera? but this is surely a typographical error.]

(fe $\sim$ )fera-? Oenale.

Out-comparisons:

horun Fehan Tetun. cliff.

rai hourun, horun East Tetun. precipice, chasm. [irr. from PRM: $* \mathrm{a}=u$ correspondence] (Mo:88)

werne Kisar. cliff.

*feto Morph: *feto-k. PRM. man's sister, female. Etym: *bətaw 'sister (man speaking)'. [irr. from PRM: * $\mathrm{t}>$ $? / V_{-}$V for third and fourth uses in Termanu]

feto-k (2) tua feto (3) fe?o (4) ana fe?o-k Termanu. 1) sister of a man. 2) the female, that is, the fruit-bearing lontar palm. (J:134) 3) sister, used to address a sister, young girl, or young woman. Also used as a friendly word when speaking about her. (J:132f)

4) daughter, girl, young daughter.

feto-? (2) tua feto Korbafo.

feto-k (2) tua feto Bokai.

feto-? (2) tua feto Bilbaa.

feto-? (2) tua feto Rikou.

feto-k (2) tua feto $B a^{\prime} a$.

feto-k Tii.

feto-? (2) tua feto Dengka.

feto-? (2) tua feto Oenale. feto|? (2) feto Ro'is Amarasi. 1) sister (of man). 2) female, feminine.

feto-f (2) feto Kotos Amarasi. 1) sister (of man). 2) female, feminine.

$<$ feto $>$ Molo. sister. (M:117)

feto|? Kusa-Manea. sister.

Out-comparisons:

bata Semau Helong. sister (male speaking).

'feto (2) 'ofeto-n East Tetun.

1) woman. 2) sister, cousin. Borrowed from: Meto feto (shown by irr. $*_{2}=e$ correspondence, expect $o$ ). (Mo:33)

*fetu Morph: *ka-fetu. CERM. trap, snare.

fe $\sim \mathbf{f e t u}-\mathbf{k}$ Termanu. kind of trap (like hi hi?i-k), which is placed on the ground. (J:134)

na-k|fetu (2) k|fetu-s Kotos Amarasi. 1) set a kfetus trap. 2) snare trap for catching wild chickens made by bending part of a tree down to the ground so that when a chicken steps on it, it flips out.

na-k|fetu Molo. sprung back into its original position. (M:203)

*feu Morph: *feu-k. PRM. in-law. Doublet: *beu1. Etym: *baqəRu 'new, fresh; recent(ly)'. [Form: PMP *b $>*_{\mathrm{f}}$ (rather than $* \mathrm{~b}=* \mathrm{~b}$ as in the doublet *beu1 may have been due to this term always occurring as the second member of a compound and thus intervocalic.] [Semantics: Only used in the phrases for 'daughter-in-law' and 'son-in-law'. The semantic association between 'new' and 'in-law' is connected with the fact that from someone who has just married into a family is the newest member of that kin group.]

$\begin{array}{ccc}\text { mane_feu-k } & \text { (2) } & \text { feto_feu-k } \\ \text { Tегтапи. } & \text { 1) } & \text { son-in-law. }\end{array}$

2) daughter-in-law. (J:134) 
mana/feu-? Korbafo. child-in-law (ambiguous between male and female).

mana/feu-k Bokai. child-in-law (ambiguous between male and female).

mana/feu-? Bilbaa. child-in-law (ambiguous between male and female).

mana/feu-? (2) feto/feu-? Rikou.

mone_feu-k (2) feto_feu-k $B a^{\prime} a$.

mone_feu-k (2) feto_feu-k Tii.

mone_feu-s (2) feto_feu-s Dengka. mone_feu-s (2) feto feu-s Oenale. moen fe?u, moen feu-f Kotos Amarasi. son-in-law, opposite sex sibling's son.

moen fe?u Molo. son-in-law. (M:117) Out-comparisons:

${ }^{\circ}$ manhiu Funai Helong. son-inlaw, opposite sex sibling's son. Borrowed from: either Rote or Meto, as shown by irr. ${ }^{*} \mathrm{R}$ $>\varnothing$ compared with regular *maRuqanay $>$ blanen 'brother of woman' and balu 'new'.

mane foun (2) feto foun East Tetun. 1) son-in-law, nephew.

2) daughter-in-law, niece. (Mo:36)

*fia PRM. wild taro, elephant's ear or itching taro. Alocasia species. Etym: *biRaq.

fia Termanu. yam. Usage: poetic. (Fox 1991:256)

fia Dengka. kind of aquatic plant. (J:696)

fia-l Molo. kind of wild tuber. (M:118) Out-comparisons:

fia East Tetun. variety of yam, edible but care must be taken in cooking otherwise it will irritate the mouth and tongue to cause swelling (a member of the Arum family). (Mo:34) *fiti1 Rote. jerk up, flip upwards with great force. Doublet: *bitiz. Etym: *bitik 'snare, noose trap; spring up suddenly, jerk up (as a fishing line or noose trap)'.

fiti-k (2) na-ka-fi fiti-k (3) ma-kafi fiti-k, ma-ka-fi fiti ana-k (4) fiti Termanu. 1) go upwards with great strength, or go back to one end. $\mathbf{a i}=\mathbf{a}$ fiti-k one end of the piece of wood goes upwards because someone stood on the other end (or it did this because after someone bent it they released the other end). 2) go as high up as possible. 3) the outermost point. 4) come up with a jerk, like a fishing rod. (J:137)

fiti-? Korbafo.

fiti-k Bokai.

fiti-? Rikou.

fiti-k $B a^{\prime} a$.

fiti-k Tii.

fiti-? Dengka.

fiti-? Oenale.

*fiti2 Rote. kick. Etym: *bintiq 'calfkicking contest'.

fiti Termanu. kick, give a kick, stick the leg out sideways (also of a horse). (J:137)

fiti Korbafo.

fiti Bokai.

fiti Bilbaa.

fiti Rikou.

fiti $B a^{\prime} a$.

fiti Tii.

fiti Dengka.

*fitis CERM. carry something hanging from the hand. Doublet: *bifi, *biti. Etym: *bitbit 'pull at body part; hold something dangling from the fingers'. [irr. from PRM: * ${ }_{\mathrm{t}}>$ ? in Rote and Kusa-Manea] [Form: The parallel irregular sound change in both Rote and Kusa-Manea suggests a doublet at the level of PRM; *fiti and *fi?i.] 
fiPi Termanu. pinch with the tip of the fingers or fingernails. From that: hold fast with the tips of the fingers. (J:135)

fi?i Korbafo.

fi?i $B a^{\prime} a$.

fiPi Tii.

n-fiti Kotos Amarasi. carry an item hanging below the waist, typically hanging from a rope or strap.

n-fiti Molo. one carries something hanging from a rope. (M:121)

fii? Kusa-Manea. carry an item hanging below the waist. [irr. from PRM: *t > ?] [Form: metathesised form of (currently unattested) *fi?i.]

\section{Out-comparisons:}

biPit (2) fi?it East Tetun. 1) lift by the fingers. (Mo:14) 2) remove with the points of the fingers, to lift up suspended in the paw or claw. Usage: Luka village. (Mo:34)

tiwi Bima. carry something hanging from the hand. [Sporadic: consonant metathesis ${ }^{*} \mathrm{WVt}>$ $t V w$.$] (Jonker 1893:105)$

*foe1 PRM. white spots. [irr. from PRM: $* \mathrm{f}>p$ in Amarasi (also Funai Helong)] [History: The Funai Helong and Amarasi forms are probably borrowings as both have irr. ${ }^{*} \mathrm{f}>p$. Both forms may come from Bolok Helong or Semau Helong that has undergone $\mathrm{a} * \mathrm{f}>p$ sound change.]

foe-k Termanu. white (albino) spot or spots on the hand or foot of a person, or on other places on an animal. (J:139)

foe Korbafo.

foe-k Bokai.

foe Bilbaa.

foe Rikou.

(muti/)foe-k $B a^{\prime} a$.

muti/foe-k Tii.

foe Dengka.

foe Oenale. pe/muti(-s) Kotos Amarasi. tinea. mut/foe, met/foe Meto. (J:139)

Out-comparisons:

poemuti Funai Helong. tinea.

*foe 2 CER. crocodile. See: *beis. Etym:

*buqaya. [Form: Through the pathway

*buqaya $>* *$ buaya $>* *$ boya $>* *$ boe

$>*^{*}$ foe in which all the intermediate steps are regular.]

foe $L a n d u$. crocodile. (own field notes)

foe $B a^{\prime} a$. crocodile. [Note: This form is given with a note that this form occurs in other dialects, but which other varieties are not specified.] (J:697)

Out-comparisons: voe Hawu. (J:697)

*foes PRM. move, struggle.

foe Termanu. get up in a metaphorical sense. tasi $=\mathbf{a}$ foe the sea is turbulent (J:139)

foe Korbafo.

foe Bokai.

foe Bilbaa.

foe Rikou.

foe $B a^{\prime} a$.

foe $T i$.

foe Dengka.

foe Oenale.

n-foe Kotos Amarasi. move around a lot, struggle hard.

n-foe Molo. severe (disease). (M:124)

*fo?a Rote. get up, wake up.

fo?a Termanu. get up. (J:138)

fo?a Korbafo.

fo?a Bokai.

foa Bilbaa.

foa $L a n d u$. get up. (own field notes)

fo?a Rikou. [Form: Nako et al. (2014) gives foa.]

fo?a $B a^{\prime} a$.

fo?a $T i$.

foa Dengka. sally forth.

foa Oenale. sally forth.

Out-comparisons:

buka Semau Helong. wake up, awaken. 
*fo?i PRM. lever (v.), turn. [irr. from PRM: *? $>\varnothing$ in Termanu, Korbafo, Bokai, and Ba'a]

foi Termanu. tilt by means of a lever, or with a crowbar or machete, which one sticks in the ground and then raises earth. (J:139f)

foi Korbafo.

foi Bokai.

foi Bilbaa.

foi Rikou.

foi $B a^{\prime} a$.

foi Tii.

fo?i Dengka.

fo?i Oenale.

n-fo?i, n-fo?e Kotos Amarasi. lever.

<an-foi> (2) 2-fo?i Molo. 1) turns

(e.g. ground). 2) key. (M:125)

Out-comparisons:

fokit East Tetun. pull along abruptly, jerk along. (Mo:35)

vuki $H a w u$. tilt with a lever, grub around (in dirt). (J:139)

*foo Morph: *kai_foo-k. PRM. moringa tree. Moringa oleifera. [Sporadic: *VV-k $>$ *VV? $>$ V?V in Meto (perceptual metathesis).] [Form: initial *kai probably from *kaiu 'tree, plant, wood'.]

kai/foo-k, ka/foo-k Termanu. moringa tree, the fruits of the moringa tree. $(\mathrm{J}: 215)$

kai/foo-? Korbafo.

kai/foo-k Bokai.

kai foo-? Bilbaa.

ai/foo-? Rikou.

kai/foo-k $B a^{\prime} a$.

kai/foo-k Tii.

ai/foo-? Dengka.

ai/foo-? Oenale.

ai/foo-? Dela. moringa.

uut hau_?|fo?o Ro'is Amarasi. moringa leaves.

uta_?|fo?o Kotos Amarasi. moringa leaves. [Form: utan = 'vegetable'.]

$<$ hau fo' $>$ Molo. moringa tree. Moringa oleifera. (M:124)
Out-comparisons:

${ }^{\circ}$ uut hau foo? Funai Helong. moringa leaves. Borrowed from: Ro'is Amarasi (shown by first two elements).

ut_poo Semau Helong. moringa leaves.

*fora PRM. grate, scour.

fola Termanu. scour, scrub, file, e.g. the fingers. (J:141)

fola Korbafo.

fola Bokai.

fola Bilbaa.

fora Rikou.

fola $B a^{\prime} a$.

fora Tii.

fola Dengka.

fora Oenale.

n-fona (2) ?-fona-? Kotos Amarasi.

1) grate, rasp. 2) grater.

n-fona (2) ?-fona-? Molo. 1) sharpen (knife). 2) rasp, grater. (M:127)

\section{Out-comparisons:}

hola Semau Helong. sharpen sword or knife.

pəla Hawu. [irr. from PRM: $p=$ $\left.* \mathrm{f} ; \partial=*_{\mathrm{o}}\right](\mathrm{J}: 141)$

*foti PRM. race back and forth.

foti Termanu. a) run or jump back and forth, e.g. deer in the wood. b) dance, dance of men, also perform the king's dance. c) horses running and jumping, do a dance, which takes place on the occasion of a kuus party. (J:142)

foti Korbafo.

foti Bokai.

foti Bilbaa.

foti Rikou.

foti $B a^{\prime} a$.

foti $T i$.

foti Dengka.

foti Oenale.

n-foti Kotos Amarasi. back and forth.

n-foti Molo. racing, dances. (M:128) 
*fua1 Rote. carry a burden, load. Etym: *buhat 'stand up, arise, emerge, begin, carry; cargo; take something; take a wife'.

fua Termanu. lay on something, load, have as a burden, carry. (J:143)

\section{fua Korbafo.}

fua Bokai.

fua Bilbaa.

fua Rikou.

fua $B a^{\prime} a$.

fua Tii.

fua Dengka.

fua Oenale.

*fuar PMeto. traditional religion, traditional beliefs. [History: No entry for fua or a similar form with a similar meaning has been found in Morris (1984), this term is probably thus a loan from Meto into Fehan Tetun.]

fuan (2) fua-t Ro'is Amarasi.

1) traditional worship/religion.

2) objects or matters associated with traditional religion.

n-fua (2) fua-t Kotos Amarasi.

1) traditional worship/religion.

2) objects or matters associated with traditional religion.

n-fua Molo. sacrifice, used in the meaning of an?onen $=$ 'calls upon, invokes'. (M:129)

n-fua (2) uma -fu?a Timaus.

1) worship. 2) house of traditional religion.

Out-comparisons:

ai_fuan, afuan Fehan Tetun. divination. [Semantics: Therik (2004:122) gives afuan as 'spear divination'.]

*fudi $P R M$. persuade.

fu fudi Termanu. cajole, entice, seduce. (J:145)

fu fudi Korbafo.

fu fudi Bokai.

fu fudi Rikou.

fu fudi $B a^{\prime} a$.

fu fudi $T i$. fu fudi Dengka.

fu fudi Oenale.

n-furi Kotos Amarasi. persuade, beg, coax.

n-fuli (2) <fuli> Molo. 1) persuade.

2) bait. (M:132)

*fudga PRM. foam, foaming. Etym: *bujəq 'foam, bubbles, lather, scum, froth; to foam, to bubble; foam at the mouth; fond of talking; type of white bead' (Blust and Trussel (ongoing) provide the third Termanu form as evidence for PCEMP *budeq 'sponge' and the first two forms for PMP *bujəq. However, the retention of PCEMP $* \mathrm{~d}$ as $d$ in Termanu would be irregular and the complete semantics of tasi_fude, which includes 'foam', shows that this third form is also from PMP *bujəq. PCEMP *budeq is probably spurious.). [irr. from PMP: $*_{\partial}=*_{\partial} /$ q $\#($ expect $* \mathrm{e})$ ] [minority from PMP: $* \mathrm{j}>*_{\mathrm{d}}$ (expect $\left.* \mathrm{~d}\right)$ ] [Sporadic: $*_{\partial}>e / \mathrm{C}+$ palatal_ in all except Kusa-Manea.] [Form: The correspondences for the medial consonant are not the same as either $*_{\mathrm{d}}$ or $*_{\mathrm{d}}$. Reconstructing medial $*_{\mathrm{d}} \mathrm{s}$ has the potential to both explain these (otherwise) irregular correspondences, as well as explaining the final vowel correspondences. We can propose that regular final $*_{\partial}>*_{\text {a }}$ took place in wRM, followed by sporadic raising of $* \mathrm{a}>e$ under the influence of preceding palatal $* d$ s, followed by the change of $* \mathrm{~d}_{3}>* \mathrm{~d}>r>l$. This raising has not taken place in Kusa-Manea, thus indicating that two forms *fude (from *fudze with raising of final $* \mathrm{a}>e$ ) and *fuda (from *fudza without raising of the final vowel) probably existed in PMeto.]

\section{na-fu fude (2) fude-k (3) tasi}

fude Termanu. 1) foaming. 2) foam. (J:145) 3) sea foam, also: sponge. (J:144) 
na-fu fude Korbafo.

na-fu fude Bokai.

na-fu fude Bilbaa.

na-fu fude Rikou.

na-fu fude $B a^{\prime} a$.

na-fu fude $T i i$.

na-fu fule Dengka.

na-fu fure Oenale.

fure-? Dela. foam.

fuiri-f $R o$ 'is Amarasi. foam.

2|furi|?, ?|furi-f Kotos Amarasi. bubble, foam. [Sporadic: vowel height harmony $* \mathrm{e}>i / \mathrm{uC}$ in Amarasi.]

$<$ fule $>$ (2) <na-fule > Molo. 1) foam.

2) it foams. (M:132)

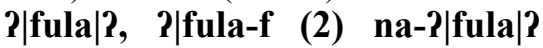
Timaus. 1) foam. 2) foaming. [Form: regular final $* \mathrm{e}>a$.]

fa fura-f Kusa-Manea. foam.

Out-comparisons:

budat ['boret] Funai Helong. foam.

bulat Semau Helong. foam, froth.

furin East Tetun. foam (of water), froth (of mouth). (Mo:38)

nawuri Kisar. froth, foam at mouth.

*fue PRM. legumes, beans. Etym: *buay (Helong, Tetun, Kemak, and Tagalog attest PMP *buday or *bujay.). [Sporadic: vowel height harmony $* \mathrm{u}$ $>o$ /_e in some Meto.]

fu fue Termanu. generic name of beans and legumes. (J:146)

fu fue Korbafo.

fu fue Bokai.

fu fue Bilbaa.

fu fue Rikou.

fu fue $B a^{\prime} a$.

fu fue Tii.

fu fue Dengka. mung beans, green grams. (J:146)

fu fue Oenale.

foe Ro'is Amarasi. mung beans, green grams. foe Kotos Amarasi. mung beans, green grams.

fue, fua Amanuban. beans.

foe-l Fatule'u. beans (generic).

fue Molo. legumes (generic). (M:130)

fa foa Kusa-Manea. beans. [Form: regular $*_{\mathrm{e}}>a / \mathrm{V}_{-} \#$ in Upper Manulea.]

Out-comparisons:

bula Semau Helong. bean, nut. [irr. from PMP: *ay > a]

fore East Tetun. bean, plants with pods and edible seeds. (Mo:35)

hure Kemak. mung bean, green gram.

bue Sika. mung bean, green gram. (Pareira and Lewis 1998:28)

mbue Ende. bean, pea.

wue Manggarai. kind of small bean. Phaeseolu ?angularis. (Verheijen 1967:768)

kebui Hawu. (J:146)

kaboe (2) buwe Bima. 1) mung bean. (Ismail et al. 1985:48)

2) kind of long bean. (Jonker 1893:12)

buwe Bugis. long beans. (Masse 2013:96)

tambue Pamona. kind of edible bean of which the leaves are also eaten. Phaseolus species. [Note: language of central Sulawesi ISO 639-3 [pmf].] (Adriani 1928:796)

bulai Tagalog. [Note: language of the Philippines ISO 639-3 [tgl].] (J:146)

*fufu1 PMeto. peak of the skull, ridgepole of house. Doublet: *fumbu. Etym: *bubun 'fontanelle; crown of the head; ridge of the roof; ridge of a mountain, peak; deck of a boat; cover the ridgepole with thatch'.

fufu-n Ro'is Amarasi. peak of the skull.

fufu-f Kotos Amarasi. peak of the skull, ridgepole of house. 
fufu-f Molo. skull, crown, top of head. (M:130)

Out-comparisons:

fuhur East Tetun. fontanelle. (Mo:35)

*fufu2 Morph: *ka-fufu-k. PRM. weevil. Etym: *bukbuk3 'weevil that infests wood, bamboo, and rice; dust produced by the boring of this insect'.

fufu-k Termanu. rice worm, woodworm, rotten. (J:146)

fufu-? Korbafo.

fufu-k Bokai.

fufu-? Bilbaa.

fufu-? Rikou.

fufu-k $B a^{\prime} a$.

fufu-k Tii.

fufu-? Dengka.

fufu-? Oenale.

?|fufu|? Kotos Amarasi. weevil.

<fufuk> Molo. black beetle with a boring snout, with which it drills holes in corn, rice and wood. (M:130)

Out-comparisons:

hupu Semau Helong. black corn beetle. [irr. from PMP: *b $>h$ /\#_; $\mathrm{b}>p / \mathrm{V} \_\mathrm{V}$ (possibly with consonant metathesis from earlier **puhu, but initial *b > $p$ would still be irregular)]

fuhuk East Tetun. weevil that eats grain. (Mo:36)

vubu Hawu. [Form: Jonker's entry is ambiguous between $<\mathrm{b}>[\mathrm{b}]$ and $<\mathrm{b}>[\mathrm{b}]].(\mathrm{J}: 146)$

*fuil $n R M$. pour water on, douse (as a fire). Etym: *buqi (PCEMP).

fui Termanu. pour on, douse. (J:146)

fui Korbafo.

fui Bokai.

fui Bilbaa.

fui Rikou.

fui $B a^{\prime} a$.

fui $T i i$.

n-fui Kotos Amarasi. pour on, sprinkle on.

\section{Out-comparisons:}

fui East Tetun. pour liquids, pour out, empty, pour water upon, water, irrigate. (Mo:37)

*fui2 PRM. wild. Etym: *bukij 'mountain; forested inland mountain areas'. [irr. from PMP: * $\mathrm{k}>\varnothing$ ] [History: The loss of the medial consonant in *bukij (even in languages that otherwise retain $* \mathrm{k}$ ) is common in the greater Timor area, as is the semantic shift to 'wild'. That the same semantic shift is accompanied by the same irregular sound change in a number of languages indicates these forms have spread by contact.]

fui-k Termanu. the opposite of ae-k $[\mathrm{OE}=$ tame $]$, wild in the expression. (J:146)

fui-? Korbafo.

fui-k Bokai.

fui-? Bilbaa.

fui-? Rikou.

fui-k $B a^{\prime} a$.

fui-k Tii.

fui-? Dengka.

fui-? Oenale.

fui Ro'is Amarasi. wild.

fui Kotos Amarasi. wild.

fui Molo. wild. (M:131)

\section{Out-comparisons:}

huin Semau Helong. wild, untamed.

fuik East Tetun. wild, savage, untamed. (Mo:37)

hui Ili'uun. forest, used only in connection with beings living in the forest, e.g. hahi hui, 'wild boar'. (dJ:118)

hui Dhao. wild.

*fuiz Morph: *na-fui. Rote. wash (hands). Etym: *buRiq.

na-fui oe Termanu. wash the hands.

(J:146)

na-fui oe Korbafo.

na-fui oe Bokai.

na-fui oe Bilbaa.

na-fui oe Rikou. 
na-fui oe $B a^{\prime} a$.

na-fui oe $T i i$.

(na-fui oe ?) Oenale.

${ }^{\circ}$ n-boe Kotos Amarasi. wash. Borrowed from: probably Helong, but there are phonological irregularities no matter whether we posit borrowing from Helong into Meto or vice-versa. I tentatively identify Helong as the source language as it usually preserves initial $* \mathrm{~b}=b$.

${ }^{\circ}$ an-boe hae-n Molo. washes one's feet. (M:78)

\section{Out-comparisons:}

boe Semau Helong. wash. [irr. from PMP: *R $>\varnothing$ (expect $l)$, vowel lowering]

*fuka Rote. dig or work the ground around a plant. Doublet: *huka. Etym: *buka 'open, uncover, expose' (Blust and Trussel (ongoing) reconstruct PCEMP *buqal 'levered up; uprooted' on the basis of Rembong boal 'levered up', Termanu fu?a, Tetun fu?a 'pull out roots and dirt together' and Maori hua 'lever; raise with a lever; steer, paddle'. Bilbaa fu fuka with medial $k$ shows that any connection between putative *buqal and the Rote forms is spurious. Likewise, medial $?$ in Tetun attests earlier $* \mathrm{k}$ not $* \mathrm{q}$.). Pattern: k-8/9. [Semantics: While the sound changes from PMP *buka are regular, I am not completely confident that the required semantic shift is justified.]

(fu) fu?a Termanu. a) dig or work the ground around a plant. b) also used for: fertilisation of the ground. (J:144)

fu fu?a Korbafo.

fu fu?a Bokai.

fu fuka Bilbaa.

fu fu?a Rikou.

fu fu?a $B a^{\prime} a$.

fu fu?a Tii.

fu fu?a Dengka.

\section{Out-comparisons:}

boka (2) voka Hawu. 1) open, undo, reveal, confess, begin; dismantle, tear apart. 2) work open the ground, work with a spade. (J:144)

*fula Rote. white. Etym: *bula[n/R] 'unnaturally white, albino'.

fula (2) fula-k Termanu. 1) be(come) white. 2) white. (J:146f)

fula Korbafo.

fula Bokai.

fula Bilbaa.

fula Rikou.

fula $B a^{\prime} a$.

fula Tii.

fula Dengka.

fula Oenale.

fula-? Dela. silver, white (used for skin colour and colour of leaves that are drying up).

*fule $n R M$. wrap around.

fule Termanu. twist around. mene a fule manu the snake wraps itself around the chicken (J:147)

fule Korbafo.

fule Bokai.

fule Bilbaa.

fule Rikou.

fule $B a^{\prime} a$.

(fule ?) $\mathrm{Tii}$.

n-funa Kotos Amarasi. go around, wrap around.

n-fuun Molo. go throughout. [Form: metathesised form of n-funa.] (M:132)

*fuli Rote. cowrie shell. Cypraea mauritiana. Etym: *buliq.

fuli Termanu. a) kind of shell. b) shells or pieces of lead used to weight a net. (J:147)

fuli Korbafo.

fuli Bokai.

fuli Bilbaa.

fuli Rikou.

ful $B a^{\prime} a$.

fuli Tii. 
meo_fuli (2) fuli Dengka. 1) kind of shell. 2) shells or pieces of lead used to weight a net. (J:147)

ndai fuli (2) fuli Oenale. 1) kind of shell. 2) shells or pieces of lead used to weight a net. ( $\mathrm{J}: 147$ )

Out-comparisons: vovuri (2) vuri Hawu. (J:147)

*fuloat PRM. kind of tropical chestnut tree. Sterculia urceolata. [irr. from PRM: *a $>o$ in wRM, glottal stop insertion in Meto]

faloa Termanu. kind of tree. Sterculia urceolata. [Semantics: Scientific identification comes from Middelkoop (1972:123) who has Molo ' $<$ flolo $>=$ kind of tree; Sterculia urceolata Smith; Rote faloa'.] (J:124)

faloa Korbafo.

faloa Bokai.

faloa Bilbaa.

faloa Rikou.

faloa $B a^{\prime} a$.

faloa $T i$.

fuloo Dengka.

fuloo Oenale.

filoo Dela. kind of tree.

fno?ot Kotos Amarasi. kind of tropical chestnut tree. Sterculia urceolata.

*fumbu Morph: *ka-fumbu-k. PRM. crown of the head, ridgepole, peak. Doublet: *fufu. Etym: *bubuy 'fontanelle; crown of the head; ridge of the roof; ridge of a mountain, peak; deck of a boat; cover the ridgepole with thatch'. [irr. from PMP: *b > *mb] [irr. from PRM: ${ }^{*} \mathrm{f}>p$ in Meto (sporadic assimilation?); $* \mathrm{f}>h$ in Oenale]

fupu-k (2) na-fupu Termanu.

1) crown of the head. 2) be hot. (J:149)

fupu-? (2) na-fupu Korbafo. fupu-k (2) na-fupu Bokai. fupu-? (2) na-fupu Bilbaa. fupu-? (2) na-fupu Rikou. (2) na-fumpu $B a^{\prime} a$.
(2) na-fumbu Tii. humbu-? Oenale.

maans=ii na-2|pupu $R o^{\prime}$ is Amarasi. midday, noon. [Form: maans=ii = 'sun'] [Semantics: Literally '(when) the sun peaks'.]

maans=ee na-?|pupu (2) ? pupu-n Kotos Amarasi. 1) midday, noon. 2) house ridge.

<ume pupu-n> Molo. ridge of a house. (M:457)

Out-comparisons:

muhuy Semau Helong. peak, ridge cap.

bßəyu Dhao. ridgepole.

*funi1 PRM. hide, conceal. Etym: *buni.

funi (2) na-funi Termanu. 1) hide oneself. 2) hide something. (J:148)

funi (2) na-funi Korbafo.

funi (2) na-funi Bokai.

funi (2) na-funi Bilbaa.

funi (2) na-funi Rikou.

funi (2) na-funi $B a^{\prime} a$.

funi (2) na-funi Tii.

(2) na-Pa-funi Dengka.

na-Pa-funi-? (2) ma-Pa-funi-?

Dela. 1) hide something from someone. 2) hidden.

<na-funi> Molo. hides something. [Note: Middelkoop identifies this as a loan from Rote, though the reason for this is not clear.] (M:133)

Out-comparisons:

huni Semau Helong. hide.

hakfunin Fehan Tetun. hide or conceal oneself. (Mo:54)

*funi2 Rote. placenta, afterbirth. Etym:

*tabuni. [irr. from PRM: $* \mathrm{f}>h$ in wRote]

funi-k Termanu. after birth, placenta. ( $\mathrm{J}: 148)$

funi-? Korbafo.

funi-k Bokai.

funi $L a n d u$. afterbirth, placenta. (own field notes)

funi-k $B a^{\prime} a$. 
funi-k Tii.

huni-? Dengka.

huni-? Oenale.

*funu PnMeto. betel-pepper. Etym: *burun 'inferior kind of betel nut' (Blust and Trussel (ongoing) reconstruct this on the basis of cognates in Mongondow, Pamona, and Tetun. They note it may be a chance resemblance.).

funu Amanuban. betel-vine fruit. Usage: Kusi village.

\section{Out-comparisons:}

furu East Tetun. creeper with leaves similar to the betelpepper. (Mo:38)

huru roon Galolen. betel. Usage: Talur dialect. (Hinton 2000:120)

buru Welaun betel-vine fruit.

huru Kemak. betel-vine fruit.

*fura Rote. trim lontar palm leaves. [irr. from PRM: *a $>i$ in wRote]

fula so $\sim$ soga (2) fu $\sim$ fula-k Termanu.

1) cut off the edge of young lontar palm leaves. 2) cutting off; the tool for cutting off: a leaf vein folded in half against which one holds a knife. (J:147)

fula Korbafo.

fula Bokai.

fula Bilbaa.

fura Rikou.

fula $B a^{\prime} a$.

fura $T i i$.

fuli Dengka.

furi Oenale.

Out-comparisons:

hula Helong. (J:147)

* futu PRM. bundle up, tie, bind. Doublet:

*mbutu. Etym: *butu 'group,

crowd, flock, school, bunch, cluster'

(PCEMP).

futu-s Termanu. yarn tied in bundles for weaving. (J:149)

futu-? Korbafo.

futu-k Bokai.

futu-? Bilbaa. futu-s Rikou.

futu-s $B a^{\prime} a$.

futu-s Oenale.

n-futu Ro'is Amarasi. tie together.

n-futu (2) ?-futu-? (3) na-?-futu-?

Kotos Amarasi. 1) bind. 2) band, sash. 3) wear a sash.

n-futu (2) ?-futu-? (3) ?-futu-? (4) futu-s Molo. 1) bind. 2) girdle. 3) wears a girdle. 4) bound thread ready to be dyed. (M:133)

\section{Out-comparisons:}

butu Semau Helong. tie, tie up.

futu East Tetun. tie into a bundle, bundle up. (Mo:38)

futu (2) fuut Mambae, South. 1) gather, come together. 2) accompany, together with. (Grimes et al. 2014b:19)

wuku Kisar. tie.

*fuu PRM. blow. Doublet: *sabuu. Etym: *buu (PCEMP. Blust and Trussel (ongoing) provide the Termanu form as evidence for both PCEMP *buu and PMP *qombus 'blow hard; snort, pant'. The connection between *qombus and fuu is spurious. (The expected regular reflex would be *epu.) The Termanu form is, furthermore, their only eastern evidence for positing *qombus at PMP rather than PWMP.).

fuu Termanu. blow, blow with the mouth. (J:143)

fuu Korbafo.

fuu Bokai.

fuu Bilbaa.

fuu Rikou.

fuu $B a^{\prime} a$.

fuu Tii.

fuu Dengka.

fuu Oenale.

n-fuu Kotos Amarasi. blow.

n-fuu (2) ?-fu?u Molo. 1) blows (e.g. wind). 2) harmonica. (M:128)

\section{Out-comparisons:}

huu Semau Helong. blow.

huu East Tetun. blow on. (Mo:89) 
*ha Morph: *na-ha. PRM. eat. Etym: *kaən. [irr. from PMP: *k $>* \mathrm{~h}$ (expect $\left.*_{\mathrm{k}}={ }^{*} \mathrm{k}\right)$ ] [Form: This is one of the few monosyllabic roots reconstructed to PRM. Similarly, it is the only monosyllabic verb root in the Rote-Meto languages. ${ }^{*} \mathrm{~h}$ develops as though it were a medial consonant in all daughters, thus providing evidence that this root obligatorily took vocalic agreement prefixes in PRM. The verbal inflection is irregular (and historically more conservative) in the Nuclear Rote languages with the prefixes having vowels other than /a/. (The segmentation in the entries below follows their historical analysis for these languages.) The wRM forms are regular for a verb that takes vocalic prefixes (see §2.6.5).]

-2a Termanu. eats. Morph: 1SG (?) u-?a, 2SG mu-?a, 3SG na-?a, 2PL/1PL.EXCL mi-Pa, 1PL.INCL ta-2a, 3PL la-2a. (J:368)

-2a Korbafo. Morph: 1SG ku-?a, 2SG mu-?a, 3SG na-?a, 2PL/1PL. EXCL mi-?a, 1PL.INCL ta-?a, 3PL la-?a.

-2a Bokai. Morph: 1SG ku-?a, 2SG mu-?a, 3SG na-?a, 2PL/1PL. EXCL mi-?a, 1PL.INCL ta-?a, 3PL la-?a.

-a Bilbaa. Morph: 1SG ku-a, 2SG mu-a, 3SG na-a, 2PL/1PL.EXCL mi-a, 1PL.INCL ta-a, 3PL la-a.

-a Landu. eat. Morph: 3SG na-a. [Note: Forms for other persons are currently unknown.] (own field notes)

-a Rikou. Morph: 1SG (?)u-a, 2SG mu-a, 3SG na-a, 2PL/1PL.EXCL mi-a, 1PL.INCL ta-a, 3PL ra-a. -a Oepao. eat. Morph: 3SG na-a, 1PL. INCL ta-a. [Note: Forms for other persons are currently unknown, but are probably the same as Rikou.] (own field notes)

-2a Ba'a. Morph: 1SG (?)u-?a, 2SG mu-?a, 3SG na-?a, 2PL/1PL. EXCL mi-?a, 1PL.INCL ta-?a, 3PL la-?a.

-Pa Lole. Morph: 1SG (2)u-?a, 2SG mu-?a, 3SG na-?a, 2PL/1PL. EXCL mi-?a, 1PL.INCL ta-?a, 3PL la-?a.

-2a Tii. Morph: 1SG (?)u-?a, 2SG mu-?a, 3SG na-?a, 2PL/1PL. EXCL mi-?a, 1PL.INCL ta-?a, 3PL ra-?a.

-a Dengka. Morph: 1SG (?)u-a, 2SG mu-a, 3SG na-a, 2PL/1PL.EXCL mi-a, 1PL.INCL ta-a, 3PL la-a.

-a Oenale. Morph: 1SG Pu-a, 2SG mu-a, 3SG na-a, 2PL/1PL.EXCL mi-a, 1PL.INCL ta-a, 3PL ra-a.

-a Dela. eat. Morph: 1SG Pu-a, 2SG mu-a, 3SG na-a, 2PL/1PL.EXCL mi-a, 1PL.INCL ta-a, 3PL ra-a.

-ha Ro'is Amarasi. eat (soft food). Morph: 1SG ku-ha, 2SG mu-ah, 3SG na-ah, 2PL/1PL.EXCL mi-ah, 1PL.INCL ta-ah. [Form: Not all forms have been attested unmetathesised, but 1SG ku-ha, 3SG na-ha, and 3PL na-ha-n (the last two in Kotos below) indicate that metathesis is fully productive with this verb.]

-ha Kotos Amarasi. eat (soft food). Morph: 1SG ?u-ah, 2SG mu-ah, 3SG na-ha, 2PL/1PL.EXCL mi-ah, 1PL.INCL ta-ah, 3PL na-ha-n.

-ha Molo. eat. Morph: 3SG na-ha. (M:51) 
-ah Kusa-Manea. eat. Morph: 1PL. INCL ta-ah. [Form: metathesised form of ta-ha.]

Out-comparisons:

kaa Semau Helong. eat.

haa East Tetun. eat. (Mo:101)

-Pon, -Pan Kisar. eat. Morph: 1SG o?on, 2SG/2PL/1PL.EXCL mo?on, 3SG na?an, 1PL.INCL ka?an, 3PL ra?an.

*haa1 CER. lontar thorn.

beba_haa-k Termanu. the edge of the branching thorn of the beba; also in pana haa-k. (J:150)

\section{Out-comparisons:}

haa Semau Helong. lontar stem.

*haa2 PRM. four. Etym: *opat. [irr. from

$P M P:{ }^{*} \boldsymbol{\partial}>\varnothing$ with doubling of the final vowel to create a disyllable] [Form: External witnesses suggest earlier **paat. The Welaun correspondence indicates $\mathrm{EVC}>\mathrm{ACV}$ metathesis of *opat $>* *$ pəat.]

haa Termanu. four. (J:150)

haa Korbafo.

haa Bokai.

haa Bilbaa.

haa Rikou.

haa $B a^{\prime} a$.

haa Tii.

haa Dengka.

haa Oenale.

haa Ro'is Amarasi. four.

haa Kotos Amarasi. four.

haa|2 Molo. four. (M:134)

Out-comparisons:

aat Semau Helong. four.

haat East Tetun. four. (Mo:39)

paat Kemak. four.

hoat Welaun. four.

faat Mambae, South. four. (Grimes et al. 2014:17)

ihaat Galolen. four. wo?akka Kisar. four.

*habu PnMeto. cloud, fog. Etym: *kabut 'fog, haze, mist; indistinct, blurry'. [Note: Reconstructed with initial $* \mathrm{~h}$ (not ${ }^{*} \mathrm{k}$ ) as this is a Proto-Nuclear Meto form. Thus, I am proposing PRM *k $>$ Proto-Nuclear Meto $* \mathrm{~h}$ in this form.] [minority from PMP: *b $(>$ $* 6)=* b / V \_V($ expect $\left.* f)\right]$ [Form: If this form were *kabu in PRM it would exemplify pattern k-3/4 for initial *k.] habu Kusa-Manea. cloud, fog.

*hade $P R M$. rice in the field; rice plant. Etym: *pajay. [minority from PMP: ${ }^{*} \mathrm{j}$ $>* \mathrm{~d}($ expect $* \mathrm{~d})$ ] [Sporadic: $* \mathrm{~h}>\varnothing$ in wRM.]

hade Termanu. rice, the plant and the crop, the grains as long as they have not yet been husked. (J:151)

hade Korbafo.

hade Bokai.

hade Bilbaa.

hade Rikou.

hade $B a^{\prime} a$.

hade Tii.

ale Dengka.

are Oenale.

are Dela. rice plant.

aan/Poe|k Ro'is Amarasi. wet rice field. [Form: The final element is from oe 'water' with $\mathbf{k}$ presumably from nominal suffix *-k.]

ain/Poe|k Kotos Amarasi. wet rice field.

ane Amanuban/Amanatun. field rice.

ane (2) aan/ Poe|k Molo. 1) paddy rice. 2) wet rice field. (M:22)

ane (2) aen/oa|k Kusa-Manea. 1) field rice. 2) wet rice field. [Form: no medial glottal stop.]

Out-comparisons:

ale Semau Helong. field rice.

hare East Tetun. rice (plant and unhusked grain). (Mo:77)

are Dhao. rice on stalk; grain plant.

are Hawu. rice on stalk; grain plant.

*hae $P R M$. breathe.

hae (2) hae, hai Termanu. 1) breathe.

2) rest, stop. (J:152)

hae (2) hae, hai Korbafo. 
hae (2) hae, hai Bokai.

hae (2) hae, hai Bilbaa.

hae (2) hae, hai Rikou.

hae (2) hae, hai $B a^{\prime} a$.

hae (2) hae, hai Tii.

hae (2) hae, hai Dengka.

hae (2) hae, hai Oenale.

n-hae Kotos Amarasi. physically tired, exhausted.

\section{Out-comparisons:}

kae, hkae Semau Helong. tire, weary. [irr. from PRM: ${ }^{*} \mathrm{~h}=(h)$ $k$ correspondence]

a ae, ae Dhao. breath, stop.

*hae|? PMeto. foot/leg. Doublet: *ei. Etym: *kakay (Wolff 2010:862) (Reconstructed with the doublets *waqay and *qaqay.). [Note: Reconstructed with initial $* \mathrm{~h}($ not $* \mathrm{k})$ as this is a Proto-Meto form. Thus, I am proposing PRM $* \mathrm{k}>$ PMeto $* \mathrm{~h}$ in this form.] [Sporadic: *VV-k $>$ *VV? > V?V in Amarasi (perceptual metathesis).] [Form: If this form is regular from *kakay it exemplifies pattern $\mathrm{k}-3 / 4$ for initial $* \mathrm{k}$ and pattern k-8 for medial $* \mathrm{k})$.]

hae-f Ro'is Amarasi. foot/leg. ha?e, hae-f Kotos Amarasi. foot/leg. hae-k Molo. leg. (M:134)

Out-comparisons:

kae_fola $H a w u$. foot, leg.

*hai1 Morph: *hai-k. Rote. container woven from lontar leaves. Etym: **pai (pre-RM).

hai-k Termanu. bucket woven from lontar leaves. (J:154)

hai-? Korbafo.

hai-k Bokai.

hai-k Bilbaa.

hai-? Rikou.

hai-k $B a^{\prime} a$.

hai-k Tii.

hai-? Dengka.

hai-? Oenale.

Out-comparisons:

pai Semau Helong. basket. fai Bolok Helong. basket.

pai Hawu. plate (woven from lontar leaves).

*haiz PRM. stingray. Etym: *paRih 'skate, stingray (generic); a constellation'.

hai Termanu. (sting)ray. (J:153)

hai Korbafo.

hai Bokai.

hai Bilbaa.

hai Rikou.

hai $B a^{\prime} a$.

hai Tii.

hai Dengka.

hai Oenale.

hai|f Meto. (J:153)

Out-comparisons:

ai Helong. [irr. from PMP: $* \mathrm{R}>\varnothing$ (expect $l)](\mathrm{J}: 153)$

*ha(?)i Rote. take.

hai (2) hai (3) hai (4) hai (5) hai (6) ha?i Termanu. 1) dismiss. 2) take out in certain expressions. 3) bring in the expressions ala hai $\mathbf{o e}=\mathbf{a}$ leo dae neu 'someone brings the water to the lower regions' and manakolu-la hai soo 'the harvesters have brought (the ears of corn) to the big mat'. 4) decamp, leave, in the expression ala hai leo dea leu 'they leave, they go to the field to stay there temporarily during the harvest season'. 5) as a separate word usually haik e.g. hai(k) ndai, (bufu) 'pull up a scoop-net (or a trap), take something out of the water'. 6) take, fetch, take away. (J:153f)

hai (2) ha?i (3) hai (4) hai (5) hai (6) ha?i Korbafo.

(2) hai (3) ha?i (4) hai (5) hai (6) ha?i Bokai.

hai (3) hai (4) hai (5) hai (6) hai Bilbaa.

(2) hai (3) hai (4) hai (5) hai (6) (hai ?) Rikou.

hai (2) hai (3) ha?i (4) hai (5) hai

(6) ha?i $B a^{\prime} a$. 
hai (2) hai (3) ha?i (4) hai (5) hai (6) ha?i Tii.

hai (2) ha?i (3) ha?i (4) hai (6) ha?i Dengka.

hai (3) hai (4) hai (6) ha?i Oenale. Out-comparisons:

əgo Hawu. take. [Note: Jonker (1908:154) gives agi 'take out', which may be from Malay angkit.]

ahi Bima. take something out of somewhere, take away. (Jonker 1893:1)

*halu Morph: *halu-k. PRM. pestle. Etym: *qaholu. [minority from PMP: ${ }^{*} \mathrm{q}>* \mathrm{~h}$ ] [irr. from PRM: $* \mathrm{~h}>\varnothing$ in Rote]

alu-k Termanu. pestle. (J:8)

alu-? Korbafo.

alu-k Bokai.

alu-? Bilbaa.

alu-? Rikou.

alu-k $B a^{\prime} a$.

alu-k Tii.

alu-? Dengka.

alu-? Oenale.

alu-? Dela. pounder.

haunu|k Ro'is Amarasi. pestle.

hanu|k Kotos Amarasi. pestle.

hanu Amanuban/Amanatun. pestle.

hanu Kopas. pestle.

hanu Kusa-Manea. pestle.

Out-comparisons:

alu Semau Helong. pestle, pounder.

alu(k) East Tetun. pestle for grinding grain. (Mo:3)

*hambu PRM. overtake, catch up with.

[History: Blust and Trussel (ongoing) reconstruct Proto-Philippine *qabut 'reach, overtake, catch up with'.]

hapu Termanu. overtake. (J:162)

hapu Korbafo.

hapu Bokai.

hapu Bilbaa.

hapu Rikou.

hampu $B a^{\prime} a$. hambu Tii.

hambu Dengka.

hambu Oenale.

n-hapu Molo. overtakes, finds. [Note: Jonker (1908:162) specifies hapu as being from Taebenu.] (M:140)

Out-comparisons:

hapu Semau Helong. get, obtain, able.

abßu Dhao. get, find, receive; able, can, be able to; exist, be, there are.

abu (2) abo Hawu. 1) get, find, encounter (pl.). 2) get, find, encounter (sg.).

*hanas Morph: *ma-hanas, *na-hana.

PRM. warm, hot. Etym: *panas.

hanas (2) na-ma-hana (3) na-

hana Termanu. 1) hot, warm of water and everything which can be heated up, also of the body; warmth. 2) be warm, such as water or the body. 3) warm up. (J:158f)

hana-? Korbafo.

hana-k Bokai.

hana-? Bilbaa.

hanas Rikou.

hanas $B a^{\prime} a$.

hanas Tii.

hanas Dengka.

hanas Oenale.

m|anas Ro'is Amarasi. sun.

m|anas (2) na-hana (3) k/hanas

Kotos Amarasi. 1) sun, midday.

2) cook. 3) drought.

m|anas (2) na-hana Molo. 1) sun.

2) cooks. (M:304)

m|anas Kusa-Manea. hot.

[Semantics: maputu? is also 'hot'.

The semantic difference between manas and maputu? is currently unclear.]

Out-comparisons:

manas East Tetun. hot, warm, burning; pungent, caustic, hot to the taste; $n$. heat. (Mo:138)

manha Kisar. hot. 
*hani1 Rote. bait, feed (animals). Etym: *panin 'bait; fodder; to feed animals'.

hani-k Termanu. bait, bait for fishing.

(J:161)

hani-? Korbafo.

hani-k Bokai.

hani-? Bilbaa.

hani-? Rikou.

hani-k $B a^{\prime} a$.

hani-k Tii.

hani-? Dengka.

ha hani-? Oenale.

hani Dela. feed animals.

Out-comparisons:

hani Semau Helong. feed the chickens.

ani Hawu. bait, chicken-feed. $(\mathrm{J}: 161)$

<pàni> (2) <pàningu> Kambera. 1) feed especially for chickens. 2) bait. (On:398)

pənin Sika. give food to chickens. (Pareira and Lewis 1998:158)

*hani2 Morph: *hani-k. PnRote. kind of shell, turtle shell. Etym: **napi (pre$\mathrm{RM}$ ). [Sporadic: consonant metathesis $*_{n V p}>*_{p} \mathrm{Vn}>*_{\mathrm{hVn}}$.]

hani-k (2) kea hani-k (3) poe hani-k Termanu. 1) kind of shell.

2) the shell of a turtle. 3) the shell/ shuck of a shrimp. (J:161)

hani-? Korbafo.

hani-k Bokai.

hani-? Rikou.

hani-k $B a^{\prime} a$.

(hani-k ?) Tii.

Out-comparisons:

<nepi> Kambera. scale. (On:317)

$<$ napi> Anakalang. [Note: also in Mamboru, Weyewa, and Kodi.]

$<$ napu $>$ Lewa.

*hano Morph: *ka-hano. PRM. fungus infection which produces light patches on the skin: Tinea flava or Pityriasis. Etym: *panaw. ha hano Termanu. spots of a lighter colour on the skin. (J:161)

ha hano Korbafo.

ha hano Bokai.

ha hano Bilbaa.

ha hano Rikou.

ha hano $B a^{\prime} a$.

ha hano Tii.

hano Dengka.

hano Oenale.

?|hano Kotos Amarasi. tinea.

$<$ hano $>$ Molo. rash on the face.

(M:139)

Out-comparisons:

ano Helong. (J:161)

*hanga PwRote. hand span. See: *tenga.

Etym: **panga (pre-RM).

hayga Dengka. span. (J:699)

hayga Oenale. span. (J:699)

Out-comparisons:

<ăga> Hawu. (J:621)

<pangga> Kambera. span (from thumb to middle-finger), to measure that distance. (On:400)

paga Sika. span. (Pareira and Lewis 1998:155)

*hao PRM. feed. Etym: **pao (pre-RM).

hao Termanu. eat with the hand, put a bite (of food) in one's mouth. (J:161)

hao Korbafo.

hao Bokai.

hao Bilbaa.

hao Rikou.

hao $B a^{\prime} a$.

hao Tii.

hao Dengka.

hao Oenale.

n-hao Ro'is Amarasi. feed.

n-hao Kotos Amarasi. feed.

Out-comparisons:

pao Bima. mouthful, make mouthfuls, place into the mouth. (Jonker 1893:79)

*hapi PRM. pinch, clamp. Doublet:

*kabi. Etym: *kapit 'pinch, press between; fasten thatch together with 
slats in roofing a house'. [irr. from PMP: $* \mathrm{k}>* \mathrm{~h}$ ] [minority from PMP: ${ }^{*} \mathrm{p}=* \mathrm{p}$ ] [irr. from PRM: ${ }^{*} \mathrm{~h}>h, \varnothing$ in Oenale]

hapi Keka. pinch. (J:699)

hapi $B a^{\prime} a$.

na-ka-hapi Tii.

hapi Dengka.

hapi (2) api Oenale. 1) pinch. (J:699)

2) clamps. (J:678)

n-hapi Kotos Amarasi. clasp, entreaty.

n-hapi Molo. cuts through the skin with a clamp. (M:134)

*hara Morph: *hara-k. PRM. voice, sound.

hala-k Termanu. voice, noise of a person or animal. (J:156)

hala-? Korbafo.

hala-k Bokai.

hala-? Bilbaa.

hara-? Rikou.

hala-k $B a^{\prime} a$.

hara-k Tii.

hala-? Dengka.

hara-? Oenale.

hana-f (2) na-hana Ro'is Amarasi.

1) voice. 2) make a sound.

hana-f (2) na-hana Kotos Amarasi.

1) voice, sound, news. 2) make a sound, hum, resound (like a bell).

hana-f (2) na-haan Molo. 1) sound.

2) resound. (M:138)

\section{Out-comparisons:}

bhala, khala Funai Helong. voice, sound.

fala Semau Helong. taste; voice, sound.

hala Bolok Helong. voice, sound.

*haru Morph: *haru-k. PRM. shoulder. [irr. from PRM: $* \mathrm{~h}>\varnothing$ in Rote]

alu-k Termanu. shoulder of people and animals. (J:8)

alu-? Korbafo.

alu-k Bokai.

alu-? Bilbaa.

aru-? Landu. shoulder. (own field notes) aru-? Rikou.

alu-k $B a^{\prime} a$.

aru-k Tii.

alu-? Dengka.

aru-? Oenale.

aru-? Dela. shoulder.

haunu-f Ro'is Amarasi. shoulder.

hanu-f Kotos Amarasi. shoulder.

hanu-k Amfo'an. shoulder.

hanu-f Kusa-Manea. shoulder.

Out-comparisons:

adu Semau Helong. shoulder.

*hatahori PRM. man, person. Doublet:

*ata. Etym: *qaRta + *qudip 'person + living' (Charles Grimes pers. comm.). [Note: Osmond and Ross (2016b:47) reconstruct POc *[qa]ta-maquri 'living person' noting it '... spans a large piece of Oceania, but it has few reflexes and may reflect parallel innovations'. Kemak has atamoas 'human being', which is similarly a compound of a reflex of *qaRta with moas 'living'.] [minority from PMP: $* \mathrm{q}>* \mathrm{~h} ; * \mathrm{q}>* \mathrm{~h}$ ] [Sporadic: $* \mathrm{~h}>\varnothing$ in wRM.] [Form: Loss of medial $* \mathrm{~h}$ in Meto is probably due to historic antepenultimate vowel reduction with subsequent simplification of the resulting consonant cluster; e.g. *hatahori $>* *$ ataholi $>* *$ atholi $>$ pre-Meto $* *$ atoli $>$ Proto-Meto *atoni.]

hataholi Termanu. person. (J:164f)

hataholi Korbafo.

hataholi Bokai.

hataholi $B a^{\prime} a$.

hataholi Lole. (Zacharias et al. 2014)

hatahori Tii.

atahori Oenale.

atahori Dela. person.

atoni|? Ro'is Amarasi. man, person.

atoni|? Kotos Amarasi. man, person. [Semantics: By default this term refers to males, but can be used more generally to refer to 'person'.] atoni-ds Amfo'an. man, person. 
$<$ atoni> Molo. human, man. (M:34) atoin Kusa-Manea. person.

Out-comparisons:

${ }^{\circ}$ atuli Semau Helong. person, people. Borrowed from: Meto before $* 1>n$.

*hauk PRM. soothe. na-ka-ha hau-k (2) hau-k Termanu. 1) calm down, soothe, e.g. a crying child. 2) quieten down, stop crying (e.g. like a small child). (J:166)

na-ka-ha hau? Korbafo. na-ka-ha hau-k Bokai. na-ha hau-? Rikou. na-ka-ha hau-k $B a^{\prime} a$. na-ka-ha hau-k Tii. na-Pa-ha hau-? Oenale. (na-)hauk Meto. stop crying. (J:166) *hedu Morph: *hedu-k. PRM. gall bladder, bitter. Etym: *qapəju 'gall, gall bladder, bile'. [minority from PMP: $* \mathrm{j}>* \mathrm{~d}($ expect $* \mathrm{~d})$ ] [Sporadic: $* \mathrm{~h}>\varnothing$ in wRM.] [Form: PwRM *meru can be reconstructed. It is from earlier **ma-hedu.]

hedu-k (2) ma-ka-hedu-k Termanu. 1) gallbladder. 2) bitter. (J:169)

hedu-? (2) ma-ka-hedu-? Korbafo. hedu-k (2) ma-ka-hedu-k Bokai. hedu-? Bilbaa.

hedu=na Landu. gallbladder. (own field notes)

hedu-? (2) ma-hedu-? Rikou. hedu-k (2) ma-ka-hedu-k $B a^{\prime} a$. hedu-k (2) ma-ka-hedu-k Lole. (Zacharias et al. 2014)

hedu-k Tii.

elu-? (2) m|elu Dengka. eru-? (2) m|eru Oenale. eru-? (2) m|eru Dela. 1) gallbladder. 2) bitter.

eunu-f $R o$ 'is Amarasi. gallbladder. enu-f (2) m|enu|? Kotos Amarasi. 1) gallbladder. 2) bitter. <enu> (2) m|enu|? Molo. 1) gall bladder. (M:102) 2) bitter. (M:319) enu-f (2) m|enu|? Kusa-Manea. 1) gallbladder. 2) bitter.

Out-comparisons:

ilu-n Semau Helong. gall bladder. horun Fehan Tetun. gall, if you vomit till vomit is blue, this stuff comes from the horun.

na?an hourun East Tetun. bile. (Mo:88)

Peru Peru-n Kisar. gallbladder.

*hedis Rote. sick, pain. Doublet: *meras. Etym: *hapəjis 'smarting, stinging pain'.

hedis (2) na-ma-hedi Termanu. 1) pain, sickness, painful. 2) be or become sick. (J:168)

hedi-? Korbafo.

hedi-k Bokai.

hedi-? Bilbaa.

(2) na-ma-hedi $L a n d u$. s/he's sick. (own field notes)

hedis Rikou.

hedis $B a^{\prime} a$.

hedis Tii.

hedis Dengka.

hedis Oenale.

hedis (2) na-ma-hedi-? Dela.

1) illness, sickness. 2) be sick.

Out-comparisons:

ili Semau Helong. sick, pain.

*hee1 CERM. lobster.

poe hee-k Termanu. kind of big lobster. (J:166)

poe_hee-? Korbafo.

poe_hee-? Bilbaa.

kpoe hee oo Ro'is Amarasi. lobster. poe hee Kotos Amarasi. lobster.

Out-comparisons:

een Semau Helong. lobster, crayfish.

*hee2 Rote. press out. Etym: *pəRəq 'squeeze out juice, wring out water'. [irr. from PRM: $\varnothing>? \sim \varnothing$ in Termanu and Bokai] [Form: regular *a $>e / \mathrm{q} \#$. 
Forms with medial $?$ are perhaps due to influence from *ke?e and/or reflexes of PMP * gəmgəm.]

he?e, hee Termanu. press, press out. (J:169)

hee Korbafo.

he?e, hee Bokai.

hee Bilbaa.

hee Rikou.

hee $B a^{\prime} a$.

hee Tii. [Form: A putative alternate form he?e is given with question mark.]

hee, ee Dengka.

hee Oenale.

Out-comparisons: hele Helong. (J:169)

*hela $n R M$. pull. [irr. from PRM: *1 $>r / l$ in Meto]

hela Termanu. pull, drag, drag along. (J:170)

hela Korbafo.

hela Bokai.

hela Bilbaa.

hela Rikou.

hela $B a^{\prime} a$.

hela Tii.

n-hera Kotos Amarasi. pull.

n-hela Molo. pulls. (M:144)

Out-comparisons:

pela Semau Helong. pull.

*helu Rote. bend. Doublet: *fenu, *felu.

Etym: *bəluk. [irr. from PMP: *b $>* \mathrm{~h}$ ]

helu Termanu. bend something, bent back to the point of breaking. (J:172)

helu Korbafo.

helu Bokai.

helu Bilbaa.

helu Rikou.

helu $B a^{\prime} a$.

helu Tii.

helu Dengka.

helu Oenale.

Out-comparisons:

hilu Semau Helong. curve, bend. [irr. from PMP: *b $>h / \#$ (possibly a loan from Rote)] *hena $P R M$. hope.

na-ma-hena

(2) dale $=$ na

hena hena Termanu. 1) hope, wish, expect, trust. 2) her/his heart is full of hope, $s /$ he is full of hope. ( $\mathrm{J}: 173)$

na-ma-hena Korbafo.

na-ma-hena Bokai.

na-ma-hena Bilbaa.

na-ma-hena Rikou.

na-ma-hena $B a^{\prime} a$.

na-ma-hena Tii.

na-ma-hena Dengka.

na-ma-hena Oenale.

na-mhena Meto. (J:173)

Out-comparisons:

'namhena Semau Helong. Borrowed from: Meto. (J:173)

*hene PRM. climb. Etym: *panahik. [Sporadic: antepenultimate $*_{a}>* *$ o $>*$ e.]

hene Termanu. in the other varieties of Rote still means 'climb' but in Termanu only in the phrase hene (noo): 'climb a coconut tree to pick the fruit'. Also in metaphorical senses: climb = 'increase, grow'. (J:174)

hene Bokai.

hene Bilbaa.

hene Rikou.

hene $B a^{\prime} a$.

hene Tii.

hene Oenale.

*henu CERM. full. Etym: *ponuq.

henu-k Termanu. full. (J:176)

henu-? Korbafo.

henu-k Bokai.

henu-? Bilbaa.

henu-? Rikou.

henu-k $B a^{\prime} a$.

na-henu $R o^{\prime} i s$ Amarasi. be full, fill up.

na-henu Kotos Amarasi. be full, fill up.

$<$ henu> Molo. full. (M:145)

na-heun Kusa-Manea. fill, full. 
Out-comparisons:

inu Semau Helong. full.

benu Kemak. full.

penu Kisar. full.

*henuh PRM. beads. Etym: **penuk (pre-RM). [Sporadic: ${ }^{*} \mathrm{~h}>\varnothing$ in wRM; vowel height harmony *e > $i$ / $\mathrm{Cu}$ in wRM] [Form: Blust and Trussel (ongoing) reconstruct PMP *hinuq, and PAN *SiNuq. The eastern correspondences point to **penuk, and it may be that we have to reconstruct a doublet to $\mathrm{P}(\mathrm{CE}) \mathrm{MP}$. If so, the wRM reflexes could reflect $P M P *$ hinuq (but with irregular final $* \mathrm{q}>h$ as retained by Meto), while the other Rote reflexes reflect **penuk.]

henu Termanu. bead, beads. (J:176)

henu Korbafo.

henu Bokai.

henu Bilbaa.

henu Rikou.

henu $B a^{\prime} a$.

henu Tii.

inu Dengka.

inu Oenale.

inu Dela. beads.

inuh Kotos Amarasi. beads.

inuh Molo. bead. (M:161)

inuh Kusa-Manea. necklace, beads.

Out-comparisons:

henu East Tetun. wear on the neck, anything worn on the neck. (Mo:85)

enu Ili'uun. bead. (dJ:114)

pinu (2) pinu e-wadun Buru.

1) carry with strap over shoulder (e.g. hunting pouch).

2) necklace. [Form: wadun $=$ 'nape of neck'.] (Grimes and Grimes 2020:745)

penu Ende. hang on the neck.

*hendam PRM. wild pandanus. Etym: * pandan 'pandanus'. [irr. from PMP: $*_{\mathrm{a}}>*_{\mathrm{e}} ; *_{\mathrm{n}}>*_{\mathrm{m}}$ ] [Sporadic: ${ }^{*} \mathrm{~h}>\varnothing$ in wRM; $\varnothing>$ ? /\# in Dela-Oenale.] [Form: The initial ? in Dela-Oenale may point to an earlier initial $* * k$. Additional evidence for this comes from Ili'uun ketfan. The source of this initial $* * k$ could be the nominal prefix *ka-.]

hena-k Termanu. pandanus, also: pineapple. (J:174)

hena-k Bokai.

hena-? Bilbaa.

henda-? Landu. pandanus. (own field notes)

henda-? Rikou.

hera-? Oepao. (own field notes)

hena-k $B a^{\prime} a$.

henda-k Lole. (Zacharias et al. 2014)

henda-k Tii.

(?)enda-? Dengka.

Penda-? Oenale.

Penda-? Dela. pandanus.

eram, erem Ro'is Amarasi. wild pandanus.

ekam Kotos Amarasi. wild pandanus.

ekam Molo. pandanus. (M:99)

ekam Kusa-Manea. wild pandanus. Usage: Upper Manulea village.

ekom Kusa-Manea. wild pandanus. Usage: Uabau' village. [Sporadic: *a > / m (compare PMP *maqitəm > PMeto *metam > metom 'black').]

Out-comparisons: edan Semau Helong. pandanus. ketfan Ili'uun. pandanus. [irr. from PMP: *p $>\left({ }^{*} \varnothing\right)>k$ (compare *pusəj > kusan, *hutək > gutan, and *baqbaq $>$ kahan)] (dJ:121)

*henge PRM. hang (rope/cord), tie. [History: possibly connected with PMP *hikət.]

heye Termanu. tie (general). (J:174)

heye Korbafo.

hene Bilbaa.

heke Rikou.

henge $B a^{\prime} a$.

henge Tii.

henge Dengka. 
na-?|heke (2) P-heke-? Kotos Amarasi. 1) hang/suspend around the neck. 2) necklace.

na-? |heke Molo. garland with a cord. (M:143)

Out-comparisons:

eki Kisar. living beings that hang from something, e.g. bat hanging from branch, people hanging from a branch.

*hengu PRM. eat something hard. Etym: *pagut 'snap at with the mouth' (Blust and Trussel (ongoing) note regarding their reconstruction: 'The semantics of this comparison leave something to be desired.' They also reconstruct the root *-gut 'gnaw'.). [irr. from PMP: *a > ${ }^{*} \mathrm{e}$ [Sporadic: ${ }^{*} \mathrm{~h}>\varnothing$ in Meto.]

heju Termanu. nibble on something hard; bite a piece of something with the teeth. (J:175)

henu Korbafo.

henu Bokai.

heyu Bilbaa.

heku Rikou.

hengu $B a^{\prime} a$.

hengu Tii.

hengu Dengka.

hengu Oenale.

n-eku Kotos Amarasi. eat; implies eating something hard such as sugar, coconut, bread, cake.

n-eku Molo. eats. (M:98)

Out-comparisons:

<pànggitu> Kambera. chew on. [Form: The final vowels in the forms from languages of Sumba is epenthetic.] (On:401) <pànggutu> Lewa. $<$ pàgutu $>$ Anakalang. $<$ pangguta $>$ Mamboru.

*heo Morph: *he heo. Rote. move.

he heo-k Termanu. he turns himself, rotates himself. (J:178)

he heo-? Korbafo.

he heo-k Bokai.

he heo-? Rikou. he heo-k $B a^{\prime} a$.

he heo-k Tii.

he heo-? Dengka.

he heo-? Oenale.

Out-comparisons:

he heon Semau Helong. move.

*hesu PnRote. fart. Etym: ** pəsu (pre$\mathrm{RM}$ ). [irr. from PRM: * $\mathrm{h}>\emptyset$ in Central East Rote]

esu Termanu. fart, wind. (J:116)

esu Korbafo.

esu Bokai.

esu Bilbaa.

esu Rikou.

esu $B a^{\prime} a$.

hesu Tii.

Out-comparisons:

nisu Semau Helong. fart.

hosu, housu East Tetun. expel flatus through the anus, break wind, fart. (Mo:88)

pəhu Hawu.

$<$ kapíhu $>$ Kambera. wind, flatulence, pass wind. (On:165)

$<$ kapísu $>$ Anakalang.

$<$ kapàsu $>$ Mamboru.

$<$ pou $>$ Weyewa.

$<$ pàsu $>$ Kodi.

potfu Bima. wind, pass wind. (Jonker 1893:82)

*həndi Rote. finish, finished. Etym: *qəti 'stop, end, finish, complete; finished, used up'. [irr. from PMP: *t $>$ *nd (also in Helong)] [minority from PMP: ${ }^{*} \mathrm{q}>{ }^{*} \mathrm{~h}$ ] [Form: ${ }^{*} \mathrm{nd}>n / \mathrm{o}_{-}$in Tii and Rikou.]

heni Termanu. out or gone, disappeared or drained away. (J:175)

heni Korbafo.

heni Bokai.

heni Bilbaa.

heni Rikou.

heni $B a^{\prime} a$.

heni Tii.

hendi Dengka.

hendi Oenale.

Out-comparisons:

hidi Semau Helong. finish, end. 
*hia PRM. want. Etym: *pian 'want, desire, wish or long for'. [irr. from PRM: $*_{\mathrm{a}}>i$ in Rote; $*_{\mathrm{ia}}>\left(*_{\mathrm{e}}\right)>a$ in Kusa-Manea] [Form: Medial *ia is required to account for $e$ in Amarasi.]

hii Termanu. want, be inclined to, desire, wish, consent. (J:179)

hii Korbafo.

hii Bokai.

hii Bilbaa.

hii Rikou.

hii $B a^{\prime} a$.

hii Tii.

hii Dengka.

hii Oenale.

hii Dela. like.

he Kotos Amarasi. irrealis marker; want, will. [Note: hena and henati? appear to have the same functions.]

ha Kusa-Manea. irrealis marker.

*hida PRM. how much, how many.

Doublet: *hida. Etym: *pija 'interrogative of quantity: how much?, how many?; adjective of indefinite quantity: some, several, a few'. [minority from PMP: $* \mathrm{j}>* \mathrm{~d}$ (expect $* d)$ ]

hida Termanu. how much/many? (J:182)

hida Korbafo.

hida Bokai.

hida Bilbaa.

hida Rikou.

hida $B a^{\prime} a$.

hida Lole.

hida Tii.

hira Oenale.

hira Dela. several, how many, some, how much.

hian Kusa-Manea. how much/many? [Form: metathesised form of (currently unattested) *hina.]

Out-comparisons:

ila Semau Helong. how many?, several.

hira East Tetun. how much, how many? (Mo:86) *hida PwRote. few, reduce. Doublet: *hida. Etym: *pija 'interrogative of quantity: how much?, how many?; adjective of indefinite quantity: some, several, a few'. [irr. from PRM: * $\mathrm{h}>\varnothing$ in Dengka] ida-? (2) na-ma-ida Dengka. 1) few. 2) reduce. (J:705)

na-ma-hida Oenale. reduce. (J:702) hida(k) Dela. few.

*hine PwRM. know. [Sporadic: vowel height harmony $*_{\mathrm{e}}>i / \mathrm{iC}_{-}$in some Meto]

na-hine Dengka. know. (J:702)

na-hine Oenale. know. (J:702)

na-hini Ro'is Amarasi. know.

na-hini, na-hine (2) ma-hine-?

Kotos Amarasi. 1) know. Usage:

The form na-hini is much more common than na-hine.

2) knowledge, wisdom.

na-hiin (2) $<$ hine-n $>$ Molo.

1) knows. 2) knowledge. (M:148)

na-hini Kusa-Manea. know.

*hiri PRM. choose, select; to pick out. Etym: *piliq. [irr. from PMP: *1 $>* \mathrm{r}$ ] [irr. from PRM: $* \mathrm{i}>e$ in Rote] [Sporadic: ${ }^{*} \mathrm{~h}>\varnothing$ in Meto; diphthongisation in Meto: $*_{\mathrm{i}}>$ ai.]

hele Termanu. choose, select. (J:170)

hele Korbafo.

hele Bokai.

hele Bilbaa.

here Rikou.

hele $B a^{\prime} a$.

here Tii.

hele Dengka.

here Oenale.

<anaini> Molo. one chooses stones in a row for the sequence, in which one tells a story or investigates a matter. (M:11)

\section{Out-comparisons:}

huli Semau Helong. choose, select, appoint, pick; select out from a bunch. [irr. from PMP: $* \mathrm{i}>u$ ] 
hili East Tetun. pick up from the ground; to arrange. (Mo:86)

pidi $H a w u$. choose, select, appoint, set aside.

*hitu PRM. seven. Etym: *pitu.

hitu Termanu. seven. (J:185)

hitu Korbafo.

hitu Bokai.

hitu Bilbaa.

hitu Rikou.

hitu $B a^{\prime} a$.

hitu Tii.

hitu Dengka.

hitu Oenale.

hitu Ro'is Amarasi. seven.

hitu Kotos Amarasi. seven.

hitu Molo. seven. (M:149)

hiut Kusa-Manea. seven.

Out-comparisons:

itu Semau Helong. seven.

hitu East Tetun. seven (7). (Mo:86)

itu Kemak. seven.

(ha?)itu Ili'uun. seven. (dJ:119)

*hoi PRM. dry in the sun. Doublet: *fai.

Etym: *waRi 'day, sun'. [irr. from

PMP: Ø $>* \mathrm{~h}$ ] [Sporadic: vowel height harmony $* \mathrm{i}>e / \mathrm{o}_{-}$in Amarasi.] [Form: Tetun provides evidence that the unexpected initial $h$ in the reflexes could be retention of a causative prefix; e.g. ${ }^{* *}$ pa-waRi.]

hoi Termanu. expose something to the heat of the sun, dry something in the sun. (J:186)

hoi Korbafo.

hoi Bokai.

hoi Bilbaa.

hoi Rikou.

hoi $B a^{\prime} a$.

hoi Tii.

hoi Dengka.

hoi Oenale.

n-hoe Ro'is Amarasi. dry in the sun.

n-hoe Kotos Amarasi. dry in the sun.

n-hoi Molo. dry in the sun, scorch. (M:151)

hoi Kusa-Manea. dry in sun.

\section{Out-comparisons:}

${ }^{\circ}$ hui? Semau Helong. dry in sun. Borrowed from: Meto (shown by irr. $* \mathrm{R}>\varnothing$, expect $* \mathrm{R}>l$ ). [Form: regular Helong midvowel raising before/after a high vowel $*_{0}>u /$ i.]

hawai Fehan Tetun. dry by placing in the sun.

habai, hauai East Tetun. expose in the sun, dry in the sun. (Mo:39, 83)

noe Hawu. dry in sun.

*hoka PRM. call up, invite. Pattern: k-5.

hoka Termanu. call up, convene for public work. (J:187)

hoka Korbafo.

hoka Bokai.

hoka Bilbaa.

ho?a Rikou.

hoka $B a^{\prime} a$.

hoka Tii.

hoka Dengka.

hoka Oenale.

n-hoka Ro'is Amarasi. call up.

n-hoka Kotos Amarasi. invite, call up.

<hoka> Molo. invite, first announcement of an invitation. (M:151)

Out-comparisons:

hoka Helong. (J:187)

*holas PRM. naked. [Sporadic: *h $>\varnothing$ in Meto.]

ma-ka-hola-k (2) hola hola, tao hola (3) na-ka-hola Termanu. 1) naked, someone who is usually naked. 2) naked, be unclothed. 3) bare; bare oneself, mainly unknowingly while asleep. (J:188)

ma-ka-hola-? Korbafo.

ma-ka-hola-k Bokai.

(2) hola hola Bilbaa. ma-ka-hola-k $B a^{\prime} a$.

hola Lole. naked. (Zacharias et al. 2014)

ma-ka-hola-k Tii. 


$$
\begin{gathered}
\text { ma-Pa-hola-? } \\
\text { Dengka. }
\end{gathered}
$$

ma-?a-hola-?

Oenale.

monos Ro'is Amarasi. naked.

muti?_monas (2) n-?afa?_monas

Kotos Amarasi. 1) openly. 2) stark naked. [Form: n-Pafa? = 'clean, shaved, naked'.]

n-monas (2)<amonak> Molo. 1) be naked. (M:xxxiii, 88) 2) naked. (M:129)

Out-comparisons:

ke?ula Hawu. [irr. from PRM: $* 1=r$ correspondence; $*_{\mathrm{O}}=u$ correspondence] (J:188)

holar Sika. naked. (Pareira and Lewis 1998:78)

*holu1 PRM. hug, embrace. Etym: *pəluk 'bend, curve'. [irr. from PMP: *o > ${ }^{*} \mathrm{o}$ ] [irr. from PRM: *1 $>\varnothing$ in Meto; ${ }^{*} \mathrm{u}$ $>o$ in Meto]

holu Termanu. hug, embrace. (J:190)

holu Korbafo.

holu Bokai.

holu Bilbaa.

holu Rikou.

holu $B a^{\prime} a$.

holu Lole.

holu Tii.

holu Dengka.

holu Oenale.

holu Dela. hug.

n-hoo Kotos Amarasi. hug, embrace, put one's arm around.

n-hoo Molo. hug. (M:149)

*holu2 Rote. help, assist. [History:

Possibly connected with PMP *tulun and/or Malay tolong, though this would require irregular $* \mathrm{t}>* \mathrm{~h}$.]

holu Termanu. help, assist. (J:190)

holu Korbafo.

holu Bokai.

holu Bilbaa.

holu $B a^{\prime} a$.

(holu ?) Tii.

holu Dengka.

\section{Out-comparisons:}

huluy Semau Helong. help, assist. [Form: regular Helong midvowel raising before/after a high vowel $*_{\mathrm{o}}>u$ /_u.]

*horis PRM. life, living. Doublet: *mori. Etym: *qudip 'live'. [irr. from PMP: $* \mathrm{u}>*_{\mathrm{o}}$ ] [minority from PMP: * $\mathrm{q}$ $>* \mathrm{~h}$ ] [Form: expected *p $>s$ /_\# (§3.5.1.3).]

holis Keka. living. (J:703)

horis Oenale. living. (J:703)

ka-hoinis (2) na-honi? Ro'is Amarasi. 1) living one. 2) give birth to.

?|honis (2) na-honis (3) na-honi? (4) honi? (5) a-mahoni-t Kotos Amarasi. 1) life. 2) give birth to.

3) be born. 4) genetic (of relation).

5) parent, clan elders.

$<$ honi> (2) oe Phonis (3) nahoni? (4) au mahoni-k Molo. 1) live. 2) spring that never dries up. 3) bears (child). 4) my parent. (M:152)

Out-comparisons:

nulis (2) nuli Semau Helong. 1) life. 2) live.

horis Fehan Tetun. living.

houris East Tetun. alive, with life. (Mo:188)

Pori Pori Kisar. life, foliage.

*hosu PRM. loosen. [Form: The Rote forms with initial $m$ probably come from earlier **ma-hosu.] [History: Jonker (1908:496) also gives Termanu, Korbafo, Bokai, Bilbaa, and Rikou posu 'loosen, come off, slip', which is probably related and points to earlier **posu. This term is related to a network of formally and semantically similar terms including Termanu, Korbafo, Bokai podu 'slip, slip off, loosen' (Jonker 1908:489), Termanu, Korbafo, Bokai, Ba'a odu 'pull on, put on, pull something on, put something on' (Jonker 1908:452), and all Rote olu 
'pull on, put on' (Jonker 1908:457), Termanu, Korbafo, Bokai, Bilbaa, Tii kosu 'remove skin, loosening of the skin'. Among these terms, the first could have reinforced irregular retention of pre-RM $* \mathrm{p}=p$ in posu.]

m|osu Termanu. be loose. (J:362)

m|osu Korbafo.

m|osu Bokai.

m|osu Bilbaa.

m|osu Rikou.

m|osu $B a^{\prime} a$.

m|osu Tii.

m|osu Dengka.

m|osu Oenale.

n-osu Kotos Amarasi. pull out.

n-osu (2) n-hosu (3) na-t|hosu

Molo. 1) draws (sword). (M:408)

2) draws (sword). (M:154) 3) come loose. (M:154)

*hotu PRM. burn. See: *mbutu 'hot, burning'. [Sporadic: ${ }^{*} \mathrm{~h}>\varnothing$ in Meto] hotu Termanu. burning, set on fire. (J:191)

hotu Korbafo.

hotu Bokai.

hotu Bilbaa.

hotu Rikou.

hotu $B a^{\prime} a$.

hotu Tii.

hotu Dengka.

hotu Oenale.

n-otu Ro'is Amarasi. burn.

n-otu Kotos Amarasi. burn.

n-otu (2) ma?-otu-? Molo. 1) burns.

2) hot. (M:409)

n-out Kusa-Manea. burn.

Out-comparisons:

otot Semau Helong. hot.

hotu Waima'a. firewood.

*huka Rote. open. Doublet: *fuka. Etym: *buka 'open, uncover, expose'.

Pattern: k-6. [irr. from PMP: $* \mathrm{~b}>* \mathrm{~h}$ ]

huka Termanu. open. (J:195)

huka Korbafo.

huka Bokai.

huka Bilbaa.

huka $B a^{\prime} a$. huka Tii.

hu?a Dengka.

hu?a Oenale.

Out-comparisons:

boka Semau Helong. open. [irr. from PMP: *u $>$ o]

*huPe PnMeto. eucalyptus. Etym: **pue (pre-Meto). [History: Several AlorPantar languages have likely cognates of this form, e.g. Kabola has pu?.]

hu?e-l Amfo'an. eucalyptus tree.

hu?e Molo. eucalyptus. Eucalyptus alba. [Form: Other varieties of Meto are known to have ho?e.] (M:154)

Out-comparisons:

poe Kemak. eucalyptus.

oe Welaun. eucalyptus.

*hulu PRM. first, in front. Doublet: *ulu. Etym: *qulu 'head; top part; leader, chief; headwaters; handle of a bladed implement; prow of a boat; first, firstborn'. [minority from PMP: $* \mathrm{q}>* \mathrm{~h}$ ] [irr. from PRM: $* \mathrm{~h}>\varnothing$ in Rikou]

na-ka-hulu-k Termanu. be in front, first, be the first. (J:196)

na-ka-hulu-k Bokai.

na-ulu Rikou. first. (own field notes)

na-ka-hulu-k $B a^{\prime} a$.

na-ka-hulu-k Tii.

na-Pa-hulu-? Dengka.

na-Pa-hulu-? Oenale.

na-hunu Ro'is Amarasi. go first, be first.

na-hunu Kotos Amarasi. go first, be first, at first.

na-hunu Molo. go in front of. (M:156)

*humək PRM. smile, be joyful. [Sporadic: *ə $>e / \sigma_{-} \#$ in wRote (perhaps $* \partial>*_{\mathrm{a}}>e / \#$ ).]

hume Termanu. smile. (J:197)

hume Korbafo.

hume Bokai.

hume Bilbaa.

hume Rikou.

hume $B a^{\prime} a$.

hume Tii. 
hume - hume, humel Dengka.

humek Oenale.

humek Dela. smile.

humu-f (2) huma? Ro'is Amarasi.

1) face. 2) kind, type.

huma-f (2) huma? (3) na-huma

(4) n-huma?_moe Kotos Amarasi. 1) face. 2) kind, type.

3) beautiful. 4) smile, be joyful, be glad.

huma-n (2) n-huma? moe Molo.

1) his form, his face. 2) smiles, chuckles. (M:156)

humu? Kusa-Manea. face.

*hundi PRM. banana. Etym: *punti. [minority from PMP: *nt $>*^{*}$ nd (expect $\left.{ }^{*} \mathrm{t}\right)$ ] [Sporadic: ${ }^{*} \mathrm{~h}>\varnothing$ in Meto.]

huni Termanu. banana. (J:197)

huni Korbafo.

huni Bokai.

huni Bilbaa.

hundi Landu. (own field notes)

hundi Rikou.

huri Oepao. (own field notes)

huni $B a^{\prime} a$.

hundi Tii.

hundi Dengka.

hundi Oenale.

uri Ro'is Amarasi. banana.

uki Kotos Amarasi. banana.

uki Molo. banana. (M:663)

uki Kusa-Manea. banana.

Out-comparisons:

hudi East Tetun. banana. (Mo:88)

*hunga PRM. chop with force, chop big branches. Etym: *pu(y)kaq 'break off'.

[Sporadic: ${ }^{*} \mathrm{~h}>\varnothing$ in Meto.]

huya Termanu. chop with an axe or machete firmly held with both hands. (J:197)

huka Rikou.

huyga $B a^{\prime} a$.

huyga Tii.

huyga Oenale.

uka-l Molo. chop big branches. [Form: insertion of $/ 1 /$ after /a/ to mark a third person object.] (M:585)

\section{Out-comparisons:}

<púnggu> Kambera. chop, chop down, fell. [Note: also in Mangili.] (On:423)

<ponggu> Mamboru. [Note: also in Lewa.]

<pogu> Anakalang.

<ponggo> Kodi. [Note: also in Weyewa.]

pongo Bima. chop, chop with an axe, split or cut with an axe. (Ismail et al. 1986:121)

*husə Morph: *husə-k. PRM. navel. Etym: *pusəj 'navel, umbilicus; midpoint or centre of something'. [irr. from PRM: * $\mathrm{h}>$ ? in most Rote] [Sporadic: $* \mathrm{~h}>\varnothing$ in Meto.] [Form: The initial $?$ in wRote possibly from earlier $* \mathrm{k}$, also attested by Ili'uun kusan. These forms are perhaps via intermediate *ka-husə-k.]

(?)use-k Termanu. navel. (J:671)

(?)use-? Korbafo.

(?)use-k Bokai.

(?)use-? Bilbaa.

?use-? Rikou.

(?)use-k $B a^{\prime} a$.

huse-k Lole. (Zacharias et al. 2014)

huse-k Tii.

(?)usa-? Dengka.

?usa-? Oenale.

?usa-? Dela. belly button, navel.

usu-f Ro'is Amarasi. navel, belly button.

usa-f Kotos Amarasi. navel, belly button; shoot, sprout, off-shoot.

$<$ usa> Molo. navel. (M:591)

usa-f Kusa-Manea. navel.

Out-comparisons:

husar, husor East Tetun. umbilicus, navel. (Mo:89)

huso Waima'a. navel.

ohor-ne Kisar. navel.

kusan Ili'uun. navel. [irr. from $P M P: * \mathrm{p}>(* \varnothing)>k$ (compare *paydan $>$ ketfan, *hutək > gutan, and $*$ baqbaq $>$ kahan)] (dJ:122) 
əhu Hawu. navel.

*huti CERM. provoke, incite.

[Form: There is also a variant

*huhi found outside wRote, e.g.

Termanu na-ka-hu hu?i-k, Bilbaa na-ka-hu hui-? and others (Jonker 1908:195).]

huti, na-ka-hu huti-k Termanu. provoke. na-ka-hu huti-k au nonook=a busa he provokes me like I was a dog (J:198)

$<$ n-huit(a)> Molo. incite. a|n-huit asu incites a $\operatorname{dog}(\mathrm{M}: 154)$

*hutu Morph: *na-hutu. $n R M$. rub, smear.

na-hutu (2) na-la-hu hutu Termanu. 1) rub, rub in, besmear oneself or one another. 2) pulverise by rubbing. (J:199)

$\underset{\text { Korbafo. }}{\text { na-hutu (2) na-la-hu } \sim \text { hutu }}$

na-hutu (2) na-la-hu hutu Bokai.

na-hutu (2) na-la-hu hutu Bilbaa. na-hutu (2) na-la-hu hutu Rikou.

(2) na-la-hu hutu $B a^{\prime} a$. na-hutu (2) na-la-hu hutu Tii.

n-huta Kotos Amarasi. rubs, smears (e.g. oil).

n-huut Molo. smears (e.g. blood). (M:157)

*huu Morph: *huu, *huu-k, *ma-huu-k. PRM. base of a tree; cause; source, origin; beginning. Etym: *puqun 'base of a tree; cause; source, origin; beginning; foot of a hill or mountain; first wife; model or example (to be copied); expression for the mother's brother'. [Sporadic: ${ }^{*} \mathrm{~h}>\varnothing$ in Meto; $* \mathrm{VV}-\mathrm{k}>* \mathrm{VV}$ ? $>\mathrm{V}$ ?V in Meto (perceptual metathesis).]

huu-k (2) huu (3) ma-huu-k (4) na-ta-huu, mete/huu-n Termanu. 1) trunk of a tree. 2) cause reason. 3) having a trunk. 4) starting from, from. (J:193f)

huu-? (2) huu Korbafo.

huu-k (2) huu Bokai.

huu-? (2) huu Bilbaa.

huu-? (2) huu Rikou.

huu-k (2) huu $B a^{\prime} a$.

huu-k (2) huu Tii.

huu-? (2) huu Dengka.

huu-? (2) huu Oenale.

uu-f Ro'is Amarasi. source, tree trunk. u?u (2) uu-f (3) ma?-u?u (4) na?$\mathbf{u u}-\mathbf{b}=\mathbf{0 o - n}$ Kotos Amarasi. 1) tree counter. 2) source, beginning. 3) based on. 4) go back to; finds its source in. Lit: '3-base-TR=REFL3SG.GEN'.

uu|? (2) uu-f Molo. 1) tree counter. 2) trunks. (M:583)

\section{Out-comparisons:}

uun Semau Helong. bamboo.

huun East Tetun. base, foot, bottom, the lower part of flank; the beginning, the source; the trunk of any tree. (Mo:89)

\section{I - i}

*ia PRM. this, here. Etym: *ia

ia Tii. 'demonstrative pronoun and adverb: this, here; that, there'.

ia Termanu. this, here. (J:199)

ia Korbafo.

ia Bokai.

ia Bilbaa.

ia Rikou.

ia $B a^{\prime} a$.

ia Dengka.

ia Oenale.

ia Dela. this, here.

ai Ro'is Amarasi. here, this.

ia (2) =ii Kotos Amarasi. 1) here, this. 2) 1DET, definiteness marker for things (physically or metaphorically) near the speaker. 
ii Amanuban. here.

iin, ii Fatule'u. here.

ii Molo. this. (M:158)

ii Amfo'an. here.

ain, iin Timaus. here.

ia Kusa-Manea. here.

Out-comparisons:

ia Semau Helong. here.

*idu Rote. nose, kiss. Etym: *ijun 'nose'.

[Sporadic: $\varnothing>$ ? /\#_ in Dela-Oenale.]

idu_ai-k (2) paña_idu Termanu.

1) back of the nose. 2) nostrils. (J:200)

idu-? Bilbaa. nose. (own field notes)

idu-? Landu. nose. (own field notes)

idu-? Rikou. nose. (own field notes)

idu-? Oepao. nose. (own field notes)

idu $B a^{\prime} a$. kiss. [Note: In Timor 'kissing' is to rub noses.] (J:705)

idu-k Lole. nose. (Zacharias et al. 2014)

idu-k (2) idu Tii. 1) nose. 2) kiss. (J:705)

(?)idu Dengka. kiss. (J:705)

Pidu Oenale. kiss. (J:705)

Pidu Dela. kiss.

Out-comparisons:

ilu Semau Helong. nose.

ilu-r Kemak. nose. Usage: Lemia, Atsabe, Saneri, and Diirbati dialects.

ilug-aạ Kemak. nose. Usage: Kutubaba dialect.

iliguur Kemak. nose. Usage: Leolima dialect. [Form: The correct morphological analysis of this form is unclear. It could be ilig-uur or iliguu-r. Either way, $g$ is a retention of PMP * $\mathrm{y}$.]

iluk-aat Welaun. nose.

*ifa PRM. lap. Etym: *riba. [irr. from PMP: *r $>\varnothing($ expect *r)] [Sporadic: diphthongisation $* \mathrm{i}>a i$ in Meto; $\varnothing>$ ? /\#_in Dela-Oenale.]

ifa (2) ifa-k Termanu. 1) hold in the lap. 2) lap. (J:200)

ifa Korbafo. ifa Bokai.

ifa Bilbaa.

ifa Rikou.

ifa $B a^{\prime} a$.

ifa Tii.

(?)ifa Dengka.

Pifa Oenale.

(2) Pifa-? Dela.

na-?aifa|? Ro'is Amarasi. carry in the lap. Usage: poetic, only currently known in the parallelism na-skau =ma_naPaifa? metaphor 'cares for people' where na-skau $=$ 'hold in the arms, hug'. [Form: automatic glottal stop insertion between $\mathrm{CV}$ - prefix and \#V-initial stem.]

ifa-n (2) aifa-f (3) n-aifan (4) a-fafa-t $=m a$ a-mn-aifa-t Molo. 1) intestinal fat. (M:158) 2) lap. 3) held in the lap (said of a child who has the same character as its mother). 4) the one who cares and holds (us) in his lap. (M:9)

\section{Out-comparisons:}

iha Semau Helong. lap.

*iha PnRote. sister-in-law. Etym: *hipaR 'sibling-in-law (probably of the same sex only)'. [irr. from PRM: *h $>$ ? $/ V_{-}$V in wRote (expect $\left.\varnothing\right) ;{ }^{*} \varnothing>h$ in Tii and Lole] [Sporadic: $\varnothing>h$ /\#_V? in Termanu, Korbafo, Bokai, Ba'a and Lole.] [History: The irregularities in wRote, Tii, and Lole indicate that this term is a borrowing in these lects.]

hi?a Termanu. sister-in-law, used mutually by sisters. (J:182)

hi?a Korbafo.

hi?a Bokai.

ia Bilbaa.

ia Rikou. (own field notes)

hi?a $B a^{\prime} a$.

hi?a Lole. sister-in-law (male speaking). (Zacharias et al. 2014)

hi?a Tii. 
hi?a Dengka.

hi?a Oenale.

Out-comparisons:

iha Semau Helong. cousin, in-law.

*ika Morph: *ika-k. PRM. fish. Etym:

*hikan. Pattern: k-10.

i?a-k Termanu. fish. (J:200)

i?a-? Korbafo.

iPa-k Bokai.

ika-? Bilbaa.

ika-? Landu. fish. (own field notes)

ika-? Rikou.

ika-? Oepao. fish. (own field notes)

i?a-k $B a^{\prime} a$.

iPa-k Tii.

ia-? Dengka.

Pu?u_ia-? Dela. all kinds of fish. [Form: $\mathbf{P u}$ ?u is the normal word for 'fish' in Dela.]

ika|? Ro'is Amarasi. fish.

ika|? Kotos Amarasi. fish.

ika|? Molo. fish. (M:159)

ika|? Kusa-Manea. fish.

Out-comparisons:

ikan Semau Helong. fish.

ikan East Tetun. fish. (Mo:90)

i?a Dhao. fish.

*ikə Rote. snare, noose. Etym: *hikət 'tie, bind, attach to by tying'. Pattern: $\mathrm{k}-6{ }^{\prime}\left({ }^{*} \mathrm{k}>\varnothing\right.$ in Bilbaa, ${ }^{*} \mathrm{k}>$ ? in Ba'a; expect $* \mathrm{k}=k$ in both). [irr. from PRM: $*_{\partial}>a$ in Termanu, Korbafo, Ba'a, and Tii] [Sporadic: $\varnothing>h$ /\#_V? in Bilbaa, Rikou and Ba'a.] [Form: *o $>(* \mathrm{e})>i$ in Bilbaa can be explained by the prohibition against the vowel sequences ie and uo.] [History: Rote *i?i-k 'kind of liana suitable for tying' may also be from PMP *hikət. Reflexes of Rote *i?i-k are as follows: Termanu, Bokai, Tii, i?i-k Korbafo, Rikou, Dengka i?i-?, Bilbaa ii-?, and Ba'a hi?i-k.]

ika (2) i ika-k, ika-k Termanu.

1) snare with a noose attached to a stick. 2) snare, noose, which is tied to a stick and used to catch wild animals (and chickens, etc.). (J:200f)

ika (2) i ika-? Korbafo.

ike (2) i ike-k Bokai.

na-ka-hi hii (3) hi-hii Bilbaa.

hi?e (2) hi hi?e Rikou.

(2) hi hi?a-k $B a^{\prime} a$. [Note: Jonker (1908:200) gives the Ba'a equivalent of Termanu ika as ike, but on page 705 he indicates that this is not certain.]

ike (2) i ike-k, ike-k Tii.

(?)iia (2) (?)iia-t Dengka. PiPa (2) ?i Pi?a-t Oenale.

Pi?a Dela.

*iko Morph: *iko-k. PRM. tail. Etym:

*ikuR. Pattern: k-5. [Sporadic: ${ }^{*} \mathrm{u}>$ $*_{\mathrm{o}}$ / *R\#.]

iko-k Termanu. tail of a four-footed animal or of a fish. (J:201)

iko-? Korbafo.

iko-k Bokai.

iko-? Bilbaa.

iko=na $L a n d u$. tail. (own field notes)

iko-? Rikou.

iko-k $B a^{\prime} a$.

iko-k Tii.

iko-? Dengka.

iko-? Oenale.

iko-? Dela. tail.

iuku-f Ro'is Amarasi. tail.

iku-f Kotos Amarasi. tail. [Sporadic: vowel height harmony $*_{0}>u / i C$ in Amarasi and Kusa-Manea.]

iko-f Molo. tail. (M:159)

iku-f Kusa-Manea. tail.

Out-comparisons:

ikun Semau Helong. tail.

iku-n East Tetun. tail, buttocks; final. (Mo:90)

hi?on Kemak. tail.

*ila Rote. mole, freckle, birthmark. Etym: *qila 'any natural mark on human skin: birthmark, freckle, mole'. 
i ila Termanu. spots on the skin or the body, including: i ila ngeok [OE = 'black spot'] freckles, and i ila pilas $[\mathrm{OE}=$ 'red/brown spot'] birthmark. (J:201)

i ila Korbafo.

i ila Bokai.

ila Bilbaa.

ila Rikou. [Form: My consultants gave vowel initial i ila.]

ila $B a^{\prime} a$.

ila Tii.

ila Dengka.

ila Oenale.

ila Dela. mole.

*imun PMeto. kind of biting flying insect; midge, mosquito.

imun Ro'is Amarasi. kind of small white mosquito whose bite is worse than a normal mosquito.

imun Kotos Amarasi. kind of small white mosquito whose bite is worse than a normal mosquito.

<imu> Molo. flies. (M:160)

Out-comparisons:

əmu Central Nage. mosquito with a horizontally striped black-and-white body. (Forth 2016:332)

xəmu, səmu, əmu Ngadha. mosquito. [History: The variant forms suggest Proto-Central Flores *kləmu, as discussed by Elias $(2018: 84,115)$. Thus the similarity between the Meto forms and Central Flores forms may be due to chance.] (Arndt 1961)

*ina Morph: *ina-k. PRM. mother, mother's sister. Etym: *ina. [Sporadic: diphthongisation $*_{\mathrm{i}}>a i$ in Meto.]

ina-k Termanu. woman, a female human being; mother. (J:202f)

ina-? Korbafo.

ina-k Bokai.

ina-k Bilbaa.

ina-? Landu. woman. (own field notes) ina-? Rikou.

ina-k $B a^{\prime} a$.

ina-k Tii.

ina-? Dengka.

ina-? Oenale.

ina-? (2) ina-n Dela. 1) female human. 2) mother.

ina? Ro'is Amarasi. mother.

aina-f, aina? Kotos Amarasi. mother. aina?, oin Molo. mother. (M:88)

ena-f Baikeno. mother. (Charles E.

Grimes pers. comm.)

ene? Kusa-Manea. mother.

Out-comparisons:

ina Semau Helong. mother.

ina-n (2) inan East Tetun.

1) mother, maternal aunt.

2) female (animals). (Mo:90)

ina Hawu. mother, mother's older sister (maternal aunt), father's brother's wife.

*inu Morph: *n-inu. PRM. drink. Etym: *inum.

n-inu Termanu. drinks. (J:397)

n-inu Korbafo.

n-inu Bokai.

n-inu Bilbaa.

n-inu Rikou.

n-inu $B a^{\prime} a$.

n-inu Tii.

n-inu Dengka.

n-inu Oenale.

n-inu Dela. drink.

n-inu Ro'is Amarasi. drink.

n-inu Kotos Amarasi. drink.

n-inu Molo. drink. (M:160)

Out-comparisons:

n-inu Semau Helong. drink.

*inus CER. certain kind of bird called burung angin in Kupang Malay.

inus Termanu. kind of bird called burung angin (wind bird) in Kupang. (J:205)

inu-? Korbafo.

inu-k Bokai.

inu-? Bilbaa.

inus $B a^{\prime} a$. 
Out-comparisons:

maun_inus Helong. (J:205)

*ingu $P R M$. country, land.

inu Termanu. in the proper sense means 'country, place', but also used in the game of te tena-k (Malay congkak), in which the holes which belong to one side are called inu (those holes that belong to the opposite side are called inu fe?e-k, 'foreign land'). (J:204)

inu Korbafo.

inu Bokai.

inu Bilbaa.

iku Rikou.

ingu $B a^{\prime} a$.

ingu Tii.

ingu Dengka.

ingu Oenale.

ingu Dela.

iku Kotos Amarasi. place of rest in the centre of the fields for the spirits of the rice and corn. 'The new seeds, which are called peen iku [corn $i k u$ ] and maak iku [rice $i k u$ ], are made with a ritual in the field. There are no restrictions as to which seeds from home should be planted. A planting of peen iku is placed in the middle of the field, and the same affair occurs with rice seed which is called maak iku. They place a border (nakat) around it using a sign (?soko) made from a coconut which has been drained of water. This mark is meant to inform people that the crop of corn and rice in the middle of the field is like a house of rest for the spirit of the corn and the spirit of the rice. This marker is called iku. The iku is intended to be a house of rest for the spirits of the rice and corn which come from all four corners of the globe.' (Heronimus Bani, unpublished typescript).

\section{Out-comparisons:}

inu Funai Helong. village, island. inu Semau Helong. village. [Semantics: Jonker (1908:204) gives the meaning of Helong inu as 'country, village'.]

*isa1 PRM. tie together. [Note: Those languages with only one form given use it for both the Termanu senses unless otherwise indicated.] [irr. from PRM: *a $>e$ in several lects and senses] [Sporadic: diphthongisation $*_{\mathrm{i}}$ $>a i$ in Meto.]

isa (2) ise (3) ise-k Termanu. 1) tie something so that it stays hanging. 2) tie something with a rope, tie a rope around something so that it can be picked up or hung up. (J:205) 3) the loop, or the part of a rope, by which something is picked up or hung. [Semantics: Some Rote varieties have different forms for each of these senses, and some varieties have only one form which covers both senses.] (J:206)

isa (2) ise Korbafo.

ise Bokai.

isi (2) (ise) Bilbaa.

ise Rikou.

isa (2) isa $B a^{\prime} a$.

ise Tii.

(?)isa Dengka.

Pisa Oenale.

Pisa Dela. tie with a rope, tie something so that it hangs.

na-Paisa Kotos Amarasi. tie something so that there is a handle which hangs out. [Form: automatic glottal stop insertion between CVprefix and \#V-initial stem.]

aisa-t Molo. four or eight corn cobs tied/hung together in pairs. (M:11)

aisi-t Timaus. tied bundle. [Form: regular assimilation of $* a$ in final closed syllables.]

*isa2 Morph: *n-isa. PRM. utterly, the final point. 
n-isa Termanu. s/he kills. (J:398)

n-isa Korbafo.

n-isa Bokai.

n-isa Bilbaa.

n-isa Rikou.

n-isa $B a^{\prime} a$.

n-isa Tii.

n-isa Dengka.

n-isa Oenale.

n-isa Kotos Amarasi. completely, totally, the logical end point; win.

n-isa Molo. death; wins. (M:161)

Out-comparisons:

isi Semau Helong. too, very.

*isi Morph: *isi-k. Rote. contents, fruit flesh. Etym: *isi? 'flesh (of humans, animals, fruits, tubers); reside; blade of a knife; inhabitants, residents'.

isi=na, isi-k Termanu. contents of something; flesh, muscles; the contents as the main part of something. (J:206)

isi=na Korbafo.

isi=na Bokai.

isi=na Bilbaa.

isi=na Rikou.

isi=na $B a^{\prime} a$.

isi=na Tii.

isi-? Dengka.

isi=na Oenale.

isi-? Dela. flesh, contents.

\section{Out-comparisons:}

isi-n Semau Helong. contents.

isi-n East Tetun. the body or torso; the product, the internal part, the contents, the useful part, a layer; a keen cutting edge of a knife, etc. (Mo:91)

*ita Morph: *n-ita. PRM. see; try, attempt. Etym: *kita2. [irr. from PMP: $\left.*_{\mathrm{k}}>\varnothing / \#\right]$

n-ita (2) n-ita-k Termanu. 1) sees.

2) mainly used in more metaphorical senses including those where 'see' $=$ attempt. (J:398f)

n-ita Korbafo. n-ita Bokai.

n-ita Bilbaa.

n-ita Rikou.

n-ita $B a^{\prime} a$.

n-ita Tii.

n-ita Dengka.

n-ita Oenale.

n-ita (2) n-ita-? Dela. 1) see.

2) suppose.

n-ita Ro'is Amarasi. see; try.

n-ita Kotos Amarasi. see, look at; try, see if.

n-ita Molo. see. (M:239)

iat Kusa-Manea. see.

Out-comparisons:

n-eta Semau Helong. see.

*iu PRM. shark. Etym: *qihu.

iu Termanu. shark. (J:207)

iu Korbafo.

iu Bokai.

iu Bilbaa.

iu Rikou.

iu $B a^{\prime} a$.

iu Tii.

iu Dengka.

iu Oenale.

iu Dela. shark.

iu Ro'is Amarasi. shark.

iik_iu Kotos Amarasi. shark.

iu Molo. shark. (M:163)

Out-comparisons:

iu Semau Helong. shark.

uu Fehan Tetun. shark, some long thin sea animal, about 1-2 m long, 5 ' wide. It is claimed that it also lives in the wee knuuk (underground water), along with crocodile. [irr. from PMP: $*_{\mathrm{i}}>u$ ] [Form: East Tetun has uud 'large whale' (Morris 1984:76), and if this is cognate with Fehan Tetun uu, it would indicate that these forms are not from PMP *qihu.] 
*kaa1 Rote. bite. Etym: *kaRat. Pattern: $\mathrm{k}-1 / 2 / 3$.

kaa Termanu. take something between the teeth, bite off, bite, also said of snakes and birds. (J:208)

kaa Korbafo.

kaa Bokai.

kaa Bilbaa.

Paanan Landu. bite. [Form: The correct morphological analysis of this form is unclear.] (own field notes)

Paa Rikou.

Paa Oepao. bite. (own field notes)

kaa $B a^{\prime} a$.

kaa Dela. bite.

*kaa2 PRM. crow (the bird). Pattern: k-1.

[History: Blust and Trussel (ongoing) reconstruct *wakwak which may be cognate.] [Semantics: onomatopoeia.] kaa Termanu. crow (the bird). (J:208) kaa Korbafo.

kaa Bokai.

kaa Bilbaa.

kaa Rikou.

kaa $B a^{\prime} a$.

kaa Tii.

kaa? Dengka.

kaa? Oenale.

koor_kaa? Ro'is Amarasi. crow.

koor_kaa? metan Kotos Amarasi. crow.

kool_kaa? Molo. crow. (M:164)

Out-comparisons:

kakalo Semau Helong. crow.

kaoa East Tetun. crow (bird). (Mo:101)

*ka|benu PMeto. fly. Doublet: *mbena.

Etym: *bəRnaw (Blust and Trussel (ongoing) reconstruct both *bəRyaw and *banaw.). [irr. from PMP: *aw $>$ ${ }^{*} \mathrm{u}($ expect $* \mathrm{o})$ ] [minority from PMP: $* \mathrm{~b}>(* \mathrm{~b})>* \mathrm{~b}$ ]

kbenu Ro'is Amarasi. fly.

kbenu Kotos Amarasi. fly. akbenu Kusa-Manea. fly.

*kabi PRM. clamp. Doublet: *hapi. Etym: *kapit 'pinch, press between; fasten thatch together with slats in roofing a house'. Pattern: k-3. [irr. from PMP: *p $>* 6$ ]

kabi Termanu. clamp, squeeze with pliers, press with a press. (J:209)

kabi Korbafo.

kabi Bokai.

kabi Bilbaa.

Pabi Rikou.

kabi $B a^{\prime} a$.

kabi Tii.

(?)abi Dengka.

Pabi Oenale.

Pabi Dela. squeeze the lontar palm flowers with a squeezer made of the rib of a lontar palm leaf.

<abi> (2) n-habi (3) <kiba habi> Molo. 1) clamp. (M:3) 2) clamping. (M:134) 3) kind of ant which bites.

(M:134)

n-Paib Kusa-Manea. pinch, clamp.

Out-comparisons:

habit East Tetun. squeeze between two things, put in a splint. (Mo:40)

*kade Morph: *kade-k. Rote. charcoal. Etym: *qajəy. Pattern: $\mathrm{k}-1^{\prime}\left({ }^{*} \mathrm{k}>\right.$ $?$ in $\mathrm{Ba}$ a, expect $\left.{ }^{*} \mathrm{k}=k\right)$. [minority from PMP: *ə $>*_{\mathrm{e}} / \#$ \# (expect $*_{\partial}>$ $a$ in wRote, possibly $\left.\left.{ }^{*} \partial>*_{a}>e\right)\right]$ [Sporadic: $* \mathrm{a}>* \mathrm{e} / * \mathrm{C}+$ palatal_.] [Form: The initial $* \mathrm{k}$ may be from the PRM nominal prefix *ka-.]

(ha?i) kade-k Termanu. charcoal. (J:211)

kade-k Bokai.

kade-? Bilbaa.

Pade-? Rikou.

(?)ade-k $B a^{\prime} a$.

kade-k Tii.

kade-? Dengka.

kade-? Dela. burned. 


\section{Out-comparisons:}

alen Semau Helong. charcoal.

arne Kisar. charcoal. (Rinnooy 1886:169)

*kae PRM. cockatoo. Pattern:

k-1. [History: Clark (2011:321)

reconstructs Proto Papuan Tip

*wakeke $\sim$ *kakawe which may be cognate.] [Semantics: onomatopoeia.]

ka kae Termanu. cockatoo. (J:211)

ka kae Korbafo.

ka kae Bokai.

ka kae Bilbaa.

ka kae Rikou.

ka kae Dengka.

koor kae Ro'is Amarasi. Yellow Crested Cockatoo.

koor kae muti? Kotos Amarasi. Yellow Crested Cockatoo.

kool kae, kae muti? Molo. cockatoo. (M:167)

Out-comparisons:

ka kae, ka kai East Tetun. cockatoo (bird). (Mo:96)

*kahi1 PRM. pull towards oneself. Pattern: k-2b.

ka?i Termanu. pull towards oneself. (J:214)

ka?i Korbafo.

(?)ai Rikou. [Form: Whether the initial glottal stop is underlying or epenthetic is not clear. I could not elicit this form from my consultants.]

ka?i $B a^{\prime} a$.

ka?i Tii.

(?)ai Dengka.

Pai Oenale.

Pai Dela.

n-?ai Kotos Amarasi. push down.

n-Pai (2) na-?ai-b=on (3) na?ai-b-a? Molo. 1) pushes down.

2) withdraws. 3) moves someone sideways. (M:8)

Out-comparisons:

kahi Helong. ( $\mathrm{J}: 214)$

*kahi2 Rote. count. Pattern: k-2/3. ka?i Termanu. count, calculate. ( $\mathrm{J}: 214)$

ka?i Korbafo.

ka?i $B a^{\prime} a$.

ka?i Tii.

(?)a (?)ai Oenale.

Out-comparisons:

kahi, kasi Semau Helong. count, one, a.

*kahin PRM. stop, prevent. Pattern: $\mathrm{k}-2 \mathrm{a}$. [irr. from PRM: ${ }^{*} \mathrm{k}>\eta$ in Bilbaa] ka?i Termanu. stop, prevent. (J:214)

ka?i Bokai.

na-sa-nai Bilbaa.

Pai Rikou. [Form: My consultants gave na-sa-?ai.]

ka?i $B a^{\prime} a$.

ka?i Tii.

(?)ai Dengka.

Pai Oenale.

Pai Dela. rebuke.

na-kain-a? Kotos Amarasi. forbid.

Out-comparisons:

kain Semau Helong. prohibit, forbid.

hakahik East Tetun. prohibit, prevent, retain, hold, to not allow the action of any practice. (Mo:49)

*kahu Rote. kind of fish. Pattern: k-1. [irr. from PRM: ${ }^{*} \mathrm{~h}>$ ? in Rikou (expect $* \mathrm{~h}>\varnothing)$ ] [Semantics: vague semantics weaken reconstruction.]

ka?u Termanu. kind of ocean fish. (J:224)

ka?u Korbafo.

ka?u Bokai.

kau Bilbaa.

ka?u Rikou.

ka?u $B a^{\prime} a$.

ka?u Tii.

kau Dengka.

kau Oenale.

Out-comparisons:

khau Helong. (J:224)

*kai1 Rote. stiff, awkward. Pattern: k-2/3. 
na-ma-kai (2) bala/kai-k Termanu.

1) have a stiff or tired feeling, like someone who has been sitting for, lying on one side or carrying something for a long time, often combined with sota. (J:214) 2) stiff, hard, strong, powerful. (J:26)

na-ma-kai (2) bala/kai-? Korbafo. na-ma-kai (2) bala/kai-k Bokai. na-ma-kai (2) bala/kai-? Bilbaa. na-ma-?ai (2) bara/ai-? Rikou. na-ma-kai (2) bala/kai-k $B a^{\prime} a$. na-ma-kai (2) bara/kai-k Tii.

(2) bala?ai-? Dengka.

Out-comparisons:

kalkait (2) kain Semau Helong. 1) stiff, awkward. 2) tighten. [Note: Jonker (1908:213) gives Helong balakaik and bkain.]

kai Ili'uun. fixed, stiff, hard, taut, stuck fast; avaricious. (dJ:120)

*kai 2 PRM. hook. Etym: *kawit. Pattern: k-1.

kai Termanu. hook, be hooked. (J:212)

kai Korbafo.

kai Bokai.

kai Bilbaa.

Pai (2) Pa Pai Rikou. 1) hook, be hooked. (J:212) 2) hook which is affixed to one's waist from which buckets can be hung while ascending a lontar palm. (own field notes)

kai $B a^{\prime} a$.

kai Tii.

kai Dengka.

kai Oenale.

P-ka?i Kotos Amarasi. kind of hook with a sharp angle, these used to be put on the saddle of a horse and people would hang goods from them for transportation. [Note: $\operatorname{tanu}=$ 'fish-hook'.]

<nak'ai> Molo. fetches towards oneself with a hook. (M:169)

\section{Out-comparisons:}

kait Semau Helong. fish hook.

*kai/ou PRM. Casuarina tree. Casuarina species. Etym: *qaRuhu 'shore tree Casuarina equisetifolia'. Pattern: $\mathrm{k}-2 \mathrm{~b}^{\prime}\left({ }^{*} \mathrm{k}>?\right.$ in Tii, expect $\left.{ }^{*} \mathrm{k}=k\right)$. [irr. from PMP: *a $>*_{\mathrm{o}}$ (sporadic assimilation)] [Sporadic: $* \mathrm{k}>$ ? /\#_ in Tii.] [Form: initial *kai probably from *kaiu 'tree, plant, wood'.]

kai/ou Termanu. the cemara tree (in Kupang pohon kasuwari[s]). (J:216f)

kai/ou Korbafo.

kai/ou Bokai.

kai_ou Bilbaa.

kai/ou Landu. Casuarina tree. (own field notes)

(?)ai/ou Rikou.

kai/ou $B a^{\prime} a$.

(?)ai/ou Tii.

(?)ai/ou Dengka.

(?)ai/ou Oenale.

kaidzo?o Ro'is Amarasi. Casuarina.

Paidło?o Kotos Amarasi. Casuarina tree. [Form: expected epenthetic consonant in *VVV Final ?o disappears when modified. This also occurs for naiso?o 'onion'.]

Paioo, Paiyoo Amanuban, South. Casuarina.

Pudzau Kopas. Casuarina tree. Usage: Usapisonba'i village.

Paidgoo Kopas. Casuarina tree. Usage: Bone village.

Padzau Molo. Casuarina. Casuarina junghuhniana. [Form: Middelkoop (1972:4) transcribes this <adjau>. Meijer Drees (1950) gives the Molo form as <adjáo>.] (M:4, Meijer Drees 1950:1)

Paidzau Amfo'an. Casuarina tree.

Paroo-gw Timaus. Casuarina tree. Usage: Sanenu village.

Puro? Timaus. Casuarina tree. Usage: Oekona' village. 
Paioo Kusa-Manea. Casuarina tree.

Out-comparisons:

ka/keu Fehan Tetun. Casuarina tree (e.g. found at the beach and near the river).

ka/keu East Tetun. Casuarina tree. (Mo:97)

k'au Waima'a. Casuarina. Casuarina junghuhniana.

gou Kemak. Casuarina tree.

ai hou Welaun. Casuarina tree.

$<$ kajú $>$ Kambera. kind of Casuarina tree. Casuarina junghuhniana. [Note: Another name for a (possibly different) kind of Casuarina tree is $<$ kajiu $>$ for which Lewa has kadiu, indicating that Kambera ds may be from palatalisation of earlier $* d$ and that this term is not cognate.] [Form: $\langle\mathrm{j}\rangle=$ [d]17 (On:137)

*kais Morph: *ma-kais. PRM. sour. Pattern: k-3.

ma-keis (2) ma-kei Termanu. 1) sour. 2) become sour, has become sour. (J:228)

ma-kei-? Korbafo.

ma-kei-? Bokai.

ma-kei-? Bilbaa.

ma-Peis Rikou.

ma-keis $B a^{\prime} a$.

ma-keis Tii.

ma-Peis Dengka.

ma-Peis Oenale.

ma-Pei Dela. sour.

<ma|hai> Molo. sour. (M:297)

*kaiu PRM. tree, plant; wood. Etym: *kahiw. Pattern: k-4. [Note: Several names of specific trees in Rote lects begin with a (fossilised) reflex of *kaiu. These reflexes of *kaiu are not always identical to the reflexes given here.]

ai Termanu. tree, stem, wood. (J:5)

ai Korbafo.

ai Bokai. kai Bilbaa.

ai Landu. tree, wood. (own field notes)

ai Rikou.

ai $B a^{\prime} a$.

ai Tii.

hau Dengka.

hau Oenale.

hau Ro'is Amarasi. wood, tree.

hau Kotos Amarasi. wood, tree.

hau Molo. tree, plant, wood. (M:141)

hau Kusa-Manea. tree, wood.

Out-comparisons:

kai Semau Helong. wood, tree.

ai East Tetun. tree, bush, shrub, plant, vegetable; stick, wood, timber, firewood. (Mo:2)

kau Habun. tree. [Note: language of east Timor ISO 639-3 [hbu].]

(Dawson 2014)

ai Kemak. plant, wood.

au Kisar. wood.

afu Hawu. tree, wood.

afu Dhao. tree, wood.

*kai/usu Rote. ribs. Etym: *Rusuk. Pattern: k-2/3. [irr. from PRM: * $\varnothing>$ $h$ in Tii] [Form: Source of initial * kai unclear, Jonker connects it with the prefix ka-.]

kai/usu-k Termanu. rib(s). (J:217)

kai/usu-? Korbafo.

kai/usu-k Bokai.

(?)ai/usu-? Rikou.

kai/usu-k $B a^{\prime} a$.

kai/husu-k Tii.

(?)ai/usu-? Dengka.

Pai/usu-? Oenale.

Pai/usu-? Dela. ribs.

Out-comparisons:

rusan Kisar. ribs.

*kaka Morph: *kaka-k. Rote. elder sibling of the same sex. Etym: *kaka. Pattern: initial k-2/3', medial k- $8^{\prime}$. [Form: The alternate Dengka form with initial $k$ is not regular under pattern $\mathrm{k}-2 / 3$, similarly medial $k$ in the alternate Dengka and Tii forms is not regular under pattern $\mathrm{k}-8$. 
These irregularities may be due to borrowing, though the nearest Rote form with medial $k$ is Bilbaa which does not neighbour either Tii or Dengka. If a borrowing hypothesis is taken to explain these irregularities Malay kakak would be the most likely source.]

ka?a-k Termanu. older brother or sister, used by brothers for brothers and sisters for sisters. (J:209)

ka?a-? Korbafo.

ka?a-k Bokai.

kaka-? Bilbaa.

Pa?a=na $L a n d u$. older sibling. (own field notes)

Pa?a Rikou.

ka?a-k $B a^{\prime} a$.

ka?a Lole. older sibling (same sex). (Zacharias et al. 2014)

kaka-k, ka?a Tii. [Form: Jonker (1908:209) gives kaka-k, Grimes et al. (2014a) gives ka?a.]

kaka-?, Pa?a Dengka. [Note: Jonker (1908:209) gives the putative Dengka form Pa?a with the note: 'also surely Dengka'.]

Pa?a Oenale.

Pa?a Dela. older sibling.

Out-comparisons:

kaka Semau Helong. older sibling. kaka Kisar. older relatives.

a?a Hawu. elder sibling same sex.

*kalati Rote. earthworm. Etym: *kaliwati. Pattern: k-1/2/3. [irr. from PMP: ${ }^{*} \mathrm{~W}>\varnothing$ ] [irr. from PRM: $\varnothing>n$ in Landu] [Sporadic: antepenultimate syllable loss in wRote.]

kalati-k (2) kelati dae Termanu.

1) worm, both earthworm and intestinal worm. 2) earthworm. (J:219)

kalati-? Korbafo.

kalati-k Bokai.

kalati-? Bilbaa.

kalnati $L a n d u$. earthworm. (own field notes) kalati-? Rikou.

sikalati-k $B a^{\prime} a$.

sikalati-k Tii.

la lati-? Dengka.

la lati-? Oenale.

Out-comparisons:

blati? Semau Helong. worm, earthworm, roundworm. [Form: Probably from the alternate PMP form *bulati.]

(k)la latik East Tetun. earthworm, intestinal worm. (Mo:108)

*kali PRM. dig. Etym: *kali. Pattern: k-3. kali Termanu. dig, excavate. (J:219)

kali Korbafo.

kali Bokai.

kali Bilbaa.

Pali Rikou.

kali $B a^{\prime} a$.

kali Tii.

(?)ali Dengka.

Pali Oenale.

Pali Dela. dig.

n-hani Ro'is Amarasi. dig.

n-hani Kotos Amarasi. dig.

n-hani Molo. dig. (M:139)

Out-comparisons:

kali Semau Helong. dig.

*kalusa PMeto. fingernail, toenail, claw.

Etym: **kilusa (pre-Meto). Pattern:

k-1/2a. [irr. from PRM: *1 $>l \sim n$ in

Kualiin Amanuban] [Form: I have posited an original trisyllable as most varieties of Meto show regular $* 1>n$, unlike PRM *kl which is reflected as $k l$ or $k r$. However, the alternate form in Kualiin Amanuban, klusa-n, points to early reduction of the antepenultimate syllable. Thus, *kalusa may have had the alternate form *klusa in PMeto.] tnusu-f Ro'is Amarasi. fingernail, toenail, claw.

knusa-f Kotos Amarasi. fingernail, toenail, claw. [Note: An alternate form for 'nail' is Ptusa-f, though knusa-f seems more common.] 
knusa-f Amanuban. fingernail, toenail, claw.

klusa-n, knusat Amanuban. fingernail, toenail, claw. Usage: Kualiin village.

\section{Out-comparisons:}

kalusun, klusun Ili'uun. nail, claw. (dJ:120)

kilusu Tugun. fingernail. (Hinton 2000:124)

*kame Rote. knead. Doublet: *ke?e, *keme, *kumu2, *ngumu. Etym: *gəmgəm. Pattern: k-2/3. [minority from PMP: *ə $>*_{\mathrm{a}} / \# \mathrm{C}$; $*_{\partial}>*_{\mathrm{e}} /$ \#]

kame (2) ka kame Termanu.

1) knead. 2) knead repeatedly. (J:220)

kame Korbafo.

kame Bokai.

kame Bilbaa.

keme Rikou.

kame $B a^{\prime} a$.

kame Tii.

(?)ame Dengka.

(?)ame Oenale.

*ka(m)i PRM. we, first person plural exclusive pronoun. Etym: *kami. Pattern: k-4. [Form: Lynch et al. (2002) state that POc *kami 'sometimes occurred as *kai'. Reflexes of putative *kai also occur outside of the Oceanic subgroup. In the Bungku-Tolaki subgroup, for example, Mead (1998:154) gives 18 isolects with kami, mami and 11 isolects with kai, mai. The Moronene language has both ikami and ikai as free pronouns. Likewise, Moronene has genitive -mami/-mai and absolutive -kami/-kai). (In Moronene the forms without medial $m$ are optionally used when certain enclitics attach to the pronoun.) Another area in which forms without medial $m$ are known to occur is in the Barrier Islands where Enggano has Pai and Mentawai has kai. Based on such forms, it seems possible to reconstruct the reduced variant *kai to PMP. Among the RM languages most nRote languages show only reflexes of *kami, while wRM, Ba'a, Tii and Lole have reflexes of *kai.]

ami Termanu. 1PL.EXCL. (J:10)

ami Korbafo.

ami Bokai.

ami Bilbaa.

ami Landu. (own field notes)

ami Rikou.

ai $B a^{\prime} a$.

ai Lole. (Zacharias et al. 2014)

ai Tii.

hai Dengka.

hai Oenale.

hai Ro'is Amarasi. 1PL.EXCL.

hai (2) =kai Kotos Amarasi. 1) 1PL. EXCL.NOM. 2) 1PL.EXCL.ACC.

hai Molo. 1PL.EXCL. (M:135)

Out-comparisons:

kami Semau Helong. 1PL.EXCL. ami East Tetun. we, 1PL.EXCL. (Mo:4)

*kamiri Rote. candlenut tree: Aleurites moluccana. Etym: *kamiri (This reconstruction is not without its problems and reflexes may have been distributed by Malay, though Blust and Trussel (ongoing) note '[Malay borrowing] is much harder to argue for Hanunóo, since the term is unknown in many Philippine languages that have borrowed much more heavily from Malay'.) Pattern: k-1. [irr. from PRM: *ri $>$ ?a in Termanu, Bokai, and Ba'a; ${ }^{*} \mathrm{k}>\eta g$ in Oenale] [Semantics: The reflexes in eastern Timor point to earlier **kamiRi.]

kami?a Termanu. candlenut. (J:220)

kami?a Bokai.

kamili Bilbaa.

(kamia ?) Rikou.

kami?a $B a^{\prime} a$.

kamiri Tii.

kamili Dengka.

ngamiri Oenale. 
Out-comparisons:

kamii(n), kmii East Tetun. tree with oily fruit, the candlenut. Aleurites Moluccana. (Mo:100)

mii Galolen. candlenut.

*kambe PRM. saliva, spittle. Etym: *kambeR (own reconstruction) (PCMP). Pattern: k-4. [irr. from PRM: *e $>u$ in Dela] [Form: I have tentatively reconstructed final $* \mathrm{e}$ (rather than *ə) as this best explains the forms in Rote-Meto, Ili'uun and central Maluku. However, it seems unlikely that ${ }^{*}$ e can account for the Central Timor forms or the alternate Tetun form kaban.] [History: May be connected with PMP *ibəR 'saliva in the mouth; drool; desire, crave, lust for'.]

ape Termanu. saliva, spittle. (J:14)

ape Korbafo.

ape Bokai.

ape Bilbaa.

ape=na Landu. saliva. (own field notes)

ape Rikou.

ape Oepao. (own field notes)

ampe $B a^{\prime} a$.

ambe Tii.

hambu oe-? Dela. saliva.

hape $R o$ 'is Amarasi. saliva.

hape Kotos Amarasi. saliva, spit.

hape Molo. saliva. (M:139)

hape Kusa-Manea. saliva.

Out-comparisons:

kapen Semau Helong. saliva, spittle.

kaba-n, kabe-n, kabuee-n

East Tetun. saliva, slobber (of animals). [Form: uee $=$ water $]$ (Mo:91)

ape(n) Ili'uun. cheek, throat. [Note: de Josselin de Jong (1947:112) also gives apore(n), apure(n) 'spittle' which is probably also related.] (dJ:112)

apar Kisar. spittle. aba Mambae, South. saliva, spittle.

(Grimes et al. 2014b:9)

aba-r Kemak. saliva, spittle.

aba-t been Welaun. saliva, spittle.

aper Kamarian. mucus. (van Ekris 1864:76)

apel Haruku. [Note: also in some varieties of Kaibobo.] (van Ekris 1864:76)

apel Nusa Laut. mucus, snot. [Note: language of Lease Islands, central Maluku ISO 639-3 [nul].] (van Hoëvell 1877:105)

*kambu $P w R M$. belly, uterus. Etym: *kambu 'lower stomach, bladder'. Pattern: k-2b.

(?)ambu-? Dengka. stomach. (J:678) Pambu-? Oenale. belly. (J:678)

Pambu-? Dela. belly, stomach.

na-kapu? Ro'is Amarasi. pregnant.

?apu-f (2) na-?apu? Kotos Amarasi.

1) womb. 2) pregnant.

Papu-f Amfo'an. side. (M:27)

Papu-f (2) < na-apu $>$ Molo. 1) uterus in general; a kind of little basket.

2) pregnant. (M:27)

na-Paup Kusa-Manea. pregnant.

Out-comparisons:

kabu-n East Tetun. stomach, abdomen (of humans). (Mo:93)

kapun Ili'uun. belly. (dJ:120)

Papun Kisar. pregnant.

*kandi PwRM. whetstone. Pattern: k-2b.

(?)andi Dengka. whetstone. (J:678)

Pandi Oenale. whetstone. (J:678)

Pandi Dela. whetstone.

kari Ro'is Amarasi. whetstone.

Paki Kotos Amarasi. whetstone.

Paki Molo. whetstone. (M:14)

\section{Out-comparisons:}

kadi East Tetun. sharpen to a keen edge (any cutting instrument). (Mo:94)

katfi Ili'uun. whetstone, grind. (dJ:120)

ati Kisar. sharpen. 
*kao PRM. scrape, scratch. Etym: *kaRaw (Blust and Trussel (ongoing) reconstruct many forms of a similar shape which mean 'scrape, scratch': PMP *kaRus, PMP * gadus, PMP * garut, PWMP *kaus, PWMP *kərud, PWMP *kərus, PMP *kaRud, PAN *karut, PWMP *aRud, PWMP *karus. Of these, the RM reflexes would be regular from *kaRaw.). Pattern: $\mathrm{k}-1 / 2 \mathrm{a}$. [irr. from PMP: $*_{\mathrm{u}}>*_{\mathrm{o}}$ ] [irr. from PRM: ${ }^{*} \mathrm{k}>\eta g$ in Dengka]

kao Termanu. scrape the hands together. (J:221)

\section{kao Korbafo.}

kao $B a^{\prime} a$.

kao Tii.

ygao Dengka. scrape, scratch. (J:743)

n-kao Kotos Amarasi. scratch.

kao Meto. scratch. (J:221)

\section{Out-comparisons:}

kau Hawu. (J:221)

$<\mathbf{k a u}>$ Kambera. scratch. (On:197)

$<$ kä̈> Weyewa.

$<$ kayo $>$ Kodi.

*kara Morph: *kara-k. $n R M$. chest.

Etym: **karas (pre-RM). Pattern: $\mathrm{k}-2 \mathrm{~b}$.

kala-? Bilbaa. breast. (J:708)

ara-? Rikou. chest. [Form: Jonker (1908:708) gives <arạ́> = araa?, my consultants gave ara-?.]

ara-? Oepao. chest. (own field notes)

kala-k Lole. chest. (Zacharias et al. 2014)

kara-k Tii. breast. (J:708)

kan/sao-f Ro'is Amarasi. chest.

Pan/sao-f Kotos Amarasi. pit of the stomach, solar plexus, 'heart' in the metaphorical sense. Usage: Occurs in parallelisms with neka-f 'feelings'.

Pan/sao-n Molo. feelings, chest. (M:145, 360)

Pa/sao-f Kusa-Manea. chest.

Out-comparisons:

kalas Funai Helong. chest. karas East Tetun. breast, the outer front part of the chest; a half fathom, measured from the tips of the outstretched fingers to the middle of the chest. (Mo:101)

kakara Dhao. chest, breast.

*karu Morph: *ka karu. Rote. scrape, rasp; scratch. Etym: *karut (Blust and Trussel (ongoing) reconstruct many forms of a similar shape which mean 'scrape, scratch': PMP *gadus, PMP * garut, PWMP *kaus, PAN *kaRus, PWMP *kərud, PWMP *kərus, PMP *kaRud, PWMP *aRud, PWMP *karus. Of these, the RM reflexes would be most regular from *karut or *gadus.). Pattern: k-1/2/3' (Dengka ? Oenale $k$ correspondence; expect both to have either $?$ for pattern $2 / 3$, or $k$ for pattern 1).

ka kalu Termanu. scratch. (J:220)

ka kalu Korbafo.

ka kalu Bokai.

ka kalu Bilbaa.

(?)a (?)aru Rikou.

ka kalu $B a^{\prime} a$.

ka karu Tii.

(?)a (?)alu Dengka.

ka karu Oenale.

Out-comparisons:

kalo Semau Helong. scratch.

haruk Kisar. scratch.

*kase PMeto. foreigner, foreign. Pattern: $\mathrm{k}-1 / 2 \mathrm{a}$. [History: This is probably a borrowing from Meto into Kemak. Waima'a has raising of final $*_{\mathrm{a}}>\mathrm{o}$ in open syllables, from which final $u$ could further be derived. Similarly, Meto has sporadic raising of $*_{a}>e$ in final open syllables. Kemak, however, does not have such sound changes thus suggesting pre-Meto **kasa with $* \mathrm{a}>e$ in Meto and subsequent borrowing into Kemak.] [Semantics: It could be that the original meaning was 'person'. This meaning occurs in 
the Meto phrase kase ?naek literally 'great foreigner' (Amarasi kaes ko?u) which is used poetically to refer to any highly honoured dignitary, including members of the Atoni ethnolinguistic group.]

kase $R o$ 'is Amarasi. foreign, foreigner. kase Kotos Amarasi. foreign, foreigner.

kase Molo. foreigner from overseas. (M:182)

kase Kusa-Manea. foreign, foreigner. Out-comparisons:

wasa-kasu Waima'a. enemy.

kase Kemak. foreigner.

*katefuan CERM. wasp, hornet. Etym: *tabuqan 'yellow-jacket wasp'. Pattern: k-2b. [irr. from PRM: *a > $e$ in Termanu and Landu] [Sporadic: consonant metathesis ${ }^{*} \mathrm{kVt}>t V k$ in Termanu and Landu.] [Form: The source of initial *kate is unclear, but reflexes of *kate- occur with at least two other biting insect terms (see the note for the Termanu reflex). Jonker connects it with Termanu kete 'itch', (probably from PMP * gatal) and this is possible.]

teke/fua-k Termanu. kind of wasp. [Form: Initial teke occurs with at least two other biting insect terms, tekefia-k 'tick', tekemela-k 'bedbug' (from *qatiməla). It also occurs with tekelaba-k 'house gecko'.] (J:614)

tekefua-? Landu. wasp. (own field notes)

katfua|? Ro'is Amarasi. kind of stinging/biting wasp. [Form: From intermediate ${ }^{* *}$ kaetfua with regular $\mathrm{CV}>\mathrm{VC}$ metathesis of the first element of a compound.]

Patfuan Kotos Amarasi. kind of stinging/biting wasp.

Patfuan Molo. wasp. (M:32)

Paetfuan Kusa-Manea. kind of stinging or biting wasp.

\section{Out-comparisons:}

tohan Semau Helong. wasp. [irr. from PMP: *a $>o$ (perhaps via intermediate consonant metathesis, *tabuqan > $* *$ taquban $>* *$ tauban)]

*kato PRM. itch, feel itchy. Doublet: *ngete. Etym: *gatəl. Pattern: k-3. [irr. from PRM: *a $>e$ in Rote] [Sporadic: $*_{\partial}>e / \sigma$ \# in wRote (perhaps $*_{\partial}>*_{\mathrm{a}}$ $>e / \#)$.

kete (2) na-kete (3) ma-kete (4) ma-kete-k Termanu. 1) biting, burning on the tongue. 2) hot, biting on the tongue; itch. 3) have an itch. 4) hot burning. (J:232f)

kete Korbafo.

kete Bokai.

kete Bilbaa.

Pete Rikou. [Form: My consultants gave na-Pete 'spicy' and ma-?ete 'itchy'.]

kete $B a^{\prime} a$.

kete Tii.

(?)ete Dengka.

Pete Oenale.

ma-?ete-? Dela. hot (spicy).

ma|hata|? Ro'is Amarasi. itchy.

ma|hata|? Kotos Amarasi. itchy.

n-ma|haat Molo. itchy. (M:140)

ma|haat Kusa-Manea. itchy.

Out-comparisons:

katen Semau Helong. itch.

katal, katar East Tetun. feel itchy, itch, sting. (Mo:103)

katal Welaun. itchy.

akal Kisar. itchy.

*kati PRM. call a dog. Etym: *kati (PCMP). Pattern: k-3' $\left({ }^{*} \mathrm{k}=k\right.$ in DelaOenale; expect ${ }^{*} \mathrm{k}>$ ?).

kati Termanu. call a dog. (J:223)

kati Korbafo.

kati Bokai.

kati Bilbaa.

(?)a (?)ati Rikou.

kati $B a^{\prime} a$.

kati Tii. 
(?)a (?)ati Dengka.

ka kati Oenale.

kati-? Dela. call a dog. (Theresia

Tamelan pers. comm. 2017)

n-hait Amanuban. calls (dog). (M:135)

n-haet (2) $\mathrm{ka}=$ ha hati Molo. 1) calls (dog). 2) in farewell poems: no longer able to be called, that is due to leaving and falling outside the reach of those who cry out. (M:135)

*kea PRM. turtle. Etym: *keRa 'hawksbill turtle' (PCEMP). Pattern: k-2a. [Sporadic: *a $>e / \#$ in wRM.]

kea Termanu. turtle. (J:225)

kea Korbafo.

kea Bokai.

kea Bilbaa.

kea $L a n d u$. (own field notes)

Pea Rikou.

kea $B a^{\prime} a$.

kea $T$ Tii.

(?)ee Dengka.

Pee Oenale.

Pee Dela. turtle.

kee Ro'is Amarasi. turtle, tortoise.

kee, kea Kotos Amarasi. turtle, tortoise.

$<\mathbf{k e} \mathbf{e}^{\prime},,<\mathbf{k e} \mathbf{a}>$ Molo. turtle. [Form: $<\mathbf{k e}^{\prime}>$ is almost certainly kee, but the correct interpretation of $<\mathbf{k e}$ 'a $>$ is not clear] (M:191)

*kees Morph: *na-kees. CERM. squeeze around the waist. Pattern: k-1/2a.

na-kee Termanu. wear around the belly. (J:225)

na-kee Korbafo.

na-ee Rikou.

na-kes kees Kotos Amarasi. have contractions.

na-kees Meto. press. (J:225)

Out-comparisons: kees Semau Helong. strangle. [Semantics: Jonker gives the meaning for Helong kees as 'tie, e.g. around the belly, also: knead'.]

*kei PRM. tickle. Pattern: $\mathrm{k}-3^{\prime}\left({ }^{*} \mathrm{k}=\mathrm{k}\right.$ in wRote and Rikou; expect *k $>$ ?).

na-la-kei Termanu. touch someone softly, e.g. with a finger or stick in order to get his attention. (J:228)

na-la-kei Korbafo.

na-la-kei Bokai.

na-la-kei Bilbaa.

ke $\sim$ kei Rikou.

na-la-kei $B a^{\prime} a$.

na-la-kei Tii. tickle. (J:710)

keis Dengka.

keis Oenale.

keis Dela. touch to get attention.

mahei Kotos Amarasi. ticklish.

mahei, mahai Meto. ticklish. (J:710)

*ke2e PnRote. knead. Doublet: *kame,

*keme, *kumu2, *ygumu. Etym:

*gəmgəm. Pattern: k-2/3. [irr. from PMP: * $\mathrm{g}>$ *?] [minority from PMP: $\left.* \mathrm{a}>*_{\mathrm{e}} / \#\right]$

kePe (2) ke ke?e Termanu. 1) pinch, knead, from that: squeeze out.

2) knead into a ball. (J:227)

ke?e Bokai.

kee Bilbaa.

(?)ee Rikou.

ke?e $B a^{\prime} a$.

ke?e Tii.

*kela Rote. leave behind. Pattern: k-4.

ela Termanu. leave over, leave behind; leave. (J:111)

ela Korbafo.

ela Bokai.

kela Bilbaa.

ela Rikou.

ela $B a^{\prime} a$.

ela Tii.

hela Dengka.

hela Oenale.

Out-comparisons: 
hela East Tetun. stay, remain, reside; to abandon, to reject; $a d v$. at rest, in the same state; particle the action is completed. [irr. from PRM: * $\mathrm{k}$ $=h$ correspondence] (Mo:85)

kera Hawu. overshoot. (J:111)

*kelas Morph: *ka-kelas. PRM. winter melon. Benincasa hispida. Pattern: k-irr. [irr. from PRM: $* \mathrm{k}>*^{*} \mathrm{gg}$ in nRote; $* \mathrm{a}>e$ Meto] [Form: This is the only known form with a nRote ${ }^{*}$ yg $=$ wRM ${ }^{*} \mathrm{~h}$ correspondence set. We could propose PRM * $\mathrm{g}$ to account for this correspondence, though in the absence of additional examples of this correspondence set I prefer not to posit an additional PRM proto-phoneme.]

ygelas Termanu. gourd, squash. (J:437)

ngela-? Korbafo.

nela-k Bokai.

jela-k Bilbaa.

kelas Rikou.

ygelas $B a^{\prime} a$.

helas Oenale.

helas Dela. pumpkin.

heens Ro'is Amarasi. winter melon.

Benincasa hispida.

?|henes Kotos Amarasi. winter melon.

Benincasa hispida.

\section{Out-comparisons:}

kelin Ili'uun. pumpkin (with red flesh and black stones). [irr. from PRM: ${ }^{*} \mathrm{a}=i$ correspondence; ${ }^{*} \mathrm{~s}$ $=\eta$ correspondence $](\mathrm{dJ}: 121)$

helas Central Manggarai. Benincasa hispida. (Verheijen 1984:46)

yelas East Manggarai. Benincasa hispida. (Verheijen 1984:46)

kelas West Manggarai. Benincasa hispida. (Verheijen 1984:46)

hala? Komodo. Benincasa hispida. (Verheijen 1984:46)

hala Bima. Benincasa hispida. (Verheijen 1984:46) *kele CERM. pant, breathe heavily. Pattern: k-3. [Note: Termanu and Tii have ngile 'heavy breathing like a sick person, panting' which may be related.]

kele kele Keka. heavy coughing. bo?o tao leo bee ndia dee lee kele kele-nemi What kind of coughing is that! You cough like you are choking. (J:710)

kele kele Korbafo. heavy breathing. (J:710)

na-Phene? Kotos Amarasi. neigh, whinny.

$<$ hene $>$ Molo. the whinny of a mating stallion. (M:145)

*keme Rote. knead. Doublet: *kame, *ke?e, *kumu2, *ngumu. Etym: *gəmgəm. Pattern: k-2/3. [minority from PMP: *ə > *e / \#]

keme (2) ke keme Termanu. 1) knead. 2) knead, hold tightly in the hand. ( $\mathrm{J}: 230)$

keme Korbafo.

keme Bokai.

keme Bilbaa.

keme Rikou.

keme $B a^{\prime} a$.

keme Tii.

(?)eme Dengka.

(?)eme Oenale.

*ke(m)i PRM. 2PL nominative, you all. Etym: *kamuyu. Pattern: k-4. [Form: The loss of medial *m in wRM, Ba'a, Tii, and Lole is parallel to the loss of medial *m in the first person plural exclusive pronoun.]

emi Termanu. 2PL. (J:113)

kemi Korbafo.

kemi Bokai.

kemi Bilbaa.

emi Landu. 2PL, you. (own field notes)

emi Rikou.

ei $B a^{\prime} a$.

ei Lole. (Zacharias et al. 2014)

ei Tii. 
hei Dengka.

hei Oenale.

hii Ro'is Amarasi. 2PL.

hii (2) =kii Kotos Amarasi. 1) 2PL. NOM. 2) 2PL.ACC.

hii Molo. 2PL. (M:147)

hei Kusa-Manea. 2PL. [Form: Usually pronounced [he:] with a single mid-high vowel in isolation.]

\section{Out-comparisons:}

mia, mi Semau Helong. 2PL.

emi, imi East Tetun. 2PL. (Mo:29)

imi Kemak. 2PL.

iim Mambae, South. 2PL. (Grimes et al. 2014b:23)

miu, mi Dhao. 2PL.

muu Hawu. 2PL.

*keni Morph: *keni-k. Rote. keel. Pattern: k-2/3.

keni-k Termanu. keel of a vessel. (J:231)

keni-? Korbafo.

keni-k Bokai.

keni-? Bilbaa.

Peni Rikou. [Note: The lack of any final glottal stop in Rikou, Oenale and Dengka may be due to a typographical error. Jonker (1908:231) gives 'D., On., R. eni'.]

keni-k $B a^{\prime} a$.

keni-k Tii.

(?)eni Dengka.

(?)eni Oenale.

Out-comparisons:

kəni Hawu. (J:231)

*kendi Morph: *ma-(sa)-kendi-k. $n R M$. slippery, smooth. Pattern: k-2b. [Form: The Meto alternates without $s$ are reflexes of *ma-kendi-k.]

masakeni Termanu. slippery, slick, polished, clean, shiny, glossy. (Fox 2016b:32)

masakendi-k Tii. clean, pure. (J:710) maskeri|? Ro'is Amarasi. slippery. mas?eki|? (2) ma?eki|? Kotos Amarasi. 1) slippery. 2) fine, smooth, flat.
maPeki|? Molo. slippery, smooth. (M:296)

*kenga Morph: *kenga-k. PRM. kind of sea-weed. Pattern: k-irr. [irr. from PRM: ${ }^{*} \mathrm{k}>\varnothing / \mathrm{P} / \#$ in $\mathrm{nRote}\left(\right.$ expect ${ }^{*} \mathrm{k}$ $=k$ in all except Rikou)]

ena-k Termanu. kind of seaweed which is eaten as a vegetable. (J:114)

ena-? Korbafo. [Note: Jonker (1908:114) gives two entries for 'T.'

(Tii) but the first is surely a mistake and should be 'K.' (Korbafo).]

eja-k Bokai.

ena-? Bilbaa.

eka-? Rikou. [Form: My consultants gave vowel initial ika doo-?. The initial part could be ika-? 'fish'.] ejga-k $B a^{\prime} a$.

enga-k Tii.

(?)eyga-? Dengka.

Penga-? Oenale.

?enga-? Dela. kind of seaweed.

keka|? Ro'is Amarasi. seaweed.

Out-comparisons:

kəka Hawu. (J:114)

*kepe PMeto. tick (parasite).

kepe Ro'is Amarasi. tick.

kepe Kotos Amarasi. tick.

Out-comparisons:

kepi Central Nage. small tick or mite that infests genital hair and embeds itself in the flesh; transferred through sexual intercourse. (Forth 2016:335)

*kera1 PRM. brother-in-law. Pattern: k-1.

kela Termanu. brother-in-law (used reciprocally by men). (J:229)

kela Korbafo.

kela Bokai.

kela Bilbaa.

Pera Rikou.

kela $B a^{\prime} a$.

kera Tii.

kela Dengka.

kera Oenale. 
ken/ba?e Ro'is Amarasi. same-sex cross-cousin, person of the same gender as the speaker who is married to the speaker's opoosite sex sibling; mate, friend. [Form: This is a historic compound of *kena + ba?e with regular metathesis of the first element to *keen with subsequent reduction of the double vowel. ba?e is the normal term for 'same-sex crosscousin' in other varieties of Meto.]

Out-comparisons:

kela Semau Helong. cousin, brother-in-law.

kela Kemak. woman's brother. Usage: Kutubaba dialect. [Semantics: Elicited from a wordlist with saudara laki-laki dari perempuan 'male sibling from a woman', the semantics should be properly checked.]

kela Welaun. same-sex crosscousin.

kera Sika. brother-in-law. (Pareira and Lewis 1998:94)

hera Bima. brother-in-law. (Jonker 1893:24)

$<$ yera> Kambera. brother-in-law, wife's brother. (On:558)

$<$ wera> Weyewa.

kesa Manggarai. brother-in-law, wife's brother, sister's husband; friend, companion (of a man). (Verheijen 1967:203)

Pedza, Pedza kera Ende. man's sister's husband, wife's brother, man's male cross-cousin.

*kera2 Morph: *ma-kera. Rote. tickle. Pattern: k-1. [History: Blust and Trussel (ongoing) reconstruct a number of similar forms: *gidik, * giri, *kidi, and *kirik.]

ma-kelas (2) ma-kela Termanu.

1) ticklish. 2) have a ticklish feeling. (J:230)

ma-kela-? Korbafo. ma-kela-? Bokai.

ma-kela-? Bilbaa.

ma-eras Rikou.

ma-kelas $B a^{\prime} a$.

ma-kera-k Tii.

ma-kelas Dengka.

Out-comparisons:

kede Semau Helong. tickles.

kakedek, kede East Tetun. tickle. Usage: Samoro village. (Mo:95)

*kerumatu Morph: *kerumatu-k. Rote. leech. Etym: *kalimatək 'jungle leech Haemadipsa species'. Pattern: k-2/3. [irr. from PMP: *ə $>* \mathrm{u}$ ] [Form: Initial *keru in Rote may be related to the original *qali-/*kali- prefix, though this requires irregular sound changes, most problematically $* \mathrm{l}>*_{\mathrm{r}}$, and $* \mathrm{i}>$ $* u$ which are not otherwise attested.] [History: Osmond (2011b:414) reconstructs Proto-Polynesian *kelemutu 'earthworm, grub' which also attests a similar initial element as well as final $* \partial>u$.]

kelumatu-k Termanu. leech; usually refers to a small kind, but can also be used for an ordinary leech. (J:230)

kelumatu-k Bokai.

kelumatu-? Bilbaa.

(?)erumatu-? Rikou. [Note: not known by my consultants.]

kelumatu-k $B a^{\prime} a$.

(kelumatu-k ?) Tii.

(?)elumutu-? Dengka.

Out-comparisons:

matak East Tetun. leech. (Mo:139)

makak Ili'uun. blood-sucker. (dJ:126)

*kesu/fani PRM. sneeze. Etym: *bañən (Blust and Trussel (ongoing) reconstruct three similar forms: *bañən, *bañan and *bəñan.). Pattern: $\mathrm{k}-2 \mathrm{~b}$. [Sporadic: vowel height 
harmony $* \mathrm{e}>i / \mathrm{Cu}$ in nRote and Ro'is Amarasi] [Form: The source of initial *kesu is currently unknown.]

kisu/fani Termanu. sneeze. (J:239f)

kisu/fani Korbafo.

kisu/fani Bokai.

kisu/fani Bilbaa.

?isu/fani Rikou.

kisu/fani $B a^{\prime} a$.

kisu/fani Tii.

(?)esu/fani Dengka.

(?)esu/fani Oenale.

Pesu/fani Dela. sneeze.

n-kius/fani, n-kis/fani Ro'is Amarasi. sneeze.

n-Peus/fani Kotos Amarasi. sneeze.

n-?eus/fani Molo. sneeze. (M:105)

Out-comparisons:

hayi Semau Helong. sneeze.

fani Fehan Tetun. sneeze.

*ketembau PRM. kind of insect.

Pattern: k-2a. [irr. from PRM: *au >

$*^{\mathrm{u}}$ in Meto; vowel metathesis in Meto

$*_{\mathrm{eCu}}>*_{\mathrm{uCe}}$ (*ketembau $>$ Proto-

Meto *ketepu > ketupe)]

ketepau-? Bilbaa.

tekepau-? Landu. tick. [Sporadic: consonant metathesis $* \mathrm{kVt}>t V k]$ (own field notes)

etepau-? Rikou.

ketempau-k $B a^{\prime} a$.

ketembau-k Tii.

(?)etembau-? Dengka.

Petembau-? Oenale.

Petembau Dela.

ketupe Kotos Amarasi. kind of beetle similar to a longhorn beetle.

*ketu PRM. break off, pluck off. Etym:

*kətuq 'pick, pluck, break off' (Blust and Trussel (ongoing) also reconstruct PAN *katun 'cut, sever' on the basis of Formosan reflexes.). Pattern: k-2a.

ketu Termanu. break off, pluck off. (J:234)

ketu Korbafo.

ketu Bokai.

ketu Bilbaa.
Petu Rikou.

ketu $B a^{\prime} a$.

ketu Tii.

(?)etu Dengka.

Petu Dela. pick fruit or harvest beans, break (e.g. rope). [Note: na-ma-Petu $=$ 'become broken (rope), stop', na-Petu-? = 'break, decide'.]

n-ketu Kotos Amarasi. cut off, cut until broken.

n-ketu Molo. breaks off. (M:203)

Out-comparisons:

kotu East Tetun. break, fracture. (Mo:117)

*ketu|k PMeto. bedbug. Pattern: k-2b.

ketu? Ro'is Amarasi. bedbug.

Petu? Kotos Amarasi. bedbug. Cimicidae species.

Petuk Kotos Amarasi. bedbug. Usage: Tais Nonof sub-dialect.

$<$ etu> Molo. bedbug. (M:105)

Peto Kusa-Manea. bedbug. [Sporadic: vowel height harmony $\left.*_{\mathrm{i}}>e / \mathrm{oC} \_.\right]$

*kənda PRM. close (v.). Pattern: k-2b. [Form: *nd $>n / \partial_{-}$in Rikou, Oepao, Landu, Lole, Tii, and wRote.]

kena Termanu. close, e.g. door. (J:231)

kena Korbafo.

kena Bokai.

kena Bilbaa.

(?)ena Landu. close (door). (own field notes)

ena Rikou.

(?)ena Oepao. (own field notes)

kena $B a^{\prime} a$.

kena Lole. close. (Zacharias et al. 2014)

kena Tii.

(?)ena Dengka.

Pena Oenale.

Pena Dela. close.

na-kera $R o^{\prime}$ is Amarasi. close.

na-Peka (2) n-Peka Kotos Amarasi.

1) close (transitive). 2) closed. 
na-Peka (2) n-Peka Molo. 1) hold together. 2) close. (M:99)

*kibo Rote. edible shellfish. Etym: *qibaw (Regarding initial PMP $* \mathrm{q}$ Blust and Trussel (ongoing) state: 'Wolio hiwo is a loan from one of the languages of the Munic group (van den Berg 1991). Although such a phonologically irregular form would not normally be cited in the main comparison, in this case it serves to disambiguate the steminitial phoneme as *q.' The Bokai and Dengka forms provide evidence for initial $* \mathrm{k}$ rather than $* \mathrm{q}$. However, the irregularities in the Rote forms caution against using them as deciding witnesses for initial ${ }^{*} \mathrm{q}$ or ${ }^{*} \mathrm{k}$ in PMP. It is also possible that the initial $k$ in Bokai and Dengka is a reflex of the nominal *ka- prefix.). Pattern: k-irr. [irr. from PMP: *Ø> ${ }^{*} \mathrm{k}$ (accretion of nominal prefix *ka- ?)] [irr. from PRM: ${ }^{*} \mathrm{~b}=b$ in Rikou and Bilbaa; ${ }^{*} \mathrm{k}$ $>\varnothing$ in all nRote except Bokai (expect ${ }^{*} \mathrm{k}=k$ in all except Rikou), Dengka $k$ Oenale $P / \varnothing$ correspondence (expect both to have $k$ or $? / \varnothing)$ ]

(?)ifo(-k) Termanu. kind of shellfish. (J:200)

(?)ifo Korbafo.

kifo Bokai.

(?)ibo Bilbaa.

(?)ibo Rikou. [Note: not known by my consultants.]

(?)ifo $B a^{\prime} a$.

(?)ifo Tii.

kifo Dengka.

(?)ifo Oenale.

*kii PnRote. left side or direction.

Doublet: *diii. Etym: *ka-wiRi.

Pattern: k-1/2/3.

kii Termanu. left, left side, north. ( $\mathrm{J}: 234 \mathrm{f})$

kii Korbafo.

kii Bokai.

kii Bilbaa.

Pii Rikou. kii $B a^{\prime} a$.

kii Lole. left, north. (Zacharias et al. 2014)

kii Tii.

*kili Morph: *ki kili. Rote. tickle. Etym:

*kilik. Pattern: k-1.

ki kili Termanu. tickle. (J:237)

ki kili Korbafo.

ki -kili Bokai.

ki kili Bilbaa.

ki $\sim$ kili $B a^{\prime} a$.

ki kili Tii.

ki kili Dengka.

ki kili Oenale.

ki kili Dela. tickle.

Out-comparisons:

hakili(k), kili East Tetun. tickle. (Mo:117)

*kilu Rote. crooked, twisted. Etym: *kiluq 'bend, curve; bent, curved, crooked'. Pattern: k-1.

kilu (ka )ka?i (2) kilu ho?e-k (3) kilu ko?e-k Termanu. 1) be or become crooked and twisted, confused. 2) crooked and bent over. 3) crooked and distorted. (J:237f)

kilu ka?i Korbafo.

kilu ka?i Bokai.

(?)ilu ai Rikou. [Form: This form is given with a note ?ilu-a?i indicating a possible alternate form with a medial glottal stop.]

kilu ka?i $B a^{\prime} a$.

kilu kai Tii.

kilu kai Dengka.

kilu kai Oenale.

Out-comparisons:

bkilu Helong. crooked. (J:237)

*kima $n R M$. giant clam. Tridacna gigas.

Etym: *kima. Pattern: k-1/2a' ${ }^{*} \mathrm{k}>$ ?

in Tii, expect $* \mathrm{k}=k$ ). [Sporadic: ${ }^{*} \mathrm{k}>$ ?/\#_in Tii.]

kima Termanu. kind of shellfish, one of the smaller kinds is used as a lamp. (J:238)

kima Korbafo.

kima Bokai. 
kima Bilbaa.

Pima Rikou.

kima $B a^{\prime} a$.

(?)ima Tii. clam.

kima? Kotos Amarasi. clam.

<kima> Molo. empty shell. (M:208)

Out-comparisons:

kima East Tetun. sea-shell. (Mo:107)

*kingi Morph: *ki kingi. PRM. cockroach. Pattern: k-irr. [irr. from $P R M:{ }^{*} \mathrm{k}>h$ in all nRote except Tii; ${ }^{*}$ yg $>r$ in Ro'is Amarasi] [Form: The Ro'is Amarasi form would be regular from earlier medial **nd. Perhaps a case of dissimilation of $* \mathrm{ng}>*_{\mathrm{nd}} / \mathrm{k}_{-}$ in Meto. Compare "kari 'kidneys'. Similarly, compare *manguz 'dry fruit skin' which shows irr. *ng $>n d$ in wRote and Tii.]

hi hini Termanu. cockroach. (J:185)

hi -hini Korbafo.

hi hini Bokai.

hi hini Bilbaa.

hi hiki Rikou.

hi hingi $B a^{\prime} a$.

hi hingi Lole. cockroach. (Zacharias et al. 2014)

(?)i (?)ingi Tii.

(?)i (?)ingi Dengka.

Pi Pingi Oenale.

Pi Pingi Dela. cockroach.

kir kiri Ro'is Amarasi. cockroach.

ik iki Kotos Amarasi. cockroach.

iki Molo. cockroach. (M:159)

*kira1 Rote. hold. Pattern: k-2/3.

kila Termanu. hold, in the metaphorical sense. (J:236)

kila Korbafo.

kila Bokai.

kila Bilbaa.

Pira Rikou. responsible for household arrangements. (J:236; own field notes)

kila $B a^{\prime} a$.

kira Tii.

(?)ila Dengka.
Pira Oenale.

ma-Pira-? (2) na-sa-Pira Dela.

1) have an agreement. 2) chokes.

\section{Out-comparisons:}

kila Semau Helong. hold.

*kira2 Rote. stingy. Pattern: k-2/3' (*k > $?$ in Tii; expect $* \mathrm{k}=k$ ). [Sporadic: ${ }^{*} \mathrm{k}$ $>$ ?/\#_ in Tii.]

kila Termanu. skimp on something. (J:237)

kila Korbafo.

kila Bokai.

kila Bilbaa.

Pira Rikou.

kila $B a^{\prime} a$.

(?)ira Tii.

(?)ila Dengka.

Pira Oenale.

Pira Dela. stingy.

Out-comparisons:

kila? Semau Helong. stingy.

*kiri1 Morph: *kiri_ei-k. Rote. little bells. Etym: *giRin 'ringing sound' (Blust and Trussel (ongoing) only give Paiwan girin 'growl' and Batak girin 'bell' as evidence for their reconstruction. They note that Paiwan $* \mathrm{R}>r$ is irregular.). Pattern: $\mathrm{k}-1 / 2 / 3^{\prime}$ (Dengka ? Oenale $k$ correspondence; expect both to have either ? for pattern $2 / 3$, or $k$ for pattern 1$)$. [minority from PMP: $* \mathrm{R}=*_{\mathrm{r}}($ expect $\varnothing)$ ] [History: Blust and Trussel (ongoing) also reconstruct Proto-Philippine *kilín 'ringing of a bell'.] [Semantics: The final element may be connected with Rote ei-k 'leg, foot' with the original meaning being 'bells on ankles' (like in Malay) with later shift to 'bells on a horse's neck'.]

kili_ei-k, kila_ei-k, kil/ei-k $\bar{T}$ ermanu. little bells on the neck of a horse. (J:237)

kili_ei-kBokai.

kili_ei-? Bilbaa.

(?)iri_ei-? Rikou.

kili_ei-k $B a^{\prime} a$. 
ki kir/ei-k Tii.

(?)i (?)ilel/ei-? Dengka.

kiri_ei-? Oenale.

Out-comparisons:

girin girin Malay. bells (on anklets), bicycle bell; various plants with seeds that rattle in their shells and which can be used for green manure.

*kiri2 PRM. sharpen to have a point.

Pattern: $\mathrm{k}-2 \mathrm{~b}^{\prime} \quad\left({ }^{*} \mathrm{k}=k\right.$ in Kotos

Amarasi, expect *k $>$ ?).

kili Termanu. sharpen to have a point. ( $\mathrm{J}: 237)$

kili Korbafo.

kili Bokai.

kili Bilbaa.

kiri Tii.

(?)ili Dengka.

Piri Oenale.

Piri Dela.

n-kini Kotos Amarasi. use a knife to remove the skin of betel nut, use a knife to sharpen to a point.

n-Pini Molo. sharpen (e.g. pencil), pointing sharply. [Form: Given as $<$ anini $>$ and $<$ atini $>$ with the glottal stop deduced from the initial vowel before a single consonant in each form. If this were a vowel initial root I would expect $<$ nini $>$ and <tini>.] (M:160)

*kisa Morph: *kisa-k. CER. single, unique. Pattern: k-1/2/3.

kisa-k Termanu. single. (J:238)

kisa-? Korbafo.

kisa-k $B a^{\prime} a$.

Out-comparisons:

kisa Semau Helong. different.

*kisi PRM. peel, remove skin. Pattern: k-irr. [irr. from PRM: ${ }^{*} \mathrm{k}>\varnothing$ in Rote except Rikou (expect other nRote * $\mathrm{k}=$ $k$, wRote $* \mathrm{k}>$ ?)] [History: Blust and Trussel (ongoing) reconstruct PCMP *isi 'peel, strip off' on the basis of the Termanu form and some Oceanic forms. They also list this cognate set as noise. The Ro'is (and Helong) forms indicate an initial $* \mathrm{k}$, though this $* \mathrm{k}$ is irregularly lost in Rote.]

na-isi (2) nisi Termanu. 1) peel, e.g. onions. (J:207) 2) peel with a knife. (J:398)

na-isi (2) nisi Korbafo.

na-isi (2) nisi Bokai.

na-isi (2) nisi Bilbaa.

na-isi (2) nisi Rikou.

na-isi (2) nisi $B a^{\prime} a$.

na-isi (2) nisi Tii.

(na-)isi Dengka.

na-isi Oenale.

n-kisi Ro'is Amarasi. peel (e.g. banana).

n-?isi Kotos Amarasi. peel (e.g. banana).

$<$ isi $>$ Molo. peel, remove the skin of fruit. (M:162)

\section{Out-comparisons:}

kisi Semau Helong. nip.

*kita PRM. 1PL.INCL. Etym: *kita1.

Pattern: k-4.

ita Termanu. 1PL.INCL. (J:207)

ita Korbafo.

ita Bokai.

ita Bilbaa.

ita $L a n d u$. 1PL.INCL, we. (own field notes)

ita Rikou.

ita $B a^{\prime} a$.

ita Tii.

hita Dengka.

hita Oenale.

hiit Ro'is Amarasi. 1PL.INCL.

hiit, hiti, hita (2) =kiit, =kiti, $=$ kita Kotos Amarasi. 1) 1PL. INCL.NOM. 2) 1PL.INCL.ACC. [Form: Unmetathesised forms with final /a/ are only (optionally) used before a consonant cluster. Other unmetathesised forms are used as expected (with a discourse function), with the exception that the alternation is not fully productive.] 
hiit Molo. 1PL.INCL. (M:149)

hita, hiat Kusa-Manea. 1PL.INCL.

Out-comparisons:

kit Semau Helong. 1PL.INCL.

ita East Tetun. we, us (inclusive of the person or person spoken to). (Mo:91)

*klaha Morph: *klaha-k. PRM. glowing coals. Etym: **klaRa (pre-RM). Pattern: k-1. [irr. from PMP: *R > *h (also in *noh and *taha)] [Form: regular *h $>\varnothing / \mathrm{a} \_\mathrm{a}$ in Rote, regular *kl $>$ Rote $k$ Meto $k r / k l$.]

ha?i_kaa-k (2) pana_kaa-k Termanu. 1) glowing coal. 2) dry snot. (J:209)

ha?i kaa-?

Korbafo. 1) charcoal.

ha?i_kaa-k (2) pana_kaa-k Bokai. ai kaa-? (2) idu kaa-? Bilbaa.

ai_?aa-? (2) idu_?aa-? Rikou.

hāii_kaa-k (2) idu_kaa-k, mpinu-kaa-k $B a^{\prime} a$.

a?i_kaa-k (2) idu_kaa-k Tii.

ai_kaa-? (2) mbana_kaa-? Dengka.

ai_kaa (2) mbana_kaa-? Oenale.

kraha|? Ro'is Amarasi. burning coals.

kraha|? Kotos Amarasi. burning coals, embers; glory.

na-klaah (2) klaha|? (3) a-klaha-t Molo. 1) emits flames. 2) flames.

3 ) the flaming one. (M:214)

Out-comparisons:

klaak Fehan Tetun. red-hot coals, red (of sunburn, coals, red hair, betel-lips).

ahi klaak East Tetun. ember or live coals. (Mo:108)

klara Ili'uun. charcoal. (dJ:121)

*kleet Morph: *na-kleet. CERM. mock, tease. Pattern: k-1. [Sporadic: glottal stop insertion in Meto.] [Form: regular $* \mathrm{kl}>$ Rote $k$ Meto $k r / k l$.] na-ke kee-k Termanu. tease someone, make someone angry, or tease a child to make it cry. [Form: It is unclear if the final consonant in Termanu is a suffix or not.] (J:225) na-kre?et Kotos Amarasi. mock.

*klou PRM. bow (e.g. bow and arrow). Pattern: k-1. [Form: regular $* \mathrm{kl}>$ Rote $k$ Meto $k r / k l$.

ko kou-k (2) kou Termanu. 1) shooting of a bow and arrow, bow. 2) shoot with a bow and arrow. (J:253)

ko kou-? Korbafo.

ko kou-k Bokai.

ko kou-? Bilbaa.

?o Pou-? Rikou.

ko kou-k $B a^{\prime} a$.

ko kou-k Tii.

ko kou-? Dengka.

ko kou-? Oenale.

krau-t Kotos Amarasi. bow.

na-klau (2) a-klau-t Molo. 1) shoots with a bow. 2) bow. (M:216)

*koa?1 PRM. Friarbird. Philemon species. Pattern: k-1. [Semantics: onomatopoeia.]

koa, koa? Termanu. kind of bird, called koak or burung siang = 'midday bird' in Kupang. (J:241)

koa, koa? Korbafo.

koa, koa? Bokai.

koa, koa? Bilbaa.

koa, koa? Rikou. Friarbird. Philemon species. [Form: My consultants only gave koa?.] (J:241; own field notes)

koa, koa? $B a^{\prime} a$.

koa, koa? Tii.

koa, koa? Dengka.

koa, koa? Oenale.

koa? (2) koa ko?u (3) koa maat me?e Ro'is Amarasi. 1) kind of bird (unsure identification).

2) Friarbird. Philemon species.

3) Green Figbird. Sphecotheres viridis. 
koa? (2) koa kiko? (3) koa ko?u Kotos Amarasi. 1) Green Figbird. Sphecotheres viridis. 2) Timor Friarbird. Philemon inornatus. 3) Helmeted Friarbird. Philemon buceroides.

kool <koa> Molo. calling bird. (M:225)

Out-comparisons:

koa? Semau Helong. koak bird.

ko?ak Fehan Tetun. bird that calls in a voice like this word. (Mo:115)

kau-ko?ak East Tetun. bird that calls in a voice like this word. (Mo:104)

koak Welaun. friarbird.

*koa?2 PRM. cry out loudly. Pattern:

$\mathrm{k}-2 \mathrm{a}^{\prime}\left({ }^{*} \mathrm{k}=k\right.$ in Rikou, expect ${ }^{*} \mathrm{k}>$ $P / \varnothing)$. [Semantics: onomatopoeia.]

ko koa Termanu. crowing, of a rooster. (J:242)

ko koa Bokai.

ko koa Bilbaa.

ko ko?oa Rikou.

ko koa $B a^{\prime} a$.

ko koa Tii.

(?)0 (?)oa Dengka. [Note: On page 242, Jonker gives the Dengka form as $<\mathbf{0 o a ̈}>$ ?0 0?a. That this is probably a typographical error is shown by the entry on page 745 in which he gives $<$ oöa $>$ ?0 ?oa.] (J:242, 745)

na-Poa Dela. cry loudly, make ?oa sound.

n-koa? Kotos Amarasi. yell out, whoop, make a loud sound without words.

n-koa? Molo. calls out loudly an announcement call. (M:225)

*koba PRM. cover. Pattern: k-2a. [irr. from PRM: $*_{\mathrm{o}}>u$ in Meto; $*_{\mathrm{a}}>o$ in Rote]

kobo Termanu. hold one's hand in front of something and thereby cover it. ( $\mathrm{J}: 243)$ kobo Korbafo.

kobo Bokai.

kobo Bilbaa.

Pobo Rikou.

kobo $B a^{\prime} a$.

kobo $T i i$.

(?)obo Dengka.

Pobo Oenale.

Pobo Dela. cover.

n-kuba Kotos Amarasi. cover.

n-kuub Molo. cover. (M:243)

*kodo PRM. bird. Pattern: d-2, k-1.

kolo/ba?o-k Termanu. kind of bird called koro ba?ok in Kupang. (J:536)

kolo/bako-? Korbafo.

kolo/baPo-k Bokai.

kolo/bako-? Bilbaa.

koro/bako-? Rikou.

kolo/ba?o-k $B a^{\prime} a$.

koro/bako-k Tii.

kolo/ba?o-? Dengka.

koro/bako-? Oenale.

koro Ro'is Amarasi. bird.

koro Kotos Amarasi. bird.

kolo Molo. bird. (M:232)

koro Kusa-Manea. bird.

Out-comparisons:

koro fawa Hawu. dove.

koloy Lamaholot, Ile Mandiri. bird. Usage: Lewoingu dialect. [Note: language of east Flores ISO 639-3 [slp].] (Klamer 2015b)

kolon Alorese. bird. (Klamer 2011:61)

kolo/ndasi Palu'e. pigeon. [Note: language of central Flores ISO 639-3 [ple].] (Donohue 2003:8)

kolo Ngadha. dove. (Djawanai 1995:Part 2, 327)

olo Oirata. quail. [Note: nonAustronesian language of Kisar Island ISO 639-3 [oia].] (Nazaruddin pers. comm. May 2017) 
*kode PRM. monkey. Pattern: k-2a. [irr. from PRM: vowel metathesis in Meto $\left.*_{\mathrm{oCe}}>e \mathrm{Co}\right]$

kode Termanu. monkey. (J:243)

kode Korbafo.

kode Bokai.

kode Bilbaa.

kode $L a n d u$. monkey. (own field notes)

Pode Rikou.

kode $B a^{\prime} a$.

kode Tii.

(?)ode Dengka.

(?)ode Oenale.

Pode Dela. monkey.

kero Ro'is Amarasi. monkey.

kero Kotos Amarasi. monkey.

kelo Amanuban. monkey. (M:196)

Out-comparisons:

kode Manggarai. crab-eating macaque. Macaca fascicularis. (Verheijen 1967:224)

kode Ngadha. monkey. (Djawanai 1995:Part 2, 356)

*kodo PRM. swallow. Pattern: k-2b. [History: Lynch (2001:339) reconstructs Proto-Meso-Melanesian *kodom that may be connected. This term is connected to a larger network of phonetically similar terms.]

kodo Termanu. swallow, gulp. (J:244)

kodo Korbafo.

kodo Bokai.

kodo Bilbaa.

Podo Rikou.

kodo $B a^{\prime} a$.

kodo Tii.

(?)odo Dengka.

Podo Oenale.

Podo Dela. swallow.

n-koro Ro'is Amarasi. swallow.

n-Poro Kotos Amarasi. swallow.

n-2olo Molo. swallows. (M:404)

Out-comparisons:

holo Semau Helong. swallow.

kakorok Dadu'a. throat. (Penn 2006:13) kodo Hawu. stay stuck in the throat. (J:244)

*koko Rote. carry in hand. Pattern: initial $\mathrm{k}-2 / 3$, medial k-8/9.

ko?o Termanu. carry on one's arm or hip, also: carry and lift something with both hands or arms. (J:250)

ko?o Korbafo.

ko?o Bokai.

koko Bilbaa.

(?)o?o Rikou.

ko?o $B a^{\prime} a$.

ko?o Tii.

(?)o?o Dengka.

Po?o Oenale.

Po?o Dela. carry with hands.

Out-comparisons:

koko Semau Helong. carry in arms.

kokkon Bugis. carry in front of oneself, for example a child, like Jesus does, to show it to the crowd. (Mathes 1874:6)

*kola Morph: *kola-?. PwRM. hole. Pattern: k-1. [irr. from PRM: *a > $o$ in wRote (positing irr. ${ }^{*} \mathrm{a}>o$ can be motivated as a sporadic case of assimilation in wRote, while alternate $*_{0}>a$ in Meto would be unmotivated)] kolo-? Dengka. hole, opening, pit. (J:712)

kolo-? Oenale. hole, opening, pit. (J:712)

kona|? Ro'is Amarasi. hole.

kona|? Kotos Amarasi. hole.

kona|? Molo. hole. (M:234)

*komba $P w R M$. pour out. Pattern: k-2b. [irr. from PRM: *a $>o$ in Dela (sporadic assimilation?)]

Pombo Dela. pour, water (plants). n-kopa? Ro'is Amarasi. pour out. n-Popa? Kotos Amarasi. pour out. n-Popan Molo. pours it entirely out. (M:407)

*kona Rote. right (side), south. Etym: *kawanan 'right (side, hand, direction)'. Pattern: k-2/3. 
kona Termanu. right, right-hand side; the south, south. (J:249)

kona Korbafo.

kona Bokai.

kona Bilbaa.

Pona Rikou.

kona $B a^{\prime} a$.

kona $T i i$.

(?)ona Dengka.

Pona Oenale.

Pona Dela. right, south.

Out-comparisons:

kanan Semau Helong. right.

kwana Fehan Tetun. right (hand side).

kuana East Tetun. right, the right side; right, of the right side. (Mo:120)

kegana, gana Hawu. right (side). Usage: Dimu dialect. [Form: The Seba and Mehara dialects have kedana.]

*koo1 Morph: *koo-k. Rote. ber tree. Ziziphus mauritiana. Pattern: k-2/3' $\left({ }^{*} \mathrm{k}=k\right.$ in Rikou; expect $\left.{ }^{*} \mathrm{k}>? / \varnothing\right)$. [Form: Kupang Malay attests a final $m$ which may have originally been present in pre-RM.]

koo-k Termanu. the ber tree, called kom in Kupang; specific kinds: koo na?u, koo sina. (J:241)

koo-? Korbafo.

koo-k Bokai.

koo-? Bilbaa.

koo-? Rikou.

koo-k $B a^{\prime} a$.

koo-k Tii.

(?)oo-? Dengka.

(?)oo-? Oenale.

Out-comparisons:

koon Helong. ( $\mathrm{J}: 241)$

koo Hawu. thorny tree species.

Can grow to a few metres tall.

Parts are eaten.

kom Kupang Malay. ber tree. Ziziphus mauritiana.

*koo2 PRM. 2SG, you. Etym: *kahu. Pattern: k-4. oо Termanu. 2SG. (J:450)

koo Korbafo.

koo Bokai.

koo Bilbaa.

oo Landu. you, 2SG. (own field notes)

oo Rikou.

oo $B a^{\prime} a$.

oo Lole. (Zacharias et al. 2014)

oo Tii.

hoo Dengka.

hoo Oenale.

hoo Dela. $2 \mathrm{SG}$.

hoo Ro'is Amarasi. 2SG.

hoo (2) =koo Kotos Amarasi. 1) 2SG. NOM. 2) 2SG.ACC.

hoo Molo. you (sg.). (M:149)

hoo Kusa-Manea. you (sg.).

Out-comparisons:

ku Semau Helong. 2SG.

oo East Tetun. you (sg.). (Mo:155)

*koro Morph: *ko koro-k. PRM.

Rainbow Bee-eater. Merops ornatus.

Pattern: $\mathrm{k}-1{ }^{\prime}\left({ }^{*} \mathrm{k}>\varnothing / 2\right.$ in Meto, expect $* \mathrm{k}=k$ ). [irr. from PRM: wRote $k=$ Meto $P / \varnothing$ correspondence]

ko kolo-k Termanu. kingfisher. (J:248)

ko kolo-? Korbafo.

ko kolo-k Bokai.

ko kolo-? Bilbaa.

20 Poro-? Rikou. Rainbow Bee-eater. Merops ornatus. (J:248; own field notes)

ko kolo-k $B a^{\prime} a$.

ko koro-k Tii.

ko kolo-? Dengka.

ko koro-? Oenale.

on ono|? Ro'is Amarasi. Rainbow Bee-eater. Merops ornatus.

on ono|? Kotos Amarasi. Rainbow Bee-eater. Merops ornatus.

*koru PRM. strip leaves or grain from branch, harvest rice. Pattern: k-3.

kolu Termanu. strip off the rice plant culm with a closed hand. ( $\mathrm{J}: 248)$

kolu Korbafo.

kolu Bokai.

kolu Bilbaa. 
Poru Rikou.

kolu $B a^{\prime} a$.

koru Tii.

(?)olu Dengka.

Poru Oenale.

Poru Dela. harvest rice, harvest mung beans.

n-honu (2) n-ono Kotos Amarasi.

1) strip leaves off a branch.

2) harvest rice.

honu Molo. remove paddy rice from the grain head. (M:153)

\section{Out-comparisons:}

n-ulu Semau Helong. strip grain off stalk with hand.

*kose Rote. rub, wipe. Pattern: k-2/3.

ko kose Termanu. rub. (J:251f)

ko kose Korbafo.

ko kose Bokai.

ko kose Bilbaa.

?o ?ose Rikou.

ko -kose $B a^{\prime} a$.

ko kose $T i i$.

(?)o (?)ose Dengka.

Po Pose Oenale.

Pose Dela. rub.

\section{Out-comparisons:}

koos Semau Helong. rub, clean, wipe.

kose East Tetun. scrape, rub, wipe, polish. (Mo:117)

kose Ili'uun. whet, sharpen. (dJ:121)

kose Dhao. rub, wipe.

*koti PRM. cut. Etym: *koti 'cut off'

(own reconstruction) (PCEMP).

Pattern: k-irr. [irr. from PRM: ${ }^{*} \mathrm{k}>\varnothing$

in nRote except Rikou (expect $* \mathrm{k}=k$ )]

[Sporadic: vowel height harmony $*_{\mathrm{i}}>$ e/oC_in Meto.]

oti Termanu. chop, cut away, e.g. shrubs, brushwood. (J:459)

oti Korbafo.

oti Bokai.

oti Bilbaa.

oti Rikou.

oti $B a^{\prime} a$.

oti Tii. (?)oti Dengka.

Poti Oenale.

Poti Dela.

n-kote Ro'is Amarasi. cut.

n-Pote Kotos Amarasi. cut.

n-Pote Molo. cuts. (M:408)

Poet Kusa-Manea. cut down.

Out-comparisons:

*koti Proto-Oceanic. cut off (hair, taro tops + ). (Osmond 1998:130)

*k|teom PMeto. sea urchin. See: *tii. Etym: **tayum (pre-RM). [Form: To account for the apparently irregular correspondence between pre-RM **tayum, PMeto *k|teom and Tetun teon we could posit vowel height harmony of $* \mathrm{u}>o / \mathrm{e}_{-}$. However, this is a rare pattern of vowel height harmony.]

k|teom Ro'is Amarasi. sea urchin.

k|teom Kotos Amarasi. sea urchin.

Out-comparisons:

tiu/Poe Semau Helong. sea urchin. [Form: regular Helong midvowel raising before/after a high vowel; *ay $>*$ e $>i$ /_u.]

teo(n) East Tetun. sea urchin. Echinus esculenta. (Mo:184)

tadzuy Komodo. sea-urchin. Echinoidea. (Verheijen 1982:126)

tadzuy Manggarai. sea-urchin. (Verheijen 1982:126)

*kua Morph: *ku kua-k. Rote. nit. Pattern: k-2/3'. [irr. from PRM: *u $>o$ in Tii and wRote]

ku kua-k Termanu. very young lice. (J:254)

ku kua-? Korbafo.

ku kua-k Bokai.

ku kua-? Bilbaa.

u ua-? Rikou.

ku kua-k $B a^{\prime} a$.

ko koa-k Tii.

(?)o (?)oa-? Dengka.

Po Poa-? Oenale. 
Po Poa-? Dela.

\section{Out-comparisons:}

kuar East Tetun. nits of lice. (Mo:120)

*kue Rote. civet. Pattern: k-2/3.

kue Termanu. kind of predator, civet. (J:255)

kue Korbafo.

kue Bokai.

kue Bilbaa.

(?)ue Rikou.

kue $B a^{\prime} a$.

kue Tii.

(?)ue Dengka.

(?)ue Oenale.

*kuku PRM. finger, toe. Etym: *kuhkuh 'claw, talon, fingernail'. Pattern: initial k-3, medial k-10.

ku?u-k (2) kuku_haa-k, kuku telu-k Termanu. 1) finger, toes. Usage: Normally in combination with lima-k ('hand, arm') or ei-k ('foot, leg'). (J:259) 2) two kinds of birds with four and three toes respectively, neither has a tail and both are called bondo in Kupang. (J:256)

ku?u (2) kuku haa-?, kuku telu-? Korbafo.

ku?u-k (2) kuku_haa-k, kuku telu-k Bokai.

kuku-? (2) kuku haa-?, kuku telu-? Bilbaa. $\overline{2}$ ) quail. (J:256; own field notes)

lima kuku-? Landu. fingernails. (own field notes)

Pu?u (2) ?u?u_haa-?, ?u?u_telu-? Rikou. 2) quail. (J:256; own field notes)

ku?u-k (2) kuku haa-k, kuku telu-k $B a^{\prime} a$.

kuku-k (2) kuku_haa-k, kuku telu-k Tii.

(?)u?u Dengka.

Pu?u Oenale.

?u?u-? Dela. fingers, toes. huku Molo. catch, grab. [Form: The existence of the alternate form heke 'catch' could indicate that Molo huku 'catch, grab' is not a reflex of *kuku. However, the sound changes are regular and the semantic shift from 'finger' $>$ 'catch, grab' is a plausible semantic shift.] (M:155)

Out-comparisons:

ku?u East Tetun. pinch, wound with the thumb-nail pressed against the forefinger; to gather with a pinching action (fruit, flowers, etc.). (Mo:122)

hu?u-t Welaun. fingernail, toenail. kaba ku?u Dhao. fingernail. kolo ku?u Hawu. fingertip.

*kukur PRM. wallow. Pattern: initial $\mathrm{k}-1$, medial k-5.

kuku Termanu. wallow in dirt or sand, said of a bird. (J:255)

kuku Korbafo.

kuku Bokai.

kuku Bilbaa.

Pu Pu?u Rikou.

kuku $B a^{\prime} a$.

kuku Tii.

kuku Dengka.

kukur Oenale.

n-kuku Kotos Amarasi. have diarrhoea without making it to the bathroom in time, wallow in the mud (e.g. of a pig or buffalo).

n-kuku Molo. wallows (also said of buffalo). (M:245)

*kumu1 PMeto. wild pigeon, wild dove.

Etym: **kumu (pre-RM). Pattern:

k-1/2a. [Semantics: onomatopoeia.]

kumu Ro'is Amarasi. all kinds of wild pigeons and wild doves.

kumu Kotos Amarasi. all kinds of wild pigeons and wild doves.

umu Amanuban. kind of dove. (M:248, 588) 
kumu (2) < umu hene> Molo. dove. (M:248) 1) kind of dove. 2) kind of dove. (M:588)

a?umab Kusa-Manea. pigeon.

Out-comparisons: manu_kumu, kumo Waima'a. wild pigeon.

*g(a)umu Proto-Oceanic. Fruit Dove. Ptilinopus species. (Clark 2011:15)

*kumu2 Morph: *ku kumu. Rote. make a fist. Doublet: *kame, *ke?e, *keme, *ygumu. Etym: *gəmgəm 'fist; hold in the fist'. Pattern: k-2/3. [irr. from $P M P: *^{\circ}>*_{\mathrm{u}}$ (sporadic assimilation to previous velar consonant and/or following labial consonant)] [History: The Tii and Oenale forms additionally mean 'knead' (Jonker 1908:714).]

ku kumu Termanu. make a fist. (J:257f)

ku kumu Korbafo.

ku kumu Bokai.

ku kumu Bilbaa.

(?)u (?)umu Rikou. [Form: My consultants gave ku kumu and kumu.]

ku kumu $B a^{\prime} a$.

ku kumu Tii. (J:704)

(?)u〜(?)umu Dengka.

Pu Pumu Oenale.

?u Pumu Dela. clench one's fist.

Out-comparisons:

kumu Helong. (J:258)

kumu East Tetun. hold in the hand. (Mo:121)

akam Kisar. squeeze, press, flatten.

*kuna $P w R M$. hide in folds of clothing.

Pattern: k-1. [Form: The Meto unmetathesised form has not yet been attested. It could be *kuna or *kunu.] ku kuna Dengka. his belly has layers or folds due to its fat. (J:714)

ku kuna Oenale. his belly has layers or folds due to its fat. (J:714) na-kuun Meto. hides something in the folds of clothing. (J:714)

*kuni Morph: *kuni-k. PRM. turmeric. Etym: *kunij. Pattern: k-4' $\left({ }^{*} \mathrm{k}=k\right.$ in Termanu, Landu, Ba'a, and Lole; expect $* \mathrm{k}>\varnothing)$.

kuni-k Termanu. turmeric. (J:258)

kuni-? Korbafo.

kuni-k Bokai.

kuni-? Bilbaa.

kuni Landu. yellow. (own field notes) uni-? Rikou.

kuni-k $B a^{\prime} a$.

kuni-k Lole. turmeric, yellow. (Zacharias et al. 2014)

uni-k Tii.

huni-? Dengka.

huni-? Oenale.

hưini|k Ro'is Amarasi. turmeric.

huni|k Kotos Amarasi. turmeric.

$<$ huki $>$ Molo. turmeric, dye in tissues. [Form: The Molo form could be related with sporadic metathesis of $*_{\mathrm{nVk}}>* *$ hukin and loss of final $n$. Jonker (1908:258) gives Meto hukim which would additionally show irr. *n $>m$.] (M:155)

\section{Out-comparisons:}

kinur Fehan Tetun. turmeric.

kinur (2) kinu East Tetun. 1) plant whose tubers are reduced to powder with the colour and taste of saffron. 2) yellow (colour). [irr. from PMP: vowel metathesis: ${ }^{*} \mathrm{uCi}$ $>i C u]$ (Mo:107)

hinu East Tetun. tree from which turmeric is made. Usage: Samoro village. [irr. from PMP: vowel metathesis: ${ }^{*} \mathrm{uCi}$ $>i C u]$ (Mo:86)

*kunu1 PMeto. breadfruit. Etym: *kuluR. Pattern: k-1/2a. [irr. from PRM: Ø> *m in Ro'is Amarasi]

kunum Ro'is Amarasi. breadfruit. 
<kunu> Molo. Cluster fig tree. Ficus glomerata. [Note: possibly unrelated.] (M:251)

\section{Out-comparisons:}

kulu? (2) kulu naunu? Funai Helong. 1) jackfruit.

2) breadfruit.

kulu East Tetun. fruiting trees in various types of Artocarpus genus. (Mo:121)

*kunu2 Rote. kind of plant. Pattern: k-irr. [irr. from PRM: * $\mathrm{k}>\varnothing / \#$ in wRote (expect $k$ or $?$ )]

kunu Termanu. used in a few plant names, as in: kunu doo, (unu loo); kunu meo-?. [Semantics: No other semantic details are given for this term.] (J:258)

kunu Korbafo.

kunu Bokai.

kunu Bilbaa.

kunu $B a^{\prime} a$.

kunu Tii.

unu Dengka.

unu Oenale.

unu hau ana? Dela. weed.

Out-comparisons:

kunu Helong. (J:258)

*kupu PMeto. fog. Pattern: k-1/2a. [History: Jonker (1908:230) compares the Meto forms with Termanu, Korbafo, Bokai kelupua 'become murky of water', Ba'a kelempua, and Dengka, Oenale elembua, but the sound correspondences and semantics are too much of a stretch for me.]

kupu Ro'is Amarasi. fog. [Form: Jonker (1908:230) gives Meto meukupa/kupa.]

kupu (2) pupu Kotos Amarasi.

1) water vapour, like in fog. 2) fog.

[Form: The second form possibly has sporadic assimilation of $* \mathrm{k}>$ $p$. Kotos Amarasi also has nipu 'fog'.]

Out-comparisons:

kuput Funai Helong. fog. api kupu Tokodede. smoke.

(Klamer 2002)

*kura Morph: *kura-k. Rote. scorpion. Etym: **sakuray (pre-RM). Pattern: $\mathrm{k}-2 / 3^{\prime}$. [History: possibly connected with PMP *quday 'shrimp, crayfish, lobster'.]

kula-k Termanu. scorpion. (J:256)

kula-? Korbafo.

kula-k Bokai.

kula-? Bilbaa.

kura-? Landu. scorpion. (own field notes)

ura-? Rikou.

kula-k $B a^{\prime} a$.

kura-k Tii.

(?)ula-? Dengka.

Pura-? Oenale.

Pura-? Dela. scorpion.

Out-comparisons:

khulan Funai Helong. scorpion.

hkulay Semau Helong. scorpion.

sakunar East Tetun. scorpion (poisonous insect). [Sporadic: consonant metathesis *rVn > $n V r$.] (Mo:165)

sa?unar Galolen. scorpion. [Sporadic: consonant metathesis *rVn $>n V r$.]

sa?uro Waima'a. scorpion. sayorne Kisar. scorpion. kəru Hawu. (J:256)

kuran Sika. lobster. (Calon 1891:318)

*kurə Morph: *ka-kurə-k. PRM. clay/ stone jar or pot. Etym: *kudən 'clay cooking pot'. Pattern: k-4.

ule-k Termanu. pot both for cooking and storing things. (J:665)

ule-? Korbafo.

ule-k Bokai.

kule-? Bilbaa.

ure-? Rikou. [Form: Whether this is vowel initial or glottal stop initial is currently unknown, but no Rikou form that fits into the first pattern for $*_{\mathrm{k}}$ (pattern 1) has an initial underlying glottal stop.] 
ule-k $B a^{\prime} a$.

ure-k Tii.

hula-? Dengka.

hura-? Oenale.

?|huna|? Kotos Amarasi. large clay or stone jar or jug.

<huun> oe Molo. clay pot which is filled with water as opposed to nai? oe 'iron pot' in which water is boiled. (M:156)

*kuru1 PRM. call chickens, summon chickens. Etym: *kur(u) 'word used to call chickens, etc.'. Pattern: k-1. [irr. from PRM: $* \mathrm{u}>o$ in all Rote except Korbafo and Tii (assimilation to following *roroo)] [Form: All varieties with lowering of $* \mathrm{u}>o$ attest a second element derivable from *roroo except for Dengka which would require irr. ${ }^{*} \mathrm{r}>d$.] [Semantics: onomatopoeia.]

kolo/loloo Termanu. call chickens, summon chickens. (J:248)

kulu kulu Korbafo.

kolo/loloo Bokai.

koro/ruruu Rikou.

kolo/loloo $B a^{\prime} a$.

kuru kuru, ku $\sim$ kuru Tii.

kolo/dodoo, ko kolo Dengka. koro/roroo Oenale.

*kuru2 Rote. wrinkly. Etym: *kurut 'curly-haired' (Blust and Trussel (ongoing) also reconstruct *kulut 'curly haired' and PWMP *korut 'shrivelled, wrinkled'.). Pattern: $\mathrm{k}-1 / 2 / 3$. [irr. from PRM: ${ }^{*} \mathrm{r}>*^{*} \mathrm{nd}$ in Tii and Ba'a] [Form: Tii and Ba'a attest *kundu, others attest *kuru, the Rikou alternate form with medial $l$ is irregular from both these forms.] [History: Blust and Trussel (ongoing) reconstruct PWMP *kərut 'shrivelled, wrinkled' on the basis of Malay kərut and Pamona koru. They give an apparent doublet *kədut, for which I cannot find a main entry. Blust and Trussel (ongoing) assign the Termanu form to PMP *kuluy 'curl, curve' where it is one of three languages attesting this reconstruction, but this reconstruction cannot account for the reflexes in other varieties of Rote.]

ku kulu (2) ku $\sim$ kulu-k Termanu.

1) be or become wrinkly.

2) wrinkle. (J:258)

ku kulu Korbafo.

ku kulu Bokai.

ku kulu Bilbaa.

(?)u (?)uru, (?)u ( (?)ulu Rikou.

ku kunu $B a^{\prime} a$.

ku kundu Tii.

ku kulu Dengka.

ku kuru Oenale.

Out-comparisons:

kurut East Tetun. wrinkled, rough, curled. (Mo:122)

*kurus PRM. chilli. Pattern: k-2b' (*k= $k$ in Kusa-Manea; expect $\left.{ }^{*} \mathrm{k}>? / \varnothing\right)$.

kulus Termanu. chilli pepper. (J:257)

kulu? Korbafo.

kulu? Bokai.

kulu? Bilbaa.

Purus Rikou.

kulus $B a^{\prime} a$.

kurus Tii.

kunus Ro'is Amarasi. chilli.

Punus Kotos Amarasi. chilli.

kunus Kusa-Manea. chilli.

Out-comparisons:

kulus Semau Helong. chilli.

${ }^{\circ}$ kunus Foho Tetun. chilli plant. Borrowed from: Meto (shown by irr. ${ }^{*} \mathrm{r}=n$ correspondence). [Note: variety of Tetun spoken in the northern part of the Tetun-speaking area of central Timor ISO 693-3 [tet].] (van Klinken 1995)

${ }^{\circ}$ kunus East Tetun. capsicum. Capsicum annuum. Borrowed from: Meto (shown by irr. *r = $n$ correspondence). (Mo:121)

kurus Kupang Malay. chilli. 
uus Welaun. chilli. [irr. from PRM: ${ }^{*} \mathrm{r}=\varnothing$ correspondence] (da Silva 2012:186)

koro Sika. chilli. (Pareira and Lewis 1998:101)

koro Ende. chilli.

ygurus Manggarai. (Verheijen 1984:14)

mburus Waerana. [Note: language of central Flores ISO 639-3 [wrx].] (Verheijen 1984:14)

*kuta PnRote. close eyes. Pattern: $\mathrm{k}-1 / 2 / 3$.

na-kuta Termanu. close the eyes out of fright, for example like someone who is afraid of being hit. ( $\mathrm{J}: 256)$

na-kuta Korbafo.

na-kuta Bokai.

(na-kuta ?) Tii.

Out-comparisons:

huta Semau Helong. close eyes. [irr. from PRM: ${ }^{*} \mathrm{k}=h$ correspondence]

*kutə Morph: *kutə-k. Rote. brains, mantle. Doublet: *kuta. Etym: *hutək 'brain, marrow'. Pattern: k-2b' $\left({ }^{*} \mathrm{k}\right.$ $>?$ in Tii, expect $* \mathrm{k}=k$ ). [irr. from PMP: $\varnothing>* \mathrm{k}$ ] [irr. from PRM: *ə > $a$ in Termanu Korbafo, and Bilbaa] [Form: The initial $* \mathrm{k}$ may be from the nominal prefix *ka- prefix.] [History: It is possible that PMP *hutək spit into a doublet in PRM: *kuta-k 'mantle' and *(k)utə-k 'brains'.]

kuta-k Termanu. the mantle of a squid. (J:258)

kuta-? Korbafo. the mantle of a squid. (J:258)

kuta-? Bilbaa. the mantle of a squid. ( $\mathrm{J}: 258)$

(?)ute-k Lole. brains. (Zacharias et al. 2014)

(?)ute-k Tii. brains. (J:777)

(?)uta-? Dengka. brains. (J:777)

Puta-? Oenale. brains. (J:777)

Puta-? Dela. brain.

\section{Out-comparisons:}

kuta Semau Helong. brain.

ka kutak East Tetun. brains, the mind, the thinking part. (Mo:98)

gutan (2) ai gutan Ili'uun. 1) brains. (dJ:115)

2) heartwood. [irr. from PMP: $* \mathrm{~h}>(* \varnothing)>g$ (compare *paydan > ketfan, *pusoj > kusan, and baqbaq > kahan)] (dJ:111)

*kutu PRM. head-louse. Etym: *kutu. Pattern: k-4.

utu Termanu. louse. (J:672)

utu Korbafo.

utu Bokai.

utu Bilbaa.

utu Landu. louse. (own field notes)

utu Rikou.

utu $B a^{\prime} a$.

utu Tii.

hutu Dengka.

hutu Oenale.

hutu Dela. lice.

hutu Ro'is Amarasi. head-louse.

hutu Kotos Amarasi. head-louse.

hutu Molo. head-louse. (M:157)

hutu Kusa-Manea. head-louse.

\section{Out-comparisons:}

kutu Semau Helong. louse.

utu East Tetun. louse. (Mo:194)

uku Kisar. fleas.

*kutus PRM. windstorm, cyclone, whirlwind. Pattern: k-irr. [irr. from PRM: ${ }^{*} \mathrm{k}>n$ in Meto (fossilised prefix?)] [Sporadic: diphthongisation $* \mathrm{u}>a u$ in most Meto.] [Form: One way to account for the initial nasal in Meto would be to reconstruct PRM *n, but this would require unexpected $*_{\eta}>$ $k$ in the Rote languages (expect $* y>n$ ) and unexpected ${ }^{*} y>n$ in Kusa-Manea $($ expect $* \eta>k)$.]

kutus Termanu. whirlwind, tornado. (J:259)

kutu-? Korbafo. 
kutu-? Bokai.

kutu-? Bilbaa.

utus Rikou.

kutus $B a^{\prime} a$.

kutus Tii.

(?)utus Dengka.

(?)utus Oenale.

nautus Ro'is Amarasi. whirlwind, tornado.

ain nautus (2) nautus, autus Kotos Amarasi. 1) storm with strong winds, windstorm, cyclone, whirlwind, tornado. 2) beetle.

ani nautus Molo. storm with strong winds. (M:357)

nutus Kusa-Manea. whirlwind, tornado.

*kuu Rote. blow the nose. See: *yguu. Pattern: k-1. [Semantics: onomatopoeia.]

na-sa-kuu (2) kuu kuu Termanu. blow hard; na-sa-kuu pana-na he blows his nose hard, e.g. to get snot out. $\operatorname{anin}=\mathbf{a}$ na-sa-kuu ko?as $=\mathbf{a}$ the strong wind blows the clouds away. (J:254) 2) blow through the nose somewhat gently. (J:253)

na-sa-kuu Korbafo.

na-sa-kuu Bokai.

na-sa-uu Rikou.

na-sa-kuu $B a^{\prime} a$. na-sa-kuu Tii.

na-sa-kuu Dengka.

*kuun PRM. kind of tall grass. Pattern: $\mathrm{k}-4{ }^{\prime}\left({ }^{*} \mathrm{k}=k\right.$ in Termanu, Ba'a, and Tii; expect $* \mathrm{k}>\varnothing)$.

ku/mea Termanu. kind of long grass, called rumput kumee in Kupang. [Form: The source of the second element in Rote is unknown. It could be related to *mea 'red'.] (J:257)

ku/mea Korbafo.

ku/mea Bokai.

ku/mee Bilbaa.

u/mee Rikou. [Form: Whether this is vowel initial or glottal stop initial is currently unknown, but no Rikou form which fits into this pattern for *k has an initial underlying glottal stop.]

ku/mea $B a^{\prime} a$.

ku/mea Tii.

hu/mee Dengka.

hu/mee Oenale.

huu_musu? Ro'is Amarasi. sword grass, alang-alang grass.

huun (2) huu_musu? Kotos Amarasi. 1) grass. 2) sword grass, alang-alang grass.

huun Molo. grass. (M:156)

\section{? - ?}

*?|fenu PMeto. candlenut. Aleurites moluccana. Etym: **ka-felu (preMeto).

fenu Ro'is Amarasi. candlenut. ?|fenu Kotos Amarasi. candlenut. ?|fenu Molo. kind of tree. Aleurites moluccana. (M:116)

fenu Kusa-Manea. candlenut.

\section{Out-comparisons:}

$<$ kawilu> Kambera. candlenut tree. Aleurites moluccana. (On:201) welu Manggarai. candlenut. (Verheijen 1984:45)

felu Rongga. candlenut. [Note: language of central Flores ISO 639-3 [ror].] (Verheijen 1984:45)

felu Ngadha. candlenut. (Verheijen 1984:45)

*?|mauka| $\mathbf{P}$ PnMeto. cuscus. See: *arum. Etym: *mansər (PCEMP). [irr. from PMP: *a $>*_{\mathrm{au}}$ ] [irr. from PRM: *a $>$ $a \sim u$ ] [Form: The best reconstruction 
of the vowels is unclear, whatever form is posited requires positing irregularities. The Ketun (Nai'oni') form appears to attest penultimate ${ }^{*}$, but if this is reconstructed it requires positing otherwise unattested $* \mathrm{a}>a u$ in all other lects. I thus posit that Ketun (Nai'oni') ?maku? attests irregular reduction of the earlier diphthong. The reconstructed diphthong *au could come from earlier $* u$ (i.e. earlier **muka) given that diphthongisation of penultimate vowels sporadically occurs in Meto. Reconstruction of final $*$ a can account for reflexes with final $u$ by assuming irregular assimilation to previous $u$ in closed syllables. Reconstruction of final $*_{\mathrm{u}}$ does not seem to be able to account for reflexes with final *a.] [History: This reconstruction is possibly consistent with PCEMP *mansər given PRM *nd $>$ PMeto ${ }^{*} \mathrm{r}>$ Proto-Nuclear Meto $k$. (Though note that Proto-Nuclear Meto *k can also be a reflex of PRM *yg or *k.) However, if the hypothesis that penultimate ${ }^{*} \mathrm{au}$ is from earlier ${ }^{*} \mathrm{u}$ is correct, then comparison of *mansər with *?|mauka| $\mathbf{P}$ would require irregular initial $* \mathrm{a}>* \mathrm{u}$.]

Pmauku? Kotos Amarasi. cuscus. Usage: Not known by all speakers. The usual word is ukum.

mauku? Amanuban, South. cuscus. Usage: Oebelo village, other known varieties of South Amanuban have ukum.

Pmaku? Ketun. cuscus. Usage: Nai'oni' village.

Pmauka? Ketun. cuscus. Usage: Taloetan village.

Pmauka? Kopas. cuscus. Usage: Masikolen, Bone, Usapisonba'i, and Oben villages.

mauka? Kopas. cuscus. Usage: Oepaha village.
Pmauku? Kopas. cuscus. Usage: Tuale'u village.

mauku?, mauka? Fatule'u. cuscus.

<mauku> Molo. cuscus.

Phalangeridae. (M:316,586)

?mauku? Timaus. cuscus.

Out-comparisons:

${ }^{\circ}$ mauka Funai Helong. cuscus. Borrowed from: Meto.

meda East Tetun. small savage marsupial animal. [irr. from PMP: *a $>$ e] (Mo:140)

k|meda Fehan Tetun. small savage marsupial animal. [irr. from PMP: *a > e] (Mo:112)

matfa Ili'uun. cuscus. (dJ:127)

madar Galolen. cuscus. (Schapper 2011:264)

maat Mambae, South. cuscus. (Schapper 2011:264)

la/mara Welaun. cuscus.

mada Kemak. cuscus. Usage: Kailaku, Lemia, Atsabe, and Saneri dialects.

${ }^{\circ}$ meda Kemak. cuscus. Usage: Leolima, Leosibe, and Diirbati dialects. Borrowed from: probably Tetun (shown by irr. $* \mathrm{a}=e$ correspondence).

*?uta $P w R M$. cut.

(?)u (?)uta Dengka. cutting, usually cutting meat. (J:777)

Pu Puta Oenale. cutting, usually cutting meat. (J:777)

Puta Dela. cut.

<naút> Meto. [Form: This is presumably na-?uut, the metathesised form of na-?uta.] (J:777)

Out-comparisons:

$\mathbf{k}^{\mathbf{2}} \mathbf{u t a}$ Waima'a. cut, slice meat into small mouth-sized pieces. 
*laa1 Morph: *laa-k. PRM. kind of tree. [Semantics: vague semantics.]

ka/laa-k Termanu. kind of tree called lalaak in Kupang. ( $\mathrm{J}: 218)$

ka/laa-? Korbafo. [Note: Bilbaa and Korbafo given as $<$ kalạ $>$ with only a single final vowel (rather than expected $<$ kalạ́ $>$ ), but this is surely a typo.]

ka/laa-k Bokai.

ka/laa-? Bilbaa.

la laa-? Rikou.

ka/laa-k $B a^{\prime} a$.

la laa-k Tii.

la laa-? Dengka.

la laa-? Oenale.

$<$ naa $>$, <apnaa $>$ Molo. kind of tree, a forest giant. Planchonia valida, Barringtonia spicata. [Form: nao is given as an alternate form of Planchonia valida in Middelkoop (1972:349).] (M:338, 26)

*laa2 PRM. temporary hut. [irr. from PRM: *1 $>r / l$ in Meto] [Sporadic: $* \mathrm{VV}-\mathrm{k}>* \mathrm{VV}$ ? $>\mathrm{V}$ ?V in Meto (perceptual metathesis).]

laa-k Termanu. temporary storage hut. (J:261)

laa-? Korbafo.

la laa-k Bokai.

laa-? Bilbaa.

laa-? Rikou.

la laa-k $B a^{\prime} a$.

laa-k Tii.

la laa-? Dengka.

laa-? Oenale.

ra?a-t (2) n-ra?an Kotos Amarasi.

1) temporary shelter; tent, hut.

2) reside somewhere temporarily.

la?a-t Molo. leaf-hut. (M:254)

Out-comparisons:

laen Fehan Tetun. hut in the fields, or at the beach, where the farmer/ fisherman can rest. [irr. from PRM: *a $=e$ correspondence] klaen East Tetun. hut on raised legs for guarding crops. Usage: Luka village. [irr. from PRM: *a $=e$ correspondence] (Mo:108)

salaak East Tetun. small hut. (Mo:108)

*laaz Rote. sail. Etym: *layaR.

laa Termanu. sail. (J:260)

laa Korbafo.

laa Bokai.

laa Bilbaa.

laa Rikou.

laa $B a^{\prime} a$.

laa Tii.

laa Dengka.

laa Oenale.

Out-comparisons:

laa Semau Helong. sail.

laan East Tetun. sails (of a boat). (Mo:98)

walara Kisar. sail.

*laa4 PnRote. fly. Etym: *layap.

laa Termanu. flying. (J:260)

laa Korbafo.

laa Bokai.

laa Bilbaa.

laa Rikou.

laa $B a^{\prime} a$.

laa Tii. [Note: given as 'also surely $\mathrm{T}$.]

*laa5 Morph: *na-laa-k, *laa-k. Rote. open the eyes. Etym: *-lat 'open the eyes wide'.

na-laa-k mata=na (2) mata

laa-k Termanu. 1) open the eyes.

2) someone who can see well (lit. someone whose eyes are open). (J:261)

na-laa-? Korbafo.

na-laa-k Bokai.

na-laa-? Rikou.

na-laa-k $B a^{\prime} a$.

na-laa-k $T i i$. 
na-laa-? Dengka.

na-laa-? Oenale.

* laba $n R M$. wind around, of plants. [irr. from PRM: $* 1>r / l$ in Meto]

na-ka-(la $)$ laba Termanu. 1) wind all the way around something, said of plants. 2) wind around, entwine, tie around. (J:262)

na-ka-laba Korbafo.

na-ka-laba Bilbaa.

na-la laba (2) na-laba Rikou.

na-ka-laba $B a^{\prime} a$.

na-ka-laba Tii.

k|raba-t Kotos Amarasi. obstacles, thorns. Usage: poetic, only in the parallel pair kisan ma krabat (kisan $=$ 'thorny weed').

n-laba (2) okan aklab laba (3) n-buun ma na-klaab Molo.

1) creeping or winding of plants. (M:254) 2) creeping cucumber. (M:254) 3) he surrounds and encloses, referring to the protective power of the chief. Usage: poetic. (M:91)

\section{Out-comparisons:}

raba $H a w u$. wind around with thorns. [Sporadic: ${ }^{*} 1=r$ correspondence.] (J:262)

*ladu PRM. palm wine. Pattern: d-2. [Sporadic: consonant metathesis **1 $\mathrm{Vr}$ $>r V l$ in Dela-Oenale and Rikou.] [Form: That it is Dela-Oenale and Rikou that show consonant metathesis rather than Tii, is indicated by other reconstructions with * $\mathrm{IVr}$ which have cognates outside of the RM languages including: *lari 'comb of rooster' and *ledo 'sun' (PMP *qaləjaw) which have undergone the same consonant metathesis * $\mathrm{IVr}>r V l$ in Dela-Oenale and Rikou.]

lalu Termanu. areng palm sap, fermented palm sap. (J:274)

lalu Korbafo.

lalu Bokai.

lalu Bilbaa. ralu Rikou.

lalu $B a^{\prime} a$.

laru Tii.

lalu Dengka.

ralu Oenale.

ralu Dela. palm-wine.

raru Kotos Amarasi. palm-wine.

lalu Molo. lontar sap in which roots which promote fermentation have been placed. (M:260)

*lafa PRM. kind of traditional cloth.

[Sporadic: $* \mathrm{a}>e / \#$ in wRM.]

lafa Termanu. well woven piece of a sarong which is tied by men around the hips and also worn as a kind of coat. (J:267)

lafa Korbafo.

lafa Bokai.

lafa Bilbaa.

lafa Rikou.

lafa $B a^{\prime} a$.

lafa Tii.

lafe Dengka.

lafe Oenale.

lafe Dela. traditional cloth worn by men.

nafe Kotos Amarasi. kind of cloth worn traditionally as a belt.

Out-comparisons:

lahan East Tetun. thread, fibre, or filament. (Mo:123)

*lafo Morph: *ka-lafo. PRM. rat, mouse. Etym: *balabaw. [irr. from PRM: initial $i \sim b i$ in Molo]

lafo Termanu. rat, mouse. (J:267f)

lafo Korbafo.

lafo Bokai.

lafo Bilbaa.

lafo Landu. mouse, rat. (own field notes)

lafo Rikou.

lafo $B a^{\prime} a$.

lafo Tii.

lafo Dengka.

lafo Oenale.

k|nafo $R o^{\prime}$ is Amarasi. mouse, rat.

k|nafo Kotos Amarasi. mouse, rat.

nafo Amanuban/Amanatun. 
ifo, bifo Molo. mouse. (M:158)

nafo Kusa-Manea. mouse, rat.

Out-comparisons:

blaho Semau Helong. mouse, rat.

laho East Tetun. rat (rodent). (Mo:123)

*laha Morph: *na-ma-laha, *laha-s. CERM. hungry. Etym: *lapaR. [Form: This is the only form in which *h /a_a is not universally lost in Rote. Instead, it follows the pattern for $* \mathrm{~h}$ between other vowels.]

na-ma-la?a (2) la?as Termanu. 1) hungry. 2) hunger, famine. (J:262)

na-ma-la?a (2) la?a-? Korbafo.

na-ma-la?a (2) la?a-? Bokai.

na-ma-laa (2) laa-? Bilbaa.

na-ma-laa $L a n d u$. hungry. (own field notes)

na-ma-laa (2) laas Rikou.

na-ma-laa Oepao. hungry. (own field notes)

na-m|naha Ro'is Amarasi. hungry.

na-m|naha Kotos Amarasi. hungry.

na-m|naha Molo. hunger. (M:325)

na-m|naah Kusa-Manea. hungry. [Form: metathesised form of na-mnaha.]

Out-comparisons:

hala?a Fehan Tetun. hungry. Usage: Betun village.

hamlaha East Tetun. be hungry. (Mo:71)

*lai Rote. run, flee; fast, quick. Etym:

*laRiw 'run, run away, flee, escape'.

na-lai (2) lai lai Termanu. 1) flee, run away. (J:270) 2) fast, quickly, speedy. (J:268)

na-lai (2) lai lai Korbafo.

na-lai (2) lai lai Bokai.

na-lai (2) lai lai Bilbaa.

na-lai (2) lai lai Rikou.

na-lai (2) lai lai $B a^{\prime} a$.

na-lai (2) lai lai Tii.

(2) lai lai Dengka.

(2) lai lai Oenale.

\section{Out-comparisons:}

lali Semau Helong. run.

lai lais East Tetun. quickly, hastily, without delay. (Mo:123)

*laia PRM. ginger. Etym: *laqia. [Sporadic: *a $>e$ /_\# in wRM (PwRM *laie).] [Form: The Rote languages have reduced this to a disyllable by deletion of the antepenultimate vowel. In Meto both initial vowels were retained with subsequent doubling of the final vowel, probably to achieve a form composed of two disyllabic feet. The Meto reflexes additionally show expected consonant insertion to break up a disallowed sequence of three vowels.]

lia Termanu. ginger. (J:310)

lia Korbafo.

lia Bokai.

lia Bilbaa.

lia Rikou.

lia $B a^{\prime} a$.

lia Tii.

lie Dengka.

naidzee Ro'is Amarasi. ginger. [History: Expected epenthetic consonant in *VVV.]

naidzeer Kotos Amarasi. ginger. [History: Final $r$ indicates that this is a borrowing from a variety of Meto with word final insertion of $l$ after Ve\#.]

naiyee? Amanuban. ginger.

naidgee-l Molo. ginger. (M:342)

Out-comparisons:

'lai/ee Semau Helong. ginger. Borrowed from: Meto before $* 1>n$ and medial consonant insertion (shown by final $*_{\mathrm{a}}$ $=e$ correspondence). [Form: Given as lai ee with white space, probably indicating that this has been reanalysed as some kind of compound.]

ai lia East Tetun. ginger. Zingibii officinale. (Mo:2) 
*lako PRM. go. Etym: *lakaw. Pattern: k-8.

la?o Termanu. go = break up, leave, go away (to land or over sea). (J:279f)

la?o Korbafo.

la?o Bokai.

lako Bilbaa.

la?o Rikou.

la?o $B a^{\prime} a$.

la?o Tii.

lao Dengka.

lao Oenale.

n-nao Ro'is Amarasi. go.

n-nao Kotos Amarasi. go.

n-nao Molo. go. (M:355)

nao Kusa-Manea. go.

Out-comparisons:

lako Semau Helong. go.

la?o East Tetun. walk, travel on foot, go. (Mo:127)

la $H a w u$. go, to, IRREALIS.

*la?i $P w R M$. male, grandfather, king. Etym: *laki 'male, masculine; man'.

manu_lai Dengka. rooster, cock. (J:716)

manu_lai Oenale. rooster, cock. (J:716)

nai?i|k Ro'is Amarasi. grandfather.

na?i-f (2) nai? (3) maun_nai (4) fe/nai (5) nai mnuki? Kotos Amarasi. 1) grandfather. [Form: The medial glottal stop is not deleted in Meto after suffixation, which shows that this glottal stop does not ultimately derive from the *-k suffix and is instead a retention of PMP *k.] 2) Mr. (used obligatorily before male names). [Form: metathesised form.] 3) rooster, cock. 4) wife of a dignitary. 5) royal son.

$<$ nai $>$ (2) <nai uuf $>$, <naidzuuf $>$

(3) <manu naif $>$ Molo. 1) prince. (M:342) 2) princes, greats of the land. (M:342) 3) rooster, cock. (M:230)

na?i Kusa-Manea. grandfather.

\section{Out-comparisons:}

lahi Semau Helong. king. [irr. from PMP: * $\mathrm{k}>$ h] [History: The irregular sound changes combined with the semantics indicate that this is a loan, but a precise donor cannot be confidently identified. Meto seems unlikely as this requires positing that the medial glottal stop went through intermediate *h that would be an irregular sound change for medial $* \mathrm{k}$ in Meto.]

${ }^{\circ}$ na?i (2) ${ }^{\circ}$ na?in Fehan Tetun.

1) an honourable term of address for nobles, or for people much younger than the speaker (e.g. one's children). 2) noble, owner, boss, player (in a game); Can be used as title. Borrowed from: Meto (shown by irr. ${ }^{*} 1=$ $n$ correspondence).

${ }^{\circ}$ naPi (2) ${ }^{\circ}$ na?in East Tetun. 1) form of address always preceding the word it qualifies, not translatable to English, but can be used instead of 'Mr', 'Your Honour', 'Your Excellency'. 2) respectful title with the same meaning as na?i placed before numerals and some pronouns when referring to people. (Mo:145)

*la?us Morph: *ka-la?us. PRM. cactus.

la?us Termanu. cactus. (J:286)

la?u-? Korbafo.

lau-? Bokai.

lau-? Bilbaa.

laus Rikou.

la?us $B a^{\prime} a$.

la?us Tii.

laus Dengka.

laus Oenale.

?|naus Molo. cactus leaves. (M:357)

Out-comparisons:

klaus Helong. cactus. (J:286) 
k(a)latun East Tetun. cactus. (Mo:98)

oplakun Kisar. pear cactus.

*lalu Morph: *lalu-k. PnRote. cock, rooster. Etym: *laluy.

manu_lalu-k Termanu. rooster. (J:274)

manu lalu-? Korbafo.

manu lalu-k Bokai.

manu lalu-? Bilbaa.

manu_lalu-? Rikou.

manu_lalu-k $B a^{\prime} a$.

manu_lalu-k Tii.

*lamat Morph: *ka-lamat. PRM. grasshopper. [irr. from PRM: $* 1=l$ in Amanuban and Kusa-Manea] [Form: The irregular retention of PRM $* 1$ as $l$ in Amanuban may be due to early reduction of the antepenultimate syllable in *ka-lamat $>* *$ klamat, with subsequent loss of the initial consonant. Compare *kalusa, with reflexes klusa-n, and knusa-n in Amanuban from Kualiin village. Irregular initial $l$ in Kusa-Manea may be due to borrowing from Kemak or antoher language with initial $l$.]

lama-k Termanu. grasshopper. (J:274)

lama-? Korbafo.

lama-k Bokai.

lama-? Bilbaa.

lama-? Landu. grasshopper. (own field notes)

lama-? Rikou.

lama-k $B a^{\prime} a$.

lama-k $T i i$.

lamat Dengka.

lamat Oenale.

k|namat Ro'is Amarasi. grasshopper.

k|namat Kotos Amarasi. grasshopper.

lamat Amanuban. grasshopper.

[Form: Middelkoop (1972:196)

also gives Amanuban $<$ nama $>$.]

lamat Kusa-Manea. grasshopper.

Out-comparisons:

klamat Funai Helong. grasshopper. klamat Semau Helong. grasshopper, locust.

l'ama Waima'a. grasshopper.

lamat Kemak. grasshopper.

*lamu CER. seaweed species. Etym: *lamut (Blust and Trussel (ongoing) include 'Rote' lamu and Ngadha lamu form as evidence for both *lamut and the PMP 'disjunct' *lamu. *lamu is only otherwise supported by Malay lamu.). [Note: Helong has similar hlamun 'moss, lichen, algae, seaweed' which is probably a reflex of PMP *lamun 'swamp grass'.]

lamu_doo-k Termanu. kind of seaweed, kind of algae. (J:275)

Out-comparisons:

klamur East Tetun. seaweed. (Mo:135)

*lani Rote. heaven, firmament, air. Etym: *lanit 'sky'. [irr. from PRM: $*_{\mathrm{n}}>\varnothing$ in all except Oenale] [Form: The irregular loss of medial *n in all varieties may be due to influence from semantically similar lain 'upper part', though this form may itself be from *lani with final ${ }^{*} \mathrm{CV}>\mathrm{VC}$ metathesis. (Jonker suggests that the final $n$ in lain is from the third person genitive enclitic $=\mathbf{n}$ ). Jonker does not record Dengka as having lain 'upper'.]

la lai Termanu. heaven, the firmament, air space. (J:269)

la lai Korbafo.

la lai Bokai.

la lai Bilbaa.

la lai Rikou.

la lai $B a^{\prime} a$.

la lai Tii.

la lai Dengka.

la lai (2) lani Oenale. 1) heaven, the firmament, air space. Usage: usually in the religious sense, otherwise lani. (J:269) 2) the firmament. (J:718)

la lai (2) lani Dela. 1) sky. 2) sky. 
*lanu Rote. tired. Etym: *layu 'vertigo' (Blust and Trussel (ongoing) include Termanu langu 'langu-dizziness: expression in ritual language for serious illness' (Fox 2016b:24) as evidence for this form, but this would require irregular retention of $* \eta$.).

na-ma-lanu Termanu. feel uncomfortably tired. (J:279)

na-ma-lanu Korbafo.

na-ma-lanu Bokai.

na-ma-lanu Bilbaa.

na-ma-lanu Tii.

lanu lanu Oenale.

Out-comparisons:

lanu (2) lanuk East Tetun. 1) intoxicate, inebriate, be drunk; poison. 2) intoxicated, drunk. (Mo:127)

melanu Hawu. dizzy. [irr. from PMP: *y>n] (J:278)

*langa Morph: *ka-langa, *ka-langa-k. PRM. head hair.

laya (2) laya-k Termanu. 1) head.

2) leader, village head, upper part. (J:276)

laya Korbafo.

laya Bokai.

laya Bilbaa.

laka-? Landu. head. (own field notes)

laka Rikou.

langa $B a^{\prime} a$.

langa Tii.

layga-? Dengka.

layga-? Oenale.

naka-f $R o$ 'is Amarasi. head, head-hair.

[Semantics: Ambiguous between

both meanings, 'head-hair' can be specified by naak bu?u.]

?|naka-f Kotos Amarasi. head; leader, boss.

P|naka-f Molo. head. (M:344)

Out-comparisons:

klaam/buu? Funai Helong. headhair. klaya Semau Helong. head-hair; prow of boat. [Note: boon $=$ 'head'.]

laka/nuri (2) laka/nuri maka, nak/nuri makan (3) laka/ nuri wi?i, naknuri wi?in Kisar. 1) coconut shell. [Note: nor or noro is the normal word for 'coconut', nuri could also be connected with *niuR, though the final vowel would be unexplained.] 2) the top half of a coconut shell, part with holes. [Form: maka = 'eye'] 3) the bottom half of the coconut shell without holes. [Form: $\mathbf{w i} \mathbf{i}=$ 'butt']

laga-na Tokodede. head, headhair. (Lekede'e Study Group 2006:15)

gala-r Kemak. head. Usage: Diirbati dialect (Manumutin desa). [Sporadic: consonant metathesis * $1 \mathrm{Vg}>\mathrm{g} V l$.]

gə̆raa-ク Kemak. head. Usage: Kutubaba dialect.

gara-r Kemak. head. Usage: other known Kemak varieties.

*lange PRM. stocks, block of wood placed around the head of an animal to prevent it from moving or suckling.

laye Termanu. stocks for criminals, block of wood tied around the neck of a buffalo or pig (or also a piece of bamboo on the neck of a dog, a coconut shell around the neck of a cat) to impede the movement of the animal. (J:278)

lane Korbafo.

lane Bokai.

laye Bilbaa.

lake Rikou.

lange $B a^{\prime} a$.

lange Tii.

lange Dengka.

layge Oenale. 
nake Kotos Amarasi. wooden stocks placed around the ankles of a criminal, wooden block placed around the neck of an animal to impede its movement.

nake Molo. stocks that are placed around the ankles of people, also a wooden triangle placed on the head of a pig or young buffalo to prevent the piglet or calf from suckling at its mother. (M:345)

\section{Out-comparisons:}

laye Semau Helong. lashed, shackled, chain, stocks.

*lari1 Morph: *lari-k. PRM. comb (of rooster). [irr. from PRM: $*_{\mathrm{i}}>e$ in Kusa-Manea] [Sporadic: consonant metathesis $* \mathrm{IVr}>r V l$ in Dela-Oenale and Rikou.]

lali-k Termanu. comb of a rooster. (J:274)

lali-? Korbafo.

lali-k Bokai.

lali-? Bilbaa.

rali-? Rikou.

lali-k $B a^{\prime} a$.

lari-k Tii.

lali-? Dengka.

rali-? Oenale.

nane-f Kusa-Manea. comb of rooster. [Note: Jonker (1908:274) gives Meto nani-f.]

\section{Out-comparisons:}

lalit Semau Helong. comb.

la larit East Tetun. rooster's comb, and crest of other birds. (Mo:125)

lari Hawu. (J:274)

lali? Bugis. chicken's comb. (Masse 2013:317)

*lari2 $P R M$. move location. [irr. from $P R M:{ }^{*} \mathrm{r}>l$ in Tii and wRote; $*_{\mathrm{r}}>l$ $\sim r$ in Oepao and Rikou] [Sporadic: consonant metathesis $* \mathrm{lVr}>r V l$ in Rikou.] [History: Possibly connected with PMP *laRiw with irregular *R > $*_{r}$, but this etymon is retained regularly as *lai 'run, fast'.] lali Termanu. move oneself, move something. (J:273)

lali Korbafo.

lali Bokai.

lali Bilbaa.

lali, na-rali Rikou.

lali, na-lari Oepao.

lali $B a^{\prime} a$.

lali Tii.

lali Dengka.

lali Oenale.

lali Dela. move.

na-nani? Kotos Amarasi. move, change.

<nani> Molo. move away. (M:349)

Out-comparisons:

lari Hawu. move location.

*larum Morph: *ka-larum. PRM. variegated fig. Ficus variegata. [Sporadic: consonant metathesis $* 1 \mathrm{Vr}$ $>$ rVl in Dela-Oenale and Rikou.] [Form: That it is Dela-Oenale and Rikou which show consonant metathesis rather than Tii, is indicated by other reconstructions with $* 1 \mathrm{Vr}$ that have cognates outside of the RM languages including: *lari 'comb of rooster' and *ledo 'sun' (PMP *qalojaw) that have undergone the same consonant metathesis * $1 \mathrm{Vr}$ $>r V l$ in Dela-Oenale and Rikou.] [Semantics: vague Rote semantics.]

lalu Termanu. kind of tree. [Semantics: Heyne (1950:755, cxlii) gives $<$ laloe loëh $>$ as Peltophorum pterocarpum.] (J:274)

lalu Korbafo.

lalu Bokai.

lalu Bilbaa.

ralu Rikou.

lalu $B a^{\prime} a$.

laru Tii.

lalu Dengka.

ralu Oenale.

?|nanum Kotos Amarasi. kind of tree. <nanum> Molo. kind of tree. Ficus variegata. (M:349) 
*lasa PRM. underlay. [irr. from PRM: *1 $>r / l$ in Meto]

lasa Termanu. anything that serves as an underlay for cutting or chopping. (J:282)

lasa Korbafo.

lasa Bokai.

lasa Bilbaa.

lasa Rikou.

lasa $B a^{\prime} a$.

lasa Tii.

lasa Dengka.

lasa Oenale.

rasa|k Kotos Amarasi. spread out leaves (with meat put on top).

n-lasan Molo. use as an underlay. (M:263)

*laso PRM. scrotum and testicles. Etym: *lasəR. [Sporadic: $\left({ }^{*} ə\right)>*_{a}>e / \#$ in Amarasi.]

lase Termanu. scrotum. (J:283)

lase Korbafo.

lase Bokai.

lase Bilbaa.

lase Rikou.

lase $B a^{\prime} a$.

lase Tii.

lasa-? Dengka.

lasa-? Oenale.

nase-n Ro'is Amarasi. testicles.

nase-f Kotos Amarasi. testicles.

$<$ nasa $>$ Molo. scrotum. (M:351)

Out-comparisons:

lasar Ili'uun. penis. (dJ:123)

*lasi1 PRM. forest, bush. [History: possibly connected with PMP *alas, though this connection would require positing irr. *a $>\varnothing$ and irregular final $i$.] lasi Termanu. wilderness, forest. ( $\mathrm{J}: 283)$

lasi Korbafo.

lasi Bokai.

lasi Bilbaa.

lasi Rikou.

lasi $B a^{\prime} a$.

lasi Tii.

lasi Dengka.

lasi Oenale. nasi Ro'is Amarasi. bush, forest. nasi Kotos Amarasi. bush, forest. nasi Molo. bush, forest. (M:352) nais Kusa-Manea. forest.

*lasi2 2 orph: *ma-lasi-k, *na-ma-lasi. PRM. old, aged.

lasi-k (2) na-ma-lasi Termanu. 1) old (especially of people and animals). 2) be or become old, of people and animals. (J:283)

lasi-? Korbafo.

lasi-k Bokai.

lasi-k Bilbaa.

lasi-? Rikou.

lasi-k $B a^{\prime} a$.

lasi-k Tii.

lasi-? Dengka.

lasi-? Oenale.

lasi-? (2) na-ma-lasi Dela. 1) old. 2) m|nasi|? Ro'is Amarasi. old, aged.

m|nasi|? (2) na-m|mnasi Kotos Amarasi. 1) old, aged. 2) be or become old/aged.

m|nasi|? Molo. old. (M:325)

m|nasi|? (2) m|nasi-k Kusa-Manea. 1) old (of fruit). 2) old, aged (of people).

*latu1 Morph: *ma-latu-k, *na-ma-latu. $P R M$. ripe.

latu-k (2) na-ma-latu Termanu. 1) ripe. 2) ripen up. (J:284)

latu-? Korbafo.

latu-k Bokai.

latu-k Bilbaa.

latu-? Rikou.

latu-k $B a^{\prime} a$.

latu-k Tii.

latu-? Dengka.

latu-? Oenale.

m|natu|? Ro'is Amarasi. ripe, cooked.

m|natu|? Kotos Amarasi. ripe, cooked.

na-m|natu Amanuban. ripe. (M:325)

Out-comparisons:

latu? Semau Helong. ripe.

keradu Hawu. [Sporadic: $* 1=r$ correspondence.] (J:284) 
*latu2 Rote. edible seaweed species. Etym: *latuq.

latu Termanu. kind of edible seaweed. ( $\mathrm{J}: 284)$

latu Korbafo.

latu Bokai.

latu Bilbaa.

latu Rikou.

latu $B a^{\prime} a$.

latu Tii.

latu Dengka.

latu Oenale.

*lea Morph: *lea-k. PnRote. cave, cavern. See: *lua|t. Etym:*lian. [irr. from PMP: $* \mathrm{i}>*_{\mathrm{e}}$ ]

lea-k Termanu. cave, cavern. (J:288)

lea-? Korbafo.

lea-k Bokai.

lea-? Bilbaa.

lea-? Rikou.

lea-k $B a^{\prime} a$. [Form: with variant lua-k.]

lea-k Tii.

Out-comparisons:

lian Semau Helong. cave.

*ledo PRM. sun, day. Etym: *qalojaw 'day'. [minority from PMP: $* \mathrm{j}>* \mathrm{~d}$ (expect $* d)$ ] [Sporadic: consonant metathesis $* 1 \mathrm{Vr}>r V l$ in Dela-Oenale.] ledo (2) ledo-k (3) le ledo-k Termanu. 1) sun. 2) day, in certain expressions. 3) daytime, during the day. (J:290f)

ledo Korbafo.

ledo Bokai.

ledo Bilbaa.

ledo Rikou.

ledo $B a^{\prime} a$.

ledo Tii.

lelo Dengka.

relo Oenale.

relo (2) re relo-? Dela. 1) sun.

2) daytime.

neno Ro'is Amarasi. day (e.g. of the week), sky. neno Kotos Amarasi. day (e.g. of the week), sky. [Semantics: Phrases such as neon sae-t 'east' (neno + rise-NMLZ') provide internal evidence for the older meaning 'sun'.]

neno Molo. sky. (M:362)

neno Kusa-Manea. sun, day. [Semantics: neno anan specifies 'sky' as opposed to 'day'.]

Out-comparisons:

lelo Semau Helong. sun, day, daylight.

loro East Tetun. the sun. (Mo:133)

ler(e) Kisar. day.

lodo Dhao. sun.

lodo Hawu. sun, day.

leo Ili'uun. sun, day; time. (dJ:124)

*lee PRM. river. [irr. from PRM: *ee > oe in wRM (possibly influence from *oe 'water')]

lee Termanu. river. (J:286)

lee Korbafo.

lee Bokai.

lee Bilbaa.

lee Rikou.

lee $B a^{\prime} a$.

lee Tii.

loe Dengka.

loe Oenale.

noe $R o$ 'is Amarasi. river.

noe Kotos Amarasi. river.

noe Molo. river. [Note: Middelkoop (1972:371) includes the parallelism noe ?nino?, koe ?nino? 'the clear river'. If koe is related to noe, it would indicate PMeto *noe. Helong has noe 'wet', which could alternately be the source of Molo koe.] (M:377)

noa Kusa-Manea. river. [Form: regular $* \mathrm{e}>a / \mathrm{V}_{-} \#$ in Upper Manulea.]

*leke Morph: *le leke. PRM. coil up. Etym: *ləkən 'coil' (Blust and Trussel (ongoing) only give a Cebuano and Buru form as supporting evidence. 
Other semantically and formally similar forms are discussed with their entry for *reken 'coiled base on which hot cooking pots are set', which is well supported.). Pattern: k-5. [minority from PMP: *ə $>*_{\mathrm{e}} /$ \# (expect $*_{\mathrm{o}}>a$ in wRote, possibly $* \partial>* \mathrm{a}>e)]$

le leke Termanu. lay down in coils, coil up. (J:293)

le leke Korbafo.

le leke Bokai.

le leke Bilbaa.

le le?e Rikou.

le leke $B a^{\prime} a$.

le leke Tii.

le leke Dengka.

*leko Rote. lure in a crafty way. Pattern: $\mathrm{k}-5^{\prime}\left({ }^{*} \mathrm{k}>\varnothing\right.$ in Rikou; expect $?$ or $k$ ).

leko Termanu. lure in a crafty way. (J:293)

leko Korbafo.

leko Bokai.

leko Bilbaa.

leo Rikou.

leko $B a^{\prime} a$.

leko Tii.

leko Dengka.

leko Oenale.

Out-comparisons:

leko Hawu. (J:293)

*leku PRM. winding, coiling. Etym: *loku? 'bend, fold, folding part of the body; curl up on the ground, of an animal'. Pattern: k-8/9' ${ }^{*} \mathrm{k}>\varnothing$ in Rikou; expect $* \mathrm{k}>$ ? or $k$ ). [irr. from PRM: *1 $>r / l$ in Meto]

na-le le?u (2) le le?u (3) le le?u-k

Termanu. 1) with winding/coils.

2) do with twists and turns. 3) do it with winding/coils. (J:309)

na-le le?u Korbafo.

na-le le?u Bokai.

na-le leku Bilbaa.

na-le leu Rikou.

na-le le?u $B a^{\prime} a$.

na-le le?u Tii.

na-le le?u Dengka. na-le le?u Oenale.

reuk/saen Ro'is Amarasi. python. Borrowed from: Rote for initial element leku or from Helong likun before $* \mathrm{e}>i$ (shown by irr. $* 1=r$ correspondence). [History: Ultimately from * reku 'winding' + sae-n 'go up'.]

riuk/saen Kotos Amarasi. python.

liuk/saen Molo. python. (M:404)

lik/saan Kusa-Manea. python. [Form: regular $* \mathrm{e}>a / \mathrm{V}_{-}$in Upper Manulea.]

Out-comparisons:

likuy (2) 'liuksay Semau Helong. 1) loop around. 2) python. Borrowed from: Meto liuksaen.

'liku/saen Fehan Tetun. python. Borrowed from: probably Meto liuksaen without CV metathesis (shown by irr. *o $=i$ correspondence, expect $o$ ). [Note: East Tetun has labak and/or foho_rai for 'python', and Tetun Belu has koes ina (Morris 1984:35, 114, 122).]

kaləku Hawu. (J:309)

*Iele Morph: *lele-k. Rote. time. [Form: Rote *loo 'stretch' also shows the correspondence of Helong $d=$ Rote $l$. lele-k Termanu. time. (J:295)

lele-? Korbafo.

lele-k Bokai.

lele-? Bilbaa.

lele-? Rikou.

lele-k $B a^{\prime} a$.

lele-k Tii.

lele-? Dengka.

lele-? Oenale.

Out-comparisons:

deden Semau Helong. night; time, moment.

*lema Morph: *ka-lema. PRM. seasnake. [Sporadic: *a $>e / \#$ in Meto.] lema-k Termanu. kind of ocean fish.

(J:296) 
lema-? Korbafo.

lema-? Rikou.

lema-k $B a^{\prime} a$.

lema-k Tii.

lema-? Dengka.

lema-? Oenale.

lema-? Dela. sea-snake.

k|neme Ro'is Amarasi. eel, sea-snake.

k|neme Kotos Amarasi. eel, seasnake. Usage: Tais Nonof subdialect.

Out-comparisons:

laleman Galolen. sea-snake.

*lemuk CER. dolphin. Etym: **lemur (pre-RM). [irr. from $P M P: \varnothing>* \mathrm{k}$ ]

lemuk Rikou. dolphin. (own field notes)

Out-comparisons:

lemur Galolen. dolphin.

lemor Batuley. dolphin. [Note: language of the Aru Islands ISO 639-3 [bay].] (Daigle 2015:258)

*lemba $P R M$. carry on a shoulder pole. Etym: *lemba (PCMP).

lepa Termanu. carry, said of one or more people who are carrying a stick that has a load at each end. (J:305)

lepa Korbafo.

lepa Bilbaa.

lepa Rikou.

lempa $B a^{\prime} a$.

lemba Tii.

lemba Dengka.

lemba Oenale.

nepa-t Kotos Amarasi. pole for carrying.

$<$ nepa $>$ Molo. pole for carrying, bar for carrying. [Form: probably 2-nepa-?.] (M:364)

Out-comparisons:

lepa Semau Helong. carry on shoulder with pole, one person with something on each end, or two or more people with one heavy thing in the middle. lebo, leba East Tetun. carry or transport any object from the ends of a pole with the centre supported by the shoulder. (Mo:128)

ai kaleba Welaun. pole for carrying.

*lena PRM. sesame. Etym: *ləyah (Zorc 1995:1128). [Sporadic: *a $>e$ /_\# in wRM]

lena Termanu. sesame. (J:296)

lena Korbafo.

lena Bokai.

lena Bilbaa.

lena Rikou.

lena $B a^{\prime} a$.

lena Tii.

lene Dengka.

lene Oenale.

nene-l Meto. sesame. (J:296)

Out-comparisons:

Iena Semau Helong. sesame. Borrowed from: Rote (shown by irr. $* \mathrm{y}=n$ correspondence).

leya Helong. sesame. (J:296)

*lendo Rote. dance.

leno Termanu. perform a sworddance, often combined with foti [OE: = 'jump around, dance'] to express 'dance' in general. (J:299)

leno Korbafo.

leno Bokai.

lendo Rikou. dance performed by women. (J:299; own field notes)

'lendo Oepao. Borrowed from: Rikou (shown by medial $n d$ ). (own field notes)

leno $B a^{\prime} a$.

lendo Tii.

lendo Dengka.

lendo Oenale.

Out-comparisons:

ledo, lendo Hawu. dance. Can be single or multiple. Mixed male and female.

*lenge $C E R$. too much.

leye Termanu. oversize, too much. (J:298) 
lege Bokai.

\section{Out-comparisons:}

ləya Hawu. left, gone, abandoned, no longer there; extreme, significantly beyond what is acceptable. [Note: Jonker (1908:298) gives Hawu from Raijua ləye.]

*lengu Rote. throw. [Note: Meto has na-neku 'lose', na-m|neku 'lost' for which the sound correspondences with the Rote forms are perfect; however, the semantic connection is far too dubious to establish these forms as cognate.]

leyu Termanu. throw, normally a small piece of wood; fall backwards. (J:299)

leyu Korbafo.

lenu Bokai.

leyu Bilbaa.

leku Rikou.

lengu $B a^{\prime} a$.

lengu Tii.

lengu Dengka.

leygu Oenale.

Out-comparisons:

ligu Hawu. (J:299)

*leo Rote. clan.

leo Termanu. clan, tribe. In Termanu there are nine clans: leo dou daya, leo inu beu-k, leo inu na?u, leo kiu kana-k, leo kota dea-k, leo masahuu-k, leo meno, leo ygofa lai-k, leo su?i. (J:301)

leo Korbafo.

leo Bokai.

leo Bilbaa.

leo Rikou.

leo $B a^{\prime} a$.

leo Tii.

leo Dengka.

leo Oenale.

Out-comparisons:

leon Helong. (J:301)

leo Fehan Tetun. hamlet.

leo East Tetun. the population, the total number of people in a place. (Mo:129) *lesu1 PRM. neck. [irr. from PRM: *s > $\varnothing$ in Meto (also Helong)] [Sporadic: vowel height harmony $* \mathrm{u}>o / \mathrm{e}_{-}$in Meto.] [Form: The main difficulty in this comparison is accounting for irregular loss of medial $*_{s}$ in both the Meto and Helong forms. There seem to be two possible explanations: (1) This comparison combines more than one cognate set; Rote lesu is not cognate with the Meto forms. (2) Medial *s was irregularly lost in Meto, from which the Helong form is a borrowing. Whatever the ultimate history of these forms, it is likely that the Helong and Meto forms are a result of borrowing before Meto *1 $>n$, though the direction of borrowing cannot yet be confidently determined.]

lesu_ha?i-k Termanu. neck in general, both sides of the neck. [Form: lesu-k by itself means 'the thinner portion of the arm at the wrist'.] (J:307)

lesu ha?i Korbafo.

lesu_ha?i-k Bokai.

lesu_kai-? Bilbaa.

lesu_ai-? Rikou.

lesu_ha?i-k $B a^{\prime} a$.

lesu_ha?i Lole. neck. (Zacharias et al. 2014)

lesu_ai-k Tii. [Form: Grimes et al. (2014a) has leusain 'neck'.]

lesu_ai-? Dengka.

lesu_ai-? Oenale.

neo-f Ro'is Amarasi. neck.

neo-f Kotos Amarasi. neck.

Out-comparisons:

leo Semau Helong. neck.

n'eo Waima'a. neck. [Form: Possibly a chance resemblance. Waima'a $n^{\text {? }}$ is not known to correspond to PRM *1. While the Waima'a form and Meto might point to pre-RM $* *$ neo 'neck', this would not account for the Helong form with initial $l$. 
*lesu2 Rote. come out, take out. Etym: *lesu (PCMP. Blust and Trussel (ongoing) also reconstruct PWMP *ləcut 'squeeze out, slip out' which could be connected.).

lesu Termanu. come out, e.g. of a hole, make something come out $=$ pull out, e.g. pull something out of a hole. (J:306)

lesu Korbafo.

lesu Bokai.

lesu Bilbaa.

lesu Rikou.

lesu $B a^{\prime} a$.

lesu Tii.

lesu Dengka.

lesu Oenale.

Out-comparisons:

$<$ luhu> Kambera. go out. (On:246)

$<$ lauhu $>$ Lewa

$<$ lausu> Anakalang.

$<$ lusa> Mamboru.

$<$ louzo> Weyewa.

$<$ loho> Kodi.

losa Bima. exit, go outside. (Jonker 1893:48)

*lete1 Morph: *ka-lete-k. PRM. mountain, hill. Etym: *letay1 'above' (PCMP).

lete-k Termanu. mountain. (J:308)

lete-? Korbafo.

lete-k Bokai.

lete-k Bilbaa.

lete-? Rikou.

le lete, lete-k $B a^{\prime} a$.

lete-k Tii.

lete-? Dengka.

lete-? Oenale.

neten $R o$ 'is Amarasi. high place.

k|nete|? Kotos Amarasi. mountain with gentle slopes on either side, hill.

<neten $>$ Molo. mountain range. (M:366)

\section{Out-comparisons:}

leten Semau Helong. mountain. leten East Tetun. above, overhead; $n$. the top, the part above. (Mo:130)

lete(n) Ili'uun. mountain. (dJ:124)

lede Hawu. mountain.

*lete2 PRM. bridge. Etym: *letay2 (PCMP).

le lete Termanu. small bridge. (J:307)

le lete Korbafo.

le lete Bokai.

le lete Bilbaa.

le lete Rikou.

le lete $B a^{\prime} a$.

le -lete Tii.

le lete Dengka.

le lete Oenale.

nete-ranan Kotos Amarasi. bridge, go-between.

$<$ kanete> Molo. bridge. (M:366)

Out-comparisons:

hleten Semau Helong. bridge, dock.

kalete Fehan Tetun. intermediary, bridge (over water), go between; in traditional courtship, interpreter, mediator (in fights), anyone who carries a message for another - usually used in courtship, but not only for that.

kla lete East Tetun. small bridge. (Mo:108)

*leu PRM. inceptive, used to ask permission to begin an activity. [Sporadic: vowel height harmony $* \mathrm{u}>o / \mathrm{e}$ in Dela.]

leu Bilba $\bar{a}$. used to ask permission to begin an activity. lako leu We're leaving now. (own field notes)

leu Rikou. used to ask permission to begin an activity. la?o leu We're leaving now. (own field notes)

leo Dela. already, now; typically used with imperatives and invitations to indicate that the action expressed by the verb should be done now or right away. 
neu Ro'is Amarasi. used to ask permission to begin an activity. au 2-bukae neu I'm going to start eating now

neu Kotos Amarasi. used to ask permission to begin an activity. Usage: In Kotos Amarasi neu is less common and considered colloquial/impolite. The more common and polite form is nai.

*londe Morph: *Ionde londe. PnRote. still standing (of water). See: *endən. [Form: *nd $>n / \partial$ in Rikou.] [History: Jonker makes comparisons with forms in Seram, e.g. Kamarian marene 'calm, quiet' (van Ekris 1864:308) but I do not find this comparison convincing given the semantics. (Kamarian marina is given as specifically 'still [of sea]', thus indicating that marene probably does not also indicate 'calm of sea'). Additionally, whether *nd would correspond regularly to $n$ in languages of Seram is unknown.]

$\mathbf{o e}=\mathbf{a}$ lene $\sim$ lene Termanu . the water is standing still, doesn't flow. (J:297)

lene lene Korbafo.

lene lene Bokai.

lene lene Rikou.

lene lene $B a^{\prime} a$.

lende Tii.

*libu CERM. swarm, teem, abound.

Doublet: *rimbu. Etym: *libut

'surround, encircle, as game'.

[minority from PMP: $* \mathrm{~b}>* 6 / \mathrm{V}_{-} \mathrm{V}$ ]

libu Termanu. swarm (of fish, people), gather together. (Fox 2016b:27)

li libu $B a^{\prime} a$. teem, abound. (J:723)

n-nibun Kotos Amarasi. surround.

n-nibun Molo. gather and meet together. (M:368)

Out-comparisons:

libur East Tetun. rejoin, assemble. (Mo:130) *lida Morph: *lida-k. PRM. wing. See: *dilas. [irr. from PRM: *a $>e \sim a$ sporadically in Termanu, Ba'a and Meto (also Kemak)] [Form: regular $* \mathfrak{d}>* * 1 / * 1$ in Meto (and Helong).]

lida-k, lide-k Termanu. wing of a bird or insect, fin of a fish. (J:310)

lida-? Korbafo.

lida-k Bokai.

lida-? Bilbaa.

lida-k, lide-k $B a^{\prime} a$.

lida-k Tii.

lida-? Dengka.

lida-? Oenale.

nini-n Ro'is Amarasi. edge.

nine|?, nini|? Kotos Amarasi. wing, edge.

nine-n, nina-n Molo. wing. (M:370) nina-f Kusa-Manea. wing.

Out-comparisons:

liras East Tetun. wing (of birds and insects). (Mo:131)

lila/faan Welaun. wing.

lila/paa-n Kemak. wing. Usage: Kailaku dialect.

lile/paa Kemak. wing. Usage: Diirbati dialect.

lili/paa-n Kemak. Usage: Kenebibi and Leolima dialects.

lili/paa Kemak. wing. Usage: Lemia and Atsabe dialects.

lira Mambae, South. wing. Usage: Letefoho village. (Fogaça 2017:234)

lila Mambae, South. wing. Usage: Betano village and Ainaro subdistrict. (Fogaça 2017:234)

lila-n Mambae, Northwest/ Central. wing. (Fogaça 2017:234)

lilar Tokodede. wing. (Klamer 2002)

*lide Morph: *lide-k. Rote. palm leaf nerve. [irr. from PRM: ${ }^{*} \mathrm{e}>e \sim a$ in Ba'a]

lide-k Termanu. nerve of a palm leaf. (J:310) 
lide-? Korbafo.

lide-k Bokai.

lide-? Bilbaa.

lide-? Rikou.

lide-k, lida-k $B a^{\prime} a$.

lide-k Tii.

lide-? Dengka.

lide-? Oenale.

\section{Out-comparisons:}

lidi Malay. ribs/veins of coconut palm fronds/leaves.

*lifu PRM. body of still water; billabong, lake. Etym: **libun (pre-RM).

lifu Termanu. a) standing still, of water. b) body of water which is still, e.g. after rain: a deep gully where there is no current. (J:311f)

lifu Korbafo.

lifu Bokai.

lifu Bilbaa.

lifu Rikou.

lifu $B a^{\prime} a$.

lifu Tii.

lifu Dengka.

lifu Oenale.

nifu (2) nefo Kotos Amarasi. 1) pool, billabong. 2) lake. [Semantics: The two words are semantically distinct, nifu is smaller than nefo. They possibly have different etymologies.]

nifu Amanuban/Amanatun. lake.

nifu (2) nefo Molo. 1) pond, lake. (M:368) 2) lake. (M:359)

\section{Out-comparisons:}

lihu Semau Helong. depth.

lihun East Tetun. dam or pond, a small body of still water. (Mo:130)

welihun Welaun. lake.

liwu Central Lembata. lake. (Fricke 2015)

$<$ kalibuku $>$ Kambera. shallow hole, cavity or hollow in a stone with still water in it. (On:145) *liha Morph: *li liha-k. Rote. centipede. Etym: *qalu-hipan (Blust and Trussel (ongoing) reconstruct PCEMP *qalipan.). [irr. from PRM: *a $>e$ in Tii (sporadic assimilation)]

li liia-k Termanu. kind of centipede which emits phosphoric light when rubbed (called kalamayar in Malay). (J:310)

li li?a-? Korbafo.

li li?a-k Bokai.

li lia-? Bilbaa.

li lias Landu. centipede. (own field notes)

li lia-? Rikou.

li li?a-k $B a^{\prime} a$.

li -li?e-k Tii.

li lia-? Dengka.

li lia-? Oenale.

Out-comparisons:

la li?an_tali Fehan Tetun. centipede.

*lii1 Morph: *na-lii, *lii-k. Rote. sound. Etym: *lin 'sound of ringing; word, speech' (Blust and Trussel (ongoing) include Termanu lii-k 'sound' as evidence for three separate reconstructions: PCMP liRi 'sound, voice', PCEMP liqə 'voice', and PMP *lin. Of these, PCMP liRi is only otherwise supported by Yamdena liri-n 'voice; language' and yé-liri 'uproar, clamour'. Unless more cognates are forthcoming, PCMP *liRi is probably spurious.).

na-lii (2) lii-k Termanu. 1) make a sound. 2) sound. (J:309)

na-lii Korbafo.

na-lii Bokai.

na-lii Bilbaa.

na-lii Rikou.

na-lii $B a^{\prime} a$.

na-lii $T i i$.

na-lii Dengka.

na-lii Oenale. 
Out-comparisons:

liin Semau Helong. sound, tune, voice.

lii Hawu. say, speak, tell; voice, sound.

*lii2 Morph: *boto_lii-k. Rote. neck. Etym: *liqəR. [irr. from PMP: *o $>*_{\mathrm{i}}$ (assimilation to previous $* \mathrm{i}$ )]

boto_lii-k Termanu. neck. (J:59)

boto lii-? Korbafo.

boto_lii-k Bokai.

boto_lii-? Bilbaa.

bo/lii-? Landu. neck. (own field notes)

bo/lii-? Rikou.

bo/lii-? Oepao. neck. (own field notes)

boto lii-k $B a^{\prime} a$.

boto_lii-k Tii.

boto_lii-? Oenale.

*lii3 Morph: *ka-lii. PnRote. shy, wild. Etym: *liaR (Dempwolff 1938:96). [irr. from PMP: *a > i] [History: Blust and Trussel (ongoing) identify several putative cognates as 'Probably a Malay loan distribution.' and this is indeed possible. However, in the case of the Rote forms, borrowing from Malay does not seem likely due to the final vowel assimilation and diphthongisation of the initial prefix. While neither process is regular in the Rote languages, they would be even more unexpected if these terms were (recently) borrowed from Malay.]

kai|lii, ka-lii (2) kai|liis (3) kai|li-lii

Termanu. 1) be or become wild or shy, e.g. a horse. 2) shy, wild.

3) make shy. (J:216,310)

ka-lii (2) kai|lii-? Korbafo.

ka-lii (2) kai|lii-k Bokai.

ka-lii (2) ka-lii-? Bilbaa.

ai|lii (2) ai|liis Rikou.

kai|lii (2) kai|liis $B a^{\prime} a$.

(2) kaliis Tii.

Out-comparisons:

melei Hawu. (J:310)

*liku1 Rote. strike, knock. Pattern: k-8/9. li?u Termanu. hit with a stick, etc. ( $\mathrm{J}: 316)$

li?u Korbafo.

li?u Bokai.

liku Bilbaa.

li?u Rikou.

li?u $B a^{\prime} a$.

li?u Tii.

li?u Dengka.

Out-comparisons:

liku Hawu. (J:316)

*liku2 PRM. back of an object. Etym:

*likud 'back'. Pattern: k-9.

li?u_dea Termanu. that which a weaver uses to support their back, on Rote usually a strip of goat or deer skin. (J:317)

li?u_dea Korbafo.

li?u_dea Bokai.

liku dea Bilbaa.

li?u_deas Rikou.

li?u dea $B a^{\prime} a$.

li?u_dea Tii.

li?u_deat Dengka.

ni?u-n Kotos Amarasi. the back part of a blade (incl. swords, knives, machetes, etc.), which is not sharp. [Note: kotif = 'back (of person)'.]

ni?u-n (2) an-ni?u (3) am-koti-f =kau, am-ni?u-f =kau Molo. 1) back of a knife or machete. 2) hit something with the back of a knife of machete. 3) you turn your back to me. Usage: parallelism. (M:374)

Out-comparisons:

likun Semau Helong. out, outside.

liku East Tetun. carry on the back (a person). (Mo:131)

*lili Morph: *lili-k. PRM. wax. Etym:

*lilin 'beeswax'.

lili-k Termanu. wax. (J:313)

lili-? Korbafo.

lili-k Bokai.

lili-? Bilbaa.

lili-? Rikou.

lili-k $B a^{\prime} a$.

lili-k Tii. 
lili-? Dengka.

lili-? Oenale.

niin|k Ro'is Amarasi. wax, candle.

nini|k Kotos Amarasi. wax, candle.

nini|k Molo. beeswax. (M:371)

nini|k Kusa-Manea. wax, candle.

Out-comparisons:

lilin East Tetun. wax. (Mo:131)

*lilis $n R M$. kind of tree. Calophyllum

teysmannii. [Semantics: vague Rote semantics.]

kai/lili-k Termanu. kind of tree. (J:216)

kai/lili-? Korbafo.

kai/lili-k Bokai.

kai-lili-? Bilbaa.

ai_lili-? Rikou.

ai/lili-k $B a^{\prime} a$.

(kai/lili-k ?) Tii.

<ninis> Amfo'an. kind of tree.

Calophyllum teysmannii. (M:371)

*lilo PRM. gold, silver. [irr. from PRM:

vowel metathesis in Meto $* \mathrm{iCo}>o C i]$

lilo Termanu. gold, silver. (J:313)

lilo Korbafo.

lilo Bokai.

lilo Bilbaa.

lilo Rikou.

lilo $B a^{\prime} a$.

lilo $T i$.

lilo Dengka.

lilo Oenale.

noni Kotos Amarasi. silver; bridewealth.

noni Molo. silver, silver coins. (M:382)

Out-comparisons:

lila (2) lil muti? (2) lil mea

Semau Helong. 1) bridewealth.

2) silver. 3) gold.

*lima1 PRM. five. Etym: *lima.

lima Termanu. five, fifth. (J:313f)

lima Korbafo.

lima Bokai.

lima Bilbaa.

lima Rikou.

lima $B a^{\prime} a$. lima Tii.

lima Dengka.

lima Oenale.

nima Ro'is Amarasi. five.

nima Kotos Amarasi. five.

nima Molo. five. (M:370)

niam Kusa-Manea. five.

Out-comparisons:

lima Semau Helong. five.

lima East Tetun. five. (Mo:131)

lima Kemak. five.

wolima Kisar. five.

ləmi Hawu. five.

*lima2 Morph: *ka-lima-k. PRM. hand/ arm. Etym: *qa-lima.

lima-k Termanu. hand, arm of a person or ape, forepaws of a crocodile. (J:314)

lima-? Korbafo.

lima-k Bokai.

lima-? Bilbaa.

lima-? Landu. hand, arm. (own field notes)

lima-? Rikou.

lima-k $B a^{\prime} a$.

lima-k Tii.

lima-? Dengka.

lima-? Oenale.

nimi-f Ro'is Amarasi. arm/hand.

?|nima-f Kotos Amarasi. arm/hand.

$<$ nima-f> Molo. hand (in general). (M:370)

2|nima-f Baikeno. arm/hand. (Charles

E. Grimes pers. comm.)

nima-f Kusa-Manea. arm/hand.

Out-comparisons:

ima Semau Helong. hand, arm. [irr. from PMP: *1> Ø]

lima-(n) East Tetun. arm, hand. (Mo:131)

limar Kemak. hand, arm.

lima Kisar. hand, arm.

*lino PRM. tree. Grewia salutaris. Etym: *qanilaw 'tree Grewia species'. [Sporadic: consonant metathesis $*_{\mathrm{nVl}}$ $>*$ IVn.] 
lino Termanu. kind tree. Grewia salutaris. (J:314) (Heyne 1950:1060,cxlviii)

lino Korbafo.

lino Bokai.

lino Bilbaa.

lino Rikou.

lino $B a^{\prime} a$.

lino Tii.

lino Dengka.

lino Oenale.

<nino> Amfo'an. small crooked tree. Antidesma ghaesembilla. [Semantics: This may be a chance resemblance given the lack of an exact semantic match between the Meto and Rote forms.] (M:371)

*lise $n R M$. shore tree with edible nuts. Terminalia catappa. Etym: *talisay. [irr. from PRM: * $>a$ in Termanu, Korbafo and Ba'a]

lisa, lise Termanu. kind of tree, the ketapang. Terminalia catappa. (J:315)

lisa, lise Korbafo.

lise Bokai.

lise Bilbaa.

lise Rikou.

lisa $B a^{\prime} a$.

lise Tii.

nisa|? Kotos Amarasi. kind of big tree the seeds of which are medicinal. [Note: ?nisa? 'gebang palm seeds (traditionally used as a kind of marble)'.]

$<$ nisa $>$ (2) $\mathbf{P} \mid$ nisa $\mid \mathbf{P}=$ ma $\mathbf{P f e n u}$ Molo. 1) kind of tree. Sterculea foetidea. (M:372) 2) medicine for women especially during pregnancy and childbirth. (M:374)

*lisum PRM. kind of plant with fruit. [Semantics: vague semantics.]

li lisu-k Termanu. kind of plant called the terong utan in Kupang, that is the wild eggplant or aubergine. [Semantics: Heyne
(1950:1345, cxlvii) gives <lilisoe olana $>$ as $\underline{\text { Solanum melongena }}=$ 'eggplant'.] (J:315)

li lisu-? Korbafo.

li lisu-k Bokai.

li lisu-? Bilbaa.

li lisu-? Rikou.

li lisu-k $B a^{\prime} a$.

li lisu-? Dengka.

li-lisu-? Oenale.

<nisum> (2) <nisume> Molo.

1) kind of tree. Ficus subglauca M.Dr. (M:372) [Semantics: This tree appears to have first been identified by Meijer Drees (1950).]

2) kind of tree. Myristica species. (M:373)

*loba $P R M$. kind of bark used to make clothing.

loba Termanu. kind of tree bark that is used for sarongs, etc., it is supplied by foreign traders. ( $\mathrm{J}: 318)$

loba Korbafo.

loba Bokai.

loba Bilbaa.

loba Rikou.

loba $B a^{\prime} a$.

loba Tii.

loba Dengka.

loba Oenale.

<noba> Molo. kind of dried bark used to make clothing mixed with $<$ baukulu $>[\mathrm{OE}=\underline{\text { Morinda }}$ citrifolia]. (M:376)

\section{Out-comparisons:}

luba Hawu. (J:318)

<loba> Kambera. kind of tree only found in the area of Parai Marapu in East Sumba. The bark and leaves are used to make a red dye for yarn and textiles. Peltophorum pterocarpum. (On:243)

*lobo Rote. tire out, hurt.

na-lobo (2) lobo Termanu. hurt, tire out. [Semantics: Both forms have the same meaning.] (J:319) 
na-lobo Korbafo.

na-lobo Bokai.

na-lobo Rikou.

na-lobo $B a^{\prime} a$.

na-lobo Oenale.

Out-comparisons:

lobo Hawu. tire oneself out. ( $\mathrm{J}: 319)$

*lode PRM. stick out. [irr. from PRM: *e $>o$ in Meto; $* 1=l$ in Meto (expect $n)]$ [Form: regular $* \mathfrak{d}>* * 1 / * 1$ in Meto.] maa $=$ na lode $\sim$ lode (2) lode-k maa maa Termanu. 1) his tongue sticks out, hangs out (e.g. like a dog that is hot). 2) stick your tongue out. (J:319)

lode lode Korbafo.

lode lode Bokai.

lole lole Bilbaa.

role role Rikou.

lode lode $B a^{\prime} a$.

lode lode Tii.

lode lode, lo lode Dengka.

lode lode Oenale.

n-lolok maa-f (2) mata-n natlolo (3) lolo-n (4) po?a-n lolo-n Molo. 1) someone sticks their tongue out. (M:284) 2) he has a bulging eye-ball. (M:xlviii) 3) neck artery. (M:284) 4) cavity in the neck, or the Adam's apple. (M:284)

*lodo Morph: *lodo-k. PRM. straight, right. [Form: regular $* \mathfrak{d}>* * 1 / * 1$ in Meto (and Helong).]

lo lodo-k Termanu. right, without curves. (J:320)

lo lodo-? Korbafo.

lo lodo-k Bokai.

lo lodo-? Rikou.

lo lodo-k $B a^{\prime} a$.

lo lodo-k Tii.

lo lodo-? Dengka.

na-m|nono, na-nono (2) m|nono|? Kotos Amarasi. 1) straighten, correct. 2) straight, correct. m|nono|? Molo. agree, fair/honest. (M:327)

m|nono|? Kusa-Manea. straight.

Out-comparisons:

lolo Semau Helong. straight; righteous, just, fair.

mololo Kisar. straight.

kelolo, lolo Hawu. straighten, stretch out.

kəlodo Lamaholot, Lewotobi. straight. [Note: language of east Flores ISO 639-3 [slp].] (Keraf 1978:296)

*loe1 Rote. go down.

loe Termanu. go down; be low. (J:320)

loe Korbafo.

loe Bokai.

loe Bilbaa.

loe Rikou.

loe $B a^{\prime} a$.

loe Tii.

loe Dengka.

Out-comparisons:

loe Hawu. lowness. (J:320)

* loe 2 PRM. listless, weak.

$\mathbf{a o}=$ na ma-sa-loe $B a^{\prime} a$. feel tired, weak or listless. [Form: $\mathbf{a o}=\mathbf{n a}=$ 'body-3GEN'.] (J:725)

ao=na ma-sa-loe Dengka. feel tired, weak or listless. (J:725)

$\mathbf{a o}=$ na ma-sa-loe Oenale. feel tired, weak or listless. (J:725)

masaloe Dela. weak.

na-noe-b-a? Kotos Amarasi. listless, apathetic, weak.

Out-comparisons:

onoe noe Semau Helong. powerless, weak, faint, soft, weak-willed, wimp. Borrowed from: Meto (shown by irr. ${ }^{*} 1=$ $n$ correspondence).

*lohas CERM. equipment, outfit, clothes. loPas Termanu. equipment. (J:318)

loa-? Bilbaa.

pake_nohas Kotos Amarasi. clothes. na-nohas Molo. equips oneself. (M:378) 
Out-comparisons:

lohas Semau Helong. ornament, decoration.

*loke PRM. hang. Pattern: $\mathrm{k}-8^{\prime}\left({ }^{*} \mathrm{k}=k\right.$ in

Dengka; expect $?$ or $\varnothing)$.

lo lo?e (2) lo?e lo?e (3) belo?e Termanu. 1) hang up, hang onto. 2) be hanging, be hanging up. 3) be hanging, be hanging up; for plural subjects. (J:321)

lo lo?e Korbafo.

lo lo?e Bokai.

lo loke Bilbaa.

lo lo?e Rikou.

lo lo?e $B a^{\prime} a$.

lo lo?e Tii.

lo loke-? Dengka.

na-k|noe Kotos Amarasi. hang, suspend. [Form: Jonker (1908:321) gives Meto noen 'hung up'.]

Out-comparisons:

loen Semau Helong. hanging, suspended, holding on.

*lole1 PRM. kind of tuber. [irr. from PRM: * $1>r / l$ in Meto $(\operatorname{expect} n) ;{ }^{*} \mathrm{e}>$ $i$ in most Meto]

lole Termanu. kind of tuber. (J:323)

lole Korbafo.

lole Bokai.

lole Bilbaa.

lole $B a^{\prime} a$.

lole Tii.

lole Dengka.

lole Oenale.

rauk rori Ro'is Amarasi. sweet potato.

rauk rori Kotos Amarasi. sweet potato.

lole Fatule'u. tuber.

loli Molo. sweet potato. (M:284)

Out-comparisons:

lole Semau Helong. taro.

*lole2 PRM. spool.

lole Termanu. a) (rotating) tool used to wind up yarn; they wind yarn on the yarn winder. b) a completely different tool, a kind of frame over which the threads are stretched out in order to be divided into boak and on which they are tied for dyeing them according to their different patterns. (J:323)

lole Korbafo.

lole Bokai.

lole Rikou.

lole $B a^{\prime} a$.

lole Tii.

lole Oenale.

?|none|? (2) na-?|none|? Kotos Amarasi. 1) pulling device (e.g. for drawing water from a well).

2) lower down with a rope.

<an-none>(2) P|none|? Molo. 1) winds up (cotton thread). 2) spool used to wind cotton thread. (M:381)

Out-comparisons:

slale Helong. [irr. from PRM: ${ }^{*} \mathrm{o}=$ $a$ correspondence] (J:323)

* Iolir Rote. roll, roll around, wallow. [Note: Jonker (1908:324) gives potential cognates in languages of South Sulawesi: Makassar doli? 'wallow', loli? 'roll (n.)', Bentong lulir 'roll', and Bugis lole 'roll up'.]

loli Termanu. wallow, roll around. ( $\mathrm{J}: 324)$

loli Korbafo.

loli Bokai.

loli Bilbaa.

loli Rikou.

loli $B a^{\prime} a$.

loli Tii.

loli (2) lolil Dengka. 1) wallow, roll around. 2) roll, like a stone. (J:324)

loli (2) lolir Oenale. 1) wallow, roll around. 2) roll, like a stone. (J:324)

Out-comparisons:

lolit (2) klolit East Tetun. 1) roll or bowl along. (Mo:132) 2) roll. (Mo:110)

*lolo1 PRM. liana, vine.

lolo kode-k Termanu. kind of creeper. (J:326)

lolo Dengka. kind of winding plant. (J:726) 
lolo_ne?et Oenale. kind of winding plant. (J:726)

nono Kotos Amarasi. kind of jungle rope, liana; descent group. 'lianas which encircle the clan and hence symbolize its fertility' (Schulte Nordholt 1971:116).

$<$ nono mofa $>$ (2) < nono panu $>$ (3) <nono siumloli> Molo. 1) kind of liana. 2) kind of liana. 3) kind of liana with a white stamen the sap of which can be used successfully to quickly heal fresh wounds. (M:384)

\section{Out-comparisons:}

lolo Helong. (J:726)

*lolo2 PRM. stretch out.

lolo Termanu. stretch out. (J:325)

lolo Korbafo.

lolo Bokai.

lolo Bilbaa.

lolo Rikou.

lolo $B a^{\prime} a$.

lolo Tii.

lolo Dengka .

lolo Oenale.

n-nono Kotos Amarasi. push string/ thread back and forth, thread string.

n-nono Molo. bifee-I an-nono mau (beti) the woman applies striped rows along the middle part of the ikat woven cloth. (M:384)

\section{Out-comparisons:}

lolo Semau Helong. separate string. Separate the string for preparation for making a pattern for weaving cloth.

lolo Hawu. stretch out the yarn. $(\mathrm{J}: 325)$

*lolo3 PRM. beam, stick.

lolo Termanu. the beams that lie across the lungus. (J:326)

lolo Korbafo.

lolo Bokai.

lolo Bilbaa.

lolo Rikou.

lolo $B a^{\prime} a$.

lolo Tii. lolo Dengka.

nono|? Kotos Amarasi. stick, rod, pole; counter for long round things (e.g. cigarettes, needles).

\section{Out-comparisons:}

lolo Semau Helong. stick, a counter for long things like: sticks, rope, chain.

lollo Kisar. stalk part of a plant.

wulu lolo Central Lembata. long wooden stick that is part of the loom. (Hanna Fricke pers. comm.)

lolo-n Central Lembata. leaf. (Fricke 2015)

*lolo4 Morph: *lolo-k. PRM. long impression in the ground; watercourse, valley.

oe_lolo-k (2) lolo-k Termanu. 1) drain, water duct. 2) path impression or groove in the ground, where the plant growth has been trodden down as the result of wildlife constantly following this path; strait. (J:326)

oe_lolo-? Korbafo.

oe_lolo-k Bokai.

oe lolo-? Bilbaa.

oe_lolo-? Rikou.

oe_lolo-k $B a^{\prime} a$.

oe_lolo-k Tii.

oe_lolo-? Dengka.

oe_lolo-? Oenale.

nono-n (2) ?|nono|f Kotos Amarasi.

1) dry area associated with water, e.g. the beach, or a river bed. [Note: Jonker (1908:326) gives Meto oe lolo? and Amarasi oe rorok.] 2) valley.

$<$ oe_nono $>(2)<$ anono kekfok am anasi kekfok $>$ Molo. 1) riverbed. 2 ) the hidden deep river valley, the dense primeval forest. (M:384)

Out-comparisons:

oelolo, oenonon (2) 'hnono Helong. 1) drain, water duct. (J:326) 2) valley. Borrowed from: Meto. 
*lombu Rote. moss, algae, duckweed. Etym: *lumbu 'plant species' (Only supported by four putative reflexes in Blust and Trussel (ongoing).). [irr. from PMP: * $\mathrm{u}>*_{\mathrm{o}}$ ]

lopu (2) lopu lee (3) lopu tasi Termanu. 1) moss. 2) duckweed. 3) algae. (J:328)

lopu Korbafo.

lopu Bokai.

lopu Bilbaa.

lopu Rikou.

lompu $B a^{\prime} a$.

lombu Tii.

lombu Dengka.

lombu Oenale.

*loo1 Rote. stretch. [Form: Rote *lele 'time' also shows the correspondence of Helong $d=$ Rote $l$.]

loo Termanu. with outstretched hand to keep, reach or indicate. ( $\mathrm{J}: 317)$

loo Korbafo.

loo Bokai.

loo Bilbaa.

loo Rikou.

loo $B a^{\prime} a$.

loo Tii.

loo Dengka.

loo Oenale.

\section{Out-comparisons:}

dooy Semau Helong. stretch, reach out.

lolo, kelolo Hawu. stretch out. [Note: Jonker (1908:317) includes this form preceded by the note 'compare' indicating that he does not consider it definitely cognate.] (J:317)

*loo2 Rote. household.

uma_loo (2) na-uma_na-loo

Termanu. 1) house in more or less metaphorical senses. 2) s/he has a household; also: establish a household, marry. (J:667)

ume loo Dela. household.

${ }^{\circ} \mathbf{l o o}$ T Kusa-Manea. village. Borrowed from: probably Tetun.

\section{Out-comparisons:}

lo?on, lo?o East Tetun. holiday house, country house. (Mo:133)

lo?o (2) tuur lo?o Fehan Tetun.

1) a few houses together, not yet a leo (hamlet). 2) live separate from other people (not in a village).

*lopo CERM. shelter, hut. Etym: *lopaw. [irr. from PMP: ${ }^{*} \mathrm{o}>{ }^{*} \mathrm{o} ;{ }^{*} \mathrm{p}=\mathrm{p}$ ] [irr. from PRM: *1 > Meto r/l] [History: The irregularities at every level indicate that this term probably spread by borrowing.]

lopo Termanu. shelter or hut made in a tree or in dry fields. (J:327)

lopo Rikou. small shelter made in a tree or in a field where someone can stay to protect crops. (own field notes)

ropo Kotos Amarasi. Timorese round social activity houses. typically with a cone shaped roof made from thatch supported on four poles and without any walls. Not commonly built in Amarasi anymore. Borrowed from: Rote (shown by irr. $* 1=r$ correspondence).

lopo Molo. round house without walls, especially as a storehouse with attic. (M:286)

\section{Out-comparisons:}

klobor, klobar East Tetun. hut with a gable roof. [irr. from PRM: $b=* \mathrm{p}$ correspondence] (Mo:110)

ləpo Sika. house. Usage: Hewa dialect/variety. (Fricke 2014:96)

* losa Rote. arrive, reach, until, up to.

losa Termanu. arrive, reach, until, up to. $(\mathrm{J}: 328)$

losa Korbafo.

losa Bokai.

losa Bilbaa.

losa Rikou.

losa $B a^{\prime} a$.

losa Tii. 
losa Dengka.

losa Oenale.

Out-comparisons:

lisu Semau Helong. arrive. [irr. from PRM: $*_{\mathrm{O}}=i$ correspondence]

loha, lohe Hawu. until. (J:328)

*lua|t $P R M$. cave, cavern. See: *lea. Etym:

*luay (Blust and Trussel (ongoing) also reconstruct formally similar PWMP *Ruqan 'hole, pit' and PMP *lubay 'hole, pit'.). [Form: Final *t in wRM could be from the nominalising suffix $t$, though why this should be attached to this form is inexplicable.]

lua-k $B a^{\prime} a$. hole, cave. [Form: with variant lea-k.] (J:727)

luat Dengka. hole, cave. (J:727)

luat Oenale. hole, cave. (J:727)

nuat $R o$ 'is Amarasi. cave.

nuat Kotos Amarasi. cave.

nuat Molo. cave. (M:389)

Out-comparisons:

uee lu?a East Tetun. well. [irr. from PMP: Ø> ?] (Mo:134)

*lua1 PRM. boiling water, boil over.

Etym: *luab 'swell up, as boiling rice; boil over'.

lua Termanu. 1) rise up of water when boiling, boiling, boiling over. (J:331)

lua Korbafo.

lua Bokai.

lua Bilbaa.

lua Rikou.

lua $B a^{\prime} a$.

lua Tii.

lua Dengka.

lua Oenale.

na-?nua Kotos Amarasi. boiling water that is bubbling away.

<na-nua> Molo. boils. (M:388)

Out-comparisons:

lua Semau Helong. boil over. *lua2 Morph: *lua-k. PRM. wide, open, openness. Etym: *luqaR 'outside, wide open spaces; loose'. [irr. from PRM: $* \mathrm{u}>o$ in Rote]

loa-k Termanu. open, wide; openness, width. (J:318)

loa-? Korbafo.

loa-k Bokai.

loa-? Bilbaa.

loa-? Rikou.

loa-k $B a^{\prime} a$.

loa-k Tii.

loa-? Dengka.

loa-? Oenale.

mai/nua|n Ro'is Amarasi. openness; opportunity. [Form: source of initial mai currently unknown.]

mai/nua|n Kotos Amarasi. openness; opportunity.

mai/nua|n Molo. wide, open. [Form: Middelkoop (1972:299) has a note: 'cf. meonuan', which presumably is an alternate form, but no separate entry is given for this form.] (M:299)

ma|nu?a-k Kusa-Manea. outside. [Sporadic: *VV-k > *VV? > VPV in Kusa-Manea (perceptual metathesis) followed by attachment of semi-productive -k suffix.]

\section{Out-comparisons:}

bluay Semau Helong. wide, broad, open.

luan East Tetun. wide, broad; vast, extensive. (Mo:134)

*lui PRM. pull off, remove.

lui Termanu. take off something that hangs. (J:332)

lui Korbafo.

lui Bokai.

lui Bilbaa.

lui Rikou.

lui $B a^{\prime} a$.

lui $T$ ii.

lui Dengka.

lui Oenale. 
n-nui Kotos Amarasi. take off (e.g. footwear).

n-nui Molo. pulls off (a horse's bridle), pulls/fetches (bark from a tree). (M:389)

\section{Out-comparisons:}

luit East Tetun. graze lightly, hardly touching; to remove or take out adroitly. (Mo:134)

*luku1 PRM. watch silently, brood. Pattern: k-9.

lu?u Termanu. brood. (J:336)

lu?u Korbafo.

lu?u Bokai.

luku Bilbaa.

lu?u Rikou.

lu?u $B a^{\prime} a$.

lu?u Tii.

lu?u Dengka.

lu?u Oenale.

n-nu?u Kotos Amarasi. watch silently, spy.

n-nu?u Molo. brood (of chicken). (M:388)

Out-comparisons:

ruku Hawu. [Sporadic: *1 $=r$ correspondence] (J:336)

*lukur Rote. bent and strengthless limb, paralysed. Etym: *lukut 'roll or crumple up'. Pattern: k-7/8' (Dengka $k$ Oenale ? correspondence, expect either $k$ in both for pattern 7, or ? in both for pattern 8). [History: Blust and Trussel (ongoing) also reconstruct PWMP *lu(y)kuq 'bend, curve' (supported by two reflexes), and POc *lukun 'bend, as an arm or leg'.] [Semantics: Jonker relates the first part of Termanu lu? la?a-k with lu?u 'brood' (*luku), lu?u 'lie down (said of animals)', and lu?u 'kneel down'. Of these, the semantic connection between the last form and 'bent' is particularly plausible.]

lu?u-k, lu?u ei-k (2) lu?u_la?a-k (3) na-ma-lu?u Termanu. 1) bent and strengthless, paralysed said of the arm or especially the legs. 2 ) = lu?u-k. 3) become bent and lame (of the legs), get bent and lame legs. (J:337)

lu?u-? (2) lu?u la?a Korbafo.

lu?u-k (2) luku laka-? Bokai.

(2) luku laka-? Rikou.

lu?u-k (2) lu?u la?a-k $B a^{\prime} a$.

lu?u-k (2) lu?u la?a-k Tii.

(2) luka_laka-? ? Dengka. [Form: Jonker explicitly states the first $a$ in this form may be a spelling error instead of $u$.]

mamalu?u Oenale.

*Iuli PRM. taboo.

luli-k Termanu. forbidden, holy. (J:323)

luli-? Korbafo.

luli-k Bokai.

luli-? Bilbaa.

luli-? Rikou.

luli-k $B a^{\prime} a$.

luli-k Tii.

luli-? Dengka.

luli-? Oenale.

nuni Kotos Amarasi. taboo.

nuni (2) na-nuni Molo. 1) taboo.

2) observes a taboo. (M:167)

\section{Out-comparisons:}

luli Semau Helong. taboo, avoided, forbidden, sacred.

luli Kisar. taboo.

luli, lulik East Tetun. prohibited, forbidden; sacred, holy. (Mo:135)

luli Tokodede. holy, sacred. (Schapper and Wellfelt 2018:109)

*lulun PRM. roll up, as a mat. Etym: *lulun (Blust and Trussel (ongoing) also reconstruct PCMP *lunu 'roll up' on the basis of the Termanu form and a Kei cognate. This reconstruction is unconvincing given that most varieties of Rote have lulu.). [Sporadic: consonant metathesis *1Vn $>*_{n} \mathrm{Vl}$ in Termanu, Bokai, and Ba'a with subsequent loss of final consonant.] 
[Form: The hypothesis of consonant metathesis to explain the otherwise irregular medial $n$ in these languages provides language internal evidence that the PRM form had a final *n.]

lunu Termanu. roll something up so it makes a roll. (J:334)

lulu Korbafo.

lunu Bokai.

lulu Bilbaa.

lulu Rikou.

lunu $B a^{\prime} a$.

lulu Tii.

lulu Dengka.

lulu Oenale.

n-nunu Kotos Amarasi. roll up.

<na-nunu> Molo. roll up (e.g. mat). (M:393)

Out-comparisons:

lulu Semau Helong. scroll, roll, waves, fold.

lulun East Tetun. roll up, wrap up. (Mo:135)

*Iunu Morph: *ka-lunu. PRM. wooden headrest, pillow. Etym: *qalunan. [irr. from PMP: *a $>*_{\mathrm{u}}$ (sporadic assimilation)] [Sporadic: consonant metathesis * $\mathrm{IVn}>n V l$ in Bilbaa, Rikou, and wRote.] [Form: The initial element has apparently been reanalysed in Rote as connected with reflexes of *kaiu 'wood'. The out-comparisons point to pre-RM **ka(r)lun[u/i].]

kai/lunu Termanu. cushion, pillow. (J:216)

kai/lunu Korbafo.

kai/lunu Bokai.

kai/nulu Bilbaa.

(?)ai/nulu-? Rikou.

kai/lunu $B a^{\prime} a$.

kai/lunu Tii.

(?)ai/nulu-? Dengka.

?ai/nulu-? Oenale.

Pai/nulu Dela. pillow.

aka/?nunu? Kotos Amarasi. pillow.

<aka/nunu>, <aika/nunu> Molo. pillow. (M:13)

\section{Out-comparisons:}

kluni (2) hluni Semau Helong. 1) pillow. 2) use pillow.

kluni, karluni East Tetun. pillow or cushion. (Mo:111, 102)

luni Kisar. pillow.

kaluni, kluni Ili'uun. head-rest, pillow. (dJ:120)

karayulu Buru. pillow, headrest. (Grimes and Grimes 2020:474)

*lusə PRM. wrap. [irr. from PRM: *s > ?

in Termanu, Korbafo, and Ba'a]

na-lu?e Termanu. wrap inside, cover. ( $\mathrm{J}: 332)$

na-lu?e Korbafo.

na-luse Bokai.

na-luse Bilbaa.

na-luse Rikou.

na-lu?e $B a^{\prime} a$.

na-luse $T i i$.

na-lusa Dengka.

na-lusa Oenale.

n-tai_nusa Kotos Amarasi. wrap. [Form: tais $=$ 'sarong', na-tai $=$ 'clothe (v.)'.]

Out-comparisons:

lose Semau Helong. wrap, cover.

*lutə Morph: *lutə-k. CERM. firebrand, burning piece of wood. Etym: *alutən.

ha?i_lute-k Termanu. burning piece of wood, a firebrand. (J:335)

ha?i_lute-? Korbafo.

ai nuta|? Kotos Amarasi. burning stick, firebrand.

nuta|? Molo. glowing stumps of firewood or torch. (M:395)

*lutu1 Morph: *ma-lutu-k, *na-lutu. $P R M$. fine, delicate, tiny.

lutu-k (2) na-lutu Termanu. 1) delicate, small. 2) make something fine, crush, from that: make into pieces, spoil, wreck, ruin. (J:335f)

lutu-? (2) na-lutu Korbafo.

lutu-k (2) na-lutu Bokai.

lutu-? (2) na-lutu Bilbaa.

lutu-? (2) na-lutu Rikou.

lutu-k (2) na-lutu $B a^{\prime} a$. 
lutu-k (2) na-lutu Tii.

lutu-? (2) na-lutu, na-mba-lutu-? Dengka.

lutu-? (2) na-lutu Oenale.

m|nutu|? (2) na-nutu Kotos Amarasi. 1) fine, tiny. 2) slice (typically something like vegetables).

na-nutu Molo. cuts (meat). (M:395)

Out-comparisons:

blutu? (3) tai lutu-n Semau Helong. 1) tiny, teeny, small. 3) intestine.

lotu(k) East Tetun. thin, slender, dainty. (Mo:134)

blutuk Sika. small, short. (Pareira and Lewis 1998:24)

*lutur Rote. pile up. Etym: **lutuR (pre-RM). [History: Schapper (2020) discusses the distribution and semantics of this cognate set throughout Linguistic Wallacea in some detail.]

lutu_batu Termanu. pile up stones, especially under a tree in order to make a resting place there; piled up heap of stones. (J:335)

lutu batu Korbafo.

lutu_batu Bokai.

lutu_batu Bilbaa.

lutu_mpatu $B a^{\prime} a$.

lutu_mbatu Dengka.

lutu_mbatu Oenale.

Out-comparisons:

lutan Helong. pile up. [irr. from $P M P: * \mathrm{u}>a](\mathrm{J}: 335)$

lutu East Tetun. hedge, fence, enclosure, circular mud wall. (Mo:135)

lutu(r) Ili'uun. pile up. (dJ:125)

lukur Kisar. rock wall.

lotar Central Lembata. pile up. (Hanna Fricke pers. comm. December 2017)

*luu1 Morph: *lu luu. Rote. castoroil plant. [Semantics: Whether the semantic connection between 'casuarina tree' and 'castor-oil-plant' is likely or not is unclear to me as a nonbotanist, but the two trees do appear to have similar seed pods. Given the existence of *kaiou being retained in Rote with the meaning 'casuarina tree', as well as geographically distant Helong and Dadu'a having this meaning, it seems most likely that the pre-Rote referent was casuarina tree with semantic shift in Rote.]

lu luu(-k) Termanu. castor-oil-plant (called damar jarak in Kupang). Ricinus communis. [Semantics: Scientific identification from Heyne (1950:928).] (J:330)

lu luu Korbafo.

lu luu Bokai.

lu luu Bilbaa.

lu luu Rikou.

lu $\sim$ luu-k $B a^{\prime} a$.

lu luu Tii.

lu luu Dengka.

lu luu Oenale.

Out-comparisons:

luu Funai Helong. casuarina tree.

luu Dadu'a. casuarina tree. (Penn 2006:98)

<lolo> Hawu. castor-oil-plant. Ricinus communis. (Heyne 1950:928)

*luu2 PRM. tears (crying). Etym: *luhəq. [irr. from PMP: *ə $>*_{\mathrm{u}}$ (sporadic assimilation)]

luu Termanu. tears. Usage: Normally compounded with oe 'water' in all Rote lects except Bilbaa. (J:330)

luu Korbafo.

luu Bokai.

luu Bilbaa.

luu Rikou.

luu $B a^{\prime} a$.

luu, luul Dengka.

luu Oenale.

nuu Kotos Amarasi. tears.

maat nuu Molo. tears. (M:387)

Out-comparisons:

luun East Tetun. tears. (Mo:135) 
*ma PRM. and. Etym: *mah. ma Termanu. and. (J:338)

ma Korbafo.

ma Bokai.

ma Bilbaa.

ma Rikou.

ma $B a^{\prime} a$.

ma Tii.

ma Dengka.

ma Oenale.

$=\mathbf{m a}$ Ro'is Amarasi. and.

=ma Kotos Amarasi. and. [Form: This connector has vowel initial forms $=\mathbf{a m a}$ or $=\mathbf{a m}$ after consonant final roots. The final vowel is often deleted yielding $=\mathbf{m}$.]

ma Molo. and. (M:295)

*maa Morph: *maa-k. PRM. tongue.

Etym: *maya (PCEMP).

maa-k Termanu. tongue. (J:338)

maa-? Korbafo.

maa-k Bokai.

maa-? Bilbaa.

maa-? Landu. tongue. (own field notes)

maa-? Rikou.

maa-k $B a^{\prime} a$.

maa-k $T i i$.

maa-? Dengka.

maa-? Oenale.

maa-f Ro'is Amarasi. tongue.

maa-f Kotos Amarasi. tongue.

maa-f Molo. tongue (in general). (M:373)

ma maa-f Kusa-Manea. tongue.

Out-comparisons:

mee Semau Helong. tongue.

*mada Rote. dry up, evaporate. Etym: *maja.

mada Termanu. dry up, dried up. (J:339)

mada Korbafo.

mada Bokai.

mada Bilbaa.

mada Rikou. mada $B a^{\prime} a$.

mada Tii.

mada Dengka.

mada Oenale.

mada-? Dela. dry.

Out-comparisons:

mara(n) East Tetun. dry, dried (not wet); $v$. to dry. (Mo:139)

*mado PRM. medicinal plant. See: *mode. [irr. from PRM: *a $>o$ in Rote (probably influence from *modo)] [Form: The evidence from Tetun favours reconstructing penultimate $*_{\mathrm{O}}$ with an irregular sound change in Meto rather than reconstructing *a with an irregular sound change in Rote.]

modo Termanu. medicine, also magic.

(J:359)

modo Korbafo.

modo Bokai.

modo Bilbaa.

modo Rikou.

modo $B a^{\prime} a$.

modo Tii.

modo Dengka.

modo Oenale.

maro Kotos Amarasi. tobacco.

malo Molo. medicine. (M:302)

Out-comparisons:

modo East Tetun. vegetables, leaves or fruit cooked as vegetables. [irr. from PRM: *a $=o$ correspondence (same as Rote)] (Mo:142)

*mae1 PRM. itchy tuber. Amorphophallus species. Etym: **maya (preRM). [Semantics: 'A few species [of Amorphophallus] are edible as 'famine foods' after careful preparation to remove irritating chemicals ... These small to massive plants grow from a subterranean tuber.'('Amorphophallus', Wikipedia. en.wikipedia.org/ wiki/Amorphophallus. Accessed 17 September 2020).] 
mae Termanu. kind of crop that causes itchiness, with thick tubers that are cooked and given to pigs as food. (J:340)

mae Korbafo.

mae Bokai.

mae Bilbaa.

mae Rikou.

mae $B a^{\prime} a$.

mae Tii.

mae Dengka.

mae Oenale.

mae Kotos Amarasi. kind of large taro which makes people itch, it can be used to feed to pigs.

lauk mae(-I) (2) Plali_mae Molo. 1) kembang bangkai $=$ because the flower emits the stink of a corpse. Amorphophallus variabilis. (M:296) 2) kind of taro. (M:260)

\section{Out-comparisons:}

maek Fehan Tetun. short tree people used to eat when very hungry; you swell up when you eat it, it is katar (itchy). It must be rendered miis $[\mathrm{OE}=$ 'insipid'] before eating. It has a flowering stem about a foot long, which is red at the top, yellow in the middle and green at the bottom.

maek East Tetun. plant with edible tubers. (Mo:136)

mai Tokodede. taro (both edible and non-edible). (Klamer 2002)

madza Bima. kind of taro. [Note: For $* \mathrm{y}>d s$ compare *bayu $>$ mbadzu 'pound' and *layaR $>$ lodza 'sail'.] (Ismail et al. 1985:85)

* mae 2 PRM. shy, ashamed, embarrassed. Etym: *ma-həyaq (PCEMP *mayaq).

mae Termanu. ashamed, embarrassed.

$$
\text { (J:340) }
$$

mae Korbafo.

mae Bokai.

mae Bilbaa. mae Rikou.

mae $B a^{\prime} a$.

mae Tii.

mae Dengka.

mae Oenale.

n-mae Kotos Amarasi. shy, ashamed, embarrassed.

mae Molo. ashamed. (M:295)

Out-comparisons:

mae Semau Helong. shame, ashamed, shy, embarrassed, bashful.

moe East Tetun. be shy, modest, demure, reserved, or coy; $n$. shyness, bashfulness, etc. [irr. from PMP: *a $>$ o] (Mo:142)

*mafo PRM. cool, shadow. [Form: Meto hafo? 'shadow' and Bima hawo 'shadow' point to earlier **kabo. The forms with initial $m$ could be reflexes of **ma-kabo.]

ma mafo Termanu. become cold, not be warm anymore, of water to the taste, etc. (J:341)

ma mafo Bokai.

ma mafo Rikou.

ma mafo $B a^{\prime} a$.

ma mafo Tii.

mafo mafo (2) mafo-? Dengka.

1) become cold, not be warm anymore, of water to the taste, etc. (J:341) 2) shadow. (J:729)

mafo (2) mafo-? Oenale. 1) become cold, not be warm anymore, of water to the taste, etc. (J:341) 2) shadow. (J:729)

na-mafo Ro'is Amarasi. shelter, took shade.

mafo|?, hafo|? Kotos Amarasi. shadow.

mafo|? Molo. shadow. (M:297)

Out-comparisons:

mahan, mahon East Tetun. shade, out of the sun. (Mo:136)

mavo (2) rai-mavo Hawu.

1) cool. 2) shadow. (J:341)

ma maho Dhao. shade. 
<maü> Kambera. shade, shelter, house; look for shade or shelter. (On:283)

$<$ mawu> Anakalang.

<mawo> Weyewa. [Note: also in Mamboru.]

<magho> Kodi.

hau Ende. shade.

mawo (2) hawo Bima. 1) cool down, no longer hot, shady. (Ismail et al. 1985:87)

2) shadow. (Ismail et al. 1985:39)

*mafu PRM. drunk. Etym: *ma-buhək. mafu-k Termanu. drunk. (J:341)

mafu-? Korbafo.

mafu-k Bokai.

mafu-? Bilbaa.

mafu-? Rikou.

mafu-k $B a^{\prime} a$.

mafu-k Tii.

mafu-? Dengka.

mafu Oenale.

n-mafu Ro'is Amarasi. drunk.

n-mafu Kotos Amarasi. drunk.

n-mafu Molo. drunk. (M:297)

Out-comparisons:

mahu Semau Helong. drunk.

* mai $n R M$. come. Etym: *ma(R)i.

mai Termanu. come, arrive. (J:341)

mai Korbafo.

mai Bokai.

mai Bilbaa.

mai Rikou.

mai $B a^{\prime} a$.

mai Lole. (Zacharias et al. 2014)

mai Tii.

Out-comparisons:

maa Semau Helong. come.

mai East Tetun. come; prep. to, for, here. (Mo:136)

mai Hawu. come.

*mali Morph: *mali-k. nRM. bitter. [irr. from PRM: $* \mathrm{i}>e$ in Meto]

mali-? Rikou.

mali-? Ba'a. bitter. (J:730)

mali-k Tii. <an-mane> Molo. brackish. [Form: This form may have a double final vowel; e.g. manee, if so it is unrelated and probably belongs under *rekət.] (M:305)

Out-comparisons:

mali?, madi? Funai Helong. bitter, salty.

mali? Semau Helong. bitter.

*malis PwRM. laugh, smile. Etym: *malip (PCEMP). [Form: expected $* \mathrm{p}>\mathrm{s}$ /_\# (\$3.5.1.3).]

mali Dengka. laugh. (J:730)

mali Oenale. laugh. (J:730)

n-mani (2) n-mainis Ro'is Amarasi.

1) laugh. 2) laugh at someone. [Form: As discussed in \$3.5.1.3, the transitive form n-manis 'laugh at' appears to attest final $* \mathrm{p}>s / \mathrm{i} \#$. The pair na-mtau 'scared' and namtaus 'scared of' (see *taku) also has transitivity marked with final $s$ and in this case the final $s$ of the transitive form may also be a reflex of the final consonant of PMP *takut.]

n-mani (2) n-manis Kotos Amarasi. 1) laugh. 2) laugh at someone.

n-mani (2) n-manis Amfo'an. 1) laugh. 2) laugh at someone.

ma main (2) $\quad$ ma $\sim$ mains $=\mathbf{a a}$ Kusa-Manea. 1) laugh. 2) laugh at someone.

Out-comparisons:

mali Semau Helong. laugh.

mari Dhao. laugh at. mari (2) mare Hawu. 1) laugh (pl.). 2) laugh (sg.).

*malus PRM. betel-pepper. [History: The non-AN Timor languages have cognates of this form. Heston (2017:82) lists: Makasae malu, Makalero malu, Fataluku maluh, and Oirata malu.] malu Termanu. betel (fruit or leaf). Usage: poetic. (Fox 2016b:31)

maunus Ro'is Amarasi. betel-pepper. manus Kotos Amarasi. betel-pepper. 
manus Molo. betel-pepper. (M:308) manus Kusa-Manea. betel-pepper.

Out-comparisons:

malus East Tetun. betel-pepper (Piper Betel) whose leaves are used for chewing with betel nut and lime (mama). (Mo:138)

maul Mambae, South. betel, can be either the leaf of the pepper. (Grimes et al. 2014b:98)

malu Ili'uun. betel-pepper. (dJ:126)

maluh, malhu Kisar. betel vine with bean-like fruit.

malus Welaun. leaf of the betel vine.

malu Central Lembata. betel vine. (Fricke 2015)

* mama PRM. chew betel nut. Etym: *mamaq 'chew without intending to swallow, as betel nut; premasticated food to give to an infant; premasticated food'.

mama Termanu. chew, especially chew betel nut. (J:344)

mama Korbafo.

mama Bokai.

mama Bilbaa.

mama Rikou.

mama $B a^{\prime} a$.

mamah Lole. chew betel nut. (Zacharias et al. 2014)

mama Tii.

mama Dengka.

mama Oenale.

mama-t Ro'is Amarasi. betel quid.

n-mama Kotos Amarasi. chew betel.

mama Molo. chew betel nut. (M:303)

Out-comparisons:

mama East Tetun. wad of bua, malus, hoo ahu (betel nut, betel-pepper, and lime), which is put in the mouth for chewing. (Mo:138)

mama Kisar. eat betel. *mamor PRM. garden, orchard. [Sporadic: $* \partial>e / \sigma \#$ in wRote (perhaps $* a>* a>e / \#) . \overline{\text { [ }}$ [Form: Final $* r($ rather than $* n)$ reconstructed on the basis of Kupang Malay mamar.]

mame-k Termanu. garden made in the bush where one plants coconuts, betel nut trees, betel pepper and so on. (J:344)

mame-? Korbafo.

mame-k Bokai.

mame-? Bilbaa.

mame-? Rikou.

mame-k $B a^{\prime} a$.

mame-k Tii.

mame-? Dengka.

mame-? Oenale.

maman Kusa-Manea. grove, orchard, garden with trees (coconut, betel nut, betel-pepper, etc.).

\section{Out-comparisons:}

maman Helong. (J:344)

mamar Kupang Malay. grove, orchard; garden with trees. (own field notes)

*mamis Rote. sweet. Etym: *ma-həmis.

mamis (2) mami Termanu.

1) insipid, sweetish, flavourless.

2) insipid, sweetish of taste, sweet or flavourless of water. (J:344)

mami-? Korbafo.

mami? Bokai.

mami-? Bilbaa.

mamis Rikou.

mamis $B a^{\prime} a$.

mamis Tii.

mamis Dengka. mamis Oenale.

*mana1 PRM. relativiser. [History: Possibly connected with PMP *maRuqanay (PRM *mane, and *mone). Particularly as reflexes of *maRuqanay have the form *mana (as a result of antepenultimate vowel reduction) in some phrases in Rote languages (see *mane).] [Semantics: In the Rote languages mana appears to 
introduce relative clauses with a verb, while foo is used for other relative clauses.]

mana Termanu. used with verbs to form compounds with verbal meaning when the verb is used attributively or independently. (Jonker 1915:106)

mana Rikou. relativiser.

mana Lole. relativiser. (Zacharias et al. 2014)

mana Tii. relativiser; that, who, which. (Grimes et al. 2014a)

mana Dela. relativiser.

mana? Ro'is Amarasi. generic counter/classifier (occurs after the noun and before the numeral). hiin aan-r=ini mana? haa

mana? Kotos Amarasi. generic counter/classifier, less often used than in Ro'is Amarasi.

mana? Molo. counter/classifier. bia mana? mese? a single cow (M:304)

*mana2 Morph: *mana-k. Rote. place. ma mana-k (2) mana-k Termanu.

1) place in which to store something or put something in.

2) used in certain expressions for ma mana-k. (J:345)

ma mana-? Korbafo.

ma mana-k Bokai.

ma mana-? Bilbaa.

ma mana-? Rikou.

ma mana-k $B a^{\prime} a$.

ma mana-k Tii.

ma mana-? Dengka.

ma mana-? Oenale.

Out-comparisons:

mana Semau Helong. place.

mna [məna] Wersing. village. [Note: non-Austronesian language of Alor ISO 639-3 [kvw].] (Schapper 2017:262) ma'na: Sawila. village. [Note: non-Austronesian language of Alor ISO 639-3 [swt].] (Schapper 2017:262)

*ma(n)at $P R M$. orphan.

ana_maa-k Termanu. orphan. (J:339)

ana_maa-? Korbafo.

ana_maa-k Bokai.

ana_maa-? Bilbaa.

ana_maa-? Rikou.

ana_maa-k $B a^{\prime} a$.

ana_maa-k Tii.

ana_maa-? Dengka.

ana_maat Oenale.

ana maat Dela. orphan.

kome? manat Kotos Amarasi. orphan. [Note: Jonker (1908:339) gives Meto aan manat.]

manat (2) <hau mana $>$ Molo. 1) tame. (M:305) 2) planted the, tame tree. (M:304)

*mane Rote. male, man. Doublet: *mone.

Etym: *maRuqanay. [History: PRM had two reflexes of *maRuqanay: *mane and *mone, both of which are attested in wRote, Ba'a, and Tii. The meaning of both forms was probably 'male, man' in PRM. Most of Rote lost *mone, Meto lost *mane, while wRote, Ba'a and Tii differentiated the semantics of the two terms.] [Semantics: That semantic expansion to include 'king' had not occurred at the level of PRM is indicated by Meto in which mone has no hint of this meaning. Instead, PRM *la?i > na?i-f has undergone a similar, though non-identical, semantic expansion. However, the wRote forms in which reflexes of *maRuqanay are combined with apparent reflexes of * Raya (that is, manae- $\mathbf{2} /$ monae-?, etc.) which have the meaning 'big, great' indicate that expansion to 'big, great' may have occurred, from which 'king' would be easily derived.] 
mane-k (2) mane (3) mane feu-k Termanu. 1) prince, king, princely. 2) male, of animals and certain plants. (J:345) 3) son-in-law. (J:134)

mane-? (2) mane(-?) (3) mana feu-? Korbafo.

mane-k (2) mane (3) mana_feu-k Bokai.

mane-? (2) mane (3) mana_feu-? Bilbaa.

mane Landu. king. (own field notes)

mane-? (2) mane (3) mana_feu-? Rikou.

mane-k (2) mane $B a^{\prime} a$.

mane-k (2) mane Tii.

mane-? (4) manae-? (5) namanae Dengka. 4) big. 5) bisu=a na-manae the wound is swollen up (J:735)

mane-? Oenale.

mane-? (4) manae-? Dela. 1) king. 4) big, important. [Form: with variant monae-?.]

Out-comparisons:

blane (2) blanen Semau Helong.

1) male (animal). 2) brother of a woman.

mane East Tetun. male, man; masculine. (Mo:139)

*maneu Morph: *maneu-k, *na-maneu. PRM. bright, light. [Sporadic: consonant metathesis $* \mathrm{mVn}>*_{\mathrm{nVm}}$ in PwRM.] [Form: PwRM *nameu.] neu-k (2) na-neu Termanu. 1) bright, brightness. 2) make clear or pure. (J:394)

neu-? Korbafo.

neu-k Bokai.

neu-? Bilbaa.

neu-? Rikou.

neu-k $B a^{\prime} a$.

neu-k Tii.

meu-? Dengka.

meu-? Oenale.

meu-? Dela. clean. nmeu (2) meu?_sine? Kotos Amarasi. 1) early morning, in two days. [Form: This form has possibly been re-analysed as verbal root with initial $n$ interpreted as the $3 \mathrm{SG}$ agreement prefix. This may be the reason initial $n$ does not occur on the second form.] 2) light (n.).

nmeu Molo. morning dawns. (M:322)

Out-comparisons:

mniu? Funai Helong. clean, pure, holy, undefiled.

niu? Semau Helong. clean, pure, holy, undefiled

*manu PRM. chicken. Etym: *manuk.

manu Termanu. fowl, chicken. (J:346)

manu Korbafo.

manu Bokai.

manu Bilbaa.

manu Rikou.

manu $B a^{\prime} a$.

manu Tii.

manu Dengka.

manu Oenale.

manu Ro'is Amarasi. chicken.

manu Kotos Amarasi. chicken.

manu Molo. chicken. (M:307)

manu Kusa-Manea. chicken.

Out-comparisons:

manu Semau Helong. chicken.

manu East Tetun. bird, a fowl (of any kind). (Mo:139)

*mangu1 Rote. tired.

manu (2) sota_manu-k Termanu.

1) tired. (Jonker 1915:53)

2) tiredness. ana ha hae huu ana mangu-naa-n seli s/he rested because s/he was very tired (J:730)

mangu $B a^{\prime} a$. tired. (J:730)

mangu Tii.

mangu Dengka.

mangu Oenale.

Out-comparisons:

<mànggilu> Kambera. exhausted, tired. [Note: also in Mangili and Lewa.] (On:272)

$<$ màgilu> Anakalang. 


$$
\begin{aligned}
& \text { <mànggula }>\text { Mamboru } . \\
& \text { <manggolo }>\text { Kodi. }
\end{aligned}
$$

*mangu 2 Rote. dry fruit skin. Etym: *ma-Rayu 'dry' (*ma-Rayu only reconstructed for PCEMP. *Rayu reconstructed for PMP). [minority from PMP: *y > *ng] [irr. from PRM: *m > $b$ in all Rote except Korbafo, Bokai, and Bilbaa; *ng > nd in wRote and Tii] [Sporadic: antepenultimate vowel reduction in Termanu, Bokai, Bilbaa, Rikou, and Dengka.] [Semantics: Ross (2003:227) reconstructs POc *maRayo/*Rayo with the meaning 'become withered (of vegetation)'.]

baya_lou-k Termanu. dry fruit skin; also the ash of the burnt skin of a fruit (normally the fruit of the nitas tress, Sterculia foetidea), which is used to make the thread for weaving a little yellow or less white. (J:29)

mayo_lou-? Korbafo.

maya_lou-k Bokai.

maya_lou-? Bilbaa.

baka_rou-? Rikou.

baygu_lou-k $B a^{\prime} a$.

bandu rou-k Tii.

banda_lou-? Dengka.

bandu_rou-? Oenale.

Out-comparisons:

mayu Hawu. be dry. (J:29)

mayo Bima. dry. (Ismail et al. 1985:86)

*masi PRM. salty, salt. Etym: *ma-qasin. masi (2) masi-k Termanu. 1) salty.

2) salt. (J:347f)

masi Korbafo.

masi Bokai.

masi Bilbaa.

masi Rikou.

masi $B a^{\prime} a$.

masi Tii.

masi Dengka.

masi Oenale.

maisi|k Ro'is Amarasi. salt.

masi|k Kotos Amarasi. salt. masi|? Molo. salt. [Note: Jonker (1908:347) gives Meto masi = 'salty'.] (M:310)

Out-comparisons:

masin Semau Helong. insipid. [Note: sila $=$ 'salt'.]

masin East Tetun. salt. (Mo:139)

*masu Morph: *masu-k. PRM. smoke.

Etym: *ma-qasu (PCMP).

masu-k Termanu. smoke of fire, (tobacco); moreover: steam of hot water, of hot viands, exhaled vapour (as visible in cold weather), vapour which rises up out of the ground, mist, also drifting dust. (J:348)

masu-? Korbafo.

masu-k Bokai.

masu-? Bilbaa.

masu-? Rikou.

masu-k $B a^{\prime} a$.

masu-k Tii.

masu-? Dengka.

masu-? Oenale.

masu| 2 Ro'is Amarasi. smoke.

masu|? Kotos Amarasi. smoke. [Note: suma? 'steam, vapour'.]

masu|? Molo. smoke. (M:389)

Out-comparisons:

ai mahun Ili'uun. smoke. (dJ:111)

ai mahu Kisar. smoke.

*mata1 Morph: *mata-k. PRM. eye,

face. Etym: *mata.

mata-k Termanu. eye, face. (J:348f)

mata-? Korbafo.

mata-k Bokai.

mata-? Bilbaa.

mata-? Landu. eye, face. (own field notes)

mata-? Rikou.

mata-k $B a^{\prime} a$.

mata-k Tii.

mata-? Dengka.

mata-? Oenale.

mata-f Ro'is Amarasi. eye.

mata-f(2) et mata-n Kotos Amarasi.

1) eye. 2) in front of. 
mata-f Molo. eye (in general), a spy. (M:312)

mata-f Kusa-Manea. eye.

Out-comparisons:

mata Semau Helong. eye.

mata-n East Tetun. the eyes. (Mo:140)

mata Welaun. eye.

mada Hawu. eye.

*mata2 Morph: *mata-k. PRM. raw, uncooked. Etym: *ma-hataq (Blust and Trussel (ongoing) make several reconstructions with similar forms and semantics.). [Sporadic: $* \mathrm{a}>e / \#$ in wRM.]

mata-k Termanu. raw, not well-done,

(rarely: not cooked). (J:350)

mata-? Korbafo.

mata-k Bokai.

mata-? Bilbaa.

ma mata-? Rikou.

mata-k $B a^{\prime} a$.

mata-k Tii.

mate-? Dengka.

mate-? Oenale.

nau|maet $R o^{\prime}$ is Amarasi. green.

n-mate (2) ma|mate? Kotos

Amarasi. 1) raw. 2) green.

mate-l Amfo' an. green, blue.

$<$ mate $>$ Molo. green, unripe, uncooked. (M:313)

\section{Out-comparisons:}

taa Semau Helong. raw, unripe, rare, crude.

matak East Tetun. green, still growing, immature; raw (not cooked), new, fresh (not stale); inexperienced. (Mo:140)

*mate PRM. die, dead; thoroughly, extremely. Etym: *matay 'die; dead'.

mate (2) mate mate Termanu. 1) die.

2) absolutely, thoroughly. (J:351)

mate Korbafo.

mate Bokai.

mate Bilbaa.

mate Rikou.

mate $B a^{\prime} a$. mate Tii.

mate Dengka.

mate Oenale.

n-mate $R o^{\prime}$ is Amarasi. die.

n-mate Kotos Amarasi. die, dead; extremely, very.

n-mate Molo. died. (M:313)

maet Kusa-Manea. die.

Out-comparisons:

mate Semau Helong. die.

mate East Tetun. die; to extinguish

(fire); to wither, die (plants); to stop, or cease (machinery); dead, stopped. (Mo:140)

made Hawu. die, dead; thoroughly, forcefully.

* mau CERM. kind of plant. [Sporadic: glottal stop insertion in Meto.] [Semantics: vague Rote semantics weakens reconstruction.]

mau Termanu. an unidentified plant or tree. Usage: poetic. [Semantics: Forms a parallel pair with pole (also an unidentified plant).] (Fox 2016b:33)

ma?u Fatule'u. grass.

ma?u Molo. grass, weed. (M:314)

* maus PRM. tame, docile, domesticated. Etym: *maRus (own reconstruction) (PCEMP).

ka-maus (2) ka-mau (3) mata=n mau mau Termanu. 1) tame, domestic. 2) be domestic. 3) he is not shy by nature (said of a person). (J:352)

ka-mau-? Korbafo.

ka-mau-k Bokai.

ka-mau-? Bilbaa.

ma maus Rikou.

ka-maus $B a^{\prime} a$.

ka-mau-k Tii.

ma maus Dengka.

ma maus Oenale.

na-mausa-b (2) n-mau Kotos

Amarasi. 1) domesticate.

2) domesticated, tame.

maus Molo. tame. (M:10) 
Out-comparisons:

hmoa Semau Helong. tame, docile; tame. [Form: Connecting this form with *maRus requires irr. ${ }^{*} \mathrm{R}>\varnothing$ (expect $l$ ) and irr. $* \mathrm{a}>o$. It may thus be a chance similarity.]

maus Fehan Tetun. tame (of animals, birds - not plants, people); can apply to wild animals too if they are unafraid of people (e.g. cuscus).

maus East Tetun. meek, mild, tame, domesticated. (Mo:140)

mhau Waima'a. meek, tame.

maru Ili'uun. tame. (dJ:127)

maru Kisar. tame animals.

mau Bima. tame, domesticated, of an animal; mild, gentle, of a person; also: accustomed to something. (Jonker 1893:51; Ismail et al. 1985:86)

maho, mahu Buru. tame, safe, comfortable, secure in the situation. (Grimes and Grimes 2020:578)

mamalu Saparua. tame, domesticated. [Note: language of Lease Islands ISO 639-3 [spr].] (van Hoëvell 1877:86, 111)

mamaru Haruku. tame, domesticated. (van Hoëvell 1877:86, 111)

(ma)maru Kamarian. soft, slow. (van Ekris 1864:306, 309)

mau Alune. [Note: language of west Seram, central Maluku ISO 639-3 [alp].] (van Ekris 1864:309)

malu Asilulu. [Note: also in Hatusua Kaibobo.] (van Ekris 1864:309)

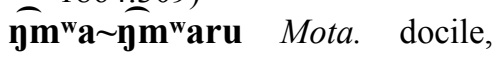
manageable, tame. [Note: language of north Vanuatu ISO 639-3 [mtt].] (Codrington and Palmer 1896:66) mom $^{\mathbf{w}} \mathbf{a u}$ Lewo. tame, quiet; heal over, of sore. [Note: language of south Vanuatu ISO 639-3 [lww].] (Malcolm Ross pers. comm. March 2017)

*mea PRM. red. Etym: *ma-iRaq. [Sporadic: *a > e/_\# in wRM; *VV? > V?V in Amarasi (perceptual metathesis).]

mea Termanu. red (only used in particular expressions): meye mea, kind of reddish snake; kuta mea, kind of red tub; dela mea, kind of red fabric. [Note: pilas = 'red'.] (J:353)

mea Korbafo.

mea Bokai.

mea Bilbaa.

mea Rikou.

mea $B a^{\prime} a$.

mea Tii.

mee Dengka.

mee Oenale.

me?e Ro'is Amarasi. red.

me?e Kotos Amarasi. red.

mee? Amanuban/Amanatun. red.

Out-comparisons:

mea Semau Helong. red, brown. [irr. from PMP: * $\mathrm{R}>\varnothing$ (expect $l$ )]

mean East Tetun. red (all shades). (Mo:140)

mea $\mathrm{Hawu}$. red, reddish-brown.

*meda Morph: *na-meda-k. Rote. wake up.

na-meda-k Termanu. wake up from sleep. (J:353)

na-meda-? Korbafo.

na-meda-k Bokai.

na-meda-? Bilbaa.

na-meda-? Rikou.

na-meda-k $B a^{\prime} a$.

na-meda-k Tii.

na-meda-? Oenale.

Out-comparisons:

melay Semau Helong. restless, stay awake (to comfort the family of the dead). 
*mee Morph: *na-ka-mee. PRM. bleat. [Semantics: onomatopoeia.]

na-ka-mee Termanu. bleat. (J:352)

na-ka-mee Korbafo.

na-ka-mee Bokai.

na-ka-mee Bilbaa.

na-mee Rikou.

na-ka-mee $B a^{\prime} a$.

na-ka-mee Tii.

na-Pa-mee Dengka.

na-Pa-mee Oenale.

na-?|mee Ro'is Amarasi. bleat, like a goat.

na-?|me?e Kotos Amarasi. bleat, like a goat.

Out-comparisons:

mee Semau Helong. sheep's cry.

*mehi Morph: *na-la-mehi. PRM. dream.

Etym: *ma-hipi (Blust and Trussel

(ongoing) reconstruct an alternate PCEMP form *mipi, presumably from *ma-hipi with deletion of the antepenultimate vowel. The reflexes in Timor attest earlier **mepi with *ahi $>*$ ai $>*^{*}$, thus $*$ ma-hipi $>* *$ maipi $>* *$ mepi. Blust and Trussel (ongoing) also reconstruct PAN *Səpi, with the PMP reflex *həpi. The only MP form given supporting PMP *həpi is Tetun mehi. However, PMP $*^{*}>o$ is regular in Tetun, thus showing that Tetun mehi is from earlier **mepi < PMP *mahipi. Whatever the validity of PAN *Səpi, putative PMP *həpi is probably spurious.). [Sporadic: consonant metathesis $* \operatorname{lm}>* \mathrm{ml}>m n$ in most Meto.] [Form: The source of the initial *la in the verbal forms is currently unknown. Jonker (1915:136f) gives a number of other forms with a fossilised la- prefix.]

na-la-me?i (2) me?i-s Termanu.

1) dream. 2) dream (n.). (J:354)

na-la-me?i Korbafo.

na-la-me?i Bokai.

na-la-mei Bilbaa. na-la-mei Landu. dream. (own field notes)

na-la-mei Rikou.

na-la-me?i $B a^{\prime} a$.

na-la-me?i Tii.

na-la-meni, na-la-mein Dengka.

na-la-mein Oenale.

na-la-mei-n (2) mei-t Dela.

1) dream (v.). 2) dream (n.).

na-mnei $R o^{\prime}$ is Amarasi. dream.

na-mnei (2) mnei-t Kotos Amarasi.

1) dream (v.). 2) dream (n.).

na-mnai, na-mnae Kopas. dream.

u-nmai? Molo. I dream. (M:348)

na-mnei Kusa-Manea. dream.

Out-comparisons:

me?i Fehan Tetun. dream.

mehi East Tetun. dream; $n$. a dream. (Mo:141)

*mela Morph: *mela-k. Rote. flea. Etym: *qatimola. [Sporadic: *a $>e$ /_\# in wRote.] [Form: compounded with teke in nRote.]

teke/mela-k Termanu. flea. [Form: Initial teke occurs with at least two other biting insect terms, tekefia-k 'tick', tekefua-k 'kind of wasp'. It also occurs with tekelaba-k 'house gecko'.] (J:614)

teke/mela-? Korbafo.

teke/mela-k Bokai.

teke/mela-? Bilbaa.

teke/mela-? Landu. flea. (own field notes)

teke/mela-k $B a^{\prime} a$.

mele-? Dengka.

mele-? Oenale.

Out-comparisons:

kmela? Funai Helong. flea. hmela Helong. (J:614)

(k)mela East Tetun. flea. (Mo:112,141)

taməla Hawu. (J:614)

*meni1 PRM. fragrant, aromatic. Etym: **məni(R) (pre-RM). [Form: Final pre- $\mathrm{RM} * * \mathrm{R}$ is attested by Dadu'a and Ende. Ende, as a member of Central Flores, has sporadic lowering 
of high vowels to mid before * $\mathrm{R}$ (Elias 2018:98f). On the other hand, Rote-Meto also should undergo $*_{\mathrm{i}}$ $>e / *$ R, thus providing evidence against final pre-RM $* * \mathrm{R}$.] [History: Possibly connected with PMP *bayəhih 'fragrant' with irr. *b > $* * \mathrm{~m}$ and sporadic antepenultimate $*_{\mathrm{a}}$ $>* *$ ə. (This sporadic change is also attested in *panahik $>$ *hene 'climb' and *sayəlaR $>$ *sena 'fry'.) If the evidence for final $* * \mathrm{R}$ in $* * \operatorname{mən} \mathrm{i}(\mathrm{R})$ were strengthened, then the connection with PMP *bayəhih would become even more tenuous.]

meni Termanu. fragrant, aromatic. (J:355f)

meni Korbafo.

meni Bokai.

meni Bilbaa.

meni Rikou.

meni $B a^{\prime} a$.

meni Tii.

meni Dengka.

ma-?a-meni-? Oenale.

foo meni Kotos Amarasi. fragrant.

foo meni Molo. fragrant. (M:318)

fu/miin Kusa-Manea. fragrant.

Out-comparisons:

minis Semau Helong. delicious, tasty, pleasant, aromatic.

meki Mambae, South. fragrant (of flowers, food). (Grimes et al. 2014b:32)

kai-kmeni Waima'a. sandalwood. ai-kmenih Dadu'a. sandalwood. (Penn 2006:36)

vuməワi Hawu. fragrant, aromatic. Pare məye Ende. kind of fragrant paddy.

* menir PnMeto. sandalwood. Sandalum album. [Form: Given the importance of sandalwood as an early item of trade, this could be an early loan between Meto and Tetun before Meto $* 1>n$.] meni Kotos Amarasi. sandalwood tree. [Note: The Meto term for 'fragrant' is foo meni the second element of which is plausibly from meni 'sandalwood'. There is independent evidence that Meto *meni is from earlier **məni(R).]

hau meni Molo. sandalwood. Sandalum album. (M:318)

Out-comparisons:

kameli(n) East Tetun. sandalwood genus. Santalum. (Mo:100)

* mendu PRM. crush, grind. [irr. from PRM: *nd $>\eta g$ in Oenale] [Form: An alternate solution would be to reconstruct *mengu and posit irr. *yg $>n d$ in Rikou and Dengka. Occam's razor currently favours *mendu with only one irregular sound change. The Kotos Amarasi form would be regular from both *mendu or *mengu. A Ro'is Amarasi reflex could decide between the two alternate reconstructions as it has *nd $>r$ but *ng $>k$.]

me mendu Tii. grind the teeth. (J:732)

na-la-me mendu Dengka. break into pieces by rubbing. (J:732)

me mengu Oenale, grind the teeth. (J:732)

n-meku Kotos Amarasi. crush.

*menge PRM. kind of red snake. [Form: Jonker (1908) connects the initial me with *mea 'red'.]

meye Termanu. kind of reddish snake. (J:355)

mege Korbafo.

meye Bilbaa.

meke Landu. snake. (own field notes)

meke Rikou.

menge $B a^{\prime} a$.

menge Tii.

menge Dengka.

menge Oenale.

uu/meke Ro'is Amarasi. kind of snake. [Form: The source of initial uu is currently unknown. It also 
is attested in Molo u?sao 'viper' given in Middelkoop (1972:592), see *sao.]

u/meke Kotos Amarasi. nonpoisonous grass snake, typically red in colour.

u/meke Molo. rat snake. (M:587)

u/meek Kusa-Manea. snake.

Out-comparisons:

${ }^{\circ}$ mege Dhao. snake (generic). Borrowed from: wRote menge.

*meo1 PRM. cat. Etym: **meoy (pre-

RM). [Semantics: onomatopoeia.]

meo Termanu. cat. (J:356)

meo Korbafo.

meo Bokai.

meo Bilbaa.

meo Rikou.

meo $B a^{\prime} a$.

meo Tii.

meo Dengka.

meo Oenale.

meo Ro'is Amarasi. cat.

meo Kotos Amarasi. cat.

meo Molo. cat. (M:319)

Out-comparisons:

meo Dhao.

meo Hawu.

meo-nao Ngadha. cat. (Djawanai 1995:Part 2, 332)

meoy Sika. cat. (Pareira and Lewis 1998:133)

meoy Manggarai. cat. (Verheijen 1967:319)

meon Balinese. cat. [Note: language of Bali ISO 6393 [ban].] (Bawa and Clynes 1995:Part 2, 332)

meoy Sasak. cat. [Note: language of Lombok ISO 639-3 [sas].] (Ali 1995:Part 2, 332)

meoy Bugis. cat. (Abas 1995:Part 2, 332)

meon Coastal Konjo. cat. [Note: language of South Sulawesi ISO 639-3 [kjc].] (Friberg 1995:Part 2, 332)
* meo2 PnMeto. warrior. [History: This term was probably spread by contact due to the cultural meaning, but the exact direction of spread cannot be confidently identified.]

meo Kotos Amarasi. warrior.

meo Molo. head-hunter. (M:318)

\section{Out-comparisons:}

meo Semau Helong. war chief; war lord.

meo East Tetun. desperado, thief, raiding warriors, etc. (Mo:141)

*meras PRM. sick. Etym: *hapojos. [irr. from PMP: *ə $>*_{\mathrm{a}}$ ] [minority from PMP: $* \mathrm{j}>*_{\mathrm{r}}\left(\right.$ expect $\left.{ }^{*} \mathrm{~d}\right)$ ] [Form: Medial $*_{j}$ and final $*^{*}$ cannot regularly account for the Keka or Helong reflexes thus indicating irregular $* *$ ma-pəjəs $>$ pre-RM **ma-hədas.]

mela_dai-k Keka. curse against an animal. (J:731)

mela Dengka. sick. (J:731)

mera Oenale. sick. (J:731)

meens Ro'is Amarasi. sickness.

menas (2) na-mena Kotos Amarasi.

1) sickness. 2) sick, painful. [Form: regular final consonant deletion to derive verb from noun.]

menas (2) na-mena Molo. 1) sickness. 2) sick. (M:318)

\section{Out-comparisons:}

heda Semau Helong. sick. Implies feverish or fluish. Not all diseases are heda.

hera Bolok Helong. sick.

moras East Tetun. sick, in poor health; ill, sick, unwell; $n$. sickness, disease, ailment. (Mo:143)

${ }^{\circ}$ moras Mambae, South. sick, pain, hurt. Borrowed from: Tetun (shown by irr. ${ }^{*} \partial=o$ correspondence). (Grimes et al. 2014b:32)

pəda $H a w u$. sick. pa pəda, papəda Dhao. sick. 
*mesa PRM. alone. Etym: *ma-əsa. [Sporadic: $* \mathrm{a}>e$ /_\# in Meto.]

mesa Termanu. alone. (J:356)

mesa Korbafo.

mesa Bokai.

mesa Bilbaa.

mesa Rikou.

mesa $B a^{\prime} a$.

mesa Tii.

mesa Dengka.

mesa Oenale.

mese? Ro'is Amarasi. one.

mese? (2) n-mese Kotos Amarasi.

1) one, single. 2) be alone.

mese? Molo. one. (M:319)

mese? Kusa-Manea. one.

Out-comparisons:

mesa Semau Helong. one, as.

mesa(k) East Tetun. only, alone, solely. [irr. from PMP: *ə $>e$ (expect $o$ )] (Mo:141)

*metam PMeto. black, civet. Etym: *maqitəm 'black; deep blue'. [Semantics: Balle and Cameron (2014) ascribe the etymology of Helong maat mitan 'civet' (lit. 'black eye') to the dark fur around the eyes, its nocturnal behaviour, and ability to see at night. A similar explanation for the polysemy of Meto *metam is likely.]

meten Ro'is Amarasi. black; civet. metan Kotos Amarasi. black; civet. metan Molo. black; civet. (M:321)

metom Kusa-Manea. black; civet.

[Sporadic: *a $>o$ /_m (compare

PRM *hendam $>$ PMeto *eram $>$

ekam in Upper Manulea hamlet $>$

ekom is Uabau' hamlet).]

\section{Out-comparisons:}

mitan Funai Helong. black. [Note: maat mitan 'civet' lit. 'black eye'.]

mitay Semau Helong. black. [Note: maat mitan 'civet' lit. 'black eye'.]

metan East Tetun. black. [Note: laku = 'civet'.] (Mo:141) *meti PRM. low, of the tide. Etym: *maqati.

meti Termanu. low tide, tide in general, the part of a beach that is dry at low tide. (J:357)

meti Korbafo.

meti Bokai.

meti Bilbaa.

meti Rikou.

meti $B a^{\prime} a$.

meti Tii.

meti Dengka.

meti Oenale.

n-meiti Kotos Amarasi. dry up (of water, including rivers).

n-meti Molo. dried up, low-tide. (M:322)

Out-comparisons:

miti Semau Helong. dry up, recede.

moti East Tetun. dry up, cease running (a current of water). (Mo:143)

meki Kisar. low tide.

*meto PwRM. dry.

meto-? Dengka. dry, e.g. like wood.

meto-? Oenale. dry, e.g. like wood.

(J:733)

meto Dela. be(come) dry.

meto|? Ro'is Amarasi. dry.

meto|? (2) n-meto Kotos Amarasi.

1a) dry. 1b) indigenous, native,

Meto. The second sense probably comes from phrases such as atoin paah meto? 'people of the dry land'. 2) be(come) dry.

meto|? Molo. dry. (M:322)

ma meto|? Kusa-Manea. dry.

*midu Rote. saliva, spit out. Doublet: *ninu. Etym: *qizuR 'saliva, spittle' (PWMP). [irr. from PMP: $*_{\mathrm{z}}>$ *d $($ expect $* \mathrm{~d})$ ] [irr. from PRM: vowel metathesis in nRote $* \mathrm{iCu}>u C i]$ [Form: The source of initial $* \mathrm{~m}$ in *midu is not clear. It may be from the prefix *ma- though initial *ma- 
qi would regularly yield me. Kedang has iyu? $\sim$ miyu? attesting an optional initial $*^{m}$ (Samely 1991:221), and some varieties of Lamaholot have a form attesting earlier *təmidu: e.g. Lamalera təmiro, Lewokukun tomidzu (Keraf 1978).] [History: Rote * midu is cognate with PMeto *paninu and both are ultimately probably connected with *qizuR, but it is not possible to reconcile the initial $* \mathrm{~m}=$ *pay correspondence and combine them into one set attesting a single PRM reconstruction.]

mudi Termanu. spit, spit out. (J:364)

mudi Korbafo.

mudi Bokai.

mudi Bilbaa.

mudi Rikou.

mudi $B a^{\prime} a$.

mudi Tii.

milu Dengka. saliva, spit out. (J:364, 733)

miru Oenale. saliva, spit out. (J:364, 733)

Out-comparisons:

ilu Hawu. saliva, spittle.

m-miri (2) miri miri Onin.

1) spit (imperative form). (Smits

and Voorhoeve 1992:118)

2) saliva. [Note: language of the

Bomberai Peninsula ISO 639-3

[oni].] [Form: regular PMP $*_{\mathrm{u}}$

$>i$ / (C)\#, but irregular $* \mathrm{R}>$

$\varnothing($ expect $* \mathrm{R}>r)$.] (Smits and Voorhoeve 1992:94)

*mii Morph: *ka-mii. PRM. urine. Etym: *kəmiq. [irr. from PRM: *ii $>$ oe in nRote (probably influence from oe 'water')]

moe Termanu. make water, urinate.

$$
\text { (J:360) }
$$

moe Korbafo.

moe Bokai.

moe Bilbaa.

moe Rikou.

moe $B a^{\prime} a$.

moe Tii. mii Dengka. piss. (J:733)

mii Oenale. piss. (J:733)

mii (2) miis Dela. 1) urinate. 2) urine.

k|mii Ro'is Amarasi. urine.

k|mii (2) na-k|mi?i Kotos Amarasi.

1) urine. 2) urinate.

mii Amanuban/Amanatun. urine.

mii-ds Kopas. urine.

mii-ds Fatule'u. urine.

$<$ mi $>$ Molo. urine. (M:323)

k|mii-r Timaus. urine.

mii (2) ma mii-f Kusa-Manea.

1) urine. 2) bladder.

\section{Out-comparisons:}

mii Semau Helong. urine.

mii (2) mii-n East Tetun.

1) urinate (more polite to say

li?ur besik). 2) urine. (Mo:142)

n-omi Kisar. pee.

*mina PRM. delicious, comfortable, nice; fat, grease, oil. Etym: *miñak 'fat, grease; ointment'.

mina (2) ma-mina (3) balamina Termanu. 1) oil, melted fat. $(\mathrm{J}: 357)$ 2) fatty from there = malada ['delicious'] (with which it is usually combined), tasty. 3) delicious. (J:358)

mina Korbafo.

mina Bokai.

mina Bilbaa.

mina Rikou.

mina $B a^{\prime} a$.

mina Tii.

mina Dengka.

mina Oenale.

mina? (2) na-mina Kotos Amarasi.

1) oil. 2) comfortable, nice, delicious.

mina? (2) na-miin Molo. 1) fat, oil. 2) delicious. [Form: metathesised form of na-mina.] (M:323)

(2) na-mian Kusa-Manea. delicious. [Form: metathesised form of na-mina.]

Out-comparisons:

mina Semau Helong. oil, fat. 
mina East Tetun. oil, fat, grease. (Mo:142)

namina Kisar. delicious, sweet.

miina Wetan. sweet. [Note: language of southwest Maluku, member of Luang language/ dialect cluster ISO 639-3 [lex].] (de Josselin de Jong 1987)

*miu Morph: *na-ka-miu. PRM. whistle (through the air).

na-ka-miu Termanu. whistle like a fired bullet. ( $\mathrm{J}: 358)$

na-ka-miu Korbafo. na-ka-miu Bokai. na-ka-miu Bilbaa. na-miu Rikou. na-ka-muu $B a^{\prime} a$. na-Pa-muu Dengka. na-Pa-muu Oenale. na-k|miu Meto. (J:358)

Out-comparisons:

$<$ miu $>$ (2) <kamiu $>$ Kambera . whizzing sound of a thrown stone. [Note: also in Mangili.] (On: 291, 155)

$<$ mi'u> Lewa. $<$ piu $>$ Weyewa.

*m|kaka PnMeto. open-mouthed, agape. Etym: *yana. [minority from PMP: *n $\left.\left(>*_{\mathrm{\eta}}\right)>*_{\mathrm{k}} ; *_{\mathrm{n}}\left(>*_{\mathrm{\eta}}\right)>*_{\mathrm{k}}\left(\operatorname{expect} *_{\mathrm{n}}\right)\right]$ na-m|kaka Kotos Amarasi. openmouthed, agape, speechless, amazed.

na-m|kaka Molo. open the mouth, amazed. (M:324)

*mneas PMeto. husked rice. Etym: *bəRas 'rice between harvesting and cooking; husked rice'. [Note: The Meto and Kemak forms both have irregular initial $* \mathrm{~b}>m$ and are probably borrowed before Meto underwent $*_{\mathrm{r}}$ $>* 1>n$. The direction of borrowing is unclear.] [minority from PMP: $* \mathrm{R}\left(>*_{\mathrm{r}}\right) *_{\mathrm{n}}$ ] [Sporadic: consonant metathesis *əR $>*^{*}$ ne.] [History:
Various irregularities in nearly all languages indicate that reflexes of *bəRas have spread by contact.]

mneas Ro'is Amarasi. husked rice.

mneas, mnees Kotos Amarasi. husked rice.

mnees Kusa-Manea. hulled rice.

Out-comparisons:

ael beas Semau Helong. uncooked rice. [irr. from PMP: $* \mathrm{R}>\varnothing$ (expect $l$ )]

foos East Tetun. uncooked dehusked rice. [History: This form is mostly regular from *bəRas.] (Mo:36)

resa brea Tokodede. rice plant. (Klamer 2002)

mreas, meras Kemak. rice (husked, uncooked). [irr. from $P M P: * \mathrm{~b}>m ; * \mathrm{R}>r$ (expect Ø)]

baa breas Galolen. rice (husked, uncooked). [irr. from PMP: $* \mathrm{R}>r(\operatorname{expect} \varnothing)]$

*mode Rote. tobacco. See: *mado.

mode Termanu. tobacco, both the plant and readymade for use. (J:359)

mode Korbafo.

mode Bokai.

mode Bilbaa.

mode Rikou.

mode $B a^{\prime} a$.

mode Tii.

mode Dengka.

mode Oenale.

*modo Morph: *modo-k. PRM. yellow, green. Etym: **mozo (pre-RM). [History: possibly connected with PMP *muda 'young (of fruits); immature; light (of colours)'.]

mo modo-k Termanu. yellow, also: green, blue, (in this meaning not in all varieties of Rote); pale, yellowish of the face. (J:360)

mo modo-? Korbafo.

mo modo-k Bokai. 
mo modo-? Bilbaa.

mo modo-? Rikou.

mo modo-k $B a^{\prime} a$.

mo modo-k Tii.

mo modo-? Dengka.

mo modo-? Oenale.

modo-? Dela. blue, green.

moro|? Ro'is Amarasi. yellow.

moro|? (2) k|moro-f Kotos Amarasi.

1) yellow. 2) egg yolk.

molo|? (2) k|molo|? Molo. 1) yellow.

(M:329) 2) egg yolk. (M:222)

moro? Kusa-Manea. yellow.

\section{Out-comparisons:}

hmolo Semau Helong. poisonous green snake species. [Form: Jonker (1908:360) gives Helong liti molo 'brass'.]

modok (2) samodo East Tetun.

1) yellow, green (Mo:142).

2) green tree snake. (Mo:166)

moson Ili'uun. blue, green. (dJ:128)

moson Perai. green. [Note: language of Wetar Island ISO 639-3 [wet].] (Hinton 2000:122)

moso Tugun. green. (Hinton 2000:122)

(mo )moti Roma. green. [Form: Taber (1993:418) has Roma [momote], which would be underlying mo $\sim$ moti + -e ' $3 \mathrm{SG}$ ' $\rightarrow$ momotye according to the analysis in Steven (1991).] (Steven 1991:42)

mot motni Luang. green. [Note: language of southwest Maluku ISO 639-3 [lex].] (Taber 1993:418)

moro Bima. greenish. (Jonker 1893:52)

<múru> Kambera. green, fresh, young. (On:295)

<moru> Anakalang. [Note: also in Mamboru.]

<moro $>$ Kodi. [Note: also in Weyewa.] *mofa PRM. have grey hair. Etym: *maquban.

mofa Termanu. have grey hair or become grey. (J:360)

mofa Korbafo.

mofa Bokai.

mofa Bilbaa.

mofa Rikou.

mofa $B a^{\prime} a$.

mofa $T i i$.

mofa Dengka.

mofa Oenale.

mofa? Kotos Amarasi. grey hair, white hair.

$<$ na-k $\mid$ mofa $>$ (2) $<$ ?naak mofa $>$

(3) <nono mofa> Molo.

1) greybeard. 2) kind of bird.

3) kind of liana with a white flower.

(M:327)

* mofu PRM. fall. [irr. from PRM: *f $>$ $\left(?^{*} \mathrm{~h}\right)>$ ? in Rote]

mo?u Termanu. fall, fall out. (J:363)

mo?u $B a^{\prime} a$.

mo?u Dengka.

mo?u Oenale.

n-mofu Ro'is Amarasi. fall.

n-mofu Kotos Amarasi. fall.

n-mofu Molo. falls. (M:328)

mouf (2) mofut Kusa-Manea. 1) fall.

2) drop.

Out-comparisons:

mou Mambae, South. fall (over), fall (down), fall (off). [irr. from PRM: $* \mathrm{f}=\varnothing$ correspondence $]$ (Grimes et al. 2014b:33)

mou Bima. fall. (Ismail et al. 1985:92)

*mola Morph: *mola-k. PRM. node, joint. [irr. from PRM: *1 > glottal stop insertion in Amarasi]

mola-k Termanu. joint, node. (J:361)

(mole-? ?) Korbafo.

mola-k Bokai.

mola-? Bilbaa.

mola-? Rikou.

mola-k $B a^{\prime} a$.

mola-k Tii. 
mola-? Dengka.

mola-? Oenale.

mo?o|k Kotos Amarasi. section of something long, e.g. the section between the joints of a finger or bamboo. Usually refers to something that is a tube shape.

$<\operatorname{moa}(\mathbf{n})>$ Molo. joint, node. (M:527,545,586)

*mone PRM. male, man. Doublet: *mone. Etym: *maRuqanay 'male'. [History: PRM had two reflexes of *maRuqanay: *mane and *mone. Both of which are attested in wRote, Ba'a and Tii. The meaning of both forms was probably 'male, man' in PRM. Most of Rote lost *mone, Meto lost *mane, while wRote, Ba'a and Tii differentiated the semantics of the two terms.]

mone_feu-k $B a^{\prime} a$. son-in-law. Lit: 'new male'. (J:134)

mone_feu-k Tii. son-in-law. Lit: 'new male'. (J:134)

mone Dengka. male, of animals and certain plants. ( $\mathrm{J}: 345,734)$

mone (2) monae-? Oenale. 1) male, of animals and certain plants. (J:345, 734) 2) big, many. (J:735)

mone (2) monae-? Dela. 1) male. 2) big, important. [Form: with variant manae-?.]

mone Ro'is Amarasi. husband, male.

mone (2) moen fe?u Kotos Amarasi.

1) husband, male. 2) opposite sex sibling's son, son-in-law. Lit: 'new male'.

mone Molo. husband, male. (M:330) mone Kusa-Manea. husband.

Out-comparisons:

mone Hawu. man, male.

*mone|? PMeto. outside. Doublet: *muri. Etym: *ma-udəhi 'behind, last' (Blust and Trussel (ongoing) reconstruct PCEMP *m-udi. A PMeto doublet with (regular) $* a u>o$ is required to account for the Meto forms meaning 'outside'.). [Form: The initial syllable and medial consonant are regular from *ma-udəhi > PRM *more-k. Final *əhi $>e$ is possibly irregular, though the only other reflex is *binəhiq $>$ fini 'seed'. There is a conceptual link in Atoni though between mone? outside and mone 'male' and this folketymology may have led to final $e$ rather than $i$.

mone|? Ro'is Amarasi. outside.

mone|? Kotos Amarasi. outside.

mone|? Molo. outside. (M:330)

Out-comparisons:

molin Fehan Tetun. outside, excrement, urine, defecate. [irr. from PMP: *d $>$ l]

*mono PnMeto. stupid.

n-mono Kotos Amarasi. stupid.

mono Molo. dumb. (M:331)

Out-comparisons:

mono Bolok Helong. dumb, unable to speak.

monon Ili'uun. stupid. (dJ:128)

*monu Rote. fall.

monu Termanu. fall, fall off, fall out. (J:362)

monu Korbafo.

monu Bokai.

monu Bilbaa.

monu Rikou.

monu $B a^{\prime} a$.

monu Tii.

monu Dengka.

Out-comparisons:

monu East Tetun. fall. (Mo:143)

monu Waima'a. fall.

*moo Morph: *na-ka-moo. PRM. hold in lips.

na-ka-moo Termanu. hold or take something between one's lips or in closed mouth. (J:358)

na-ka-moo Korbafo.

na-ka-moo Bokai.

na-ka-moo Bilbaa.

na-moo Rikou.

na-ka-moo $B a^{\prime} a$. 
na-ka-moo Tii.

na-?a-moo Dengka.

na-Pa-moo Oenale.

na-?|moo Kotos Amarasi. put in the mouth but not swallow.

*mopu Morph: *ka-mopu-k. PRM. old, dead palm tree. [irr. from PRM: * $\mathrm{p}>$ $\varnothing$ in Korbafo and Oenale; $* m>m b$ in Oenale] [Form: PMP *p is usually lost in Oenale and PMP ${ }^{*} p>$ ? in Korbafo. However, PRM *p is expected to be retained unchanged in both lects.]

mopu-k Termanu. an old dead and fallen down gebang or lontar palm. (J:362)

mou-? Korbafo.

mopu-k Tii.

mbou-? Oenale.

?|mopu|? Kotos Amarasi. gebang/ lontar palm trunk.

2|mopu|? Molo. dead, black lontar palm or gebang palm trunk. (M:331)

*moris PRM. live. Doublet: *horis. Etym: *ma-qudip 'living, alive'. [Form: expected $* \mathrm{p}>{ }_{s} / \#(\$ 3.5 .1 .3)$.] moli (2) molis Termanu. 1) live. 2) probably: life! health! said to a child who sneezes. (J:361)

moli Korbafo.

moli Bokai.

moli Bilbaa.

mori Rikou.

moli $B a^{\prime} a$.

mori Tii.

moli Dengka.

mori Oenale.

n-moni Ro'is Amarasi. live.

n-moni Kotos Amarasi. live.

n-moni Molo. live. (M:330)

moin Kusa-Manea. give birth.

Out-comparisons:

moris Fehan Tetun. live, be born, come about, life, lively; exist.

moris, mouris East Tetun. live, be alive, exist, be born. (Mo:144)

mori Ili'uun. live, alive, thrive, grow. (dJ:128) *mukə PRM. wild dove. Etym: *mukən 'omen dove'. Pattern: k-9. [Sporadic: $* \ni>e / \sigma \#$ in wRote (perhaps ${ }^{*} \curvearrowright>*_{a}$ $>e / \#)$.

mu?e-k Termanu. wild dove. (J:364)

mu?e-? Korbafo.

muke-? Rikou. dark green doves:

Asian Emerald Dove, Pacific

Emerald Dove. Chalcophaps indica; Chalcophaps longirostris. (J:364; own field notes)

mu?e-k $B a^{\prime} a$

muPe-k Tii.

mu?e-? Dengka.

mu?e-? Oenale.

$\mathbf{k} \mid \mathbf{m a} \sim \mathbf{m u a} \mathbf{- r}=\mathbf{a a} \quad$ Kusa-Manea. wild pigeon. [Form: metathesised form of (currently unattested) k|ma mu?a.]

Out-comparisons:

hmukan Semau Helong. dove.

lamuka(n) East Tetun. dark green dove. (Mo:127)

lamukan Welaun. Asian Emerald Dove. Chalcophaps indica.

*mulu PRM. crazy. [Sporadic: diphthongisation $* u>a u$ in Meto.]

ka-mulu-s (2) mulu-k (3) na-mulu Termanu. 1) crazy. 2) insanity.

3) be or become crazy. (J:366)

ka-mulu-? Korbafo.

ka-mulu-s Bokai.

ka-mulu-? Bilbaa.

ma-mulu-? Rikou.

ka-mulu-s $B a^{\prime} a$.

ka-mulu-k Tii.

ma-mulu-? Dengka.

ma-mulu-? Oenale.

na-maunu Kotos Amarasi. crazy.

na-maunu Molo. rages, is crazy.

(M:316)

*mumu1 Rote. hold in the mouth and suck. Etym: *mulmul.

mumu Termanu. hold something in the mouth and suck on it, suck on a solid object. ( $\mathrm{J}: 366)$

mumu Korbafo. 
mumu Bokai.

mumu Bilbaa.

mumu Rikou.

mumu $B a^{\prime} a$.

mumu Tii.

mumu Dengka.

*mumu2 Morph: *na-ka-mumu.

Rote. gargle, rinse the mouth. Etym:

*muRmuR.

na-ka-mumu Termanu. he rinses his mouth out with water, also: he gargles. (J:366)

na-ka-mumu Korbafo.

na-ka-mumu Bokai.

na-ka-mumu Bilbaa.

na-mumu Rikou.

na-ka-mumu $B a^{\prime} a$.

na-ka-mumu Tii.

na-Pa-mumu Dengka.

na-Pa-mumu Oenale.

Out-comparisons:

mumun Semau Helong. gargle.

*munde Morph: *ka-munde. PRM. citrus tree. Etym: *muntay. [minority from PMP: *nt $>*$ nd $($ expect $* \mathrm{t})]$

mune Termanu. pomelo. (J:366)

mune Korbafo.

mune Bilbaa.

dero munde Landu. kind of citrus. (own field notes)

munde Rikou.

${ }^{\circ}$ munde Oepao. Borrowed from: Rikou munde (shown by irr. *nd $=n d$ correspondence). (own field notes)

mune $B a^{\prime} a$.

munde Tii.

munde Dengka.

munde Oenale.

muri Ro'is Amarasi. lime (fruit).

?|muki? Kotos Amarasi. lime (fruit). <muke> Molo. lime. (M:333)

*muri PRM. youngest, last, west. Doublet: *mone|?. Etym: *ma-udəhi 'behind, last' (PCEMP *m-udi).

muli-k (2) muli Termanu. 1) youngest child; young of people, animals, and plants. 2) west. (J:365) muli-? (2) muli Korbafo.

muli-k (2) muli Bokai.

muli-? (2) muli Bilbaa.

muri-? (2) muri Rikou.

muli-k (2) muli $B a^{\prime} a$.

muri-k (2) muri Tii.

muli-? (2) muli Dengka.

muri-? (2) muri Oenale.

muini|f Ro'is Amarasi. youngest.

muni|f (2) na-muni Kotos Amarasi.

1) youngest (child). 2) back, end.

muni|f (2) na-muni Molo. 1) young.

2) come behind. (M:330)

muni|f Kusa-Manea. young.

Out-comparisons:

mudi (2) hmudin Semau

Helong. 1) follow, from behind, go along with. 2) youngest, last in a series.

*muse1 Morph: *muse-?. PwRM. seed, pip. Etym: **musa (pre-RM). [Sporadic: vowel height harmony ${ }^{*} \mathrm{e}>i$ /uC_ in Ro'is Amarasi.] [Form: Positing earlier **musa with final $* \mathrm{a}>e$ in wRM can account for the wRM and Tetun forms based on attested sound changes. However, it cannot account regularly for the Hawu form. Earlier $* *$ muse could possible account for the Hawu form, but cannot account regularly for the Tetun form.] boa_muse-? Oenale. kidney. [Semantics: In other Rote languages 'kidney' is most often expressed by a compound of boa 'fruit' with the word for 'pip, seed', e.g. in Termanu boa de?e-k = 'kidney'. muse-? is not (currently) known to have any independent meaning in Oenale and the normal word for 'pip' is deke-?.] (J:683, 53)

musi|? Ro'is Amarasi. seed, pip, stone. Out-comparisons:

musan East Tetun. seed, pip, grain, etc. (Mo:144) 
lamuhi Hawu. seed (tree). [Note: vini $=$ 'seed (generic for seeds planted by humans)'.]

musi Ngadha. kidney. (Djawanai 1995:Part 2, 508)

*muse2 Morph: *muse-k. CER. white civet. Etym: *musay 'civet cat and similar small predatory mammals of the family Viverridae'. [irr. from PMP: $*_{\mathrm{a}}>*_{\mathrm{e}}$ ] [Form: The Rote forms would be regular from **musəy.]

mu muse-k Termanu. the white civet. (J:366)

mu muse-? Korbafo. mu muse-k $B a^{\prime} a$.

* musi PRM. suck.

na-sa-musi (2) musi Termanu.

1) suck, suck up. 2) suck, suck up. (J:366)

na-sa-musi Korbafo.

na-sa-musi Bokai.

na-ka-musi Bilbaa.

na-sa-musi Rikou.

na-sa-musi $B a^{\prime} a$.

na-sa-musi $T i i$.

musi Dengka.

na-sa-musi Oenale.

musi Dela. suck.

n-musi Kotos Amarasi. suck.

n-musi Molo. sucks. (M:335)

*musu PRM. enemy. [History: The Rote-Meto forms may be borrowings from Malay musuh. Blust and Trussel (ongoing) reconstruct *busuR that may be irregularly related.]

musu Termanu. enemy, also: personal enemy, hostile, war. (J:367)

musu Korbafo.

musu Bokai.

musu Bilbaa.

musu Rikou.

musu $B a^{\prime} a$.

musu Tii.

musu Dengka.

musu Oenale.

musu Kotos Amarasi. enemy.

musu Molo. enemy. (M:335)
Out-comparisons:

musu Semau Helong. enemy, opponent.

muhu (2) pemuhu Hawu.

1) enemy. 2) fight each other, war against each other, be at odds, become enemies.

*muta PRM. vomit. Etym: *um-utaq (PCEMP *mutaq).

muta (2) muta-s Termanu. 1) vomit

(v.). 2) vomit (n.). (J:367)

muta Korbafo.

muta Bokai.

muta Bilbaa.

muta Rikou.

muta $B a^{\prime} a$.

muta Tii.

muta Dengka.

muta Oenale.

n-muta Kotos Amarasi. throw up, said of a small child.

n-muut Molo. throws up (baby). li?ana? mee? an-muut the baby throws up milk (M:335)

Out-comparisons:

muta Semau Helong. vomit.

muta East Tetun. vomit. (Mo:144)

madu Hawu. vomit.

*muti Morph: *muti-k. $n R M$. white. Etym: *ma-putiq.

muti/foe-k $B a^{\prime} a$. white (albino) spot or spots on the hand or foot of a person, or on other places on an animal. (J:139)

muti/foe-k (2) kue_muti-k Tii. 1) white (albino) spot or spots on the hand or foot of a person, or on other places on an animal. (J:139)

2) white civet. (J:735)

muti|? Ro'is Amarasi. white.

muti|? (2) k/muti-f Kotos Amarasi.

1) white. 2) egg white.

muti|? Molo. white. (M:336)

muti|2 Kusa-Manea. white.

Out-comparisons:

muti? Semau Helong. white. 
mutin, mutik East Tetun. white (colour). (Mo:144)

* muut PRM. kind of insect. [History: Osmond (2011b:416) reconstructs Proto-Polynesian *mū 'flying insect', the reflexes of which designate mainly moths and dragonflies.] [Semantics: likely onomatopoeia, vague Rote semantics weaken reconstruction.]

mu $\sim$ muu Termanu. kind of insect that appears in the fruit of the kaa-k (fig-tree). (J:363)

mu muu Korbafo. mu muu Bokai.

mu muu Bilbaa.

mu muu Rikou.

mu muu $B a^{\prime} a$.

mu muu Tii.

mu muu Dengka.

mu muu Oenale.

muut Ro'is Amarasi. mosquito. [Semantics: Generic term for normal mosquitoes.]

muut Kotos Amarasi. kind of small white mosquito whose bite is worse than a normal mosquito.

MB - mb

* mbaa $n R M$. meat, flesh. [irr. from PRM: *aa > ee in Fatule'u]

paa Termanu. meat, flesh. (J:459)

paa Korbafo.

paa Bokai.

paa Bilbaa.

paa Landu. meat. (own field notes)

paa Rikou.

paa Oepao. meat. (own field notes)

mpaa $B a^{\prime} a$.

mbaa Lole. (Zacharias et al. 2014)

mbaa Tii.

pee-k Fatule'u. meat, flesh.

<pa'> Molo. a piece of meat which

is offered to spirits at the end of

a ritual meal. [Form: probably

[paa?].] (M:410)

Out-comparisons:

ompa? Sumbawa. flesh, fish. [Note: language of Sumbawa ISO 639-3 [smw].] (Mbete 1990:401, 403)

ompa? Sasak. meat, fish (as food as opposed to meat). [Note: language of Sasak Island ISO 639-3 [sas].] (Goris 1938:92)

*mbada Morph: *mbada-k. PRM. short in height, squat and compact in build, of a person. Doublet: *pande. Etym: *pandak. Pattern: d-2. [irr. from PMP: $* \mathrm{p}>*_{\mathrm{mb}},{ }^{*} \mathrm{nd}=* \mathrm{~d}$ ] pala_mata-k Termanu. close proximity. [Form: The Rote forms may also connected with Meto paumaka-?, haumaka-? 'near'. The second element could be related to *mata 'eye' with irr. *t $>k$ in Meto. (Reflexes of *mata also mean 'in front' in Rote and Meto.) Alternately, the second element could originally have been *maka with irr. ${ }^{*} \mathrm{k}>t$ in Rote due to influence from *mata.] (J:465f)

pala_mata-k Bokai.

mpala_mata-k $B a^{\prime} a$.

mbala_mata-? Dengka.

mbara mata-? Oenale.

para|? Ro'is Amarasi. short.

para|? Kotos Amarasi. short, typically used for trees.

pala|? Molo. short. (M:415)

pa para|? Kusa-Manea. short.

Out-comparisons:

kbadak Fehan Tetun. short (e.g. of people); width.

badak East Tetun. short, brief. (Mo:7)

badak Dadu'a. be short. (Penn 2006:85)

bada Mambae, South. short, low. (Grimes et al. 2014b:13) 
*mbae Rote. swell up, swollen. Etym: *baRəq 'abscess, boil, swelling on the body'. [minority from PMP: *b > *mb] [Form: regular *a > e/_q\#.]

pae Termanu. swell up, swollen up, only used (at least in Termanu) in combination with peta. (J:462)

pae Korbafo.

pae Bokai.

pae Bilbaa.

pae Rikou.

mpae $B a^{\prime} a$.

mbae Tii.

mbae Dengka.

mbae Oenale.

Out-comparisons:

hale Semau Helong. swell, swollen.

bai Hawu. (J:462)

*mbai CERM. rotten. Etym: *baRiw 'beginning to spoil, tainted (of food left uneaten too long)' [Form: Jonker also gives Tii na-sa-pai but indicates that this is questionable. If confirmed, this would indicate $\mathrm{nRM}$ *pai.]

na-sa-pai Termanu. mouldy, bad/ rotten. [Form: forms without initial sa- are currently unattested.] (J:463)

ma-sa-pai Korbafo.

ma-sa-pai Bokai.

na-sa-mpai, ma-sa-mpai $B a^{\prime} a$.

n-pai Kotos Amarasi. begin to rot. pai Molo. bad/rotten. (M:412)

Out-comparisons:

(vou-)bai Hawu. mouldy. (J:463)

mbai Bima. rotten. (Ismail et al. 1985:87)

*mbaki PRM. beetle. Pattern: k-9' (*k= $k$ in Bokai; expect $* \mathrm{k}>$ ?). [irr. from PRM: $* \mathrm{i}>e$ in Meto]

pa?i Termanu. kind of beetle that eats

coconuts. (J:463)

pa?i Korbafo.

paki Bokai.

paki Bilbaa. pa?i Rikou. kind of grub that is found in the gebang palm or lontar palm, similar to ba bate-? (see *bate) but can't be eaten. (J:463; own field notes)

mpa?i $B a^{\prime} a$.

mba?i Tii.

mba?i Dengka.

mba?i Oenale.

pa?e Ro'is Amarasi. beetle.

pa?e Kotos Amarasi. beetle. Usage: Tais Nonof sub-dialect.

Out-comparisons:

(mo)kebaki Hawu. beetle. (J:463)

* mbala PRM. co-wife, concubine.

pala (2) na-pala Termanu. 1) cowife; that is, the second wife of a man in comparison to the first; have or take a co-wife. 2) have concubines, said of a man. na-pala telu lesak he has three concubines (J:465)

pala Korbafo.

pala Bokai.

pala Bilbaa.

pala Rikou.

mpala-k $B a^{\prime} a$.

mbala Tii.

mbala-? Dengka.

mbala Oenale.

pana? (2) n-pana? Kotos Amarasi.

1) co-wife, concubine. 2) be polygamous, engage in polygamy.

$<$ in anmeu pana $>$ Molo. he lives polygamously. (M:418)

pana? Amfo'an. co-wife, concubine.

* mbalu PRM. cover, enclose. Doublet: *balu3. Etym: *balun 'bind, bundle, wrap in cloth; death shroud; cloth(ing)'. [minority from PMP: $* \mathrm{~b}>* \mathrm{mb}$ ]

palu Termanu. cover, enclose in a garment or similar. (J:466)

palu Korbafo.

palu Bokai.

mpalu $B a^{\prime} a$.

mbalu Tii. 
mbalu Dengka.

ma-?a-mbalu-? Dela. covered. <an-pano> (2) <pan/felo> Molo. 1) binds closely. anpano asu fefan someone tightly ties up the mouth of the dog. (M:421) 2) rope around a horse's mouth. (M:420)

*mbana Morph: *mbana-k. PRM. tip, end; nose.

pana-k Termanu. nose (of person or animal). (J:467)

pana-? Korbafo.

pana-k Bokai.

pana-? Bilbaa.

idu_pana-? Rikou. face. (own field notes)

mpana-k $B a^{\prime} a$.

mbana-k Tii.

mbana-? Dengka.

mbana-? Oenale.

pana-f $R o$ 'is Amarasi. nose.

pana-f Kotos Amarasi. nose; the extremity of something, e.g. point, fingertips, peninsula.

pana-f Molo. nose (in general), cape, promontory. (M:419)

*mbao Rote. mango. Etym: *qambawan 'large mango Mangifera odorata' (PWMP). [irr. from PRM: ${ }^{*} \mathrm{mb}>p$ in Dengka]

pao Termanu. mango tree and fruit. (J:468)

pao Korbafo.

pao Bokai.

pao Bilbaa.

pao Rikou.

mpao $B a^{\prime} a$.

mbao Tii.

pao Dengka. [Note: given as 'also D. idem'.]

mbao Oenale.

*mba|raa Morph: *mba|raa-k. PRM. old (things). Etym: *daqan 'old, ancient'. [Form: The Rote languages attest an earlier element *mba (which is attested as semi-productive verbal prefix) while Meto attests earlier stative prefix *ma-. Furthermore, the reflexes of PMP $* \mathrm{~d}$ are regular for medial $* \mathrm{~d}$, thus indicating that the initial *mba element was added before PRM. The best solution currently appears to be to posit PRM *mba|raa with irr. *mb > $m$ in Meto motivated by pressure from the stative prefix *ma-, thus pre-Meto **maraa.]

palaa-k (2) na-palaa Termanu. 1) old (of things, etc.). 2) be or become old. (J:465)

palaa-? Korbafo.

palaa-k Bokai.

palaa-? Bilbaa.

paraa-? Rikou.

mpalaa-k $B a^{\prime} a$.

mbaraa-k Tii.

mbalaa-? Dengka.

mbaraa-? Oenale.

m|naa|? Ro'is Amarasi. old, previous, former.

m|naa|? Kotos Amarasi. old, previous, former.

m|naPa-k Kusa-Manea. old, previous former. [Sporadic: *VV-k $>* \mathrm{VV}$ ? $>$ V?V in Kusa-Manea (perceptual metathesis) followed by attachment of semi-productive -k suffix.]

Out-comparisons:

blaan Semau Helong. old.

*mbasa PwRM. slap. Etym: **mbasaR (pre-RM). [History: possibly connected with PMP *sambak with consonant metathesis, though the final consonants of the pre-RM form would also need to be accounted for.]

mba mbasa? Dengka. name of a medicinal plant of which the leaf makes a noise when it is broken off. Called daun pukul tangan (hand hitting leaf) in Kupang. (J:750, 640) mbasa (2) mba mbasa? Oenale. 1) give a slap. (J:750) 2) name of a medicinal plant of which the leaf 
makes a noise when it is broken off. Called daun pukul tangan (hand hitting leaf) in Kupang. (J:750, 640)

n-pasa Kotos Amarasi. slap.

n-pasa Molo. hit with a flat hand.

(M:424)

Out-comparisons:

papas Semau Helong. slap. [irr. from PRM: initial syllable]

basa East Tetun. hit with the open hand, slap. (Mo:10)

basa, baas Mambae, South. slap (open hand). (Grimes et al. 2014b:12f)

paas Ili'uun. strike (with the hand). (dJ:132)

pahar Kisar. slap.

pahak Sika. slap. Usage: Hewa dialect/variety. [irr. from PRM: * $\mathrm{mb}=p$ correspondence (expect b)] (Klamer 2015a)

*mbau1 Morph: *ka-mbau-k. PRM. kind of beam. [Sporadic: glottal stop insertion in Amfo'an.]

pa pau-k Termanu. kind of beam under the roof, outside the actual house. (J:471)

pa pau-? Korbafo.

pa pau-k Bokai.

pa pau-? Bilbaa.

pa pau-? Rikou.

dii mpa mpau-k $B a^{\prime} a$. pillar for a front gallery, the 'stup' pillar. (J:471)

mba mbau-k Tii.

mba mbau-? Dengka.

?|pa?u-f Amfo'an. stair joint; the place where the horizontal part meets the vertical part.

* mbau2 PRM. stab, prick, pound. Etym: *bayu 'pound rice'. [minority from PMP: *b $>* \mathrm{mb}]$

pau Termanu. stab, prick. (J:470)

pau Korbafo.

pau Bokai.

pau Bilbaa.

pau Rikou. mpau $B a^{\prime} a$.

mbau Tii.

mbau Dengka.

mbau Oenale.

na-pau Ro'is Amarasi. pound (rice).

na-pau Kotos Amarasi. pound, stab.

na-pau Molo. pound, stab. (M:427)

ta-pau Kusa-Manea. pound.

Out-comparisons:

hai Semau Helong. plant, stick into ground; pound, pierce, stab impale. [History: from *bayu.]

fai East Tetun. pound, the action for removing the husk from whole grain and polishing rice with a tapered wooden stick (alu) driven in and out of a tapered hole in a wooden block (nesun), or any similar action. [History: from *bayu.] (Mo:31)

pai Ili'uun. pound, thresh. [History: from *bayu.] (dJ:132)

*mbeda PRM. put down. [irr. from PRM:

$* \mathrm{mb}>b \sim p$ in Meto]

peda Termanu. set, lay, lay something down, store something in, put something in. (J:472)

peda Korbafo.

peda Bokai.

peda Bilbaa.

peda Rikou.

mpeda $B a^{\prime} a$.

mbeda Tii.

mbeda Dengka.

mbeda Oenale.

n-pera (2) na-bera Kotos Amarasi.

1) throw down stones. 2) put down, drop.

na-pela, na-bela Molo. put down. (M:56, xxxix)

*mbe?u $n R M$. lie down.

na-na-pe?u-k Termanu. lie, lie down. (J:482)

na-ka-pe?u-? Korbafo.

na-na-pe?u-k Bokai.

na-pe?u-? Bilbaa. [Form: My consultants gave na-peu 'lie down'.] 
na-pe?u-? Rikou. [Form: My consultants gave na-peu 'lie down'.]

na-ka-mpe?u-k $B a^{\prime} a$. na-ka-mbe?u-k Tii.

pe?u-n Kotos Amarasi. sleepiness, typically used to refer to someone who hasn't fully woken up yet. iin peu?-n=ee $k a=$ na-mneuk $=\mathbf{f a}$ fe? s/he hasn't fully woken up yet

n-pe?u-g Amfo'an. sleepy.

<n-piu> (2) <masapeu> Molo. 1) doze. 2) nodding of the head while dozing. (M:437)

peu? Kusa-Manea. sleep. [Form: metathesised form of pe?u.]

*mbela Morph: *mbela-k. PRM. Job's tears. [Note: Jonker (1912:21) gives Loura (language of Sumba, ISO 6393 [lur]) bəla 'millet' (Dutch gierst) as cognate, but I have been unable to confirm the existence of this in the (very sparse) available sources for Loura.] [History: This is the only PRM crop term that does not appear reconstructible to a higher node. Given this, and the fact that both sorghum and corn (which this term designates in daughter languages) are not native to this region, this term probably originally designated a native plant with later semantic shift as new crops were assimilated to the category of old crops. The most likely candidate is Job's tears, Coix lacryma-jobi, of which a wild subspecies is present in the region. Verheijen (1984:14) states, concerning Job's tears: 'The wild native subspecies agrestis of which the stony seeds with a very tiny pip are still used as beads, greatly resembles the cultivated subspecies ma-yuen. It is very probable that the introduced edible species was named after the wild plant, which then fell into the background. I am inclined to surmise that in this way names from substratum languages were saved. That may explain the enormous diversity of names in east Indonesia and the Philippines.' PRM *mbela appears to be such an example of a substratum term which was retained after the shift to an Austronesian language.]

pela-k (2) pela hii-k Termanu. 1) maize (the plant and its fruit). (J:477) 2a) maize of native soil, native corn (called jagung Rote $[\mathrm{OE}=$ 'sorghum']). (J:477) $2 \mathrm{~b})$ sorghum, that is native maize, simply/only maize, ordinary maize. [Form: hii-k = 'simply, only'.] [Semantics: Fox (1991:250) states: 'At present pela can refer to three different plants. pela hii-k, 'true pela', refers to sorghum; pela hii dele ngeo-k, 'black-flecked pela' refers to Job's tears; while pela or pela sina refers to maize. On Roti [sic] it is clear that maize when it was introduced was culturally assimilated to the category of 'sorghum'. It is also conceivable that at an earlier period when sorghum was introduced, it was assimilated to the category of Job's tears. Thus this category, pela, may subsume three stages of an agricultural progression: pela $[\mathrm{A}]$ ('Job's tears') > pela [B] ('sorghum') > pela [C] ('maize').'] (J:181)

pela-? (2) pela hii-? Korbafo.

pela-k (2) pela hii-k Bokai. pela-? (2) pela dae-? Bilbaa. [Form: dae-? = 'soil, land, earth'.]

pela-? (2) pela dae-? Rikou. mpela-k (2) mpela hia-k $B a^{\prime} a$. mbela-k (2) mbela hie-k Tii. mbela-? (2) mbela dae-? Dengka. [Form: Jonker gives the note 'Bilbaa, Dengka, Rikou have dae-?' implying that Dengka does not have expected mbela lae-?.] 
mbela-? (2) mbela hie-? Oenale. pena|? Ro'is Amarasi. corn, maize. pena|? (2) peen mina? Kotos Amarasi. 1) corn, maize. 2) sorghum. [Form: The second element could be connected with $\operatorname{mina}$ ? $=$ 'oil' or $\operatorname{mina}=$ 'comfortable, nice, delicious'.] pena|? Molo. maize. (M:431) pena|? Kusa-Manea. corn, maize. *mbena Rote. fly. Doublet: *kalbenu. Etym: *bəRnaw (Blust and Trussel (ongoing) reconstruct both *bəRyaw and *banaw.). [irr. from PMP: *aw $>$ $* \mathrm{a}$ [minority from PMP: $* \mathrm{~b}>* \mathrm{mb}$ ] [Sporadic: *a $>$ e/ \# in wRote] [Form: Helong attests earlier **mbena.]

pena Termanu. fly. (J:479) pena Korbafo. pena Bokai. pena Bilbaa.

pena-? Landu. fly. (own field notes) pena Rikou. mpena $B a^{\prime} a$. mbena Tii. mbene Dengka. mbene Oenale.

Out-comparisons:

lael_peya Helong. kind of fly. (J:479)

*mbesik PRM. throw down. [irr. from PRM: $* \mathrm{i}>e$ in Meto]

pesi Termanu. throw down, fling down. (J:480)

pesi Korbafo.

pesi Bokai.

pesi Bilbaa.

pesi Rikou.

mpesi $B a^{\prime} a$.

mbesi(k) Tii.

mbesi-? Dengka.

mbesi-? Oenale.

n-pesek Kotos Amarasi. throw down something flat, like a playing card.

Out-comparisons: pəhi Hawu. throw. [irr. from PRM: ${ }^{*} \mathrm{mb}=p$ correspondence] (J:480)

*mbeta CERM. wet. [Form: The Meto unmetathesised form has not yet been attested. It could be *na-peta or *na-pete.]

ma-ka-peta-k Termanu. still moist, not yet properly dry. (J:481)

ma-peta-? Rikou.

ma-ka-mpeta-k $B a^{\prime} a$.

na-peet Kotos Amarasi. moisten, wet, satiate.

na-peet Molo. wet. (M:435)

*mbetak PRM. swollen, sore.

peta Termanu. swell up, swollen. (J:481)

peta Korbafo.

peta Bokai.

peta Bilbaa.

peta Rikou.

mpeta $B a^{\prime} a$.

mbeta Tii.

mbeta Dengka.

mbeta Oenale.

mbeta Dela. ache, sore.

n-petak Kotos Amarasi. sore, tired, exhausted.

n-petak Molo. stuffed, ascites (the accumulation of fluid in the peritoneal cavity, causing abdominal swelling), swollen. (M:436)

Out-comparisons: petan Semau Helong. swell. peken Kisar. swollen.

*mbii PRM. pull taut.

na-ka-pii (2) ei_pi pii-k Termanu. 1) tense, tight. 2) the Achilles tendon. (J:483)

na-ka-pii Korbafo.

na-ka-pii Bokai.

na-ka-pii Bilbaa.

na-pii Rikou.

na-ka-mpii $B a^{\prime} a$.

na-ka-mbii $T i i$.

na-ka-mbii Dengka. 
na-ka-mbii Oenale.

pii_koete-f Ro'is Amarasi. back part of the lower leg, from the back of the knee to the Achilles' tendon.

na-2-pii (2) n-pii (3) pii_?ote-f Kotos Amarasi. 1) tie. 2) pull tight.

3) back part of the lower leg, from the back of the knee to the Achilles' tendon.

<na-pii> (2) < pii> (3) pii_?ote-n (4) < pii-n enu> Molo. 1) tautens, pulls tight. 2) the bend of the knee.

3 ) because that is where the tendons of a sacrificial buffalo are severed.

4) knee nerves. (M:437)

*mbiko PRM. kind of coniferous tree. Podocarpus rumphii. Pattern: k-6.

piko Termanu. a) kind of tree that yields good wood for timber. [Semantics: 'Podocarpus rumphii is a valuable timber tree where it attains large sizes with a clear, straight bole. Its wood is used as round wood for masts, spars, and poles, in house construction as beams, in highgrade construction for flooring, joinery and other carpentry, for furniture and cabinet work, veneer, make boxes, and for match sticks. In traditional use it was sought after for (dugout) canoes, used in coastal house construction, for household utensils, and wood carving. It is not known to be in cultivation, either as a forestry plantation tree or as an ornamental tree; the species is present in a few tropical botanic gardens and arboreta.' (Farjon 2017:920).] (J:484) b) kind of tree; a forest tree with thick bark and small but thick leaves. (Bark can be used for dyeing.) (Fox 2016b:46)

piko Korbafo.

piko Bokai.

piko Bilbaa.

piko Rikou.

mpiko $B a^{\prime} a$. mbiko Tii.

mbi?o Dengka.

$<$ hau pio $>$ Miomafo. kind of tree, rare on Ambon but occurring in the village of Ema, from which the name Lignum Emaniom comes in the high stony mountains of Laitimor. Also rare in Timor. Podocarpus rumphii. (M:439)

* mbila PRM. blaze, emit light. Etym: *bilak 'shine, glitter'. [minority from PMP: *b $>* \mathrm{mb}$ ]

pila (2) pila-s Termanu. 1) flaming, burning. (J:484) 2) red. (J:485)

pila (2) pila-? Korbafo.

pila (2) pila-k Bokai.

pila (2) pila-? Bilbaa.

pila (2) pilas $R i k o u$.

mpila (2) mpila-s $B a^{\prime} a$.

mbila (2) mbila-s Tii.

mbila (2) mbila-s Dengka.

mbila (2) mbila-s Oenale.

n-pina Ro'is Amarasi. blaze, emit light.

n-pina Kotos Amarasi. blaze, emit light; be glorious.

n-pina Molo. flaming, shines. (M:438)

Out-comparisons:

bilan East Tetun. cook. (Mo:15)

*mbinu PRM. snot. [History: possibly connected with PMP *hinus, though the initial $* m b$ is unexplained. Blust and Trussel (ongoing) reconstruct * pipus based on a comparison of Rote (presumably Termanu) pinu and a Cebuano form pinús-pinús. The Tii, Dengka and Oenale forms with initial $m b$ in this cognate set indicate that, unless more cognates are forthcoming, the comparison of Termanu pinu and Cebuano pinús-pinús is sheer chance (PMP $* \mathrm{p}>m b$ is never attested in western Rote). Blust and Trussel (ongoing) $d o$ correctly note that the normal reflex of $* \mathrm{p}$ in Rote is $h$. However, in support of their etymology 
for Rote pinu from * pinus they note that in some forms it is reflected as $p$. They give *papan > papa 'plank, board' and *picik > pisi 'to sprinkle, spray (water)' as examples. In each of these examples the cognates in all Rote varieties, including Tii, Dengka and Oenale have $p$, with the exception of Ba'a which has $m p$. These forms thus do not show the same sound correspondences as *mbinu 'snot'. Instead, both these words are loans. ( $p$ is often borrowed in Ba'a as $m p$. .)] pinu Termanu. snot. (J:486) pinu Korbafo. pinu Bokai. pinu Bilbaa.

pinu Rikou. mpinu $B a^{\prime} a$. mbinu Tii. mbinu Dengka. mbinu Oenale.

pinu Kotos Amarasi. runny snot. <pinu> Amfo'an. snivel. Usage: poetic. (M:439)

*mbisa Morph: *mbisa-k. Rote. kind of basket made from lontar leaves.

pisa-k Termanu. kind of basket made from lontar leaves. ( $\mathrm{J}: 486$ )

pisa-k Korbafo.

pisa-k Bokai.

pisa-k Bilbaa.

pisa-? Rikou.

mpisa-k $B a^{\prime} a$.

mbisa-? Dengka.

mbisa-? Oenale.

Out-comparisons:

kebiha Hawu. (J:486)

*mboes Morph: *ka-mboes. PRM. shrimp, prawn.

poe-k Termanu. shrimp, lobster. (J:489)

poe-? Korbafo.

poe-k Bokai.

poe-? Bilbaa.

poe-? Landu. shrimp, lobster. (own field notes) poe-? Rikou.

mpoe-k $B a^{\prime} a$.

mboe-k Tii.

mboe-? Dengka.

mboe-? Oenale.

k|poes Ro'is Amarasi. shrimp, prawn.

poes (2) na-k|poe Kotos Amarasi.

1) shrimp, prawn. 2) go shrimping,

look for shrimp.

poes Molo. river shrimp. (M:444)

poe-k Kusa-Manea. shrimp.

Out-comparisons:

boek East Tetun. shrimp, prawns. (Mo:16)

wa?ihe Kisar. lobster. [irr. from PMP: *mbo > wa]

voe Hawu. (J:489)

*mbonu Morph: *ka-mbonu-k. $n R M$. thick hair, mane.

po ponu-k Termanu. the mane of a horse, also said of a pig. (J:495)

po ponu-? Korbafo.

po ponu-k Bokai.

po ponu-? (2) na-po ponu Bilbaa.

2) the hair is luxuriant. (J:755)

po ponu-? Rikou.

mpo mponu-k $B a^{\prime} a$.

mbo mbonu-k Tii.

pounu-f Ro'is Amarasi. eyebrows, eyelashes, feathers.

ponu-f Kotos Amarasi. moustache.

?|pono-f Molo. horse's mane, goat hair worn by a dancer around their ankles. (M:448)

*mboo Morph: *mboo-k. PRM. hole, wide open. [Sporadic: glottal stop insertion in Meto.] [History: Blust and Trussel (ongoing) reconstruct PMP *buhay 'hole, pit', which may be irregularly connected.]

poo poo (2) poo-k (3) na-po $\sim$ poo

(4) na-poo-k Termanu. 1) wide open, yawning. 2) hole, cavity.

3) keep open continuously. 4) open (the mouth). (J:487)

poo poo (2) poo-? Korbafo.

poo poo (2) poo-k Bokai. 
poo poo (2) poo-? Bilbaa. poo poo (2) poo-? Rikou. mpoo $\sim$ mpoo $B a^{\prime} a$. mboo mboo (2) mboo-k Tii.

mboo mboo Dengka. mboo $\sim$ mboo Oenale.

po?o (2) po?o-f Ro'is Amarasi. 1) hole. 2) throat. [Note: Jonker (1908:487) gives Meto puu in the etymology notes for Termanu poo-poo.]

pa po?o-f Kusa-Manea. throat.

Out-comparisons:

boo Hawu. hole. (J:487)

*mbori PRM. pour on.

poli Termanu. pour, pour out, also: pour oneself out, e.g. like rain. (J:494)

poli Korbafo.

poli Bokai.

poli Bilbaa.

pori Rikou.

mpoli $B a^{\prime} a$.

mbori Tii.

mboli Dengka.

mbori Oenale.

n-poni Kotos Amarasi. pour over something, flush.

n-poni Molo. irrigate, water (e.g. plants). (M:448)

*mboro PRM. palm leaves.

polo-k Termanu. palmiet, young leaves of a palmiet, young leaves of a coconut, lontar palm, etc. (J:494)

polo-? Korbafo.

polo-k Bokai.

polo-? Bilbaa.

polo-? Rikou.

mpolo-k $B a^{\prime} a$.

mboro-k Tii.

mboro-? Oenale.

pono Ro'is Amarasi. sugar palm leaf.

pono Kotos Amarasi. sugar palm leaf. pono Molo. lontar palm leaves. (M:448)

Out-comparisons:

pola Semau Helong. palm leaves.
* mbosi PRM. release, set loose. [irr. from PRM: *s $>$ ? in most Rote]

po?i Termanu. release; loosen, escape.

(J:490)

po?i Korbafo.

po?i Bokai.

poi (2) posi Bilbaa. 2) slips away, becomes loose. (J:755)

po?i Rikou. [Form: Jonker indicates that this could actually be poi without a medial glottal stop. Nako et al. (2014) give po?i taata 'free, released'.]

mpo?i $B a^{\prime} a$.

mbo?i Tii.

mbo?i Dengka.

mbo?i Oenale.

na-t|posi? Kotos Amarasi. come loose.

Out-comparisons:

${ }^{\circ}$ natpusi Semau Helong. Borrowed from: Meto. (J:490)

habusik, husik East Tetun. untie, loosen, leave loose. (Mo:41)

kapisu Kamarian. loose, loosened. [Note: also in some varieties of Kaibobo.] (van Ekris 1864:99)

pusi Asilulu. [Note: also in Lusa Laut.] (van Ekris 1864:99)

posi Kaibobo. Usage: Piru dialect. (van Ekris 1864:99)

* mbou PRM. blanket, cloth. [Sporadic: $* \mathrm{VV}-\mathrm{k}>* \mathrm{VV} ?>\mathrm{V}$ ?V in Meto (perceptual metathesis).]

pou (2) pou-k Termanu. 1) woman's skirt, sarong. 2) the hair-like fibres between the stem and the leaf of different palm trees. (J:498)

pou (2) pau-? Korbafo.

pou (2) pau-k Bokai.

pou Bilbaa.

pou (2) pau-? Rikou. mpou (2) mpou-k, mpau-k $B a^{\prime} a$.

(2) mbou-? Oenale.

po?u Kotos Amarasi. scarf.

po?u Molo. blanket. (M:451) 


\section{Out-comparisons:}

kaboon Fehan Tetun. blanket.

*mbuah PRM. betel nut, areca palm. Doublet: *bua. Etym: *buaq 'fruit; areca palm and nut; grain; berry; seed; nut; endosperm of a sprouting coconut; kidney; heart; finger; calf of the leg; testicle; various insects; scar tissue; roe; bud; flower; blossom; bear fruit; words, speech, or songs; meaning, contents of discussion; numeral classifier for roundish objects; buttock; Adam's apple; nipple of the breast; button; marble; tattooing'. [minority from PMP: $* \mathrm{q}>* \mathrm{~h} ; * \mathrm{~b}>* \mathrm{mb}$ ] [Form: Irregular $* b>* m b$ is attested in many languages of the region for reflexes of *buaq meaning 'betel nut' and *mb can be reconstructed to proto-TimorBabar, perhaps even to a higher node.] pua Termanu. betel nut, areca, both the fruit and the tree. (J:499)

pua Korbafo.

pua Bokai.

pua Bilbaa.

pua $L a n d u$. betel nut. (own field notes)

pua Rikou.

mpua $B a^{\prime} a$.

mbua Tii.

mbua Dengka.

mbua Oenale.

puah Ro'is Amarasi. betel nut.

puah Kotos Amarasi. betel nut, areca palm.

puah Molo. both the areca palm and its fruit. Areca catecha. (M:452)

puah, puha Kusa-Manea. betel nut. [Form: The form puha ['pohe] may be due to reanalysis of puah as a metathesised form given that Kusa-Manea does not have assimilation of $/ \mathrm{a} /$ after metathesis. This is also attested in noah, noha 'coconut'.]

Out-comparisons:

pua Semau Helong. betel. bua East Tetun. betel nut, areca. Areca catechu. (Mo:18)

boo Kemak. betel nut, areca nut.

buu Mambae, South. betel nut, areca nut. (Grimes et al. 2014b:14)

bua Galolen. betel nut. pua Ili'uun. betel nut. (dJ:133) poo Kisar. areca nut.

*mbuat PwRM. spread out yarn. [Form: Final $t$ could be the nominaliser -t.] mbu $\sim$ mbuat Oenale. the two layers of the yarn spread out in front of the weaving. (J:756)

puat Kotos Amarasi. round wood, over the entire length of which weft thread is rolled.

puat Molo. round wood, over the entire length of which weft thread is rolled. (M:452)

\section{Out-comparisons:} pu puat Helong. (J:757)

*mbui PRM. quail, bird. Etym: *puyuq 'quail' (PWMP). [irr. from PMP: *p > *mb] [Form: Irregular $* \mathrm{p}>m b$ is also attested in Palu'e.]

manu_pui Termanu. bird, in general. $(\mathrm{J}: 347)$

manu_pui(-?) Korbafo.

manu_pui Bokai.

manu_pui-? Bilbaa.

man_pui-? Landu. bird. (own field notes)

manu_pui-? Rikou.

mpui-k $B a^{\prime} a$.

mbui-k Lole. (Zacharias et al. 2014)

mbui-k Tii.

mbui-? Dengka.

manu_mbui-? Oenale.

pui Kotos Amarasi. quail.

pui Molo. partridge and meko $=$ kind of bird that lives in the reeds. (M:453)

\section{Out-comparisons:}

mbuu Palu'e. quail. [Note: language of central Flores ISO 639-3 [ple].] (Donohue 2003:12) 
*mbuku1 PRM. mushroom, toadstool. Pattern: k-6.

puku Termanu. mushroom. (J:501)

puku Korbafo.

puku Bokai.

puku Bilbaa.

puku Rikou.

mpuku $B a^{\prime} a$.

mbuku Tii.

mbu?u Dengka.

mbu?u Oenale.

pu?u Kotos Amarasi. mushroom, toadstool.

$<$ pu'>, < $\mathbf{a}^{\prime} \mathbf{p u}$ 'e $>$ Molo. mushroom that comes up when the field is full of corn. [Form: The second form probably has the nominal determiner $=$ ee enclitic attached.] (M:451)

pu?u Kusa-Manea. mushroom, toadstool.

\section{Out-comparisons:}

buu/manu Semau Helong. mushroom. [irr. from PRM: $* \mathrm{mb}=b$ correspondence (expect $p) ;{ }^{*} \mathrm{k}=\varnothing$ correspondence]

*mbuku2 Rote. bent, hunchback. Etym: *bukuq 'bend, bent, bowed'. Pattern: $\mathrm{k}-5$. [minority from PMP: *b $>* \mathrm{mb}$ ]

puku (2) puku-k (3) (lete) pu puku-k Termanu. 1) bent of back. 2) hunchbacked. 3) hill. (J:502)

puku (3) pu puku-? Korbafo. puku (3) pu puku-k Bokai. puku (3) pu puku-? Bilbaa. puku (3) pu puku-? Rikou. mpuku (3) mpu mpuku-k $B a^{\prime} a$. mbuku (3) mbu mbuku-k Tii. mbuku (3) mbu mbuku-? Dengka. (3) mbu mbuku-? Oenale.

\section{Out-comparisons:}

leten pupuku Helong. (J:502)

buku Hawu. (J:502)

*mbule Morph: *mbule-k. PRM. grain head. Etym: *buliR '(entire) stalk of bananas; ear of grain'. [minority from
PMP: *b $>* \mathrm{mb}$ ] [Sporadic: $* \mathrm{i}>*_{\mathrm{e}}$ / *R\#] [Form: The Tetun, Helong and Kisar reflexes are regular from initial *b.]

pule-k Termanu. ear of grains, grasses. (J:502)

pule-? Korbafo.

pule-k Bokai.

pule-? Bilbaa.

pule-? Rikou.

mpule-k $B a^{\prime} a$.

mbule-k Lole. (Zacharias et al. 2014)

mbule-k Tii.

mbule-? Dengka.

mbule-? Oenale.

pune|? Kotos Amarasi. grain head.

pune|? Molo. corn-cob. (M:532)

Out-comparisons:

bulin Semau Helong. grain head.

fulin East Tetun. head (of grain, rice, etc.). (Mo:37)

wurna Kisar. corn ears.

*mbulə PwRM. wind around, twist around. [Note: All Rote lects (except Bilbaa) also have pole 'bind around, tie around, e.g. the muzzle of an animal' (Jonker 1908:493).] [Sporadic: *ə > $e / \sigma_{-} \#$ in wRote (perhaps $* \partial>*_{\mathrm{a}}>e$ /_\#).]

mbule Dengka. twist around. (J:147) mbule Oenale. twist around. (J:147)

n-puna Kotos Amarasi. wind around.

n-puna (2) na-puun Molo. 1) wraps around. 2) walks around. (M:455)

*mbumbu Rote. expand, swollen.

pupu Termanu. expand, like rice when cooked. (J:504)

pupu Korbafo.

mpumpu $B a^{\prime} a$.

mbumbu Dengka.

mbumbu Oenale.

Out-comparisons:

bubu East Tetun. swell. (Mo:18)

*mbune PRM. tree with edible fruit. Antidesma bunius. Etym: *buRnay (PWMP). [minority from PMP: *b > *mb] 
pune Termanu. kind of tree with small edible fruits. (J:503)

pune Korbafo.

pune Bokai.

pune Bilbaa.

pune Rikou.

mpune $B a^{\prime} a$.

mbune Tii.

mbune Dengka.

mbune Oenale.

<pune/klia>, <puni/klian> Molo.

kind of plant with glossy leaves.

Dillenia pentagyna. [Note:

Middelkoop (1972:351) gives

$<$ nasi $>$ as the term for Antidesma

bunius.] (M:455)

*mbunut Morph: *ka-mbunut. PRM. coconut husk, coir. Etym: *bunut. [minority from PMP: *b $>*^{*} \mathrm{mb}$ ] [Form: The Helong, Tetun and Welaun reflexes all also attest initial $*$ mb for pre-RM.]

punu-k Termanu. bark, fibrous husk

of a coconut. (J:504)

punu-? Korbafo.

punu-k Bokai.

punu-? Bilbaa.

punu-? Landu. hard inner shell of a coconut. (own field notes)

punu-? Rikou.

mpunu-k $B a^{\prime} a$.

mbunu-k Lole. (Zacharias et al. 2014)

mbunu-k Tii.

mbunut Dengka. [Form: final $t$ in Dengka and Oenale analysable as nominaliser -t.]

mbunut Oenale.

?|punu-f (2) na-?|punu|? Kotos Amarasi. 1) coconut husk. 2) loud sound like 'boom, boom' this is the sound that an old coconut makes when it falls from a tree (also bombs, etc.).

a-Plotos na-?|punu|? Molo. the thunder rattles. (M:456)

\section{Out-comparisons:}

punut Semau Helong. shell, husk. bunuk East Tetun. the durable external woody shell of palm trees used extensively in Timor as a building material for houses because of its strength and durability when split into long straight lengths. (Mo:19)

kabunut Welaun. hard inner coconut shell.

kebunu Hawu. (J:504)

*mburuk PRM. rotten. Etym: *buRuk. [minority from PMP: $* \mathrm{~b}>* \mathrm{mb} ; * \mathrm{R}=$ *r (expect Ø)]

pulu-k Termanu. old, rotten, stinking, whatever is rotten. ( $\mathrm{J}: 503)$

pulu-? Korbafo.

pulu-k Bokai.

pulu-? Bilbaa.

puru-? Rikou.

mpulu-k $B a^{\prime} a$.

mbulu-k Lole. (Zacharias et al. 2014)

mburu-k Tii.

mbuluk Dengka.

mburu-? Oenale.

n-punu Ro'is Amarasi. rotten.

n-punu Kotos Amarasi. rot, decay, fall apart.

<punu> Molo. rotten, bad. (M:456)

*mbusər PRM. sweat. [Sporadic: *ə > $(* a)>e$ /_\# in Meto.]

puse Termanu. sweat. (J:504)

puse Korbafo.

puse Bokai.

puse Bilbaa.

puse Rikou.

mpuse $B a^{\prime} a$.

mbuse Tii.

mbusa (2) na-Pa-mbusa Dengka.

1) sweat. 2) stuffy. (J:757)

mbusa (2) na-?a-mbusa Oenale.

1) sweat. 2) stuffy. (J:757)

mbusar Dela. sweat.

puus, puse (2) n-puus Kotos Amarasi. 1) sweat. 2) sweating. [Form: The unmetathesised 
form of puus has not yet been attested. It could be pusa or pusu. Middelkoop (1972:457) gives the Amarasi parallelism a-n-pusun $=$ ma maskeet 'instils fear and fright', which indicates that the unmetathesised form is *pusu with final $u$.]

puus (2) n-puus Molo. 1) sweat. 2) sweats. [Form: Jonker (1908:504) gives Meto puse-l.] (M:457)

Out-comparisons:

kabßəsu Dhao. [Form: regular vowel metathesis in Hawu and Dhao.]

kebəhu Hawu. (J:504)

pusə? Bugis. sweat. (Masse 2013:77)

bussay Makassar. feel stuffy due to heat. (Cense 1979:150)

*mbusu Morph: *mbusu-k. $n R M$. thigh. [irr. from PRM: * $>\varnothing$ in nRote]

puu-k Termanu. thigh (of person). (J:499)

puu-? Korbafo.

puu-k Bokai.

puu-? Bilbaa.

puu-? Landu. thigh. (own field notes)

puu-? Rikou.

mpuu-k $B a^{\prime} a$.

mbuu-k Tii.

pusu-f Ro'is Amarasi. thigh.

pusu-f Kotos Amarasi. thigh.

pusu-f Molo. thigh. (M:458)

pusu-f Kusa-Manea. thigh.

*mbutu1 PRM. hot, burning. See: *hotu 'burn'.

putu (2) na-putu Termanu.

1) burning, scorching. 2) scorch something. (J:505)

putu Korbafo.

putu Bokai.

putu Bilbaa.

putu Rikou.

mbutu Tii.

mbutu Dengka. mbutu Oenale.

ma|putu|? Ro'is Amarasi. hot.

n-putu (2) putu|? (3) ma|putu|?

Kotos Amarasi. 1) burn. 2) burnt up (wood), charcoal. 3) hot.

n-putu Molo. is on fire, burnt off. (M:459)

puut (2) ma|putu|? Kusa-Manea.

1) charcoal. 2) hot.

Out-comparisons:

otot Semau Helong. hot. [irr. from PRM: ${ }^{*} \mathrm{u}=o$ correspondences]

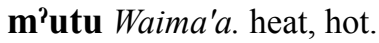

<mutunu> Kambera. burn (transitive and intransitive), on fire. [Form: The final vowel is epenthetic.] (On:295)

$<$ mutu $>$ Kodi.

poto-t Buru. hot. (Grimes and Grimes 2020:756)

mpoto Kayeli. fever. [Note: language of Buru Island ISO 639-3 [kzl].] (Charles E. Grimes pers. comm.)

pətu Keo. hot, cook, sick. [Note: language of central Flores ISO 639-3 [xxk].] (Baird 2002:572)

*mbutur Rote. tie up in a bundle. Doublet: *futu. Etym: *butu 'group, crowd, flock, school, bunch, cluster' (PCEMP). [minority from PMP: *b > *mb]

pu putu Termanu. tie up in a bundle. (J:505)

pu putu Korbafo.

pu putu Bilbaa.

pu putu Rikou.

mpu mputu $B a^{\prime} a$.

mbu $\sim$ mbutu Tii.

mbu mbutu Dengka.

Out-comparisons:

butuk East Tetun. sheaf, bundle, $v$. hang many things together. (Mo:19)

* mbuu PRM. make a noise. [irr. from PRM: ${ }^{*} \mathrm{u}>o$ in Meto] [Semantics: onomatopoeia.] 
puu Termanu. shout, make a noise. (J:498)

puu Korbafo.

puu Bokai.

puu Bilbaa.

puu Rikou.

mpuu $B a^{\prime} a$. mbuu Tii.

mbuu Dengka.

mbuu Oenale.

na-poo Kotos Amarasi. make a sound.

na-poo Molo. rolls (thunder), pops (rifle). (M:442)

\section{$\mathbf{N}-\mathbf{n}$}

*naa1 PRM. that, there. Etym: *-na 'distal spatio-temporal deixis: that, there; then'.

naa (2) ana, -n Termanu. 1) demonstrative pronoun, that there. (J:367) 2) non-emphatic form of the third person singular pronoun. (J:12) (Jonker 1915:332ff)

naa (2) ana Korbafo.

naa (2) ana Bokai.

naa (2) ana Bilbaa.

naa (2) ana Rikou.

naa (2) ana $B a^{\prime} a$.

naa (2) ana Tii.

naa (2) ana Dengka.

naa (2) ana Oenale.

naa Kotos Amarasi. zero person demonstrative.

*naa2 Morph: *naa-k. PRM. woman's brother. Etym: *ñaRa 'brother (woman speaking)'. [irr. from PRM: *a $>o$ in Meto] [Sporadic: *VV-k $>* \mathrm{VV}$ ? $>$ V?V in Meto (perceptual metathesis).] naa-k Termanu. brother, in relation to a sister. (J:368)

naa-? Korbafo.

naa-k Bokai.

naa-? Bilbaa.

naa-? Rikou.

naa-k $B a^{\prime} a$.

naa-k Tii.

naa-? Dengka.

naa-? Oenale.

nao-f Ro'is Amarasi. brother of woman. nao-f, na?o Kotos Amarasi. brother of woman.

na?o Kusa-Manea. brother.

Out-comparisons:

naan East Tetun. brother, cousin (only used by women to their brothers and male cousins). (Mo:145)

nara(n) Ili'uun. man's sister, a woman's brother. (dJ:130)

*naaz Rote. Papua New Guinea rosewood. Pterocarpus indica. Etym: *naRa. [Semantics: Meijer Drees (1950) gives Meto $<$ náo $>,<$ na $>$ and $<$ apnà $>$ as Planchonia valida and Barringtonia spicata, and $<$ matáni $>$ as Pterocarpus indica.]

naa Termanu. kind of tree called kayu merah (red wood) in Kupang. (J:368)

naa Korbafo.

naa Bokai.

naa Bilbaa.

naa Rikou.

naa $B a^{\prime} a$.

naa-k Tii.

naa Dengka.

naa Oenale.

Out-comparisons:

ai naa East Tetun. the tree that produces rosewood; rosewood tree, a good timber for furniture. Pterocarpus indicus. (Mo:2, 145) 
*naa4 Morph: *naa-k. Rote. gebang palm fibres. Etym: *qanahaw 'sugar palm: Arenga spp.'. [irr. from PMP: *aw > *a] [Form: The initial parts of some of the Rote terms are analysable as reflexes of *ekut 'palm fibres, woven ring from palm fibres'.]

eke naa-k Termanu. the outermost hard part of a young gebang palm (called tula pato), which is processed (called dusi) and separated from the useless inner part (called tula tei-k) and used to make a kind of rope or string called tali_eke/naa-k (in Kupang called tali heknaak), which is used to make various things (lapik). (J:110)

eke_naa-? Korbafo.

eke_naa-k Bokai.

heke/na-? Bilbaa.

(henaa-? ?) Rikou.

heke/naa-k $B a^{\prime} a$.

aki/naa-k Tii.

da?i/naa Dengka.

da?i/naa Oenale.

Out-comparisons:

naa (2) naa tais East Tetun. 1) palm trees of various kinds. 2) thread similar to that produced by Piassava palm. (Mo:145)

*nada PMeto. gums. Doublet: *ngadas. Etym: *yadas 'palate'. [minority from PMP: $* \mathrm{~d}=* \mathrm{~d}$ (expect $* \mathrm{~d}>* \mathrm{r}>* 1>$ $*_{\mathrm{n}}$ ]

nara-f $R o$ 'is Amarasi. gums.

nara-f Kotos Amarasi. gums.

nala-n Amanuban. palate.

$<$ nala> Molo. palate, uvula. (M:342) nara-f Kusa-Manea. gums.

*nade Morph: *ka-nade. PRM. taro, grass. Pattern: d-2. [irr. from PRM: ${ }^{*} \mathrm{e}>i$ in wRM, Ba'a, Termanu and Bokai] [History: possibly connected with Malay keladi, though $l>n$ in Rote would be unexplained.] [Semantics: The semantic connection between Rote and Meto is dubious.]

nali Termanu. kind of grass. (J:378)

nale Korbafo.

nali Bokai.

nale Bilbaa.

(nale ?) Rikou.

nali $B a^{\prime} a$.

nare Tii.

nali-? Dengka.

nari-? Oenale.

nari|? Ro'is Amarasi. taro.

?|nari|? Kotos Amarasi. taro.

P|lali-ds Amfo'an. taro.

P|lali Molo. taro. Colocasia antiquorom. (M:260)

*nado Rote. look upward. Etym: *yadaq (PWMP). [irr. from PMP: *a $>*_{\mathrm{o}}$ (also Ende)] [minority from PMP: $* \mathrm{~d}>* \mathrm{~d}$ ] nado Termanu. look up, look upwards. (J:371)

nado Bokai.

nado Bilbaa.

nado Rikou.

nado $B a^{\prime} a$.

nado Tii.

olo/nado Dengka.

oro/nado Oenale.

Out-comparisons:

yada Semau Helong. look up, come before. [Note: Jonker (1908:371) gives Helong yala.] [irr. from PMP: $* \mathrm{~d}=d$ /V_V (expect $l)]$

tanaat East Tetun. look up at (anything on a higher elevation); to look closely at someone expecting to be given something. [Note: Given the irregular final consonant and vowel correspondences, this may be a chance resemblance.] (Mo:180)

yadu, yado Ende. bend oneself back. 
*nadza Morph: *nadza-k. Rote. name. Etym: *yajan. [minority from PMP: *j $>*_{\mathrm{d}}($ expect $* \mathrm{~d})$ ] [Sporadic: $* \mathrm{a}>e$ /C+palatal_in nRote.] [Form: Given that the Meto forms are not inheritances from *nadza-k, the correspondence sets are consistent with both $*$ ds (see *fudzo 'foam') or *d. Reconstruction of medial $* d$ provides no explanation for the change of final $* a>e$ in Nuclear Rote. By reconstructing medial *ds we can propose that this vowel change is due to the previous palatal consonant before the change of $* d s>d / d$. An alternate reconstruction which can account for these reflexes would be **nado, but this requires positing otherwise unattested final PMP *a to PRM *o.]

nade-k Termanu. name. (J:370)

nade-? Korbafo.

nade-k Bokai.

nade-? Bilbaa.

nade $=$ na $L a n d u$. name. (own field notes)

nade-? Rikou.

nade-k $B a^{\prime} a$.

nade-k Lole. name. (Zacharias et al. 2014).]

nade-k Tii.

nala-? Dengka.

nara-? Oenale.

nara $=$ na Dela . name.

'kana-f Ro'is Amarasi. name. Borrowed from: Helong yala, before $* 1>n$ (shown by irr. $* \eta>$ $k$ correspondence, combined with identical semantics).

'kana-f Kotos Amarasi. name, clan.

${ }^{\circ}$ kana-f Molo. name. (M:178)

'kana-f Kusa-Manea. name.

Out-comparisons:

yala Semau Helong. name, tribe, clan, people group.

naran East Tetun. name. (Mo:152)

kala Mambae, South. name.

(Grimes et al. 2014b:24) gala-n Kemak. name.

nean Ili'uun. name, named, be named. (dJ:130)

*nae PRM. big, large. Etym: *Raya. [irr. from PMP: ${ }^{*} \mathrm{R}>{ }^{*} \mathrm{n}$ ] [irr. from PRM: $* \mathrm{e}>i$ in Kusa-Manea] [Form: One possible source of the unexpected $*_{n}$ in PRM is historic compounding of reflexes of *maRuqanay (*mone or *mane) with *Raya $>* *$ ae. Such a form is still attested in Hawu and may be the source of Dela-Oenale monae-? (see *mone). The Dela-Oenale, Dengka and Meto forms without initial mo or ma could then be subsequent reanalysis of ma as a prefix. However, this explanation does not account for the Bima form nae which also has unexpected initial $n$, suggesting that the irregular appearance of this consonant is much earlier than PRM.]

na-ma-nae Bilbaa. bisu=a na-ma-nae the wound has swollen up (J:735)

na-anae (2) ma-nae-? (3) nama-nae Dengka. 1) become big. 2) big. 3) bisu=a na-ma-nae the wound has swollen up (J:735)

nae (2) mo/nae-? Oenale. 1) big, many. (J:735) 2) big. [Form: Historic compound of mone 'man' and *Raya $>$ *nae (or perhaps earlier **ae.] (J:734)

nae-? (2) mo/nae-?, ma/nae-? Dela. 1) many, much. 2) big, important.

na-?|nae Ro'is Amarasi. grow.

na-?|nae (2) ?|nae|k (3) ?|nae|f (4) kbenu_?|naes Kotos Amarasi. 1) grow. 2) great, auspicious. 3) old man. 4) big fly.

?|nae|k Amfo'an. big.

na-?|nae Molo. big, eldest. (M:340)

bi/nai|? Kusa-Manea. big. [irr. from PRM: *e > i] [Form: source of initial bi currently unknown.] 


\section{Out-comparisons:}

mone ae Hawu. large, big, significant, important.

nae Bima. big, mature, grand. (Jonker 1893:58)

*nafu Rote. to anchor. Etym: *nabuq 'fall'.

nafu (2) nafu-k Termanu. 1) anchor (v.). 2) anchor. Usage: used less in Termanu, used more in Ba'a. (J:373)

nafu Korbafo.

nafu Bokai.

nafu Bilbaa.

nafu Rikou.

nafu $B a^{\prime} a$.

nafu Tii.

nafu Dengka.

nafu Oenale.

Out-comparisons:

penavu Hawu. anchor.

*nafu|? PnMeto. body hair, feather. Etym: **rafu (pre-Meto).

nafu? Amanuban/Amanatun. body hair, feathers. (M:340)

nafu? Amfo'an. feathers.

nafu? Kusa-Manea. hair.

Out-comparisons:

rahun Fehan Tetun. feather. (Mo:158)

ravuk Central Lembata. body hair, feather. (Fricke 2015)

*nahe Morph: *ka-nahe-k. CERM. mat. See: *nehi. Etym: *hapin 'liner, layer, insulation, padding; sleeping mat'. [irr. from PMP: $* \mathrm{i}>*_{\mathrm{e}}$; ${ }^{*} \varnothing>$ $*_{\mathrm{n}}$ ] [irr. from PRM: vowel metathesis in Meto $* \mathrm{eCa}>$ aCe] [Form: I have reconstructed *nahe with penultimate *a rather than *neha on the basis of the external evidence from Tetun (and PMP). While Meto has more instances of vowel metathesis than the Rote languages, it would be difficult to explain the connection between the Meto and Tetun forms as a result of borrowing due to the semantic difference. Thus, *neha would require two independent instances of vowel metathesis. *nahe, on the other hand, only requires one instance of vowel metathesis in CERM.]

ne?a-k Termanu. mat. (J:385)

ne?a-? Korbafo. [Note: Jonker (1908:385) gives two forms marked K., but the second of these is surely a typographical error for R. = Rikou.]

ne?a-k Bokai.

nea Bilbaa.

nea-? Rikou.

?|nahe|k Kotos Amarasi. mat.

<nahe> Molo. mat. (M:341)

nahe Kusa-Manea. mat.

Out-comparisons:

knehe? Funai Helong. mat.

nehe Semau Helong. mat, sleeping mat.

na?e Fehan Tetun. spread out.

nahe East Tetun. spread out, unfold (mat, towel, etc.). (Mo:146)

*nako Rote. steal. Etym: *nakaw. Pattern: $\mathrm{k}-5^{\prime}\left({ }^{*} \mathrm{k}>\right.$ ? in Tii and Dengka; expect $* \mathrm{k}=k)$.

na-ma-nako (2) nako Termanu.

1) steal. 2) theft. (J:374)

na-ma-nako Bokai.

na-ma-nako Bilbaa.

(2) na?o Rikou. thief, burglar. [Note: Jonker (1908:374) gives Rikou (na-ma-nao ?).] (Nako et al. 2014) na-ma-nako $B a^{\prime} a$.

(2) na?o (3) nako/daa Lole. 2) thief. 3) thief, steal. (Zacharias et al. 2014)

na-ma-na?o Tii.

na-ma-na?o Dengka.

na-ma-nako Oenale.

Out-comparisons:

nako Semau Helong. steal, rob.

hamna?o East Tetun. rob, steal, pilfer, plunder. (Mo:71)

mena?o Hawu. steal, rob. 
*nama PRM. creep. [Form: Helong attests earlier initial $* 1$. Initial $*_{n}$ in PRM could be sporadic assimilation to the following nasal.]

nama, nama nama Termanu.

creep, of a worm or insect. (J:379)

nama nama Korbafo.

nama nama Bokai.

nama nama Bilbaa.

nama nama Rikou.

nama nama $B a^{\prime} a$.

nama nama $T$ ii.

nama nama Dengka.

nama nama Oenale.

$<$ aknama $>$ Molo. creeping of a plant.

(M:346)

Out-comparisons:

naklama Semau Helong. tiptoe, sneak. [irr. from PRM: *n $=l$ correspondence]

*namo1 Morph: *ka-namo. $n R M$. senna (kind of tree). Senna timoriensis.

na namo Termanu. kind of healing plant called akar pele in Kupang. [Semantics: Heyne (1950:748) gives Timor Malay kayu pelen as Senna timoriensis.] (J:379)

na namo Korbafo.

na namo Bokai.

na namo Bilbaa.

na namo $B a^{\prime} a$.

na namo Tii.

k|namo|? Kotos Amarasi. senna. Senna timoriensis. [Form: Middelkoop (1972:347) gives $<$ tefu namo $>$ 'wild sugarcane' and $<$ namo $>$ 'chair of banana and sugarcane' which may be related.] [Semantics: Identification from Heyne (1950:748) who has 'Timor' $<$ kĕnamoh $>$.]

*namor PRM. coast, beach. Etym: *namaw 'sheltered water: deep place in a river; cove, harbor, lagoon'.

namo Termanu. coast, beach. (J:379) namo Korbafo.

namo Bokai. namo Bilbaa.

namo Rikou.

namo $B a^{\prime} a$.

namo Tii.

namo Dengka.

namo Oenale.

namo Molo. area around a village. (M:347)

Out-comparisons:

namon East Tetun. mouth of river, port. (Mo:151)

namo(n) Ili'uun. earth, field, garden, place, world. (dJ:129)

*namba Morph: *ka-namba-k. $n R M$. leaves covering fruit.

napa-k Termanu. the leaves which cover the fruit of palm trees. (J:380)

napa Korbafo.

napa-k Bokai.

napa Bilbaa.

napa Rikou.

namba-k Tii.

ma-k|napa-? Kotos Amarasi. squeezed together, conjoined.

uki k|napa|? Molo. the leaves of the heart-shaped banana blossom. (M:222)

*nana Morph: *nana-k. Rote. pus. Etym:

*nanaq.

nana-k Termanu. pus. (J:380)

nana-? Korbafo.

nana-k Bokai.

nana-? Bilbaa.

nana-? Rikou.

nana-k $B a^{\prime} a$.

nana-k Tii.

nana-? Dengka.

nana-? Oenale.

*naye Rote. swim. Etym: *nayuy. [irr. from PMP: *uy $>*_{\mathrm{e}}($ expect $\left.* \mathrm{i})\right]$ [minority from PMP: $*_{\eta}=*_{\eta}$ (required for Termanu and Korbafo)] [irr. from PRM: * $\mathrm{n}>\varnothing$ in most nRote (reanalysis as agreement?)] [History: The Rote forms are possible borrowings from Helong. This could explain $\eta$ in Termanu and Korbafo. Final *uy > 
$e$ would also be irregular in Helong, but proposing that the Rote forms are borrowings from Helong requires positing irregular sound changes in one less instance.]

(?)ane Termanu. swim. (J:12)

(?)aye Korbafo.

(?)ane Bokai.

(?)ane Bilbaa.

(?)ane Landu. swim. (own field notes)

Pane Rikou. [Form: My consultants gave na-?ane 's/he swims'.]

(?)ane $B a^{\prime} a$.

nane Tii.

nane Dengka.

nane Oenale.

Out-comparisons:

nayen Semau Helong. swim.

nani East Tetun. swim; climb (of plants). (Mo:152)

*naru PRM. long, length. Doublet:

*daru. Etym: *anaduq (Reflexes of

the doublet *adaduq $>$ *daru are (apparently) not found in wRote. This indicates that the Meto forms are probably inheritances of *anaduq $>$ *naru even though they would also be regular from *adaduq).

ma-nalu (2) nalu (3) nalu-k, manalu-k Termanu. 1) long. 2) long. 3) length. (J:378)

ma-nalu Korbafo.

ma-nalu Bokai.

ma-nalu Bilbaa.

naru-? Rikou. tall, long. (Nako et al. 2014)

ma-nalu $B a^{\prime} a$.

ma-nalu Lole. long. (Zacharias et al. 2014)

ma-naru Tii.

nalu-? Dengka. long, tall. (J:378, 736)

naru-? Oenale. long, tall. (J:378, 736)

naru-? Dela. tall, long.

m|nanu|? Ro'is Amarasi. long, length, deep, depth.

m|nanu|? Kotos Amarasi. long, length, deep, depth. m|nanu|? Molo. long. [Note: No headword given for mnanu? but many examples of this word occur in other entries.] (M:583)

\section{Out-comparisons:}

naruk East Tetun. long, lengthy, tall, lofty. (Mo:152)

*nase Morph: *ka-nase. PRM. fish, Bluespot Mullet. Moolgarda seheli. Etym: *kanasay. [Form: The initial *ka of the PMP form was probably reanalysed as the nominal prefix in PRM.]

nase Termanu. kind of fish, the ikan belanak. (J:381)

nase Korbafo.

nase Bokai.

nase Bilbaa.

nase Rikou.

nase $B a^{\prime} a$.

nase Tii.

nase Dengka.

nase Oenale.

k|naes Ro'is Amarasi. mullet. [Note: Jonker (1908:381) gives unmetathesised Meto knasa which would probably attest irr. *e > a.] [Form: metathesised form of (currently unattested) *knase.]

\section{Out-comparisons:}

hnase Helong. ( $\mathrm{J}: 381)$

knase (2) manu knase East Tetun. 1) fish. 2) hen or rooster whose spots are like those of the fish. (Mo:113)

*nasi PRM. jackal jujube (kind of fruit tree). Ziziphus oenoplia. [Form: Helong attests earlier *y.]

asi/nasi Termanu. kind of tree. Ziziphus oenoplia. (J:15; Heyne 1950:1003, cxv)

asi/nasi Korbafo. kai_nasi Bilbaa. ai_nasi Rikou. asi/nasi $B a^{\prime} a$. asi/nasi $T i i$. nga/nasi Dengka. jga/nasi Oenale. 
<asnási> Meto. Ziziphus timoriensis. (Meijer Drees 1950:1)

Out-comparisons:

<kaingasi> Helong. Ziziphus oenoplia. (Heyne 1950:1003, cxv)

ai knase East Tetun. tree with sticky gum. (Mo:113)

*nasu PRM. cook by boiling. Etym: *nasu.

nasu Termanu. boil. (J:381)

nasu Korbafo.

nasu Bokai.

nasu Bilbaa.

nasu Rikou.

nasu $B a^{\prime} a$.

nasu Tii.

nasu Dengka.

nasu Oenale.

n-nasu Kotos Amarasi. cook greens by boiling.

n-nasu Molo. cooks. n-nasu utan one cooks uncut greens (M:352)

*natu Morph: *natu-k. PRM. ovary, gamete. Etym: *natuq 'ovary of an oviparous animal'.

natu-k, (2) manu natu-k, (3) manu tolo natu-k Termanu. 1) the pollen of flowers, etc. 2) the beginnings of an egg in the body of a chicken. 3) egg yolk. (J:382)

natu-? Korbafo.

natu-k Bokai.

natu-? Bilbaa.

natu-? Rikou.

natu-k $B a^{\prime} a$.

natu-k Tii.

natu-? Dengka.

natu-? Oenale.

natu-f Kotos Amarasi. ovaries.

Out-comparisons:

natu? Semau Helong. roe.

keradu Hawu. egg yolk. (J:382)

*natu|n PRM. hundred. Etym: *sapa-Ratus. [Form: Inherited via intermediate **yatus. While the final *n may be a fossilised suffix, which suffix it would be is unclear.] natun Termanu. hundred. (J:382)

natun Korbafo.

natun Bokai.

natun Bilbaa.

natun Rikou.

natun $B a^{\prime} a$.

natun Tii.

natun Dengka.

natun Oenale.

nautun Ro'is Amarasi. hundred.

natun Kotos Amarasi. hundred.

natun Molo. hundred. (M:355)

natun Kusa-Manea. hundred.

Out-comparisons:

yatus Semau Helong. hundred.

atus East Tetun. hundred. [Form:

Not inherited via intermediate

**yatus.] (Mo:5)

rahu Kisar. hundred. [irr. from PMP: *t $>h$ (possibly via irregular medial *s)]

yasu Dhao. hundred.

yahu Hawu. hundred.

*nee1 PRM. six. Etym: *onəm. [irr. from $P M P:{ }^{*}$ ə $>\varnothing / \#$ ( or final $* \mathrm{VC}>\mathrm{CV}$ metathesis)]

nee Termanu. six. (J:383)

nee Korbafo.

nee Bokai.

nee Bilbaa.

nee Rikou.

nee $B a^{\prime} a$.

nee Tii.

nee Dengka.

nee Oenale.

nee Ro'is Amarasi. six.

nee Kotos Amarasi. six.

$<$ ne> Molo. six. (M:358)

nee Kusa-Manea. six.

Out-comparisons:

eney Semau Helong. six.

neen East Tetun. six. [irr. from PMP: *o $>e($ expect $o)]$

(Mo:153)

neem, neme, həneem, eneem Kemak. six.

inam Welaun. six.

ineen Galolen. six. 
ha-neen Ili'uun. six. (dJ:94, 130)

fa-nen Tugun. six. (Hinton 1991:63)

woneme Kisar. six.

əna Hawu. six.

*nee2 PRM. quiet, still, at rest. Etym: *qonəy. [irr. from PMP: *ə $>\varnothing$ with doubling of the final vowel to create a disyllable, or medial $\mathrm{VC}>\mathrm{CV}$ metathesis]

nee nee (2) na-ma-nee Termanu.

1) still, quiet. 2) ana sunu na-ma-nee $s /$ he is fast asleep, $s /$ he is deep asleep ( $\mathrm{J}: 384)$

nee nee (2) na-ma-nee Korbafo. nee nee (2) na-ma-nee Bokai. nee nee (2) na-ma-nee Bilbaa.

(2) na-ma-nee Rikou.

nee nee (2) na-ma-nee $B a^{\prime} a$.

nee nee (2) na-ma-nee $T i i$.

nee nee (2) na-ma-nee Dengka.

nee nee (2) na-ma-nee Oenale.

na-m|nee Kotos Amarasi. calm.

Out-comparisons:

hanook East Tetun. keep quiet, or silent, shut up. (Mo:76)

*nehi Rote. mat. See: *nahe. Etym: **nepi (pre-RM). [History: Blust and Trussel (ongoing) reconstruct PMP *təpiR which is the source of Hawu dəpi and Dhao dzəpi. However, initial $*$ t cannot regularly account for initial $n$ in the Rote or Bima forms.]

ne?i (2) ne ne?i-k Termanu. 1) lie on top of something. ana ne?i ne?a-k esa he lies on a mat. (J:386)

2 ) the action of lying on something; mat, bag functioning as a pillow or mattress (Dutch bultzak). (J:387)

ne?i Korbafo.

ne?i Bokai.

nei Bilbaa.

ne?i (2) ne $\sim$ ne?i-k $B a^{\prime} a$.

(2) ne ne?i-k Lole. mat. (Zacharias et al. 2014)

ne?i (2) (ne $)$ ne?i-k Tii.

(2) ne nei-? Dengka.

(2) ne nei-? Oenale.
(2) ne nei-? Dela. mat, rug.

Out-comparisons:

nepi Bima. mattress, bag functioning as a pillow or mattress (Dutch bultzak). (Jonker 1893:60)

*neko Morph: *neko-k. $n R M$. abomasum (the fourth and final stomach compartment in ruminants that secretes rennet). Pattern: k-5/6.

neko-(k) Termanu. one of the parts of the stomach of a ruminant, the abomasum, in Kupang perut lingkar. (J:387)

neko-k Tii. neko-n Meto. (J:387)

*nembə Morph: *nembə-k. Rote. hard, fixed. Etym: *təbəl 'thick, of objects; dense, of crowds'. [irr. from PMP: *t $>*_{\mathrm{n}} ; *_{\mathrm{b}}>*^{*} \mathrm{mb}$ ] [irr. from PRM: *n $>$ $m$ in Bilbaa and Rikou]

nepe-k Termanu. hard, fixed. (J:391) nepe Korbafo. nepe-k Bokai. mepe-? Bilbaa. mepe-? Rikou. nempe-k $B a^{\prime} a$. nemba-? Oenale.

*nene1 PRM. press, push. [irr. from PRM: ${ }^{*} \mathrm{n}>$ ? in Rote except Bilbaa and Rikou] ne?e Termanu. push. (J:386)

ne?e Korbafo.

ne?e Bokai.

nene Bilbaa.

nene Rikou.

ne?e $B a^{\prime} a$.

ne?e Tii.

ne?e Dengka.

n-nene Kotos Amarasi. press, push. na-nene Molo. presses on. (M:362)

*nene2 Rote. hear, listen. [History: Perhaps connected with PMP *dəyəR, though this requires irregular initial $*_{\mathrm{d}}>*_{\mathrm{n}}$ and final $*_{\partial}>*_{\mathrm{e}}$ (expect final $*_{\partial}=* \partial$ ). Furthermore, ${ }^{*}$ dəyəR is retained regularly in Oenale and Meto (see *rena).] 
na-ma-nene (2) nene nene

(3) ne nene Termanu. 1) hear.

2) listen. 3) listen. (J:388)

na-ma-nene Korbafo.

na-ma-nene Bokai.

na-ma-nene Bilbaa.

na-ma-nene Rikou.

na-ma-nene $B a^{\prime} a$.

ni-mi-nene Lole. (J:737)

na-ma-nene, i-mi-nene Tii. (J:388, 705)

na-ma-nene Dengka.

na-ma-nene Oenale.

*neo Rote. shy, jealous.

neo Termanu. shy, be scared. (J:390)

neo Korbafo.

neo Bokai.

na-neo Bilbaa.

neo Rikou.

neo $B a^{\prime} a$.

neo Lole. suspicious. (Zacharias et al. 2014)

neo Tii.

neo Dengka.

na-ma-neo Oenale.

Out-comparisons:

neo Semau Helong. jealous.

*neru Morph: *na-ma-neru. Rote. warm oneself. [irr. from PRM: ${ }^{*} \mathrm{e}>i$ in several Rote lects (sporadic assimilation)] [Form: Reconstruction of penultimate ${ }^{*}$ e requires irr. ${ }^{*} \mathrm{e}>i$ in at least three separate cases: West Rote (Oenale, Dengka), central Rote (Termanu, Bilbaa), and Hawu. However, this sound change can be motivated as a case of sporadic assimilation to the following high vowel and/or an earlier palatal nasal (as attested in Hawu). Reconstruction of penultimate *e would require unmotivated $*_{i}>e$ in at least two cases: eastern Rote (Korbafo, Bokai, Rikou) and Ba'a.]

na-ma-nilu Termanu. warm oneself. (J:397)

na-ma-nelu Korbafo.

na-ma-nelu Bokai. na-ma-nilu Bilbaa.

na-ma-neru Rikou.

na-ma-nelu $B a^{\prime} a$.

na-ma-niru $T i i$.

na-ma-nilu Dengka.

na-mba-niru Oenale.

Out-comparisons:

mepiru ai Hawu. warm self over fire.

*nesa $n R M$. same, alike. [History: This is almost certainly connected with *esa 'one'.]

na-ka-nesak Termanu. compare, equate. (J:391)

na-ka-nesak Bokai.

na-ka-nesak $B a^{\prime} a$.

na-ka-nesak Tii.

na-m|nesa Kotos Amarasi. same.

na-nesa (2) na-m|nesa Molo.

1) agree with one another. 2) alike.

(M:364)

Out-comparisons:

hanesa-n East Tetun. be the same, equal, or identical, be alike; $n$. similar; $a d v$. similarly, equally, of the same type, shape, make, or size. (Mo:75)

*nesu Morph: *nesu-k. PRM. mortar. Etym: *losuy. [irr. from PMP: *1 > *n] [irr. from PRM: *n $>\varnothing$ in most Meto (also Helong)] [Form: Outcomparisons point to pre-RM **yəsun, perhaps with metathesis of initial and final consonants.]

nesu-k Termanu. mortar. (J:392)

nesu-? Korbafo.

nesu-k Bokai.

nesu-k Bilbaa.

nesu-? Rikou.

nesu-k $B a^{\prime} a$.

nesu-k Tii.

nesu-? Dengka.

nesu-? Oenale.

eusu|k Ro'is Amarasi. mortar.

esu|k Kotos Amarasi. mortar.

esu|? Molo. mortar. (M:104)

nesu|k Kusa-Manea. mortar. 


\section{Out-comparisons:}

isuy Helong. mortar. ( $\mathrm{J}: 392)$

nesun East Tetun. mortar, part of a tree trunk with a hollow at one end, used in conjunction with alu for de-husking grain; $v$. to pound or grind grain, tapioca, etc. [irr. from PMP: *o $>e$ (expect $o$ )] (Mo:154)

knehun Ili'uun. mortar (for ricepounding). (dJ:121)

nesug Kemak. mortar. Usage: Saneri and Leosibe dialects.

kosun Welaun. mortar.

yətfu Dhao. mortar and pestle.

*neta Morph: *na-ma-neta. Rote. choke. Etym: **ma-neta (pre-RM).

na-ma-neta Termanu. choke, unable to take a breath. (J:392)

na-ma-neta $B a^{\prime} a$.

na-ma-neta $T i i$.

na-ma-neta Dengka.

na-ma-neta Oenale.

Out-comparisons:

mejita Hawu. (J:392)

*nihe Morph: *nihe-k. Rote. ant. Etym:

*nipay 'snake'. [irr. from PRM: *e >

$a$ in Ba'a; ${ }^{*} \mathrm{e}>i$ in Korbafo and East

Rote (sporadic assimilation?)]

ne?e-k Termanu. ant. (J:386)

ni?i-? Korbafo.

nii-? Bilbaa.

nii-? Landu. (own field notes)

nii-? Rikou.

nii-? Oepao. (own field notes)

ni?a-k $B a^{\prime} a$.

niPe-k Lole. ant. (Zacharias et al. 2014)

ni?e-k Tii.

nie-? Dengka.

nie-? Oenale.

Out-comparisons:

ne?ek Fehan Tetun. ant.

nehek East Tetun. ant (of many

varieties). (Mo:153)

nee Kisar. snake. *nihis Morph: *ma-nihis. PRM. thin. Etym: *nipis 'thinness (of materials)'. [irr. from PRM: $* \mathrm{i}>a$ in Meto; ${ }^{*} \mathrm{~h}>$ ? /V_V in wRote (expect Ø)]

ni?is (2) na-ma-ni?i Termanu. 1) thin. 2) become thin. (J:396) ni?i-? Korbafo.

nii-? Bilbaa.

niis Landu. thin. (own field notes)

niis Rikou.

ni?is $B a^{\prime} a$.

niPis Lole. thin. (Zacharias et al. 2014)

ni?is Tii.

ni?is Dengka.

ni?is Oenale.

mainihas Kotos Amarasi. thin. [Note: I also have manihis as an alternate form in my dictionary but I don't know the source of this form and when I checked it with my main Amarasi consultant he was mystified by it.]

mainihas Molo. thin. (M:xxx)

manihis Timaus. thin. [Form: regular assimilation of $*$ a in final closed syllables.]

Out-comparisons:

mnihis Funai Helong. thin.

nihis Semau Helong. thin.

ni?is Fehan Tetun. thin (not thick), sensitive (of a person).

mihis East Tetun. thin, slender (slices, etc.). (Mo:142)

*niit Morph: *ka-niit. PRM. crab. [Form:

The Tetun forms point to earlier **kanipis, though positing these forms as cognate requires irr. ${ }^{*} p>\left({ }^{*} h\right)>\varnothing$ in Termanu, Ba'a, Bokai, Korbafo and Tii.]

nii-k, iPa nii-k Termanu. crab. (J:396)

nii-? Korbafo.

nii-k Bokai.

nii-? Bilbaa.

nii-? Rikou.

nii-k $B a^{\prime} a$.

nii-k Tii. 
niit, nii-? Dengka. [Note: Both Dengka forms flagged as 'also surely Dengka'.]

niit Oenale.

k|niit Ro'is Amarasi.

k|niit Kotos Amarasi. crab.

k|niit Molo. horn (instrument). (M:223)

Out-comparisons:

kniit Funai Helong. crab.

hniit Semau Helong. crab.

ni?is Fehan Tetun. freshwater crab. [irr. from PRM: *Ø $=?$ correspondence; $* \mathrm{t}=s$ correspondences (perhaps influenced by ni?is 'thin')]

nihis East Tetun. small crab of quiet water. Usage: Luka and Ue Keke villages. [irr. from PRM: $* \varnothing=h$ correspondence; $* \mathrm{t}=s$ correspondences (perhaps influenced by mihis 'thin')] (Mo:154)

mihis East Tetun. a variety of crab. Usage: Bubu Susu village. [irr. from PRM: ${ }^{*} \mathrm{n}=m$ correspondence] (Mo:154)

kaniik Kupang Malay. (J:396)

*nimba Rote. kind of fish. [irr. from PRM: *a $>e$ in most Rote]

nipa Termanu. kind of sea fish called ikan nipe in Kupang. (J:398)

nipe Korbafo.

nipe Bokai.

nipe Bilbaa.

nipe Rikou.

nimpa $B a^{\prime} a$.

nimbe Tii.

nimbe Dengka.

nimbe Oenale.

Out-comparisons:

nipe Semau Helong.(J:398)

*nini Morph: *nini-k. Rote. mosquito. Etym: *ñikñik 'tiny biting insect: gnat, sandfly'.

nini-k Termanu. mosquito. (J:397)

nini-k Korbafo. nini-? Bokai.

nini-? Bilbaa.

nini-? Landu. mosquito. (own field notes)

nini-? Rikou.

nini-k $B a^{\prime} a$.

nini-k Tii.

nini-? Dengka.

nini-? Oenale.

Out-comparisons:

kniki? Funai Helong. mosquito.

niki?, hmiki Semau Helong. mosquito.

ninik East Tetun. small mosquito with a painful sting. (Mo:154)

nini Hawu. kind of small mosquito. (J:397)

*nisi Morph: *nisi-k. PRM. tooth, teeth. Etym: *yis(y)i(s) 'grin, show the teeth'.

nisi-k Termanu. tooth. (J:398)

nisi-? Korbafo.

nisi-k Bokai.

nisi-? Bilbaa.

nisi-? Landu. (own field notes)

nisi-? Rikou.

nisi-? Oepao. (own field notes)

nisi-k $B a^{\prime} a$.

nisi-k Tii.

nisi-? Dengka.

nisi-? Oenale.

nisi-f Ro'is Amarasi. tooth.

nisi-f Kotos Amarasi. tooth.

nisi-k Molo. tooth. (M:372)

nisi-f Kusa-Manea. tooth.

Out-comparisons:

sii Semau Helong. tooth. [irr. from $P M P: * n i>\varnothing]$

kisaa-t Welaun. teeth. [irr. from $P M P: *$ is $>\varnothing$, apparently from intermediate $* *$ jis]

*nitas PRM. hazel sterculia tree. Sterculia foetidea.

nitas Termanu. kind of tree also called nitas in Kupang, the wood of this tree is used in carpentry, amongst other things for coffins, the fruit 
of this tree (the nita boa-k) yields a kind of resin: dama nitas, the ashes of the dried husks are also used. (J:399f)

nita-? Korbafo.

nita-? Bokai.

nita-? Bilbaa.

nitas Rikou.

nitas $B a^{\prime} a$.

nitas Tii.

nitas Dengka.

nitas Oenale.

$<$ nitas $>$ Molo. kind of tree. Sterculia foetidea. (M:374)

\section{Out-comparisons:}

nitas, ai knitas East Tetun. tree with good timber. Sterculia foetidea. (Mo:153)

nita Sika. Sterculia foetidea. (Verheijen 1984:67)

nita? Komodo. Sterculia foetidea. (Verheijen 1984:67)

nintap Manggarai. Sterculia foetidea. (Verheijen 1984:67)

litap East Manggarai. Sterculia foetidea. (Verheijen 1984:67)

litat Kepo'. Sterculia foetidea. [Note: language of central Flores ISO 639-3 [kuk].] (Verheijen 1984:67)

litat Rajong. Sterculia foetidea. [Note: language of central Flores ISO 639-3 [rjg].] (Verheijen 1984:67)

*nitu PRM. spirit. Etym: *qanitu 'ghost, ancestral spirit; nature spirit; corpse; owl; various plants'.

nitu Termanu. spirits of the ancestors; demon. (J:400)

nitu Korbafo.

nitu Bokai.

nitu Bilbaa.

nitu Rikou.

nitu $B a^{\prime} a$.

nitu Tii.

nitu Dengka.

nitu Oenale. nitu Kotos Amarasi. spirit of dead person, corpse.

nitu Molo. corpse and spirit. (M:374)

*nodo Rote. crawl, creep. Doublet:

*rodok.

nodo nodo Termanu. crawl forward, usually said of children; push forward on one's behind. (J:402)

nodo nodo Korbafo.

nodo nodo Bokai.

nodo nodo Bilbaa.

nodo nodo Rikou.

nodo nodo $B a^{\prime} a$.

nodo $\sim$ nodo Tii.

nodo nodo used in place of lodo for the crawling of snakes, etc. Dengka.

nodo nodo Oenale. used in place of lodo for the crawling of snakes, etc.

*noe PRM. melt.

noe Termanu. melt, dissolve; melt something. (J:402)

noe Korbafo.

noe Bokai.

noe Bilbaa.

noe Rikou.

noe $B a^{\prime} a$.

noe $T i i$.

noe Dengka.

noe Oenale.

na-m|noe Kotos Amarasi. melt.

na-m|noe, na-t|noe (2) na-noe-b Molo. 1) melted. 2) someone melts something. (M:377)

\section{Out-comparisons:}

noe(m) (2) noe Semau Helong. 1) melt. 2) destroyed, damaged, shattered, smashed, crushed.

*noh PRM. coconut. Etym: *niuR. [irr. from PMP: * $\mathrm{i}>\varnothing ; * \mathrm{u}>*_{0} ; * \mathrm{R}>$ *h (also in *klaha and *taha)] [irr. from PRM: $\varnothing>a$ in Meto (also Kemak)] [History: The connection between PMP *niuR and PRM *noh involves several irregularities. Firstly, final ${ }^{*} \mathrm{~h}$ must be reconstructed to account for the Meto reflexes as Meto usually 
retains *h finally while it is lost in Rote (cf. *dilah, *mbuah, *sikəh, *henuh, and *nunuh). PRM *h could plausibly, though irregularly, be derived from $* \mathrm{R}$ as the change $* \mathrm{R}>* \mathrm{~h}$ is attested in two other forms (cf. *klaha, *taha). Secondly, the lowering of $* u>$ $*_{0}$ must be accounted for. While lowering of high vowels to mid before $* \mathrm{R}$ is sporadically attested in the Rote-Meto languages, it is not attested in other languages of the Timor region included in the out-comparisons that also have irr. ${ }^{*} \mathrm{u}>o$ in this form (e.g. Ili'uun iku 'tail' $<*$ ikuR, telu $<*$ qatəluR 'egg'). This indicates that $* \mathrm{u}>o$ is an irregular development before the time of PRM. Thirdly, the second vowel $a$ must be accounted for in Meto. This could be an irregular insertion in PMeto in order to create a disyllable, though we would probably expect that the original single vowel would have been doubled, as in the Rote languages and several other languages of the region. (Helong has nian, which also has irregular insertion of $a$, though the retention of $*_{\mathrm{i}}=i$ in Helong indicates that it is not directly connected with the Meto form.) Finally, reflexes of PMP *niuR that do not attest irr. $*_{\mathrm{u}}>o$ are also found in the greater Timor region. Examples include Tetun nuu, Welaun nuu, Leti nura and Dhao jiu. This indicates that the precursor to irregular *noh probably occurred alongside regular reflexes of PMP *niuR in this region.]

noo Termanu. coconut (tree and fruit). (J:400)

noo Korbafo.

noo Bokai. noo Bilbaa.

noo Landu. coconut. (own field notes)

noo Rikou.

noo $B a^{\prime} a$.

noo Tii.

noo Dengka.

noo Oenale.

noah Ro'is Amarasi. coconut.

noah Kotos Amarasi. coconut.

noah Molo. coconut. (M:376)

noah, noha Kusa-Manea. coconut. [Form: The form noha ['nohe] may be due to reanalysis of noah as a metathesised form given that Kusa-Manea does not have assimilation of /a/ after metathesis. This is also attested in puah, puha 'betel nut'.]

\section{Out-comparisons:}

noo Galolen. coconut.

noo Mambae, South. coconut.

(Grimes et al. 2014b:35)

noo Ili'uun. coco-tree. (dJ:130)

noor Tugun. coconut. (Hinton 2000:120)

nor Kisar. coconut.

noa Kemak. coconut. Usage: Kutubaba and Leolima dialects. [Form: Final $a$ in Kemak may be an irregular vocalisation of earlier final $* \mathrm{R}$. Compare *qauR > Kemak oa $\sim$ ua 'bamboo'.]

nua Kemak. coconut. Usage: other known varieties of Kemak.

*noko Rote. shake. Pattern: k-5. [irr. from PRM: ${ }^{*} \mathrm{o}>u$ in second sense in Korbafo, Bokai, and Bilbaa]

noko noko (2) na-ka-no noko

Termanu. 1) shake, move in a shaking manner. 2) shake something. (J:405)

(2) na-ka-nu nuku Korbafo. noko noko (2) na-ka-nu $\sim$ nuku Bokai.

(2) na-ka-nu nuku Bilbaa. noko noko Rikou. 
noko noko (2) na-ka-no noko $B a^{\prime} a$.

noko noko (2) na-ka-no noko Tii.

noko noko Dengka.

Out-comparisons: noko, kenoko Hawu. shake. (J:405)

*nono1 PnMeto. sorcery. [Form: If these forms are indeed related, they probably point to earlier $* *$ nodo(h).]

$<$ le?u nono> Molo. fertility magic. (M:384)

Out-comparisons:

in nodo Funai Helong. sorcerer, witch.

nodoh Semau Helong. sorcery.

*nono2 PnMeto. family group.

nono (2) nono-t Kotos Amarasi.

1) kind of jungle rope, liana; descent group. 'lianas which encircle the clan and hence symbolize its fertility' (Schulte Nordholt 1971:116). 2) family, clan group. nono-t Molo. origin group. (M:384)

\section{Out-comparisons:}

noro Buru. origin group, kin group, clan. (Grimes and Grimes 2020:663)

*nori Morph: *na-nori. PRM. teach, learn.

na-noli Termanu. learn, instruct. (J:406)

na-noli Korbafo.

na-noli Bokai.

na-noli Bilbaa.

na-nori Rikou.

na-noli $B a^{\prime} a$.

na-nori $T i i$.

na-noli Dengka.

na-nori Oenale.

na-noni? Ro'is Amarasi. learn, teach.

na-noni? Kotos Amarasi. learn, teach. na-noin-a? Molo. points out. (M:379) Out-comparisons:

hanorin Fehan Tetun. teach, instruct, indoctrinate. hanourin East Tetun. teach, instruct, indoctrinate. (Mo:76) nori Kisar. coax, persuade, woo. nori (2) noir Mambae, South. 1) teaching, lesson. 2) teach, instruct. (Grimes et al. 2014:35) nori Dhao. teaching.

*noto Morph: *noto noto. PRM. slow.

noto noto Termanu. slow (walking). (J:407)

noto noto Korbafo.

noto noto Bokai.

noto noto Bilbaa.

noto noto Rikou.

noto noto $B a^{\prime} a$.

noto noto $T i i$.

noto noto Dengka.

not noto? Kotos Amarasi. slow, taking some time; e.g. describes the situation of a person very early in the morning when they have just gotten out of bed, they are still sleepy and just sitting around without having started doing anything.

Out-comparisons: noto notok Fehan Tetun. quiet. noto Mambae, South quiet, silent. (Grimes et al. 2014b:36)

*nudu Morph: *nudu-k. PwRM. outer mouth region. Etym: *yusuq 'nasal area; snout'. [irr. from PMP: * $\mathrm{s}>* \mathrm{~d}$ ] [Sporadic: ${ }^{*} \mathrm{n}>r / l$ in most varieties of Meto (except Ro'is Amarasi and optionally Kusa-Manea).] [Form: Irregular $*_{S}>* d$ may have been through intermediate $*_{\mathrm{z}}$ [d]]. Irregular $*_{\mathrm{s}}>*_{\mathrm{z}}$ is also found in Celebic for this form, e.g. Balaesang yudu, Dampelas yudzu (Himmelmann 2001:160f). However, while the reflexes in southwest Maluku also have irregularities in this consonant, these cannot be attributed to *z. Instead, they attest irregular $*_{\mathrm{s}}>*_{\mathrm{d}}$.]

nudu-? Dengka. lip, beak of bird. (J:739) 
nudu-? Oenale. lip, beak of bird. (J:739)

nuru-n Ro'is Amarasi. lip.

ruru-f Kotos Amarasi. outer mouth region.

lulu-f Molo. lip(s). (M:292)

nuru-f, ruru-f Kusa-Manea. lips.

Out-comparisons:

nuru Ili'uun. mucus, slime. (dJ:148)

\section{nuran (2) nuran wulla}

(3) nurhe Kisar. 1) mouth.

2) moustache. 3) snot.

nuru Luang. mouth. [Note: language of southwest Maluku ISO 639-3 [lex].] (Taber 1993:424)

nur-e Central Marsela. mouth. (Taber 1993:424)

*nu?a PRM. scabies. Etym: *nuka 'wound'.

[Sporadic: *a $>$ e/_\# in Meto.]

nu?a Termanu. scabies. (J:408)

nu?a Korbafo.

nu?a Bokai.

nua Bilbaa.

nu?a Rikou.

nu?a $B a^{\prime} a$.

nu?a Tii.

nu?a Dengka.

nu?e Amanuban. wound. (M:389)

Out-comparisons:

nuka East Tetun. ulcer, atrophic sore (difficult to heal, possibly due to poor nutrition). (Mo:155)

no?o Kisar. wound, sore, cut.

nua West Damar. wound. [Note: language of southwest Maluku ISO 639-3 [drn].] (Chlenov and Chlenova 2008:154)

*numbu Morph: *na-numbu. Rote. grow, germinate, sprout. Doublet: *tumbu. Etym: *tu(m)buq. [irr. from PMP: $*_{\mathrm{t}}>*_{\mathrm{n}}$ (also in Helong and Central Lembata)]

na-nupu Termanu. sprouting, germination of seeds, fruits. (J:409)

na-nupu Korbafo.

na-nupu Bokai. na-nupu Bilbaa.

na-nupu Rikou.

na-numpu $B a^{\prime} a$.

na-numbu Tii.

na-numbu Dengka.

na-numbu Oenale.

Out-comparisons:

nupu Semau Helong. grow, shoot. nubu Central Lembata. grow.

(Fricke 2015)

*nunuh PRM. banyan tree. Etym:

*nunuk.

nunu-? Bilbaa. banyan. (J:739)

nunu-? Rikou.

nunu-k Tii.

nunu_londa-? Dengka. aerial roots of the banyan tree. [Note: liti-? = 'banyan tree'.] (J:739)

nunu_londa-? Oenale. aerial roots of the banyan tree. [Note: liti bibi-? = 'banyan tree'.] (J:739)

nunah Ro'is Amarasi. banyan tree.

nunuh Kotos Amarasi. banyan tree.

nunuh Molo. banyan tree. Ficus species. (M:393)

Out-comparisons:

nunu? beas Semau Helong. banyan.

*nuya $P R M$. kind of flowering tree. Cordia species. Etym: **nuyan (preRM).

kai/nuna-k Termanu. kind of tree, people usually put the afterbirth in a certain kind of basket and hang it from this tree. $(\mathrm{J}: 216)$

kai/nuna-? Korbafo.

kai/nuna-k Bokai.

kai_nuna-? Bilbaa.

ai_nuna-? Rikou.

ngai/nuna-k $B a^{\prime} a$.

nuna-k Tii.

nunga-? Dengka.

nunga-? Oenale.

nuk/ba?i Kotos Amarasi. kind of tree.

nun/ba?i, nuk/ba?i, kuk/ba?i (2) kuk/bai? tasi, nuk/bai? tasi (3) nuk/bai? < nono> (4) nun/bai? fui (5) ?|nuna fui Molo. 1) kind of 
tree. Cordia species. [Form: Forms with initial and medial $k$ probably show sporadic assimilation of $* n>$ *y /_Vy. (Compare *nura 'young' for similar assimilation.)] (M:390, 391, 245) 2) kind of tree. Cordia subcordata. (M:390, 245) 3) kind of tree. Cordia dichotoma. (M:390)

4) kind of tree. Cordia obliqua. (M:391) 5) kind of tree with a soft trunk. Cordia obliqua. (M:391)

\section{Out-comparisons:}

tatasi_nunay Helong. Cordia obliqua. (Heyne 1950:1307, $\operatorname{ccxx}$ )

ai nunan East Tetun. tree with good red timber. Cordia subpubescens. (Mo:155)

*nusa PRM. island, state. Etym: *nusa.

nusa-k Termanu. island; land, landscape, state; city. (J:409)

nusa-? Korbafo.

nusa-k Bokai.

nusa-? Bilbaa.

nusa-? Rikou.

nusa-k $B a^{\prime} a$.

nusa-k Tii.

nusa-? Dengka.

nusa-? Oenale.

nusa Timaus. island.

nusa Meto. (J:409)

Out-comparisons:

nusa Semau Helong. kingdom, nation, state, country, island.

nusa East Tetun. island. (Mo:155)

*nuu1 Morph: *nuu-k. Rote. bird's nest.

manu_nuu-k Termanu. kind of

basket made from lontar leaves for hens to lay their eggs in. (J:408)

manu_nuu-? Korbafo.

manu_nuu-k Bokai.

manu_nuu-? Bilbaa.

manu_nuu-? Rikou.

manu_nuu-k $B a^{\prime} a$.

manu_nuu-k Tii.

manu_nuu-? Dengka.

manu_nuu-? Oenale.

\section{Out-comparisons:}

hnoo Semau Helong. nest. [irr. from PRM: ${ }^{*} \mathrm{u}=o$ correspondence]

snoo Bolok Helong. nest.

knuuk East Tetun. nest, den, lair (of animals and birds). (Mo:114)

*nuur CERM. property, possession. Etym: *anu 'unnamed thing'.

nuu Termanu. property, possession. (J:407)

nuu Korbafo.

nuu Bokai.

nuu Bilbaa.

nuu Rikou.

=nu Kotos Amarasi. used to mark an otherwise unexpressed plural possessum with VV final pronouns.

*nuus Rote. squid. Etym: *nuəs (Blust and Trussel (ongoing) comment regarding their reconstruction: 'The search for a PMP term for 'squid' is frustrating, since there are many words with this meaning that show greater than chance phonetic similarity, but few that exhibit recurrent sound correspondences, a situation that obtains even within the Oceanic group'.). [irr. from PMP: $*_{\partial}>\mathrm{u}$ (sporadic assimilation)] [History: Pawley (2011:200) reconstructs POc *nusa and *nus. PCEMP *nus could account for the Timor reflexes with doubling of the vowel to fulfil the disyllabic requirement for content words.]

(iPa) nuus Termanu. squid. (J:408)

nuu-? Korbafo.

nuu-? Bokai.

nuu-? Bilbaa.

nuus Rikou.

nuus $B a^{\prime} a$.

nuus $T i i$.

nuus Dengka.

nuus Oenale.

Out-comparisons:

nuus Semau Helong. squid. 
*ndake1 PnRote. climb, ascend. Etym: *dakih. Pattern: k-7/8/9/10. [irr. from PMP: *d $>*_{\mathrm{nd}}$; $*_{\mathrm{i}}>*_{\mathrm{e}}$ (influence from *sakay $>$ *sake 'go up')]

nda?e Termanu. climb (a mountain). (J:412)

nda?e Korbafo.

la?e Bokai.

na-lake Bilbaa.

ra?e Rikou.

nda?e $B a^{\prime} a$.

nda?e Tii.

*ndake 2 Rote. betel leaves. Pattern: $\mathrm{k}-8 / 9^{\prime}\left({ }^{*} \mathrm{k}>\varnothing\right.$ in Oepao, ${ }^{*} \mathrm{k}>?$, Ø in Rikou; expect only $* \mathrm{k}>$ ? in both). [irr. from PRM: *nd $>d$ in Termanu, Korbafo, Bokai, Landu, and Ba'a] [Form: Assimilations of ${ }^{*} \mathrm{nd}>d$ in first sense are due to this term being compounded with reflexes of *doo-k 'leaf', compare reflexes of *ndiki 'ear' and *bife 'lips'.]

da?e doo-k Termanu. betel, betelpepper, both the plant and the leaf. (J:69)

da?e_doo-? Korbafo.

daPe_doo-k Bokai.

lake_doo-? Bilbaa. [Form: My own field notes have Bilbaa leke doo-? 'sirih' = 'betel leaves/pepper'.]

dake_doo-? Landu. betel leaves/ pepper. (own field notes)

ra?i_doo-?, rai_doo-? Rikou. [Form: Jonker (1908:69) has medial glottal stop, Nako et al. (2014) does not.]

rii_doo-? Oepao. betel leaves/pepper. (own field notes)

da?e doo-k $B a^{\prime} a$.

nda?e_doo-k Lole. betel-pepper.

(Zacharias et al. 2014)

nda?e doo Tii.

nda?e Dengka.

nda?e Oenale.

nda?e Dela. betel leaves. *ndake_bu?u Morph: *ndake_bu?u-k. Rote. wild basil. Pattern: $\mathrm{k}-6^{\prime}\left({ }^{*} \mathrm{k}>\right.$ ? in Ba'a and Tii; expect ${ }^{*} \mathrm{k}=k,{ }^{*} \mathrm{k}>\varnothing$ in Rikou; expect *k $>$ ?).

ndake bu?u, nda?e bu?u Termanu. wild basil, called fleskrois in Kupang. (J:413)

ndake_bu?u Korbafo.

lake bü?u Bokai.

leke_buu Bilbaa.

(rae bu?u ?) Rikou.

nda?e_bu?u $B a^{\prime} a$.

nda?e_bu?u Tii.

nda?e buu Dengka.

nda?e buu Oenale.

*ndaki Morph: *ka-ndaki. PRM. kind of aquatic snake or leech. Pattern: k-5. [irr. from PRM: *nd $>r$ in Meto (expect $k)]$

ndaki Termanu. kind of water snake called ular ndaki in Kupang Malay. (J:413)

ndaki Korbafo.

laki Bilbaa.

ra?i Rikou.

ndaki $B a^{\prime} a$.

ndaki Tii.

ndaki Oenale.

?|rake|? Kotos Amarasi. leech.

<lake> Molo. leech. (M:257)

ra rake|? Kusa-Manea. leech.

*ndaru PRM. cut. [irr. from PRM: *r $>l$ in Rikou (sporadic dissimilation?)]

ndalu Termanu. cut off, cut the end off something. (J:414)

ndalu Korbafo.

lalu Bokai.

ralu Rikou.

ndalu $B a^{\prime} a$.

ndaru Tii.

ndalu Dengka.

ndaru Oenale.

n-ranu Ro'is Amarasi. cut a field.

n-kanu Kotos Amarasi. cut a field. 
$<$ kanu $>$ (2) <anPote kanu> Molo.

1) overgrown garden. 2) he cuts down the wood in a new garden. (M:180)

*ndau Rote. needle. Etym: *zaRum. [minority from PMP: ${ }^{\mathrm{z}}>{ }^{*}$ nd (expect $\left.{ }^{*} \mathrm{~d}\right)$ ] [History: This is probably an early loan, though the source language has not been identified.]

nda ndau-k Termanu. needle. (J:415)

nda ndau-? Korbafo.

la lau-k Bokai.

la lau-? Bilbaa.

ra rau-? Rikou.

nda ndau-k $B a^{\prime} a$.

nda ndau-k Tii.

nda ndau-t Dengka.

nda ndau-t Oenale.

\section{Out-comparisons:}

lauy Semau Helong. needle. [irr. from PMP: $* \mathrm{R}>\varnothing($ expect $l)]$

daun East Tetun. needle. (Mo:23)

*ndefa PRM. landslide. See: *feraz. [History: Interference/collapse with *fera2, which is likely related via consonant metathesis. A possible etymon for *ndefa is PWMP *robaq [rəbaq] 'collapse, fall down, as a house' with doublets *robas and *Rəbaq (Blust and Trussel ongoing).] ndefa (2) na-ndefa Termanu.

1) landslide of earth of stones from a mountain into a chasm; collapse. 2) drop, make something fall. [Semantics: Both senses are verbs.] (J:416f)

ndefa Korbafo.

lefa Bokai.

lefa Bilbaa.

ndefa $L a n d u$. cliff, ravine, landslide. (own field notes)

refa Rikou.

ndefa $B a^{\prime} a$.

ndefa Tii.

ndefa Dengka. ndefa (3) nde $\sim$ ndefa-? Oenale.

3) ravine. ( $\mathrm{J}: 130)$

ndefa-? Dela. push over.

refe|k (2) n-refa Ro'is Amarasi.

1) ravine, cliff, gap. 2) be affected or destroyed by a landslide.

kefa|n Kotos Amarasi. ravine, cliff, gap.

$<$ kefa $>$ Molo. gorge, steep cliff. (M:192)

*ndelat PRM. lightning. [Form: Final *t finds external support from Ili'uun. The final $t$ in Meto may have been reanalysed as a nominalising suffix.] [History: This could be connected with PWMP *kidəlat 'lightning' (Blust and Trussel ongoing) with irr. ${ }^{*} \mathrm{~d}>{ }^{*}$ nd. However, the only evidence given for PWMP *kidəlat 'lightning' is Tagalog kidlát and Malay kilat. The Malay form is also given as evidence for PMP *kilat 'lightning' (from PAN *likaC) which is much better supported than putative *kidəlat.] [Semantics: The semantic shift from 'lightning' to 'gun' which is required for some of the forms given here is reasonable and still attested by some of the forms in Meto. The Tetun and Mambae forms could be borrowings from Meto after *nd $>$ $*_{\mathrm{r}}>k$ and before $* 1>n$, borrowings from Malay kilat 'lightning', and/or inheritances of PMP *kilat 'lightning'. It has been proposed that the Atoni expansion was driven by their early acquisition of guns and maize (Fox 1988), thus Meto being the ultimate donor language is reasonable.]

ndela-s (2) na-ndela Termanu.

1) lightning. 2) lightning flashes; also glitter, sparkle, shine. (J:417)

ndela-? Korbafo.

lela-k Bokai.

lela-? Bilbaa.

ndela-s Landu. lightning. (own field notes)

rela-s Rikou. 
na-rela Oepao. lightning. (own field notes)

ndela-s $B a^{\prime} a$.

ndela-s Tii.

ndela-s Dengka.

ndela-s Oenale.

renet (2) ${ }^{\circ}$ makenet $R o$ 'is Amarasi.

1) firearms, weapons. 2) be at war. Borrowed from: Kotos Amarasi (shown by irr. $*_{\text {nd }}=k$ correspondence, expect $r$ ).

kenat (2) ma-kenat (3) na-kena Kotos Amarasi. 1) firearms, weapons. 2) be at war. 3) thunder and lightning that is very loud and typically makes people scared. [Note: The normal word for 'lightning' is rima-t from root rima 'flash, shine'.]

kenat (2) ma-kenat (3) keen neno

Molo. 1) firearms. 2) be at war.

3) thunderclap. [Form: neno = 'sky'.] (M:198)

kenat Kusa-Manea. firearm, gun.

Out-comparisons:

'lelat Semau Helong. fire-arm, weapon, gun, rifle. Borrowed from: probably Meto after *nd $>*^{*}$ and before $* 1>n$.

${ }^{\circ}$ kilat Fehan Tetun. firearm of any type: gun, cannon. Borrowed from: perhaps pre-Meto **kelat (after $* \mathrm{nd}>* \mathrm{r}>k$, but before $* 1$ $>n$ ) perhaps also influenced by Malay kilat.

${ }^{\circ}$ kilat East Tetun. firearms, any type of gun. (Mo:107)

${ }^{\circ}$ kilat Mambae, South. gun. (own field notes)

dzilat Ili'uun. lightning. (dJ:114)

noho til tila (2) Oilak Kisar. 1) thunder. [Note: noho = 'island, land, earth, world, environment, weather', noho ler lere $=$ 'lightning'.] 2) gun. *ndeli Morph: *ka-ndeli. PRM. ring. [irr. from PRM: *nd $>r / l$ in Nuclear Meto (expect $k$, indicates PMeto *ka-deli)] ndeli (2) na-ka-ndeli Termanu. 1) ring. 2) wear a ring, have a ring. (J:417)

nde ndeli Keka.

ndeli Korbafo.

le leli Bokai.

le leli Bilbaa.

nde $\sim$ ndeli $L a n d u$. ring. (own field notes)

re reli Rikou.

re $\sim$ reli Oepao. (own field notes)

ndeli $B a^{\prime} a$.

ndeli Tii.

ndeli Dengka.

ndeli Oenale.

k|reni Ro'is Amarasi. ring.

k|reni Kotos Amarasi. ring.

ka|leli Molo. ring for finger. (M:174)

Out-comparisons:

kadeli East Tetun. finger ring. (Mo:93)

kadeli Dhao. ring.

teli Kisar. ring.

kdyeli Roma. ring. (Steven 1991:37)

*ndesi PRM. nettle.

ndesi Termanu. kind of nettle (called daun keser in Kupang); another kind of plant of which the crushed leaves are used as a kind of putty. (J:418)

ndesi Korbafo.

lesi Bokai.

lesi Bilbaa.

resi Rikou.

ndesi $B a^{\prime} a$.

ndesi Tii.

ndesi Dengka.

ndesi Oenale.

<kese> (2) < kese asu> Molo. 1) nettle. 2) kind of nettle that causes a severe and persistent skin condition when touched. (M:201) 
*ndia PnRote. third person pronoun, demonstrative pronoun. Etym: *si-ia '3sg. personal pronoun: he, she, it'. [irr. from PMP: ${ }^{*} \mathrm{~s}>*^{*}$ nd (but compare Malay dia 'he, she, it' with irr. ${ }^{*} \mathrm{~s}>d$ )] ndia Termanu. a) demonstrative pronoun; this, that. b) third person singular pronoun; he, she, it, mainly used for emphasis (otherwise ana), always used as a possessive pronoun. (J:419)

ndia Korbafo.

lia Bokai.

lia Bilbaa. 3SG, relativiser.

ndia Landu. 3SG pronoun. (own field notes)

ria Rikou.

ria Oepao. 3SG pronoun. (own field notes)

ndia $B a^{\prime} a$.

ndia Lole. proximal, this, here, now, 3SG. (Zacharias et al. 2014)

ndia $T i i$.

*ndika $P w R M$. stick to, cling. Pattern:

$\mathrm{k}-7$. [irr. from PRM: $* \mathrm{a}>i$ in some Meto (also Tetun); *nd $>r$ in KusaManea (expect $k)$ ]

ndika Dela. cling, stick.

na-ki?i Kotos Amarasi. cling, stick.

n-kii? (2) <ka na-kia fa> Molo.

1) stuck. 2) not stuck, not grafted. (M:205)

ma?ria? Kusa-Manea. sticky. [Form: metathesised form of (currently unattested) *ma?ri?a.]

Out-comparisons:

ha-kriPit Fehan Tetun. stick to (e.g. prickle sticks to clothes).

kri?ik East Tetun. straight, stiff. (Mo:119)

*ndiki Morph: *ndiki-k. Rote. ear. Pattern: k-7. [Form: Assimilations of $*_{\text {nd }}>d$ in first sense are due to this term being compounded with reflexes of *doo-k 'leaf', compare *ndake_ doo-k $>$ da?e_doo-k 'betel' and *bife $>$ difa_doo-k' 'lips'.] ndi?i-k (2) di?i_doo-k Termanu. 1) the edges of a leaf that has small veins and is good for making a bucket, the edges are trimmed off. (J:419) 2) ear. (J:87)

ndi?i-? (2) di?i_doo-? Korbafo.

li?i-k (2) di?i_doo-k Bokai.

liki-? (2) liki doo-? Bilbaa.

(2) ndiki_doo-? Landu. (own field notes)

ri?i-? (2) rii_doo-? Rikou.

(2) rii_doo-? Oepao. (own field notes)

ndi?i-k (2) di?i_doo-k $B a^{\prime} a$.

(2) ndi?i doo-k $\bar{L}$ ole. (Zacharias et al. 2014)

ndi?i-k (2) ndi?i doo-k Tii.

ndiki-? (2) ndi?i-? Dengka. 1) edge of a palm leaf. (J:741) 2) ear.

ndiki-? (2) ndiki-?, ndiki roo-? Oenale. 1) edge of a palm leaf. 2) ear. (J:741)

ndiki-? Dela. ear.

*ndoo PRM. straight, straighten.

ndoo-s (2) na-ka-ndoo (3) nama-ndoo Termanu. 1) straight, right. 2) go straight on, continue. 3) be or become straight (also in metaphorical sense), make straight. (J:420f)

ndoo-? Korbafo.

loo-k Bokai.

loo-? Bilbaa.

roo-s Rikou.

ndoo-s $B a^{\prime} a$.

ndoo-s Tii.

ndoo-s Dengka.

ndoo-s Oenale.

na-ndoo Dela. make straight, stretch.

na-koo Kotos Amarasi. stretch out (e.g. body to sleep).

na-koo Molo. stretches. mu-koo nuku-m stretch out your hand (M:225)

\section{Out-comparisons:}

loos East Tetun. right, correct, exact, straight, erect, vertical; truly, correctly, exactly. [irr. from 
PRM: *nd $=l$ correspondence (expect $d$ )] [Form: Kemak has loson which, if connected with the Tetun form, would further indicate that this Tetun form is not (regularly) cognate with PRM *ndoo.] (Mo:133)

*ndoro PRM. walk around. [irr. from PRM: *nd $>r$ in Oenale; *nd $>n$ in Meto (probably via intermediate *r, given Dela-Oenale intermediate rolo)] ndolo Termanu. go around. (J:423) ndolo Korbafo.

lolo Bokai.

lolo Bilbaa.

rolo Rikou.

roro Oepao. (J:423, 726)

ndolo $B a^{\prime} a$.

ndoro Tii.

ndolo Dengka.

rolo Oenale. (J:423, 726)

rolo Dela. wander, walk aimlessly.

n-non nono Kotos Amarasi. walk around.

nono Molo. goes around. (M:384)

Out-comparisons:

loloh Semau Helong. walk around. only used for farms.

lodo Hawu. go. [Sporadic: consonant metathesis $* \mathrm{dVl}>$ lVd.]

yaro (2) rero Bima. 1) go back and forth, go everywhere. (Jonker 1893:66) 2) go around everywhere. (Jonker 1893:86)

reron, ririn Sika. restless, go around. (Pareira and Lewis 1998:172)

*ndoto PRM. hyacinth bean. Lablab purpureus. [irr. from PRM: *nd $>k$ in Ro'is Amarasi (expect $r$ )]

ndoto Termanu. 1) kind of climbing plant called arbila in Kupang. (J:424) 2) arbila. Dolichos lablab/ Lablab vulgaris. (Fox 1991:260)

ndoto Korbafo.

loto Bokai. loto Bilbaa.

ndoto $L a n d u$. hyacinth bean.

(own field notes)

roto(s) Rikou.

rotos Oepao. (own field notes)

ndoto $B a^{\prime} a$.

ndoto Tii.

ndoto Dengka.

ndoto Oenale.

koot kase Ro'is Amarasi. kidney beans. Lit: 'foreign koto?'.

koto? Kotos Amarasi. hyacinth beans. $<$ koto $>$ Molo. kinds of beans. (M:238) kotu-gw Timaus. hyacinth beans. [Form: vowel final root koto.]

koto Kusa-Manea. hyacinth beans.

Out-comparisons:

${ }^{\circ}$ koto East Tetun. variety of bean. Borrowed from: Meto koto (shown by initial *nd $=k$ correspondence). (Mo:117)

'koto Waima'a. broad beans. Borrowed from: ultimately Meto koto, perhaps via Tetun.

'koto Mambae, South. bean. Borrowed from: ultimately Meto koto, perhaps via Tetun. (Fogaça 2017:236)

*ndou Morph: *ka-ndou-k. PRM. nape of the neck. [Form: *nd develops as an initial consonant in Rote.]

ka|ndou-k Termanu. nape of the neck. (J:221)

ka|ndou-? Korbafo.

ka|lou-k Bokai.

ka|lou-? Bilbaa. (J:221,742)

ka|ndou-? Landu. nape of the neck. (own field notes)

ka|rou-? Rikou.

ka|rou-? Oepao. (own field notes)

ka|ndou-k $B a^{\prime} a$.

ka|ndou-k, ndo ndou-k Tii.

ka|ndou-? Dengka.

ndo(u) ndou-? Oenale. (J:221,742)

k|roo-n Ro'is Amarasi. nape of the neck. 
ko/tore-f Kotos Amarasi. nape of the neck. [Form: historic compound of *ka-ndou + *tode.]

P|koo-n Amanuban. nape of the neck.

P|koo-n (2) $\mathbf{p} \mid$ ko/tole-k Molo. 1) the nape of his neck. 2) the protuberance on the back of my head. [Form: historic compound of *ndou + *tode.] (M:225)

P|koo-f Timaus. nape of the neck.

*ndui PRM. draw/scoop water.

ndui Termanu. scoop with a pot, etc. (J:426)

ndui Korbafo.

lui Bokai.

lui Bilbaa.

rui Rikou.

ndui $B a^{\prime} a$.

ndui Tii.

ndui Dengka.

ndui Oenale.

n-rui Ro'is Amarasi. draw water.

n-kui (2) 2-ku?i Kotos Amarasi.

1) draw water. 2) dipping bucket.

n-kui oe (2) <kui> Molo. 1) draw water with a container or tin.

2) water scoop. (M:245) n-kui Kusa-Manea. draw water.

*nduna Morph: *ka-nduna-k. PRM. nest. [irr. from PRM: *a $>u$ in nRote (sporadic assimilation); $*_{\text {nd }}>k$ in Ro'is Amarasi (expect $r$ )]

ndunu-k Termanu. nest of all sorts of animals, in compounds one normally says: ne?e ndunu, (lafo ndunu, bafi ndunu), ant's nest, (rat's nest, wild pig's nest). (J:427)

ndunu-? Korbafo.

lunu-k Bokai.

lunu-? Bilbaa.

ndunu-? Landu. nest. (own field notes)

runu-? Rikou.

runu-? Oepao. (own field notes)

ndunu-k $B a^{\prime} a$.

ndunu-k Tii.

nduna-? Dengka.

nduna-? Oenale.

kuna|? Ro'is Amarasi.

P|kuna|? Kotos Amarasi. nest (of bird).

P|kuna|? Molo. nest, pouch (of sarong, of marsupial). (M:249)

\section{D - y}

*nano PwRM. plait, braid. [irr. from PRM: $*_{\mathrm{y}}>h$ in wRote] [Form: It is not clear what initial consonant can account for wRote $h$, (most) Meto $k$, and Amanuban $n$. I have reconstructed $*^{*} y$ as this best accounts for the Meto reflexes.]

hano Dengka. plait, braid. (J:699)

hano Oenale. plait, braid. (J:699)

na-kano Ro'is Amarasi. plait.

na-kano, n-kane Kotos Amarasi. plait, weave. [Form: The form n-kane with final $e$ may be due to influence from PRM *ane.]

na-nano Amanuban. plait, weave. na-kano Molo. plait, braid. (M:180) na-kaon Kusa-Manea. braid, plait.

\section{Out-comparisons:}

yana Central Nage. plait, weave. (Forth 2016:336)

*naper PRM. beckon with hand. [irr. from PRM: * ${ }^{*}>k$ in Ba'a (probably expect $\eta g$ )] [History: Blust and Trussel (ongoing) reconstruct PWMP *ambay 'wave back and forth', PMP *kaway 'wave the hand or arms; call by waving', and PMP *kapay 'flutter the wings'. Wolff (2010:838) reconstructs PMP *abay stating: 'In S[outh] Phil[ippines] and N[orth] Sul[awesi] this form was prefixed with *ka-.'] 
ygape-k Termanu. beckon, wave. (J:433)

ygape-? Korbafo.

yape-k Bokai.

yape-? Bilbaa.

kape-? Rikou.

kape-k $B a^{\prime} a$.

ygape-k Tii.

kapel Dengka.

kaper Oenale.

kaper Dela. wave to get someone's attention.

n-napen Kotos Amarasi. beckon someone with one's hand.

n-napi, n-nape Molo. beckons. [Note: Jonker (1908:433) gives Meto ainape, nape naper.] (M:35)

Out-comparisons:

kahi East Tetun. beckon. (Mo:95) gape Hawu. signal by hand, summon by hand motion.

* yato $P R M$. string together, sew together.

nato Termanu. insert, string together. (J:381)

nato Korbafo.

nato Bokai.

nato Bilbaa.

nato Rikou.

nato $B a^{\prime} a$.

nato Tii.

nato Dengka.

nato Oenale.

n-katon Kotos Amarasi. poke and sew with a leaf stem to connect leaves or fish.

n-kato (2) < kato $>$ Molo. 1) inserts. 2) fork. (M:186)

Out-comparisons:

noto Semau Helong. thread the needle. [irr. from PRM: *n $=n$ correspondence; $* \mathrm{a}=o$ correspondence]

*yilu PRM. tamarind, sour. Etym: *yilu 'painful sensation in teeth, as from eating something sour'. [minority from
PMP: * ${ }^{\mathrm{y}}=*_{\mathrm{y}}$ ] [irr. from PRM: *1 > $\varnothing$ in all Meto except Kusa-Manea in Meto forms meaning 'tamarind'] ni nilu_na?u (2) ni nilu_dae loo-k (3) ni nilu_tasi Termanu. 1) tamarind. 2) star-fruit. 3) kind of seaweed called tambrín laut [OE $=$ 'sea tamarind'] in Kupang. [Semantics: No definition given for ninilu when not compounded. My fieldwork on Rote consistently has ni nilu as 'tamarind tree'.] (J:396f)

ni nilu Korbafo.

ni nilu Bokai.

ni nilu Bilbaa.

ni nilu Landu. tamarind. (own field notes)

ni nilu Rikou.

ni nilu $B a^{\prime} a$.

ni nilu Tii.

ni nilu Dengka.

ni nilu Oenale.

niu Ro'is Amarasi. tamarind.

kiu (2) mai?ninu? Kotos Amarasi. 1) tamarind. 2) sour, acidic; sharp pains in the body.

kiu (2) kiu ma-tabi-? (3) <maininu> Molo. tamarind. Tamarindus indica. (M:199)

2) star fruit. Averrhoa carambola. (M:208) 3) sour. (M:299)

ka kinu Kusa-Manea. tamarind.

Out-comparisons:

yidu?, yilu? Funai Helong. sour. yilu Semau Helong. sour.

*ninu PMeto. spit out. Doublet: *midu. Etym: *qizuR 'saliva, spittle' (PWMP). [minority from PMP: $*_{\mathrm{z}}>\left({ }^{*} \mathrm{~d}\right)>*_{\mathrm{n}}$ (expect $*_{\mathrm{z}}>*_{\mathrm{d}}>*_{\mathrm{d}}$ )] [Form: The source of initial $p a$ in Kusa-Manea is unclear. It may be due to historic compounding.] [History: PMeto * ninu is cognate with Rote *midu and both are ultimately probably connected with *qizuR, but it is not possible to straightforwardly reconcile the initial 
${ }^{*} \mathrm{~m}=*_{\mathrm{n}}$ correspondence and combine them into one set attesting a single PRM reconstruction.]

na-ninu Ro'is Amarasi. spit.

na-kinu Kotos Amarasi. spit.

na-kinu, na-kinu-t Molo. spits. (M:210)

na-kinu Timaus. spit.

pakiun Kusa-Manea. spit.

Out-comparisons:

taniru East Tetun. spit. (Mo:180)

anilu Welaun. spit. [irr. from

PRM: ${ }^{*} \mathrm{y}=n$ correspondence

(We expect $* \eta=k$, thus Welaun

anilu may be a borrowing from an unidentified source)]

* yoli Morph: * oli-k. PRM. canine tooth, tusk. Etym: **yoli (pre-RM).

noli(-k) Termanu. canine tooth, tusk. Also metaphorically: the end point, the end of a row of people. (J:405)

noli-? Korbafo.

noli-k Bokai.

noli-(?) Bilbaa.

noli-(?) Rikou.

noli-k $B a^{\prime} a$.

noli-k Tii.

noli-? Dengka.

noli-? Oenale.

niis koni-f (2) n-koni Kotos Amarasi. 1) canine tooth.

2) copulate. [Semantics: For the semantic link between 'canine tooth' and copulate, see the Molo entry.]

koni-f (2) n-koni (3) ma-koni-?

Molo. 1) canine tooth, tusk, during the circumcision ritual in the phallic sense of penis. 2) have sexual intercourse. 3) unlimited sexual intercourse, practise promiscuity. (M:235)

koni-f Kusa-Manea. molars.

Out-comparisons:

nilin Helong. (J:405)

neli Waima'a. molar.

oli Hawu. (J:405)
$<$ uli $>$ Kambera . canine tooth.

[Note: also in Mangili,

Anakalang, and Mamboru.]

(On:513)

$<\mathbf{u l u}>$ Lewa.

$<$ ule> Kodi. [Note: also in

Weyewa.]

neri Kamarian. tusks of a pig. (van Ekris 1864:315)

neri Saparua. tusk (of a pig). [Note: also in Nusa Laut language of Lease Islands ISO 639-3 [spr].] (van Hoëvell 1877:105)

neri-ne Asilulu. tusk (of a pig). [Note: also in Haruku.] (van Hoëvell 1877:105)

neli Hitu. tusk (of a pig). [Note: language of Ambon Island ISO 639-3 [htu].] (van Hoëvell 1877:105)

*yura PRM. young of plants. Etym: *yuda. [minority from PMP: * $\mathrm{y}=* \mathrm{n}$ ] [Sporadic: *a $>e$ /_\# in wRM except for Kusa-Manea. [Form: The Meto reflexes are probably best explained by positing that $* \eta$ was retained as $* \eta$ into PMeto. PMeto * yune then underwent consonant metathesis to **nuye followed by subsequent regular PMeto $*_{\eta}>k$. The full hypothesised pathway is thus PMP * yuda > PRM *yura $>$ PwRM * yure $>* *$ yule $>$ PMeto *yune $>* *$ nuye $>$ nuke. The Meto forms with two ' $k$ 's probably attest sporadic assimilation of $*_{n}>*_{\eta} /$ Vn before $* \eta>k$. Thus, ${ }^{* *}$ nune $>* *$ yune. (Compare *nuya 'Cordia species' for similar assimilation.) The Rote forms show regular PRM $*_{y}>n$.]

nula-k (2) nula Termanu. 1) young, unripe fruit. (J:409) 2) woods, forest. (J:408)

nula-? (2) nula Korbafo.

nula-k (2) nula Bokai.

nula-? (2) nula Bilbaa. 
(2) nura $L a n d u$. forest. (own field notes)

nura-? (2) nura Rikou.

(2) nura Oepao. forest. (own field notes)

nula-k (2) nula $B a^{\prime} a$.

nura-k (2) nura Tii.

nule-? Dengka.

nure-? Oenale.

mai|nuki|? Ro'is Amarasi. young (of fruit). mai|nuke|?, mai|nuki|?

(2) m|nuki| $\mathbf{2}$ Kotos Amarasi.

1) young (of fruit). 2) young (of

fruit/people). Usage: poetic.

mai|kuke|?, ma|kuke|? Molo. young of fruit, new moon. (M:301, xlvi)

noa ma|kuka|? Kusa-Manea. young green coconut.

Out-comparisons:

nurak East Tetun. young, immature, delicate, lush (of plants). (Mo:155)

\section{DG - ng}

*ygadas PRM. palate, gills, throat. Doublet: *nada. Etym: *yadas 'palate' (Osmond (2011a:130) reconstruct POc *gara 'gills' which could be cognate, instead of PMP * yadas. POc *gara would be from PMP **(g,k)a(d,r)a). Pattern: d-2. [irr. from PMP: * $\mathrm{d}=$ *d] [minority from PMP: * $\mathrm{y}>*^{*} \mathrm{gg}$ ] [Sporadic: $* \mathrm{a}>e /$ \# in Meto.]

ngala_bote-k Termanu. external throat. (J:430)

yga ngalas $B a^{\prime} a$. gills of a fish. (J:742)

ygara-? (2) ygara_bote-? Oenale. 1) gills of a fish. (J:742) 2) external throat. (J:742)

ygara-? Dela. throat.

?|kaere-f Ro'is Amarasi. palate.

?|kare-f Kotos Amarasi. palate.

?|kael uti-n Amanuban. uvula.

P|kale-n Molo. fraenulum of tongue, tongue web. [Note: Jonker (1908:742) gives Meto kunkalas 'palate, gills'. The first part of this would be connected with kun kunu-f, which means 'cheek' in some varieties of Meto.] (M:13, 174)

Out-comparisons:

hegara Hawu. palate, gills. (J:742) *ygae $P w R M$. cry, weep.

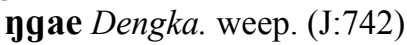

ygae Oenale. weep. (J:742)

n-kae Ro'is Amarasi. cry, weep.

n-kae Kotos Amarasi. cry, weep.

n-kae Molo. weep, cry. (M:167)

n-kaa Kusa-Manea. cry. [Form: regular $*_{\mathrm{e}}>a / \mathrm{V}_{-} \#$ in Upper Manulea]

*ygaem PR.M. country almond. Terminalia catappa.

ygae-k Termanu. kind of tree with edible fruit. (J:429)

ygae-? Korbafo.

nae-k Bokai.

yae-? Bilbaa.

kae-? Rikou.

ngae-k $B a^{\prime} a$.

ygae-k Tii.

ygae-? Dengka.

ygae-? Oenale.

kaem Molo. kind of tree. Terminalia catappa. (M:167)

Out-comparisons:

kaen Fehan Tetun. type of big fruit tree, with big leaves, the fruit is boiled before eating.

kaen East Tetun. tree with edible fruit. (Mo:94) 
*ygafat $P R M$. light (not heavy). [irr. from PRM: *ng $>k \sim n$ in Meto (indicates PMeto *ma?nafa? with irr. ${ }^{*} \mathrm{ng}>*^{*} \mathrm{y}$ where we expect $* \mathrm{k}$ )]

ygafa-k Termanu. quick, fast at running, usually with the added meaning of: maintaining a fast pace for a long time while running; speed. (J:429)

ygafa-? Korbafo.

yafa-k Bokai.

yafa-? (2) yafa-? Bilbaa. 2) light, not heavy. (J:742)

kafa-? (2) kafa-? Rikou. 2) light, not heavy. (J:742)

ngafa-k $B a^{\prime} a$.

ygafa-k Tii.

ngafa-? (2) ngafa-? Dengka. 2) light, not heavy. (J:742)

ygafa-? (2) ygafat Oenale. 2) light, not heavy. (J:742)

(2) ygafat Dela. light, not heavy.

ma?|kafa|? Ro'is Amarasi. light, not heavy.

ma?|kafa|? Kotos Amarasi. light, not heavy.

$<$ n-makafa $>$ (2) <manafa $>$, <makaf> Molo. 1) he feels fit. (M:299) 2) light, easy. (M:299, 304)

Out-comparisons:

kahan Semau Helong. lightweight; easy, simple.

kawa Kamarian. light. [Note: also in Haruku and Kaibobo from Tihulale village.] (van Ekris 1864:100)

*ygafur PRM. powder.

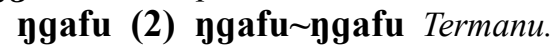

1) shake out, beat out (dust, etc.).

2) flutter (e.g. a sail or flag). (J:430)

ngafu Korbafo.

yafu Bokai.

nafu Bilbaa.

kafu Rikou.

ngafu $B a^{\prime} a$.

ngafu Tii.

ngafu Dengka.

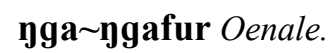

ygafur Dela. shake off.

kafu? Kotos Amarasi. powder or flakes which fall off something bit by bit, e.g. from the skin or a clump of something.

kafu? Molo. skin powder. (M:168)

*ngaha $P w R M$. no, not. [Form: regular $* \mathrm{~h}>\varnothing / \mathrm{a} \_\mathrm{a}$ in Rote.]

yga Dengka. not. Usage: pre-predicate negator. (J:742)

$\mathbf{k a}=$ Ro'is Amarasi. NEGATOR. Usage: The normal negator in Ro'is is mae? however, $\mathbf{k a}=$ sporadically occurs as a negator, particularly in parallel pairs, and is a tag question particle in the same way as it is in Kotos Amarasi.

ka=, kahaf, kaah Kotos Amarasi. NEGATOR. Usage: The proclitic $\mathbf{k a}=$ combines with an enclitic $=\mathbf{f a}$ in (prescriptive) Kotos Amarasi.

ka= Molo. in denial. (M:264)

*ngai PRM. pick at, scratch.

ygai Termanu. pick out, mainly pick one's ears. ( J:431)

ygai Korbafo.

nai Bokai.

yai Bilbaa.

kai Rikou.

ygai $B a^{\prime} a$.

ygai Tii.

ngai Dengka.

ygai Oenale.

n-kai Kotos Amarasi. scratch.

n-kai Molo. one scrapes. (M:168)

*ygala Morph: *nga mgala. PRM. agati, vegetable hummingbird. Sesbania grandiflora. [Sporadic: *a $>e$ /\# in Dela, Dengka and Meto.] [History: Blust and Trussel (ongoing) reconstruct Proto-Philippine * gala 'the almasiga tree: Agathis celebica' on the basis of Ilokano gala and Tagalog gala-gala. They note that the Ilokano form may be a Tagalog loan.] 
yga ygala Termanu. kind of tree called pohon gala-gala in Kupang. (J:430)

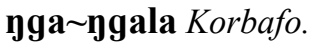

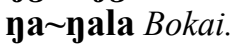

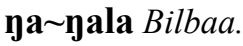

ka kala Rikou.

yga ygala $B a^{\prime} a$.

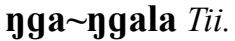

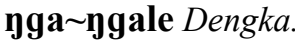

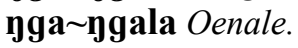

yga ngale Dela. (Thersia Tamelan pers. comm. February 2018)

?|kane Kotos Amarasi. vegetable hummingbird, agati. Sesbania grandiflora.

P|kane Fatule'u. vegetable hummingbird, agati. Sesbania grandiflora.

P|kane Molo. tree that is 5-10 metres high and has a short life-span. A beverage against thrush can be prepared from the decoction of the rough and somewhat sticky bark. Sesbania grandiflora. (M:14, 179)

\section{Out-comparisons:}

kala East Tetun. tree. Sesbania grandiflora. [Note: ai turi is given as identical.] (Mo:98)

*ygango PRM. water spinach. Ipomoea aquatica. [irr. from PRM: *ng $>\varnothing$ /\#_ in Korbafo] [History: Ultimately a borrowing from another source, e.g. compare similar Malay kangkung. Blust and Trussel (ongoing) say regarding the Malay form: 'Although a precise donor language is difficult to identify, a Chinese source appears likely.']

ygayo_dano Termanu. kind of plant, water spinach. [Form: dano= 'lake',] (J:432)

ayo_dano Korbafo.

yayo_dano Bokai.

yayo_dano Bilbaa.

kako_dano Rikou.

ygango_dano $B a^{\prime} a$. ygaygo_dano Tii.

ngajgo-? Dengka.

ngango-? Oenale.

ygango Dela. water cress.

uut kako Kotos Amarasi. water spinach.

Out-comparisons:

hkako (2) hkako klehen Semau Helong. 1) kind of seaweed.

2) palm species.

*ygarasa Morph: *ngarasa-k. Rote. part of the back.

karasa-? Rikou. the part of the back behind the shoulders. (J:708)

ngarasa-? Oenale. back, or part of the back. (J:743)

Out-comparisons:

keraha Hawu. slope, side. [Semantics: Jonker (1908:708) gives this as 'side of the body'.]

$<$ karaha $>$ Kambera. edge, side, flank. [Note: also in Kodi.] (On:183)

$<$ karasa $>$ Anakalang. [Note: also in Mamboru.]

*ngari $P R M$. sow, scatter.

ygali Termanu. scatter, strew. (J:431)

ngali Korbafo.

nali Bokai.

yali Bilbaa.

kari Rikou.

ygali $B a^{\prime} a$.

ggari Tii.

ygali Dengka.

ygari Oenale.

n-kani? (2) kan kani?, sab/kani?

Kotos Amarasi. 1) sow (seeds).

2) drizzle (rain).

kani-n Amanatun. outpoured portion. Usage: poetic. (M:179)

\section{Out-comparisons:}

kari East Tetun. scatter; to fling with the throwing hand. (Mo:101)

gari Dadu'a. sow. (Penn 2006:102)

ari Kisar. broadcast seed, scatter seed randomly.

*ngasi Rote. shout, speak. 
ggasi Termanu. crying, whining or howling scream. (J:433)

ygasi Korbafo.

yasi Bokai.

yasi Bilbaa.

kasi Rikou.

ggasi $B a^{\prime} a$.

ngasi Tii.

ngasi Dengka.

ngasi Oenale.

ngasi Dela. yell.

Out-comparisons:

kais Kisar. holler out, pig squeal.

gase Mambae, Northwest. speak. (Fogaça 2017:249)

kase Mambae, South/Central. speak. (Fogaça 2017:249)

ggahi Bima. speak, say, word, speech. [irr. from PRM: *s $=$ $h$ correspondence (probably expect $s$, though reflexes of intervocalic *s are somewhat unclear)] (Jonker 1893:67)

yasi Ende. get angry, complain. [Note: Jonker (1908:433) gives Ende nahi 'speak'.]

*ygebo CERM. cut into pieces. [irr. from PRM: ${ }^{*} \mathrm{o}>i$ in Meto] [Form: Final $*_{\mathrm{o}}$ has been tentatively reconstructed as $*_{0}>i$ in Meto can be motivated as an instance of sporadic assimilation to the previous vowel while alternate $*_{\mathrm{i}}>o$ in nRote appears unmotivated.]

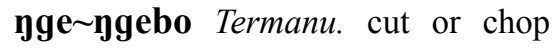
into pieces. (J:436)

nge ngebo Korbafo.

ne nebo Bilbaa.

ke kebo Rikou.

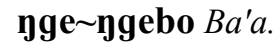

kebi? Kotos Amarasi. pieces of a whole.

kebi? Molo. cut into chunks. (M:192)

*ngede Rote. frog, croaking of a frog. [Note: With the exception of Termanu, the second forms in this entry follow the entry for Korbafo.] [irr. from PRM: ${ }^{*} \mathrm{e}>a$ in Molo; ${ }^{*} \mathrm{~d}>l$ in Termanu; ${ }^{*} \mathrm{gg}$ $>k$ in Bokai and Dengka] [History: Semau Helong has klete which is not regularly cognate, but is phonetically similar. Funai Helong has klatkee?.] [Semantics: likely onomatopoeia.]

ygede (2) lodo_ygele-k Termanu. 1) croaking of a frog, also used for the crying of a newly born child. (J:436) 2) frog. (J:320)

ngede (2) ngede oe Korbafo. 1) croaking of a frog, also used for the crying of a newly born child. (J:436) 2) frog. (J:743)

kede kede Bokai. croaking of a frog, also used for the crying of a newly born child. (J:436)

yede (2) yede oe, yed/oe Bilbaa.

kede Rikou.

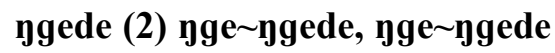
oe $B a^{\prime} a$.

(2) yge ygede Lole. 2) frog. (Zacharias et al. 2014)

ngede (2) yge ngede Tii.

kede $\sim$ kede (2) yge ngede Dengka. ngede (2) ygede Oenale.

(2) ngede Dela. 2) frog.

kala/be?o Molo. frog. [Form: Initial kala is optional, e.g. be?o also occurs in isolation meaning 'frog'.] (M:59)

Out-comparisons:

kere Kemak. frog. [irr. from PRM: $*_{y g}=k$ correspondence; expect g)]

kere/dokon Mambae, Northwest. frog. Usage: Barzatete subdistrict. (Fogaça 2017:235)

keda/lokon Mambae, Northwest. frog. Usage: Railaco subdistrict. (Fogaça 2017:235)

kaidi/lokon Mambae, Central. frog. Usage: Laulara subdistrict. (Fogaça 2017:235)

kele? Bunak. frog. [Note: nonAustronesian language of central Timor ISO 639-3 [bfn].] (Schapper 2009:174) 
*ygela1 Morph: *ygela-k. Rote. earwax. Etym: *tanila 'ear' (Blust and Trussel (ongoing) reconstruct the doublet talina with consonant metathesis. *talina is the most common form outside of Taiwan. Nonetheless, Helong attests *tanila, lending credibility to the notion that the Rote forms here are irregularly connected to *tanila). [irr. from PMP: $* \mathrm{i}>*_{\mathrm{e}}$ ] [minority from PMP: * ${ }^{\mathrm{y}}>*_{\mathrm{ng}} \mathrm{g}$

di?i_doo ygela-k Termanu. ear dirt, earwax. (J:437)

ngela-? Korbafo.

yela-k Bokai.

yela-? Bilbaa.

kela-? Rikou.

ngela-k $B a^{\prime} a$.

ngela-k Tii.

ygela-? Dengka.

ngela-? Oenale.

Out-comparisons:

knila Funai Helong. ear.

hyila Semau Helong. ear.

talina-r Idate. ear. (Klamer 2002)

liga-r Kemak. ear.

lika-t Welaun. ear.

*ygela2 Morph: *ygela-k. Rote.

eucalyptus; gum tree.

ygela-k Termanu. kind of tree which yields good timber, called gelang in Kupang. (J:437)

ngela-? Korbafo.

yela-k Bokai.

yela-? Bilbaa.

kela-? Rikou.

ngela-k $B a^{\prime} a$.

ngela-k Lole. gum tree, eucalyptus.

(Zacharias et al. 2014)

ngela-k Tii.

ygela-? Dengka.

ngela-? Oenale.

Out-comparisons:

kelan Funai Helong. eucalyptus.

*ngengo Morph: *na-ngengo. PRM.

shake. Etym: *gərgər 'shake, shiver, tremble'. [irr. from PMP: *ə $>*^{*}$ ] [irr. from PRM: *o $>o \sim u$ in Meto] [Form: Irregular final $*_{\partial}>o$ is also seen in Bugis gego? (Mathes 1874:53) and Makassar gengo (Cense 1859:68)] na-ngeno Termanu. shake. (J:438)

na-ngeyo Korbafo.

na-neyo Bokai.

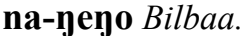

na-keko Rikou.

na-ngengo $B a^{\prime} a$.

na-ngengo $\mathrm{Ti}$.

na-ngengo Dengka.

na-ygeygo Oenale.

<na-keku>, <na-keko>(2) <anaka

keku> Molo. 1) moves back and forth. 2) shake-head, said of someone with nervous disorder as a result of which he keeps shaking his head from front to back and from left to right. (M:194)

*ygeo Morph: *ngeo-k. Rote. black.

ngeo-k Termanu. black. (J:438)

ngeo-? Korbafo.

neo-k Bokai.

yeo-? Bilbaa.

$\mathbf{k e o}=\mathbf{a} L a n d u$. black. (own field notes)

keo-? Rikou.

keo-? Oepao. black. (own field notes)

நgeo-k $B a^{\prime} a$.

ngeo-k Tii.

ngeo-? Dengka.

ygeo-? Oenale.

*ygete PRM. pinch, nip. Doublet: *kato. Etym: *gatəl (Reconstructed with the doublets *getil and *ketil.). [minority from PMP: *ə $>*_{\mathrm{e}} / \ldots$ \# (expect $*_{\mathrm{o}}>a$ in wRote, possibly $\left.*_{\partial}>*_{\mathrm{a}}>e\right)$ ] [irr. from PRM: * ${ }_{\mathrm{t}}>$ ? /V_V in Termanu, Korbafo, Ba'a, and Tii]

nge?e Termanu. pinch, like a lobster. (J:436)

gge?e Korbafo.

yete Bokai.

nete Bilbaa.

gge?e $B a^{\prime} a$.

yge?e Tii.

ngete Dengka. 
ygete Oenale.

kete, ete Molo. bite off. [irr. from PRM: *ng $>\varnothing$ in second Molo form] (M:202)

*ygeu PRM. shave. [irr. from PRM:

*yg $>y g \sim k$ in Dela-Oenale]

ngeu Termanu. scrape. (J:440)

ygeu Korbafo.

neu Bokai.

neu Bilbaa.

keu Rikou.

ygeu $B a^{\prime} a$.

ygeu Tii.

ygeu Dengka.

Peu Oenale.

ygeu, Reu Dela. shave.

n-keu Ro'is Amarasi. shave.

n-keu Kotos Amarasi. shave.

n-keu Molo. scrapes his tongue. (M:203)

keu Kusa-Manea. scrape, shave.

*ngia PRM. parakeet. [irr. from PRM:

$\varnothing>t / V_{-} \mathrm{V}$ in Meto] [Semantics: onomatopoeia.]

ngia Termanu. turaco, lourie (kinds of parrot). [Note: Turacos and louries appear to be only found in Africa. This word probably designates a similar Timorese bird identified/ named thus by Jonker.] (J:441)

ngia Korbafo.

nia Bokai.

nia Bilbaa.

kia Rikou. Olive-shouldered Parrot. Aprosmictus jonquillaceus. [Semantics: My consultants gave kia-? for a photo of an Oliveshouldered Parrot.]

ngia $B a^{\prime} a$.

ngia Tii.

ygia Dengka.

ygia Oenale.

kita Molo. kind of parakeet. (M:211)

Out-comparisons:

man gea Buru. small parrot or parakeet. (Grimes and Grimes 2020:585) *ygii Morph: *ngii-k. PRM. bunch of fruit. [History: Jonker (1908:440) gives Tetun Dili kiu, which may be cognate, but the final $u$ of which would be irregular.]

ngii-k Termanu. blossom bunch of a palm or banana tree, bunch of coconuts of bananas. (J:440)

ggii-? Korbafo.

nii-k Bokai.

nii-? Bilbaa.

kii-? Rikou.

ngii-k Tii.

ygii-? Dengka.

ygii-? Oenale.

$<\mathbf{k i}>$ Molo. stem with a bunch of fruit on it (e.g. betel nut). (M:205)

\section{Out-comparisons:}

<nggai> Kambera. bunch. (On:353)

<nggi> Lewa.

<nggí> Mamboru.

<nggi'i > Weyewa.

<nggiyo $>$ Kodi .

*ngirat Rote. kind of shrub. [Semantics: vague semantics.]

ngila-k Termanu. kind of shrub. (J:441)

ygila-? Korbafo.

jila-k Bokai.

yila-? Bilbaa.

kira-? Rikou.

ygila-k $B a^{\prime} a$.

ngira-k Tii.

ngilat Dengka.

ngirat Oenale.

*ygiro Morph: *ygi ngiro. CERM. lower head area. [History: Osmond and Ross (2016a:112) reconstruct POc *k(i,e)ju 'back of head, base of skull, occiput, nape' and Proto New Guinea Oceanic *g(i,e)ju, which are phonetically and formally similar to the PRM form. However, the final vowel correspondences are irregular and the initial PRM *ng $=$ POc ${ }^{*} \mathrm{k}$ correspondence may also be irregular. Something like PCEMP **gijo may 
indeed be possible, however in the absence of cognates in other areas of Wallacea, I prefer not to make such a reconstruction at this point.]

\section{(lesu ha?ik) ngi ngilo=na}

Termanu. the lower part of the neck, where it is attached to the body and on which it turns. (J:441)

ngi ngilo=na Korbafo.

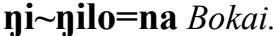

ki kiro=na Rikou.

ngi ngilo=na $B a^{\prime} a$.

kiunu-f Ro'is Amarasi. cheek.

?|kinu-f Kotos Amarasi. cheek.

kino-n Amanuban. sideburns.

kino-f (2) $\mathbf{P | k i n u - f ~ ( 3 ) ~ < k i n u > ~}$ Molo. 1) loose rope hanging down from a horse's head; also: whiskers.

2) portion of the loop of a rope around the mouth of a horse that in a tied state runs to the bottom.

2) whiskers. (M:210)

*ygoa Morph: *ygoa-k. Rote. stupid.

ygoa-k Termanu. mute; dumb, foolish; muteness; stupidity, astonishment. (J:443)

ygoa-? Korbafo.

noa-k Bokai.

yoa-? Bilbaa.

koa-? Rikou.

ygoa-k $B a^{\prime} a$.

ygoa-k Tii.

ngoa-? Dengka.

ygoa-? Oenale.

Out-comparisons:

goa Dhao. stupid.

goa Hawu. stupid. [Note: Given with the note: 'probably borrowed' (from Rote).] (J:443)

*ngois PRM. eel.

ygois Termanu. kind of ocean fish called ikan gois in Kupang. (J:445)

ygoi-? Korbafo.

yoi-k Bokai.

yoi-? Bilbaa.

kois Rikou. ocean eel. (J:445; own field notes) ygois $B a^{\prime} a$.

ngois Tii.

ygois Dengka.

ggois Oenale.

kois Ro'is Amarasi. eel.

Out-comparisons:

kois East Tetun. water snake. (Mo:101)

kois Galolen. earthworm.

*ngo?u CERM. big, great.

leyou Bilbaa. the domain of Rikou on the eastern part of Rote. (own field notes)

ri/kou, rai/kou Rikou. the domain of Rikou on the eastern part of Rote. [Form: raikou is reported by some of my consultants to be the original form.] [History: A possible etymology is *dae 'land' combined with an otherwise lost reflex of *ngou meaning 'big, great'. One hypothesis is that the term originally referred to the Timor mainland and later underwent semantic shift (for whatever sociopolitical reasons) to the eastern Rote domain of Rikou. However, in the absence of corroborating evidence this etymology is highly speculative. Note that Tetun rai (from PMP *daRəq) means 'earth, soil, ground; land estate, kingdom; the world'. If this is the source of the initial element, how it was borrowed into the Rote languages is unclear.] (own field notes)

ko?u Ro'is Amarasi. big, great.

ko?u Kotos Amarasi. big, great. [History: In his entry for ko?u Middelkoop (1972:239) has: 'Renggo'u = Rai nggo'u the great prince, now a place name'. He apparently takes rai as meaning 'prince' by analogy with the first part of the second member of the Amarasi doublet uis ko?u, nai? ko?u 'the great lord'.] 
*ygola Rote. monitor lizard, flying lizard. ngola Termanu. kind of flying lizard. (J:445f)

ygola Korbafo. kind of flying lizard. (J:445f)

yola (2) yala/fao Bokai. 1) kind of flying lizard. (J:445f) 2) kind of lizard. (J:742)

yala/fao Bilbaa. kind of lizard. $(\mathrm{J}: 742)$

kara/fao Rikou. kind of lizard. (J:742) ngola $B a^{\prime} a$. monitor lizard. (J:445f)

jgola Dengka. monitor lizard. (J:445f)

\section{Out-comparisons:}

lagura Hawu. monitor lizard. (J:445)

<lawora> Kambera. monitor lizard. Varanus salvtor. (On:236)

$<$ laghora $>$ Kodi.

yora Rongga. big lizard. [Note: language of central Flores ISO 639-3 [ror].] (Arka et al. 2007:42)

*ngomi Morph: *ngomi ngomi.

PnRote. beard. Etym: *gumi(s) 'moustache, beard' (Reconstructed with the doublet *kumis.). [irr. from $P M P: * \mathrm{u}>*_{\mathrm{o}}$ ]

ngomi ngomi Termanu. have a thick beard or a big goatee. (J:447)

nomi nomi Bokai.

komi komi Rikou. ngomi ngomi Tii.

*ygoro PRM. snout, nose, snore. See: *ygoroz 'snore'. Etym: *yodok (own reconstruction) (PCEMP). [minority from PMP: *y > *ng]

ygo ygolo-k (2) ygolo_mei-k (3) ygolo ygolo (4) na-sa-ngolo Termanu. 1) muzzle, snout of a pig, horse, buffalo, dog, etc., mouth of some fish, beak, bill of a bird; proboscis of a mosquito, etc.; also a vulgar word for the mouth of a person. 2) beard, also moustache of a person, goatee of a goat. (J:445)

3) make a snoring sound, snore.

4) make a snoring sound, snore. (J:445f)

ygo ygolo-? (2) ygolo_mei-?

(3) ygolo ngolo Korbafo.

no polo-k (2) nolo_mei-k

(3) yolo polo Bokai.

(2) yolo_mii-? (3) yolo nolo Bilbāa.

(2) (koro ?) (3) koro koro Rikou. ngo ngolo-k (2) ygolo_mei-k

(3) ygolo ngolo $B a^{\prime} a$.

(ygo)ygoro-k (2) ygoro_mei-k

(3) ygoro ngoro Tii.

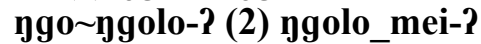

(3) ygolo ngolo Dengka.

(3) ygoro ygoro Oenale.

(4) na-sa-ngoro Dela. snore.

kono-f (2) na-kono Ro'is Amarasi.

1) sideburns, beard, moustache.

2) snore.

kono-f (2) na-kono Kotos Amarasi.

1) beard. 2) snore.

$<$ kono $>$ (2) na-kono

(3) < n-konolot> Molo. 1) beard.

(M:235) 2) barks. 3) snores.

(M:236)

Out-comparisons:

koon Semau Helong. snore.

kour Kisar. (dog) bark at something. [irr. from PMP: $*_{\mathrm{o}}>u$ ]

kagoro Bima. make a sound like a snoring noise. (Ismail et al. 1985:53)

* yoro norok (2) * yorok ProtoOceanic. 1) channel above upper lip. (Osmond and Ross 2016a:125) 2) snore. (Osmond et al. 2003:48)

noro? Malay. snore.

*ygoti Morph: *ngoti-k. PRM. back.

ngoti_ha?i-k Termanu. the lowest part of the back, the area of the tailbone. (J:448)

yoti_ha?i-k Bokai. 
noti-? Bilbaa.

koti_ai-? Rikou.

ygoti_ha?i-k $B a^{\prime} a$.

ygoti_a?i-k, ngoti-k Tii.

ygoti-? Dengka.

ygoti-? Oenale.

koiti-f Ro'is Amarasi. back, behind.

koti-f Kotos Amarasi. back (body); behind (location).

koti-f (2) n-baikoti Molo. 1) behind. (M:174) 2) turns one's back towards someone. (M:42)

\section{Out-comparisons:}

kotuk Fehan Tetun. back (of person); behind.

kotuk East Tetun. back, loins. (Mo:117)

kodo Hawu. back.

*ygout PRM. thorn. [Note: Ro'is and

Kotos Amarasi have kaut 'papaya' which would be an almost perfect formal match for the first Rote forms, but the semantic connection is too farfetched.]

ngau-k (2) ngou-k Tегтапи.

1) thorn on a tree, living thorn. (J:434) 2) thorn, mainly a single loose thorn. (J:448)

ygau-? (2) ygou-? Korbafo.

yau-k (2) you-k Bokai.

yau (2) you-? Bilbaa.

kou=na Landu. thorn. (own field notes)

kau-? (2) kou-? Rikou.

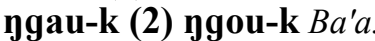

ygau-k (2) ygou-k Tii.

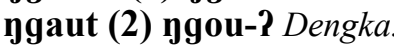

ggaut (2) ygou-? Oenale.

ygaut (2) ma-ngou-? Dela. 1) thorn. 2) thorny.

$<$ ka'o $>$ Molo. agave, aloe. Furcroea gigantea. (M:181)

Out-comparisons:

ai koon Welaun. thorn.

kau Hawu. (J:434)

*ngua Rote. freshwater turtle. [History: The comparison between this form and Malay kura-kura 'tortoise' is striking, though the initial consonants cannot be regularly reconciled. We could posit PMP *kuRa with irr. *k > *yg. Dempwolff (1938:83) reconstructs ${ }^{*} \mathrm{ku}[1] \mathrm{a}{ }^{\prime} \quad\left({ }^{*} \mathrm{ku}(\mathrm{r}) \mathrm{aq}\right.$ in Blust's transcription) 'milt, tortoise' giving Toba-Batak, Javanese, and Malay cognates as evidence. Blust and Trussel (ongoing) attribute these forms (and others) to being loans from Malay, noting that their explanation assumes that borrowing into Lampung and Sundanese (each with kuya) took place before $* \mathrm{R}>y$. The gloss in Sundanese is 'freshwater turtle', thus matching the Rote glosses here.]

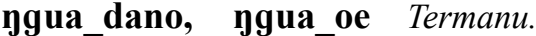
freshwater turtle. [Form: dano $=$ 'lake', oe = 'water'.] (J:449)

ngua_dano Korbafo.

yua_dano Bokai.

yua_dano Bilbaa.

kua_dano Rikou.

ngua_dano $B a^{\prime} a$.

ngua dano Tii.

ygua_dano Dengka.

ygua_dano Oenale.

*ygumu $n R M$. make a fist. Doublet: *kame, *ke?e, *keme, *kumu2. Etym: *gəmgəm 'fist; hold in the fist'. [irr. from PMP: $*_{\partial}>*_{\mathrm{u}}$ (sporadic assimilation to previous velar consonant and following labial consonant)]

na-fa-ngumu Termanu. make a fist. (J:449)

na-fa-numu Bokai.

na-fa-numu Bilbaa.

na-la-kumu Rikou.

na-fa-ngumu $B a^{\prime} a$.

na-fa-ngumu, na-sa-ngumu Tii.

n-kumu Kotos Amarasi. squeeze, press, wring out, like getting coconut milk from a coconut.

n-kumu Molo. squeeze, palpate. (M:248)

kuum Kusa-Manea. squeeze. 
*yguru $P R M$. drone, growl, make a steady constant noise. Etym: * guru(q) 'noise, tumult'. (Both *guru and *guru(q) are reconstructed as 'disjuncts' without any difference in semantics.) [Semantics: onomatopoeia.]

$\begin{array}{rrr}\text { ggulu ngulu } & \text { (2) } & \text { na-sa- } \\ \text { ngu } \sim \text { ggulu } & \text { (3) } & \text { na-ngulu }\end{array}$ Termanu. 1) constantly droning, grumble at someone. 2) growl, like a dog or cat. 3) thunder (of sky), roar (of sea), rumble (of stomach), also used to describe the noise that someone makes when they run fast (not everyone understands this word). (J:449)

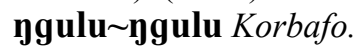

yulu pulu (3) na-yulu Bokai.

julu qulu Bilbaa.

kuru kuru Rikou.

ngulu ngulu (3) na-ngulu $B a^{\prime} a$.

nguru nguru (2) na-sa-

ngu nguru Tii.

ygulu ngulu Dengka. yguru pguru (2) na-sangu mguru Oenale.

na-kunut Kotos Amarasi. sigh, a sign that someone is bored or disappointed.

na-kunut Molo. growls (e.g. dog). (M:251)

*nguu CERM. make a constant monotonous sound, howl (of wind), blow, drone. See: *kuu. [Semantics: onomatopoeia.]

na-nguu Termanu. howling of the wind. hataholi=la la-nguu mai soo the droning of the people comes nearer (J:448)

na-yguu Korbafo.

na-nuu Bokai.

na-yuu Bilbaa.

ku/truu Ro'is Amarasi. owl.

ku/tru?u Kotos Amarasi. owl.

$<$ ku> (2) ku/tluu (3) ku/tlui Molo. 1) owl, ghost bird. (M:242) 2) owl, ghost bird. (M:242) 3) owl. (M:403)

\section{O - o}

*oe PRM. water. Etym: *wahiR. [Sporadic: $* \mathrm{i}>* \mathrm{e} /{ }^{*} \mathrm{R} \#$ ]

oe Termanu. water, fluid in general. (J:452)

oe Korbafo.

oe Bokai.

oe Bilbaa.

oe Rikou.

oe $B a^{\prime} a$.

oe Tii.

oe Dengka.

oe Oenale.

oe (2) oe-? Dela. 1) water. 2) liquid.

oe Ro'is Amarasi. water.

oe Kotos Amarasi. water.

oe Molo. water. (M:398)

oa Kusa-Manea. water. [Form: regular $*_{\mathrm{e}}>a / \mathrm{V}_{-}$in Upper Manulea dialect]

\section{Out-comparisons:}

ui Semau Helong. water. [irr. from $\left.P M P:{ }^{*} \mathrm{~W}>u(\operatorname{expect} p)\right]$

wee, ue Fehan Tetun. water.

uee (2) bee East Tetun. 1) water (Mo:192). 2) water. Usage: in the interior uee is more commonly used [than bee], but both are equally understood everywhere. (Mo:12)

bea Kemak. water.

eer Mambae, South. water, liquid.

(Grimes et al. 2014b:16)

oir Kisar. water.

eer Ili'uun. water, river, place where there is water. $(\mathrm{dJ}: 114)$

*oha Morph: *oha-s. PnRote. cloud. [Form: Medial $* \mathrm{~h}$ is perhaps from earlier $*$ p, e.g. $* *$ kopa. The Tii and 
Landu forms with initial $s$ and $s i$ respectively indicate some kind of historic compound. The initial $k / 2$ in the other Rote forms may be from the nominal prefix *ka-. Reconstruction of a stem -oha which was combined with an initial element is supported by Tetun which appears to attest something like kal + ohan.] [History: This reconstruction is perhaps connected with PMP *awan 'atmosphere, space between earth and sky' through a pathway *awan $>* *$ owan $>$-oha, as suggested by Jonker (1915:35). However, this requires otherwise unattested $*_{\mathrm{W}}>* \mathrm{~h}$, as well as initial *\#aw $>*_{0}$. The regular outcome of *away would be **ao(n).]

ko?as Termanu. cloud. (J:242)

ko?a Korbafo.

ko?a Bokai.

koa Bilbaa.

sioa-? Landu. (own field notes)

Poa-? Rikou. (J:746)

ko?as $B a^{\prime} a$.

ko?as Lole. cloud. (Zacharias et al. 2014)

so $\sim$ so?a-k Tii. cloud. (J:764)

Out-comparisons:

kalo?an Fehan Tetun. cloud.

kalohan East Tetun. cloud. (Mo:99)

kova Central Lembata. cloud. [irr. from PRM: $v=h$ correspondence] (Fricke 2015)

*ohi Morph: *n-ohi. nRM. almost. [Sporadic: $\varnothing>h$ /\#_V? in Korbafo.] [Form: The initial $n$ in most Rote forms is probably a historic third person prefix but has become fossilised in most varieties.]

no?i Termanu. at the point of, almost.

[Form: The Termanu form does not take person agreement, e.g. au no?i 'I almost'.] (J:403)

ho?i Korbafo.

no?i Bokai. noi Bilbaa.

P-oi, n-oi Rikou. [Form: Given as 'oi, noi, etc.' probably implying that the Rikou form takes person agreement.]

no?i Tii.

n-oi Kotos Amarasi. nearly.

n-oi Molo. to. (M:400)

*oka1 Morph: *oka-k. CERM. pen, corral. Pattern: k-6/9. [Sporadic: consonant metathesis $* \mathrm{kVf}>*$ ?Vf $>$ $f V$ ? in Ro'is.]

oka-? Bilbaa. pen for cattle. (J:746)

oka-? Rikou. pen for cattle. (J:746)

o?o|f, ofa? Ro'is Amarasi. pen corral. Usage: o? of in Buraen, ofa? in Tunbaun.

o?o|f Kotos Amarasi. pen, corral.

o?a|f, o?o|f Molo. corral, cage, jail. (M:397,406)

o?a|f Kusa-Manea. pen, corral.

Out-comparisons:

okat Semau Helong. corral, barnyard.

oka Dhao. field, garden, yard; pen, corral.

<oka> Kambera. corral, fence. (On:383)

*oka2 Morph: *oka-k. Rote. root. Doublet: \#ba?at. Etym: *wakaR.

Pattern: k-5.

oka-k (2) na-oka Termanu. 1) root.

2) have roots, rooted. (J:455)

oka-? Korbafo.

oka-k Bokai.

oka-? Bilbaa.

oka=na Landu. root. (own field notes)

oka-? Rikou.

oka-k $B a^{\prime} a$.

oka-k Tii.

(?)oka-? Dengka.

Poka-? Oenale.

Poka-? (2) na-Poka Dela. 1) roots.

2) grow roots.

Out-comparisons:

akar Ili'uun. root. (dJ:112) 
*oken Morph: *n-oken. PRM. call someone to come here. Pattern: k-6.

n-oke Termanu. calls, requests. (J:404)

n-oke Bokai.

n-oke $B a^{\prime} a$.

n-oke Tii.

n-o?e Dengka.

n-o?e Oenale.

n-o?e Dela. ask, call.

n-o?en Kotos Amarasi. call someone to come here; to summon, beckon.

n-oPen Molo. calls. (M:399)

Out-comparisons:

noken Semau Helong. call, refer to, greet, address.

*ombu PRM. female animal.

upu-k Termanu. female, of very young animals. (J:670)

opu-? Korbafo.

upu-k Bokai.

opu-? Bilbaa.

opu-? Rikou.

ompu-k $B a^{\prime} a$.

ombu-k Tii.

ombu-? Dengka.

ombu-? Oenale.

opu Kotos Amarasi. female animal which has not yet given birth.

$<$ bidgael opu $>$ (2) < fafi opu > Molo.

1) buffalo cow. 2) sow. (M:407)

*001 Morph: *n-oo. PRM. with. Etym:

$* *_{\mathrm{o}} \mathrm{Ro}$ (pre-RM). [irr. from PRM: Ø>

$k$ in Meto]

n-oo Termanu. s/he is with. (J:400)

n-oo Korbafo.

n-oo Bokai.

n-oo Bilbaa.

n-oo Rikou.

n-0o $B a^{\prime} a$.

n-0o Tii.

n-0o Dengka.

n-oo Oenale.

n-oo Dela. bring (someone), with.

n-ook Ro'is Amarasi. is with, accompanies. n-ok, n-oka Kotos Amarasi. is with, accompanies (M-form, U-form).

n-ok Molo. come along. (M:400)

Out-comparisons:

nol Semau Helong. and, with.

ho East Tetun. prep. with; conj. and, also (the latter only when between two nouns). (Mo:86)

n-oro, n-or Kisar. with, and.

*002 PRM. bamboo (generic). Etym: *qauR 'type of large bamboo'. [Sporadic: $\varnothing>$ ? /\#_in Dela-Oenale.] oo Termanu. bamboo, also the name of a big grassy plain at Pariti (a place name). (J:450)

oo Korbafo.

oo Bokai.

oo Bilbaa.

oo Landu. bamboo. (own field notes)

oo Rikou.

oo $B a^{\prime} a$.

oo Tii.

(?)oo Dengka.

Poo Oenale.

Poo Dela. bamboo.

oo Ro'is Amarasi.

oo Kotos Amarasi. bamboo (generic).

oo bena? Kopas. kind of bamboo. Schizostachyum blumei. [Note: In Kopas, Amfo'an and Fatule'u kaka? is the generic term for 'bamboo'.]

oo Molo. bamboo. (M:397)

oo Kusa-Manea. bamboo.

Out-comparisons:

au East Tetun. bamboo. (Mo:5)

oa Kemak. bamboo (generic).

oor Mambae, South. bamboo.

(Grimes et al. 2014b:36)

oo Galolen. bamboo.

our Kisar. bamboo.

*osa PnMeto. price, value. See: *sosa 'buy, sell'.

osa-f (2) ma-Posa-? Kotos Amarasi.

1) price. 2) expensive.

osa Molo. price. (M:407) 


\section{Out-comparisons:}

osa Semau Helong. value, price, worth, cost.

osan Fehan Tetun. money includes old coins and modern paper money.

osan East Tetun. money, precious metal. (Mo:156)

osa Ili'uun. goods, property, cloth. (dJ:131)

osa Kemak. money.

*osi Rote. garden in a village. Etym:

**wasi (pre-RM).

osi Termanu. garden or plantation in or nearby a village. (J:458)

osi Korbafo.

osi Bokai.

osi Bilbaa.

osi $B a^{\prime} a$.

osi Tii.

osi Dengka.

osi Oenale.

Out-comparisons:

asi Kemak. garden. wasi Asilulu. forest garden. (van Hoëvell 1877:50)

wasi Buru. a) grove, orchard.

b) field (abandoned), garden (unused), fallow land. (M:479) [Form: Has the variant form wase with the second sense.] (Grimes and Grimes 2020:996)

*otas PRM. kind of insect which eats thatch/string. [irr. from PRM: $*_{\mathrm{o}}>e$ in Meto]

otas Termanu. kind of worm that eats the roof covering of new houses in the rainy season. (J:459)

ota-k Bokai.

ota-? Bilbaa.

otas Rikou. [Note: not known by my consultants.]

otas $B a^{\prime} a$.

otas Tii.

otas Dengka.

otas Oenale.

otas Dela.

etas Kotos Amarasi. kind of louse that eats string.

\section{$\mathbf{P}-\mathbf{p}$}

*pado Rote. octopus.

pado Termanu. octopus. (J:462)

pado Korbafo.

pado Bokai.

pado Bilbaa.

pado Rikou. octopus. (J:462; own field notes)

mpado $B a^{\prime} a$.

pado Tii.

pado Dengka.

pado Oenale.

pado Dela. octopus.

Out-comparisons:

kapafu Dhao. octopus.

kepafo Hawu. (J:462) *paha PnMeto. split, divide. Doublet: *faka1, *faka2. Etym: *bakaq. [irr. from PMP: * $\mathrm{k}>* \mathrm{~h}$ ] [minority from PMP: *b $>* \mathrm{mb} * \mathrm{p}$ (Tetun has regular $* \mathrm{mb}>b$, thus attesting pre-Meto *mb)] n-paha Kotos Amarasi. split, divide. n-paha Molo. cuts. (M:411) Out-comparisons:

baka Fehan Tetun.

*pande Rote. short in height, squat and compact in build, of a person. Doublet: *mbada. Etym: *pandak. [irr. from PMP: *a $>* \mathrm{e}$ ] [minority from PMP: $* \mathrm{p}=* \mathrm{p}]$

pane pane Termanu. small, short in stature, kind of small. (J:467) pane $\sim$ pane Korbafo. pane pane Bokai. 
pane pane Bilbaa.

pane pane $B a^{\prime} a$.

pande $\sim$ pande $T i i$.

pande pande Dengka.

pande $\sim$ pande Oenale.

*papa CERM. guard, protect. [irr. from PRM: * $\mathrm{p}>f$ in Meto] [Form:

Alternately, we could reconstruct

*mbafa with medial $*_{f}$ and posit irregular fortition of $* \mathrm{f}>p$ in Rote.]

papa Termanu. occasionally monitor animals that are secured in the field or bush. (J:468)

papa Rikou.

n-pafa? Kotos Amarasi. protect.

<n-pafa $>$ Molo. takes care of. (M:410)

\section{Out-comparisons:}

papa_piara Semau Helong. look after, take care.

*paru PRM. grate. Etym: *parud 'rasp, file'. [minority from PMP: ${ }^{*} \mathrm{p}=* \mathrm{p}$ ]

palu (2) pa palu-k Termanu.

1) grate. 2) grating, grater. [Semantics: All examples in Jonker (1908) refer to coconuts.] (J:466)

palu Korbafo.

palu Bokai.

palu Bilbaa.

paru Rikou.

palu $B a^{\prime} a$.

paru Tii.

palu Dengka.

paru Oenale.

panu|? Ro'is Amarasi. hard inner coconut shell.

?|panu|? Kotos Amarasi. hard inner coconut shell.

<panu> Molo. half a coconut shell. (M:421)

?|panu|? Kusa-Manea. hard inner coconut shell.

*peni Morph: *ka-peni-k. PRM. underlay, saddle.

peni-k Termanu. native saddle made from gebang palm leaves tied onto fabric rags, used both to ride on or to put loads on. (J:480) peni-? Korbafo.

peni-k Bokai.

peni-? Bilbaa.

peni-? Rikou.

peni-k $B a^{\prime} a$.

peni-? Dengka.

peni-? Oenale.

?|peni|? Kotos Amarasi. base, pad, sandals, saddle.

<peni> Molo. saddle. (M:432)

Out-comparisons:

beni Fehan Tetun. cover; put a cloth saddle on a horse; spread dirt on a log bridge. [irr. from PRM: * $\mathrm{p}=b$ correspondence]

peni Hawu. (J:480)

*petun PnMeto. Rough Bamboo; Giant

Bamboo. Dendrocalamus species. Etym: *botuy 'bamboo of very large diameter, probably Dendrocalamus species'. [minority from PMP: *b > $* \mathrm{mb}>{ }^{*} \mathrm{p}$ ] [Form: Initial *p in PMeto is from earlier *mb. Irregular $* b>* m b$ is attested in many languages of the region for reflexes of *botun and *mb can be reconstructed to proto-TimorBabar, perhaps even to a higher node.] petu Kopas. bamboo.

petu, petun Molo. kind of large bamboo. Dendrocalamus asper. (M:436)

petun Baikeno. bamboo. (Charles E. Grimes pers. comm.)

petu Kusa-Manea. kind of large bamboo. Dendrocalamus asper.

\section{Out-comparisons:}

betun East Tetun. variety of thick bamboo. [Form: Tetun *mb > $b$ and $* \mathrm{~b}>f$ are regular, hence this form also attests initial *mb.] (Mo:14)

betu Dadu'a. bamboo. (Penn 2006:92)

petuy Ili'uun. kind of bamboo. (dJ:133) 
*pio PnMeto. garlic. Etym: **mbio (preMeto). [History: This form may have been distributed by contact, though this would have been early as the reflexes of *mb are regular.]

kar/peo Kotos Amarasi. garlic. [Sporadic: vowel height harmony $*_{\mathrm{i}}>$ e/_o.] [Form: source of initial kar currently unknown.]

pio Amanuban. garlic.

peo Molo. onions. (M:434)

\section{Out-comparisons:}

kbiu Foho Tetun. garlic. [Note: variety of Tetun spoken in the northern part of the Tetunspeaking area of central Timor ISO 693-3 [tet].] [Sporadic: vowel height harmony $*_{\mathrm{o}}>u$ ii_.] (own field notes)

*piru PRM. sling. [irr. from PRM: * $\mathrm{r}>\varnothing$ $\sim n$ in Meto; ${ }^{*} \mathrm{p}>b \sim f$ in Meto]

pilu (2) pi $\sim$ pilu-k Termanu. 1) sling

(v.). 2) throwing in a slinging manner, a sling or a piece of wood used to throw in a slinging manner. (J:485)

pi pilu-? Korbafo.

pi piru-? Rikou.

mpi mpilu-? $B a^{\prime} a$.

pi p pi?u-k Tii.

pi p pilut Dengka.

pi pirut Oenale.

P|fi?u Kotos Amarasi. sling.

biut, fiku, finu Molo. sling. (M:72, $119,120)$

\section{Out-comparisons:}

tali (fa )firun, fa firuk East Tetun. sling. [irr. from PRM: $* \mathrm{p}=f$ correspondence $]$ (Mo:30, 34)

*poka PRM. swollen, paunch. Pattern: $\mathrm{k}-10^{\prime}\left({ }^{*} \mathrm{k}>?, k\right.$ in Termanu and Oenale; expect only * $\mathrm{k}>$ ?). [irr. from PRM: *a $>o$ in first Rote forms]

tei_po?o-k (2) po poka-k Termanu. 1) stomach (of people and some animals). hataholi tei po?o madema someone with a 'deep stomach', a glutton (J:495)

2) hataholi ei po pokak someone whose feet have swollen up due to sickness (J:492)

tei_po?o-? Korbafo.

tei_po?o-k Bokai.

tei_poko-? Bilbaa.

tei_poko-? Rikou.

tei_mpo?o-k $B a^{\prime} a$.

tei po?o-k Tii.

tei_poko-? (2) po po?at Oenale.

2) kind of fish that swells up. (J:754)

n-pook Ro'is Amarasi. fat. [Form: metathesised form of n-poka.]

n-poka Kotos Amarasi. fat.

n-pook (2) fafi a-poka-t (3) <meen poko> Molo. 1) fat. 2) a fat pig. 3) kind of leprosy which causes swelling. [Form: meen metathesised from menas 'sickness'.] (M:445)

*poke Rote. blind. Pattern: k-5.

poke (2) poke-k (3) po?e Termanu.

1) be or become blind. 2) blind, a blind person. (J:492) 3) = poke-k, mainly as a swearword. (J:490)

poke Korbafo.

poke Bokai.

poke Bilbaa.

poke Rikou.

mpoke $B a^{\prime} a$.

poke Tii.

poke Dengka.

poke Oenale.

Out-comparisons:

<poki> Kambera. blind. [Note: also in Mangili, Anakalang, and Mamboru.] (On:418)

$<$ poku> Lewa.

peke Perai. blind. [Note: language of Wetar Island ISO 639-3 [wet].] (Hinton 2000:125)

pok pok Sekar. blind. [Note: language of the Bomberai Peninsula ISO 639-3 [skz].] 
[Form: possibly a chance resemblance.] (Smits and Voorhoeve 1992:128)

*poko CER. plop. Pattern: k-5/6.

[Semantics: onomatopoeia.]

poko poko Termanu. plop, make a plopping noise by something that falls. (J:492)

\section{Out-comparisons:}

kepo?o Hawu. (J:492)

*ponia $P w R M$. sacrifice. [irr. from PRM:

*p $>f$ in Meto]

ponia Dengka. sacrifice. (J:755)

fnia-t Molo. poured out sacrifice. (M:123)

*pupu PRM. blowpipe blow through

a blow pipe. Etym: *putput 'puff, blow, expel air rapidly, as in using a blowgun'. [minority from PMP: *p = ${ }^{*} \mathrm{p} ;{ }^{*} \mathrm{p}={ }^{*} \mathrm{p}$ ] [irr. from PRM: ${ }^{*} \mathrm{p}>f$ in most nRote /\#]

fupu Termanu. blow through a blowpipe. (J:149)

fupu Korbafo.

fupu Bokai.

fupu Bilbaa.

fupu Rikou.

fumpu $B a^{\prime} a$.

pupu Tii.

pupu Dengka.

pupu Oenale.

puput Molo. a) bamboo from which flutes are made. (M:457)

b) blowpipe. (M:479)

Out-comparisons:

pupu Helong. (J:149)

\section{$\mathbf{R}-\mathbf{r}$}

*raho $n R M$. three-stone hearth, fireplace. Etym: *dapuR 'hearth'. [minority from PMP: *d $>*_{\mathrm{r}} / \#_{-}($expect $\left.* \mathrm{~d})\right]$ [Sporadic: $\left.* \mathrm{u}>*_{\mathrm{o}} /{ }_{-} \mathrm{R} \#.\right]$

la?o Termanu. cooking place, the three stones on which a pot is placed to cook the food, trivet, or the space between it. Also fireplace in general. ( $\mathrm{J}: 279)$

la?o Korbafo.

la?o Bokai.

lao Bilbaa.

rao Rikou.

la?o $B a^{\prime} a$.

rapo Tii.

auf nao Kotos Amarasi. ash in the hearth.

nao Kopas. ash from the three-stone hearth.

\section{Out-comparisons:}

avu rao Hawu. ash.

rao Dhao. earth oven. Made from clay for cooking clay pots. *ra?i Rote. tie. Etym: *Rakit 'lay long objects side by side; raft'. [minority from PMP: $\left.{ }^{*} \mathrm{R}={ }^{*} \mathrm{r}(\operatorname{expect} \varnothing)\right]$

la?i Termanu. tie bamboo or betel nut together in a certain way. ( $\mathrm{J}: 271)$

la?i Korbafo.

la?i Bokai.

lai Bilbaa.

rai Rikou.

la?i $B a^{\prime} a$.

ra?i Tii.

lai Dengka.

rai Oenale.

Out-comparisons:

'lai Semau Helong. Borrowed from: Irregular reflexes of initial * $\mathrm{R}$ in both Rote and Helong indicates borrowing. This was probably from Rote into Helong, given initial $r$ in East Rote and DelaOenale. [irr. from PMP: * $\mathrm{R}>l$ /\#] (J:271)

*ra?u PRM. scoop up in cupped hands. Etym: *rakup. [Form: Helong attests pre-RM initial $*$ d.] 
la?u Termanu. scoop up (e.g. sand, etc.) with cupped hands. (J:285)

la?u Korbafo.

la?u Bokai.

lau Bilbaa.

rau Rikou.

la?u $B a^{\prime} a$.

ra?u Tii.

lau Dengka.

rau Oenale.

n-nau Kotos Amarasi. scoop, gouge.

Out-comparisons:

daku Semau Helong. scoop.

*rates PRM. grave. [irr. from PRM: *e > $a$ in Ro'is Amarasi]

lates Termanu. grave. (J:283)

late-? Korbafo.

late-? Bokai.

late-? Bilbaa.

lates $B a^{\prime} a$.

rates $T i i$.

lates Dengka.

rates Oenale.

niut nata $R o$ 'is Amarasi. grave.

nate Kotos Amarasi. grave.

naten Molo. cairn on grave. (M:353)

nate Kusa-Manea. grave.

Out-comparisons:

rate(n) East Tetun. grave, tomb. (Mo:159)

rata, rate Waima'a. cemetery, tomb.

rate Dadu'a. grave. (Penn 2006:13)

dare (2) rai dare Hawu. 1) bury, covered; establish as law, command, order, set as regulation. 2) graveyard, cemetery. [Sporadic: consonant metathesis *rVd $>d V r$.]

raten Sika. grave, tomb. (Pareira and Lewis 1998:169)

$<$ reti> Kambera. grave. (On:438)

$<$ rate $>$ Kodi. grave.

$<$ rati $>$ Mamboru. grave.

$<$ ratu $>$ Lewa. grave.

rade Bima. grave. (Jonker 1893:83) rate Bima. grave. Usage: Kolo dialect. (Jonker 1893:83)

*ree Rote. wave. [Note: The forms with final $i i$ may be historically unrelated to those with final ee.]

lee-k (2) lii Termanu. 1) oblong tracks (e.g. of tortoise on sand, boat in water). (J:286) 2) wave, waves. (J:309)

lee-? (2) lii Korbafo.

lee-k (2) lii Bokai.

lee-? (2) lii Bilbaa.

ree Rikou.

lee-k (2) lii $B a^{\prime} a$.

ree-k (2) rii Tii.

lee Dengka. wave, waves. (J:309)

ree, rii Oenale. wave, waves. (J:309)

ree Dela. wave.

Out-comparisons:

leen Semau Helong. wave. [Note: Jonker (1908:286) gives Helong lee(n) for the second Rote meaning.]

*reha PRM. fathom, arm span. Etym: *dəpa. [minority from PMP: $* \mathrm{~d}>*_{\mathrm{r}}$ /\#_(expect*d)] [Sporadic: *a $>$ e/_\# in wRM.]

le?a Termanu. fathom. (J:288)

le?a Korbafo.

le?a Bokai.

lea Bilbaa.

rea Rikou.

le?a $B a^{\prime} a$.

re?a Tii.

lee Dengka.

ree Oenale.

nehe Kotos Amarasi. fathom.

nehe Molo. an arm span. in an-nehe nuku-n he stretches his arms out (M:360)

Out-comparisons:

dea Semau Helong. fathom.

ro?a Fehan Tetun. unit of length, from one outstretched hand to the other (about 1.5 metres); can be measured using a tali (rope). 
roha East Tetun. fathom, the length of the extended arms; $v$. to measure by fathoms. (Mo:162)

ree Kisar. arm width.

* rekət Morph: *ma-ka-rekət. PRM. tart, brackish. Pattern: k-9.

ma-ka-lePe-k Termanu. tart. (J:292)

ma-ka-le?e Korbafo.

ma-ka-le?e-k Bokai.

ma-ka-leke Bilbaa.

ma-re?e Rikou.

ma-ka-le?e-k $B a^{\prime} a$.

ma-ka-re?e Tii.

ma-Pa-le?a Dengka.

ma-Pa-re?a Oenale.

ma?|ne?at Kotos Amarasi. suffering.

ma?|ne?at Molo. tart, sour; fig. trouble and sorrow, tribulation, distress. [Note: Jonker (1908:292) gives Meto makne?et.] (M:305)

\section{Out-comparisons:}

mneken Helong. (J:292)

rəkət Central Lembata. (Fricke 2015)

dəkət Lamaholot, Ile Ape. [Note: language of Lembata Island ISO 639-3 [slp].] (Keraf 1978:264)

bərəkət Lamalera. [Note: language of Lembata Island ISO 639-3 [lmr].] (Keraf 1978:264)

*rena PwRM. hear, listen. Etym: *dəyəR. rena Oenale. hear. ( $\mathrm{J}: 721)$

rena Dela. hear, listen.

n-nena Ro'is Amarasi. hear.

n-nena Kotos Amarasi. hear.

n-nena Molo. hear. (M:361)

nean Kusa-Manea. hear.

Out-comparisons:

rona East Tetun. hear, listen, pay attention to. (Mo:162)

dene Waima'a. hear, believe. terne Kisar. hear. *reo PRM. turn around.

(na)-leo Termanu. turning around something. (J:302)

(na)-leo Korbafo.

(na)-leo Bokai.

(na)-leo Bilbaa.

na-reo Rikou.

(na)-leo $B a^{\prime} a$.

na-reo Tii.

(na)-leo Dengka.

na-reo Oenale.

n-neo Molo. loops, winds around. ma?u an-neo niis ane the weed loops, winds itself around the field rice, choking [Form: Jonker (1908:302f) gives nero and neo/ neok = 'turn something', as well as na-knero and na-kneo = 'turn'.] (M:363)

*rifu|n PRM. thousand. Etym: *Ribu. [minority from PMP: $* \mathrm{R}=*_{\mathrm{r}}$ (expect Ø)] [Form: The origin of final ${ }^{*} n$ is currently unclear, though it is attested widely outside of Rote-Meto.]

lifun Termanu. thousand. (J:312)

lifun Korbafo.

lifun Bokai.

lifun Bilbaa.

rifun Rikou.

lifun $B a^{\prime} a$.

rifun $T i i$.

lifun Dengka.

rifun Oenale.

niufun Ro'is Amarasi. thousand.

nifun Kotos Amarasi. thousand.

Out-comparisons:

lihu Semau Helong. thousand.

rihun East Tetun. thousand.

(Mo:161)

riwun, riwan Kisar. thousand.

*rimbu Rote. teem, abound. Doublet:

*libu. Etym: *libut 'surround, encircle, as game'. [irr. from PMP: *1 $>*$ r; *b $>* \mathrm{mb}]$

ri rimbu Tii. teem, abound. (J:724)

rimbu-rimbu Oenale. teem, abound.

(J:724) 
*rigin Morph: *ma-rinin, *ma-ka-rinin. PRM. cold. Etym: *dinin. [minority from PMP: *d $>* \mathrm{r} \#$ /_ (expect $* \mathrm{~d})$; $*_{\mathrm{y}}=*_{\mathrm{y}}$ ] [Form: Medial PRM $*_{\mathrm{y}}$ is required to account for Meto $k \sim$ n.] [History: Termanu dinis $=$ 'dew' (Jonker 1908:88) may be connected, as suggested by Jonker, though this would require irregular initial $* \mathrm{~d}=$ $d$. Additionally, Tii has denis which would attest irr. $* \mathrm{i}>e$.]

ma-ka-lini Termanu. be cold. (J:314)

ma-ka-lini Korbafo.

ma-ka-lini Bokai.

ma-ka-lini Bilbaa.

ma-rini Rikou.

ma-ka-lini $B a^{\prime} a$.

ma-ka-rini $T i i$.

ma-Pa-lini Dengka.

ma-Pa-rini Oenale.

mainikin Ro'is Amarasi. cold. [Note: mainirin was identified by my main Kotos Amarasi consultant as a Ro'is Amarasi form but was not known by any of my Ro'is consultants. If this form does exist, it would point to earlier *madindin and indicate that a doublet should be reconstructed to PRM.]

mainikin Kotos Amarasi. cold.

manikin, <mainini> Amanuban/ Amanatun. cold and coldness. [Note: My Amanuban and Amanatun data only has manikin, mainini is from Middelkoop (1972:298).] (M:298; own field notes)

<mainiki> Molo. cold and coldness. (M:298)

manikin Kusa-Manea. cold.

Out-comparisons:

blinin Semau Helong. cold, cool, chilly, fresh.

malirin East Tetun. cold, cool; $n$. cold; $a d v$. coldly, without enthusiasm; $v$. to be cold or cool. [irr. from PMP: *d > $(* \mathrm{r})>l$ (possibly sporadic dissimilation)] (Mo:137)

rinna Kisar. cold.

merini Hawu. cold, cool.

*rodok PRM. crawl, creep. Doublet: *nodo. [Form: regular $* d>* * 1 / * 1$ in Meto (and Helong) e.g. * rodok $>$ $* *$ lodok $>* *$ lolok $>$ nonok.]

lodo Termanu. crawl, crawl forward, like a snake. (J:319)

lodo Korbafo.

lodo Bokai.

lodo Bilbaa.

rodo Rikou.

lodo $B a^{\prime} a$.

rodok Tii.

rodo? Oenale. crawl, crawl forward, like a snake.

n-nonok Kotos Amarasi. slither, crawl (on chest/belly). [History: Both the semantics and the final $k$ indicate that the Meto reflexes belong under this headword rather than similar *nodo.]

n-nonok Molo. flows, crawls, leaks out. (M:384)

Out-comparisons:

lolo Semau Helong. crawl.

rodo Hawu. (J:319)

*roko Morph: *na-ka-roko. Rote. rattle. Pattern: k-5. [Semantics: onomatopoeia.]

na-ka-loko Termanu. rattling, as for instance the kernels of some fruit when shaken, also used for the sound that the contents of an old coconut makes when shaken. (J:322)

na-ka-loko Korbafo.

na-ka-loko Bokai.

na-ka-loko Bilbaa.

na-ro roko Rikou.

na-ka-loko $B a^{\prime} a$.

na-ka-roko Tii.

na-Pa-loko Dengka. 
Out-comparisons:

kerəko Hawu. (J:322)

*royga Rote. pen, corral.

loya Termanu. hutch, close in a hutch.

loya Korbafo.

loya Bokai.

loya Bilbaa.

roka Rikou.

longa $B a^{\prime} a$.

longa Lole. corral, barn, stable, stall. (Zacharias et al. 2014)

royga Tii. caged, trapped. (Grimes et al. 2014a)

longa Dengka.

royga Oenale.

Out-comparisons:

doka Hawu. garden, corral.

*rose $P R M$. rub, wipe.

lose Termanu. rub, rub into, rub off, wipe off. ( $\mathrm{J}: 328)$

lose Korbafo.

lose Bokai.

lose Bilbaa.

rose $R$ ikou.

lose $B a^{\prime} a$.

rose Tii.

lose Dengka.

rose Oenale.

n-nose Kotos Amarasi. wipe away, erase.

n-nose (2) $\quad$ ?-nose-? Molo. 1) wipe away (e.g. tears). 2) rag, handkerchief. (M:386)

\section{Out-comparisons:}

rose Kemak. rub.

<rúhi>, <rúhu> Kambera. rub, polish, clean. (On:448)

roho Hawu. (J:328)

blosok (2) berosok Sika. 1) rub. (Fricke 2014:85) 2) rub. Usage:

Hewa dialect/variety. (Keraf 1978:292)

roso Ende. file.

*rou Morph: *rou-k. Rote. skin. [Note:

While the Out-comparisons are formally similar, they point to earlier **1 that would not regularly develop into PRM *r. Furthermore, the semantic match between 'skin' and 'sarong', while not implausible, is also not entirely convincing. Thus, they are probably chance resemblances.]

lou-k Termanu. a) skin, hide, leather, bark, fruit peel, shell. b) anything in which things can be stored, a bag, barrel, etc. (J:329f)

lou-? Korbafo.

lou-k Bokai.

lou-? Bilbaa.

rou-? Landu. skin. (own field notes)

rou-? Rikou.

lou-k $B a^{\prime} a$.

lou-k Lole. skin. (Zacharias et al. 2014) rou-k Tii.

lou-? Dengka.

rou-? Oenale.

Out-comparisons:

<lä̈> [lau] Kambera. skirt, sarong. (On:234)

$<$ lawu> Anakalang.

$<$ lawo> Kodi.

dawo Ende. female sarong.

*ruan PMeto. village. [Note: Blust and Trussel (ongoing) reconstruct PAN *kuan on the basis of an Amis form and Meto kuan. Ro'is Amarasi ruan with initial $r$ shows that this reconstruction is a result of chance similarity.] [irr. from PRM: ${ }^{*} \mathrm{r}>l$ (expect $k$ ) in poetic Molo form (possibly archaic retention with $* \mathrm{r}>l$ after $* \mathrm{r}>k$ )] [Form: perhaps from earlier **nduan.] [History: May be connected with Rongga, Ngadha, Lio, and Ende nua 'village'.] ruan $R o$ 'is Amarasi. village.

kuan Kotos Amarasi. village.

kuan (2) luan Molo. 1) village. (M:243) 2) village. na-?uul ees Saneploon bian, hai lua Ploo hai m-ak fauknais literally, 'it is raining in the coastal plains below Camplong, but we in distant villages say it is the dry season', a metaphor for a complaint submitted 
in Kupang against a king, while in the inland people prefer stupid kings. Word-level: 3-rain COP Camplong side 1PL.EXCL village far 1PL.EXCL 1PL.EXCL-say drought. Usage: poetic.

* ruku Rote. bent over. Etym: *duku 'bend over, stoop'. Pattern: k-5. [minority from PMP: *d $>*$ r $/ \#$ (expect $* \mathrm{~d})]$

luku luku Termanu. bent over, bent. (J:332)

luku luku Bokai.

luku luku $B a^{\prime} a$.

(luku luku ?) Tii.

luku luku Dengka.

ruku ruku Oenale.

Out-comparisons:

ru?u Hawu. (J:332)

*rumu Rote. press. [History: Blust and Trussel (ongoing) identify the Termanu form as a reflex of PMP *lumu 'soft', but initial *1 cannot account for Rote varieties with initial $r$, which regularly comes from $*$ r.]

lumu Termanu. knead something softly. (J:334)

lumu Korbafo.

lumu Bokai.

lumu Bilbaa.

rumu Rikou.

lumu $B a^{\prime} a$.

rumu Tii.

lumu Dengka.

rumu Oenale.

Out-comparisons:

lumu Semau Helong. press.

* rumbi Rote. mantle of a squid.

lumpi-k $B a^{\prime} a$. the mantle of a squid. (J:728f)

lumbi-? Dengka.

rumbi-? Oenale.

Out-comparisons:

rupu, rupe Kisar. huge octopus or squid that is big enough to wrap around a sailboat; also referred to as ghosts of the ocean. *ruyirai Rote. whale. [irr. from PRM: ${ }^{*} \mathrm{u}>o$ in Rikou] [History: Jonker (1908:334) tentatively suggests this may be a borrowing from Hawu.]

lunilai Termanu. whale. (J:334)

lunilai Bokai.

rokirai Rikou.

lungilai $B a^{\prime} a$.

rungirai Tii.

luygilai Dengka.

rungirai Oenale.

Out-comparisons:

lunirai Hawu. whale. (J:334)

*rutus $P R M$. rust.

na-lu lutu (2) lu lutu-k Termanu.

1) rusting. 2) rust. (J:336)

na-lu lutu Korbafo.

na-lu lutu Bokai.

na-lu lutu Bilbaa.

na-ru rutu Rikou.

na-lu lutu $B a^{\prime} a$.

na-ru rutu Tii.

na-lu lutu Dengka.

na-ru rutu Oenale.

nutus Kotos Amarasi. rust.

nutus Molo. rust. (M:327)

Out-comparisons:

rotus Fehan Tetun. rust (of iron).

rutu Hawu. rust.

rutu Sika. rusty. (Pareira and Lewis 1998:177) 


\section{$\mathbf{S}-\mathbf{S}$}

*-s $P w R M$. people group suffix.

-s Dengka. sometimes the same as Meto at the end of the names of people groups: sina-sius = sina-siu, Chinese. (J:757)

-s Dela. people group suffix. [Note: Examples include: dela-s $=$ 'Dela person', ndao-s = 'Dhao person', tii-s = 'Tii person', and sonobai-s $=$ 'Atoni'.]

-s, -as Kotos Amarasi. people group suffix. Usage: -s is used after vowel final stems and CVC\# final stems (for which it replaces the final $\mathrm{C}$ ), -as is used with VVC\# final stems.

-s, -as Molo. Jonker rightly identifies the 'inorganic' final -s on the names of people groups that end in a vowel. When they end in a consonant they take the suffix -as. [Note: Strange as it may seem, this is the description given by Middelkoop of this suffix. He is probably referring to Jonker (1906).] (M:xxix)

*saa PwRM. what?

saa Dengka. what? (J:757f)

saa Oenale. what? (J:757f)

$\mathbf{s a a} \mathbf{2 n}=\mathbf{a a}$ Ro'is Amarasi. what.

saa? Kotos Amarasi. what?

$<$ sa'> Molo. what. (M:462)

na-sa?a Kusa-Manea. why?

Out-comparisons:

saa Semau Helong. what.

saa East Tetun. what. (Mo:163)

*sabake Morph: *sabake-k, *na-sabake.

$n R M$. branch. Pattern: k-10.

baPe-k (2) na-ba?e Termanu. 1) big branch of a tree. 2) have big branches. (J:20)

ba?e Korbafo.

ba?e-k Bokai.

bake-? Bilbaa.

bake-? Landu. forked branch. (own field notes) bake-? Rikou.

ba?e-k $B a^{\prime} a$.

ba?e-k Tii.

sbake|? Ro'is Amarasi. forked branch.

sbake|? (2) na-sbake Kotos Amarasi. 1) forked branches. 2) grow (forked) branches.

<sbake> Molo. forked branch. (M:478)

*sabuu CERM. blown up, inflated; smoke. [irr. from PRM: *uu $>$ oo in Meto]

bu buu Termanu. a) inflated, blown up. suye $=$ na bu buu his cheeks are inflated, perhaps from blowing, perhaps because he has something in his mouth lafa=na bu buu his sarong is inflated (e.g. because of the wind). b) also used of the swelling up of the cheeks while smoking. ana bu buu modo he smokes tobacco. (J:60)

na-sboo (2) sboo-t Kotos Amarasi. 1) smoke (cigarette). 2) cigarette.

*sadu PRM. wig, false hair. Pattern: d-2. salu Termanu. false hair, false plait. (J:518)

salu Korbafo.

salu Bokai.

salu Bilbaa.

saru Rikou.

salu $B a^{\prime} a$.

saru Tii.

salu Dengka.

saru Oenale.

salu? Meto. [Note: Jonker (1908:518) gives Amarasi farus.] (J:518)

Out-comparisons: salu? Helong. (J:518)

ruharu Hawu. mane of a horse. (J:518)

*sada PRM. cut, peel.

sa $\sim$ sada Termanu. cut into discs. (J:507f)

sa $\sim$ sada Korbafo. 
sa sada Bokai.

sa $\sim$ sada Bilbaa.

sa sada Rikou.

sa sada $B a^{\prime} a$.

sa $\sim$ sada Tii.

sa $\sim$ sada Dengka.

n-sara Kotos Amarasi. peel fruit with a knife.

n-sala Molo. peels. (M:471)

Out-comparisons:

sadat Semau Helong. peel.

*sadi Rote. as long as.

sadi Termanu. only, as long as, if only, provided that. (J:508)

sadi Korbafo.

sadi Bokai.

sadi Bilbaa.

sadi Rikou.

sadi $B a^{\prime} a$.

sadi Tii.

sadi Dengka.

sadi Oenale.

Out-comparisons:

sadi Semau Helong. as long as.

hadi Hawu. as long as, provided that.

*sadodo PRM. slide, slip. Pattern: d-2. [irr. from PRM: ${ }^{*} \mathrm{~d}>n d$ in Oenale] [Sporadic: consonant metathesis $* \mathrm{dVr}$ $>*_{r} V d$ in Bilbaa, Rikou and Landu; consonant metathesis $* 1 \mathrm{Vn}>* 1 \mathrm{Vn}$ in Molo.]

dolo Termanu. slide, glide. (J:98)

dolo Korbafo.

dolo Bokai. slide, skid.

lodo Bilbaa. slide, skid. (J:725)

na-rodo-? Landu. slippery. (own field notes)

rodo Rikou. slip, skid. (J:725)

dolo $B a^{\prime} a$.

doro Tii.

dolo Dengka.

ndoro Oenale.

na-sroro Kotos Amarasi. slide.

<n-sikanolo>, <n-sakanolo> (2)

n-sinolok Molo. 1) slide. (M:xliii)

2) slips, slides. (M:497)

\section{Out-comparisons:}

sarodok, sadorok East Tetun. slip, slide or glide. (Mo:167, 164)

hedodo, hedodi Hawu. slide. (J:98)

*safe CERM. catchbirdtree, birdcatcher tree, birdlime tree. Pisonia alba. [Semantics: Hoola van Nooten et al. (1880) say of Pisonia alba: 'The leaves of Pisonia sylvestris and $P$. alba are used as a vegetable. Pisonia alba is known as Moluccan cabbage and is an albino form of the lettuce tree (Pisonia grandis R.Br.)'. Their picture is of a plant with large white (or green) leaves. This matches well the description of the plant given by Jonker (1908:511). Pisonia sylvestris appears to be a synonym of Pisonia alba.]

safe Termanu. kind of tree, called sayur bulan ('moon vegetable') in Kupang, the leaves are eaten as vegetables. Pisonia alba. (J:511; Heyne 1950:610, cxviii)

$<$ safe $>$ Molo. kind of tree the leaves of which are use at a desecration. (M:465)

*saha1 Morph: *saha-k. nRM. whetstone. Etym: *hasaq 'whet, sharpen'. [irr. from PMP: *a $>\varnothing]$ [minority from PMP: $* \mathrm{q}>* \mathrm{~h}$ ] [Form: regular $* \mathrm{~h}>$ $\varnothing$ /a_a in Rote. The Waima'a outcomparison indicates earlier *hasaq $>$ **saqa.]

saa-k Termanu. whetstone for knives and machetes. (J:507)

saa-? Korbafo.

saa-k Bokai.

saa-? Bilbaa.

saa-k $B a^{\prime} a$.

saa-k Tii.

saha|k Kotos Amarasi. large whetstone.

n-saha (2) sahan Molo. 1) scrapes, makes smooth. 2) large, round whetstone. (M:466) 


\section{Out-comparisons:}

sa?a Waima'a. sharpen.

*saha2 Morph: *na-saha. PRM. carry on the shoulders. Etym: *pasaqan. [minority from PMP: $* \mathrm{q}>* \mathrm{~h}$ ] [Form: regular $* \mathrm{~h}>\varnothing / \mathrm{a} \_\mathrm{a}$ in Rote.]

na-saa Termanu. carry on or over one's shoulder. (J:506)

na-saa Korbafo.

na-saa Bokai.

na-saa Bilbaa.

na-saa Rikou.

na-saa $B a^{\prime} a$.

na-saa $T i i$.

na-saa Dengka.

na-saa Oenale.

na-saha Kotos Amarasi. carry an item by placing it on one's shoulder or back.

*sai1 $n R M$. flow.

sai Termanu. make flow away, e.g. water that is standing still. (J:512)

sai Korbafo.

sai Bokai.

sai Rikou.

sai $B a^{\prime} a$.

(sai ?) Tii.

na-sai Kotos Amarasi. flow.

Out-comparisons:

sai Funai Helong. flow.

*sai2 Rote. open.

sai Termanu. 1) cut open the belly of a slaughtered animal. 2) open something, such as a pot or bag, and begin to use the contents, open something. (J:512)

sai Korbafo.

sai Bokai.

sai Bilbaa.

sii_bati (2) sai Rikou. [Semantics: sii bati is equivalent to Termanu sense (1), sai is equivalent to Termanu sense (2).]

sai $B a^{\prime} a$.

sai Tii.

sai Dengka.

sai Oenale.

\section{Out-comparisons:}

sai Semau Helong. open, unveil, reveal.

*saiz Rote. torn up.

sai sai (2) sai Termanu. 1) totally torn. 2) torn. (J:513)

sai $\sim$ sai Korbafo.

sai sai Bokai.

sai $\sim$ sai $B a^{\prime} a$.

sai $\sim$ sai $T i i$.

sai sai Dengka.

Out-comparisons:

sait Semau Helong. torn.

*saka PRM. thigh, buttocks. Pattern: k-irr. [irr. from PRM: ${ }^{*} \mathrm{a}>i$ in much of Nuclear-Rote; ${ }^{*} \mathrm{k}>h / \mathrm{V}$ V in Meto] [History: Blust and Trussel (ongoing) reconstruct Proto-Philippine *sáka 'leg of a fowl (?)'.]

saki/bolo-k Termanu. rear haunch of an animal. (J:514)

saki/bolo-? Korbafo.

saki/bolo-k Bokai.

saka/bolo-? Bilbaa. thigh, rear haunch of an animal. (J:758)

saki/bolo-? Rikou.

saki/bolo-k $B a^{\prime} a$.

saka_bolo-k Tii.

saka-? Dengka. thigh of a person or animal. (J:758)

saka-? Oenale.

saka-? Dela. thigh.

saha-n Amanuban. thigh, buttocks.

saha-k Molo. thigh. (M:466)

Out-comparisons:

sakan East Tetun. hip (of people), flank (of animals). (Mo:164)

sakar Galolen. thigh.

*sake PRM. go up, ascend, rise. Etym: *sakay 'climb, ascend, rise up'. Pattern: k-8.

sa?e Termanu. climbing, rising (intransitive); have gone up, is up; sit down, of birds. (J:509)

sa?e Korbafo.

sa?e Bokai.

sake Bilbaa. 
sa?e Rikou.

sa?e $B a^{\prime} a$.

sa?e Tii.

sae Dengka.

sae Oenale.

n-sae Ro'is Amarasi. ascend.

n-sae Kotos Amarasi. go up, ascend, climb up.

n-sae Molo. rises, ascent. (M:464)

saa Kusa-Manea. go up. [Form: regular $*_{\mathrm{e}}>a / \mathrm{V}_{-} \#$ in Upper Manulea.]

\section{Out-comparisons:}

sake Semau Helong. rise, ascend.

sa?e East Tetun. climb on, mount; to rise up, ascend. (Mo:164)

ha?e Hawu. ascend, climb, go up, embark, get on.

*sakiki Morph: *na-sakiki. PRM. brush teeth. Etym: *kiskis 'shave, scrape off' (Blust and Trussel (ongoing) also reconstruct the doublet *gisgis 'rub, scrape against', which is reflected as 'brush (one's teeth)' in Tausug in the Philippines). Pattern: initial k-2a, medial k-6.

na-sa-kiki, kiki (2) kiki-k Termanu.

1) brush (the teeth); rub something used as medicine on one's teeth.

2) kind of brush made from palm leaf stalks used to clean the bucket that is used for tapping palm trees. (J:236)

na-sa-kiki Korbafo.

na-sa-kiki Bokai.

na-sa-kiki $B a^{\prime} a$.

(na-sakiki ?) Tii.

na-sa-Pi?i Oenale.

na-sa-Pi?i Dela. use tobacco to rub one's teeth after chewing betel nut. (own field notes)

na-skiki Ro'is Amarasi. brush one's teeth.

na-skiki Kotos Amarasi. brush one's teeth.

<skiki> (2) na-skiki Molo.

1) toothbrush. 2) brushes

(the teeth). (M:236) ta-skiik Kusa-Manea. brush the teeth.

Out-comparisons:

kikin Semau Helong. shave (head). [Note: Jonker (1908:236) gives Helong kiki, skiki 'brush, sweeper'.]

sakiki nehan Fehan Tetun. brush the teeth. (Mo:165)

*sakoro Morph: *na-sakoro. PRM. sip. Pattern: k-1. [irr. from PRM: ${ }^{*} \mathrm{r}>\varnothing$ in all Rote except Oenale]

na-sa-koo Termanu. sip, drink with sips. (J:241)

na-sa-koo Korbafo.

na-sa-koo Bokai.

na-sa-koo Bilbaa.

na-sa-oo Rikou.

na-sa-koo $B a^{\prime} a$.

na-sa-koo Tii.

na-sa-koo Dengka.

na-sa-koro Oenale.

na-sakono Meto. (J:241)

*sala PRM. wrong, mistake, incorrect. Etym: *salaq 'wrong, in error (of behaviour); miss (a target); mistake, error, fault'.

sala Termanu. wrong, incorrect, mistaken. (J:514)

sala Korbafo.

sala Bokai.

sala Bilbaa.

sala Rikou.

sala $B a^{\prime} a$.

sala Tii.

sala Dengka.

sala Oenale.

n-sana Kotos Amarasi. make a mistake, be wrong.

n-sana Molo. is wrong. (M:473)

Out-comparisons:

sala Semau Helong. wrong, fault, mistake, sin.

sala East Tetun. err, make a mistake, be wrong, be in error, sin; $n$. error, mistake, sin, crime, blame. (Mo:165) 
*sale $n R M$. sorry, contrition.

sale Termanu. contrition, repentance. (J:517)

sale Korbafo.

sale Bokai.

sale Bilbaa.

sale Rikou.

sale $B a^{\prime} a$.

sale Tii.

sane-l=oo-n Molo. be sorry. (M:474)

*sali PnRote. pour, fill. Etym: *salin 'pour from one vessel into another; translate, interpret' (PWMP. Blust and Trussel (ongoing) also reconstruct PMP *saliR 'flow, of water', which is where they place the Termanu cognate, but if this were correct we would expect the final vowel in Rote to lower before loss of final *R.).

sali Termanu. put a liquid somewhere, pour into. (J:517)

sali Korbafo.

sali Bokai.

sali Bilbaa.

sali Rikou.

sali $B a^{\prime} a$.

sali Tii.

Out-comparisons:

sali Semau Helong. pour, fill.

salin East Tetun. empty, spill out. (Mo:165)

hali Hawu. fill (liquid), transfer container, pour.

*salili Morph: *na-salili, *salili-k. PRM. armpit. Etym: **salili (pre-RM).

na-sa-lili (2) lili_bolo-k Termanu.

1) carry something in the armpit/ under the arm while it hangs by a band from the shoulder. 2) armpit. [Form: The second part of the Rote nominal forms all mean 'hole'.] (J:313)

na-sa-lili (2) lili_poo-? Korbafo. na-sa-lili (2) lili_bolo-k Bokai. na-sa-lili (2) lili_poo-? Bilbaa. na-sa-lili (2) lili_bolo-? Rikou. na-sa-lili (2) lili_bolo-k $B a^{\prime} a$. na-sa-lili (2) lili_bolo-k Tii.

na-sa-lili (2) lili kolo-? Dengka. na-sa-lili (2) lili_ndola-? Oenale. snini-f Ro'is Amarasi. armpits.

snini-f (2) na-snini Kotos Amarasi.

1) armpits. 2) carry under arm with strap around shoulder.

snini-n Molo. armpit. (M:507)

snini-f Kusa-Manea. armpit.

Out-comparisons:

slili Helong. armpits. (J:313)

kalili, klilin East Tetun. armpit. (Mo:110)

kahalilin Ili'uun. armpit. (dJ:119)

hililla Kisar. armpit.

lila-r Kemak. armpit.

saliri Bima. armpit. (Jonker 1893:91)

salili Sumbawa. [Note: language of Sumbawa ISO 639-3 [smw].] (J:313)

*saluku Morph: *na-saluku. PnRote. gather under the wings. Pattern: k-5/6. na-sa-lu luku Termanu. gather under the wings. (J:332)

na-sa-lu luku Korbafo.

na-sa-lu luku Bokai.

na-sa-lu luku Bilbaa.

na-sa-lu lu?u Rikou.

na-sa-lu luku $B a^{\prime} a$.

na-ka-lu lu?uk Tii.

Out-comparisons:

herugu Hawu. (J:332)

*sambat Morph: *ka-sambat. PRM. bucket.

samba-k Tii. bucket, specifically a kind of big bucket. (J:759)

sambat Dengka.

sambat Oenale.

sambat Dela. leaf bucket.

?|sapa|? Kotos Amarasi. leaf bucket made from lontar leaves.

Out-comparisons: sapat Semau Helong. bucket. [Note: Jonker (1908:759) gives Helong ksapat.] 
(k)naban, kanaban East Tetun. palm leaf basket. [irr. from PRM: ${ }_{\mathrm{s}}=n$ correspondence] (Mo:165)

haba Hawu. pail, container made from young lontar palm leaves. [Semantics: Used as palm wine container, or for irrigating fields by hand.]

$<$ hamba> Kambera. bucket made from lontar leaves. (On:60)

$<$ saba $>$ Anakalang.

$<$ samba> Mamboru.

*sambi $P R M$. remove bark.

sapi Termanu. cut the bark off a tree with a machete or axe, remove the bark of a tree in such a way. (J:522)

sapi Korbafo.

sapi Bokai.

sapi Bilbaa.

sapi Rikou.

sampi $B a^{\prime} a$.

sambi Tii.

sambi Dengka.

sambi Oenale.

sambi Dela. remove coconut shell.

n-sapi Kotos Amarasi. cut at slanted angle, cut chips off; e.g. to remove the shell of a coconut from its flesh.

n-sapi Molo. trims, shave off until smooth. (M:476)

\section{Out-comparisons:}

sabir Fehan Tetun. use knife to remove bark (from wood).

*sambudas $P R M$. sow, scatter; scattered about; splash water on something. Etym: **sa(m)bura[t/s] (pre-RM). Pattern: d-2. [irr. from PRM: *mb > $p$ in Oenale and Tii] [History: Blust and Trussel (ongoing) reconstruct a number of forms that are formally and semantically similar to the form I have placed in the etymology field: *sa(m) buR, *sabuD, *saq(ə)buR, *buras. None of these can quite account for the RM reflexes, though (of course) there are also problems with my own tentative reconstruction.] na-sa-pula Termanu. squirt or squirt out with the mouth (stronger than na-sa-puu). (J:502)

na-sa-pula Korbafo.

na-sa-pula Bokai.

na-sa-pula Bilbaa.

na-sa-pura Rikou.

na-sa-mpula $B a^{\prime} a$.

na-sa-pura Tii.

na-sa-mbula, mbulas Dengka.

puras Oenale.

puras, purak Dela. spit.

na-spura?, n-puran Kotos Amarasi. spurt water.

<na-spula $>$ (2) $\quad<$ n-pula $>$, n-pulan (3) <Oe Pula> Molo. 1) bubbles up (water). 2) spits on, pushes out of the mouth. 3) source where water spurts out, the name of springs, one is at Kupang the other is at Kauniki. (M:453)

\section{Out-comparisons:}

buras Sika. spray/spout (medicine) from the mouth. (Pareira and Lewis 1998:30)

hura Kamarian. spit something out, like medicine. [Note: also in Haruku and Kaibobo from Tihulale.] (van Ekris 1864:92)

hula Asilulu. spit something out, like medicine. (van Ekris 1864:92)

samburat Javanese. [Note: language of Java ISO 639-3 [jav].] (J:502)

*sana|? PMeto. bunch of fruit. Etym: *saya 'bifurcation, fork of a branch'. saan_oo Ro'is Amarasi. stick insect. noa_sana|? (2) saan_oo Kotos Amarasi. 1) bunch of coconuts. [Note: noah = 'coconut'.] 2) stick insect. [Form: regular final $\mathrm{CV} \rightarrow$ $\mathrm{VC}$ metathesis of first element of a compound combined with $\mathbf{0 o}=$ 'bamboo'.]

sana|? Molo. bunch of fruit or flowers. (M:473) 


\section{Out-comparisons:}

saya Semau Helong. strong forked branch.

ai sanak East Tetun. branch, bough, or fork of tree, a spar or pole. (Mo:166)

dzaya Hawu. branch. [irr. from $\left.P M P: *_{\mathrm{s}}>d_{3}\right]$

*sanahulu Rote. ten. Etym: *sa-napuluq. [Sporadic: $* \mathrm{~h}>\varnothing$ in wRote.] sanahulu (2) hulu Termanu. 1) ten.

2) multiple of ten. (J:196)

sanahulu (2) hulu Korbafo.

sanahulu (2) hulu Bokai.

sanahulu (2) hulu Bilbaa.

sanahulu (2) hulu Rikou.

salahunu (2) hulu Ba'a. [Sporadic: consonant metathesis ${ }^{*} n . . .1>n$...l. $]$

sanahulu (2) hulu Tii.

salahunu (2) nulu Dengka. [Note: Dengka salahunu is given as 'probably'.] [Sporadic: consonant metathesis *n...1>n...l.]

sanahulu (2) nulu Oenale.

Out-comparisons:

smulu Funai Helong. ten.

hyulu Semau Helong. ten.

syulu Bolok Helong. ten.

sanulu East Tetun. ten. (Mo:166)

heyuru Hawu. ten.

*sanasə PRM. breath. [irr. from PRM: *ə

$>u$ in some Rote (probably sporadic assimilation of antepenultimate vowel to stressed vowel in some cases)] [History: The closest resemblance in PMP is *yəsyəs.]

nase_bu?u-k Termanu. cheek of people and animals. [Form: bu?u-k = 'joint, node'.] (J:381)

nase_bu?u-k Bokai. cheek of people and animals. (J:381)

nase_buku-?, nasu_buku? Bilbaa. bump of the cheekbone, cheekbone.

nasa_bu?u-? Rikou. cheek of people and animals. (J:381)

nasu-k $B a^{\prime} a$. cheek. (J:736)

nasu-k Lole. cheek. (Zacharias et al. 2014) nasu-k Tii. cheek. (J:736)

nasu-? Dengka. cheek. (J:736)

nasu-P Oenale. cheek. (J:736)

snasa-f (2) na-snasa Kotos Amarasi.

1) breath. 2) take a break, rest, breathe.

snasa-f (2) na-snasa Molo. 1) breath in general. 2) rest. (M:505)

\section{Out-comparisons:}

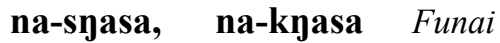
Helong. breathe.

hyasa Semau Helong. breath.

*sanu Rote. coconut with soft flesh.

noo_sanu Termanu. kind of coconut of which the flesh remains soft. (J:520)

noo_sanu Korbafo.

noo_sanu Bokai.

noo_sanu Bilbaa.

noo_sanu Rikou.

noo_sanu $B a^{\prime} a$.

noo sanu Tii.

noo_sanu Dengka.

noo_sanu Oenale.

Out-comparisons:

hayo Hawu. (J:520)

<hangu> Kambera. soft, e.g. of the flesh of fruit. (On:67)

<sangu> Anakalang. [Note: also in Mamboru.]

<zangu> Weyewa.

*sandiit $n R M$. an insect that makes a loud noise, either a cicada or cricket. [irr. from PRM: *nd $>k$ in Ro'is; *nd $>$ $r$ in Kusa-Manea; ${ }^{*}$ nd $>t$ in Termanu and Korbafo (onomatopoeia and/or assimilation to previous $t$ ); *nd $>d$ in East Rote] [Form: Onomatopoeia may be the source of some of the irregularities.]

toko_tii-k Termanu. kind of cricket. (J:640)

toko_tii Korbafo.

toko lii Bokai.

(tee) di dii Landu. kind of cricket. (own field notes) 
toko/dii, di dii Rikou. cricket. (own field notes)

toko_ndi?i-k $B a^{\prime} a$.

(toko_tii-k ?) Tii.

skiit ${ }^{\prime} o^{\prime} i s$ Amarasi. cicada.

skiit Kotos Amarasi. cicada.

ani_kra riit=aa Kusa-Manea. cicada.

Out-comparisons:

kniit Funai Helong. cicada. [irr. from PRM: *nd $=n$ correspondence]

hnii(t) ulan Semau Helong. cicada. [irr. from PRM: *nd = $n$ correspondence]

da $\sim$ dii_derok East Tetun. variety of cicada. Usage: Luka village. (Mo:20)

*sangenger PRM. surprise, startle, frighten.

ngene (2) na-ngene(-k) Termanu.

1) frighten, terrify. 2) surprise, startle. (J:437)

ngene Korbafo.

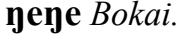

neye Bilbaa.

keker=asa $L a n d u$. (own field notes)

keke Rikou.

ngenge $B a^{\prime} a$.

ngenger Tii.

ngenge Dengka.

ngenger Oenale.

ngenger (2) na-ngenge-? Dela.

1) surprised, suddenly. 2) make surprised.

na-skeke Ro'is Amarasi. surprised, suddenly.

na-skeke Kotos Amarasi. surprised, startled, suddenly.

na-skeke Molo. scared, frightened. (M:501)

Out-comparisons:

${ }^{\circ}$ nahkeka Semau Helong. immediately, suddenly. Borrowed from: probably Meto na-skeke (shown by irr. *yg = $k$ correspondence, expect $\eta$ ). *sayguma Morph: *sayguma-k. PRM. hermit crab. Etym: *[q/k]uman. [irr. from PRM: *yg $>k$ in wRote] [Form: The source of initial *sang is currently unknown. It could be connected with Rote *sayga 'look for' given that hermit crabs look for their shell, but this is highly speculative. The wRote forms probably reflect *kuman without any additional element.]

yguma-k Termanu. kind of animal with a shell, called bai-kumbang [OE $=$ 'hermit crab'] in Kupang. (J:449)

yguma-? Korbafo.

yuma-? Bilbaa.

kuma-? Landu. hermit crab. (own field notes)

kuma-? Rikou.

yguma-k $B a^{\prime} a$.

yguma-k Tii.

kuma-? Dengka.

?uma-? Oenale.

?uma-? Dela.

skuma|? Ro'is Amarasi. hermit crab.

skuma|? Kotos Amarasi. hermit crab.

*sa01 PRM. marry. Etym: *qasawa

'spouse: husband, wife'.

sao Termanu. marry, get married, be married, both men and women. (J:521)

sao Korbafo.

sao Bokai.

sao Bilbaa.

sao Rikou.

sao $B a^{\prime} a$.

sao Tii.

sao Dengka.

sao Oenale.

n-sao (2) n-mat/sao Ro'is Amarasi.

1) marry. 2) marry.

n-sao (2) n-mat/sao (3) mat/ sao-s Kotos Amarasi. 1) marry, got married. 2) get married, wed. [Semantics: n-matsao focuses more on the actual wedding ceremony, while n-sao on the resulting state.] 3) wedding, 
marriage. [Form: Initial mat in matsao could be from the reciprocal prefix mak with irregular assimilation of the final consonant. In modern Amarasi the normal form of the reciprocal prefix is ma with the form mak being found before some, but not all, roots that begin with $/ \mathrm{t} /$.]

n-sao (2) n-mat/sao (3) sao-m Molo. 1) marry. 2) wed. 3) spouse. (M:477)

Out-comparisons:

safa Funai Helong. spouse. sapa (2) sapan Semau Helong.

1) marry, wed. 2) spouse.

*sao2 Morph: *kai/sao. PRM. green viper. Etym: *sawa 'python'. [Form: Initial *kai probably from *kaiu 'tree, plant, wood'.]

kai/sao Termanu. kind of poisonous snake. (J:217)

kai/sao Korbafo.

kai/sao Bokai.

kai/sao $B a^{\prime} a$.

kai/sao Tii.

ai/sao Dengka.

ai/sao Oenale.

ai/sao Dela.

sao Ro'is Amarasi. green viper.

P/sao Kotos Amarasi. green viper.

u?/sao, a?/sao Molo. poisonous green adder. [Form: Initial $u$ is probably cognate with initial $u$ in umeke (see *menge). Any original independent meaning of this element is unknown.] (M:592)

sao Kusa-Manea. viper.

Out-comparisons:

sa/mea East Tetun. the general name for all snakes. (Mo:166)

*sara PRM. loose, spread out.

sala sala Termanu. loose, free, not bound. (J:516)

sala sala Korbafo.

sala sala Bokai.

sala sala Bilbaa. sara sara Rikou.

sala sala $B a^{\prime} a$.

sara sara Tii.

sala sala Dengka.

sara sara Oenale.

$<$ na-siitb $=$ oo-n =am na-

saan $=00-n>$ Molo. they spread out in all directions. (M:473)

*saraa PRM. light, shine. [irr. from PRM: $*^{r}>l$ in nRote]

na-sa-laa (2) bula-k na-sa-laa (3) bula ma-sa-laa-k Termanu. 1) shine, be light, be bright; appear, show up. 2) the moon waxes, grows. 3) waxing/growing moon. $(\mathrm{J}: 261)$

(2) na-sa-laa Korbafo. na-sa-laa (2) na-sa-laa Bokai.

(2) na-sa-laa Bilbaa.

na-sa-laa (2) na-sa-laa Rikou.

na-sa-laa (2) na-sa-laa Tii.

(2) na-sa-la laa Dengka.

na-sa-raa

(2) na-sa-ra raa

Oenale.

snaa-f (2) snaa-t=ee n-sae Kotos Amarasi. 1) light, sunbeam, colour.

2) dawn from the perspective of the sun.

<snaa $>$ (2) fe? asnaa-t=ee Molo. 1) gleam, shining. 2) it begins to become light. (M:504)

*sarait Morph: *na-sarait. PRM. lean.

na-sa-lai (2) (ai) la lai-s Termanu.

1) lean; make something lean, lean something against something.

2) something against which a dead person leans. (J:270)

na-sa-lai Korbafo.

na-sa-lai Bokai.

na-sa-lai Bilbaa.

na-sa-rai Rikou.

na-sa-lai $B a^{\prime} a$.

na-sa-rai $T i i$.

na-sa-lai Dengka.

na-sa-rai Oenale.

na-snait Kotos Amarasi. lean on.

na-snait Molo. leans something against something. (M:505) 


\section{Out-comparisons:}

nahlae Semau Helong. lean, hope, rely. [irr. from PRM: *i $>$ e] [Form: Jonker (1908:270) gives Helong naslae.]

hakrai East Tetun. support, prop, or lean. (Mo:173)

*sarakaen PRM. sand. Pattern: k-irr. [irr. from PRM: *a $>o$ in most nRote; $* \mathrm{k}>\varnothing$ in Lole, Tii and Meto (expect *k $=k)]$ [Form: The irregular loss of * $\mathrm{k}$ in Meto and (optionally) in Lole and Tii is probably due to antepenultimate vowel reduction to intermediate **sarkae with subsequent deletion medial $* \mathrm{k}$ to resolve the consonant cluster.] solokae-k Termanu. sand. (J:561) solokae-? Korbafo.

solokae-k Bokai.

solokae-? Bilbaa.

soro?ae-? Landu. sand. (own field notes)

soro?ae-? Rikou.

soro?ae-? Oepao. sand. (own field notes)

solokae-k $B a^{\prime} a$.

salakae-k, salae-k Lole. [Note: salakae-k is the only form given by Jonker (1908:759). Zacharias et al. (2014) gives only and salae-k.]

sarakae-k, sarae-k Tii. [Note: sarakae-k is the only form given by Jonker (1908:561). Grimes et al. (2014a) gives both sarakae-k and sarae-k.]

sala?ae-? Dengka.

saraPae Oenale.

snaen Ro'is Amarasi. sand.

snaen Kotos Amarasi. sand.

snaan Kusa-Manea. sand. [Form: regular $*_{\mathrm{e}}>a / \mathrm{V} \#$ in Upper Manulea.]

Out-comparisons:

${ }^{\circ}$ slaen Funai Helong. sand. Borrowed from: PMeto *slaen (shown by irr. ${ }^{*} \mathrm{k}=\varnothing$ correspondence).

ohlaen Semau Helong. sand. sidayik Idate. sand. (Klamer 2002)

sraek Lakalei. sand. [Note: language of east Timor ISO 639-3 [lka].] (Klamer 2002)

salae Dhao. beach, shore, sand, coast.

lahalae Hawu. sand.

sarae Bima. sand. (Jonker 1893:94)

sirain Kamang. sand. [Note: nonAustronesian language of Alor ISO 639-3 [woi].] (Schapper 2010)

*sare Morph: *na-sare. PnRote. facing somewhere.

na-sale Termanu. turn oneself to face somewhere, be located opposite something. (J:517)

na-sale Korbafo.

na-sale Bokai.

na-sale Bilbaa.

na-sare Rikou.

na-sale $B a^{\prime} a$.

na-sare Tii.

Out-comparisons:

salo Semau Helong. face, across from, front on. [irr. from PRM: $* \mathrm{e}=o$ correspondence]

*sasi PRM. overflow.

sasi Termanu. overflow, spill over. (J:524)

sasi Korbafo.

sasi Bokai.

sasi Bilbaa.

sasi Rikou.

sasi $B a^{\prime} a$.

sasi Tii.

sasi Dengka.

sasi Oenale.

sasi Meto. (J:524)

Out-comparisons:

sasi Helong. (J:524)

*sau1 $n R M$. bite.

sau Termanu. bite into something, bite something off. (J:524f)

sau Korbafo.

sau Bokai. 
sau $B a^{\prime} a$.

sau Tii.

n-sau Ro'is Amarasi. bite.

n-sau Kotos Amarasi. bite.

n-sau Molo. bites. (M:478)

Out-comparisons:

sau Semau Helong. bite.

*sau2 PRM. pick. [Sporadic: *VV-? > VPV in Meto (perceptual metathesis).] sau Termanu. pick. (J:524)

sau Korbafo.

sau Bokai.

sau $B a^{\prime} a$.

sau-? Dengka.

n-sa?u Molo. strips leaves off a plant or branch. (M:477)

*saus Rote. comb. Etym: *sau. [irr. from $P M P: * a u=* a u($ expect *oo)]

sau-k (2) sau Termanu. 1) comb.

2) combing. (J:524)

sau-? Korbafo.

sau-k Bokai.

sau-? Bilbaa.

sau-? Rikou.

sau-k $B a^{\prime} a$.

sau-k Tii.

sau-t Dengka.

sau-t Oenale.

*seda Rote. kind of red coral used as bead.

(henu) seda_sada, (henu) seda ndao (2) seda Termanu. 1) kinds of coral called muti-salah in Kupang. [Note: Jonker states that this term only occurs in certain phrases: seda_sada (sada has no independent meaning), seda Ndao $(\mathbf{N d a o}=$ name of an island $)$, seda Ndao dae huu-k all of which can also be preceded by henu 'bead'. The equivalents of these phrases in other varieties of Rote are not given.] (J:527) 2) classifier for muti-salah (henu), hence: a muti-salah bead. The muti-salah are reddish (glass) beads of great value. (Fox 2016b:49) seda Korbafo.

seda Bokai.

seda Bilbaa.

seda $B a^{\prime} a$.

seda Dengka.

Out-comparisons:

(wona) hida Hawu. (J:527)

<hàda> Kambera. bead. [Note: also in Mangili, Lewa, and Kodi.] (On:38)

<sàda> Anakalang.

*sedo PRM. mix.

se $\sim$ sedo-k, sedo-k Termanu.

1) mixing of dry things. (J:527f)

se $\sim$ sedo-? Korbafo.

se $\sim$ sedo-k Bokai.

se $\sim$ sedo-? Bilbaa.

se $\sim$ sedo-? Rikou.

se $\sim$ sedo-k $B a^{\prime} a$.

se $\sim$ sedo-k Tii.

se $\sim$ sedo-? Dengka.

se $\sim$ sedo-? Oenale.

n-sero? Ro'is Amarasi. mix.

n-sero? Kotos Amarasi. mix.

<n-selo> Molo. mixes. (M:483)

*see1 PwRM. address.

me?u_see Dengka. address. (J:733)

na-see Kotos Amarasi. take leave, excuse one's self, warn.

*see2 PRM. who? Etym: *sai.

see Termanu. who? (J:525)

see Korbafo.

see Bokai.

see $B a^{\prime} a$.

see Tii.

see Dengka.

see Oenale.

seka Dela. who.

se/kau Ro'is Amarasi. who.

se/kau Kotos Amarasi. who.

se/kau Molo. who. (M:483)

see Kusa-Manea. who.

Out-comparisons:

asii, sii Semau Helong. who. [irr. from PMP: *ai > ii]

see East Tetun. who. (Mo:169) 
*sefe Rote. paddle. Etym: *bəRsay 'canoe paddle; paddle a canoe'. [Sporadic: consonant metathesis $* \mathrm{fVs}>*_{\mathrm{sVf}}$.] sefe Termanu. paddle ( $\mathrm{v}$. and n.). (J:529)

sefe Korbafo.

sefe Bokai.

sefe Bilbaa.

sefe Rikou.

sefe $B a^{\prime} a$.

sefe Tii.

sefe Dengka.

sefe Oenale.

*sefi PRM. set loose, untie. [irr. from PRM: *f $>\left(?^{*} \mathrm{~h}\right)>?$ in Termanu, Korbafo and Bokai]

se?i Termanu. loosen that which is tied. (J:529)

se?i Korbafo.

se?i Bokai.

sefi Bilbaa.

sefi Rikou.

sefi $B a^{\prime} a$.

sefi $T i i$.

sefi Dengka.

sefi Oenale.

n-sefi Kotos Amarasi. untie.

n-sefi Molo. sets free (a horse). (M:480)

*seke PRM. force, stuck. Pattern: k-10.

na-ka-se?e, se?e (2) se?e se?e

Termanu. 1) squeezed/stuck into something. 2) completely trapped in something; squeezed, very cramped. (J:528)

na-ka-se?e Korbafo.

na-ka-se?e Bokai.

na-ka-seke, seke rseke Bilbaa.

na-seke Rikou.

na-ka-se?e $B a^{\prime} a$.

na-ka-se?e $T i i$

na-?a-se?e Dengka.

na-Pa-se?e Oenale.

na-?|seke|? Kotos Amarasi. force, put pressure on, press to do.

$<$ na-seke $>,<$ n-seke $>$ Molo. forced.

(M:481)

\section{Out-comparisons:}

naseke Helong. forces. ( $\mathrm{J}: 528)$

*seko $n R M$. fish (v.). Pattern: k-5/6'

$\left({ }^{*} \mathrm{k}>\varnothing\right.$ in Rikou; expect $?$ or $\left.k\right)$.

seko Termanu. fish in the sea with a scoop net. (J:530)

seko Korbafo.

seko Bokai.

seko Bilbaa.

seo (se?o ?) Rikou.

seko $B a^{\prime} a$.

seko Tii.

n-seko Kotos Amarasi. hunt.

n-seko Timaus. hunt.

Out-comparisons:

seko Semau Helong. traditional fish trap.

heko Hawu. small kind of net. (J:530)

*se?i PRM. roast

se?i Termanu. dry roast. (J:530)

se?i Bokai.

sei Bilbaa.

se?i Rikou.

se?i $B a^{\prime} a$.

se?i Tii.

se?i Dengka.

se?i Oenale.

n-se?i Kotos Amarasi. smokes, roasts.

n-se?i Molo. smokes (meat). (M:481)

Out-comparisons:

həワi Hawu. bake. (J:530)

*sela Morph: *sela-k. Rote. big, gross, coarse. Etym: *solaR. [Sporadic: *a > e/\# in wRote.]

sela-k Termanu. coarse, large. (J:531) sela-? Korbafo.

sela-k Bokai.

sela-? Bilbaa.

sela-? Rikou.

sela-k $B a^{\prime} a$.

sela-k Tii.

sele-? Dengka.

sele-? Oenale.

\section{Out-comparisons:}

bseda? Funai Helong. rough. 
sela? Semau Helong. sore, strained, rough. [Note: Jonker (1908:531) gives Helong $<$ bsela $>$.]

*selə PRM. plant (v.).

sele Termanu. stick in the ground, plant. (J:581)

sele Korbafo.

sele Bokai.

sele Bilbaa.

sele Rikou.

sele $B a^{\prime} a$.

sele Tii.

sela Dengka.

sela Oenale.

n-sena Kotos Amarasi. plant.

n-seen Molo. plant. [Form: metathesised form of n-sena.] (M:483)

n-sena Kusa-Manea. plant.

Out-comparisons:

həle Hawu. plant.

*selut PRM. replace, exchange. [History:

Blust and Trussel (ongoing) reconstruct Proto-Philippine * sulit, which could be connected via vowel metathesis.]

selu-k (2) na-seluk Termanu.

1) exchange. 2) swap with someone. (J:535)

selu-? Korbafo.

selu-k Bokai.

selu-? Bilbaa.

selu-? Rikou.

selu-k $B a^{\prime} a$.

selu-k Tii.

selut Dengka.

selut Oenale.

n-senu|? Kotos Amarasi. replace.

<n-senu> Molo. reciprocates. ina

n-seun banin kau he gives me a gift in return. (M:485)

\section{Out-comparisons:}

silu Semau Helong. change clothes.

haselu East Tetun. repay; to take revenge; to swap or exchange. (Mo:80)

selu Ili'uun. exchange. (dJ:136) ntfelu (2) selu Bima. 1) exchange.

2) something that replaces something else, substitute. Usage: Kolo dialect. (Jonker 1893:121)

$<$ hilu> Kambera. replace, reimburse. (On:91)

sulih, silih, sulur Javanese. [Note: language of Java ISO 639-3 [jav].] (J:535)

ma-silur Balinese. exchange, exchanged. [Note: language of Bali ISO 639-3 [ban].] (Kersten 1984:538)

sulle Bugis. replace. (Masse 2013:360)

*sembo PRM. barter, swap, exchange.

sepo Termanu. go and sell or swap consumables outside the capital for salt, tobacco, etc., peddle consumables outside the capital. (J:539)

sepo Korbafo.

sepo Bokai.

sepo Bilbaa.

sepo Rikou.

sempo $B a^{\prime} a$.

sembo Tii.

sembo Dengka.

sembo Oenale.

na-sepo Meto. (J:539)

*seya PRM. fry. Etym: *sayəlaR 'stirfry, cook in a frying pan without oil'. [irr. from PMP: *laR $>\varnothing]$ [minority from PMP: $*_{\mathrm{y}}=*_{\mathrm{y}}$ ] [Sporadic: antepenultimate *a $>*_{\text {e.] }}^{\text {[Form: }}$ Medial $*_{y}$ is required to account for Meto $k$. Mills (2010:285) also draws attention to Leti sekra with irregular medial $* y>k$ rather than expected $n$.] se $\sim$ sena Termanu. fry or bake meat. (J:537)

se $\sim$ sena Korbafo.

se $\sim$ sena Bokai.

se $\sim$ sena Bilbaa.

se sena Rikou.

se $\sim$ sena $B a^{\prime} a$. 
se $\sim$ sena Tii.

se $\sim$ sena Dengka.

se sena Oenale.

na-seka Timaus. fry.

ta-seek Kusa-Manea. fry. [Form: metathesised form of (currently unattested) *ta-seke.]

\section{Out-comparisons:}

neyan Semau Helong. fry. [irr. from PMP: * $\mathrm{s}>n$ ]

sona East Tetun. roast, fry. (Mo:173)

*seygi PRM. snap off, harvest.

seni Termanu. break (Latin frangi), as a stick, etc.; break (Latin frangere). (J:537)

seji Korbafo.

seni Bokai.

seji Bilbaa.

seki Rikou.

sengi $B a^{\prime} a$.

semgi Tii.

seygi Dengka.

sengi Oenale.

n-seki Kotos Amarasi. harvest corn.

n-seki (2) na-t|seki Molo. 1) harvests (corn). 2) snapped off, of a branch. (M:482)

ta-seik Kusa-Manea. harvest corn. [Form: Phonetically this is a single mid-high vowel [ta'se:k] in my notes.]

Out-comparisons:

sinin Semau Helong. pick corn.

*seo $n R M$. whisper.

na-se $\sim$ seo Termanu. murmur, whisper. (J:538)

na-se $\sim$ seo Korbafo.

na-se $\sim$ seo Bokai.

na-se $\sim$ seo Bilbaa.

na-se $\sim$ seo Rikou.

na-se $\sim$ seo $B a^{\prime} a$.

na-se $\sim$ seo $T i i$.

na-?se?o Kotos Amarasi. whisper. <na-seo> Molo. whispers. (M:485)

*seru PRM. weaving sword (part of the loom). Etym: **sodu(t). selu Termanu. the sabre shaped beam of the loom. (J:535)

selu Korbafo.

selu Bokai.

selu Bilbaa.

seru Rikou.

selu $B a^{\prime} a$.

seru Tii.

se $\sim$ seru Oenale.

senu Ro'is Amarasi. the long beam in the loom that the weaver pulls towards themself by holding on each end, it doesn't move smoothly but lurches as it is pulled.

senu Kotos Amarasi. the long beam in the loom that the weaver pulls towards themself by holding on each end, it doesn't move smoothly but lurches as it is pulled.

\section{Out-comparisons:}

silu Helong. (J:535)

souru, soru East Tetun. weave (cloth). (Mo:174)

seru Waima'a. weave.

pehədu Hawu. (J:535)

surit Central Lembata. weaving sword. (Hanna Fricke pers. comm.)

*sesə PRM. stuff, cram in; be crowded.

Etym: *səksək. [Form: The Meto unmetathesised form has not yet been attested. It could be *na-?sesa or *na-?sese.]

sese Termanu. tamp, cram, impress.

sese Korbafo.

sese Bokai.

sese Bilbaa.

sese Rikou.

sese $B a^{\prime} a$.

sese Tii.

sesa Dengka.

sesa Oenale.

na-Psees Kotos Amarasi. crowded, tight.

Out-comparisons:

həhi, kehəhi Hawu. narrow. (J:539) 
*seti PRM. tight, tightly packed.

na-ka-seti Termanu. close together, tightly packed together, as e.g. planks. (J:541)

na-ka-seti Korbafo.

na-ka-seti Bokai.

na-ka-seti Bilbaa.

na-seti Rikou.

na-ka-seti $B a^{\prime} a$.

na-ka-seti $T i i$.

na-Pa-seti Dengka.

na-Pa-seti Oenale.

na-?|seti|? (2) n-seit=oo-n Kotos Amarasi. 1) force one's way (e.g. out, through). 2) involve oneself in (e.g. other people's business).

\section{Out-comparisons:}

siti Semau Helong. push through.

seti East Tetun. put a wedge or chock under. (Mo:171)

*seu $P R M$. pick fruit.

seu Termanu. pick, of fruit. (J:541)

seu Korbafo.

seu Bokai.

seu Bilbaa.

seu Rikou.

seu $B a^{\prime} a$.

seu Tii.

seu Dengka.

seu Oenale.

n-seu Kotos Amarasi. pick (of fruit, etc.).

n-sio Amanuban/Amanatun. picks (fruit).

n-seu (2) na-t|seu Molo. 1) picks (fruit). 2) fallen off, of fruit. (M:488)

seu Kusa-Manea. pick fruit (like coconuts, mangoes).

Out-comparisons:

siku? Semau Helong. pick, strip off.

*səru PRM. meet, greet. Etym: **səru (pre-RM). [irr. from PRM: *ə $>o$ in Rote]

na-so $\sim$ solu Termanu. meet. (J:561)

na-so solu Korbafo. na-so solu Bokai.

na-so $\sim$ solu Bilbaa.

na-so $\sim$ soru Rikou.

na-so $\sim$ solu $B a^{\prime} a$.

na-so $\sim$ soru $T i i$.

na-so $\sim$ solu Dengka.

na-so $\sim$ soru Oenale.

n-senu Kotos Amarasi. greet, meet.

n-senu Molo. go to meet. too anpoi

neem he nsenu usif the people

come from outside to meet the king

(M:485)

Out-comparisons:

hasoru Fehan Tetun. meet, e.g. person in house greets a visitor, meet on the street, visitor greets a person in the house; can be meeting on the street without talking to one another.

hasouru East Tetun. be opposed to, go against; encounter, meet (two people meeting face to face when going in opposite directions); prep. against. (Mo:80)

səli Central Lembata. meet. [irr. from PRM: * $\mathrm{u}=i$ correspondence] (Hanna Fricke pers. comm.)

*səu PRM. kind of tree. Alstonia villosa. [irr. from PRM: *o $>o$ in nRote] [Semantics: 'Alstonia trees are used in traditional medicine. The bark of the Alstonia constricta and the Alstonia scholaris is a source of a remedy against malaria, toothache, rheumatism and snake bites. The latex is used in treating coughs, throat sores and fever.' ('Alstonia', Wikipedia. en.wikipedia.org/wiki/Alstonia. Accessed 17 September 2020).]

sou Termanu. kind of tree the bark of that is used as a healing agent. (J:565)

sou Korbafo.

sou Bokai.

sou Bilbaa. 
sou Rikou.

sou $B a^{\prime} a$.

(sou ?) Tii.

seu-? Dengka. (J:762)

seu-? Oenale. (J:762)

hau seu Molo. kind of tree. Alstonia villosa. (M:488)

*sida PRM. rip.

sida Termanu. rip. (J:543)

sida Korbafo.

sida Bokai.

sida Bilbaa.

sida Rikou.

sida $B a^{\prime} a$.

sida Tii.

sida, side Dengka.

sida, side Oenale.

$<$ n-sila $>$ na-klati? (2) < aksilaksila $>$ Molo. 1) rips into small pieces. [Form: na-klati? = 'destroy'.] 2) completely torn. (M:495)

\section{Out-comparisons:}

$<$ hira> Kambera. rip. [Note: also in Mangili and Lewa.] (On:94)

$<$ sira $>$ Anakalang. [Note: also in Mamboru.]

$<$ ira $>$ Weyewa.

$<$ hirya $>$ Kodi.

*sifi PRM. braid, plait.

sifi Termanu. the name of a particular kind of braiding. (J:544)

sifi Korbafo.

sifi Bokai.

sifi Bilbaa.

sifi Rikou.

sifi $B a^{\prime} a$.

sifi Tii.

sifi Dengka.

sifi Oenale.

n-sifi Kotos Amarasi. braiding work.

n-sifi Molo. inserts, braids. bifee an-sifi <poni> the woman repairs a basket by inserting new strips of lontar leaves (M:490)

\section{Out-comparisons:}

həbi Hawu. braid (a chair). (J:544)
* sii1 CER tie up, snare. [History: Blust and Trussel (ongoing) reconstruct ProtoPhilippine *siluq 'noose, snare; net'.]

sii Termanu. tie up a chick (or a pig) with a certain kind of noose so that its foot is not wounded. ( $\mathrm{J}: 542)$

\section{Out-comparisons:}

hii Hawu. snare. (J:542)

*sii2 Morph: *sii-k. Rote. shellfish. [History: Blust and Trussel (ongoing) reconstruct *sisi and *sisuq (with doublet *sisiq) 'small snail or periwinkle'.]

si $\sim$ sii-k Termanu. normally in: sii bi?i-k, sii hedu, sii mina, kinds of shellfish, the general name of which is si $\sim$ sii-k. (J:542)

si $\sim$ sii-? Korbafo.

si $\sim$ sii-k Bokai.

si sii-? Bilbaa.

si sii-? Rikou.

si $\sim$ sii-k $B a^{\prime} a$.

si $\sim$ sii-k Tii.

la?i_sii-? Dengka.

lai_sii-? Oenale.

Out-comparisons:

sii Ili'uun. shell-fish. [Note: sii_suhun = 'shell', sii_ogo $=$ 'signalling-shell (which is blown)'.] (dJ:137)

*sika Rote. open, uncover. Doublet:

*singa. Etym: *sinkab. Pattern: $\mathrm{k}-5$. [minority from PMP: $* \mathrm{nk}>* \mathrm{k}$ ] [Sporadic: *a>el_\# in wRote.]

sika Termanu. open something wide, hold open. (J:544)

sika Korbafo.

sika Bokai.

sika $B a^{\prime} a$.

sike Dengka.

sike Oenale.

*sikəh PRM. lath, rod (part of the loom). Pattern: k-irr. [irr. from PRM: *o > a in Termanu and Ba'a; ${ }^{*} \mathrm{k}>\varnothing$ in Oenale and Meto (given other Rote $k$ we expect $* \mathrm{k}>k$ or ? in both)] [Sporadic: $* ə>e / \sigma_{-} \#$ in wRote (perhaps *ə $>* \mathrm{a}$ $>e / \#)$. 
sika-k Termanu. name of part of the underside of the loom, a piece of bamboo used to make the pieces of fabric taut and to keep them apart from each other. (J:544)

si sike-? Korbafo.

sike-k Bokai.

sike-? Bilbaa.

si?e-? Rikou.

sika-k $B a^{\prime} a$.

si?e-k Tii.

sike-t Dengka.

sie-t Oenale.

siah Kotos Amarasi. name of a thin split bamboo slat used in weaving.

sia-l Molo. name of a thin split bamboo slat used in weaving. 'Two vertically placed laths (sial) behind these serve to raise the even and the odd threads in turn.' (Schulte Nordholt 1971:42). [Note: Jonker (1908:544) gives siel.] (M:489)

\section{Out-comparisons:} sia Helong. (J:544)

*siku Morph: *siku-k. PRM. elbow. Etym: *siku. Pattern: k-7. [Sporadic: antepenultimate vowel assimilation in wRote.]

si?u-k Termanu. elbow. Usage: usually compounded with lima 'arm'. (J:549)

si?u-? Korbafo.

si?u-k Bokai.

siku-? Bilbaa.

lima si?u-? Landu. elbow. (own field notes)

siku-? Rikou.

si?u-k $B a^{\prime} a$.

si?u-k Tii.

siki_buku-? Dengka. elbow. (J:763)

siki_buku-? Oenale. elbow. (J:763)

siu?u-f Ro'is Amarasi. elbow.

si?u-f Kotos Amarasi. elbow.

siku-n Kopas. elbow. Usage: Tunfe'u hamlet, other varieties of Kopas have si?u-n.

siPu-f Molo. elbow. (M:500) si?u-f Kusa-Manea. elbow.

\section{Out-comparisons:}

sikun Semau Helong. elbow.

siku-n East Tetun. elbow, corner, angle. (Mo:171)

*simo PRM. receive. [irr. from PRM:

*m $>*$ mb in Rote]

sipo Termanu. receive, accept. (J:547)

sipo Korbafo.

sipo Bokai.

sipo Bilbaa.

sipo Rikou.

simpo $B a^{\prime} a$.

simbo Tii.

simbo Dengka.

simbo Oenale.

n-simo, n-simu Kotos Amarasi. receive. Usage: poetic, only in the parallel pair $\mathbf{n}$-sium =ma n-toup.

n-simo Molo. receives. (M:496)

siam Kusa-Manea. receive. [Form: metathesised form of (currently unattested) *sima. It is possible that the unmetathesised form is *simo and that $o$ dissimilates to $a$ after high vowels in Kusa-Manea, but this would go against the attested raising of final mid vowels in words such as uim $\sim$ umi 'house' $<*$ ume $<*$ uma.]

\section{Out-comparisons:}

simu Semau Helong. receive, greet arrival.

simu East Tetun. receive, take, accept; to welcome; to respond. (Mo:172)

simu Waima'a. receive, accept, welcome.

sium Mambae, South. receive, accept, agree. (Grimes et al. 2014b:41)

həmi, həme Hawu. receive (sg.), receive (pl.).

səmi, həme Dhao. receive.

simo Ende. receive.

himo Sika. receive. (Pareira and Lewis 1998:76) 
*sina Morph: *sina-k. PRM. light (n.). Etym: *sinaR 'ray of light; to shine'. [Sporadic: *a $>e$ /_\# in wRM.]

bula sina-k Termanu. moonlight. (J:546)

bula_sina-k Keka. in bright moonlight. [Note: Given with a note that this form is found in Keka and others'.] (J:763)

fula sine-? Dela. moonlight.

meu?_sina|? Ro'is Amarasi. light, brightness.

meu?_sine|? (2) sine|?, sina|? Kotos Amarasi. 1) light (n.). 2) four days from now. [Note: Also occurs in the parallel pair na-toon $=$ ma nasine? 'tell, make known'.]

n-sine|k (2) huma-n an-sine $\mid k$ Molo. 1) light, not cloudy, at dawn or moonrise. 2) one looks fresh (after bathing or recovery from illness). [Note: huma-n = 'face, type'.] (M:497)

ma?sian Kusa-Manea. brightness, light.

\section{Out-comparisons:}

rai (nak)sinak East Tetun. return to good weather with sun shining. (Mo:172)

*sinara?e PRM. kingfisher with white underbelly and blue back and head. There are two such species in Timor; the Sacred Kingfisher and the Collared Kingfisher. Todiramphus chloris, Todiramphus sanctus. [irr. from PRM: $*_{\mathrm{i}}>\mathrm{u}$ in Bilbaa; ${ }^{*} \mathrm{r}>l$ in Dela; $* \mathrm{e}>\varnothing$ in Dela (if the putative reflex is cognate)] [Sporadic: glottal stop insertion in Kotos Amarasi (first syllable); antepenultimate vowel reduction in all reflexes.] [Form: I have reconstructed initial $*_{i}$ on the basis of Rikou and Lole with $i$, as well as Amfo'an, which attests a front vowel $e$. Under this hypothesis Bilbaa has undergone irr. $*_{i}>u$ while Dela, Ro'is Amarasi, and Kotos Amarasi have reduced this vowel to the default unstressed vowel $a$. This form also has ante-penultimate vowel reduction in most lects with subsequent coalescence of the resulting consonant cluster in all lects apart from Lole.]

sulae Bilbaa. Collared Kingfisher. Todiramphus chloris. (own field notes)

sirae Rikou. Collared Kingfisher. Todiramphus chloris. (own field notes)

sinla?e Lole. kingfisher. (own field notes)

sanala-? Dela. kind of small bird with blue belly and yellow neck (but not the Collared Kingfisher). [Note: This Dela form may not be cognate but is included here because of the phonetic and semantic similarity.] (Thersia Tamelan pers. comm. December 2017)

sanae Ro'is Amarasi. Sacred Kingfisher, Collared Kingfisher. Todiramphus chloris; Todiramphus sanctus.

sa?na?e|k Kotos Amarasi. Sacred Kingfisher, Collared Kingfisher. Todiramphus chloris; Todiramphus sanctus.

senae-l Amfo'an. Sacred Kingfisher, Collared Kingfisher. Todiramphus chloris; Todiramphus sanctus.

*sinor Rote. maggots.

sino-k Termanu. maggots from fly eggs in a wound. (J:547)

sino-? Korbafo.

sino-k Bokai.

sino-? Bilbaa.

sino-? Rikou.

sino-k $B a^{\prime} a$.

sino Tii.

sino-? Dengka.

sinor Oenale.

Out-comparisons: sinun Helong. (J:547) 
*singa CER. open, uncover. Doublet: *sika. Etym: *sinkab.

sina Bilbaa. open wide. (J:763)

*singada? PwRM. scold. Pattern: d-2. [Form: Lack of known reflexes in nRote means the medial consonant is ambiguous between $* \mathrm{~d}$ s and $* \mathrm{~d}-2$. nRote $l / r$ would support $* \mathrm{~d}-2$, while $d / d$ would support *ds.]

asinga ngara? Dela. scold severely. na-skara? Kotos Amarasi. snarl, rebuke, mad, angry, shouting mad.

*sio PRM. nine. Etym: *siwa. [Sporadic: vowel height harmony $*_{\mathrm{i}}>e$ /_o in some Meto.]

sio Termanu. nine. (J:547)

sio Korbafo.

sio Bokai.

sio Bilbaa.

sio Rikou.

sio $B a^{\prime} a$.

sio Tii.

sio Dengka.

sio Oenale.

seo Ro'is Amarasi. nine.

seo Kotos Amarasi. nine.

sio? Amanuban/Amanatun. nine.

seo? Amfo'an. nine.

seo, sio Molo. nine. (M:485)

seo Timaus. nine.

sio Kusa-Manea. nine.

Out-comparisons:

sipa Funai Helong. nine. [irr. from $\left.P M P:{ }^{*} \mathrm{w}>p(\operatorname{expect} f)\right]$

sipa Semau Helong. nine.

siwi Fehan Tetun. nine.

sia East Tetun. nine. (Mo:171)

sibe Kemak. nine.

siwi Welaun. nine.

heo Hawu. nine.

*sira PRM. they. Doublet: *ra. Etym:

*si-ida.

sila Termanu. plural form of ndia [OE $=3 \mathrm{SG}] .(\mathrm{J}: 545)$

sila Korbafo.

sila Bokai. sila Bilbaa.

sira Landu. 3PL, they. (own field notes)

sira Rikou.

sila $B a^{\prime} a$.

sira Tii.

sila Dengka.

sira Oenale.

siin, sini, sina Ro'is Amarasi. they.

siin, sini, sina Kotos Amarasi. 3PL.NOM, 3PL.ACC. [Form:

Phonetically the metathesised form siin almost always has a single short vowel [sin]. The unmetathesised form sina is only used before consonant clusters.]

siin Molo. 3PL. (M:496)

sian Kusa-Manea. 3PL.

Out-comparisons:

sira East Tetun. they; when placed after a noun indicates plural. (Mo:172)

hira Ili'uun. they, their, them. (dJ:118)

*sisi PwRM. flesh, meat. Etym: *həsi. [irr. from PMP: $\varnothing>*_{\mathrm{s}} ; *_{\partial}>*_{\mathrm{i}}$ (sporadic assimilation)]

sisi Dengka. meat, flesh. (J:763)

sisi Oenale. meat, flesh. (J:763)

sisi-n Ro'is Amarasi. meat, flesh.

sisi-f Kotos Amarasi. meat, flesh.

sisi Molo. meat, flesh. (M:499)

Out-comparisons:

sisi Semau Helong. meat.

sisi Idate. meat. (Klamer 2002)

sisi Midiki. meat. (Dawson 2014)

${ }^{\circ}$ sisi Dhao. meat. Borrowed from: probably wRote sisi (shown by irr. ${ }^{*} \varnothing=s$ and irr. ${ }^{*} \partial=i$ correspondences).

sisi Tokodede. meat. (Klamer 2002)

siis Mambae, South. meat. (Grimes et al. 2014b:41) 
*soda Morph: *soda-k. PRM. space. Pattern: d-irr. [irr. from PRM: *d > $d$ in wRote (expect $* \mathrm{~d}>r / l)$ ] [Form: Alternately, we could reconstruct *soda and posit irr. ${ }^{*} d>n$ in Meto.] soda-k (2) uma=a $\operatorname{soda}=$ na, uma soda-k (3) dae soda-k (4) soda $\sim$ soda Termanu. 1) space in time. 2) open space or piece of land. 3 ) open space of ground free from weeds; but also free unmanaged land that can be built on. (J:553f) 4) have an opportunity, have time. (J:552f)

soda-? (2) soda-? (4) soda $\sim \operatorname{soda}$ Korbafo.

soda-k (2) soda-k (4) soda $\sim$ soda Bokai.

soda-? (2) soda-? (4) soda $\sim$ soda Bilbaa.

soda-? (2) soda-? (4) soda $\sim$ soda Rikou.

soda-k (2) soda-k (4) soda $\sim$ soda $B a^{\prime} a$.

soda-k (2) soda-k (4) soda $\sim \operatorname{soda}$ Tii.

soda-? (2) ume soda-?, ume $=$ a so $\sim$ soda $=$ na (4) $\quad$ soda $\sim$ soda Dengka.

soda-? (2) soda-? (4) soda $\sim \operatorname{soda}$ Oenale.

soda Dela. yard.

sona-f (2) na-?sona? Kotos Amarasi.

1) space that belongs to someone; midst, realm, by extension; kingdom, country, palace. 2) widen. sona? (2) na-?sona? (3) sona-f Molo. 1) space. 2) clear (the way). 3) palace. (M:513)

*soda Rote. sing.

soda Termanu. sing. (J:552)

soda Korbafo.

soda Bokai.

soda Bilbaa.

soda Rikou.

soda $B a^{\prime} a$.

soda Tii. soda Dengka.

soda Oenale.

Out-comparisons:

hoda-kefeka Hawu. shout praise. [Note: Jonker (1908:552) gives

Hawu hoda 'singing on a ship'.] soda Ende. singing on a vessel. (J:552)

*soe PRM. disaster.

soe Termanu. disaster, impending disaster, compare silaka with which this word is often compounded: soe silaka. (J:554)

soe Korbafo.

soe Bokai.

soe Bilbaa.

soe Rikou.

soe $B a^{\prime} a$.

soe Tii.

soe Dengka.

soe Oenale.

n-soe Kotos Amarasi. suffer loss.

soe-l Meto. (J:554)

Out-comparisons: soe Helong. (J:554)

*soeneru PRM. leaf umbrella. [irr. from $P R M$ : vowel metathesis in Meto $* \mathrm{eCu}$ $>u C e]$

suneru Tii. kind of umbrella made from a big sewn gebang-palm leaf. (J:541)

soenelu-? Dengka.

soeneru-? Oenale.

soeneru-? Dela. rain cape from gebang leaves. Usage: archaic.

snuna? Fatule'u. umbrella made from a leaf.

snuna?, snune? Molo. umbrella made from gebang palm leaves. (M:508)

Out-comparisons:

salurin Fehan Tetun. old-style umbrella, made of akar [sago palm] leaf, and held over the head.

salurik East Tetun. palm leaf used as an umbrella. (Mo:165) 
*soi PRM. open, ransom, pay off.

soi Termanu. 1) open (v.). 2) pay a debt, ransom. (J:556)

soi Korbafo.

soi Bokai.

soi Bilbaa.

soi Rikou.

soi $B a^{\prime} a$.

soi $T i i$.

soi Dengka.

soi Oenale.

n-soi (2) na-soitan (3) na-tsoi

(4) na-soin Kotos Amarasi.

1) ransom, pay debt. 2) open something. 3) opened. 4) opened.

n-soi (2) na-soin (3) na-soitan Molo. 1) ransoms. 2) open. 3) open (the door). (M:510)

\section{Out-comparisons:}

sui Semau Helong. ransom, redeem, pay off.

soi East Tetun. redeem, pay off; to acquire, win, to possess; rich, well-to-do. (Mo:173)

soi Waima'a. buy.

ho?i Sika. redeem. (Pareira and Lewis 1998:77)

so?i Ende. buy. (J:556)

*soka Rote. sack made from palm leaves.

Pattern: k-5. [History: possibly connected with Dutch zak.]

soka Termanu. sack of gebang or lontar palm leaves. (J:557)

soka Korbafo.

soka Bokai.

soka Bilbaa.

so?a Rikou.

soka $B a^{\prime} a$.

soka? Dengka.

soka? Oenale.

Out-comparisons:

hoka Hawu. (J:557)

sokal Kupang Malay. (J:557)

sokatol Saparua. kind of box

(Malay tatumbu). [Note: language of Lease Islands ISO 639-3 [spr].] (van Hoëvell 1877:55) *soke PRM. scoop up, pick out. Pattern: k-8/9' (Kotos Amarasi Ø Molo ? correspondence; expect either $\varnothing$ in both for pattern 8 or ? in both for pattern 9).

so?e Termanu. 1) use a coconut shell or lalik (kind of small basket) to scoop solids such as rice, salt, etc. 2) coconut spoon. (J:555)

so?e Korbafo.

so?e Bokai.

soke Bilbaa.

so?e Rikou.

so?e $B a^{\prime} a$.

so?e Tii.

so?e Dengka.

so?e Oenale.

n-soe Kotos Amarasi. pick out, raise something up in a container.

<amso'e main le'ot> Molo. using the hand, scoop the finely pounded rice out of the hole in the rice pounder. (M:509)

\section{Out-comparisons:}

soke (2) soet Semau Helong. 1) scoop. 2) pick out.

sukit (2) sui East Tetun.

1) remove, extract (with any tool or implement). 2) remove, extract, withdraw. (Mo:175)

sukke Bugis. pry out. (Masse 2013:358)

passukki? (2) anndzukki?

Makassar. 1) bamboo stick with a hook used to remove fruit from a tree or a bucket from the well. 2) remove fruit from a tree with a stick of bamboo with a hook, use a hook to remove a bucket from a well. (Cense 1979:726)

*sokum PRM. galangal. Kaempferia Galanga. Pattern: k-6. [irr. from PRM: $*_{\mathrm{o}}>i$ in Meto] [History: Jonker (1908) suggests this is from Kupang Malay koncur (compare Malay kencur) with consonant metathesis, but this doesn't explain the final $m$ in Meto.] 
sokus Termanu. kind of medicinal root, called kencur in Javanese, it is offered for sale on Rote by foreigners. ( $\mathrm{J}: 558)$

soku-? Korbafo.

soku Bokai.

soku Bilbaa.

so?us Rikou.

sokus $B a^{\prime} a$.

soku Tii.

so?u Dengka.

so?u Oenale.

sikum Meto. galangal. Kaempferia Galanga. (Heyne 1950:494, ccviii)

Out-comparisons:

sukuy Helong. galangal. Kaempferia Galanga. (Heyne 1950:494, ccxi)

*so?i Morph: *so?i-t. PnMeto. comb.

so?i-t Kotos Amarasi. comb (n.).

Out-comparisons:

sui (2) sa $\sim$ suit East Tetun. 1) comb, smooth with a comb or any similar action (Mo:174). 2) comb, a hair comb for retaining the hair in place. [Sporadic: vowel height harmony $*_{0}>u /$ i.j (Mo:168)

sui Waima'a. comb.

*soo PRM. sew. Etym: **sauR (pre-RM). [History: Blust and Trussel (ongoing) reconstruct PCMP *sora, including Meto as one of their attestations. The cognates in Timor and Flores appear to be better explained by *sauR, with no final vowel and $* \mathrm{R}[\mathrm{r}]$ instead of $* \mathrm{r}[\mathrm{r}]$. The final vowel in Blust and Trussel's putative *sora would only be supported by Leti sora, Wetan ora, and Kemak sora. However, the Luangic languages (including Leti and Wetan) are known to added final vowels to historically consonant final stems (Blevins and Garret 1998:542f), thus rendering their evidence moot. The Kemak form provides better evidence for final $*$ a, but this goes against the evidence of many languages in the region, including Rote-Meto, which would be expected to retain $* \mathrm{a}=a$. Furthermore, if $* \mathrm{R}$ is reconstructed rather than $*$ r, than $* \mathrm{R}>r$ is irregular in Kemak as we expect $* \mathrm{R}>\varnothing$.]

soo Termanu. sew. In Termanu usually in a compound as seu_soo. (J:550) soo Korbafo.

soo Bokai.

soo Bilbaa.

soo Rikou.

soo $B a^{\prime} a$.

soo Tii.

soo Dengka.

soo Oenale.

n-soo Kotos Amarasi. sew.

n-soo Molo. sews. (M:508)

Out-comparisons:

soo Semau Helong. sew. Borrowed from: probably Meto or Rote given $* * \mathrm{R}>\varnothing($ expect $* \mathrm{R}>l)$.

hour Kisar. sew.

sora Kemak. sew.

saur Central Lembata. sew. (Fricke 2015)

a-sor Uruangnirin. sew. [Note: language of the Bomberai Peninsula ISO 639-3 [urn].] (Visser 2019)

*soro PRM. spoon, ladle, scoop. Doublet: *suru. Etym: *sudu. [irr. from PMP: $*_{\mathrm{u}}>*_{\mathrm{o}}$ ] [irr. from PRM: $*_{\mathrm{r}}>n \sim k$ in Meto (compare similar *təlo $>$ teno?/ teko? 'egg')]

solo Termanu. scoop, e.g. dirt out of water. (J:559)

solo Korbafo.

solo Bokai.

solo Bilbaa.

soro Rikou.

solo $B a^{\prime} a$.

soro Tii.

solo Dengka.

soro Oenale.

?|sono|? Kotos Amarasi. spoon.

$<$ a'soko> Molo. spoon. (M:511) 
P|soko|? Timaus. spoon.

*sosa PMeto. buy. See: *osa 'price, value'.

n-sosa (2) na-?sosa? Ro'is Amarasi.

1) buy. 2) sell.

n-sosa (2) na-?sosa? Kotos Amarasi.

1) buy. 2) sell.

n-sosa (2) na-?sosa? Molo. 1) buy.

2) sell. (M:516)

soas Kusa-Manea. buy.

Out-comparisons:

sosa Semau Helong. buy.

sosa East Tetun. buy. (Mo:174)

*soso Rote. peel.

soso Termanu. cut the meat as close as possible to the bones. (J:564)

soso Korbafo.

soso Bokai.

soso Bilbaa.

soso Rikou.

soso $B a^{\prime} a$.

soso Tii.

soso Dengka. peel. (J:564, 765)

soso Oenale. peel. (J:564, 765)

Out-comparisons:

soso Bugis. peel or de-husk with a knife. (Mathes 1874:763)

soso Makassar. peel, de-husk.

(Mathes 1859:612)

*sua1 PnRote. accuse.

sua Termanu. accuse someone, usually falsely accuse someone. (J:567)

sua Korbafo.

sua Bokai.

sua Rikou.

sua $B a^{\prime} a$.

sua $T i i$.

Out-comparisons:

suan kali Semau Helong. gossip, provocateur; someone who tries to find faults in others to bring them to everyone's attention.

*sua2 PnMeto. rafter, roof-spar. Etym: *sukəd 'prop, support; to prop up or support'. [irr. from PMP: *k $>\varnothing / \mathrm{u}$ ] sua-f Kotos Amarasi. roof spar.

(Cunningham 1964:37, 44)

sua-f Molo. rafter. (M:518)

Out-comparisons:

sukan Helong. rafter. (J:95)

*sua3 Morph: *ka-sua-k. PRM. digging stick. Etym: *suaR 'lift up with a lever, lever up, root up' (Blust and Trussel (ongoing) also reconstruct doublets *sual and *suat).

ai_su $\sim$ sua-k Termanu. pointed stick used to work the ground, a kind of agricultural tool. (J:568)

ai_su $\sim$ sua-? Korbafo.

ai_su sua-k Bokai.

kai__su $\sim$ sua-? Bilbaa.

ai_su sua-? Rikou.

ai_su $\sim$ sua-k $B a^{\prime} a$.

ai_su sua-k Tii.

hau_su sua-? Dengka.

hau_su $\sim$ sua-? Oenale.

?|suak (2) na-?|sua Kotos Amarasi.

1) digging stick. 2) dig with digging stick.

$<$ suan> Molo. digging stick, plank stick. (M:519)

\section{Out-comparisons:}

ksuan Funai Helong. crowbar, digging stick.

suan Semau Helong. crowbar, digging stick.

ai suak (2) au suak besi East Tetun. 1) digging stick, used for weeding and digging in the garden. 2) an iron digging stick, used as above. (Mo:175)

*suba PRM. bury. [Semantics: The semantic shift from 'bury' to 'engrossed' is likely (e.g. compare English phrases such as She's buried in her book). However, if the Dela form is cognate and its semantics are older, then the Rote forms are probably not cognate with Meto.]

suba Termanu. totally engrossed in something, so that one forgets everything else. (J:568) 
suba Korbafo.

suba Bokai.

suba Bilbaa.

suba Rikou.

suba $B a^{\prime} a$.

suba Tii.

suba Dengka.

suba Oenale.

suba-k, suba suba Dela. silent, idle.

n-suba Ro'is Amarasi. bury.

n-suba Kotos Amarasi. bury.

n-suba Molo. bury, submerge. (M:519)

suub Kusa-Manea. bury. [Form: metathesised form of (currently unattested) *subu.]

\section{Out-comparisons:}

subal, subar East Tetun. hide, conceal. [Semantics: The semantics of the Tetun form are not a very good match, and thus it may be a chance resemblance.] (Mo:175)

*sufu PRM. cool in water. Etym: *səbuh 'douse a fire, extinguish a fire with water; to hiss, as water on fire'. [irr. from PMP: *ə $>*^{*} \mathrm{u}$ (sporadic assimilation)]

ma-ka-sufu-k (2) sufu (2) na-sufu Termanu. 1) cold. 2) cool down. 3) be cold. (J:571)

ma-ka-sufu-? Korbafo.

ma-ka-sufu-k Bokai.

ma-ka-sufu-? Bilbaa.

ma-sufu-? Rikou.

ma-ka-sufu-k $B a^{\prime} a$.

ma-ka-sufu-k Tii.

ma-?a-sufu-? Dengka.

ma-?a-sufu-? Oenale.

ma-Pa-sufu Dela. be cold (food).

sufu? Kusa-Manea. cool down, put out a fire.

\section{Out-comparisons:}

suhu Semau Helong. put out fire with water. [Note: Jonker (1908:571) gives Helong $\operatorname{suhu(n)}$ 'to shower'.] suhu East Tetun. immerse in water, quench or harden (hot steel). (Mo:175)

sowo Bima. cool/fresh, cold. (Ismail et al. 1985:147)

həßо Sika. put out (fire). (Pareira and Lewis 1998:75)

*suhat Rote. kind of comb. Etym: *suat (PRM suhat would be regular from PMP * supat. Blust and Trussel give the following forms as evidence for their reconstruction with no medial consonant: Binukud and Mansaka suwat, Blaan (Sarangani) swat, Manam ruat-i, and 'Rote' $<$ sua $>$.).

su?a-k Termanu. kind of comb worn as decoration by people from Timor, not worn by people from Rote. (J:568)

su?a-? Korbafo.

su?a-k Bokai.

sua-? Bilbaa.

su?a-k $B a^{\prime} a$.

suat Dengka.

suat Oenale.

*suhu Morph: *suhu-k. Rote. boundary, border. Etym: *supu.

su?u-k Termanu. edge, side, shore.

(J:578)

su?u-? Korbafo.

su?u-k Bokai.

suu-? Bilbaa.

suu-? Rikou.

su?u-k $B a^{\prime} a$.

suu-? Dengka.

suu-? Oenale.

Out-comparisons:

suut Semau Helong. edge. [Note: Jonker (1908:578) gives Helong suut, ksuut.]

huu Hawu. tip, end.

*suku Morph: *suku-k. Rote. breadfruit. Etym: *sukun. Pattern: k-7.

su?u-k Termanu. breadfruit tree; its fruit. (J:578)

su?u Korbafo.

su?u-k Bokai. 
suku-? Bilbaa.

suku-? Rikou.

su?u-k $B a^{\prime} a$.

su?u-k Tii.

suku-? Dengka.

suku-? Oenale.

*sulə PRM. insert, plug. Etym: *sulə[n/d].

[Sporadic: $*_{\partial}>e / \sigma_{\#} \#$ in wRote (perhaps $*_{\partial}>* a>e / \#$ ).]

sule Termanu. close something with a stopper, put a stopper in something to close it. (J:573)

sule Korbafo.

sule Bokai.

sule Bilbaa.

sule Rikou.

sule $B a^{\prime} a$.

sule Tii.

sule Dengka.

sule Oenale.

n-suun Molo. corks. [Note: Jonker (1908:573) gives unmetathesised nominalised Meto suna-t.] (M:523)

*suma PRM. steam, vapour. [History: Blust and Trussel (ongoing) reconstruct PWMP *s $<$ um $>$ ebuh 'to hiss or steam, of water touching a fire' which is formally and semantically similar.]

suma Termanu. hold in hot steam, cook in steam, steaming. (J:574)

suma Korbafo.

suma Bokai.

suma Bilbaa.

suma Rikou.

suma $B a^{\prime} a$.

suma Tii.

suma Dengka.

suma? (2) n-suma Kotos Amarasi.

1) steam, vapour. 2) steaming.

suma? Molo. steam from boiling water, etc. (M:523)

*sumanə Morph: *sumanə-k. PRM. soul of a living being; soul of the rice plant. Etym: *sumanəd. [Form: Antepenultimate $*_{\mathrm{u}}$ (high back rounded vowel) is probably required to account for Landu $i$ (high vowel), as well as the alternate Termanu from with antepenultimate $o$ (back rounded vowel).]

samane-k, somane-k Termanu. life spirit. (J:519; Fanggidaej 1892:556)

samane-? Korbafo.

samane-k Bokai.

samane-? Bilbaa.

simane-? Landu. (own field notes)

samane-? Rikou.

samane-k $B a^{\prime} a$.

samane-k Tii.

mana-? Dengka.

samana-? Oenale.

smana-f Kotos Amarasi. spirit or soul of a person, spirit of the rice plant.

smana-f Molo. spirit. (M:504)

Out-comparisons:

smayin Funai Helong. spirit, soul; forehead.

hmāin Semau Helong. spirit, soul; forehead.

hemaya Hawu. spirit, soul. Spirit of both the living and the dead. What remains and continues on after the body dies.

*sunu Morph: *su sunu. Rote. kind of sea fish.

su $\sim$ sunu Termanu. kind of sea fish. (J:576)

su $\sim$ sunu Korbafo.

su $\sim$ sunu Bokai.

su $\sim$ sunu Bilbaa.

su sunu Rikou.

su $\sim$ sunu $B a^{\prime} a$.

su $\sim$ sunu Tii.

su sunu Dengka.

su $\sim$ sunu Oenale.

Out-comparisons:

sunu Kamarian. a certain fish (ikan papua). [Note: also in Kaibobo.] (van Ekris 1865:118)

*sungə Morph: *sungə-k. PRM. cheek hollow. [Sporadic: *ə $>e / \sigma_{-} \#$ in wRote (perhaps $*_{\partial}>*_{\mathrm{a}}>e^{\bar{l}}$ \#).] 
[Form: The Meto unmetathesised form has not yet been attested. It could be *suka- or *suku-.]

suye-k (2) na-sune Termanu. 1) the inner part of the cheek. 2) hold something in the mouth between the cheeks. (J:575)

sune-? Korbafo.

sune-k Bokai.

suye-k Bilbaa.

suke-? Landu. cheek. (own field notes)

suke-? Oepao. cheek. (own field notes)

suyge-k Tii.

sunge-? Dengka.

sunge-? Oenale.

suk $\sim$ suuk-n=aa Ro'is Amarasi. cheekbone. [Note: This form was given (unprompted) by one Ro'is consultant, but was completely unknown by all other Ro'is consultants.]

*sura Morph: *sura-k. PRM. horn.

Doublet: *sure. Etym: *suja 'pitfall or trail spikes made of sharpened bamboo'. [History: Blust and Trussel (ongoing) reconstruct both PCMP *sula and *sulan 'horn' on the basis of Termanu sula and Buru sula-n. This reconstruction is problematic as the other Rote languages clearly attest PRM $*_{r}$ (which also accounts regularly for Termanu $l$ ). Additionally, the putative Buru term sula-n is not found in any published sources I have access to. Instead they give sodi-n (Grimes and Grimes 2020:864) or soden (Hendriks 1897:93). Hoogervorst (2016:568) identifies Sanskrit śūla [Ju:la] 'a spear or lance, an offensive weapon' as the source of putative PCMP *sula. While Sanskrit śūla [Ju:la] may be the source of PRM * sura, this would not account straightforwardly for medial *r. Jonker (1908:573) compares the Rote forms to tulane from Asilulu in Ambon (Hoëvell 1877:69). But this form would not show regular sound correspondences. Among languages of Ambon, Stresemann (1927:37) only records $t$ as a reflex of *s in Amahei, and then only before non-high vowels.] su $\sim$ sula-k Termanu. horn of a buffalo, of a deer, etc. (J:573)

su $\sim$ sula-? Korbafo.

su sula-k Bokai.

su $\sim$ sula-? Bilbaa.

su $\sim$ sura-? Landu. horn. (own field notes)

su sura-? Rikou.

su sula-k $B a^{\prime} a$.

su sura-k Tii.

su sula-? Dengka.

sura-? Oenale.

sunu-f Ro'is Amarasi. horn.

suna-f Kotos Amarasi. horn, antennae (of insects).

suna-f Molo. horn of livestock. (M:523)

suna-f Kusa-Manea. horn.

Out-comparisons:

sulu? Semau Helong. horn. [irr. from PRM: *a $=u$ correspondence]

*sure Morph: *sure-k. PRM. pointed weapon; caltrop, sword. Doublet: *sura. Etym: *suja 'pitfall or trail spikes made of sharpened bamboo'. [minority from PMP: $* \mathrm{j}>*_{\mathrm{r}}$ (expect *d)] [Sporadic: $* \mathrm{a}>*_{\mathrm{e}} /{ }^{*} \mathrm{C}+$ palatal_.] sule-k Termanu. something pointed, like a thorn, which is used as a caltrop (foot-trap); place a caltrop. (J:574)

sule-? Korbafo.

sule-k Bokai.

sule-? Bilbaa.

suri-? Rikou.

sule-k $B a^{\prime} a$.

sure-k Tii.

sule-? Dengka.

sure-? Oenale.

suni|? Kotos Amarasi. sword, fighting sword. Particularly the long curved fighting sword found on Timor. 
[Sporadic: vowel height harmony $*_{\mathrm{e}}>i / \mathrm{uC}$ in Meto.] [Form: Final $* \mathrm{e}>i$ on Timor mainland combined with semantic shift to 'sword' suggests diffusion/borrowing though the direction of diffusion remains to be determined.]

suni|? Molo. sword. (M:524)

suni|? Kusa-Manea. machete.

\section{Out-comparisons:}

suli? Semau Helong. sword. long thin fighting sword. May be about a metre in length, and width described as 'between 2-3 fingers'.

surik East Tetun. sword with a long curved sharp blade which is sheathed in a scabbard when not in use. (Mo:176)

surik Mambae, South. sword, long curved fighting sword found on Timor, single blade. (Grimes et al. 2014b:42)

*suru PRM. spoon, ladle, scoop. Doublet: *soro. Etym: *sudu.

sulu (2) sulu-k Termanu. 1) scoop up with a spoon. (J:574) 2) spoon. (J:574)

sulu Korbafo.

sulu Bokai.

sulu Bilbaa.

suru Rikou.

sulu $B a^{\prime} a$.

suru Tii.

sulu Dengka.

suru Oenale.

<sunu> Amanuban. spoon. (M:511)

sa $\sim$ sunu|? Kusa-Manea. spoon.

\section{Out-comparisons:}

sulu Semau Helong. spoon, scoop.

suru East Tetun. extract or take out with a spoon or ladle, to perform any similar action. (Mo:175)

huru Kisar. spoon.

kaba huru Hawu. spoon, ladle. *susi $P R M$. investigate, walk around. [irr. from PRM: $* \mathrm{i}>u$ in Bilbaa and Rikou] [Semantics: The semantic connection between the Meto terms and Rote terms is unclear to me, but Jonker (1908:576) gives the Meto forms as connected and it's better to give all the (potential) data than to sweep things under the carpet.]

susi Termanu. investigate a matter, inquire into something. (J:576)

susi Korbafo.

susi Bokai.

susu Bilbaa.

susu Rikou.

susi $B a^{\prime} a$.

susi Tii.

susi Oenale.

n-susi Kotos Amarasi. walk around without any particular destination in mind, go to someone's house and hang out without any plan.

n-susi Molo. one crawls back through something. (M:525)

*susu1 PRM. tax. [Semantics: Given the semantics this may be a borrowing, though the kingdoms of Timor did have traditional systems of tribute.]

susu Termanu. apply taxes, tax. (J:577)

susu Korbafo.

susu Bokai.

susu Bilbaa.

susu Rikou.

susu $B a^{\prime} a$.

susu Tii.

susu Dengka.

nusu Oenale.

n-suus Molo. earn (money). (M:524)

Out-comparisons:

susut Semau Helong. tax.

*susu2 PRM. female breast; udder. Etym:

*susu. [irr. from PRM: $*_{\mathrm{s}}>$ ? in

Termanu, Korbafo, Bokai and Ba'a]

su?u Termanu. female breast. (J:577)

su?u Korbafo.

su?u Bokai. 
susu Bilbaa.

susu Rikou.

su?u $B a^{\prime} a$.

susu Tii.

susu(-?) Dengka.

susu Oenale.

susu Ro'is Amarasi. breast.

susu-f Kotos Amarasi. breast.

susu Molo. milk, breasts (of woman).

(M:525)

\section{Out-comparisons:}

susu Semau Helong. breast.

susu-n East Tetun. breast (of women), udder (of animals). (Mo:177)

*suti PRM. nautilus, nautilus shell.

[Semantics: I have reconstructed this term with the meaning 'nautilus' following the meaning in Rote rather than the meaning in Ro'is Amarasi as the people in Rote have a much stronger connection to the sea than the Atoni and it thus seems more likely that terms in Rote referring to the sea would be more conservative. This meaning is also probably partly retained in the Kotos Amarasi and Molo reflexes.]

suti Termanu. nautilus, nautilus shell.

(J:577; Fox 2016b)

suti Korbafo.

suti Bokai.

suti Bilbaa. nautilus, nautilus shell.

(J:577; own field notes)

suti Rikou. nautilus, nautilus shell.

(J:577; own field notes)

suti $B a^{\prime} a$.

suti Tii.

suti Dengka.

suti Oenale.

suti $R o$ 'is Amarasi. sea snails/shellfish that are oval shaped and have a wide opening, often with a distinctive lip which runs the length of the shell: conches, volutes. [Note: benkae $=$ 'nautilus'.] suti Kotos Amarasi. little round plate upon which the woman has the spool twirl in order to wind the cotton thread. [Semantics: Regarding the semantic connection between the terms in Rote and the Kotos Amarasi and Molo terms, Fox (2016a:43) states: 'Suti, the nautilus shell, becomes the container for dye, particularly indigo dye; and Bina, the bailer shell, becomes the base on which the spindle for winding thread is turned. The two shells are ritual icons for the processes of preparing a cloth for weaving.']

suti Molo. little round plate upon which the woman has the spool twirl in order to wind the cotton thread. (M:526)

*suu1 PRM. scrape the ground.

suu Termanu. dig or scoop out with the hands, also dig out in general. (J:566)

suu Korbafo.

suu Bokai.

suu Bilbaa.

suu Rikou.

suu $B a^{\prime} a$.

suu Tii.

suu Dengka.

suu Oenale.

na-k|suu (2) n-suu Molo.

1) scratches (ground), makes scratches (on the ground), scrabbles out. 2) scrabbles out. (M:518)

Out-comparisons:

su?u East Tetun. mine, fossick, dig a mine shaft. (Mo:177)

*suu2 PRM. carry on head. Etym: *suqun.

suu Termanu. put or carry on one's head. (J:566)

suu Korbafo.

suu Bokai.

suu Bilbaa.

suu Rikou.

suu $B a^{\prime} a$. 
suu Tii.

suu Dengka.

suu Oenale.

n-suu Kotos Amarasi. carry on one's

head. n-suu Molo. carry on one's head.

(M:518)

suu Kusa-Manea. carry on head.

\section{$\mathbf{T}-\mathbf{t}$}

*taa1 PRM. track, footprint. Etym: *tapak 'palm of the hand, sole of the foot' (PWMP). [irr. from PMP: * $\mathrm{p}>\varnothing$ $\left({ }^{*} \mathrm{p}>\varnothing\right.$ is regular in wRote, Bilbaa, Rikou and Meto but we would expect ${ }^{*} \mathrm{p}>?$ in the other Rote languages)] [Sporadic: *a $>e$ /_\# in Meto.]

ta -taa Termanu. make an impression with the hand or foot, leave tracks behind. (J:580)

ta taa Korbafo.

ta taa Bokai.

ta taa Bilbaa.

ta taa Rikou.

ta $\sim$ taa $B a^{\prime} a$.

ta $\sim$ taa Tii.

ta $\sim$ taa Dengka.

n-tae Kotos Amarasi. look down.

<taè> Molo. look down, trace/track down. [Form: Jonker (1908:580) gives Meto ta?en with the meaning 'identical [to the Rote meaning] and: agree upon'.] [Semantics: The meaning 'trace/track down' appears to be the semantic link between 'track' and 'look down'.] (M:527)

\section{Out-comparisons:}

tahe Semau Helong. sign.

*taa2 Rote. unhusked rice. See: *eto.

Etym: *qata 'rice husk, rice bran'. [irr. from PMP: *ə $>\varnothing$ with doubling of the final vowel to create a disyllable]

(hade) taa-k Termanu. unhusked rice grains under the husked grains. (J:581)

taa-? Korbafo.

taa-k Bokai.

ka|taa-? Bilbaa. taa-? Rikou.

taa-k $B a^{\prime} a$.

taa-k Tii.

taa-? Dengka.

taa-? Oenale.

*taa3 PnRote. negative marker: no, not. Etym: *taq.

taa Termanu. non-existent, no, not. [Form: Phrases such as $<$ ana-ták $>$ ana taa-k 'childless' provide evidence for the double vowel.] (J:578)

taa Korbafo.

taa Bokai.

taa Bilbaa.

taa Rikou.

taa $B a^{\prime} a$.

taa Tii.

Out-comparisons:

ka Kisar. not.

*taa4 Morph: *taa-k. Rote. endure. Etym: *taqan 'hold back, keep in reserve'. [Form: Final $k$ cannot be straightforwardly analysed as synchronic suffix in Korbafo, Bilbaa and Dengka.]

na-taa-k=ana (2) na-ka-ta taa-k

Termanu. 1) endurance. 2) endure. (J:581)

na-taa|k=ana Korbafo.

na-taa-k=ana Bokai.

na-taa $\mid \mathbf{k}=$ ana Bilbaa.

na-taa- $\mathbf{P}=$ ana Rikou.

na-taa-k=ana $B a^{\prime} a$.

na-taa-k=ana $T i i$.

na-taa $\mid k=$ ana (2) na-Pa-ta $\sim$ taa-?

Dengka.

(2) na-?a-ta taa-? Oenale. 
*tabu PRM. tread, step.

tabu Termanu. put the foot somewhere, step, tread. (J:582)

tabu Korbafo.

tabu Bokai.

tabu Bilbaa.

tabu Rikou.

tabu $B a^{\prime} a$.

tabu Tii.

tabu Dengka.

tabu Oenale.

tabu Ro'is Amarasi. time.

tabu Kotos Amarasi. time.

n-tabo Amfo'an. treads. (M:527)

tabu Molo. clock, hour. (M:527)

Out-comparisons:

tabu Waima'a. time, watch.

tabu Galolen. time.

*tadengus $P R M$. kind of dove, probably

Rose-crowned Fruit-Dove. Ptilinopus

regina. Pattern: d-2. [irr. from PRM: vowel metathesis in Kusa-Manea

$\left.*_{\mathrm{eCu}}>*_{\mathrm{uCe}}>u C i\right]$

rekus Landu. Rose-crowned FruitDove. Ptilinopus regina. (own field notes)

rekus Rikou. Rose-crowned FruitDove. Ptilinopus regina. (own field notes)

lengus Dengka. dove; in Dengka species include: lengu lasi, lengu ma?amuu, lengu manu ina. (J:722)

rengus Oenale. dove. (J:722)

kuum_treukus Ro'is Amarasi. Rosecrowned Fruit-Dove. Ptilinopus regina. [Form: kumu $=$ 'wild dove'.]

ra rukis Kusa-Manea. wild doves. [Sporadic: vowel height harmony $*_{\mathrm{e}}>i / \mathrm{uC}$ (alternately $*_{\mathrm{e}}>i / \mathrm{Cu}$ before vowel metathesis).]

*tadu Morph: *la-tadu-k. CER. opposite.

la-tadu-k Termanu. sit opposite one another. (J:585)

la-tadu-k Bokai.

la-tadu-k $B a^{\prime} a$.
Out-comparisons:

ntando (2) satando (3) tando

Bima. 1) facing one another.

(Ismail et al. 1985:103)

2) face towards. (Ismail et al.

1985:143) 3) forward, front

part; face towards. (Ismail et al. 1985:151)

*tae $n R M$. praying mantis. [Sporadic: consonant metathesis ${ }^{*} \mathrm{rVt}>t V r$ in Nuclear Meto; glottal stop insertion in Meto] [Form: The source of the initial element in Meto currently unknown. This element also has irregular Ro'is $k=$ Nuclear Meto $r$ correspondence. This is the reverse pattern to what would be expected for PMeto *r.]

telu_tae Termanu. kind of shrimplike creature. (Fox 2016b:13, 55)

aka?ta?e Ro'is Amarasi. praying mantis.

ata?ra?e Kotos Amarasi. praying mantis.

ata?la?e Molo. praying mantis. (M:31)

Out-comparisons:

'akatae Semau Helong. praying mantis. Borrowed from: Ro'is Amarasi.

astatae Welaun. praying mantis.

*tafa Rote. sword, machete.

tafa Termanu. sabre, sword. (J:586)

tafa Korbafo.

tafa Bokai.

tafa Bilbaa.

tafa Rikou.

tafa $B a^{\prime} a$.

tafa Tii.

tafa-? Dengka.

tafa-? Oenale.

Out-comparisons:

taha East Tetun. machete, a jungle knife. (Mo:178)

kawa Kisar. machete. 
*taha Morph: *na-taha. PRM. answer. Etym: **taRa (pre-RM). [irr. from $P M P: * \mathrm{R}>* \mathrm{~h}$ (also in *noh and *klaha)] [Form: regular $* \mathrm{~h}>\varnothing$ /a_a in Rote.]

na-taa Termanu. answer. (J:580f)

na-taa Korbafo.

na-taa Bokai.

na-taa Bilbaa.

na-taa Rikou.

na-taa $B a^{\prime} a$.

na-taa $T i i$.

na-taa Dengka.

na-taa Oenale.

na-taha Kotos Amarasi. answer.

ta-taah Kusa-Manea. answer.

Out-comparisons:

tala Semau Helong. answer.

hataa Fehan Tetun. respond, answer, give a reply to. (Mo:80)

hataan East Tetun. respond, answer, give a reply to. (Mo:81)

*tahi Rote. winnow. Etym: *tapi. [irr. from PRM: * $\mathrm{t}>d$ in nRote]

da?i Termanu. winnow. (J:71)

da?i Korbafo.

da?i Bokai.

dai Bilbaa.

dai Rikou.

da?i $B a^{\prime} a$.

da?i Tii.

tai Dengka.

tai Oenale.

Out-comparisons:

tahin Semau Helong. winnow.

*tai1 Morph: *tai-k. PRM. belly, stomach, guts. Doublet: *tai2. Etym: *taqi 'faeces, excrement'. [irr. from PRM: $* \mathrm{a}>e$ in Ro'is Amarasi and all Rote lects except Tii (sporadic assimilation to following $i$ )]

tei-k Termanu. belly, intestines. (J:612f)

tei-? Korbafo.

tei-k Bokai.

tei-? Bilbaa.

tei-? Landu. belly. (own field notes) tei-? Rikou.

tei-? Oepao. belly. (own field notes)

tei-k $B a^{\prime} a$.

tai-k Tii.

tei-? Dengka.

tei-? Oenale.

tei Dela. stomach.

tei-f Ro'is Amarasi. belly, stomach.

tai-f Kotos Amarasi. belly, stomach, guts.

tai-n Molo. intestines. (M:623)

Out-comparisons:

tain Semau Helong. stomach, abdomen, belly.

*tai2 PRM. faeces, excrement. Doublet:

*tai1. Etym: *taqi. [irr. from PRM:

$*_{\mathrm{a}}>e$ in lects except Tii (sporadic assimilation to following $i$ )]

tei Termanu. faeces, excrete. (J:612f)

tei Korbafo.

tei Bokai.

tei Bilbaa.

tei Landu. faeces. (own field notes)

tei Rikou.

tei Oepao. faeces. (own field notes)

tei $B a^{\prime} a$.

tai Tii.

tei Dengka.

tei Oenale.

tei Dela. faeces.

tei Ro'is Amarasi. faeces.

tei (2) na-te?i Kotos Amarasi.

1) faeces. 2) excrete.

tei Molo. faeces. (M:623)

Out-comparisons:

tai Funai Helong. excrement.

tai Semau Helong. faeces.

tee-n East Tetun. excrement, dregs, residue. (Mo:183)

*tai3 Rote. stick to.

tai Termanu. adhere. (J:587)

tai Korbafo.

tai Bokai.

tai Bilbaa.

tai Rikou.

tai $B a^{\prime} a$.

tai Tii. 
tai Dengka.

tai Oenale.

Out-comparisons:

tai Semau Helong. hang, suspend.

*tairua CERM. half. [irr. from PRM: *t

$>h$ in nRote] [Form: second part from

*dua 'two'.]

hailua Termanu. half full. (J:155)

hailua Korbafo.

hailua Bokai.

hailua Bilbaa.

hairua Rikou.

n-tainua (2) tainua Kotos Amarasi.

1) halve. 2) half. Usage: somewhat archaic, stena? from Malay sətəna is the usual term in my data.

Out-comparisons:

tailuan Semau Helong. halfheartedly.

*tais PRM. cloth, sarong. Etym: *tapis 'loincloth (?)'. [irr. from PMP: * $\mathrm{p}>\varnothing$ (Meto $\varnothing$ could be regular from *p, but we would still expect ? in Termanu)]

tai_sai-k Termanu. cloth, a torn piece of stuff, a nappy. Usage: still used in Dengka and Oenale, as in Meto, in disuse elsewhere in Rote. (J:589)

tais Dengka. pants. (J:768)

tais Oenale. pants. (J:768)

tais Ro'is Amarasi. clothing.

tais Kotos Amarasi. sarong, clothes.

tais Molo. sarong, skirt. (M:532)

tais Kusa-Manea. cloth.

Out-comparisons:

tais East Tetun. cloth of indigenous manufacture. (Mo:178)

*taku PRM. fear. Etym: *takut. Pattern: k-8.

$\begin{array}{rrr}\text { na-ka-ta ta?u (2) } & \text { ta?u-s (3) } \\ \text { ma-ka-ta ta?u-k } & \text { Termanu. }\end{array}$

1) frighten, threaten. 2) fear.

3) someone (a person, demon, etc.)

which causes fright. (J:604f)

na-ka-ta $\sim$ ta?u Korbafo.

na-ka-ta ta?u Bokai.

na-ka-ta taku Bilbaa.

na-ta taPu Rikou. na-ka-ta ta?u $B a^{\prime} a$.

na-ka-ta $\sim$ ta?u-k Tii.

na-Pa-ta ta?u-? (2) na-tau-? (3)

na-ma-tau Dengka. 1) frighten, threaten. (J:604) 2) frighten. (J:769f) 3) be afraid. (J:769f)

na-Pa-ta ta?u-? (2) na-tau-? (3) na-ma-tau Oenale. 1) frighten, threaten. (J:604) 2) frighten. (J:769f) 3) be afraid. (J:769f)

- (2) - (3) na-ma-tau Dela. 3) becomes afraid, becomes worried.

na-m|tau Ro'is Amarasi. is scared.

na-m|tau (2) na-m|tau|s Kotos Amarasi. 1) scared. 2) scared of. [Form: The final $s$ in the transitive form na-mltaus 'scared of' may be a reflex of the final consonant reconstructed for PMP *takut, thus $* \mathrm{t}>s$. The nominalising suffix - $\mathrm{t}$ in Meto has the allomorph -s after stems which contain a $t$ (Edwards 2020:455f) and thus the putative change of $*_{\mathrm{t}}>s$ in this form may be a case of dissimilation from word initial $*$ t. Note also that the pair n-mani 'laugh' and n-manis 'laugh at' (see *malis) also has transitivity marked with final $s$. In this case, the final consonant of the transitive form also appears to be a reflex of the final consonant of PMP *malip (see §3.5.1.3).]

na-m|tau (2) na-m|tausan (3) n-haka|tau (4) ma|taus Molo. 1) scared. 2) scared of. (M:332) 3) scare (someone). 4) those who are scared. (M:542)

Out-comparisons:

hata?uk East Tetun. be afraid, fear, dread. (Mo:183)

-ka?uk Kisar. afraid. meda?u Hawu. afraid.

*talada PRM. middle, centre. [irr. from PRM: $* \mathrm{t}>k$ in Dengka] [Form: regular ${ }^{*} \mathfrak{d}>* * 1 / * 1$ in Meto (and Helong).] 
talada Termanu. middle. (J:590)

talada Korbafo.

talada Bokai.

talada Bilbaa.

talada Rikou.

talada $B a^{\prime} a$.

talada Tii.

kalada-? Dengka.

talada-? Oenale.

talada-? Dela. centre.

tnana|? Ro'is Amarasi.

tnana|?, tnana-f Kotos Amarasi. middle, waist.

au tnana-k Molo. my interior. (M:561)

tnana|? Kusa-Manea. middle.

Out-comparisons:

hlala Semau Helong. middle, centre.

tlala Bolok Helong. middle, centre.

klara-n East Tetun. middle, the centre. (Mo:109)

klalan Galolen. middle.

telora Hawu. middle.

Ploray Sika. inside, in the middle. (Pareira and Lewis 1998:124)

hatalae Kamarian. among, in the middle. [Note: also in Kaibobo and Haruku.] (van Ekris 1864:85)

hatarale Kaibobo. Usage: Piru village. (van Ekris 1864:85)

hatalea Kaibobo. Usage: Hatusua village. (van Ekris 1864:85)

samtarae Alune. [Note: language of west Seram, central Maluku ISO 639-3 [alp].] (van Ekris 1864:85)

hatalea, haalea Nusa Laut. [Note: language of Lease Islands, central Maluku ISO 639-3 [nul].] (van Ekris 1864:85)

*tales Rote. taro. Colocasia esculenta. Etym: *tales. [minority from PMP: *ə $>*_{\mathrm{e}}$ /_\# (expect $*_{\mathrm{\rho}}>a$ in wRote)] tale Termanu. 1) kind of water plant with big leaves. (J:591) 2) taro. (Fox 1991:257)

tale Korbafo.

tale Bokai.

tale Bilbaa.

tale Rikou.

tale $B a^{\prime} a$.

ta tale-k Tii.

ta tales Dengka.

tale Oenale.

Out-comparisons:

talas East Tetun. an aroid plant with highly prized edible tubers. (Mo:179)

*tali PRM. rope, cord, twine, string. Etym: *talih.

tali Termanu. rope. (J:591)

tali Korbafo.

tali Bokai.

tali Bilbaa.

tali Rikou.

tali $B a^{\prime} a$.

tali Tii.

tali-? Dengka.

tali-? Oenale.

tani Ro'is Amarasi. rope.

tani Kotos Amarasi. rope.

tani Molo. rope. (M:538)

tani Kusa-Manea. rope.

Out-comparisons:

tali Semau Helong. rope, cord, string, twine, strand.

tali(n) East Tetun. rope, cord, string, etc. (Mo:179)

*talin PRM. money. [History: This could be a borrowing, but a likely source language has not been identified.]

tali_doi-k Termanu. money in general.

(J:591)

tali_doi-? Korbafo.

tali_doi-k Bokai.

tali_doi-k Bilbaa.

tali doi-? Rikou.

tali doi-k $B a^{\prime} a$.

tali doi-k Tii.

tali_doi-? Dengka. 
tali_doi-? Oenale. tanin Meto. (J:591)

Out-comparisons:

talin Helong. ( $\mathrm{J}: 591)$

*tama1 PMeto. enter. Etym: *tama 'enter, penetrate; bold, of persons'.

n-tama Ro'is Amarasi. enter.

n-tama Kotos Amarasi. enter.

an-tama Molo. go inside. (M:536)

n-tama Timaus. enter.

Out-comparisons:

tama Semau Helong. enter, go in.

tama East Tetun. enter, introduce, penetrate. (Mo:179)

*tama2 Rote. appropriate, suitable, right; fit together. Etym: *tama(q) (Blust and Trussel (ongoing) reconstruct both *tamaq and *tama as 'disjuncts' with almost identical semantics. The Termanu form is included as evidence for both.).

tama Termanu. fit together well. papa-k=ala tama matalolole the planks fit well together. (J:592)

tama Korbafo.

tama Bokai.

tama Bilbaa.

tama Rikou.

tama $B a^{\prime} a$.

tama Tii.

tama Dengka.

tama Oenale.

*tamae Rote. bedbug. Etym: **tamayuy (pre-RM). [Form: I have reconstructed antepenultimate PRM *a primarily on the basis of external evidence and the fact that this is the most common antepenultimate vowel in PRM. This means proposing antepenultimate *a $>i$ in Landu. This sound change finds some support from PRM sumanə-k 'soul' > Landu simane-?, which also shows a shift of an antepenultimate vowel to $i$. In both words this may be sporadic assimilation to the previous apical consonant.]

mae-k Termanu. bedbug. (J:340)

mae-? Korbafo. mae-k Bokai.

mae-? Bilbaa.

timae-? Landu. bedbug. (own field notes)

mae-? Rikou.

mae-k $B a^{\prime} a$.

mae-k Tii.

mai-? Dengka.

mai-? Oenale.

Out-comparisons:

kmaen Funai Helong.

hmaen bedbug. Helong. (J:340)

(ta)madzuy Alorese. bedbug. (Moro 2016)

tomadzuy Central Lembata. bedbug. (Fricke 2015)

madzu Central Nage. bedbug. Cimex lectularius. (Forth 2016:335)

main Sika bedbug. (Pareira and Lewis 1998:128)

madzun Manggarai. bedbug. Cimex rotundatus. (Verheijen 1967:304)

*tamo CERM. ancestral name. [irr. from PRM: * $>$ > in Meto]

tamo-k Termanu. name established by divination; name of protecting ancestor. (Fox 2016b:53)

tama-f Kotos Amarasi. name someone after a deceased relative.

au tama-k Molo. the name of my grandfather after whom I must be called. (M:536)

Out-comparisons:

<tamu> Kambera. name, namesake. [Note: also in Mangili, Lewa, Anakalng and Mamboru.] (On:464)

<tamo> Kodi. [Note: also in Weyewa]

tamo Ende. name-sake, namefellow.

tamo Tolaki. name. [Note: language of Southeast Sulawesi ISO 639-3 [lbw].] (own field notes) 
san/tamo Kulisusu. namesake; name that two people choose in secret for each other but don't utter in public. [Note: language of Southeast Sulawesi ISO 639-3 [vkl]] [Form: initial sanfrom *isa 'one'.] (David Mead pers. comm. April 2016)

*tamu Morph: *na-tamu. PRM. close the mouth, chew. Etym: *tamu (PCEMP. Blust and Trussel (ongoing) only give Motu tamu-tamu 'smack the lips while eating' and Uruava tamu 'eat' and the Termanu form as evidence for their reconstruction.).

na-tamu (2) tamu tamu Termanu.

1) close the mouth suddenly. bafi $=\mathbf{a}$ na-tamu bafa-n the pig closes its mouth with a smack, kaiboi-k kima=a na-tamu bafa-na the clam suddenly closes itself 2) make a smacking sound while eating, like a pig. (J:593)

na-tamu Korbafo.

na-tamu Bokai.

na-tamu Bilbaa.

na-tamu Rikou.

na-tamu $B a^{\prime} a$.

na-tamu Tii.

na-tamu Dengka.

na-tamu Oenale.

na-tamu Kotos Amarasi. chew.

na-tamu Molo. chews. mu-tamu

koe koe chew your food properly

(M:227)

na-tamu Kusa-Manea. chew.

*tamba1 CER. throw.

tapa Termanu. throw, toss. (J:599)

tapa Korbafo.

tapa Bokai.

tapa Bilbaa.

tapa Rikou.

Out-comparisons:

taba East Tetun. stone, throw stones, chase away with stones; to break into fragments; grind, crush. (Mo:177) toba Bima. trow, hurl, hurl at someone. (Jonker 1893:105)

taha Kamarian. stab, throw. [Note: also in Kaibobo and Nusa Laut.] (van Ekris 1864:119)

kaha Haruku. (van Ekris 1864:119)

tfawa Alune. [Note: language of west Seram, central Maluku ISO 639-3 [alp].] (van Ekris 1864:119)

anna?ba? Makassar. throw a small round object at something (e.g. marble or candlenut). [Form: root $=$ ta?ba?.] (Cense 1979:744)

*tambar PRM. mend, patch. Etym: *tambal (Blust and Trussel (ongoing) reconstruct a number of formally and semantically similar forms including: *tambəj 'tie up, bind tightly', and *tambəl 'patch'.).

tapa Termanu. stick, cleave. (J:599f) tapa Korbafo.

tapa Bokai.

tapa Bilbaa.

tapa Rikou.

tampa $B a^{\prime} a$.

tamba Tii.

tamba Dengka.

tamba Oenale.

n-tapa Molo. binds (wound). [Form: Jonker (1908:600) gives Meto na-ktapa, na-ktape.] (M:539)

Out-comparisons:

tapa Semau Helong. connect, attach, stick to; patch.

tabar Fehan Tetun. join, go/be together with, mix, meet in one place from separate places; patch (clothes).

*tambele PRM. suspend, hang.

pele pele (2) pe pele (3) pele Termanu. 1) to be hung up while spread out. (J:478) 2) hang something up while it is spread out. (J:478) 3) spread out, spread 
(of news or rumour). In ordinary language, this has a physical sense of 'of spreading a piece of cloth, or a sail, of hanging it up'. (Fox 2016b:45)

pele $\sim$ pele Korbafo.

pele pele Bokai.

pele $\sim$ pele Bilbaa.

pele $\sim$ pele Rikou.

mpele $\sim$ mpele $B a^{\prime} a$.

mbele $\sim$ mbele Tii.

mbele mbele (2) na-ta-mbele

Dengka. 1) spread out, suspended.

(J:478) 2) fly. (J:752; Fox 2016b:45)

mbele mbele (2) na-ta-mbele

Oenale. 1) spread out, suspended. (J:478) 2) fly. (J:752; Fox 2016b:45)

na-tpene $R o$ 'is Amarasi. fly (v.).

na-kpene Kotos Amarasi. fly (v.).

na-tpene Amanuban. fly (v.).

Out-comparisons:

tabele East Tetun. hang, dangle; hanging, dangling. (Mo:177)

*tana|? PnMeto. thorn. Etym: **tara-k (pre-Meto).

tana? Kusa-Manea. thorn.

Out-comparisons:

tarak, taran East Tetun. thorn. (Mo:180)

ai taran Kemak. thorn.

$<$ tara $>$ Kambera. thorns, spines; pandanus leaf. (On:482)

karna Kisar. cock's spur.

*tana1 PRM. cover.

ta tana (2) tana Termanu.

1) cover something with a lid, close something. (J:593) 2) put something somewhere so that it is covered up a little. (J:593)

ta tana Korbafo.

ta tana Bokai.

ta tana Bilbaa.

ta $\sim$ tana Rikou.

ta $\sim \operatorname{tana} B a^{\prime} a$.

ta $\sim$ tana Tii.

ta $\sim$ tana Dengka.

ta tana Oenale.

na-taan Meto. (J:593)

\section{Out-comparisons:}

tuya Semau Helong. close, cover. [irr. from PRM: $* \mathrm{a}=u$ correspondence]

ketana Hawu. lid. (J:593)

<tanga> Kambera. lid, cover which fits on top. (On:474)

*tana2 Morph: *tana-k. Rote. crispy, dried out. Etym: **tana (pre-RM).

tana-k Termanu. dry, snappy, crisply baked or fried. (J:593)

tana-? Korbafo.

tana-k Bokai.

tana-? Bilbaa.

tana-? Rikou.

tana-k $B a^{\prime} a$.

tana-k Tii.

tana-? Dengka.

tana-? Oenale.

Out-comparisons:

tana Semau Helong. stiff, withered, crispy, dried out.

*tana3 PMeto. ask, inquire. Doublet: *tane. Etym: *utaña. [History: PRM had two reflexes of *utaña: *tane and *tana. Both are still attested in Molo.] na-tana $R o^{\prime} i s$ Amarasi. ask.

na-tana Kotos Amarasi. ask na-tana Molo. queries. (M:537)

*tane1 Rote. mud. Etym: *tanəq 'earth, soil, land'. [Form: regular*ə> e/_q\#.] tane Termanu. mud. (J:594)

tane Korbafo.

tane Bokai.

tane Bilbaa.

tane Rikou.

tane $B a^{\prime} a$.

tane Tii.

tane Dengka.

tane Oenale.

*tane2 Morph: *na-tane. PRM. ask, inquire. Doublet: *tana. Etym: *utaña. [Sporadic: $* \mathrm{a}>*_{\mathrm{e}} /{ }^{*} \mathrm{C}+$ palatal_.] [History: PRM had two reflexes of *utaña: *tane and *tana. Both are still attested in Molo.]

na-tane Termanu. ask, pose a question, query. (J:594) 
na-tane Korbafo.

na-tane Bokai.

na-tane Bilbaa.

na-tane Rikou.

na-tane $B a^{\prime} a$.

na-tane $T i i$.

na-tane Dengka.

na-tane Oenale.

ma-tane-n Molo. question oneanother. (M:537)

*tanee PRM. contain (liquid).

na-ta-nee Termanu. contain something, both liquids and other things. (J:384f)

na-ta-nee Korbafo.

na-ta-nee Bokai.

na-ta-nee Bilbaa.

na-ta-nee Rikou.

na-ta-nee $B a^{\prime} a$.

na-ta-nee $T i i$.

na-ta-nee Dengka.

na-ta-nee Oenale.

na-tnee Molo. contains. (M:562)

Out-comparisons:

tenae Hawu. contain (liquid).

*tani PnRote. weep, cry; mourn. Etym:

*tanis.

na-ma-tani Termanu. manahelo=a na-ma-tani the poet recited in a complaining tone [Semantics: This form is given without a definition and with a note that in (unspecified) other varieties of Rote the meaning is 'weep'.] (J:595)

na-ma-tani Korbafo.

na-ma-tani Bokai.

na-ma-tani Bilbaa. na-ma-tani Tii.

*tande Morph: *ma-tande, *tande-k. PRM. sharp. Etym: *tazom. [minority from PMP: $*_{\mathrm{z}}>*_{\text {nd }}($ expect $* \mathrm{~d})$; *o $>*_{\mathrm{e}}$ /_\# (expect $*_{\partial}>a$ in wRote, possibly $\left.* \partial>*_{\mathrm{a}}>e\right)$ ] [History: Blust and Trussel only give cognates in Taiwan and western MP languages. This, combined, with the irregular sound changes which must be posited, may indicate that this form is not a direct inheritance from PMP, but a subsequent borrowing.]

ma-tane (2) tane-k (3) na-matane Termanu. 1) sharp, pointy. 2) sharp, pointy; sharpness. 3) be or become sharp; sharpen. (J:594)

ma-tane Korbafo.

ma-tane Bokai.

ma-tane Bilbaa.

ma-tande Rikou.

ma-tane $B a^{\prime} a$.

ma-tande Tii.

tande-? Dengka.

tande-? Oenale.

*tanga PRM. jasmine tree, Indian cork tree. Millingtonia hortensis.

taya Termanu. kind of tree the leaves of which strongly resemble those leaves of the moringa tree Moringa oleifera. [Semantics: 'Kind of tree (with thick bark, fine leaves with white, sweet smelling flowers and excellent hard wood used for building)' (Fox 2016b:54).] (J:595)

tana Korbafo.

taya Bokai.

taya Bilbaa.

tayga $B a^{\prime} a$.

tajga Tii.

tayga Dengka.

tanga Oenale.

$<$ hau taka> Amfo'an. kind of tree. Millingtonia hortensis. [Note: Middelkoop lists this form as occurring in Amfo'an, Beboki, Amarasi and Miomafo. Beboki also has the variant <taeka>. Middelkoop's entry is almost certainly from Meijer Drees (1950:17) who gives $<$ (hau) tàka>.] (M:533)

*tao PRM. put, place, do. Etym: *taRuq 'store, put away for safekeeping, hide valuables; to place a bet in gambling; lay an egg'. [irr. from PMP: ${ }^{*} \mathrm{u}>{ }^{*} \mathrm{o}$ ] 
tao Termanu. set, lay, place, store, put in, place (a bet), etc.; do, act, make, cause. (J:595)

tao Korbafo.

tao Bokai.

tao Bilbaa.

tao Rikou.

tao $B a^{\prime} a$.

tao Tii.

tao Dengka.

tao Oenale.

n-tao Ro'is Amarasi. put.

n-tao Kotos Amarasi. put, do, cast a spell.

n-tao (2) tao-s Molo. 1) sets. 2) deeds. (M:542)

Out-comparisons:

talu Semau Helong. put; guarantee.

tau East Tetun. place, put, set. (Mo:183)

*tara Morph: *ta tara. PRM. adze. Etym: *taRaq 'hewing with an adze'. [minority from $P M P: * \mathrm{R}={ }^{*} \mathrm{r}$ (expect Ø)]

ta tala Termanu. adze, that which is worked with an adze. (J:590)

ta tala Korbafo.

ta tala Bokai.

ta $\sim$ tala Bilbaa.

ta tara Rikou.

ta tala $B a^{\prime} a$.

ta tara Tii.

ta $\sim$ talas Dengka.

ta taras Oenale.

tan tana Meto. (J:590)

*taruku PRM. chiton. Etym: *taduku (own reconstruction) (PCEMP).

Pattern: k-9.

lu?u Termanu. kind of edible mollusc without a shell that is found between rocks in seawater. (J:337)

lu?u Korbafo.

luku Bilbaa.

ruku-? Landu. chiton. [History: Jonker (1908) gives Rikou rutu as potentially cognate, but Landu has sarutu-? = 'sea urchin' which is a more likely cognate for this Rikou form. Thus, Rikou rutu and Landu sarutu-? are probably not reflexes of *taruku.] (own field notes)

lu?u $B a^{\prime} a$.

ru?u Tii.

ru?u Oenale.

tnu?u Ro'is Amarasi. chiton.

Out-comparisons:

kruku Waima'a. sticky sea creature in the rocks by the edge of the sea.

*tadruku Proto-East Oceanic. chiton. (Pawley 2011:197)

*tasa Morph: *ma-tasa-k. PRM. ripe, cooked. Etym: *tasak.

tasa-k (2) na-ma-tasa Termanu.

1) cooked, boiled (e.g. rice).

2) be(come) cooked. (J:601f)

tasa-? Korbafo.

tasa-k Bokai.

tasa-? Bilbaa.

tasa-? Rikou.

tasa-k $B a^{\prime} a$.

tasa-k Tii.

tasa-? Dengka.

tasa-? Oenale.

m|tasa|? (2) me?e m|tasa|?

Kotos Amarasi. 1) cooked, ripe.

2) maroon.

a-m|tasa|? Amfo'an. red.

m|tasa|? Molo. red. (M:332)

Out-comparisons:

tasa Semau Helong. cooked.

tasak, tasan East Tetun. mature, ripe; edible, cooked. (Mo:181)

madasa Dhao. ripe, mature.

*tasi PRM. sea, ocean. Etym: *tasik 'sea, saltwater'.

tasi Termanu. sea, ocean. (J:601)

tasi Korbafo.

tasi Bokai.

tasi Bilbaa.

tasi Rikou.

tasi $B a^{\prime} a$.

tasi Tii.

tasi-? Dengka. 
tasi Oenale.

tasi-? Dela. sea.

tasi Ro'is Amarasi. sea, ocean.

tasi Kotos Amarasi. sea, ocean.

tasi Molo. sea, ocean. (M:540)

tasi Kusa-Manea. sea, ocean.

Out-comparisons:

tasi Semau Helong. sea.

tasi East Tetun. sea, ocean. (Mo:181)

kahi Kisar. salt water, sea.

*tata Rote. clap, beat, hack. Etym:

*tabtab.

tata Termanu. split. tata ai split wood, chop wood (J:602)

tata Korbafo.

tata Bokai.

tata Bilbaa.

tata Rikou.

tata $B a^{\prime} a$.

tata Tii.

tata Dengka.

*tato $n R M$. boy, older sibling. [irr. from

PRM: * $\mathrm{t}>$ ? /V_V in most of Rote]

ta?e_ana-k Termanu. boy, youngster, of about twelve years old. (J:586)

ta?e_ana-? Korbafo.

taPe_ana-k Bokai.

tate_ana? (2) tate Bilbaa. 2) boy. $(\overline{\mathrm{J}}: 769)$

tate_ana? (2) tate Rikou. 2) boy. (J:769)

ta?e_ana-k $B a^{\prime} a$.

taPe ana-k Tii.

tata-f Ro'is Amarasi. same-sex older sibling.

tata-f Kotos Amarasi. same-sex older sibling.

tata-f oli-f (2) an-ma?-oil tata $=$ n

(3) tata-n Molo. 1) older and younger brothers. 2) younger and older brothers or younger and older sisters with respect to one another. 3) older colt or male calf in respect to a later birth. (M:541) tata|? Kusa-Manea. female older sibling. [Note: to?o = "male older sibling'.]

\section{Out-comparisons:}

tate, tata (2) kaka Kisar. 1) older sibling. [irr. from PRM: $* \mathrm{t}=$ $t$ correspondence (expect $k$ )]

2) older siblings.

*tati Rote. cut, chop.

tati Termanu. chop with a machete, cut with a sword. (J:602)

tati Korbafo.

tati Bokai.

tati Bilbaa.

tati Rikou.

tati $B a^{\prime} a$.

tati Tii.

tati Dengka.

tati Oenale.

Out-comparisons:

dati Semau Helong. cut.

*taum Morph: *ka-taum. PRM. indigo plant and dye. Etym: *taRum.

tau-k Termanu. the indigo plant, indigo; also: dark blue. (J:604)

tau-? Korbafo.

tau-k Bokai.

tau-? Bilbaa.

tau-? Rikou.

tau-k $B a^{\prime} a$.

tau-k, tau doo Tii.

tau-? Dengka.

tau-? Oenale.

?|taum Kotos Amarasi. indigo plant, a short tree whose leaves are used to dye cloth black.

$<$ taum> Molo. indigo. Indigofera spec. (M:543)

taum=aa Kusa-Manea . kind of plant mixed with mineral lime. ao to make a black dye.

Out-comparisons:

talun Helong. indigo. Ingofera spec. (Heyne 1950:770, ccxvii)

*taun PRM. year. Etym: *taqun 'year, season'. [irr. from PRM: $* \mathrm{a}>e$ in nRote] [Form: PwRM *toon.] 
teu-k Termanu. year. (J:627)

teu-? Korbafo.

teu-k Bokai.

teu-? Bilbaa.

teu-? Rikou.

teu-k $B a^{\prime} a$.

teu-k Tii.

too(-?) Dengka.

too Oenale.

toon Ro'is Amarasi. year.

toon Kotos Amarasi. year.

toon Molo. year. (M:570)

toan Kusa-Manea. year. [irr. from PRM: *o > a] [Form: Kusa-Manea toan may not be a direct inheritance from PRM *taun. Instead, it may be a borrowing from Tetun tonan 'year'. If so, the form here would be the metathesised form of (currently unattested) *tona.]

\section{Out-comparisons:}

taun Semau Helong. year.

*tea PRM. arrive, until, the point that. [irr. from PRM: *a > $e$ in all Rote and some Meto (sporadic assimilation)] [Form: I have reconstructed final $*$ a rather than $*$ e as $* \mathrm{a}>e$ can be motivated as sporadic assimilation while the reverse sound change would be unmotivated.] tee Termanu. come, arrive. (J:607)

te?e Keka. reach. (J:770)

tee Korbafo.

tee Bokai.

tee Bilbaa.

tee Rikou.

tee $B a^{\prime} a$.

tee Dengka.

n-tea Ro'is Amarasi. arrive, until, to the point that.

n-tea, n-tee Kotos Amarasi. arrive, until, to the point that. Usage: tea has 36 examples in my corpus while tee has 29 examples.

n-tee, n-tia Molo. enough, arrives. (M:544, 554)

n-tee, ntea Timaus. arrive, until, to the point that. tea Kusa-Manea. arrive.

\section{Out-comparisons:}

to?o East Tetun. arrive, reach; suffice, be enough; enough, sufficient; to, until, as far as. (Mo:188)

tii Waima'a. until.

*teas PRM. heartwood of a tree, hard, durable core of wood; ironwood tree. Etym: *təRas. [Sporadic: *a $>e / \#$ in wRM.]

tea, teas Termanu. core of wood. (J:608)

tea Korbafo.

tea Bokai.

tea Bilbaa.

tea Rikou.

tea $B a^{\prime} a$.

tea Tii.

tee-? Dengka.

tee-? Oenale.

teas Kotos Amarasi. hard centre of tree trunk.

teas, tees Molo. hard core of treetrunk. (M:544)

teas Kusa-Manea. hard.

\section{Out-comparisons:}

telas Semau Helong. beam, strong. Sense of structurally sound, not corrupted by mould or rot.

toos East Tetun. hard, durable; stiff, difficult to open; stubborn. (Mo:188)

*tebes PRM. true. [irr. from PRM: *6 $>$ ? in Termanu and Bokai]

(te?e ) te?e Termanu. in truth, in reality, truly. (J:77)

(tebe )tebe Korbafo.

(te?e )te?e Bokai.

(tebe $)$ tebe, te $\sim$ tebe-? Bilbaa.

(tebe $\sim$ )tebe, te $\sim$ tebes $=$ a Rikou .

(tebe $\sim$ )tebe, te $\sim$ tebes $=\mathbf{a} B a^{\prime} a$.

(tebe )tebe, te $\sim$ tebe-k Tii.

(tebe $\sim$ )tebe, te $\sim$ tebes $=$ a Dengka.

(tebe $\sim$ )tebe, te $\sim$ tebes $=$ a Oenale.

te tebes (2) tebe tebes Dela.

1) true. 2) truly. 
tebe (2) na-?tebe Kotos Amarasi. 1) true, earnest. 2) true, earnest.

teeb (2) < nateb> Molo. 1) yes, it is true. 2) confirms, accords. (M:544)

\section{Out-comparisons:}

tebes Semau Helong. true, right.

tebes East Tetun. certainly, truly, in truth. (Mo:183)

*tebi PRM. break into pieces. Etym: *təbiq 'split off, break off a piece, as in breaking off a section of betel nut' (PWMP). [minority from PMP: *b > $*_{6} / \mathrm{V}_{-} \mathrm{V}$ ] [irr. from PRM: ${ }^{*} \mathrm{~b}>b \sim$ ? in Termanu, Korbafo and Bokai]

tebi (2) te?i Termanu. 1) chipped, crumbled at the edge. (J:608) 2) break something, break into pieces with the fingers. (J:613)

tebi (2) te?i Korbafo.

tebi (2) te?i Bokai.

tebi Bilbaa.

tebi (2) tebi Rikou.

tebi $B a^{\prime} a$.

tebi Tii.

tebi Dengka.

n-tebi Kotos Amarasi. break up into pieces (e.g. bread).

n-tebi Molo. crumble into pieces. (M:544)

\section{Out-comparisons:}

teben Semau Helong. stubby, short, chop. [irr. from PRM: *i $=e$ correspondence]

tohi(k) East Tetun. chip off little pieces. (Mo:187)

*tede Rote. crush with fingernail. Etym: *tindos 'crush lice with the fingernails'. [irr. from PMP: *nd > $*_{\mathrm{d}} ; *_{\mathrm{i}}>*_{\mathrm{e}}$ (also in eastern varieties of Malay)] [minority from PMP: *o $>*_{\mathrm{e}}$ /_\# (expect *ə> $>a$ in wRote, possibly $\left.*_{\partial}>*_{\mathrm{a}}>e\right)$ ] [Form: Some eastern Malays (e.g. Kupang Malay, Ambon Malay) have tendes 'press' also with $*_{\mathrm{i}}$ $>$ e. Hawu also shows $* \mathrm{i}>e$.] tede Termanu. flatten, whether between the fingernails or between a fingernail and a hard object. (J:609)

tede Korbafo.

tede Bokai.

tede Bilbaa.

tede Rikou.

tede $B a^{\prime} a$.

tede Tii.

tede Dengka.

tede Oenale.

Out-comparisons:

təda Hawu. (J:609)

<tidihungu> Kambera. press, press down. <tidihungu wutu $>$ crush lice (On:493)

$<$ tiduhungu $>$ Lewa.

$<$ tiḍasungu $>$ Anakalang.

$<$ tede $>$ Weyewa.

$<$ katidihyo $>$ Kodi.

*tee Rote. spear. Etym: **təRə (pre-RM).

tee Termanu. spear. (J:605)

tee Korbafo.

tee Bokai.

tee Bilbaa.

tee Rikou.

tee $B a^{\prime} a$.

tee Tii.

tee Dengka.

tee Oenale.

\section{Out-comparisons:}

kere Kisar. spear.

tera Wetan. spear. [Note: language of southwest Maluku, member of Luang language/dialect cluster ISO 639-3 [lex.]] (de Josselin de Jong 1987)

too Welaun. spear.

*tefe CER. broken, tired.

tefe Termanu. weary (actually: 'broken'). tefe basa au luyu laya so $\sim$ solu-n my knees and my shins are very weary (literally: broken), I'm very tired (J:612)

tefe Korbafo.

tefe Bokai. 
tefe Bilbaa.

tefe Rikou. piece, broken. (J:770)

Out-comparisons:

tehen Semau Helong. break apart, snap.

*tefu PRM. sugarcane. Saccharum officinarum. Etym: *təbuh.

tefu Termanu. sugarcane. (J:612)

tefu Korbafo.

tefu Bokai.

tefu Bilbaa.

tefu Landu. sugarcane. (own field notes)

tefu Rikou.

tefu $B a^{\prime} a$.

tefu Tii.

tefu Dengka.

tefu Oenale.

tefu Ro'is Amarasi. sugarcane.

tefu Kotos Amarasi. sugarcane.

tefu Molo. sugarcane. Saccharum afficinarum. (M:545)

tefu Kusa-Manea. sugarcane.

Out-comparisons:

tihu Semau Helong. sugarcane. touhu East Tetun. sugarcane.

Saccharum officinarum. (Mo:189)

keu Kisar. sugarcane.

*teka PRM. call, greet. Pattern: k-10.

na-te?a Termanu. greet in passing, say goodbye. (J:608)

na-te?a Korbafo.

na-te?a Bokai.

na-teka Bilbaa.

na-te?a Rikou.

na-te?a $B a^{\prime} a$.

na-te?a Tii.

na-tea Dengka.

na-tea Oenale.

n-teka Ro'is Amarasi. call, refer to as.

n-teka Kotos Amarasi. call, refer to as.

n-teka (2) <a'teka> (3) <nateka $>$ Molo. 1) names. 2) riddle. 3) someone tells a riddle. (M:546)

Out-comparisons:

teka Semau Helong. tell, inform. *teke Morph: *ka-teke. PRM. gecko. Etym: *təktək. Pattern: k-5. [minority from PMP: *ə > *e / \# (expect *ə > $a$ in wRM, possibly $*^{*}>* \mathrm{a}>e$ in wRM)] [Semantics: onomatopoeia.] teke Termanu. gecko. (J:614) teke Korbafo.

teke Bokai.

teke Bilbaa.

te?e Rikou.

teke $B a^{\prime} a$.

teke Tii.

teke Dengka.

teke Oenale.

teke Ro'is Amarasi.

?|teke Kotos Amarasi. gecko.

?|teke Molo. kind of tree lizard. (M:547)

Out-comparisons:

ktoke? Funai Helong. calling gecko. [irr. from PMP: *ə $>o$ (expect $e$ )]

toke? Semau Helong. gecko.

teki East Tetun. gecko lizard often found living in houses. [irr. from PMP: *o $>e($ expect $o)]$ (Mo:183)

*tekə PRM. staff, walking stick. Etym: *təkən 'downward pressure; bamboo punting pole'. Pattern: k-8.

te $\sim$ tePe-k (2) te $\sim$ tePe Termanu.

1) staff, walking stick; the use of a walking stick. (J:610f) 2) use a walking stick.

te $\sim$ te?e-? Korbafo.

te te?e-k Bokai.

te teke-? Bilbaa.

te te?e-? Rikou.

te $\sim$ te?e ai-k $B a^{\prime} a$.

te $\sim$ te?e ai Tii.

te tea-s Dengka.

te $\sim$ tea-s Oenale.

te tea-s Dela. walking stick, staff.

tea|s Kotos Amarasi. walking stick, staff.

tee|s, tea|s Molo. staff. (M:544, 552) 
Out-comparisons:

tnikan Funai Helong. staff.

tikan (2) hnikan Semau Helong.

1) support, use a walking stick.

2) staff, walking stick, rod.

ai kato?an Welaun. staff, pole.

*telu PRM. three. Etym: *təlu.

telu Termanu. three. (J:615)

telu Korbafo.

telu Bokai.

telu Bilbaa.

telu Rikou.

telu $B a^{\prime} a$.

telu Tii.

telu Dengka.

telu Oenale.

tenu Ro'is Amarasi. three.

tenu Kotos Amarasi. three.

tenu Molo. three. (M:550)

Out-comparisons:

tilu Semau Helong. three.

tolu East Tetun. three. (Mo:183)

telu Waima'a. three.

wokelu Kisar. three.

*tema1 CERM. eagle. [Sporadic: *a $>e$ /_\# in Meto.]

te-tema Termanu. kite (bird); fly down on something like a kite. (J:617)

te -tema Korbafo.

te tema Bokai.

te tema Bilbaa.

te tema Rikou.

teme Ro'is Amarasi. kite.

teme Kotos Amarasi. eagle.

teme Molo. hawk. (M:548)

Out-comparisons:

tem/lusi Helong. (J:617)

*tema2 Morph: *ka-tema-k,

*teme teme. PRM. whole, entire.

[Sporadic: *a > e/\# in wRM.]

tema tema (2) ka-tema-k

Termanu. 1) intact, whole, in its entirety. 2) intact, entirely. (J:616)

tema tema (2) ka-tema-? Korbafo.

tema tema (2) ka-tema-k Bokai.

tema tema (2) ka-tema-? Bilbaa. tema tema (2) ka-tema-? Rikou.

tema tema (2) ka-tema-k $B a^{\prime} a$.

tema tema (2) ka-tema-k Tii.

teme teme, teme-? Dengka.

teme teme, teme-? Oenale.

2|teme Kotos Amarasi. closed, sealed; entire, whole.

<teme> Molo. inaccessible (forest), virgin, virginal, full (moon), receptive (heart). (M:548)

Out-comparisons:

yae ktema? Funai Helong. cooked corn.

tema Semau Helong. whole.

naktomak East Tetun. be complete; completed, entire. (Mo:188)

ke keme Kisar. whole.

tema Ili'uun. all, together, whole, complete. (dJ:138)

ketəme Hawu. whole. (J:616)

təmak, təmaワ Sika. unbroken, whole. (Pareira and Lewis 1998:193)

*temə Morph: *na-temə. PRM. accustomed to. Etym: *təmən. (Dempwolff 1938:135) (Reconstructed with final $*$ a, but this seems unable to account for the reflexes of the final vowels in a number of languages. Blust and Trussel (ongoing) list cognates under their 'noise' section.).

na-teme Termanu. used to, accustomed to, usual. (J:618)

na-teme Korbafo.

na-teme Bokai.

na-teme Bilbaa.

na-teme Rikou.

na-teme $B a^{\prime} a$.

na-teme $T i i$.

na-tema Dengka.

na-tema Oenale.

na-teem Meto. (J:618)

Out-comparisons:

teman Helong. (J:618)

toman East Tetun. be in the habit of, accustom. (Mo:188) 
tima Hawu. often, normally, customarily.

*temba Rote. sardine. Etym: *tamban (PWMP). [irr. from PMP: $* \mathrm{a}>*_{\mathrm{e}}$ ] [History: Possibly a borrowing from Malay tembay. Jonker also gives Makassar and Bugis tembay.]

iPa tepa Termanu. kind of small ocean fish that is like a sardine, called ikan tembang in Kupang. (J:623)

tepa Korbafo.

tepa Bokai.

tepa Bilbaa.

tepa Rikou.

tempa $B a^{\prime} a$.

temba Dengka.

temba Oenale.

*tena1 PRM. sink, submerge. [Note: Blust and Trussel (ongoing) reconstruct *təñəj 'sink, set (sun)' on the basis of the Termanu reflex (glossed 'sink, set (of the sun)') and a Cebuano reflex. But a regular reflex Termanu of this form would have a final $e$ rather than a.]

tena (2) tena-k (3) te tena-k (4) na-tena (5) na-ka-tena-k Termanu. 1) sinking, usually said of a ship when it is full of water; sink (transitive). 2) more definitely: drown. 3) sinking, etc.; pit, in which something has to sink, e.g. animals to catch them. 4) sink (transitive). 5) lower (something down). (J:619)

tena (2) tena-? Korbafo.

tena (2) tena-k Bokai.

tena (2) tena-? Bilbaa.

tena (2) tena-? Rikou.

tena (2) tena-k $B a^{\prime} a$.

tena (2) tena-k Tii.

tena (2) tena Dengka.

tena Oenale.

n-tena $R o$ 'is Amarasi. sink slowly like the sun.

n-tena Kotos Amarasi. sink slowly, like things in water.

\section{Out-comparisons:}

denes (2) dene Semau Helong.

1) submerge, drown. 2) set, go down. [irr. from PRM: *t $=d$ correspondence; $* \mathrm{a}=e$ correspondence]

*tena2 PRM. alight, land. [irr. from PRM: $* \mathrm{t}>n$ in Kotos Amarasi] [Sporadic: ${ }^{* a}$ $>e$ /_\# in Meto]

tena Termanu. alight, touch the ground, land on the ground; also, be landed (of a bird). (J:619)

tena Korbafo.

tena Bokai.

tena Bilbaa.

tena Rikou.

tena $B a^{\prime} a$.

tena Tii.

tena Dengka.

n-nene Kotos Amarasi. press, land. tene Meto. (J:619)

*tenas PnMeto. calm down, quieten. Etym: *tənəy 'calm, still, as the surface of water' (PWMP).

na-?|tena|? Kotos Amarasi. calm down, quieten.

<na-tena $>$ Molo. become quiet. (M:548)

Out-comparisons:

tene Semau Helong. stop, cease.

*tene PRM. kind of mangrove, with bark used for dyeing. Ceriops species. Etym: *təyəR. [irr. from PMP: *ə > *e (expect $* \partial>a$ in wRM, possibly *ə $>$ $* \mathrm{a}>e$ in wRM)]

tene Termanu. kind of tree that grows on the beach, it has good wood and yields a red dye. (J:620)

tene Korbafo.

tene Bokai.

tene Bilbaa.

tene Rikou.

tene $B a^{\prime} a$.

tene Tii.

tene Dengka.

tene Oenale. 
tene Molo. small tidal forest tree. Ceriops tagal. (M:549)

*teni PMeto. again.

n-teni? Ro'is Amarasi. again.

teni?, n-teni Kotos Amarasi. again.

n-teni Molo. again. (M:549)

Out-comparisons:

teni East Tetun. again, afresh. (Mo:184)

teni Waima'a. again.

*tenu PRM. weave (cloth, baskets). Etym: *tonun.

tenu Termanu. weave. (J:623)

tenu Korbafo.

tenu Bokai.

tenu Bilbaa.

tenu Rikou.

tenu $B a^{\prime} a$.

tenu Tii.

tenu Dengka.

tenu Oenale.

n-tenu Kotos Amarasi. weave.

n-tenu Molo. weave. (M:550)

Out-comparisons:

tinu Semau Helong. weave.

kenna Kisar. weave (cloth).

*tendə Morph: *tendə-k. PRM. ribcage, lungs.

tene-k Termanu. the ribcage of a pig, the ribs together. (J:621)

tene-? Korbafo.

tene-k Bokai.

tene-? Bilbaa.

tende dui-? Landu. ribs. (own field notes)

tende-? Rikou.

tere-? Oepao. ribs. (own field notes)

tene-k $B a^{\prime} a$.

tende-k Tii.

tenda-? Dengka. chest. (J:771)

tenda-? Oenale. chest. (J:771)

tenda-? Dela. chest.

tere-f $R o^{\prime}$ is Amarasi. lungs.

teka-f Kotos Amarasi. lungs.

teek noo-n (2) teek fua-n

Amanuban. 1) lungs. 2) heart.

teka-n Amanatun. heart. teka-k Amfo'an. heart.

teka-n Molo. heart muscle. (M:546)

*tenga PnRote. hand span. See: *hanga.

tena Termanu. span; measure with spans. (J:621)

tema Korbafo.

tena Bokai.

teya Bilbaa.

teka Rikou.

tenga $B a^{\prime} a$.

tenga Tii.

*teri Morph: *oo_teri-k. Rote. giant bamboo. Dendrocalamus species. Etym: *tərin 'bamboo species'.

oo_teli-k Termanu. the biggest kind of bamboo. (J:615)

oo_teli-? Korbafo.

oo_teli-k Bokai.

oo_teli-? Bilbaa.

oo_teri-? Rikou.

oo_teri-k Tii.

oo_teli-? Dengka.

oo_teri-? Oenale.

*tesa $\bar{C} E R M$. setting (of sun/moon).

tesa Bilbaa. the setting of the sun or moon. (J:771)

neon n-tees Kotos Amarasi. west. Lit: 'sun sets'.

n-tesan Molo. decline. (M:552)

*teta PRM. cut into small pieces, mince.

[Sporadic: *a $>$ e/_\# in Molo.]

te $\sim$ teta-ta $\sim$ tata Termanu. cut into small pieces, mince. (J:625)

te teta-ta tata Korbafo.

te teta-ta tata Bokai.

te teta-ta tata Bilbaa.

te teta-ta tata Rikou.

te teta-ta tata $B a^{\prime} a$.

te teta lutu? Dengka.

te $\sim$ teta-ta $\sim$ tata Oenale.

n-teta Kotos Amarasi. cut across something, dismantle, separate.

n-tete Molo. minces. (M:553)

Out-comparisons: teta (2) tetas Semau Helong. 1) cut off, amputate, chop off, lop off. 2) cut. 
tetak East Tetun. crumble into pieces; to chop at with a cutting tool. (Mo:184)

*tete Morph: *ka-tete. CERM. dam, dyke. [Semantics: I have reconstructed the meaning 'dam, dyke' as this accounts for the semantics in both Hawu and the Rote lects. The shift to 'reef' appears to have spread by contact between Bokai, Rikou, Amarasi and Helong (with subsequent shift of 'reef' to 'ridge' in Amarasi). This scenario seems more likely than the alternate in which shift to 'dam, dyke' occurred in Hawu and other Rote lects. Note also that Jonker (1908:670) also gives unu-k/-? as 'reef which is visible at low tide' for all Rote languages (including Oepao) except Bokai and Rikou.]

tete (2) na-ka-tete Теrmanu. 1) dyke, dam. 2) dam up. (J:626) tete Korbafo.

- (2) - (3) tete-k Bokai. 3) reef that is visible at low tide. ( $\mathrm{J}: 772)$

tete Bilbaa.

tete (2) - (3) tete-? Rikou. 3) reef which is visible at low tide. (J:772)

k|tete|? Kotos Amarasi. ridge.

Out-comparisons:

teten Semau Helong. reef.

titi Hawu. dam. (J:626)

*tetu PRM. upright, midday. [Form: The reflexes meaning 'midday' outside of RM reflect something like $* *$ dətu or **ndətu.]

na-tetu (2) ledo=a na-matetu (3) tetu tetu (4) tetu-k Termanu. 1) upright, put upright, put something the right way up. 2) the sun stands high, it is midday. 3) completely upright. 4) in metaphorical senses: perfect, completely in order; perfection. (J:626f)

na-tetu (2) na-ma-tetu Korbafo. na-tetu (2) na-ma-tetu Bokai. na-tetu (2) na-ma-tetu Bilbaa. na-tetu (2) na-ma-tetu Rikou. na-tetu (2) na-ma-tetu $B a^{\prime} a$.

na-tetu (2) na-ma-tetu Tii.

na-tetu (2) na-ma-tetu Dengka.

na-tetu (2) na-ma-tetu Oenale.

na-tetu (2) na-m|tetu Kotos

Amarasi. 1) stand upright.

2) upright.

na-tetu (2) manas na-m|tetun Molo. 1) is upright, stands upright.

2) the sun is in its zenith. (M:553)

Out-comparisons:

titu (2) lelo ditu Semau Helong.

1) straight. 2) midday.

loro natutun East Tetun. noon. (Mo:133)

nətu lodo Hawu. midday.

ləro dətu (2) dətū Sika. 1) midday. 2) flat area. (Pareira and Lewis 1998:37)

(.əra) rətu (2) ndətu Ende. 1) midday. (J:626) 2) flat place, level ground.

*teu Morph: *ka-teu. PRM. pigeon, dove. Etym: **lakateRu (pre-RM).

ka|teu Termanu. kind of pigeon, wild pigeon. (J:223)

ka|teu Korbafo.

ka|teu Bokai.

ka|teu Bilbaa.

ka|teu Rikou.

ka|teu $B a^{\prime} a$.

teu Tii.

teu Dengka.

teu Oenale.

too/tiu Kotos Amarasi. kind of bird like a White-necked Myna. [Sporadic: vowel height harmony $* \mathrm{e}>i$ / u.]

Out-comparisons:

tilu? Semau Helong. dove, pigeon.

lakateu East Tetun. dove. (Mo:124)

laktyeru Leti. turtle-dove. [Note: language of southwest Maluku, member of Luang language/ dialect cluster ISO 639-3 [1ti].] (van Engelenhoven 2004:419) 
lakateun Kamarian. turtle-dove. [Note: also in Kaibobo and Haruku.] (van Ekris 1864:104)

rakateun Asilulu. [Note: also in Lusa Laut.] (van Ekris 1864:104)

*təlo Morph: *təlo-k. PRM. egg. Etym: *qatəluR 'egg; testicle'. [irr. from PRM: *ə $>o$ in nRote; $* 1>n \sim k$ in Nuclear Meto (perhaps partly via intermediate irregular PMeto $*_{1}>*_{\mathrm{r}}$ before $*_{\mathrm{r}}>k$ )] [Sporadic: $*_{\mathrm{u}}>*_{\mathrm{o}}$ /_R\#.]

tolo-k Termanu. egg. (J:641)

tolo-? Korbafo.

tolo-k Bokai.

tolo-? Bilbaa.

tolo-? Landu. egg. (own field notes)

tolo-? Rikou.

tolo-k $B a^{\prime} a$.

tolo-k Lole. egg. (Zacharias et al. 2014)

tolo-k Tii.

telo-? Dengka.

telo-? Oenale.

teno|? Ro'is Amarasi. egg.

teno|?, teko|? Kotos Amarasi. egg.

teko|? Molo. egg. (M:550)

teno|? Kusa-Manea. egg.

Out-comparisons:

tilun Semau Helong. egg.

tolu-n (2) tolon East Tetun. 1) an egg. 2) the germ of seeds. (Mo:188)

thelu Waima'a. egg.

telon Kemak. egg.

*tiam PRM. oyster. Etym: *tiRəm. [irr. from $P M P: * \partial>* a$ (expect $* \partial>e$ in nRote)]

ti tia-k Termanu. oyster, including the creature, also ti $\sim$ tia isi-k for the creature and also ti - tia lou-k for the shell. (J:629)

ti - tia-? Korbafo.

ti tia-k Bokai.

ti tia-? Bilbaa.

ti tia-? Rikou. ti tia-k $B a^{\prime} a$.

ti tia-k Tii.

ti tia-? Dengka.

ti tia-? Oenale.

tiam Meto. (J:629)

Out-comparisons:

tian Helong. (J:629)

*tiba Morph: *ka-tiba-k. PRM. bamboo container.

tiba-k Bokai.

tiba-k $B a^{\prime} a$. bamboo container. (J:772)

tiba-k Tii.

tiba-? Dengka.

tiba-? Oenale.

?|tiba|? Kotos Amarasi. small tube shaped container for mineral lime.

*tido $P R M$. kind of tuber.

tido (2) tido-k Termanu. 1) kind of plant with oblong, egg-shaped fruits. 2) ina tei tido-k a woman with a slim figure (J:629)

tido (2) tido-? Korbafo.

tido (2) tido-k Bokai.

tido (2) tido-? Bilbaa.

tido (2) tido-? Rikou.

tido (2) tido-k $B a^{\prime} a$.

tido (2) tido-k Tii.

tido (2) tido-? Dengka.

tido (2) tido-? Oenale.

tiro|k Kotos Amarasi. kind of wild tuber that cannot be eaten, it causes an itch.

$<$ tilo $>$ Molo. kind of sweet potato with a stem which sticks up. (M:557)

*tii Morph: *tii-k. Rote. sea urchin.

See: *k|teom. Etym: **tiRi (pre-

RM). [Note: Although the reflexes here are similar to those under *k|teom (**tayum), they cannot be straightforwardly combined as the sound correspondences are not regular and both Tetun forms tii and teon would be unexplained if this were done.] [History: Possibly connected with PWMP *təRi 'kind of small fish', but the semantic shift seems unlikely.] 
tii-k Termanu. kind of small sea creature with long spines differentiated into tii hade-k, a white kind, and tii bete-k, a red kind. (J:628)

tii-? Korbafo.

tii-k Bokai.

tii-k $B a^{\prime} a$.

tii-k Tii.

tii-? Dengka.

tii-? Oenale.

tii-? Dela. sea urchin.

Out-comparisons:

tii East Tetun. sea urchin. Echinus esculenta. (Mo:185)

tiri Fordata. sea urchin. [Note: language of the Tanimbar Islands ISO 639-3 [frd].] (Drabbe 1932:175)

tir Kei. sea urchin. Echinus esculentus. [Note: language of the Kei Islands ISO 639-3 [kei].] (Geurtjens 1921)

*ti(?)o Rote. goatfish, family Mullidae. Etym: *tiqaw. [Form: Whether or not the Termanu form attests a medial glottal stop affects whether this should be reconstructed or not. Similarly, depending on the form of possible cognates in other Rote languages it may be possible to posit medial ${ }^{*} \mathrm{~h}$ instead of *?.]

tio (2) iPa ti?o Termanu. 1) kind of small but ritually important fish; Bar-Tail Goat Fish. Mullidae: Upeneus tragula. (Fox 2016b:56)

2) kind of ocean fish. (J:632)

*tila Morph: *tila-k. PRM. vagina. Etym: *tila.

tila-k Termanu. vagina. (J:630)

tila-? Korbafo.

tila-k Bokai.

tila-? Bilbaa.

tila-? Rikou.

tila-k $B a^{\prime} a$.

tila-k Tii.

tila-? Dengka. tila-? Oenale.

tini-f $R o$ 'is Amarasi. vagina.

tina-f Kotos Amarasi. vagina.

tina|? Molo. vagina, private parts of a woman or female animal. (M:558)

*timi Morph: *timi-k. PRM. chin, jaw. Etym: *timid. [irr. from PRM: $* \mathrm{i}>u$ in

Amanuban and Kusa-Manea]

timi-k Termanu. chin, jaw. (J:630)

timi-? Korbafo.

timi-k Bokai.

timi-? Bilbaa.

timi dai-? Landu. chin. (own field notes)

timi-? Rikou.

timi-k $B a^{\prime} a$.

timi-k Tii.

timi-? Dengka.

timi-? Oenale.

tai_timu-n Amanuban. chin.

tool timi-n Timaus. chin.

timi-f Meto. (J:630)

ta timu-f Kusa-Manea. chin.

Out-comparisons:

timir East Tetun. beard, whiskers, chin. (Mo:186)

*timu1 CER. east wind. Etym: *timuR.

ani timu Termanu. east wind. (J:630)

*timu2 Morph: *ka-timu-k. PRM. cucumber. Cucumis sativa L. Etym: *qatimun.

ti -timu-k Termanu. papaya (tree and fruit). (J:630)

ti - timu-? Korbafo.

ti-timu-? Rikou.

ti timu-k $B a^{\prime} a$.

(ti timu-k ?) Tii.

ti timu-? Dengka.

ook_tiumu|k Ro'is Amarasi. cucumber.

oka_?|timu|k Kotos Amarasi. cucumber.

ook_<timo>, okan_<timo> Molo. watermelon. (M:401)

Out-comparisons: saah-timun, tium-takan Helong. (J:630) 
*tina Rote. dry field which is replanted every year. Etym: **tinaR (Mills 2010:285). [irr. from PRM: *a $>e$ in Termanu, Korbafo, Rikou, Oenale, and Dela]

tina, tine Termanu. dry field or plantation that is cleaned and replanted every year. (J:630)

tina, tine Korbafo.

tina Bokai.

tina Bilbaa.

tine Rikou.

tina $B a^{\prime} a$.

tina Tii.

tine Dengka.

tine Oenale.

Out-comparisons:

tinan East Tetun. year, the commencement of the rainy season (usually in November) to the beginning of the next rainy season. (Mo:186)

kirna Kisar. garden.

tiran Roma. garden. (Steven 1991:51)

tina West Damar. garden. [Note: language of southwest Maluku ISO 639-3 [drn].] (Chlenov and Chlenova 2008:145)

ti-ol Dawera-Daweloor. garden. [Note: language of the Babar Islands, southwest Maluku ISO 639-3 [ddw].] [Form: regular $\left.*_{y}>\varnothing\right]$ (Chlenova 2002:170)

tikan Welaun. year.

*tino PRM. peer, mirror. Doublet: *tiro. Etym: *tindaw 'see in the distance'. [Note: I have placed the Meto forms here rather than under *tiro as the medial $*_{n}$ provides a potential motivation for irregular initial $* \mathrm{t}>n$.] [irr. from PMP: *nd $>* \mathrm{n}$ ] [irr. from PRM: $* \mathrm{t}>n$ in Meto]

ti tino (2) ti tino-k (3) ti tino-k

(4) ti tino Termanu. 1) peep, peer, e.g. through a hole, but also peering in general. 2) mirror. 3) peeping, peering, peeking. 4) go see, go visit. (J:631f)

ti tino (2) ti - tino (4) ti $\sim$ tino Korbafo.

ti-tino (2) ti tino-k (4) ti-tino Bokai.

ti tino (2) tino_ao (4) ti - tino Bilbaa.

ti tino (2) tino_ao (4) ti $\sim$ tino Rikou.

ti tino (2) (ti-tino-s ?) (4) ti - tino $B a^{\prime} a$.

ti tino (4) (ti -tino ?) Tii.

ti-tino Oenale.

?|ninu|? Kotos Amarasi. glass. [Sporadic: vowel height harmony $*_{\mathrm{o}}>u / \mathrm{iC}$ in Amarasi.]

?nino? (2) noe ?nino?, koe ?nino? Molo. 1) mirror. 2) the clear river. (M:371)

*tinga Morph: *tinga-k, *na-tinga. PRM. heel. Etym: *tikəd. [irr. from PMP: *k $>$ *ng; *ə > *a]

ei_tina-k (2) na-tina (3) tina Termanu. 1) heel. 2/3) put the heels down firmly or put them in something. [Semantics: The meanings for na-tina and tina are given as identical.] (J:631)

ei_tina-? (2) na-tina Korbafo.

ei_tina-k (2) na-tina Bokai.

ei_tina-? (2) na-tina Bilbaa.

ei tika-? (2) na-tika Rikou.

ei_tinga-k (2) na-tinga $B a^{\prime} a$.

ei_tinga-k (2) na-tinga Tii.

ei_tinga-? (2) na-tinga Dengka.

2a) put the heels down firmly or put them in something. 2b) kick.

ei_tinga-? (2) na-tinga Oenale. 2a) put the heels down firmly or put them in something. 2b) kick.

tiki-f Ro'is Amarasi. heel.

tika-f (2) na-tika Kotos Amarasi. 1) heel. 2) kick or stamp with the heel. 
tika-n (2) <an-tika loto $>$ (3) $<$ bikase> na-tiik Molo. 1) heel. 2) tumbles over the ground. 3) the horse kicks backwards. (M:556)

*tiri Morph: *tiri tiri. Rote. drip. Etym: *tiRis 'drip, ooze through, leak'. [minority from PMP: $* \mathrm{R}={ }^{*} \mathrm{r}$ (expect Ø)]

tili tili Termanu. flow by drops. (J:630)

tili tili Korbafo.

tili tili Bokai.

tili tili Bilbaa.

tiri - tiri Rikou.

tili tili $B a^{\prime} a$.

tiri tiri Tii.

tili tili Dengka.

tiri tiri Oenale.

*tiro Rote. mirror, visit. Doublet: "tino.

Etym: *tindaw 'see in the distance'.

[irr. from PMP: *nd $>*$ r]

tiro ao Rikou. mirror. (Nako et al. 2014)

ti tilo-s $B a^{\prime} a$. mirror. (J:632)

tilo Lole. look in on. (Zacharias et al. 2014)

ti tiro (2) tiro Tii. 1) mirror. (J:632)

2) visit. (Grimes et al. 2014a)

ti tilo_ao Dengka. mirror. (J:632)

tiro_ao Oenale. mirror. (J:632)

*tisa $n R M$. pour. [irr. from PRM: *a $>e$ in

Bokai, Tii and Rikou; *a $>i$ in Bilbaa and Meto]

ti -tisa-k Termanu. the overhanging part of the roof from which water drips. (J:633)

ti tisa-? Korbafo.

ti tise-k Bokai.

ti tisi-? Bilbaa.

ti tise-? Rikou.

ti tisa-k $B a^{\prime} a$.

ti $\sim$ tise-k Tii.

n-tisi (2) na-tisi (3) na-m|tisi Kotos Amarasi. 1) pour. 2) fill, complete. 3) complete.

n-tesi, n-tosi, n-tisi (2) na-m|tisi Molo. 1) pour. 2) complete. (M:559)

\section{Out-comparisons:}

tiis Semau Helong. pour.

tisi East Tetun. empty, spill, pour (liquids). (Mo:186)

*titi Rote. drip. Etym: *titis 'drip, ooze'.

titi Termanu. drip. (J:633)

titi Korbafo.

titi Bokai.

titi Bilbaa.

titi Rikou.

titi $B a^{\prime} a$.

titi Tii.

titi Dengka.

*tobi PRM. hot, heated up. [irr. from PRM: * $\mathrm{i}>e$ in Meto]

ma-tobi-k (2) na-tobi (3) tobi Termanu. 1) hot. 2) be hot. 3) burn, scorch. (J:637)

ma-tobi-? Korbafo.

ma-tobi-? Bilbaa.

ma-tobi-? Rikou.

ma-tobi-k $B a^{\prime} a$.

ma-tobi-k Tii.

ma-tobi-? Dengka.

ma-tobi-? Oenale.

na-tobe Meto. steam cooked. (J:637)

*todi PRM. protuberance, stick out. [Sporadic: consonant metathesis * $\mathrm{tVd}$ $>d V t$ in $\mathrm{Ba}$ a for the first sense; vowel height harmony $*_{\mathrm{i}}>e / \mathrm{oC}$ for first sense in nRote and Meto.] [Form: The Meto forms are Historic compound of *ka-ndou 'nape of the neck' + *todi.] tode (2) todi (3) nisi todi-k Termanu. 1) stick out lengthwise. 2) stick out (said of teeth). 3) tooth that sticks out. (J:637)

tode (2) todi Bokai. 1) stick out lengthwise. 2) stick out (said of teeth).

(2) todi Bilbaa.

dote (2) todi $B a^{\prime} a$. 1) stick out lengthwise. 2) stick out (said of teeth).

(2) todi Tii.

(2) todi Dengka. 
ko/tore-f Kotos Amarasi. nape of the neck.

P|ko/tole-k Molo. the protuberance on the back of my head. (M:225)

*tofa1 $n R M$. weed (field).

tofa Termanu. weed (v.). (J:638)

tofa Korbafo.

tofa Bilbaa.

tofa Rikou.

tofa $B a^{\prime} a$.

tofa Tii.

n-tofa Ro'is Amarasi. weed, remove weeds.

n-tofa Kotos Amarasi. weed, remove weeds.

$<$ tofa> Molo. weeding knife. (M:566)

Out-comparisons:

topa Semau Helong. weed.

*tofa2 PRM. quarrel.

tofa Tii. quarrel. (Grimes et al. 2014a)

na-tofa Dengka. dispute, quarrel. (J:773)

na-tofa Oenale. dispute, quarrel. (J:773)

n-tofa Kotos Amarasi. quarrel.

n-tofan Molo. have a dislike of. ho m-tofan kau you have a dislike of me (M:566)

*toka Rote. impede, against. Pattern: $\mathrm{k}-8 / 9$.

to?a Termanu. but up against something, rebound, be stopped, impeded, obstructed. (J:635)

to?a Korbafo.

to?a Bokai.

toka Bilbaa.

to?a Rikou.

to?a $B a^{\prime} a$.

to?a Tii.

to?a Dengka.

to?a Oenale.

Out-comparisons:

toka Semau Helong. support, prop up.

toka Hawu. gate (of fence). [Note: Jonker (1908:635) gives Hawu toka, toke 'stutten' = 'support'.] tuki Bima. support. (Jonker 1893:107)

tuke Sika. support. (Pareira and Lewis 1998:201)

*toki PRM. dig out. Pattern: k-8.

to?i Termanu. bore out, chisel out. (J:639)

to?i Korbafo.

to?i Bokai.

toki Bilbaa.

to?i Rikou.

to?i $B a^{\prime} a$.

to?i Tii.

to?i Dengka.

n-toi Kotos Amarasi. dig out.

Out-comparisons:

tuki Semau Helong. dig out, peck, adze.

*toko Rote. beat, knock. Etym: *tuktuk 'knock, pound, beat; crush'. Pattern: k-6. [irr. from PMP: * $\mathrm{u}>{ }^{*} \mathrm{o}$ ]

toko Termanu. beat, knock. (J:639)

toko Korbafo.

toko Bokai.

toko Bilbaa.

to?o Rikou.

toko $B a^{\prime} a$.

toko $T$ ii.

to?o Dengka.

to?o Oenale.

*to?is CERM. horn (instrument).

to?i-k Termanu. triton shell, also a horn of a buffalo on which to blow, also a musical instrument made from lontar leaves. (J:639)

to?i-? Korbafo.

to?i-k Bokai.

toi-? Bilbaa.

to?is Rikou.

to?i-k $B a^{\prime} a$.

toi?is Ro'is Amarasi. horn.

to?is Kotos Amarasi. horn (musical instrument), trumpet.

to?is Amanuban/Amanatun. blow on a horn. (M:567) 
to?is Molo. horn (instrument). [Semantics: This apparently only occurs in Molo in the parallel pair to?is ma kniit.] (M:223)

*to?o PRM. man, male. Doublet: *tou. Etym: *tau. [irr. from PMP: $\varnothing>*$ ?] [History: The original meaning of forms combined with huu- (see *huu) 'base, source, origin, beginning' was probably 'man'. Further evidence for this comes from Oenale which has tou huu-? 'maternal uncle' (see *tou) in which the first element is identical to the word for 'man'. Thus, for instance, Termanu to?o huu-k was was probably originally 'man of origin'. The woman is the source of life in Timorese thinking, and thus the mother's brother has an important role as the representative of the wife-giving maternal relatives.]

to?o-k (2) to?o huu-k Termanu. maternal uncle. Usage: loses the final $k$ in the vocative, etc. in the same way as fv;ama-k 'father'. (J:644)

to?o Korbafo.

to?o-k Bokai.

too-?, too huu-? Bilbaa. [Note: too-? comes from my own field notes, too huu-? from Jonker (1908:644).]

to?o=na $L a n d u$. (own field notes)

to?o Rikou.

to?o Oepao. (own field notes)

to?o-k $B a^{\prime} a$.

to?o Lole. (Zacharias et al. 2014)

to?o-k Tii.

too huu-? Dengka.

to?o Dela. uncle, mother's brother.

too? Kusa-Manea. male older sibling, older brother. [Note: tata|? $(<*$ tatə $)=$ 'female older sibling'.] [Form: possibly the metathesised form of (currently unattested) *to?o.]

*toygo $n R M$. meet together.

na-togo Termanu. meet someone or something, meet together. (J:643) na-tojo Bilbaa.

na-toko Rikou.

na-toygo $B a^{\prime} a$.

na-tongo $\mathrm{Tii}$.

na-toko (2) noe toko-n Molo.

1) meet. 2) meeting place or confluence of two rivers. (M:568)

*tou PRM. person. Doublet: *to?o. Etym:

*tau 'person, human being'. [irr. from

$P M P: * \mathrm{a}>*_{\mathrm{o}}$ (sporadic assimilation)]

[irr. from PRM: *u $>o$ in Meto

(probably motivated by the rarity of the sequence ou)]

tou-k Termanu. man (in opposition to woman). (J:645)

tou-? Korbafo.

tou-k Bokai.

tou-? Bilbaa.

tou-? Landu. man. (own field notes)

tou-? Rikou.

tou-k $B a^{\prime} a$.

tou-k Tii.

tou-? Dengka.

tou-? (2) tou huu-? Oenale. 1) man (in opposition to woman). (J:645)

2) maternal uncle. (J:644)

tou-? Dela. male (only used for humans).

too Ro'is Amarasi. citizenry, populace.

too Kotos Amarasi. citizenry, populace.

too Molo. people. (M:564)

too Kusa-Manea. citizenry, populace.

*tua1 Rote. big, size. Etym: *tuqah 'old, of people; mature, as fruit'.

ma-tua (2) tua (3) na-ma-tua (4) na-ka-tu tua (5) ma-ka-tuas Termanu. 1) big, size. 2) used in place of matua in compounds. 3) become big. 4) (make big), from that to make arrogant, usually said of spoilt children who always get their own way. 5) finally, ultimately. (J:647f)

ma-tua (5) ma-ka-tua-s Korbafo.

ma-tua (5) ma-ka-tuas Bokai.

ma-tua (5) ma-ka-tua-s Bilbaa. 
ma-tua Rikou.

ma-tua (5) ma-ka-tuas $B a^{\prime} a$.

(5) ma-ka-tua-s Tii.

(5) ma-Pa-tua Dengka.

(5) ma-Pa-tua Oenale.

Out-comparisons:

tuan Semau Helong. big, large, huge.

tuan East Tetun. elderly, advanced age. (Mo:189)

*tua2 PRM. lontar palm. Borassus flabellifer. Etym: *tuak 'palm wine'.

tua Termanu. the fan palm or lontar palm; the juice of the lontar palm. (J:647)

tua Korbafo.

tua Bokai.

tua Bilbaa.

tua Rikou.

tua $B a^{\prime} a$.

tua Tii.

tua-? Dengka.

tua-? Oenale.

tua|? Kotos Amarasi. lontar palm.

tua|? Molo. lontar palm. Borassus flabellifer. (M:572)

\section{Out-comparisons:}

tua Semau Helong. lontar juice, lontar tree.

tua East Tetun. an alcoholic drink; a palm from which palm juice is extracted. Borassus flabellifer. (Mo:189)

due Hawu. lontar palm.

*tua3 Morph: *tua-k. PRM. lord, master. Etym: *qatuan. (Both Blust and Trussel (ongoing) and Wolff (2010:960) reconstruct the meaning as 'deity', but I do not consider this well supported by the non-Oceanic reflexes. Instead, 'lord' was probably the original meaning with expansion to 'deity' in POc and occasionally also in other languages. It is also unclear to me whether initial *qa is supported outside of Oceanic.) lama/tua-k Termanu. lord, master, word of address for princes and officials. (J:275)

lama/tua-? Korbafo.

lama/tua-k Bokai.

lama/tua-? Bilbaa.

rama/tua-? Rikou.

lama/tua-k $B a^{\prime} a$.

lama/tua-k Tii.

lama/tua-? Dengka.

lama/tua-? Oenale.

tua-n (2) tua|f (3) tua $R o$ 'is Amarasi. 1) lord, master, owner, self. 2) individual, person. 3) sir, madam; yes, a discourse particle used to acknowledge the listener.

tua-n (2) tua|f (3) tua Kotos Amarasi. 1) lord, master, owner, self. 2) individual, person. 3) sir, madam; yes, a discourse particle used to acknowledge the listener.

uim tua-f (2) ma-?usi-? =ma matua-? Molo. 1) the owner, lord of the house. 2) have a prince and a lord. (M:573)

Out-comparisons:

lamtua Semau Helong. master, owner.

tuak Fehan Tetun. mother's brother, father's sister's husband.

am tuak East Tetun. grandfather. (Mo:189)

*tudui nRM. owl. Pattern: d-2. [Form:

The first element in Meto reflexes is from *yguu 'howl'.] [History: Clark (2011:331) reconstructs POc *drudru(r,R) 'owl'.] [Semantics: onomatopoeia.]

tu turui-? Rikou. owl. (J:775)

tu tului-k Lole. owl. (J:775)

tu $\sim$ turui-k Tii. owl. (J:775)

ku/truu Ro'is Amarasi. owl.

ku/tru?u Kotos Amarasi. owl.

ku/tlui, ku/tluu Molo. owl. (M:242, 403) 
*tudu PRM. point at, point out; give directions. Etym: *tuzuq. [irr. from PRM: * $\mathrm{t}>r$ in Meto (likely sporadic assimilation; *tudu $>$ PMeto *dudu)] na-tudu Termanu. point, show, draw to someone's attention, have someone see. (J:650)

na-tudu Korbafo.

na-tudu Bokai.

na-tudu Bilbaa.

na-tudu Rikou.

na-tudu $B a^{\prime} a$.

na-tudu $T i i$.

na-tudu Dengka.

na-tudu Oenale.

na-ruru-? (2) k/ruru-f $R o$ 'is Amarasi. 1) show, point out to.

2) finger, toe.

n-ruru (2) na-ruru-? (3) k|ruru-f Kotos Amarasi. 1) designate.

2) show, appoint, establish.

3) finger, toe.

n-lulu Molo. points something out with curled up lips. (M:650)

Out-comparisons:

tulu Semau Helong. point, show, designate.

hatudu East Tetun. show, indicate, point out, direct. (Mo:83)

pe-fufu Hawu. designated, selected, appointed (pl.).

*tufa PRM. plant with roots that are pounded and put in rivers to stun fish. Derris elliptica. Etym: *tuba. [Sporadic: $* \mathrm{a}>e /$ \# in wRM.]

tufa Termanu. certain plant the roots of which are used to daze fish. (J:650)

tufa Korbafo.

tufa Bokai.

tufa Bilbaa.

tufa Rikou.

tufa $B a^{\prime} a$.

tufa Tii.

tufe Oenale.

tufe Molo. climbing plant the roots of which are used to daze fish. Derris elliptica. (M:573) *tufu PRM. punch. Etym: *tumbuk 'punch, hit, pound'. [irr. from PMP: ${ }^{*} \mathrm{mb}>\left({ }^{*} \mathrm{~b}\right)>*_{\mathrm{f}}$ ] [irr. from PRM: ${ }^{*} \mathrm{u}>$ $a$ in Rote; ${ }^{*} \mathrm{t}>t \sim n d$ in Termanu, Ba'a and Dengka; $*_{\mathrm{t}}>t \sim n$ in Bilbaa]

tufa, ndufa Termanu. hit with the fist, punch. (J:650, 426)

tufa Korbafo.

tufa Bokai.

tufa, nufa Bilbaa.

tufa Rikou.

tufa, ndufa $B a^{\prime} a$.

tufa Tii.

tufa, ndufa Dengka.

n-tufu Kotos Amarasi. punch.

n-tufu Molo. hit with the fist. (M:574)

Out-comparisons:

tupu Ili'uun. hit, strike. (dJ:140)

*tuil PRM. line, carve. [irr. from PRM: *t $>d$ in Rote]

dui-k Termanu. stripe, put stripes. (J:105)

dui-? Korbafo.

dui-k Bokai.

dui-? Rikou.

dui-k $B a^{\prime} a$.

dui-k Tii.

dui Dengka.

n-tui Ro'is Amarasi. write.

n-tui Kotos Amarasi. write, carve.

n-tui Molo. writes. (M:574)

Out-comparisons:

tuis Semau Helong. carve, chisel, sculpt; line.

tui (2) tuik East Tetun. 1) scratch a line; to scratch with a fingernail or any similar object. 2) line, scratch, sore, or mark; thread, yarn. (Mo:190)

*tuiz Rote. tui tree, mangrove trumpet tree. Dolichandrone spathacea. Etym: *tui.

tui Termanu. a) kind of tree with light wood that is used to make floating wood for fishnets. (J:652) b) kind of large tree that grows beside rivers and lakes. (Fox 2016b:58) 
tui Korbafo.

tui Bokai.

tui Bilbaa.

tui Rikou.

tui $B a^{\prime} a$.

tui Tii.

tui Dengka.

tui Oenale.

*tuin CERM. follow. Etym: **tuir (pre$\mathrm{RM})$.

tui Termanu. follow a track. (J:650f)

na-tuin Ro'is Amarasi. follow, because.

na-tuin Kotos Amarasi. follow, because of.

na-tuin Molo. follow. (M:575)

Out-comparisons:

tuin Helong. follow an example. (J:650)

tuir East Tetun. follow, come behind; to follow, to imitate; to follow, have the same opinion as; to follow, obey the orders of...; to follow, to support. (Mo:190)

toi, tfui Ili'uun. follow, following, according to, because. (dJ:139)

nui Hawu. follow a track. (J:650)

sui Kamarian. follow, go along with. [Note: also in Alune and most varieties of Kaibobo.] (van Ekris 1865:117)

kui Haruku. (van Ekris 1865:117)

*tuka Morph: *tuka-?. PwRM. short, truncated. Pattern: k-5. [irr. from PRM: *a $>u$ in wRote (sporadic assimilation?)]

tuku-? Dengka. truncated, blunt. (J:775)

tuku-? Oenale. truncated, blunt. ( $\mathrm{J}: 775)$

tuka|? Kotos Amarasi. short, cut short.

tuka|2 Molo. short. (M:575)

Out-comparisons:

tuk/leke Semau Helong. dwarf, stunted. *tuke Morph: *tuke-k. PRM. bamboo vessel. Etym: *tukil (PWMP). Pattern: k-5. [Note: Jonker (1908:652) identifies the reflexes as being borrowings from Bugis tokka, but it is hard to explain final RM $e$ under this hypothesis.] [irr. from PMP: $*_{\mathrm{i}}>*_{\mathrm{e}}$ ]

tuke-k Termanu. container made from a bamboo node used to store tobacco, sugar, milk or similar things. (J:652)

tuke-? Korbafo.

tuke-? Bilbaa.

tuke-? Rikou.

tuke-k $B a^{\prime} a$.

(tuke-k ?) Tii.

tuke-? Dengka.

tuke-? Oenale.

tuke|? Kotos Amarasi. piece of bamboo used as a water container.

tuke|? Molo. bamboo container. (M:575)

Out-comparisons:

au toka Fehan Tetun. bamboo container. (Mo:187)

*tuku1 Rote. scull, row. Pattern: k-6.

tuku Termanu. scull, row. (J:652)

tuku Korbafo.

tuku Bokai.

tuku Bilbaa.

tu?u Rikou.

tuku $B a^{\prime} a$.

tuku Tii.

tu?u Dengka.

tu?u Oenale.

Out-comparisons:

tuku Semau Helong. paddle, pole (a boat), punt (a boat), push with pole. Usage: archaic.

tuku Hawu. row.

tuku Sika. scull. (Pareira and Lewis 1998:201)

*tuku2 Rote. throw. Pattern: k-8/9.

tu?u Termanu. throw. (J:661)

tu?u Korbafo.

(tu?u ?) Bokai.

tuku Bilbaa. 
tu?u Rikou.

tu?u $B a^{\prime} a$.

tu?u Tii.

tu?u Dengka.

tu?u Oenale.

Out-comparisons:

tuku Hawu. (J:661)

<tuku> Kambera. toss, throw. (On:505)

*tula PRM. gebang palm. Corypha utan.

[Sporadic: *a $>e$ /_\# in wRM.]

tula Termanu. gebang palm. (J:652)

tula Korbafo.

tula Bokai.

tula Bilbaa.

tula Rikou.

tula $B a^{\prime} a$.

tula Tii.

tule Dengka.

tule Oenale.

tune, tuni Kotos Amarasi. gebang palm. [Sporadic: vowel height harmony $* \mathrm{e}>i / \mathrm{uC}$.

tune Molo. gebang palm. (M:577)

Out-comparisons:

kluti? Funai Helong. gebang palm. [irr. from PRM: *a $>$ i] [Sporadic: consonant metathesis $* 1 \mathrm{Vt}>t V l$.

kluti? Semau Helong. gebang palm.

*tuli Rote. stop by to visit when travelling. Etym: *tuluy.

tuli Termanu. drop in somewhere while passing by, "come roaring along'. (J:653)

tuli Korbafo.

tuli Bokai.

tuli Bilbaa.

tuli Rikou.

tuli $B a^{\prime} a$.

tuli Tii.

tuli Dengka.

tuli Oenale.

Out-comparisons:

tuli Semau Helong. layover, stop by.

duli Hawu. (J:653) *tuma PRM. clothes louse. Etym: *tumah. [Sporadic: *a $>e$ /_\# in wRM.]

tuma Termanu. louse, clothes louse. (J:654)

tuma Korbafo.

tuma Bokai.

tuma Bilbaa.

tuma Rikou.

tuma $B a^{\prime} a$.

tuma Tii.

tume Dengka.

tume Oenale.

tume Ro'is Amarasi.

tume Kotos Amarasi. clothes louse.

tume Amanuban. body louse.

tume Molo. clothes louse. (M:578)

Out-comparisons:

ktuma? Funai Helong. clothes louse.

*tumbi Rote. kind of tree that has soft wood.

tupi Termanu. kind of tree that has soft wood. (J:657)

tupi Korbafo.

tupi Bokai.

tupi Rikou.

tumpi $B a^{\prime} a$.

tumbi Tii.

tumbi Dengka.

tumbi Oenale.

Out-comparisons:

tuwi Bima. kind of soft wood. (Ismail et al. 1985:158)

*tumbu CERM. heap, abundant. Doublet: *numbu. Etym: *tu(m)buq 'grow, germinate, sprout'. [irr. from PRM: *mb $>p \sim b$ in Meto (forms with medial $b$ are possibly from *tubuq with irr. $\left.{ }^{*} \mathrm{~b}=b\right)$ ]

tupu $\sim$ tupu (2) tu tupu-k Termanu.

1) somewhere full of it, present in abundance. 2) heap, mass. (J:657)

tupu tupu Korbafo.

tupu tupu Bokai.

tupu tupu Bilbaa.

tumpu tumpu $B a^{\prime} a$. 
na-?|tupu, na-?|tubu (2) $\mathbf{P} \mid$ tubu Kotos Amarasi. 1) heap up, pile up. 2) hill.

a-2|tubu Molo. hill. (M:573)

ta tubu Kusa-Manea. mountain.

*tuna PRM. freshwater eel. Etym: *tuna.

[Sporadic: $* \mathrm{a}>e$ /_\# in wRM]

tuna Termanu. eel. (J:654)

tuna Korbafo.

tuna Bokai.

tuna Bilbaa.

tuna Rikou. freshwater eel. (J:654; own field notes)

tuna $B a^{\prime} a$.

tuna Tii.

tune Dengka.

tune Oenale.

tune, tuni Kotos Amarasi. eel, seasnake. [Sporadic: vowel height harmony $* \mathrm{e}>i / \mathrm{uC}$.

tune Molo. eel. (M:577)

Out-comparisons:

tuna Helong. (J:654)

tuna East Tetun. conger eel. Congridae. (Mo:190)

thuno Waima'a. eel.

*tuni PRM. press.

tuni Termanu. press. (J:655)

tuni Korbafo.

tuni Bokai.

tuni Bilbaa.

tuni Rikou.

tuni $B a^{\prime} a$.

tuni Tii.

tuni Dengka.

tuni Oenale.

tuni Meto. (J:655)

*tunu1 PRM. stumble, stub one's toe. [irr. from PRM: *u $>a$ in Koto Amarasi; *n $>n d$ in Rikou and Landu] [Form: The Meto forms are from *na-sa-tunu, while the Rote are forms from *na-ka-tunu.]

na-ka-tunu Termanu. bump against something with the foot, stumble. (J:656)

na-ka-tunu Korbafo. na-ka-tunu Bokai. na-ka-tunu Bilbaa.

na-tundu Landu. (own field notes)

na-tundu Rikou.

na-ka-tunu $B a^{\prime} a$.

na-ka-tunu $T i i$.

na-Pa-tunu Dengka.

na-Pa-tunu Oenale.

na-stunan Kotos Amarasi. stub one's toe.

na-stuun Molo. stumbles. (M:517)

Out-comparisons:

tunun Semau Helong. stumble.

kedune Hawu. (J:656)

$<$ tunjuru> Kambera. stub the tow, stumble. (On:509)

$<$ tujuru> Anakalang.

$<$ katunjura $>$ Mamboru.

$<$ kantunura $>$ Weyewa.

tune (2) maatune Kamarian.

1) step, stamp with the foot or heel. [Note: also in Kaibobo, Nusa Laut, and Asilulu.] (van Ekris 1864:127) 2) stub the toe. [Note: also in Haruku and some varieties of Kaibobo.] (van Ekris 1864:303)

tahatune Kaibobo. stub the toe. (van Ekris 1864:303)

*tunu2 PRM. roast, grill. Etym: *tunu.

tunu Termanu. fry, bake without fat. (J:656)

tunu Korbafo.

tunu Bokai.

tunu Bilbaa.

tunu Rikou.

tunu $B a^{\prime} a$.

tunu Tii.

tunu Dengka.

tunu Oenale.

n-tunu? Kotos Amarasi. roast, grill.

$<$ tunu> Molo. roast. (M:579)

tuun Kusa-Manea. burn.

Out-comparisons:

tunu Semau Helong. burn, roast.

tunu East Tetun. roast, bake (in an oven or over a fire). (Mo:190) 
*turis PRM. pigeon pea. Cajanus cajan. Etym: *tuduy 'agati Sesbania glandiflora' (Fox 1991:258) (Blust and Trussel (ongoing) reconstruct *tudiq for Sesbania grandiflora, however Fox (1991) gives a number of reflexes from western languages including Sunda turuy and Madurese toroy which indicate final *uy.). [irr. from PMP: $\varnothing>*^{*}$ ] [ [Form: source of final *s unclear.] [Semantics: The seed pods of Sesbania grandiflora are edible. This appears to be the basis for the semantic link between Sesbania grandiflora and pigeon peas.]

tulis Termanu. pigeon peas. Cajanus cajan. (J:653)

tuli-? Korbafo.

tuli-? Bokai.

tuli-? Bilbaa.

turis Landu. pigeon peas. (own field notes)

turis Rikou.

tulis $B a^{\prime} a$.

turis Tii.

tulis Dengka.

turis Oenale.

tuinis Ro'is Amarasi. pigeon pea.

tunis Kotos Amarasi. pigeon pea.

tunis Molo. pigeon peas. Cajanus Cajan. (M:580)

Out-comparisons:

tulis Helong. ( $\mathrm{J}: 653)$

turis Fehan Tetun. pigeon pea: bush 1-2 metres tall with yellow pea flowers, dark green beans 3' long, cooked with rice. It grows mainly in the hills.

turis, tunis East Tetun. bush with a fruit pod similar to pea in looks and taste when tender. (Mo:191)

turiana Kisar. cashews.

tori Hawu. [irr. from PRM: *t = $d$ correspondence (expect $d)]$ (J:653) ulis Welaun. pigeon pea. [irr. from PRM: $*_{\mathrm{t}}=\varnothing$ correspondence (expect $t)]$

*turu PRM. overflow, leak. Etym: *tuduq 'leak, drip, as a leaky roof; a drop of water'. [irr. from PRM: *r $>r \sim n$ in Meto]

tu tulu Termanu. overflow, flow off around the edge while pouring. (J:653)

tu $\sim$ tulu Korbafo.

tu tulu Bokai.

tu tulu Bilbaa.

tu turu Rikou.

tu tulu $B a^{\prime} a$.

tu $\sim$ turu Tii.

tu $\sim$ tulu Dengka.

tu $\sim$ turu Oenale.

turu Kotos Amarasi. shower.

oe tunu Amanuban. aqueduct, that is a piece of bamboo or half a hollowed areca stem, placed in a water source on a higher level from which the water flows. (M:579)

oe tulu Molo. aqueduct, that is a piece of bamboo or half a hollowed areca stem, placed in a water source on a higher level from which the water flows. (M:579)

oa ta tunu|? Kusa-Manea. bamboo with which to shower.

\section{Out-comparisons:}

tudu Semau Helong. leak. [irr. from PMP: $* \mathrm{~d}=d($ expect $l)]$

turu East Tetun. drip, fall in drips or drops. (Mo:191)

noro Hawu. leak through, permeate. (J:653)

*tusi1 PRM. split gebang palm leaf. [irr. from PRM: $* \mathrm{t}>d$ in Rote] [Form: Alternately we could reconstruct *dusi and propose irr. ${ }^{*} d>t$ in Meto and Helong. Under this hypothesis either the Helong or Meto form would be a borrowing.] 
dusi Termanu. cut the outside part of a young gebang palm leaf off from the middle part with a knife. (J:108)

dusi Korbafo.

dusi Bokai.

dusi Bilbaa.

dusi Rikou.

dusi $B a^{\prime} a$.

dusi Tii.

dusi Dengka.

dusi Oenale.

tuis muti? (2) tuis molo? Molo.

1) the split pale underside of a gebang palm leaf that is used for thread in ikat weaving. 2) split yellowish underside of gebang palm leaf from which ropes for horses are made. the stem is tusi = 'divide'; which in compounds undergoes metathesis of the final syllable. (M:581)

Out-comparisons:

tusi Helong. (J:108)

*tusiz PRM. rub.

tusi Termanu. rub and pinch, rub and press. (J:657)

tusi Korbafo.

tusi Bokai.

tusi Bilbaa.

tusi Rikou.

tusi $B a^{\prime} a$.

tusi Tii.

tusi Dengka.

tusi Oenale.

n-tuis Kotos Amarasi. massage, rub.

n-tusi Molo. rub. (M:581)

Out-comparisons:

tusi Semau Helong. massage.

tusi Fehan Tetun. rub sacred betel on someone (e.g. so he becomes a fukun elder).

*tute PRM. join. [irr. from PRM: *e $>i$ in Nuclear-Rote; ${ }^{*} \mathrm{e}>a$ in Meto (and Helong)]

tuti Termanu. join, join on, set a piece. (J:658)

tuti Korbafo. tuti Bokai.

tuti Bilbaa.

tuti Rikou.

tuti $B a^{\prime} a$.

tuti Tii.

tute Dengka.

tute Oenale.

n-tuta Kotos Amarasi. continue, join.

Out-comparisons:

tutan Semau Helong. join, connect, chain, link.

*tutu PRM. beat, pound. Etym: *tuktuk 'knock, pound, beat; crush'.

tutu Termanu. hitting, pounding on something with the fist or with an object. (J:659)

tutu Korbafo.

tutu Bokai.

tutu Bilbaa.

tutu Rikou.

tutu $B a^{\prime} a$.

tutu Tii.

tutu Dengka.

tutu Oenale.

n-tutu Kotos Amarasi. pound, beat.

tutu Molo. pounds (iron). (M:582)

Out-comparisons:

tutu Semau Helong. pound.

tutu (2) tuku East Tetun. 1) peck (birds); to prod with the end of an object (Mo:191). 2) hit, strike, hammer (with an implement). (Mo:190)

*tuu1 Morph: *tuu-f. PMeto. knee. Etym: *tuhud.

tuu-f Ro'is Amarasi. knee.

tuu-f Kotos Amarasi. knee.

<tu'> Molo. knee. (M:572)

tuu-f Kusa-Manea. knee.

Out-comparisons:

tuur East Tetun. knee. (Mo:191)

rutuu Hawu. knee.

*tuu2 Morph: *tuu-k. PnRote. dry. Etym:

*tuquR 'evaporate, dry up'.

tuu-k Termanu. dry. (J:646)

tuu-? Korbafo.

tuu-k Bokai. 
tuu-? Bilbaa.

tuu-? Rikou.

tuu-k $B a^{\prime} a$.

tuu-k Tii.

Out-comparisons:

tuu Semau Helong. dry. [Note: Jonker (1908:646) gives Helong ptuu.]

vomedu?u Hawu. dry of beans. $(\mathrm{J}: 646)$

*tuur nRM. sit. [Form: Reflexes of *ng in Rote develop as though they were word medial. Meto final $n$ attests an earlier final consonant. Tetun tuur shows that this was $r$, with subsequent (regular) ${ }^{*} \mathrm{r}>*_{1}>n$ in Meto, and loss of the final consonant in nRote.]

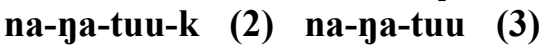
tu tuu-s (4) tu tuu-k Termanu. 1) sit oneself down, sit, sit in a physical sense. 2) sit in a figurative sense. 3) a seat of piled stones under a tree, e.g. as is used in a huus festival, these seats are made for the spirits of dead people. 4) seat, chair. (J:647)

na-na-tuu-? Korbafo.

na-na-tuu-k Bokai.

na-ka-tuu-? Bilbaa.

na-tuu-? Rikou.

na-nga-tuu-k $B a^{\prime} a$.

na-nga-tuu-k Tii.

n-tuun=oo-n Ro'is Amarasi. sit. Lit: 'seat oneself'. Usage: poetic.

n-tuun=oo-n Kotos Amarasi. sit. Usage: poetic.

Out-comparisons:

tuur East Tetun. sit down; to reside, inhabit; to settle; to rest or be resting. (Mo:191)

tuur Ili'uun. stay, live somewhere. (dJ:140)

\section{$\mathbf{U}-\mathbf{u}$}

*uas PRM. jicama. Pachyrhizus erosus. [Sporadic: * $\varnothing>$ ?/\# in Oenale.] uas Termanu. kind of tuber also called uas in Kupang. (J:662)

ua-? Korbafo.

ua-? Bokai.

ua-? Bilbaa.

uas Rikou.

uas $B a^{\prime} a$.

uas Tii.

(?)uas Dengka.

Puas Oenale.

Puas Dela.

uas Kotos Amarasi. jicama.

uas Molo. kind of herb that winds to the left, the tubers are eaten peeled.

Pachyrhizus erosus. (M:584)

Out-comparisons:

uas Fehan Tetun. edible root plant.

*uat PRM. vein, tendon, muscle, palm lines. Doublet: *urat. Etym: *uRat 'artery, blood vessel, blood vein; muscle; nerve; sinew; tendon; fibre; vein of a leaf; grain of wood; strand (of thread, rope); fishing line; root'.

ua-k Termanu. vein, tendon, muscle. (J:662)

ua-? Korbafo.

ua-k Bokai.

ua-? Bilbaa.

ua-? Rikou.

ua-k $B a^{\prime} a$.

ua-k Tii.

ua-? Dengka. a) vein, tendon, muscle.

(J:662) b) palm line. (J:776)

ua-? Oenale.

ua-? Dela. vein.

ua-n Ro'is Amarasi. palm lines.

ua-f (2) uat Kotos Amarasi. 1) the lines on the palm of one's hands. 2) blue vein, especially of an animal's liver (traditionally used in divination). [Note: keo-f $=$ 'vein, artery, blood vessel'.] 
ua-n (2) uat Amanuban. 1) the lines on the palm of one's hands. 2) veins.

$<$ ua $>$ Molo. veins in the hand; lot, age. (M:583)

Out-comparisons:

ulat, udat Funai Helong. veins.

ulat Semau Helong. strand, sinew, vein, tendon, nerve, artery; fortune, fate, luck, profit; divination, palm reading.

uan (2) uat Fehan Tetun. 1) fortune. 2) veins, grain of wood.

uat East Tetun. veins, artery, nerves, tendons. (Mo:192)

orok Kisar. veins.

*udan PRM. rain. Etym: *quzan. [Sporadic: $\varnothing>$ ? /\#_ in Dela-Oenale.] udan (2) uda Termanu. 1) rain.

2) raining. (J:662)

uda Korbafo.

uda Bokai.

uda Bilbaa.

uda Rikou.

udan $B a^{\prime} a$.

udan Tii.

(?)udan Dengka.

Pudan Oenale.

Pudan Dela. rain.

urun, uurn Ro'is Amarasi. rain.

uran (2) na-?ura Kotos Amarasi.

1) rain. 2) rain (v.). [Form: automatic glottal stop insertion between CV-prefix and \#V-initial stem.]

ulan Molo. rain. (M:587)

uran Kusa-Manea. rain.

Out-comparisons:

ulan Semau Helong. rain.

udan East Tetun. rain. (Mo:192)

usa Kemak. rain.

uus Mambae, South. rain. (Grimes et al. 2014b:46)

usan Galolen. rain.

usan Ili'uun. rain, rainy season.

(dJ:141) okon Kisar. rain.

ofi Dhao. rain. [irr. from PMP: $* u>i]$

əfi Hawu. rain. [irr. from PMP: $* u>i]$

*ue PRM. rattan. Calamus species. Etym:

*quay. [irr. from PRM: *e $>e \sim a$ in

Meto]

ue Termanu. rattan. (J:663)

ue Korbafo.

ue Bokai.

ue Bilbaa.

ue Landu. rattan. (own field notes)

ue Rikou.

ue $B a^{\prime} a$.

ue Tii.

ue Dengka.

ue Oenale.

ue Dela. rattan.

ue, ua Amanuban. rattan.

ue Amanatun. rattan.

ua-l Fatule'u. rattan.

agoe-l Amfo'an. rattan.

ue Molo. rattan. (M:574)

ua Kusa-Manea. rattan. [Form: Final $a$ is also in Uabau' in which ${ }^{*} \mathrm{e}>a$ $/ \mathrm{V}$ does not occur.]

Out-comparisons:

u/latu Funai Helong. rattan.

ui_latu Semau Helong. rattan.

hue Kemak. rattan. [irr. from PMP: $\varnothing>h]$

oe Welaun. rattan.

*ufi PRM. purple yam, greater yam. Dioscorea alata. Etym: *qubi 'yam Dioscorea alata'.

ufi Termanu. tuber. (J:664)

ufi Korbafo.

ufi Bokai.

ufi Bilbaa.

ufi Rikou.

ufi $B a^{\prime} a$.

ufi Tii.

ufi Dengka.

ufi Oenale.

ufi Dela. tuber. 
rauk_ufi Ro'is Amarasi. greater yam. Dioscorea alata.

rauk_ufi Kotos Amarasi. kind of long yam.

laku ufi Amanuban. kind of red tuber. (own field notes)

Out-comparisons:

uhi Helong. (J:664)

uhi East Tetun. creeper with a single tuber. (Mo:193)

*-uki PRM. have, own, exist, wealth.

Pattern: k-9. [Form: The initial consonant correspondences in Rote and Meto cannot be reconciled. The remaining three segments show regular correspondences.]

su?i Termanu. riches, treasures; wealth. (J:571)

su?i Korbafo.

su?i Bokai.

suki Bilbaa.

su?i Rikou.

su?i $B a^{\prime} a$.

su?i Tii.

su?i Dengka.

su?i Oenale.

n-mu?i (2) mui?i|t (3) n-ma?|mu?i

(4) n-ha?mu?i Ro'is Amarasi.

1) have, own, exist. 2) domestic animal. 3) poor. 4) suffer.

n-mu?i (2) mu?i|t (3) ma?|mu?i

(4) n-ha?mu?i Kotos Amarasi.

1) have, own, exist. 2) domestic animal. 3) poor. 4) torment, torture, oppress.

n-mu?i Molo. possess. (M:332)

Out-comparisons:

kmukit (2) muki Funai Helong.

1) (domestic) animal. 2) rich.

muki (2) hmuki (3) hmukit Semau Helong. 1) exist, is, are, have, own. 2) wealth, possessions. 3) (domestic) animal.

mukit East Tetun. be lacking, become poor; poor, needy, etc. (Mo:144) mukit Dhao. animal. (Charles Grimes pers. comm.)

*ule Rote. wring, wring out. Etym: *pulos 'twist, wring'. [irr. from PMP: *p $>\varnothing$ $($ expect $* \mathrm{~h}) ; *^{\circ}>*_{\mathrm{e}} / \#$ (expect $*_{\mathrm{o}}>a$ in wRote, possibly $\left.\left.*_{\partial}>*_{\mathrm{a}}>e\right)\right]$

ule Termanu. wring, wring out. (J:665)

ule Korbafo.

ule Bokai.

ule Bilbaa.

ule Rikou.

ule $B a^{\prime} a$.

ule Tii.

ule Dengka.

ule Oenale.

*ulə Morph: *ulə-k. PRM. worm, caterpillar. Etym: *quləj 'maggot, caterpillar, larva of a metamorphosing insect'. [Form: Meto kauna? is probably from *ka-ulo-k with the nominal prefix *ka-.]

ule-k Termanu. worm, caterpillar in general, also: intestinal worms or maggots in a wound. (J:665)

ule-? Korbafo.

ule-k Bokai.

ule-? Bilbaa.

ule-? Rikou.

ule-k $B a^{\prime} a$.

ule-k Tii.

ula Dengka.

ula Oenale.

ula Dela. caterpillar.

ka|una|? Ro'is Amarasi. creature; snake.

ka|una|? Kotos Amarasi. 1) creature. kauna? is a life form category for all creatures whose primary means of locomotion is perceived of as crawling or walking. The other life forms are koro 'birds' and ika? 'fish'. 2) snake, worm.

ka|una|? Molo. insect. (M:188)

ka|una|? Kusa-Manea. grub, caterpillar.

Out-comparisons:

ulas Semau Helong. maggot, grub, caterpillar. 
ular East Tetun. worm, caterpillar, larva. (Mo:193)

orre Kisar. worms, e.g. worms found in corn.

otu Hawu. maggot.

*uli Rote. rudder. Etym: *qulin 'rudder; steer (a boat)'.

uli Termanu. rudder of a vessel. (J:666)

uli Korbafo.

uli Bokai.

uli Bilbaa.

uli Rikou.

uli $B a^{\prime} a$.

uli Tii.

uli Dengka.

uli Oenale.

na-uli Dela. control, drive (a ship).

Out-comparisons:

ulin Semau Helong. rudder.

*ulu PRM. front, head hair. Doublet: *hulu. Etym: *qulu 'head; top part; leader, chief; headwaters; handle of a bladed implement; prow of a boat; first, first-born'.

ulu-k (2) ulu Termanu. 1) in front.

2) head hair. (J:666)

ulu-? (2) ulu Korbafo.

ulu-k (2) ulu Bokai.

ulu-? (2) ulu Bilbaa.

ulu-? (2) ulu Rikou.

ulu-k (2) ulu $B a^{\prime} a$.

ulu-k (2) ulu Tii.

ulu-? (2) ulu Dengka.

ulu-? (2) ulu Oenale.

unu|? Ro'is Amarasi. at first, past time.

unu|? Kotos Amarasi. earlier, olden days.

un unu|? Molo. in the beginning. (M:588)

Out-comparisons:

ulu(n) East Tetun. head (of anything); the upper part; a position of leadership, a leader. (Mo:193)

ulu-t Welaun. head.

ulu/wakun Kisar. head. *uma PRM. house. Etym: *Rumaq. [Sporadic: *a $>e$ /_\# in wRM; vowel height harmony $* \mathrm{e}^{>} i / \mathrm{uC}$ in Amarasi and Kusa-Manea.]

uma Termanu. house. (J:667)

uma Korbafo.

uma Bokai.

uma Bilbaa.

uma Rikou.

uma $B a^{\prime} a$.

uma Tii.

ume Dengka.

ume Oenale.

ume Dela. house, hut, building.

umi Ro'is Amarasi. house, building.

umi, ume Kotos Amarasi. house, building.

ume Molo. house. (M:587)

umi Kusa-Manea. house.

Out-comparisons:

uma Semau Helong. house.

uma East Tetun. house, dwelling place; lair (of animals); cocoon (of insects). (Mo:193)

ruma Ili'uun. granary. (dJ:136)

rom Kisar. house, clan.

*umbu Morph: *umbu-k. PRM. grandchild. Etym: *umpu 'grandparent/ grandchild (reciprocal); ancestor'.

upu-k Termanu. grandchild. (J:670)

upu-? Korbafo.

upu-k Bokai.

upu-? Bilbaa.

$\mathbf{u p u}=\mathbf{n a} L a n d u$. grandchild. (own field notes)

upu-? Rikou.

umpu-k $B a^{\prime} a$.

umbu-k Tii.

umbu-? Dengka.

umbu-? Oenale.

umbu-? Dela. grandchild.

upu-f Ro'is Amarasi. grandchild.

upu-f, upu? Kotos Amarasi.

grandchild.

upu-f Molo. grandchild. (M:590)

upu-f Kusa-Manea. grandchild. 
Out-comparisons:

ana-upun, upu-ana Semau

Helong. grandchildren.

upu-n Kisar. grandparent, grandchild.

*unə Morph: *unə-k. Rote. scale of fish or reptile. Etym: *quhənap 'scale of fish' (PCEMP *qunəp/*qunap).

une-k Termanu. scale of a fish, or of a snake; also: scale on the feet of a rooster. (J:668)

une-? Korbafo.

une-k Bokai.

une-? Bilbaa.

une-? Rikou.

une-k $B a^{\prime} a$.

une-k Tii.

una-? Dengka.

una-? Oenale.

una-? Dela. scales of a fish or reptile.

Out-comparisons:

una? Semau Helong. scale.

*undu Rote. bore a hole, make a hole.

[irr. from PRM: ${ }^{*} \mathrm{u}>a$ in nRote]

[Form: I have reconstructed final $* u$ (rather than *a) mainly on the basis of the external evidence from Hawu.]

una (2) una ha?i Termanu. 1) hollow something out with a tool, make an opening or hole somewhere (usually with a glowing pointed piece of iron). 2) start a fire with two pieces of wood by turning one piece of wood on top of another. (J:668)

una Korbafo.

una Bokai.

una Bilbaa.

unda Rikou.

una $B a^{\prime} a$.

unda Tii.

undu Dengka.

undu Oenale.

Out-comparisons:

pudu Hawu. bore; make a fire. *ura Morph: *ura-k. Rote. palm lines, lines/sinews in the liver of a dead animal (traditionally used for divination). Doublet: *uat. Etym: *uRat 'artery, blood vessel, blood vein; muscle; nerve; sinew; tendon; fibre; vein of a leaf; grain of wood; strand (of thread, rope); fishing line; root'. [minority from $P M P: * \mathrm{R}=* \mathrm{r}(\operatorname{expect} \varnothing)]$

ula-k Termanu. the lines of the hand, $[\ldots]$ the lines or sinews of the liver of a dead animal. (J:664f)

ula-? Korbafo.

ula-k Bokai.

ula-? Bilbaa.

ura-? Rikou. [Note: not known by my consultants.]

ula-k $B a^{\prime} a$.

ura-k Tii.

ura-? Oenale.

\section{Out-comparisons:}

urat East Tetun. spleen (of pigs); the word used to describe the many practices in the various customs for determining the cause of any problem, or the guilty party, or in foretelling the future. (Mo:193)

*usi1 Rote. pursue, chase away. Etym: *qusiR 'pursuit (as of enemies or game)'. [irr. from PRM: * $\varnothing>h$ in Tii and Lole; ${ }^{*} \varnothing>n$ in Dengka] [Sporadic: $* \mathrm{i}>e$ / * $\mathrm{R} \#$ in Dengka.]

usi Termanu. pursue, hunt. (J:671)

usi Korbafo.

usi Bokai.

usi Bilbaa.

usi Rikou.

usi $B a^{\prime} a$.

husi Lole. chase away, expel. (Zacharias et al. 2014)

husi Tii.

nuse Dengka.

*usir PMeto. king, lord. Etym: *usi 'relative of the third ascending or descending generation' (PCMP). [History: Although Middelkoop 
(1972:592) makes a connection between this form and Javanese gusti 'lord, master', it is difficult to account for the lack of an initial consonant in Meto under a borrowing hypothesis. Furthermore, the pervasiveness of the term being used for deities that are central to the cosmology of the Atoni indicates this is unlikely to be a recently borrowed term.]

uisi-f, usi? (2) uis neno Ro'is Amarasi. 1) king. 2) God. [History: In traditional Atoni thought uis neno is the supreme god who is the source of all (see Schulte Nordholt 1972:141).]

usi-f, usi? (2) uis neno Kotos Amarasi. king, master, lord, custodian.

usif, usi? (2) usif neno Molo. 1) lord. 2) the lord of heaven. (M:592)

(2) uis neno (3) uis paah Insana. 2) the lord of heaven, the supreme god. 3) the lord of earth, the 'pendant' of uis neno. [History: 'Uis Pah [sic] is Uis Neno's pendant. They form a dual divinity, in which Uis Neno's superiority is obvious. That is not to say that Uis Pah has emanated from Uis Neno. They are two distinct entities, but are inseparable from each other one cannot exist: without the other.' (Schulte Nordholt 1972:145)]

(2) usneno Kusa-Manea. God.

Out-comparisons:

usi East Tetun. title of nobility; a former manner of address to those with the rights of royalty. (Mo:194)

osi Buru. great-grandparent, great-grandchild. (Grimes and Grimes 2020:689)

*utan PRM. vegetables. Etym: *qutan 'small, wild herbaceous plants; scrubland, bush'. uta-k, uta ai doo Termanu. all kinds of vegetables or herbs. (J:671)

uta ai doo Korbafo.

uta ai doo Bokai.

uta ai doo Bilbaa.

uta Pai doo Rikou.

uta ai doo $B a^{\prime} a$.

uta-k, uta ai doo Tii.

uta-?, uta ai doo Dengka.

uta-?, uta ai doo Oenale.

uta-? Dela. vegetables.

utu|k, uta|k Ro'is Amarasi. vegetables; pumpkin, squash.

utan Kotos Amarasi. vegetables.

utan Molo. vegetables. (M:593)

Out-comparisons:

utan Semau Helong. vegetable.

utan Ili'uun. pea, bean. (dJ:141)

Puta Keo. vegetables. [Note: language of central Flores ISO 639-3 [xxk].] (Baird 2002:583)

*uti Morph: *uti-k. PRM. penis. Etym: *qutin.

uti-k Termanu. penis. (J:671f)

uti-? Korbafo.

uti-k Bokai.

uti-? Bilbaa.

uti-? Rikou.

uti-k $B a^{\prime} a$.

uti-k Tii.

tuti-? Dengka.

uti-? Oenale.

uti-? Dela.

uti-n Ro'is Amarasi. penis.

uti-f Kotos Amarasi. penis.

$<$ uti> Molo. penis. (M:594)

Out-comparisons:

uti Semau Helong. penis.

*uu Morph: *na-yga-uu. Rote. oink. Etym: *uu 'moaning sound'. [Semantics: onomatopoeia.]

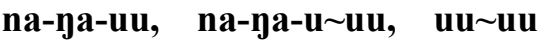
Termanu. imitation of the sound of a pig when it wants food: $\mathbf{b a f i}=\mathbf{a}$ $\mathbf{u u} \sim \mathbf{u u}$; also said of people in the sense of: grunt, grumble. (J:662) 
na-ka-u uu Korbafo.

na-ka-uu Rikou.

na-nga-u uu $B a^{\prime} a$.

na-Pa-uu Dengka.

*uut $n R M$. chaff. [History: Blust and

Trussel (ongoing) reconstruct *uta on

the basis of Kambera uta and Selaru

ut.]

uu-k Termanu. chaff, bran. (J:662)

uu-? Korbafo.

uu-k Bokai.

uu-? Bilbaa.

uu-? Rikou.

uu-k $B a^{\prime} a$.

uu-k Tii. uut Kotos Amarasi. flour.

uut (2) ut uut Molo. 1) corn that has been roasted and then crushed.

2) crunched to dust. (M:593)

\section{Out-comparisons:}

uut Helong. ( $\mathrm{J}: 662$ )

uut East Tetun. dust, any unwanted fine powder. (Mo:194)

huut Sundanese. bran. [Note: language of east Java ISO 6393 [sun].] (Coolsma 1913: 234)

oot, uot Balinese. husks, thrown away with the rice stalks. [Note: language of Bali ISO 639-3 [ban].] (Kersten 1984:432)

\section{W - W}

*wadi $P R M$. same-sex younger sibling. Etym: *huaji 'younger sibling of the same sex; younger parallel cousin of the same sex'. [Sporadic: $\varnothing>$ ? /\# in Dela-Oenale.] [Form: PwRM *odi, PnRote *fadi]

fadi-k Termanu. younger brother or sister. (J:119)

fadi-? Korbafo.

fadi-k Bokai.

fadi-? Bilbaa.

fadi=na $L a n d u$. younger sibling. (own field notes)

fadi-? Rikou.

fadi-k $B a^{\prime} a$.

fadi-k Tii.

(?)odi-? Dengka.

Podi-? Oenale.

Podi Dela. younger sibling.

ori? Ro'is Amarasi. same-sex younger sibling.

ori-f Kotos Amarasi. same-sex younger sibling.

oli-f Molo. younger brother or sister. (M:403)

ori? Kusa-Manea. same-sex younger sibling.

Out-comparisons: palin Semau Helong. younger brother/sister.

ali-n East Tetun. younger brother or sister. (Mo:3)

walin Welaun. younger sibling.

ali(n) Ili'uun. man's younger brother, father's brother's son or daughter (younger than himself), mother's sister's son or daughter (younger than himself), a woman's younger sister, father's brother's son or daughter (younger than herself), mother's sister's son or daughter (younger than herself). (dJ:112)

ari Dhao. younger sibling. ari Hawu. younger sibling.

*wani PRM. honey bee. Etym: *wani. [Form: PwRM *oni, PnRote *fani.] fani Termanu. bee, honeybee. (J:125) fani Korbafo.

fani Bokai.

fani Bilbaa.

fani Rikou.

fani $B a^{\prime} a$.

fani $T i i$.

oni Dengka. 
oni Oenale.

oni Dela. bee.

oni Ro'is Amarasi. sugar.

oni Kotos Amarasi. sugar; bee.

oni Molo. bees. (M:405)

oni Kusa-Manea. bee.

\section{Out-comparisons:}

pani Semau Helong. bee. wani Fehan Tetun. bee, wasp. uani(n) East Tetun. honey bee. (Mo:192)

wani Kisar. bee.

\#

"adulara Rote. kind of yellow wood. Borrowed from: Hawu afu 'wood' + lara 'yellow'.

adulala Termanu. kind of wood that yields a yellow dye which is used in weaving. Maclura cochinchinensis. (J:3; Heyne 1950:551, lvi)

adulala Korbafo.

adulala Bokai.

adulala Bilbaa.

adulala Rikou.

adulala $B a^{\prime} a$.

adulala $T i i$.

adulala Dengka.

adurala Oenale.

Out-comparisons:

afu + lara Hawu. wood + yellow.

\#badzu PRM. garment. Borrowed from:

Malay baju, ultimately Persian.

badu Termanu. garment; wear a garment, have a garment on. (J:19)

baruk Dela. pants.

baru Kotos Amarasi. shirt.

\#ba?at Morph: "ka-ba?at. PMeto. root. Doublet: *oka2. Etym: *wakaR. [irr. from PMP: ${ }^{*} \mathrm{w}>*^{*}$ ] [History: Probably borrowing from Helong into PMeto. The opposite direction of borrowing cannot easily explain medial $k$ in Helong. Note, however, that $\mathrm{PMP} *_{\mathrm{w}}>b$ is irregular in both Helong and Meto.]

Pba?a-f Kotos Amarasi. root.

PbaPat Amfo'an. root.

PbaPat Molo. root of a tree or plant.

(M:39)

ba?a-f Kusa-Manea. root.

\section{Out-comparisons:}

kbakat Funai Helong. root.

\#baluk Rote. boat with one or two masts and no outrigger. Borrowed from: Malay baluk, ultimately Arabic falawakat. [History: Blust and Trussel (ongoing) make a PMP reconstruction on the basis of this Rote form and Old Javanese, though they suggest the Rote form may be a loan. The Termanu form is almost certainly a Malay loan.]

(ofa) balu-k Termanu. boat with one or two masts and no outrigger. (J:29)

\#bane PRM. bowl. Borrowed from: ultimately Tamil pānnai [pa:nai] 'large earthen pot or vessel' (Hoogervorst 2016).

bane Termanu. a kind of earthenware jug with a narrow neck and wide belly, among other things used to store dyes. (J:29)

bane Korbafo.

bane Bokai.

bane Bilbaa.

bane Rikou.

bane $B a^{\prime} a$.

bane Tii.

bane Dengka.

bane Oenale.

?|fane|? Kotos Amarasi. kind of bowl traditionally made from the husk of a large pumpkin and used to carry water.

2|fane|? Molo. a plate made from pumpkin skin. (M:110) 
\#bnapa Morph: "bnapa-f. PMeto. side, ribs. Borrowed from: probably Helong into Meto before $* 1>n . b l$ is a common cluster in Helong with more than 25 attestations in Balle and Cameron (2014) while $b n$ is rare in Meto with only 7 attestations in my current Kotos Amarasi database.

bnapa-n Ro'is Amarasi. hips.

bnapa-f Kotos Amarasi. side, ribs; slope, incline.

bnapa-n Molo. side. (M:74)

Out-comparisons:

seen blapas Funai Helong. rib.

blapas Semau Helong. rib(cage); side, part; mate, partner.

\#[b/p]andut PRM. torch. Borrowed from: ultimately Malay panjut [pandzut].

banu-k Termanu. torch with wick, also: light. ( $\mathrm{J}: 30)$

banu-? Korbafo.

banu-k Bokai.

banu-? Bilbaa.

bandu-? Rikou.

baru-? Oepao. crushed seeds of the fruit of the Ceylon Oak (kusambi) used to make a torch. (own field notes)

banu-k $B a^{\prime} a$.

bandu-k Tii.

bandut Dengka.

paru Ro'is Amarasi. lamp, torch.

paku Kotos Amarasi. lamp, torch.

paku Molo. lamp. (M:414)

Out-comparisons:

hadut Semau Helong. lamp, lantern. [Note: Jonker (1908:30) gives Helong padut.]

badut East Tetun. candles made from kamii [candlenut] and other plants. (Mo:8)

badu Dadu'a. lamp. (Penn 2006:52)

patfu Ili'uun. lamp made of a fruit called too in Wetar and bintanggar in Ambon Malay. (dJ:133) watu Kisar. lamp.

\#bruuk PMeto. trousers. Borrowed from:

Dutch broek [bru:k].

bruku Ro'is Amarasi. trousers.

[Form: This form may be a result of reanalysis of bruuk as a metathesised form with back formation to derive an unmetathesised form.]

bruuk Kotos Amarasi. trousers.

Out-comparisons:

bduuk Funai Helong. trousers.

bluuk Semau Helong. trousers.

"bukae PMeto. eat, dine. Borrowed from: the direction of borrowing between Meto and Tetun is unclear though the wider semantic range in Meto may point to it being the donor. Pattern: $\mathrm{k}-6 / 9$.

n-bukae Ro'is Amarasi. eat and/ or drink, implies a social activity which is carried out with other people.

n-bukae Kotos Amarasi. eat and/ or drink, implies a social activity which is carried out with other people.

n-bukae Amfo'an. eat. [Semantics: Cannot mean 'drink'.]

bukaell (2) n-bukae Molo. 1) provisions. 2) eat. (M:88)

\section{Out-comparisons:}

bukae East Tetun. provisions for a trip, food taken on a journey. (Mo:18)

\#baba PRM. help; join with someone in doing something, do something together with someone, help someone. Borrowed from: ultimately a Chinese language, e.g. Hokkien 幫忙 pang-bâng [pantbay1], Mandarin 帮 帮 bāng bāng [baylbay1] (imperative form). (J:18)

baba Korbafo.

baba Bokai.

baba Bilbaa.

baba Rikou. 
baba $B a^{\prime} a$.

baba Tii.

baba Dengka.

baba Oenale.

n-baba Kotos Amarasi. help.

n-baba Molo. helps. (M:39)

Out-comparisons:

baba Semau Helong. follow together.

\#Ganda Rote. thing, animal. Borrowed from: ultimately Sanskrit bhānda [bia:nda] 'goods', compare Malay barang 'thing'.

bana Termanu. animal, foremost a quadruped. (J:29)

bana Korbafo.

bana Bokai.

bana Bilbaa.

banda Landu. animal. (own field notes)

banda Rikou.

bara Oepao. (J:x)

bana $B a^{\prime} a$.

banda Tii.

banda Dengka.

banda Oenale.

bare|? Kotos Amarasi. thing, stuff.

bale|? Amanatun. cargo. (M:46)

bale mnasi? Molo. heirloom. (M:46)

Out-comparisons:

berai, barai, badain Fehan Tetun. thing.

bada (2) bara Hawu. 1) animal. 2) things, belongings, possessions; material; clothes.

badza Dhao. animal.

\#basiu Rote. dish. Borrowed from:

Portuguese bacio [basiu].

basiu Termanu. kind of nonindigenous dish decorated with a flower pattern. (J:32)

basiu Korbafo.

basiu Bokai.

basiu Bilbaa.

basiu Rikou.

basiu $B a^{\prime} a$.

basiu Tii. basiu Dengka.

basiu Oenale.

Out-comparisons:

bahiu Hawu. (J:32)

\#besi PRM. iron. Borrowed from: Malay besi [bəsi] ultimately from an Indic language, such as Sanskrit vāśs [va:fi:] 'sharp pointed knife or blade', as proposed by Hoogervorst (2016:566).

besi Termanu. iron. (J:46)

besi Korbafo.

besi Bokai.

besi Bilbaa.

besi Rikou.

besi $B a^{\prime} a$.

besi Tii.

besi Dengka.

besi Oenale.

besi Kotos Amarasi. knife.

besi Molo. iron, knife. (M:60)

*bunga Rote. decorative flower. Doublet: *buna. Borrowed from: Malay bunga [buya].

buya Termanu. flower, in the sense of an ornamental flower. (J:64)

buna Korbafo.

buja Bokai.

buka Rikou.

buyga $B a^{\prime} a$.

bunga Tii.

buyga Dengka.

\#daygan PRM. trade, commerce. Borrowed from: Malay dagang.

danan Termanu. trading, trade. (J:75)

dayan Korbafo.

dayan Bokai.

dayan Bilbaa.

dakan Rikou.

daygan $B a^{\prime} a$.

daygan Tii.

daygan Dengka.

daygan Oenale.

n-rakan Ro'is Amarasi. trade, commerce.

n-rakan Kotos Amarasi. trade, commerce.

a-lakan Molo. trader. (M:18) 


\section{Out-comparisons:}

dakay Semau Helong. commerce. raka East Tetun. buy small quantities; to buy in halves, to buy a share. (Mo:158)

djagan Ili'uun. trade. (dJ:113)

"doit PRM. money. Borrowed from: Dutch duit [dœyt].

doi-k Termanu. money. (J:95)

doi-? Dela. money.

roit Ro'is Amarasi. money.

roit Kotos Amarasi. money.

loit molo? Molo. copper money. (M:282)

\section{Out-comparisons:}

duit Semau Helong. money, coin.

loit balanda Fehan Tetun. old

Dutch money. This is more valuable, as not affected by inflation. Borrowed from: Meto loit.

doi Hawu. money, currency, cash, funds.

\#dzala PRM. cast net. Borrowed from: ultimately Sanskrit jāla [ḋa:la], perhaps via Malay jala.

dala Termanu. cast-net. (J:71)

dala Korbafo.

dala Bokai.

dala Bilbaa.

dala Rikou.

dala $B a^{\prime} a$.

dala Tii.

dala Dengka.

dala Oenale.

sala Molo. kind of net. (M:471)

Out-comparisons:

sala Semau Helong. cast net.

fala Dhao. cast-net.

fala Hawu. cast-net.

\#dzinela $n R M$. window. Borrowed

from: Portuguese janela [zancla], with reported regional variant jinela, ultimately from Vulgar Latin januella diminutive of iānua 'door'.

dinela Termanu. window. (J:88) dinela Tii. window. (Grimes et al. 2014a)

eno sneer Kotos Amarasi. window. [Form: reanalysis as $\mathbf{s n e e r}=\mathbf{a a}$ ['sne:re] with the determiner $=\mathbf{a a}$ attached to a consonant final stem. eno? $=$ 'door'.]

"en PMeto. similar, like; to, towards, irrealis locative indicating a location where someone will be in the future. Borrowed from: probably Helong into Meto before *1 $>n$. [irr. from PRM: *e $>o$ in Nuclear Meto]

en Ro'is Amarasi. a) like, similar to. b) to, towards, irrealis locative indicating a location where someone will be in the future.

on Kotos Amarasi. a) like, similar to. b) to, towards, irrealis locative indicating a location where someone will be in the future.

on ii Molo. so is it, in this way. (M:405)

on Kusa-Manea. like, similar to.

Out-comparisons:

el Semau Helong. like, similar, as; to, towards.

\#etu PMeto. field. Borrowed from: the direction of borrowing between Meto and Tetun is unclear though the wider semantic range in Tetun indicates it is probably the donor.

etu Kotos Amarasi. field. Usage: poetic.

etu $A m f^{\prime}$ 'an. field. Usage: poetic.

etu Molo. land for the construction of a field for the king. (M:105)

\section{Out-comparisons:}

etun (2) liman etun (3) ai tuur etun (4) manu etun East Tetun. 1) sustenance, nourishment, provisions. 2) the portion or share or levy of food that each person has to meet at celebrations, funerals, etc. [Form: liman $=$ 'arm/ hand'.] 3) the part of the crop belonging to the king when 
the garden is made in another district or kingdom. [Form: ai tuur = 'tree stump'.] 4) the part of the crop belonging to the king when the crop is made in the proper district of the farmer. [Form: manu $=$ 'chicken, bird'.] (Mo:30)

"faduli PRM. care for. Borrowed from: Arabic faduli [fad'uli] 'curious, inquisitive', compare Malay peduli.

na-fa-duli Termanu. care for someone. (J:107)

na-fa-duli Korbafo.

na-fa-duli Bokai.

na-fa-duli Bilbaa.

na-fa-duli Rikou.

na-fa-duli $B a^{\prime} a$.

na-fa-duli $T i i$.

na-fa-duli Dengka.

na-fa-duli Oenale.

n-fairori (2) n-pairori Kotos

Amarasi. 1) care, pay attention to.

2) prepare; convalesce. [Form:

Jonker (1908:107) gives Meto

filoli, firoir.]

"fei Rote. file. Borrowed from: Dutch vijl [feil].

fei Termanu. file. ( $\mathrm{J}: 130)$

fei Korbafo.

fei Bokai.

fei Bilbaa.

fei Rikou.

fei $B a^{\prime} a$.

fei Tii.

fei Dengka.

fei Oenale.

Out-comparisons:

vei Hawu. (J:130)

*filanda Rote. European person.

Borrowed from: Malay belanda

[bolanda], ultimately from Dutch

Hollander 'person from Holland'.

filana Termanu. Dutchmen, European in general, considered more polite than olana. (J:135)

fili ana Korbafo. filana Bokai.

fila_ana Bilbaa.

filanda Rikou.

filanda Oepao. (own field notes)

filana $B a^{\prime} a$.

filanda $T i i$.

filanda Dengka.

filanda Oenale.

Out-comparisons:

balanda East Tetun. Dutchman. (Mo:9)

walate Kisar. Dutch, foreigner with white skin; foreign, manufactured.

\#fukar PMeto. spices, seasonings. Borrowed from: probably Tetun into Meto given final $r$. Final $r$ is rare in native Meto words (there are only about a dozen examples of final $r$ in native words in my Kotos Amarasi database), but not uncommon in Tetun. The presence of this word in Galolen (whether native or borrowed) also probably indicates that Tetun is the ultimate donor. Pattern: k-6/9.

fukar Kotos Amarasi. herbs, spices, seasonings. Usage: archaic.

fukal Molo. seasonings. (M:131)

fukul Timaus. herbs and spices. [Form: regular assimilation of $* \mathrm{a}$ in final closed syllables.]

\section{Out-comparisons:}

fukar East Tetun. season, spice. (Mo:37)

ai fukar Galolen. cooking spices. \#hada|k PMeto. raised platform. Borrowed from: the direction of borrowing between Proto-Meto and Helong is unclear.

harak Kotos Amarasi. raised platform. $<$ hala $>$ Molo. raised platform. (M:137)

Out-comparisons:

khaday Funai Helong. platform. hadan Semau Helong. bed. 
\#hadat $P R M$. custom, tradition. Borrowed from: ultimately Arabic 'ādat [Sa:dat] 'custom, habit'.

hada-k Termanu. custom, morals. (J:151)

harat Ro'is Amarasi. custom, tradition.

harat Kotos Amarasi. custom, tradition.

Out-comparisons:

hadat Semau Helong. custom, tradition, customary law.

hadat, adat Fehan Tetun. usage, custom. (Mo:42)

hadjak Ili'uun. custom. (dJ:155)

"horo Rote. saw. Borrowed from: ultimately probably Portuguese corte [korto].

holo Termanu. saw; cut in a sawing manner. (J:189)

holo Korbafo.

holo Bokai.

holo Bilbaa.

horo Rikou.

holo $B a^{\prime} a$.

horo Tii.

holo Dengka.

horo Oenale.

Out-comparisons:

holat Semau Helong. saw.

korat East Tetun. cut. (Mo:117)

"huta Morph: "huta-k. Rote. debt, fine.

Borrowed from: Malay hutang.

huta-k Termanu. debt, financial debt, fine. (J:198)

huta-? Korbafo.

huta-k Bokai.

huta-? Bilbaa.

huta-? Rikou.

huta-k $B a^{\prime} a$.

huta-k Tii.

huta-? Dengka.

huta-? Oenale.

\# kaba PRM. copper wire. Borrowed from:

Malay kawat. Pattern: k-1.

(tali) kafa Termanu. copper wire. (J:212) kafa Korbafo.

kafa Bokai.

kafa Bilbaa.

kafa $B a^{\prime} a$.

kafa Tii.

kafa Dengka.

kafa Oenale.

tain kaba Molo. telephone wire. (M:164)

Out-comparisons:

kaba Semau Helong. (J:212)

\#kabas PRM. cotton. Borrowed from: ultimately Sanskrit kārpāsa [ka:rpa:sa]. Reflexes of $* \mathrm{k}$ probably indicate this term probably dispersed after the break-up of PRM. Pattern: k-irr.

(?)abas Termanu. cotton shrub; the raw material, cotton; cotton fruit; yarn, thread. (J:1f)

(?)aba-? Korbafo.

kaba-? Bilbaa.

Pabas Rikou.

(?)abas $B a^{\prime} a$.

(?)abas Lole. cotton. (Zacharias et al. 2014)

(?)abas Tii.

(?)abas Dengka.

Pabas Oenale.

Pabas Dela. thread.

kabas Ro'is Amarasi. cotton.

Pabas Kotos Amarasi. cotton.

Pabas Molo. cotton plant, also cotton thread. (M:2)

Pabas Kusa-Manea. cotton.

Out-comparisons:

kabas East Tetun. cotton or cotton plant. (Mo:92)

"kadera PRM. chair. Borrowed from:

Portuguese cadeira [kadeira]. Pattern:

k-1.

kadela Termanu. chair. (J:211)

kadela Lole. chair. (Zacharias et al. 2014)

kadera Tii. chair. (Grimes et al. 2014a) 
kanreer [kand're:r] Ro'is Amarasi. chair. [Form: reanalysis as kanreer=aa ['kandre:re] with the determiner $=\mathbf{a a}$ attached to a consonant final stem.]

kanleel Molo. chair. (M:180)

Out-comparisons:

kdeda Funai Helong. chair. dela Semau Helong. chair. kader East Tetun. chair. (Mo:93)

"kado PRM. sack. Borrowed from: Malay karung/kandung. Pattern: k-2b.

ka kado (2) (ka )kado-k Termanu.

1) carry betel (etc.) in a bag/sack formed by the fold of cloth or a sarong. 2) such a bag/sack. (J:211)

ka kado Korbafo.

ka kado Bokai.

na-kado Bilbaa.

(?)a ( (?)ado Rikou.

ka kado, kali_kado $B a^{\prime} a$.

kali kado Tii.

ta?i_(?)ado Dengka.

(?)a $\sim$ (?)ado, ta?i_(?)ado Oenale.

Pkaro? Kotos Amarasi. sack.

<kalo> Molo. bag, sack. (M:175)

Out-comparisons:

karon East Tetun. a sack made from sacking; a bale; sacking or hessian; the bag at the bottom of the casting net. (Mo:102)

kado Hawu. (J:211)

"kameru|? PMeto. rice ear bug. Leptocorisa oratorius. Borrowed from: Tetun into Kusa-Manea. [Note: While it is possible to reconstruct Proto-Flores-Lembata *kəmeruy which is cognate with Tetun kamerun (thus pointing to an earlier regional Austronesian term), the absence of this term in any Rote-Meto language apart from Kusa-Manea indicates that KusaManea has borrowed this term from Tetun.]

kameru? Kusa-Manea. rice ear bug. Leptocorisa oratorius.

Out-comparisons: kamerun Fehan Tetun. small (1/2-inch-long) bug that damages rice by sucking it, so that the grain doesn't form. Leptocorisa oratorius. [Semantics: Indonesian gloss given as walang sangit $=$ 'rice ear bug'.]

kamiru East Tetun. beetle. [Semantics: This is given as identical to diru which is glossed as: 'beetle which attacks palm trees'.] (Mo:100)

kəmeruy Lamaholot, Ile Mandiri. rice ear bug. Leptocorisa oratorius. Usage: Lewoingu dialect. [Note: language of east Flores ISO 639-3 [slp].] (Klamer 2015b)

kəmoro(y) Central Lembata. rice ear bug. Leptocorisa oratorius. (Fricke 2015)

kameruy Alorese, rice ear bug. Leptocorisa oratorius. Usage: Alor Besar village. (Moro 2016, LexiRumah)

kamelin Abui. rice ear bug. Leptocorisa oratorius. [Note: non-Austronesian language of Alor ISO 693-3 [abz].] [Semantics: English gloss given as 'cockroach' in Kratochvíl (2007:467).] (Saad 2015, LexiRumah)

"kamba Rote. buffalo. Borrowed from: Malay kerbau. Pattern: k-2/3.

kapa Termanu. buffalo. (J:221)

kapa Korbafo.

kapa Bokai.

kapa Bilbaa.

Papa Landu. buffalo. (own field notes)

Papa Rikou.

kampa $B a^{\prime} a$.

kamba Tii.

(?)amba Dengka.

Pamba Oenale.

Pamba Dela. buffalo, water buffalo. 


\section{Out-comparisons:}

kabau Fehan Tetun. large domestic animal - includes cow, buffalo, horse.

karau East Tetun. water buffalo. (Mo:102)

"kapir Morph: "ka-kapir. PRM. plaited palm leaf bag or pouch. Borrowed from: Malay kampil. Etym: *kampil (PWMP. Blust and Trussel (ongoing) note that the reflexes of this form may be a Malay loan distribution.). Pattern: $\mathrm{k}-1$. [irr. from PMP: *mp $>{ }^{*} \mathrm{p} ;{ }^{*} \mathrm{l}>{ }^{*} \mathrm{r}$ ] [irr. from PRM: * $\mathrm{p}>b$ in Molo] [Form: Hawu also attests irregular medial $b$ also found in Molo.] [History: Given the irregularities in the Rote and Meto forms, the reflexes included here were probably distributed by contact.]

kapi-k Termanu. kind of basket woven from lontar leaves. (J:222)

kapi-? Korbafo.

kapi-k Bokai.

kapi-? Bilbaa.

kapi-k $B a^{\prime} a$.

kapi-? Dengka.

kapi-? Oenale.

kapi-? Dela. bag from palm leaf.

kaipir Ro'is Amarasi. braided bag typically used for holding betel nut.

?|kapi|? Kotos Amarasi. braided bag typically used for holding betel nut.

$<$ kabi $>$ Molo. four cornered betel nut basket. (M:165)

\section{Out-comparisons:}

kabir Fehan Tetun. container for betel nut.

kabi Hawu. (J:222)

"kari Morph: "kari-f. PMeto. kidneys. Borrowed from: probably an early borrowing from Helong into PMeto. Pattern: k-2b. [Form: The Meto cognates set indicates earlier medial $* *$ nd. While the Helong forms indicate earlier ** $y(g)$. This is perhaps a case of dissimilation of * $\mathrm{gg}>*_{\mathrm{nd}} / \mathrm{k}$ _ in Meto. Compare PRM *kingi ‘cockroach'.] kairi-f, kaer-n=aa Ro'is Amarasi. kidneys.

?aki-f Kotos Amarasi. kidneys.

Pake-k Kopas. lungs.

Paki-f Molo. kidneys. (M:14)

Out-comparisons:

khanin beas Funai Helong. kidneys. Usage: obsolete. [Form: beas $=$ 'seed'.]

hanin Semau Helong. kidneys.

"karu PRM. cloth bag. Borrowed from: an early borrowing from Malay karung or similar. The forms collected here probably represent several independent instances of borrowing. Pattern: k-irr.

alu \kosu Termanu. bag, purse of linen or cotton. (J:9)

alu/kosu Korbafo.

alu/kosu Bokai.

alu/kosu Bilbaa.

alu/kosu Rikou.

alu/kosu $B a^{\prime} a$.

alu/kosu Tii.

alu/kosi Dengka.

alu/kosi Oenale.

auru|k Ro'is Amarasi. small cloth bag used for holding betel nut.

aru|k, aru|? Kotos Amarasi. small cloth bag used for holding betel nut.

alu|?, alu|k (2) <alu_kosu> Molo. 1) small bag for betel-nut. 2) small whey bag which can be closed up. (M:19)

ka karu Kusa-Manea. betel-nut bag.

\section{Out-comparisons:}

olo\kohu Hawu. (J:9)

ka kalu Ili'uun. a sack or bag made of imported cloth. (dJ:120)

\#kinde PRM. spindle. Borrowed from: connected with Malay kincir 'spinning wheel; spool, reel'. Reflexes of *k probably indicate this term was not borrowed into PRM, but has a later dispersion. Pattern: k-irr. 
(?)ine Termanu. long round piece of wood which one rotates resting on the bina in order to spin yarn. (J:203)

(?)ine Korbafo.

(?)ine Bokai.

kini Bilbaa.

Pindi Landu. spindle. (own field notes)

Pinde Rikou.

Piri Oepao. (own field notes)

(?)ina(_abas) $B a^{\prime} a$.

(?)inda Lole. spinner. (Zacharias et al. 2014)

(?)inde Tii.

(?)inde Dengka.

(?)inde Oenale.

kiri Ro'is Amarasi. spool for spinning cotton thread.

Pike Kotos Amarasi. spool for spinning cotton thread.

Pike Molo. spool for spinning cotton thread. (M:159)

\section{Out-comparisons:}

kida East Tetun. spindle (for spinning thread). (Mo:40)

kinde Manggarai. spindle, tools for spinning thread. (Verheijen 1967:220)

<kindi> Kambera. spindle. (On:211)

\#k|naba|? PMeto. spider. Doublet: *lau. Etym: *lawaq. [irr. from PMP: *wa > *ba (expect $*$ o)] [History: Probably borrowing from Tetun into pre-Meto before $* 1>$ Proto-Meto ${ }^{*} \mathrm{n}$, as $*_{\mathrm{W}}>b$ is regular in East Tetun. Welaun has dabadai(n) and this may also be the source for the Meto form.]

k|naba|? Ro'is Amarasi. spider.

k|naba|? Kotos Amarasi. spider.

?|nab naba|? Molo. spider, spider web. (M:338)

naba_k|ra ra?i Kusa-Manea. spider. [Note: Kusa-Manea also has labrait which is a more recent borrowing from Tetun.]

\section{Out-comparisons:}

labadain Fehan Tetun. spider, cobweb. (Mo:122)

labadain East Tetun. spider. (Mo:122)

\#koedzabas PRM. guava. Borrowed from: Portuguese goiabas [gojaßas] (pl. of goiaba), ultimately Arawak guayabo. Pattern: k-1. [Form: In Meto the insertion of $/ \mathrm{d} / \mathrm{d} / \mathrm{can}$ be explained as a (historic) process to break up the sequence of three vowels. All three vowels are still seen in the Baikeno form. Some varieties have subsequently undergone $*_{d}>r$, or in the case of Kusa-Manea (unexpected) $* \mathrm{~d}_{3}>l$. The appearance of $/ \mathrm{d} z /$ or $/ \mathrm{d} /$ in Rote is not explicable in the same way and these forms may be from Meto. The Tetun form appears to result from a different borrowing event.]

kudzabas, kudabas Termanu. in Kupang kudzawas kind of edible fruit. ( $\mathrm{J}: 255)$

kudgawas Lole. guava. (Zacharias et al. 2014)

kuraibis Ro'is Amarasi. guava.

kudzabis, kurabis, kurabe Kotos Amarasi. guava.

kudzawas Molo. guava. (M:244)

koi-ds Amfo'an. guava.

kurabis Timaus. guava.

koedzabis Baikeno. guava. (Michael Rose pers. comm.)

koelabis Kusa-Manea. guava.

Out-comparisons:

koiabas, goiaba East Tetun. guava. (Mo:116,38)

kudzawas Kupang Malay. guava.

"kofa Morph: "kofa-k. PRM. canoe. Borrowed from: currently unidentified. Pattern: k-irr. [Form: Reflexes of $*_{\mathrm{k}}$ indicate that this term is a borrowing after the break-up of PRM.] [History: May be connected (irregularly) with PMP *qabay.]

(?)ofa-k Termanu. vessel. (J:454) 
(?)ofa-? Korbafo.

(?)ofa-k Bokai.

kofa-? Bilbaa.

(?)ofa-? Landu. canoe. (own field notes)

Pofa-? Rikou.

(?)ofa-k $B a^{\prime} a$.

(?)ofa-k Tii.

(?)ofa-? Dengka.

Pofa-? Oenale.

Pofa-? Dela. boat.

kofa|? Ro'is Amarasi. canoe, boat.

kofa|? Kotos Amarasi. canoe, boat.

Out-comparisons:

kova Hawu. boat.

kowa Ende. canoe with two supporting poles on each side. (McDonnell 2009:25)

\#koi PRM. bed. Borrowed from: Dutch kooi [ko:i], ultimately Latin cavea. Pattern: k-1.

koi Rikou. bed. (Nako et al. 2014)

koi Lole. bed. (Zacharias et al. 2014)

koi Tii. bed. (Grimes et al. 2014a)

koi Dela . bed.

koi Timaus. bed. [Form: Citation form with final consonant insertion is koor.]

Out-comparisons:

kui Semau Helong. bed.

koi Fehan Tetun. bed.

"kopi PMeto. knife. See: *dombe.

opi Ro'is Amarasi. knife.

Out-comparisons:

opi Ili'uun. sword, cutlass, chopping knife. (dJ:131, 143)

kopi Roma. knife.

opi-e Central Marsela. knife. [Note: language of the Babar Islands, southwest Maluku ISO 639-3 [mxz].] (Taber 1993:428)

"kota PRM. fortress, city. Borrowed from: ultimately Sanskrit koța [kota] 'fort', perhaps via Malay kota. Pattern: k-1. kota Termanu. fort, fortress, in particular the fort at Kupang, also Kota as the Rote name for Kupang. Also: stone fence, or wall in general. $(\mathrm{J}: 252)$

kota Dela. city, city of Kupang.

koot, kota Kotos Amarasi. city, fort. [Form: The form koot is due to reanalysis as $\mathbf{k o o t}=\mathbf{a a}$ ['ko:te] with the determiner $=\mathbf{a a}$ attached to a consonant final stem.]

koot Molo. fort. (M:238)

\#kusapi PMeto. Ceylon oak. Schleichera oleosa. Borrowed from: Hindi kusumb. Pattern: k-2b. [History: Blust and Trussel (ongoing) reconstruct PMP *kasambi?, but the tree is not native to this area.]

kusapi Ro'is Amarasi. Ceylon oak. Schleichera oleosa.

Pusapi Kotos Amarasi. Ceylon oak. Schleichera oleosa.

Pusapi Molo. kind of tree the seeds of which have oil. (M:591)

Pusapi Kusa-Manea. Ceylon oak. Schleichera oleosa.

Out-comparisons: sapi? Semau Helong. Ceylon oak.

"laisona $P R M$. shallot, eschalot, onion. Allium Ascalonicum. [irr. from PRM: $*_{\mathrm{n}}>\varnothing$ in Meto] [Sporadic: glottal stop insertion in Amarasi.] [History: Ultimately from Sanskrit rasuna, perhaps via Makassar/Bugis lasuna.]

laisona Termanu. onion. (J:271)

laisona Korbafo.

laisona Bokai.

laisona Bilbaa.

laisona Rikou.

laisona $B a^{\prime} a$.

laisona Tii.

laisona Dengka.

laisona Oenale.

naiso?o Ro'is Amarasi. eschalot, onion. Allium Ascalonicum. 
naiso?o Kotos Amarasi. eschalot, onion. Allium Ascalonicum. [Form: Final ?o disappears in compounds e.g. naiso no?o 'spring onions, scallions', same as aidzo?o 'casuarina tree'.]

\section{Out-comparisons:}

lais'one Waima'a. onion.

lahono Kisar. onion.

\#leko PMeto. good. Borrowed from: probably Helong into Meto after *1 $>n$. If this were from Proto-Meto *reko, we would not expect initial $l$ in the Waima'a out-comparison. Furthermore, Kusa-Manea does not have this form and instead has na-mria 'good'.

reko Ro'is Amarasi. good.

reko Kotos Amarasi. good.

leko Molo. be good, be healthy.

\section{Out-comparisons:}

leko Semau Helong. beautiful.

loko Waima'a. beautiful. [irr. from PRM: $e=o$ correspondence]

"mayga Rote. mango. Borrowed from: ultimately colloquial Tamil māng $\bar{a}$ [ma:yga:] (Tamil mānkkañi [ma:ykani]), perhaps via Malay mangga.

(pao) maya Termanu. the name of a big kind of mango. ( $\mathrm{J}: 346)$

mana Korbafo.

maya Bokai.

makas Rikou.

manga $B a^{\prime} a$.

manga Dengka.

manga Oenale. mango. [Semantics: Jonker (1908:346) states 'Oenale has manga in place of [Termanu] pao'.]

\section{Out-comparisons:}

makas Funai Helong. mango.

makas Semau Helong. mango.

*(m)baha Morph: \#(m)baha-k. PRM. fence. Borrowed from: ultimately Sanskrit prākāra. [History: Blust and Trussel (ongoing) reconstruct PMP *pager, but the correspondences in the east cannot be reconciled with this reconstruction.]

pa?a Termanu. fence, wooden hedge.

$$
\text { (J:460) }
$$

pa?a Bokai.

mpa?a $B a^{\prime} a$.

mba?a Lole. fence. (Zacharias et al. 2014)

mba?a Tii.

mbaa Dengka.

mbaa Oenale.

baha|k Ro'is Amarasi. fence.

baha|k Kotos Amarasi. (wooden) fence.

bahan Molo. fence made from sticks. (M:41)

bahan Kusa-Manea. fence.

\section{Out-comparisons:}

paha Semau Helong. fence.

ba?a Fehan Tetun. fence.

baha East Tetun. a circle of wall, defence or enclosure (around houses). (Mo:8)

"mei PRM. table. Borrowed from: Portuguese mesa [meza]. [Form: In most known varieties of Meto insertion of [d] occurs after /i/ before a vowel initial enclitic. Thus, Kotos Amarasi mei $+=\mathbf{a a} \rightarrow$ meedzaa ['me:dze] ['me:3e] (with mid-high [e:] not midlow $[\varepsilon:])$. It would appear that the final vowel of Portuguese mesa [meza] was reanalysed as the determiner $=\mathbf{a a}$ (cf. *aa) with the previous [z] being analysed as an inserted consonant. Such an explanation is probably not possible for Rote, thus indicating that the Rote forms were borrowed from Meto. (Note further that in Baikeno and Amfo'an /dz/ is realised as $[\mathrm{d}] \sim[3] \sim\left[\mathrm{z}^{\mathrm{j}}\right] \sim[\mathrm{z}]$.) A similar process probably took place with cognates of "ngarei 'church'.]

mei Termanu. table. (J:354)

mei Korbafo.

mei Bokai. 
mei Bilbaa.

mei Rikou.

mei $B a^{\prime} a$.

mei Tii.

mei Dengka.

mei Oenale.

mei Kotos Amarasi. table.

mei-ds Amfo'an. table.

mei Molo. table. (M:394)

mei-dts Baikeno. table. (Charles E. Grimes pers. comm.)

Out-comparisons:

midza Semau Helong. table.

mesa East Tetun. a table. (Mo:141)

"mbarani Rote. brave. Borrowed from:

Malay berani [bərani]. [Form:

Either an early loan or a case of correspondence mimicry.]

palani Termanu. brave, bravery; hero. (J:466)

palani Korbafo.

palani Bokai.

palani Bilbaa.

parani Rikou.

palani Lole. dare, daredevil. (Zacharias et al. 2014)

parani Tii. hero, brave, superb.

(Grimes et al. 2014a)

mbalani Dengka.

mbarani Oenale.

na-mba-rani Dela. brave. [Form:

The antepenultimate syllable assimilates to the quality of the agreement prefix, thus providing evidence that it has been reanalysed as a prefix; i.e. $1 \mathrm{SG} \mathbf{P u}$-mbu-rani, 2SG mu-mbu-rani, 2PL/1PL. EXCL mi-mbi-rani, 0/obviative ne-mbe-rani.]

\#aka Rote. anchor. Borrowed from: an unidentified source. Compare Persian ركن [langar], Hindi [laygar], Kambera <tanangga $>$ (Onvlee 1984:471), Bima manga (Ismail et al. 1985:85), Balinese mangar (Kersten 1984:403), and Malay jangkar [dzankar]. The ending -ayga(r) seems to be a kind of Wanderwort and may even be connected with Dutch anker [ankər], and English anchor [âkər] which are ultimately from Greek

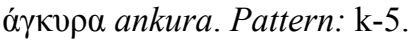

naka Termanu. anchor. (J:374)

naka Korbafo.

naka Bokai.

naka Bilbaa.

na?a Rikou.

naka $B a^{\prime} a$.

naka $T i i$.

naka Dengka.

naka Oenale.

Out-comparisons:

tenaga Hawu. [Form: Grimes, Lado et. al (2008) have Seba and Raijua penavu and Dimu penavo 'anchor'.] (J:374)

"nane Morph: "nane-f. PMeto. daughterin-law, opposite sex sibling's daughter. Borrowed from: probably Helong into Meto before $* 1>n$. But note that Helong has manhiu 'son-in-law' which is a borrowing from another source as identified by the element hiu. This is ultimately from PMP *baqəRu 'new' which is otherwise retained regularly in Helong as balu. [Note: The Rote languages have phrases meaning literally 'new woman' for 'daughterin-law', such as Termanu feto feu-k (Jonker 1908:134).]

nane-f Kotos Amarasi. daughterin-law, opposite sex sibling's daughter.

nane-f Molo. daughter-in-law. (M:348)

\section{Out-comparisons:}

nale-n Funai Helong. daughterin-law, opposite sex sibling's daughter.

nale-n Semau Helong. daughterin-law.

"nome PMeto. Venus, morning star. Borrowed from: probably Helong into Meto before $* 1>n$. [Note: Tetun 
naroma 'light' could be connected.] [Semantics: Rikou has ruu manalepa bafi literally 'the star carrying a pig' for 'Venus, morning star' (Jonker 1908:722). The other Rote languages have phrases meaning 'chicken star', such as Termanu nduu manu-k (Jonker 1908:346).]

faif noem Ro'is Amarasi. Venus, morning star. Lit: 'pig nome'.

faif nome Kotos Amarasi. Venus, morning star. Lit: 'pig nome'. [Semantics: nome has no known independent meaning.]

faif nome (2) <nome $>$ Molo. 1) the morning star. 2) a kind of plant whose leaves will gleam when the morning star shines. (M:380)

\section{Out-comparisons:}

paap lome Semau Helong. Venus, morning star. Lit: 'father lome'. [Semantics: lome has no known independent meaning.]

\#ndara Rote. horse. Borrowed from: ultimately Javanese jaran [dzaran]. [irr. from PRM: *nd $>d$ in Rikou]

ndala Termanu. horse. (J:413)

ndala Korbafo.

lala Bokai.

lala Bilbaa.

ndara $L a n d u$.

dara Rikou.

rara Oepao.

ndala $B a^{\prime} a$.

ndala Lole.

ndara Tii.

ndala Dengka.

ndara Oenale.

ndara Dela. horse.

"ngarei PRM. church. Borrowed from:

Portuguese igreja [igreza]. [Note: Jonker (1908:431) does not give the forms for each Rote language, but states: 'dialects nalei, ngarei, karei'.] [Form: In most known varieties of Meto insertion of [d] occurs after /i/ before a vowel initial enclitic. Thus,
Kotos Amarasi krei $+=\mathbf{a a} \rightarrow$ kreedzaa ['kre:d孔e] ['kre:3e] (with mid-high [e:] not mid-low [ $\varepsilon:]$ ). It would appear that the final vowel of Portuguese igreja [igreza] was reanalysed as the determiner $=\mathbf{a a}$ (cf. *aa) with the previous [3] being analysed as an inserted consonant. (A similar process probably took place with cognates of "mei 'table'.) Such an explanation is probably not possible for Rote, thus Meto may have been the donor for the Rote forms. However, proposing Meto as the intermediary for the Rote forms does not explain the 'regular' reflexes of *ng. This would appear to be a case of adaption of non-native Portuguese [g] with correspondence mimicry (Alpher and Nash 1999:14f).]

ygalei Termanu. church. (J:431)

ygalei Korbafo.

yalei Bokai.

yalei Bilbaa.

karei Rikou.

ygalei $B a^{\prime} a$.

ngalei Lole. church. (Zacharias et al. 2014)

ngarei $T i$.

ygalei Dengka.

ygarei Oenale.

krei Ro'is Amarasi. church, week.

krei Kotos Amarasi. church, week.

klei-ds, klii-ds Amfo'an. church, week.

klei Molo. church. (M:216)

"ngusi PRM. water jar. Borrowed from:

Malay guci [gutfi] 'earthenware jug', ultimately Chinese, e.g. Mandarin 骨瓷 gǔ cí [gu1tshil] 'bone china'.

ngusi Termanu. kind of jug. (J:450)

ngusi Lole. earthenware pot, clay pot. (Zacharias et al. 2014)

ngusi Tii. porcelain or earthenware jug. (Grimes et al. 2014a)

ngusi Dela. jar for water or lontar palm sugar made of clay. (Thersia Tamelan pers. comm. May 2017) 
kusi Kotos Amarasi. water jar.

kusi Molo. water vessel. (M:253)

Out-comparisons:

kusi East Tetun. a clay jar or pot. (Mo:122)

kusi Ili'uun. large earthenware jar. (dJ:122)

uhi Kisar. water jar, earth container.

"ngute Rote. cut with scissors. Borrowed from: Malay gunting [guntin].

ggute Termanu. cut with scissors. (J:450)

ggute Korbafo.

yute Bokai.

nute Bilbaa.

kute Rikou.

ngute $B a^{\prime} a$.

ggute Tii.

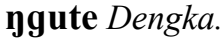

ygute Oenale.

\#omba Rote. wave, billow, swell at sea. Borrowed from: Malay ombak [omba?]. [irr. from PRM: *mb > $m$ for second sense in Termanu] [History: Blust and Trussel (ongoing) reconstruct *humbak to PWMP, but this would require irr. ${ }^{*} \mathrm{u}>o$ in Rote.] na-o opa (2) na-o oma Termanu.

1) wave, undulate. (J:458) 2) wave, undulate. (J:457)

na-o opa Korbafo.

na-o opa Bokai.

na-o opa Rikou.

na-o ompa $B a^{\prime} a$.

na-o omba Dengka.

na-o omba Oenale.

\#oras PwRM. time. Borrowed from:

Portuguese horas [oras], plural of hora

'hour, time'.

oras ia Dela. now.

oros Ro'is Amarasi. time.

oras Kotos Amarasi. time.

olas Molo. hour. (M:403)

Out-comparisons:

oras Semau Helong. time. oras East Tetun. hour, time. (Mo:156)

ors Kisar time.

\#paha PRM. chisel. Borrowed from: Malay pahat.

pa?a Termanu. chiselling. (J:461)

pa?a Korbafo.

pa?a Bokai.

paa Bilbaa.

pa?a Rikou. chisel. (Nako et al. 2014)

mpa?a $B a^{\prime} a$.

pa?a Tii.

paa Dengka.

paa Oenale.

paah Meto. chiselling. (J:461)

Out-comparisons:

pahat Semau Helong. chisel, carve.

bahat East Tetun. chisel. (Mo:8)

"papan PRM. plank. Borrowed from:

Malay papan. [History: Blust and Trussel (ongoing) reconstruct PMP * papan, but the forms here cannot be regularly derived from this.]

papa-k Termanu. plank of ordinary wood. (J:469)

papa-? Korbafo.

papa-k Bokai.

papa-? Bilbaa.

papa-? Rikou.

mpampa-k $B a^{\prime} a$.

papa-k Tii.

papa-? Dengka.

papa-? Oenale.

papan Molo. round planks or stones around the post of the lopo house which prevent mice from getting into the roof. (M:422)

"paria Morph: "paria-k. PRM. bitter melon. Momordica charantia. Borrowed from: Malay peria [poria]. [History: An early loan as indicated by regular Meto $* \mathrm{r}>* 1>n$.]

palia-k Termanu. kind of bitter cucumber. (J:466)

palia-k Bokai.

palia-k $B a^{\prime} a$. 
paria-k Tii.

palia Dengka.

paria Oenale.

pnia? Kotos Amarasi. bitter melon.

${ }^{*}[\mathbf{p} / \mathbf{b}]$ isi PRM. sprinkle. [Form: Meto and Helong show irregular initial consonants. The religious use of this word provides a cultural motivation for borrowing (see the note for the Hawu form).] [History: Blust and Trussel (ongoing) reconstruct * picik to PMP and include the Rote and Hawu forms as part of their evidence. However, (as they concede) $* \mathrm{p}>p$ would be irregular in Rote.]

pisi Termanu. sprinkle. (J:486)

pisi Korbafo.

pisi Bokai.

pisi Bilbaa.

mpisi $B a^{\prime} a$.

(pisi ?) Tii.

bisin Kotos Amarasi. sprinkle.

n-bisin Molo. sprinkles. (M:70)

Out-comparisons:

hiti? Semau Helong. splash, sprinkle, splatter.

hisik East Tetun. dust, shake out; to throw out. (Mo:86)

tebe Dhao. sprinkle, toss, cast, throw. [Sporadic: consonant metathesis: $* \mathrm{bVt}>\mathrm{t} V b$.

pihi, pihe Hawu. sprinkle. [Semantics: 'In traditional Sabu culture, this act of sprinkling can be used as a ritual at any time during one's life where one feels defiled for whatever reason, and needs to be restored to a state of ritual purity. It can be done many times. It can also be done to dedicate a new house (using sugarcane juice, or coconut milk).']

"pinga Morph: "ka-pinga-k. PRM. plate. Borrowed from: Malay pinggan, ultimately from Persian.

pina-k Termanu. dish, plate. (J:485) pina-? Korbafo.

pina-k Bokai.

pina-? Bilbaa.

bika-? Rikou.

pinga-k $B a^{\prime} a$.

pinga-k Tii.

pinga-? Dengka.

pinga-? Oenale.

2|pika|? Kotos Amarasi.

<pika> Molo. plate. (M:437)

Out-comparisons:

pinas Semau Helong. plate.

bikan East Tetun. plate. (Mo:13)

"pohat Morph: "ka-pohat. PMeto. bark of tree, husk. [History: While borrowing between Helong and Meto is likely, the direction is unclear. Amarasi Ppoho-f with medial $h$ is probably a subsequent borrowing from Helong after this term had been share between these languages.]

poho? Ro'is Amarasi. tree bark.

?|poho-f Kotos Amarasi. tree bark, egg shell, book cover.

2|po?at, ?|po?ot Kopas. tree bark.

2|po?at Molo. shell or husk of a fruit. (M:442)

2|po?a-n Amfo'an. wet tree bark. [Form: ?kuit $=$ 'dry tree bark'.] (own field notes)

P|po?o-f Timaus. tree bark.

po?at Kusa-Manea. skin, bark, fingernail, toenail.

Out-comparisons:

kpohot Funai Helong. bark, husk, shell.

pohot Semau Helong. bark.

"pukat PRM. dragnet. Borrowed from:

Malay pukat. Pattern: k-9'.

pu?a-k Termanu. dragnet. (J:499f)

pua-k Korbafo.

pu?a-k Bokai.

puka-? Bilbaa.

puka-? Rikou.

mpu?a-k $B a^{\prime} a$.

pu?a-k Tii.

pu?at Dengka. 
puPat Oenale.

pu?at Kotos Amarasi. dragnet. (M:453)

\section{Out-comparisons:}

pukat Semau Helong. dragnet.

"puli Rote. cure, heal. Borrowed from:

Malay pulih. [History: While this may ultimately be connected with PMP *pa-qudip the universal $* d=l$ correspondence and $*$ aqu $=u$ show that this is a borrowing.]

puli Termanu. cure someone, treat a sick person. (J:503)

puli Korbafo.

puli Bokai.

puli Bilbaa.

puli Rikou.

mpuli $B a^{\prime} a$.

puli Tii.

puli Dengka.

puli Oenale.

"rapa Rote. bridle. Borrowed from: Hawu into Rote.

lapa Termanu. bridle, put on a bridle. (J:281)

lapa Korbafo.

lapa Bokai.

lapa Bilbaa.

rapa $R i k o u$.

lapa $B a^{\prime} a$.

rapa $T i i$.

la lapa-t Dengka.

rapa Oenale.

Out-comparisons:

rapa Hawu. bridle. ( $\mathrm{J}: 281)$

<rapa> Kambera. bridle, reins. (On:432)

${ }^{\#}$ riti PRM. copper, bracelet. Borrowed from: ultimately Sanskrit rīti [ri:ti] 'bell-metal'. [History: This is an early loan, as shown by Meto $* \mathrm{r}>* 1>n$.]

liti Termanu. copper. (J:315)

liti Korbafo.

liti Bokai.

liti Bilbaa.

riti Rikou.

liti $B a^{\prime} a$. riti Tii.

liti Dengka.

riti Oenale.

niti Kotos Amarasi. bracelet.

niti-ds Amfo'an. bracelet.

Out-comparisons:

liti Semau Helong. bracelet.

riti East Tetun. copper, brass. (Mo:161)

"roa Morph: "roa-k. CER. room. Borrowed from: Malay ruang.

loa-k Termanu. room, chamber. ( $\mathrm{J}: 318)$

loa-? Korbafo.

loa-k Bokai.

loa-? Bilbaa.

roa-? Rikou.

Out-comparisons:

loan Helong. ( $\mathrm{J}: 318)$

"rusa PRM. deer. Borrowed from: Malay rusa. [irr. from PRM: ${ }^{*} \mathrm{r}>\left({ }^{*} 1\right)>n$ in Termanu, Korbafo, Bokai, and Ba'a]

nusa Termanu. deer. (J:409)

nusa Korbafo.

nusa Bokai.

lusa Bilbaa.

rusa Rikou.

nusa $B a^{\prime} a$.

rusa Tii.

lusa Dengka.

rusa Oenale.

ruus Kotos Amarasi. deer. [Form: reanalysis as ruus $=\mathbf{a a}$ ['ro:se] with the determiner $=\mathbf{a a}$ attached to a consonant final stem.]

luus Molo. deer. (M:293)

Out-comparisons:

lusa Semau Helong. deer.

rusa, bibi_rusa East Tetun. deer. (Mo:162)

\#sama Rote. same. Borrowed from: ultimately Sanskrit sama, perhaps via Malay sama.

sama Termanu. be the same. (J:518)

sama Korbafo.

sama Bokai.

sama Bilbaa. 
sama Rikou.

sama $B a^{\prime} a$.

sama $T i i$.

sama Dengka.

"sarani PRM. baptise. Borrowed from: Arabic nașrān̄̄ [nas'ra:ni:] 'Christians', compare Syriac nașrāya [nas'ra:ja:] 'Nazarene'. [irr. from PRM: ${ }^{*} \mathrm{n}=n / l_{-}$in Amfo'an (expect $*_{\mathrm{n}}>$ l)] [Form: The initial syllable was originally na which has been reanalysed as a third person agreement marker.] [History: This term is very old in the region. Grimes et al. (2014a) state: 'Widely used through much of eastern Indonesia in Malays and vernaculars, and its use has been established for centuries.']

salani Termanu. make someone Christian by baptising, baptise. (J:516)

sarani Rikou. baptise. (Nako et al. 2014)

salani Lole baptise. (Zacharias et al. 2014)

sarani Tii. baptise; ritual for formally initiating people as members of the Christian faith; neutral as to method (e.g. immersion or sprinkling). (Grimes et al. 2014a)

sarani Dela baptise, christen.

na-srani (2) srani|2 Kotos Amarasi.

1) baptise. 2) baptism, baptismal.

na-slani (2) slani? Amfo'an.

1) baptise. 2) baptism.

Out-comparisons:

sarani Semau Helong. baptise.

"sarombo Rote. pants, cover the body. Borrowed from: an unidentified source, possibly a language of Sulawesi give the Makassar form.

lopo (2) lopo lopo (3) uma=a nasa-lo lopo Termanu. 1) trousers.

2) he wears his sarong hanging down to the ankles like a woman's sarong. ana pake naa sidi=na lopo lopo 3) the house has a low sloping roof. (J:327)

lopo Korbafo. trousers. (J:327)

lopo Bokai. trousers. (J:327)

(2) lopo lopo, na-sa-lo lopo Bilbaa. 2) completely cover oneself or one's body. (J:727)

ropo Rikou. trousers. (J:327)

lompo (2) lompo lompo $B a^{\prime} a$.

2) completely cover oneself or one's body. (J:727)

rombo (2) rombo rombo, saro rombo Tii. 1) sarong. 2) completely cover oneself or one's body. (J:727)

(2) lombo lombo, na-salo lombo Dengka. 2) completely cover oneself or one's body. (J:727)

Out-comparisons:

sapolo Kisar. trousers, pants.

robo Hawu. cover. (J:727)

saromboy Makassar. pillowcase.

$(\mathrm{J}: 727)$

sərombon Malay. pipe, tube; hollow cylinder.

"seyge Rote. cloves. Syzygium aromaticum. Borrowed from: Malay cengkeh [tenkeh]. [Form: The first element in most forms may be connected with Malay pala 'nutmeg'.] pela/seje Termanu. cloves. (J:478) pela/sege Korbafo. pela/sene Bokai. pela/seje Bilbaa. pela/seke Rikou. pela/senge $B a^{\prime} a$. pele/senge Tii. pela/senge Dengka. senge Oenale.

${ }^{\#}$ seremere PRM. Malay gooseberry. Phyllanthus acidus. Borrowed from: Malay cermelek [termele?].

selemele(-k), selumele-k Termanu. the Malay gooseberry tree, called cermelek in Kupang. (J:532) 
sarmeri Kotos Amarasi. Malay Gooseberry.

salmele Molo. small tree the roots of which have toxic properties. Phyllantus acidus. (M:472)

"Soro Rote. push forwards. Borrowed from: Malay sorong. [History: Blust and Trussel (ongoing) reconstruct PWMP *suruy stating: 'A number of these forms may be loans (from Malay ...), but the comparison as a whole cannot easily be dismissed as a product of borrowing.' In the case of the Rote forms Jonker (1908) suggests they are borrowings from Malay and I agree, mainly due to the identical vowels which would not be explained by inheritance from * suruy.]

solo Termanu. push forwards. (J:560)

solo Korbafo.

solo Bokai.

solo Bilbaa.

soro Rikou.

solo-k $B a^{\prime} a$.

soro Tii.

solo Dengka.

soro Oenale.

\#sumba PRM. oath, pledge; curse. Borrowed from: Malay sumpah.

supa Termanu. oath, curse. (J:576)

supa Korbafo.

supa Bokai.

supa Bilbaa.

supa Rikou.

sumpa $B a^{\prime} a$.

sumba Tii.

sumba Dengka.

sumba Oenale.

n-supa Kotos Amarasi. swear.

Out-comparisons:

sumpa Semau Helong. swear, oath.

\#abe PRM. greet. Borrowed from:

Malay tabek/tabik, ultimately Sanskrit kșantavya [kşantavja] 'be pardoned, forgiven'. tabe-k Termanu. bring greetings, greetings on arrival. (J:582)

tabe Dela. shake hands.

n-tabe Kotos Amarasi. greet, shake hands with.

\section{Out-comparisons:}

tabe Semau Helong. greeting, respect. Usage: Archaic. Associated with preindependence eras. A respectful greeting to a person of high status.

tabe Fehan Tetun. greeting. (Mo:177)

\#tai PRM. weigh. Borrowed from: Malay tahil 'unit of measurement for gold'.

tai Termanu. weigh something. (J:586)

tai Korbafo.

tai Bokai.

tai Bilbaa.

tai Rikou.

tai $B a^{\prime} a$.

tai Tii.

tai Dengka.

tai Oenale.

n-tai Kotos Amarasi. weigh, evaluate, balance.

tai Molo. scales. (M:530)

Out-comparisons:

tai Semau Helong.

"tanda PRM. sign. Borrowed from: Malay tanda An early borrowing as indicated by the regular correspondences, particularly $*_{\text {nd }}>*_{\mathrm{r}}>k$ in Amarasi.

tana Termanu. sign. (J:293)

tana Korbafo.

tana Bokai.

tana Bilbaa.

tanda Rikou.

tana $B a^{\prime} a$.

tanda Tii.

tanda Dengka.

tanda Oenale.

taka Kotos Amarasi. sign.

Out-comparisons:

tada Semau Helong. sign. 
ha-tada, ha-tadak, ha-tadan

East Tetun. place a sign or mark (as a sign of ownership). (Mo:81)

"tasu PRM. fry pan. Borrowed from:

Portuguese tacho [tafu].

tasu Termanu. fry-pan. (J:602f)

tasu Korbafo.

tasu Bokai.

tasu Bilbaa.

tasu Rikou.

tasu $B a^{\prime} a$.

tasu Tii.

tasu Dengka.

tasu Oenale.

tasu Kotos Amarasi. wok.

Out-comparisons:

tasu East Tetun. small pan. (Mo:181) \#tuhas Rote. rice-cake snack cooked in a small container of woven young coconut leaves. Borrowed from: Malay ketupat. An early borrowing into Rote and Hawu as attested by the regular correspondences.

tu?as $B a^{\prime} a$. packet of rice. (J:774)

tu?as Tii.

tuPas Dengka.

tuPas Oenale.

Out-comparisons:

katupa, katufa, katuba East Tetun. rice cooked in little bags of green palm leaves. (Mo:104)

kedu?e Hawu. (J:774)

Total number of entries: 1,257 

5

English - Rote-Meto 


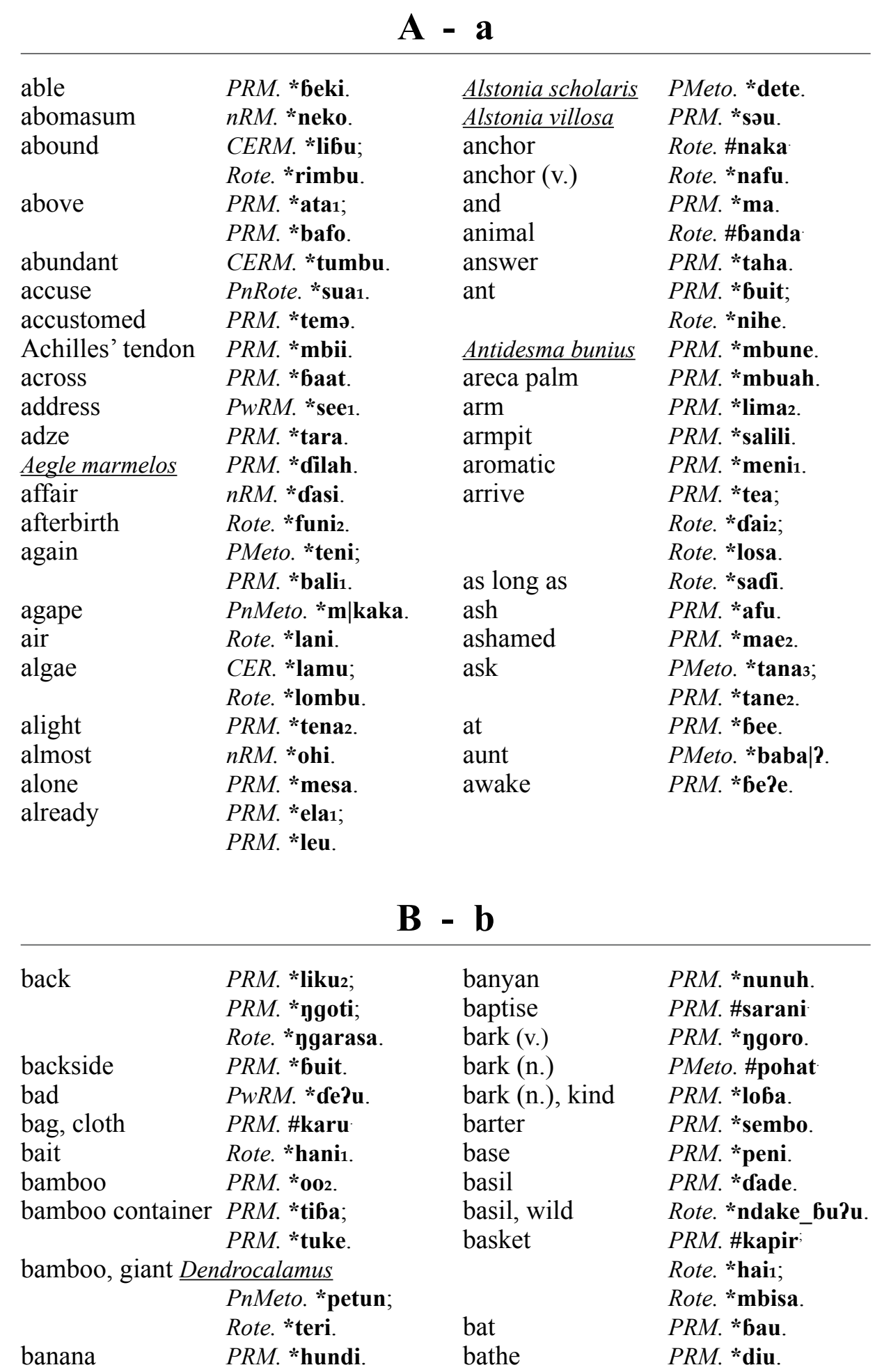




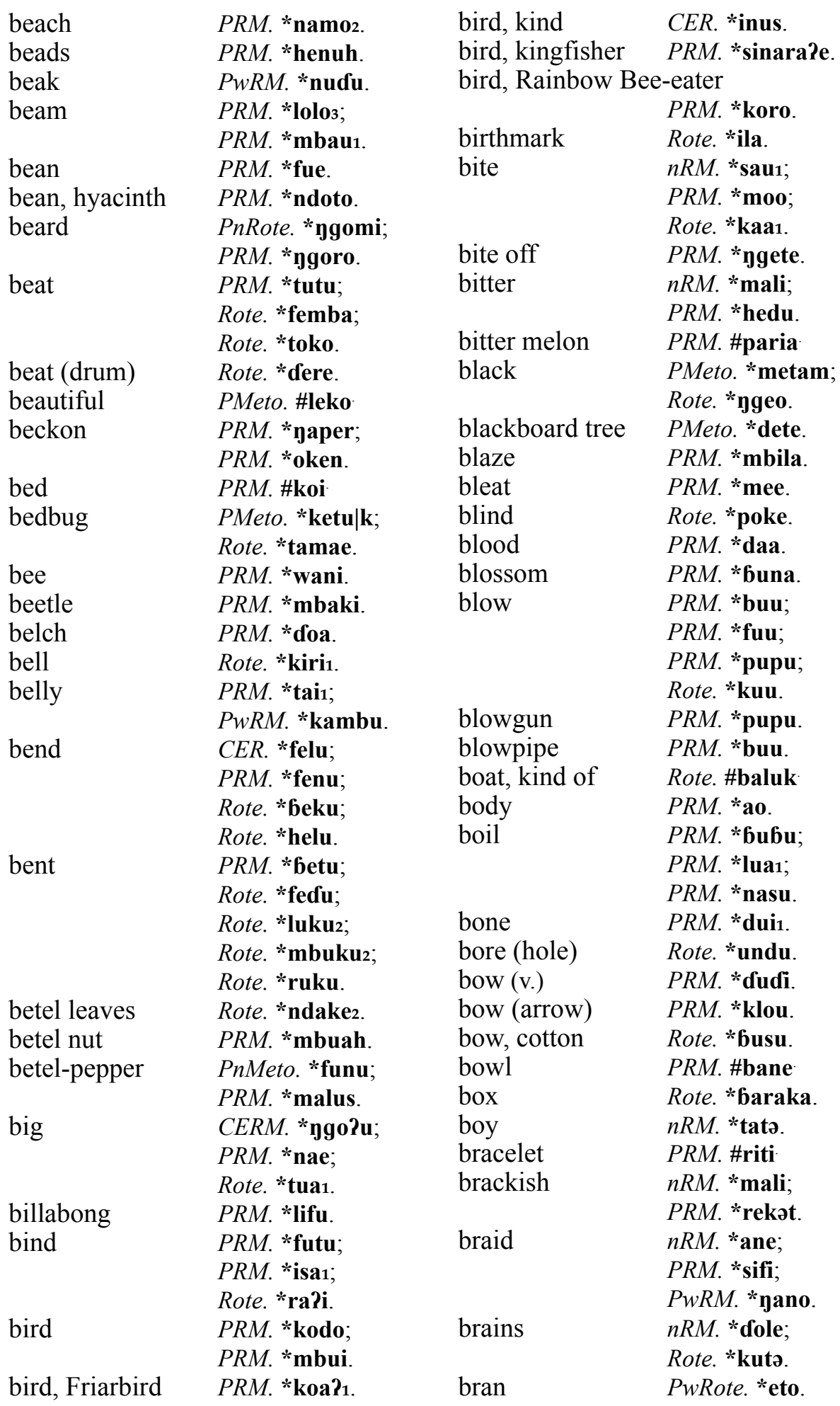




\begin{tabular}{|c|c|c|c|}
\hline branch & $n R M$. *sabake; & brother (of wor & \\
\hline & PMeto. *sana|?. & & PRM. *naa2. \\
\hline brave & Rote. \#mbarani & brother-in-law & PRM. *kera1. \\
\hline breadfruit & PMeto. *kunu1; & brother, older & $\begin{array}{l}P R M . \text { *to?o. } \\
P R M \text { *sakiki }\end{array}$ \\
\hline break & $\begin{array}{l}\text { PMeto. *fera1; } \\
\text { PRM. *tebi. }\end{array}$ & $\begin{array}{l}\text { bubble } \\
\text { bucket }\end{array}$ & $\begin{array}{l}\text { PRM. *bubu. } \\
\text { PRM. *sambat; }\end{array}$ \\
\hline break off & PRM. *ketu. & & Rote. *hai1. \\
\hline breast & $\begin{array}{l}P R M . \text { *susu2; } \\
P R M . \text { *tendə. }\end{array}$ & buffalo & $\begin{array}{l}\text { PMeto. *biae; } \\
\text { Rote. \#kamba }\end{array}$ \\
\hline breath & PRM. *sanasə. & bunch, fruit & PRM. *ngii. \\
\hline breathe & CERM. *kele; & bunch of fruit & PMeto. *sana|?. \\
\hline & $\begin{array}{l}\text { PRM. *hae; } \\
\text { PRM. *sanaso. }\end{array}$ & $\begin{array}{l}\text { bundle } \\
\text { bundle up }\end{array}$ & $\begin{array}{l}\text { PRM. *futu. } \\
\text { Rote. *mbutu2. }\end{array}$ \\
\hline bride price & Rote. *beli. & burn & PRM. *hotu. \\
\hline bridge & PRM. *lete2. & burning & PRM. * mbutu1. \\
\hline bridle & Rote. \#rapa & burp & $P R M$. *doa. \\
\hline bright & PRM. *maneu. & bury & PRM. *suba. \\
\hline bring & PRM. *əndi. & bush & PRM. *lasi1. \\
\hline broken & $C E R . *$ tefe; & buttocks & $P R M$. *saka. \\
\hline rood & $\begin{array}{l}P w R M . \text { *de?u. } \\
P R M . \text { *luku1. }\end{array}$ & buy & $\begin{array}{l}\text { PMeto. *sosa; } \\
\text { Rote. *beli. }\end{array}$ \\
\hline
\end{tabular}

\section{C - c}

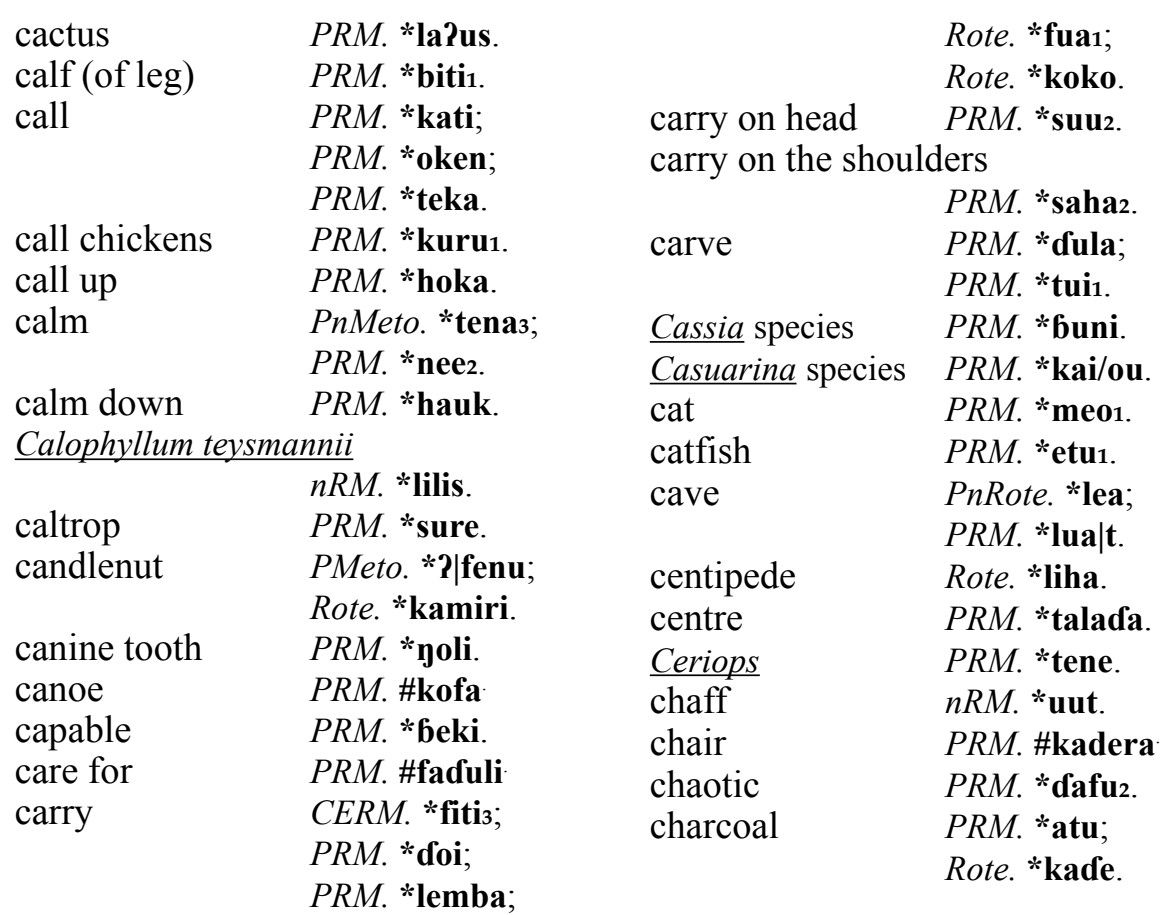




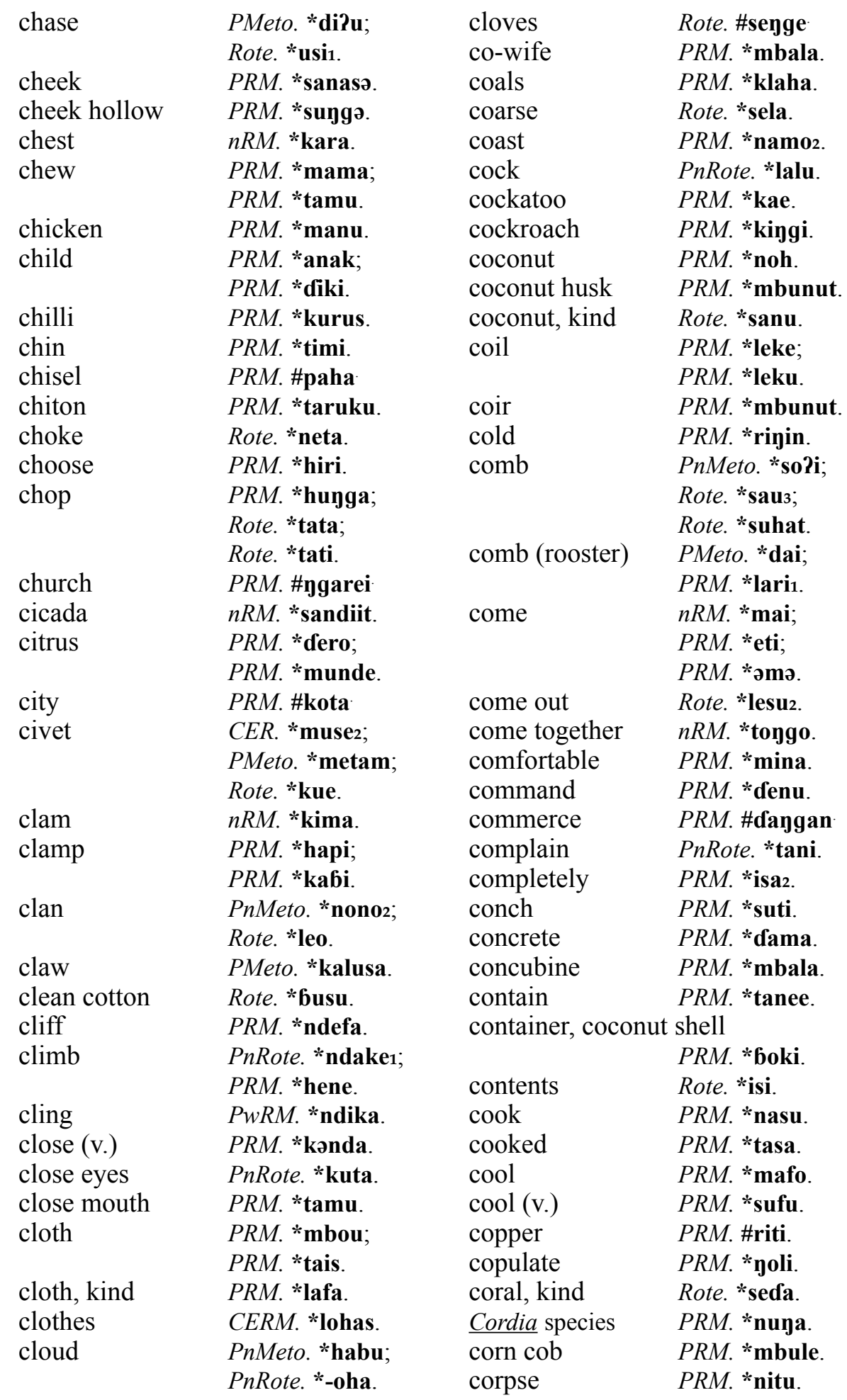




\begin{tabular}{|c|c|c|}
\hline corral & CERM. *oka1; & $\begin{array}{l}\text { crooked } \\
\text { cross }\end{array}$ \\
\hline $\cos t$ & $\begin{array}{l}\text { Rote. *royga. } \\
\text { Rote. *beli. }\end{array}$ & $\begin{array}{l}\text { cross } \\
\text { crossbeam }\end{array}$ \\
\hline cotton & PRM. \#kabas. & crouch \\
\hline cough & PRM. *boho. & crow \\
\hline count & Rote. *kahis. & crumble up \\
\hline country & PRM. *ingu. & crush \\
\hline course & PRM. *dalan. & \\
\hline cover & CER. *balus; & cry \\
\hline & $\begin{array}{l}\text { PRM. *koba; } \\
\text { PRM. *mbalu; }\end{array}$ & cry out \\
\hline & PRM. *tana1. & cucumber \\
\hline cow & PMeto. *biae. & cure \\
\hline $\mathrm{crab}$ & PRM. *niit. & current \\
\hline crab, hermit & $P R M$. *sayguma. & cuscus \\
\hline crack & Rote. *faka2. & \\
\hline cram & PRM. *sesə. & \\
\hline crawl & $\begin{array}{l}\text { PRM. *rodok; } \\
\text { Rote. *nodo. }\end{array}$ & $\begin{array}{l}\text { cushion } \\
\text { custom }\end{array}$ \\
\hline crazy & PRM. *mulu. & cut \\
\hline creature & PRM. *ulə. & \\
\hline creep & PRM. *nama. & \\
\hline cricket & $n R M$. *sandiit. & \\
\hline crisp & Rote. *tana2. & \\
\hline croak & Rote. *ngede. & \\
\hline crocodile & $C E R$. * foe 2 & \\
\hline
\end{tabular}

cut off cut vegetation cyclone
Rote. *kilu. PRM. *danga1. PRM. *baat. PRM. *dudi. PRM. *kaa2. PRM. *tebi. PRM. *mendu; Rote. *tede. $P w R M$. *ngae. PRM. *koa?2; Rote. *eki. PRM. *timuz. Rote. \#puli Rote. *faa. PMeto. *arum; PnMeto.

*?|mauka|?. PRM. *lunu. PRM. \#hadat CERM. *ygebo; $P R M$. *fandi; PRM. *koti; PRM. *ndaru; PRM. *sada; PRM. *teta; PwRM. *?uta; Rote. *fura; Rote. *tati. PRM. *ketu. Rote. *beta. PRM. *kutus.

\section{D - d}

\begin{tabular}{|c|c|c|c|}
\hline dam & CERM. *tete. & debark & PRM. *sambi. \\
\hline dance & PRM. *basoko; & debt, fine & Rote. \#huta \\
\hline & $P R M$. *foti; & decide & PMeto. *fera1. \\
\hline & Rote. *lendo. & deep & PRM. *dəma. \\
\hline dance, circle & CERM. *bone. & deer & PRM. \#rusa \\
\hline daughter-in-law & PMeto. \#nane & delicious & PRM. *mina. \\
\hline & $P R M .{ }^{*} \mathbf{f a i}$ & depending & $n R M$. *boni. \\
\hline & PRM. *ledo. & Derris elliptica & $P R M$. *tufa. \\
\hline day after tomorro & PPRM. *beni; & desire & Rote. *doki. \\
\hline & PRM. * esak. & dew & $C E R$. *ahu; \\
\hline Gay derote yester & $\begin{array}{l}\text { lay } P R M . \text { *beni; } \\
\text { PRM. *esak. }\end{array}$ & die & $\begin{array}{l}C E R M . \text { *angum } \\
P R M . * \text { mate. }\end{array}$ \\
\hline
\end{tabular}




\begin{tabular}{|c|c|c|c|}
\hline dig & $\begin{array}{l}\text { PRM. *kali; } \\
\text { Rote *fuka }\end{array}$ & dove, wild & PMeto. *kumu1. \\
\hline dig out & PRM. *toki. & draw water & $\begin{array}{l}P K M \text {. \#pukat } \\
P R M \text { *dolu. }\end{array}$ \\
\hline digging stick & PRM. *sua3. & & PRM. *ndui. \\
\hline $\operatorname{dip}$ & PRM. *dəmbə; & dream & PRM. *mehi. \\
\hline & Rote. *deta. & dried & Rote. *tana2. \\
\hline dirt & $P R M$. *daki. & dried up & PRM. * meti. \\
\hline disaster & PRM. *soe. & drink & $P R M$. *inu. \\
\hline discussion & $n R M$. *dasi. & drip & Rote. *tiri; \\
\hline dish & Rote. \#basiu & & Rote. *titi. \\
\hline distant & $P R M$. *doo. & drone & CERM. *yguu; \\
\hline distribute & CERM. *bati. & & $P R M$. *nguru. \\
\hline divide & CERM. *bati; & drown & $P R M$. *dəma. \\
\hline & PnMeto. *paha; & drunk & PRM. *mafu. \\
\hline & Rote. *faka1. & dry & PnRote. *tuuz; \\
\hline & $P R M$. *tao. & & PwRM. *meto; \\
\hline docile & PRM. *maus. & & Rote. *manguz. \\
\hline & CERM. *asu; & dry in sun & PRM. *hoi. \\
\hline & Rote. *busa. & dry season & $P R M$. *fandu. \\
\hline Dolichandrone & hacea & dry up & Rote. *mada. \\
\hline & Rote. *tuiz. & duckweed & Rote. *lombu. \\
\hline dolphin & $C E R$. *lemuk. & dugong & Rote. *duiz. \\
\hline domesticated & $\begin{array}{l}\text { PRM. *aem; } \\
\text { PRM. *maus. }\end{array}$ & dumb & $\begin{array}{l}\text { PnMeto. *mono; } \\
\text { Rote. *nqoa. }\end{array}$ \\
\hline door & $n R M$. *eno. & dust & PRM. *afu. \\
\hline douse & $n R M$. *fui 1. & dyke & CERM. *tete. \\
\hline dove & $\begin{array}{l}\text { PRM. *tadengus; } \\
\text { PRM. *teu; } \\
\text { PRM. *mukə. }\end{array}$ & & \\
\hline
\end{tabular}

\section{$\mathbf{E}-\mathbf{e}$}

eagle

ear

earth

earthworm

earwax

eat

edge

eel

eel, freshwater
CERM. *tema1.

Rote. *ndiki.

PRM. *dae.

Rote. *kalati.

Rote. *ngela1.

PMeto. \#bukae

PRM. *ha;

$P R M$. *hengu.

PRM. *lida;

Rote. "suhu.

PRM. *lema;

PRM. *ngois.

PRM. *tuna. egg

eight

elbow

embarrassed

embers

embrace

empty

enclose

endure

enemy

engrave

enter

entire

entwine
PRM. *tolo.

PRM. *falu.

PRM. *siku.

PRM. *mae.

PRM. *klaha.

PRM. *holu1.

PRM. *duman.

PRM. *mbalu.

Rote. *taa4.

PRM. *musu.

PRM. *dula.

PMeto. *tama1.

PRM. *temaz.

$n R M$. *laba. 


$\begin{array}{llll}\text { equipment } & \text { CERM. *lohas; } & \text { evaporate } & \text { Rote. *mada. } \\ & \text { Rote. *buas. } & \text { exchange } & \text { PRM. *selut; } \\ \text { Erythrina } \text { species } & \text { PRM. *deras. } & & \text { PRM. *sembo. } \\ \text { eschalot } & \text { PRM. *laisona. } & \text { excrement } & \text { PRM. *tai2. } \\ \text { ethnic group } & \text { PwRM. *-s. } & \text { exist } & \text { PRM. *-uki. } \\ \text { eucalyptus } & \text { PnMeto. *hu?e; } & \text { expand } & \text { Rote. *mbumbu. } \\ & \text { Rote. }{ }^{*} \text { mgela2. } & \text { eye } & \text { PRM. *mata1. } \\ \text { European } & \text { Rote. \#filanda } & \text { eyebrows } & n R M . * \text { mbonu. }\end{array}$

$\mathbf{F}-\mathbf{f}$

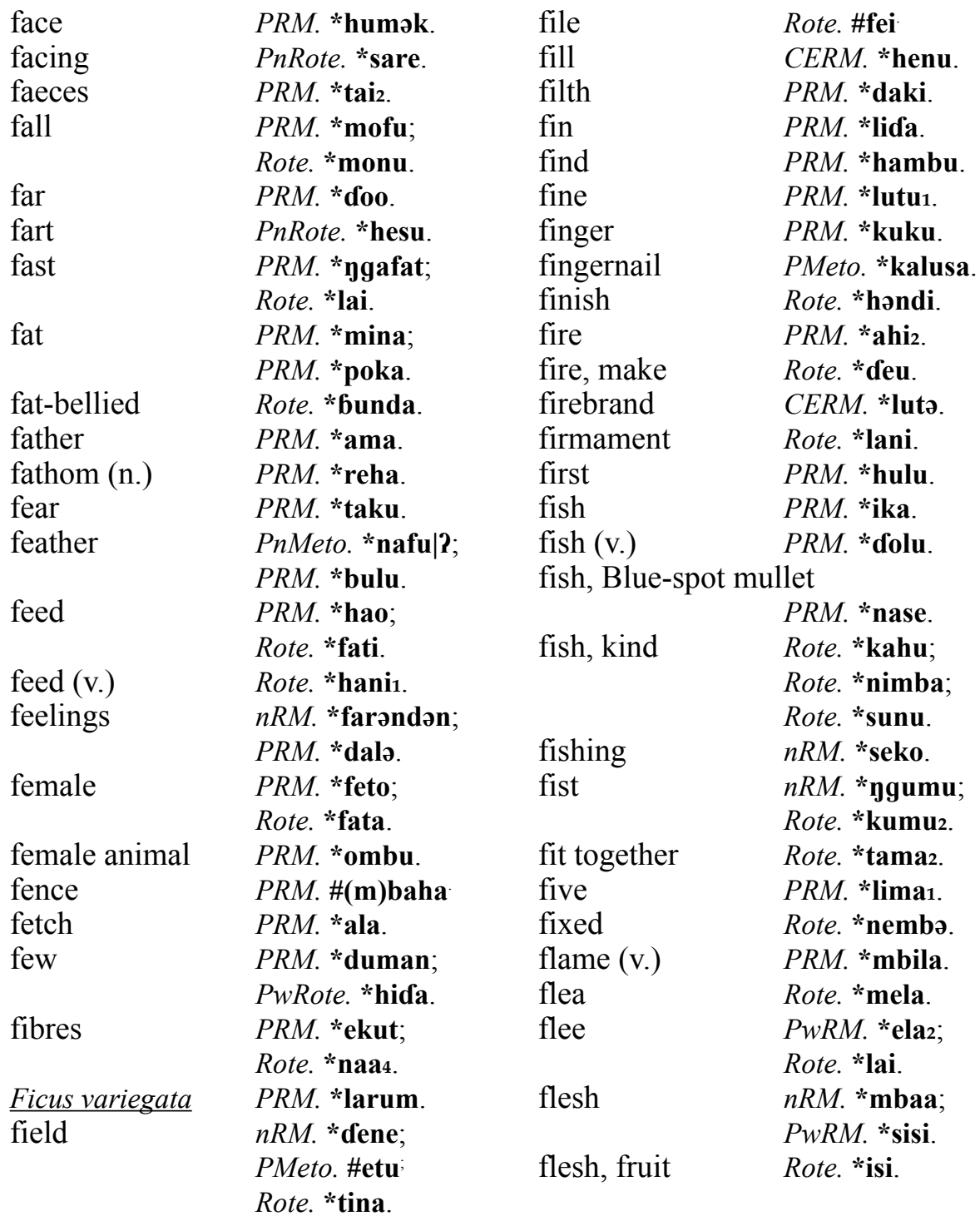




\begin{tabular}{|c|c|c|c|}
\hline flexible & $\begin{array}{l}\text { PRM. *betu; } \\
\text { Rote. *fedu. }\end{array}$ & force & $\begin{array}{l}P R M . * \text { seke; } \\
P R M . \text { *seti. }\end{array}$ \\
\hline floor & $P R M$. *afu. & forehead & Rote. *dei. \\
\hline flow & $n R M$. *sai1; & foreigner & PMeto. *kase. \\
\hline flower & $\begin{array}{l}\text { Rote. *faa. } \\
\text { PRM. *buna. }\end{array}$ & forest & $\begin{array}{l}P R M . \text { *lasi1; } \\
\text { PRM. * yura. }\end{array}$ \\
\hline flower, decorative & Rote. \#bunga & fort & PRM. \#kota \\
\hline flute & $P R M$. * feku. & fortune & PRM. *uat. \\
\hline fly & $\begin{array}{l}\text { PMeto. *ka|benu; } \\
\text { Rote. *mbena. }\end{array}$ & $\begin{array}{l}\text { four } \\
\text { foxtail millet }\end{array}$ & $\begin{array}{l}\text { PRM. *haa2. } \\
\text { Rote. *beto. }\end{array}$ \\
\hline fly (v.) & $\begin{array}{l}\text { PnRote. *laa4; } \\
\text { PRM. *tambele. }\end{array}$ & $\begin{array}{l}\text { fragrant } \\
\text { freckle }\end{array}$ & $\begin{array}{l}\text { PRM. *meni1. } \\
\text { Rote. *ila. }\end{array}$ \\
\hline flying fox & PRM. *bau. & Friarbird & PRM. *koa?1. \\
\hline foam & PRM. *fudzə. & frighten & $P R M$. *saygenger. \\
\hline fog & PMeto. *kupu; & frizzy & $n R M$. *buә. \\
\hline folded & $\begin{array}{l}\text { PnMeto. *habu. } \\
\text { PRM. *betu. }\end{array}$ & $\begin{array}{l}\text { Irog } \\
\text { from }\end{array}$ & $\begin{array}{l}\text { Rote. *ygede. } \\
\text { PRM. *əmə. }\end{array}$ \\
\hline follow & CERM. *tuin. & front & PRM. *ulu. \\
\hline fontanelle & $\begin{array}{l}\text { PMeto. *fufu1; } \\
\text { PRM. *botos; } \\
\text { PRM. *fumbu. }\end{array}$ & $\begin{array}{l}\text { front, be in } \\
\text { fruit } \\
\text { fry }\end{array}$ & $\begin{array}{l}P R M . \text { *hulu. } \\
P R M . \text { *bua. } \\
\text { PRM. *sena. }\end{array}$ \\
\hline foot & $\begin{array}{l}\text { PMeto. *hae|2; } \\
\text { Rote. *ei. }\end{array}$ & $\begin{array}{l}\text { fry pan } \\
\text { full }\end{array}$ & $\begin{array}{l}\text { PRM. \#tasu } \\
C E R M . \text { *henu. }\end{array}$ \\
\hline for & PRM. *əu. & fur & PRM. *bulu. \\
\hline
\end{tabular}

\section{G - g}

galangal

gall bladder

gamete

garden
PRM. *sokum.

PRM. *hedu.

PRM. *natu.

$n R M$. *dene;

PRM. *mamər;

Rote. *osi;

Rote. *tina.

gargle

garlic

garment

Garuga floribuna

gate

gather

gather under wings

gecko

get

get up
Rote. *mumuz.

PnMeto. *pio.

PRM. \#badzu

PRM. *beuz.

$n R M$. *eno.

PRM. *bua.

PnRote. *saluku.

PRM. *teke.

$P R M$. *ala.

$P w R M$. *fela1;

Rote. *fo?a. gills

ginger

give

glitter

go

go back

go down

go up

goat

goatee

goatfish

gold

good

gourd

grain, crushed
PRM. *ngadas.

PRM. *laia.

PRM. *fee2.

PRM. *dila2.

PRM. *eti;

PRM. *əu;

PRM. *lako.

PRM. *bali.

Rote. *loe1.

PRM. *sake.

PRM. *bibi.

PnRote. *ngomi.

Rote. *ti(?)o.

PRM. *lilo.

PMeto. \#leko

PRM. *bongoz;

PRM. *kelas.

PRM. *dio. 


\begin{tabular}{|c|c|c|c|}
\hline grain head & PRM. *mbule. & $\underline{\text { Grewia slutaris }}$ & PRM. *lino. \\
\hline grandchild & PRM. *umbu. & grey hair & PRM. *mofa. \\
\hline grandfather & $P w R M$. *la?i; & grind & PRM. *mendu. \\
\hline & Rote. *ba?i. & grope & PRM. *badoe. \\
\hline \multirow{2}{*}{$\begin{array}{l}\text { grandmother } \\
\text { grass }\end{array}$} & PRM. *bei. & ground & $P R M$. *afu; \\
\hline & $\begin{array}{l}C E R M . \text { * mau; } \\
\text { PRM. *kuun; } \\
\text { PRM. *nade. }\end{array}$ & grow & $\begin{array}{l}\text { PRM. *dae. } \\
\text { PRM. *nae; } \\
\text { Rote. *numbu. }\end{array}$ \\
\hline grasshopper & $P R M$. *lamat. & growl & PRM. *yguru. \\
\hline grate & $\begin{array}{l}P R M . \text { *fora; } \\
P R M . \text { *paru. }\end{array}$ & $\begin{array}{l}\text { grub, edible } \\
\text { guard }\end{array}$ & $\begin{array}{l}\text { PRM. *bate. } \\
C E R M . \text { *papa. }\end{array}$ \\
\hline grave & $P R M$. *rates. & guava & PRM. \#koedzabas \\
\hline great & CERM. *ngo?u. & gum & PRM. *dama; \\
\hline green & PRM. *mata2; & & PRM. *dito. \\
\hline greet & $\begin{array}{l}P R M . \text { *modo. } \\
P R M . \text { *səru: }\end{array}$ & $\begin{array}{l}\text { gum tree } \\
\text { gums }\end{array}$ & $\begin{array}{l}\text { Rote. *ngela2. } \\
\text { PMeto *nada. }\end{array}$ \\
\hline & $\begin{array}{l}\text { PRM. *teka; } \\
\text { PRM. \#tabe }\end{array}$ & gun & $P R M$. *ndelat. \\
\hline
\end{tabular}

$\mathbf{H}-\mathbf{h}$

hair

hair, body

hair, head

half

hand

hand span

hang

hang over

hard

harvest

have

he

head

head hair

head, lower

heal $n R M$. *buə;

$n R M$. *mbonu;

$P R M$. *bulu.

PnMeto. *nafu|?

PRM. *ulu.

CERM. *tairua.

PRM. "lima2.

PnRote. *tenga;

PwRote. *hayga.

$n R M$. *boni;

$P R M$. *henge;

PRM. *loke;

PRM. *tambele.

PRM. *bandae.

Rote. *nembə

PRM. *koru;

PRM. *sengi.

PRM. *-uki;

Rote. *ena.

PnRote. *ndia;

PwRM. *eni.

$P R M$. *langa.

$P R M$. *layga.

CERM. *ygiro.

Rote. \#puli heap

hear

CERM. *tumbu.

$P w R M$. *rena;

Rote. *nenez.

heart

hearth

heartwood

heaven

heavy

heel

help

PRM. *tendo.

$n R M$. *raho.

PRM. *teas.

Rote. *lani.

PRM. *berat.

$P R M$. *tinga.

PRM. \#6a6a;

Rote. *fali;

Rote. *holu2.

her $\quad P w R M$. *ee.

herd (v.) PRM. *boo1.

here

$P R M$. *ia.

Hibiscus tiliaceus

hide

PRM. *bau.

PRM. *funi1;

$P w R M$. *kuna.

hill

him

PRM. *lete1;

Rote. *mbukuz.

PwRM. *ee.

PnMeto. *deku;

PRM. *tufu;

Rote. *femba;

Rote. *liku1. 


\begin{tabular}{|c|c|c|c|}
\hline hold & Rote. *kira1. & house & PRM. *uma. \\
\hline hold in mouth & PRM. *moo. & house post & PRM. *diiiz. \\
\hline hole & PRM. *bolo1; & household & Rote. *loo2. \\
\hline & PRM. *mboo; & hover & PRM. *6andae. \\
\hline & $P w R M$. *kola. & how much? & PRM. *hida. \\
\hline hook & PRM. *kai 2. & howl & CERM. *nguu. \\
\hline hope & $n R M$. *farəndən; & hug & PRM. *holu1. \\
\hline & PRM. *hena. & hundred & $P R M$. *natu|n. \\
\hline horn & PRM. *sura. & hungry & CERM. *laha. \\
\hline horn (instrument) & CERM. *to?is. & hunt & $n R M .{ }^{*}$ seko. \\
\hline hornet & CERM. *katefuan. & hurt & Rote. *lobo. \\
\hline horse & Rote. \#ndara & hut & CERM. *lopo; \\
\hline hot & $\begin{array}{l}P R M . \text { *hanas; } \\
P R M . \text { *mbutu1; } \\
\text { PRM. *tobi. }\end{array}$ & & PRM. *laa2. \\
\hline
\end{tabular}

\begin{tabular}{|c|c|c|c|}
\hline $\begin{array}{l}\text { I } \\
\text { impede } \\
\text { in } \\
\text { incite } \\
\text { indigo } \\
\text { inflated } \\
\text { inform } \\
\text { in-law } \\
\text { insect, kind }\end{array}$ & $\begin{array}{l}\text { PRM. *au. } \\
\text { Rote. *toka. } \\
\text { PRM. *bee; } \\
\text { PRM. *dalə. } \\
\text { CERM. *huti. } \\
\text { PRM. *taum. } \\
\text { CERM. *sabuu. } \\
\text { PRM. *fada. } \\
\text { PRM. *feu. } \\
\text { PRM. *ketembau; } \\
\text { PRM. *muut; } \\
\text { PRM. *otas. }\end{array}$ & $\begin{array}{l}\text { insert } \\
\text { inside } \\
\text { insipid } \\
\text { investigate } \\
\text { invite } \\
\text { iron } \\
\text { island } \\
\text { it } \\
\text { itchy }\end{array}$ & $\begin{array}{l}\text { Rote. *duygu. } \\
\text { PRM. *dalə. } \\
\text { CER. *afa; } \\
\text { Rote. *mamis. } \\
\text { PRM. *susi. } \\
\text { PRM. *hoka. } \\
\text { PRM. \#besi } \\
\text { PRM. *nusa. } \\
\text { PRRote. *ndia. } \\
\text { PRM. *katə. }\end{array}$ \\
\hline $\begin{array}{l}\text { jar } \\
\text { jealous } \\
\text { jerk } \\
\text { jicama } \\
\text { Job's tears } \\
\text { join } \\
\text { joint }\end{array}$ & $\begin{array}{l}\text { PRM. *kurə; } \\
\text { PRM. \#ngusi } \\
\text { Rote. *neo. } \\
\text { Rote. *bitiz; } \\
\text { Rote. *fiti1. } \\
\text { PRM. *uas. } \\
\text { PRM. *dele. } \\
\text { PRM. *tamba2; } \\
\text { PRM. *tute. } \\
\text { PRM. *buku; } \\
\text { PRM. *mola. }\end{array}$ & $\begin{array}{l}\text { jump up } \\
\text { just }\end{array}$ & $\begin{array}{l}\text { Rote. *bitiz. } \\
\text { PRM. *fa. }\end{array}$ \\
\hline
\end{tabular}


$\mathbf{K}-\mathbf{k}$

\begin{tabular}{|c|c|c|c|}
\hline kapok tree & PRM. *dene. & Kleinhovia hospita & $P R M$. *bitinaa. \\
\hline keel & Rote. *keni. & knead & PnRote. *ke?e; \\
\hline kick & Rote. *fiti2. & & Rote. *kame; \\
\hline kidneys & PMeto. \#kari & & Rote. *keme; \\
\hline kill & $\begin{array}{l}\text { PRM. *dodo; } \\
\text { PRM. *isa2. }\end{array}$ & knee & $\begin{array}{l}\text { Rote. *kumu2. } \\
\text { PMeto. *tuu1. }\end{array}$ \\
\hline king & $\begin{array}{l}\text { PMeto. *usi } ; \\
\text { PwRM. *la?i; } \\
\text { Rote. *mane. }\end{array}$ & $\begin{array}{l}\text { knee cavity } \\
\text { knife }\end{array}$ & $\begin{array}{l}\text { Rote. *doka. } \\
\text { PMeto. \#kopi; } \\
\text { Rote. *dombe. }\end{array}$ \\
\hline $\begin{array}{l}\text { kingfisher } \\
\text { kiss }\end{array}$ & $\begin{array}{l}\text { PRM. *sinara?e. } \\
\text { CER. *deki; } \\
\text { Rote. *idu. }\end{array}$ & knock & $\begin{array}{l}\text { PnMeto. *deku; } \\
\text { Rote. *liku1; } \\
\text { Rote. *toko. }\end{array}$ \\
\hline kiss (nose) & $n R M$. *farəndən. & know & PwRM. *hine. \\
\hline
\end{tabular}

L - I

\begin{tabular}{|c|c|c|c|}
\hline ladder & PRM. *eda. & liana & PRM. *lolo1. \\
\hline lake & PRM. *lifu; & lick & PRM. *damei. \\
\hline & Rote. *dano. & lie down & $n R M$. * mbe?u. \\
\hline land & PRM. *dae; & lie on & Rote. *nehi. \\
\hline & PRM. *ingu. & light (adj.) & $P R M$. *ngafat. \\
\hline landslide & $\begin{array}{l}\text { PRM. *ndefa; } \\
\text { Rote. *feraz. }\end{array}$ & light (n.) & $\begin{array}{l}P R M . \text { *saraa; } \\
P R M . \text { *sina. }\end{array}$ \\
\hline lap & $P R M$. *ifa. & lightning & PRM. *ndelat. \\
\hline last & PRM. *muri. & like & PMeto. *domi. \\
\hline lath & $P R M$. *sikəh. & lime (fruit) & $P R M$. *munde. \\
\hline laugh & $P w R M$. *malis. & lime, mineral & $P R M$. *aho. \\
\hline leaf & PRM. *doo. & $\operatorname{lip}$ & $P w R M$. *nudu; \\
\hline leaf, covering & $n R M$. *namba. & & Rote. *bife. \\
\hline leaf, palm & PRM. *mboro. & listen & Rote. *nenez. \\
\hline leak & PRM. *turu. & listless & PRM. *loe2. \\
\hline lean & PRM. *sarait. & live & PRM. *moris. \\
\hline learn & PRM. *nori. & liver & PRM. *ate. \\
\hline leave & Rote. *kela. & living & PRM. *horis. \\
\hline leech & PRM. *ndaki; & lizard & $C E R$. *fae. \\
\hline & Rote. *kerumatu. & lizard, flying & Rote. *ngola. \\
\hline left & PnRote. *kii. & lizard, monitor & PRM. *baiafa; \\
\hline left (side) & $P w R M . *$ diii. & & Rote. *ngola. \\
\hline leg & PMeto. *hae|?; & load & Rote. *fua1. \\
\hline & Rote. *ei. & lobster & CERM. *hee1. \\
\hline legume & $P R M$. *fue. & long & $C E R$. *daru; \\
\hline & PRM. *dero. & & PRM. *naru. \\
\hline lever & PRM. *do?i; & long ago & PRM. *ulu. \\
\hline
\end{tabular}




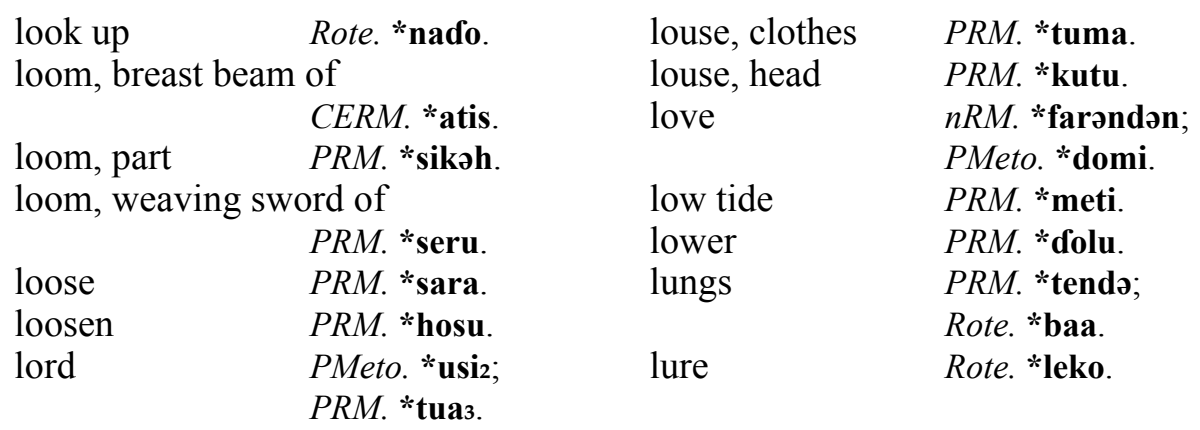

\section{M - m}

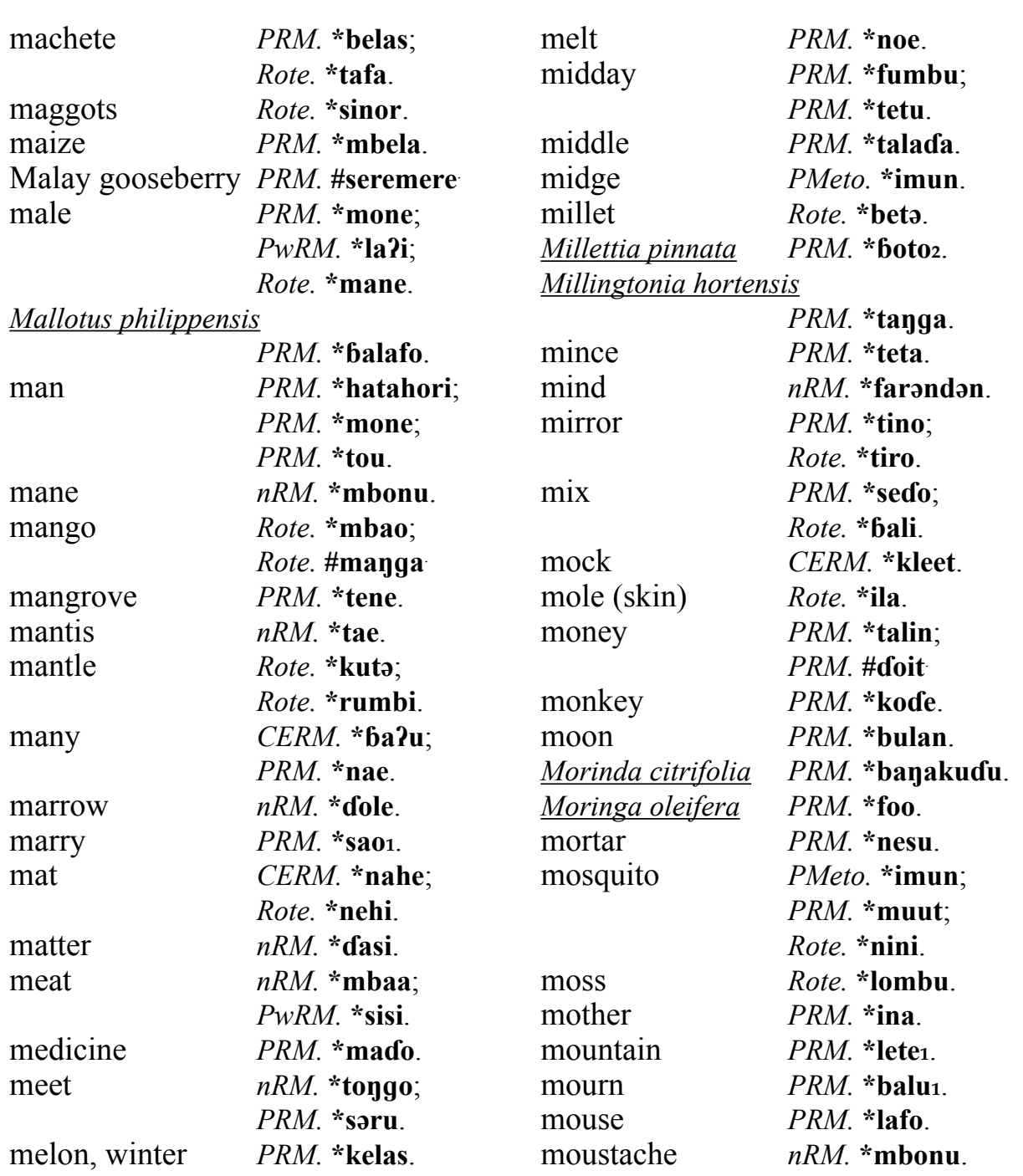




$\begin{array}{llll}\text { mouth } & \text { PMeto. *fefa; } & \text { mud } & \text { Rote. *tane1. } \\ & \text { PwRM. *nudu; } & \text { muscle } & \text { PRM. *uat. } \\ \text { Rote. }{ }^{*} \text { bafa2. } & \text { mushroom } & \text { PRM. *mbuku1. } \\ \text { move } & \text { PRM. *foes; } & \text { mute } & \text { Rote. *ngoa. } \\ & \text { Rote. }{ }^{*} \text { heo. } & \text { mutter } & \text { CERM. *boto1. }\end{array}$

move location $\quad P R M$. *lariz.

$\mathbf{N}-\mathbf{n}$

\begin{tabular}{|c|c|c|c|}
\hline nail & Rote. *fayga. & nice & $P R M$. *mina. \\
\hline nail, finger-/toe- & PMeto. *kalusa. & niece & PMeto. \#nane \\
\hline naked & PRM. *holas. & night & $P R M$. *fai. \\
\hline name & Rote. *nadza. & nine & PRM. *sio. \\
\hline name, ancestral & CERM. *tamo. & nit & Rote. *kua. \\
\hline nautilus & PRM. *suti. & no & PnRote. *taa 3 \\
\hline navel & PRM. *husə. & & $P R M . * \mathbf{f a}$ \\
\hline nearly & $n R M$. *ohi. & & $P w R M$. *ygaha. \\
\hline neck & $\begin{array}{l}\text { PRM. *boto3; } \\
\text { PRM. *lesu1; }\end{array}$ & node & $\begin{array}{l}\text { PRM. *buku; } \\
\text { PRM. *mola. }\end{array}$ \\
\hline eck, nape & $\begin{array}{l}\text { Rote. *liii. } \\
\text { PRM. *ndou. }\end{array}$ & noise & $\begin{array}{l}\text { PRM. *doto; } \\
\text { PRM. *mbuu. }\end{array}$ \\
\hline ecklace & $P R M$. *heyge. & noose & Rote. *ikə. \\
\hline eigh & CERM. *kele. & north & PnRote. *kii; \\
\hline eedle & Rote. *ndau. & & $P w R M$. *diii1. \\
\hline nerve of palm leaf & Rote. *lide. & nose & PRM. *mbana; \\
\hline & $\begin{array}{l}\text { PRM. *nduna; } \\
\text { Rote. *nuu1. }\end{array}$ & not & $\begin{array}{l}\text { Rote. *idu. } \\
\text { PnRote. *taas; }\end{array}$ \\
\hline & PRM. \#dzala & & $P w R M$. *ngaha. \\
\hline ttle & PRM. *ndesi. & now & PRM. *leu. \\
\hline
\end{tabular}

\begin{tabular}{|c|c|c|c|}
\hline oakum & Rote. *dudu. & open & $C E R$. *singa; \\
\hline ocean & PRM. *tasi. & & PRM. *fei; \\
\hline octopus & Rote. *pado. & & PRM. *soi; \\
\hline odour & PRM. *boo2. & & Rote. *huka; \\
\hline oh! & CER. *auee. & & Rote. *sai 2 \\
\hline oil & PRM. *mina. & & Rote. *sika. \\
\hline oink & Rote. *uu. & open (adj.) & PRM. *lua2. \\
\hline old & $\begin{array}{l}P R M . \text { *lasi } 2 ; \\
P R M . \text { *mba|raa. }\end{array}$ & open the eyes & $\begin{array}{l}\text { Rote. *bula; } \\
\text { Rote. *laas. }\end{array}$ \\
\hline ne & PRM. *esa. & open the mouth & PnMeto. * m|kaka. \\
\hline nion & $P R M$. *laisona. & $\begin{array}{l}\text { opposite } \\
\text { orange (fruit) }\end{array}$ & $\begin{array}{l}C E R . \text { *tadu. } \\
\text { PRM. *dero. }\end{array}$ \\
\hline
\end{tabular}




$\begin{array}{llll}\text { orchard } & \text { PRM. *mamər. } & \text { overtake } & \text { PRM. *hambu. } \\ \text { order } & \text { PRM. *denu. } & \text { owl } & \text { nRM. *tudui. } \\ \text { orphan } & \text { PRM. *ma(n)at. } & \text { owl, barn } & \text { PRM. *6akos. } \\ \text { other } & \text { Rote. *fekə. } & \text { own } & \text { PRM. *-uki; } \\ \text { outside } & \text { PMeto. *mone|?; } & & \text { Rote. *ena. } \\ & \text { PRM. *luaz. } & \text { owner } & \text { PRM. *tuas. } \\ \text { ovary } & \text { PRM. *natu. } & \text { oyster } & \text { PRM. *tiam. } \\ \text { overflow } & \text { PRM. *sasi; } & & \\ & \text { PRM. *turu; } & & \\ & \text { Rote. *faa. } & & \end{array}$

$\mathbf{P}-\mathbf{p}$

Pachyrhizus erosus packed paddle pain

palate palm, areca palm, areng palm, dead palm, gebang palm, lontar palm, trunk palm leaf stem palm line

palm wine pandanus, fragrant pandanus, wild pant pants papaya parakeet paralysed Parkia speciosa

pass patch path

pattern

peak

peel
PRM. *uas. PRM. *seti.

Rote. *sefe.

PRM. *meras;

Rote. "hedis.

PRM. *ngadas.

PRM. *mbuah.

PRM. *bole.

PRM. *mopu.

PRM. *tula.

PRM. *tua2.

PRM. *mopu.

PRM. *beba.

PRM. *uat;

Rote. *ura.

PRM. *ladu.

CERM. *6ona.

PRM. *hendam.

CERM. *kele.

Rote. \#sarombo

PRM. *timuz.

PRM. *ygia.

Rote. *luku2.

PRM. *fake.

PRM. *beni.

PRM. *tamba2.

$n R M$. *eno;

PRM. *dalan.

PRM. *dula.

$P R M$. *fumbu.

PRM. *kisi;

PRM. *sada;

Rote. *soso. peer

pen

penis

person

persuade

pestle

pick

pick at

pick out

pierce

pig

pigeon

pigeon, wild

pigeon pea

pile up

pillar

pillow

pimple

pinch

pip

Pisonia alba

place (n.)

place (v.)
PRM. *tino;

Rote. *tiro.

CERM. *oka1;

Rote. *royga.

PRM. *uti.

PRM. *hatahori;

PRM. *tou;

PRM. *tuaz.

PRM. *fudi.

PRM. *halu.

PRM. "sau2;

PRM. *seu.

PRM. *ygai.

PRM. *soke.

PnMeto. *fado.

PRM. *bafi.

PRM. *teu.

PMeto. *kumu1.

PRM. *turis.

Rote. *lutuz.

PRM. *diii2.

PRM. *lunu.

PRM. *bisu.

CER. *bibi;

PRM. *biti;

PRM. *hapi;

PRM. *kabi;

$P R M$. *ngete.

$P w R M$. *muse;

Rote. *deke.

CERM. *safe.

Rote. "mana2.

PRM. *mbeda. 


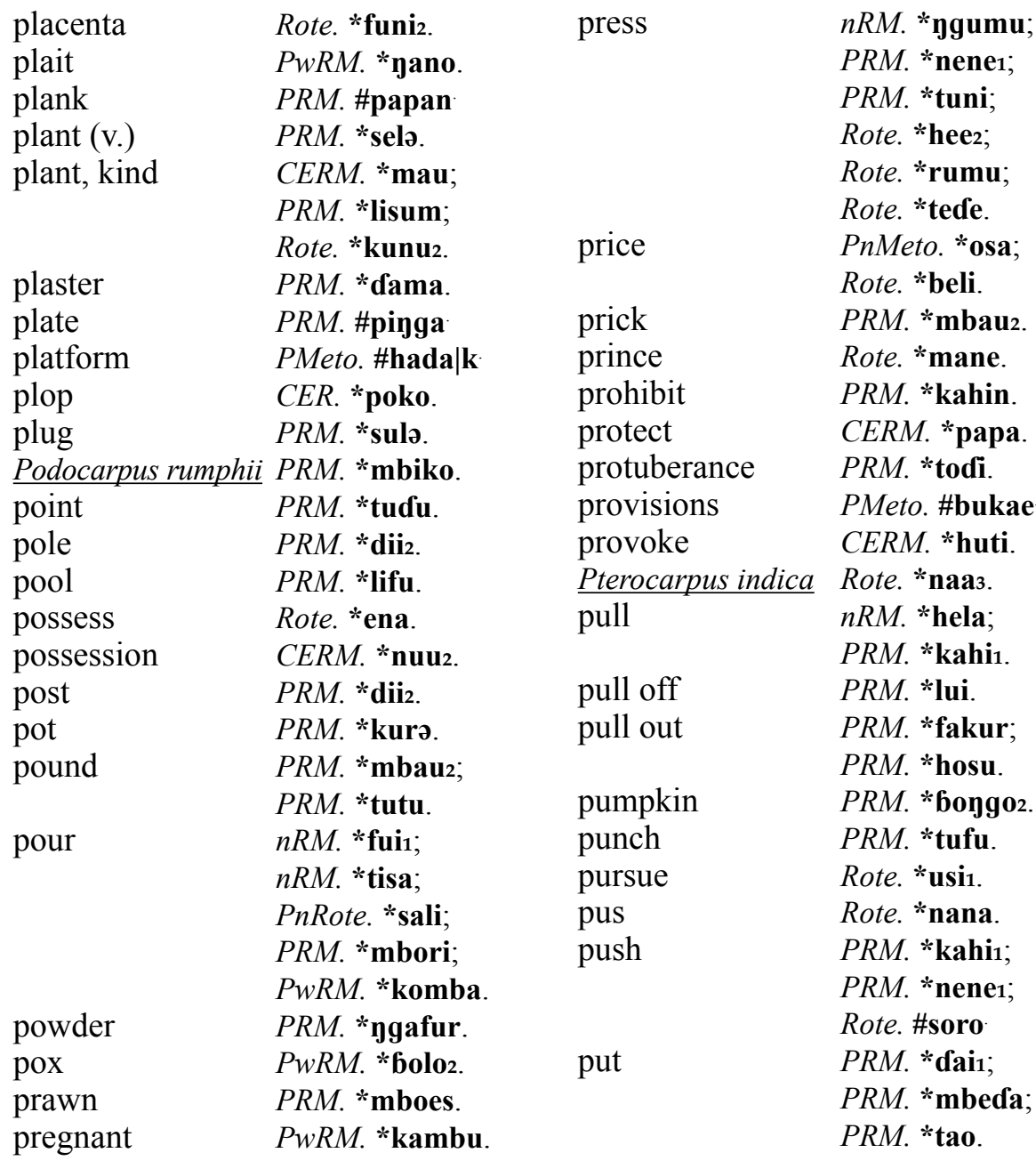

$\mathbf{Q}-\mathbf{q}$

$\begin{array}{llll}\text { quail } & P R M . * \text { mbui. } & \text { quick } & \text { PRM. *"ygafat; } \\ \text { quarrel } & \text { PRM. *tofa2. } & & \text { Rote. *lai. } \\ & \text { quiet } & \text { PRM. *nee } .\end{array}$

\section{$\mathbf{R}-\mathbf{r}$}

racing

rafter

rain

rainbow

rainy season
PRM. *foti.

PnMeto. *sua2.

PRM. *udan.

PRM. *elus.

PRM. *faat. random

ransom

rash

rat

rattan
PRM. *dafuz.

PRM. *soi.

PRM. *hano.

PRM. *lafo.

PRM. *ue. 


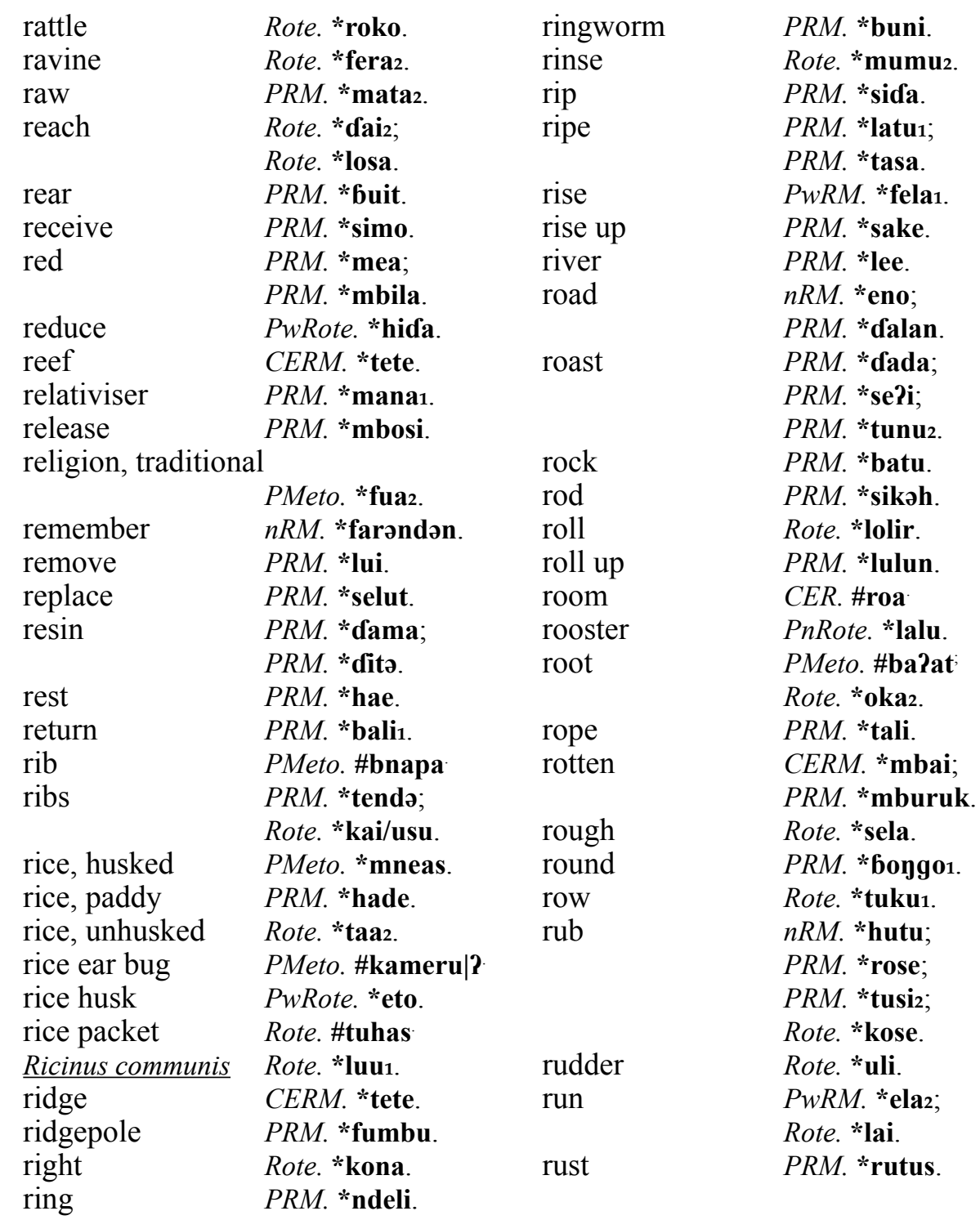

\begin{tabular}{|c|c|c|c|}
\hline sack & PRM. \#kado & salty & PRM. *masi. \\
\hline sack, kind & Rote. *soka. & same & $n R M$. *nesa; \\
\hline sacred & $P w R M .{ }^{*} \mathbf{d e} \mathbf{p}$. & & Rote. \#sama \\
\hline sacrifice & $P w R M$. *ponia. & sand & $P R M$. *sarakaen. \\
\hline saddle & PRM. *peni. & sandalwood & PnMeto. *meniz; \\
\hline sail & Rote. *laaz. & & PnMeto. *meniz. \\
\hline saliva & PRM. *kambe; & sardine & Rote. *temba. \\
\hline & Rote. *midu. & sarong & PRM. *tais. \\
\hline
\end{tabular}




saw (n.)
say
scabies
scale
scared
scatter
Schleichera oleosa
scissors
scold
scoop

scorpion

scour

scrape

scratch

scrotum

sea

sea cucumber

sea snail

sea urchin

sea-snake

seasoning

seaweed

see

seed

seethe

sell

Senna timoriensis

separate

sesame
Rote. \#horo

PRM. *fada.

PRM. *nu?a;

PwRM. *6oloz.

Rote. *uno.

PRM. *taku.

PRM. *ygari.

PMeto. \#kusapi

Rote. \#ngute

$P w R M$. *singada?.

CER. *aso;

PRM. *ndui;

PRM. *ra?u;

PRM. *soke;

PRM. *soro;

PRM. *suru.

PRM. *biti;

Rote. *kura.

PRM. *fora.

$P R M$. *kao;

PRM. *ngeu;

PRM. *suu1;

Rote. *karu.

PRM. *kao;

PRM. *ngai;

PRM. *suu1;

Rote. *karu.

PRM. *laso.

PRM. *tasi.

PRM. *banafi.

PwRM. *batus.

PMeto. *k|teom;

Rote. *tii.

PRM. *lema.

PMeto. \#fukar

CER. *lamu;

PRM. *kenga;

Rote. *latur.

PRM. *ita.

PRM. *bini;

PwRM. *muser;

Rote. *deke.

PRM. *lua1.

PMeto. *sosa.

$n R M$. *namo1.

Rote. *fekə.

PRM. *lena. $\underline{\text { Sesbania grandiflora }}$

PRM. *ngala.

seven

PRM. *hitu.

several

CERM. *ba?u.

sew

PRM. * yato;

PRM. *soo.

shadow

shake

PRM. *mafo.

CERM. *beko;

PRM. *ygengo;

Rote. *eko;

Rote. *noko.

shallot

share

shark

sharp

sharpen

shave

she

$P R M$. *laisona.

CERM. *bati.

PRM. *iu.

$P R M$. *tande.

PRM. *kiri2.

PRM. *ngeu.

PnRote. *ndia;

PwRM. *eni.

sheep PRM. *bibi.

shell

PnRote. *hani2.

shell, cowrie Rote. *fuli.

shell, kind

PRM. *suti;

Rote. *bina.

shellfish Rote. *kibo;

Rote. *sii2.

shelter CERM. *lopo.

shine

PRM. *dila2;

$P R M$. *saraa.

short

$P R M$. *mbada;

$P w R M . *$ tuka;

Rote. *pande.

shoulder PRM. *haru.

shout Rote. *ki;

Rote. *ngasi.

shrimp nRM. *tae;

PRM. *mboes.

shrub Rote. *ygirat.

shy PnRote. *liii;

PRM. *mae.

sibling, elder Rote. *kaka.

sibling, older $\quad n R M$. *tato.

sibling, younger $P R M .{ }^{*}$ wadi.

sick

PRM. *meras;

Rote. *hedis.

side

PMeto. \#bnapa

Rote. *ko. 


\begin{tabular}{|c|c|c|c|}
\hline sign & PRM. \#tanda & some & PRM. *duman. \\
\hline silver & PRM. *lilo. & soot & PRM. *atu. \\
\hline similar & PMeto. \#en & soothe & PRM. *hauk. \\
\hline $\operatorname{sing}$ & Rote. *soda. & sorcery & PnMeto. *nono1. \\
\hline $\operatorname{sink}$ & PRM. *tena1. & sorghum & PRM. *mbela. \\
\hline $\operatorname{sip}$ & PRM. *sakoro. & sorry & $n R M$. *sale. \\
\hline sister & $P R M$. *feto. & sound & PRM. *doto; \\
\hline sister-in-law & PnRote. *iha. & & PRM. *hara; \\
\hline sit & $n R M$. *tuur. & & PRM. *mbuu; \\
\hline $\operatorname{six}$ & PRM. *nee1. & & Rote. *lii1. \\
\hline size & Rote. *tua1. & sour & PRM. *kais; \\
\hline skin & Rote. *rou. & & PRM. *nilu; \\
\hline sky & PRM. *ledo. & & PRM. * rekət. \\
\hline slant & PRM. *basoko. & source & PRM. *huu. \\
\hline slap & $P w R M$. *mbasa. & sow & PRM. *ygari. \\
\hline slave & PRM. *atas. & space & PRM. *soda. \\
\hline sleep & $n R M . * \mathbf{m b e} \mathbf{u}$. & span & PnRote. *tenga; \\
\hline sleepy & $n R M . * \mathbf{m b e} \mathbf{u}$ & & PRM. * reha; \\
\hline slide & $\begin{array}{l}P R M . \text { " noto. } \\
P R M . \text { *sadodo. }\end{array}$ & & $\begin{array}{l}\text { PwRM. *dayga2; } \\
\text { PwRote. *hayga. }\end{array}$ \\
\hline sling & PRM. *piru. & speak & PRM. *fada; \\
\hline slingshot & PRM. *bitis. & & Rote. *deha; \\
\hline slippery & $n R M$. *kendi. & & Rote. *ygasi. \\
\hline slow & PRM. *noto. & spear & Rote. *tee. \\
\hline small & PRM. *anak; & speech & $n R M$. *dasi. \\
\hline & PRM. *diki; & spice & PMeto. \#fukar \\
\hline & PRM. *lutu1. & spider & PMeto. \#k|naba|? \\
\hline smell & PRM. *boo2. & & Rote. *bo/lau. \\
\hline smile & PRM. *humək. & spindle & PRM. \#kinde \\
\hline smoke & $\begin{array}{l}\text { CERM. *sabuu; } \\
P R M . * \text { masu. }\end{array}$ & spirit & $\begin{array}{l}P R M . \text { *nitu; } \\
P R M . \text { *sumanə. }\end{array}$ \\
\hline smooth & $n R M$. * kendi. & spit out & PMeto. *yinu; \\
\hline snake & $P R M$. *ndaki; & & Rote. *midu. \\
\hline & $P R M .{ }^{*} \mathbf{s a o} ;$ & spittle & PRM. *kambe. \\
\hline & PRM. *ulə. & split & PnMeto. *paha; \\
\hline snake, red & PRM. *menge. & & $P R M .{ }^{*} \mathbf{b i a}$ \\
\hline snap off & PRM. *sengi. & & PRM. *tusi1; \\
\hline snare & CER. *siii & & Rote. *faka1; \\
\hline & CERM. *fetu; & & Rote. *faka2; \\
\hline & Rote. *ikə. & & Rote. *tata. \\
\hline sneeze & $P R M$. *kesu/fani. & spool & PRM. *lole2. \\
\hline snore & PRM. *ygoro. & spoon & PRM. *soro; \\
\hline snot & PRM. *mbinu. & & PRM. *suru. \\
\hline snout & PRM. *ygoro. & spots & PRM. *foe \\
\hline so & PRM. *dee. & & PRM. *hano. \\
\hline soak & $P R M$. *endən. & spread out & $C E R .{ }^{*}$ fela2; \\
\hline soil & $P R M$. *dae. & & $P R M$. *bela; \\
\hline
\end{tabular}




\begin{tabular}{|c|c|c|c|}
\hline & $P R M$. *sara; & stop by & Rote. *tuli. \\
\hline & PRM. *tambele. & storm & PRM. *kutus. \\
\hline sprinkle & $P R M . \#[\mathbf{p} / \mathbf{b}] \mathbf{i s i} \mathbf{i}$ & straight & PRM. *lodo; \\
\hline sprout & Rote. *numbu. & & PRM. *ndoo. \\
\hline spurt & $P R M$. *sambudas. & strait & PRM. *lolo4. \\
\hline spy & PRM. *luku1. & stretch & PRM. *lolo2; \\
\hline squash & PRM. *6oygo2. & & Rote. *loo1. \\
\hline squeeze & $\begin{array}{l}C E R M . \text { *kees; } \\
n R M . \text { *ygumu. }\end{array}$ & strike & $\begin{array}{l}\text { PnMeto. *deku; } \\
\text { Rote. *femba; }\end{array}$ \\
\hline squid & Rote. *nuus. & & Rote. *liku1. \\
\hline stab & $\begin{array}{l}P R M . \text { *dodo; } \\
P R M . \text { *mbauz. }\end{array}$ & $\begin{array}{l}\text { strip leaves } \\
\text { stripe }\end{array}$ & $\begin{array}{l}P R M . \text { *koru. } \\
\text { PRM. *tui1. }\end{array}$ \\
\hline staff & PRM. *tekə. & strong & PRM. *beki. \\
\hline stairs & PRM. *eda. & struggle & $P R M$. *foe 3. \\
\hline stand & Rote. *diii. & stub & PRM. *tunu1. \\
\hline stand with & Rote. *fali. & stuck & PRM. *seke. \\
\hline & $P R M$. *fanduun. & stumble & $P R M$. *tunu1. \\
\hline $\begin{array}{l}\text { star, morning } \\
\text { startle }\end{array}$ & $\begin{array}{l}\text { PMeto. \#nome } \\
\text { PRM. *saygenger. }\end{array}$ & stupid & $\begin{array}{l}\text { PnMeto. *mono; } \\
\text { Rote. *ngoa. }\end{array}$ \\
\hline state & PRM. *nusa. & submerge & PRM. *tena1. \\
\hline steal & Rote. *nako. & suck & PRM. *musi; \\
\hline steam & $\begin{array}{l}P R M . \text { *masu; } \\
P R M . \text { *suma. }\end{array}$ & suffering & $\begin{array}{l}\text { Rote. *mumu1. } \\
\text { PRM. *rekət. }\end{array}$ \\
\hline step & PRM. *tabu. & suffocate & Rote. *neta. \\
\hline step over & PRM. *danga1. & sugarcane & PRM. *tefu. \\
\hline Sterculia foetidea & PRM. *nitas. & summon & PRM. *kati. \\
\hline Sterculia urceolata & $P R M$. *fuloat. & sun & PRM. *ledo. \\
\hline stick & PRM. *loloз. & sunbeam & PRM. *saraa. \\
\hline stick, walking & PRM. *tekə. & sunset & CERM. *tesa. \\
\hline stick out & PRM. *lode; & surprise & $P R M$. *saygenger \\
\hline stick to & $\begin{array}{l}\text { PRM. *todi. } \\
\text { PRM. *ditə; }\end{array}$ & surround & $\begin{array}{l}\text { CERM. *libu; } \\
\text { Rote. *rimbu. }\end{array}$ \\
\hline & $\begin{array}{l}P w R M . * \text { ndika; } \\
\text { Rote. *tai } 3 .\end{array}$ & $\begin{array}{l}\text { swallow } \\
\text { swan }\end{array}$ & PRM. *kodo. \\
\hline $\begin{array}{l}\text { sticky } \\
\text { stiff }\end{array}$ & $\begin{array}{l}\text { PRM. *ditə. } \\
\text { Rote. *kai1. }\end{array}$ & sway & $\begin{array}{l}C E R M . \text { *beko; } \\
P R M . \text { *basoko. }\end{array}$ \\
\hline still & PRM. *bei; & swear & PRM. \#sumba \\
\hline & PRM. * nee2. & sweat & PRM. *mbusər. \\
\hline still (water) & PnRote. *londe. & sweet & Rote. *mamis. \\
\hline stingray & $P R M$. *hai 2. & sweet potato & PRM. *lole1. \\
\hline stingy & Rote. *kira2. & swell & PRM. *mbetak; \\
\hline stink & PRM. *booz. & & PRM. *poka; \\
\hline stocks & $P R M$. *layge. & & Rote. *mbae. \\
\hline stomach & $n R M$. *neko; & swim & Rote. *naye. \\
\hline & PRM. *taii. & swollen & Rote. *mbumbu. \\
\hline & PRM. *batu. & sword & PRM. *sure; \\
\hline & PRM. *dudi. & & Rote. *tafa. \\
\hline
\end{tabular}




\section{$\mathbf{T}-\mathbf{t}$}

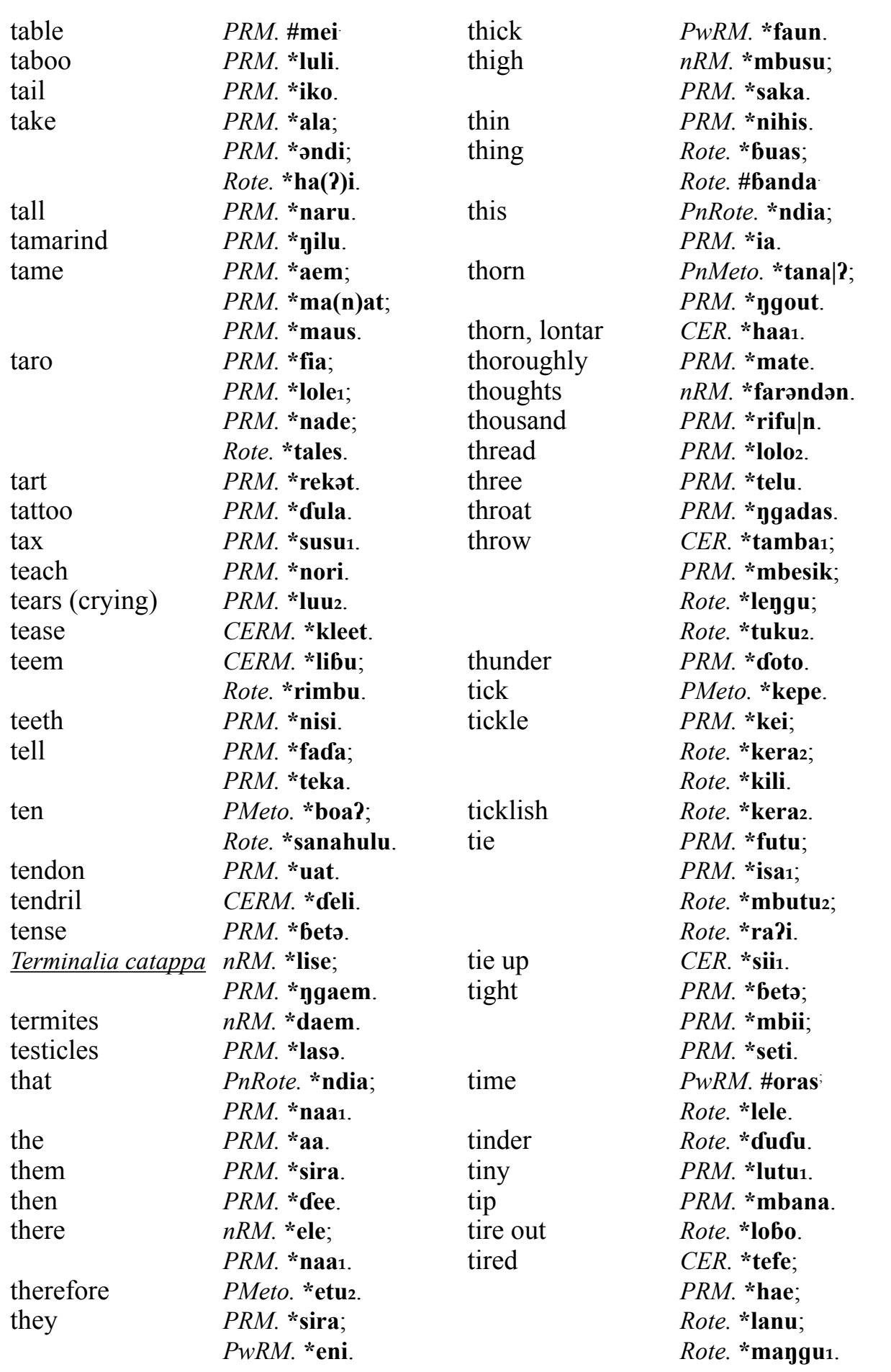




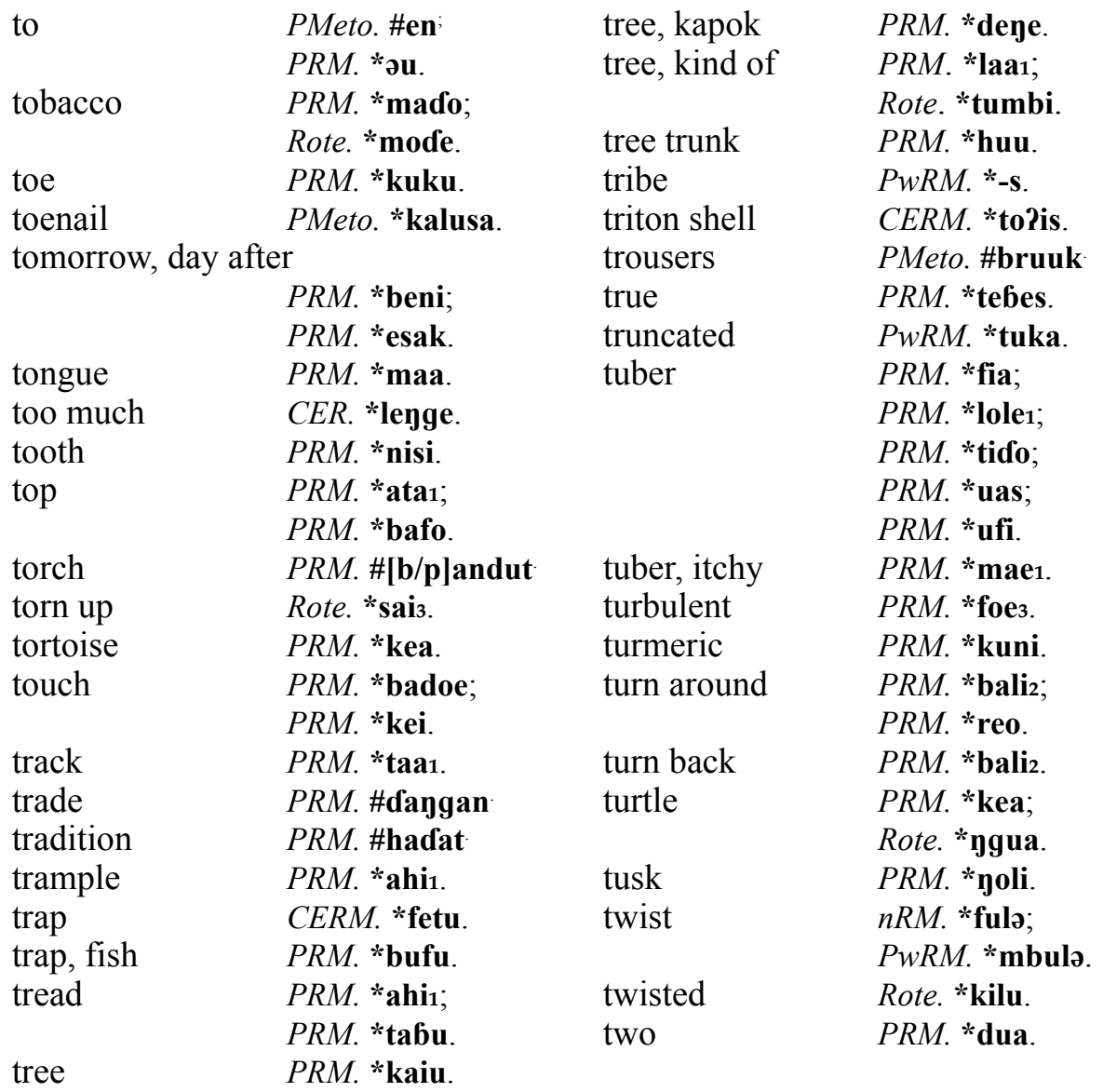

\section{$\mathbf{U}-\mathbf{u}$}

ulcer

umbrella, leaf

uncle

uncle, maternal

uncomfortable

underlay

undulate

unique

unripe

unroll

untie

until

up
PRM. *bisu.

PRM. *soeneru. PMeto. *baba|?.

PRM. *to?o.

Rote. *lanu.

PRM. *lasa.

Rote. \#omba

CER. *kisa.

PRM. *mata2;

PRM. *yura.

$C E R$. *fela2;

PRM. *bela.

PRM. *sefi.

Rote. *losa.

PRM. *ata1. up to

upright

urine

useless

uterus
Rote. *losa.

PRM. *tetu.

PRM. *mii.

PRM. *dafu1.

PwRM. *kambu. 


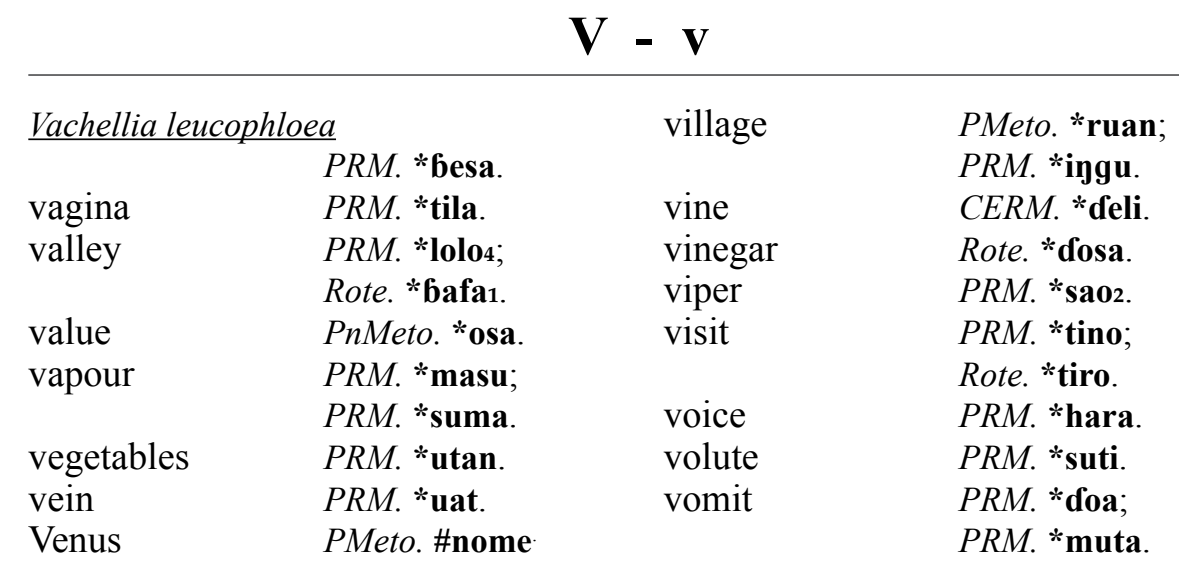

\begin{tabular}{|c|c|c|c|}
\hline wake up & $\begin{array}{l}\text { Rote. *fo?a; } \\
\text { Rote. *meda. }\end{array}$ & $\begin{array}{l}\text { wealth } \\
\text { weave }\end{array}$ & $\begin{array}{l}P R M . * \text {-uki. } \\
P R M . * \text { seru; }\end{array}$ \\
\hline walk around & $\begin{array}{l}P R M . \text { *ndoro; } \\
P R M . \text { *susi. }\end{array}$ & weed (v.) & $\begin{array}{l}P R M . \text { *tenu. } \\
n R M . \text { *tofa1. }\end{array}$ \\
\hline wall & $\begin{array}{l}\text { PnMeto. *dupi; } \\
\text { PRM. *dindi. }\end{array}$ & weep & $\begin{array}{l}\text { PnRote. *tani; } \\
\text { PwRM. * } \mathbf{g g a e .}\end{array}$ \\
\hline wallow & $\begin{array}{l}\text { PRM. *kukur; } \\
\text { Rote. *lolir. }\end{array}$ & $\begin{array}{l}\text { weevil } \\
\text { weigh }\end{array}$ & $\begin{array}{l}P R M . \text { *fufuz. } \\
P R M . \text { \#tai }\end{array}$ \\
\hline want & $P R M . *$ hia. & weight & PRM. *berat. \\
\hline warm (v.) & PRM. *dada. & west & PRM. *muri. \\
\hline warm oneself & Rote. *neru. & wet & CERM. *mbeta \\
\hline warrior & PnMeto. *meoz. & whale & Rote. *ruyirai. \\
\hline wash & $\begin{array}{l}\text { PRM. *fase; } \\
\text { Rote. *fui }\end{array}$ & $\begin{array}{l}\text { what? } \\
\text { where }\end{array}$ & $\begin{array}{l}P w R M . * \text { saa. } \\
P R M . \text { *bee. }\end{array}$ \\
\hline $\begin{array}{l}\text { wasp } \\
\text { watch }\end{array}$ & $\begin{array}{l}\text { CERM. *katefuan. } \\
\text { PRM. *luku1. }\end{array}$ & whetstone & $\begin{array}{l}n R M . \text { *saha1; } \\
P w R M . \text { *kandi. }\end{array}$ \\
\hline water & PRM. *oe. & which & $P R M$. *dee. \\
\hline water spinach & PRM. *ngango. & while & Rote. *sadi. \\
\hline $\begin{array}{l}\text { watercourse } \\
\text { watermelon }\end{array}$ & $\begin{array}{l}\text { PRM. *lolo4. } \\
\text { PRM. *timu2. }\end{array}$ & whinny & $\begin{array}{l}\text { PRM. *diii } 2 \\
\text { CERM. * kele. }\end{array}$ \\
\hline wattle (rooster) & PMeto. *dai. & whirlwind & $P R M$. *kutus. \\
\hline wave & $\begin{array}{l}\text { Rote. *ree; } \\
\text { Rote. \#omba }\end{array}$ & whisper & $\begin{array}{l}\text { CERM. *boto1; } \\
n R M . \text { *seo. }\end{array}$ \\
\hline wax & PRM. *lili. & whistle & PRM. *miu. \\
\hline way & $\begin{array}{l}n R M . \text { *eno; } \\
P R M . \text { *dalan. }\end{array}$ & white & $\begin{array}{l}n R M . \text { *muti; } \\
\text { Rote. *fula. }\end{array}$ \\
\hline we (excl.) & $P R M . * \mathbf{k a}(\mathbf{m}) \mathbf{i}$. & who? & PRM. *see2. \\
\hline we (incl.) & $\begin{array}{l}\text { PRM. *kita. } \\
\text { Rote. *ata2. }\end{array}$ & $\begin{array}{l}\text { whole } \\
\text { wide }\end{array}$ & $\begin{array}{l}P R M . \text { *tema2. } \\
P R M . \text { *lua2. }\end{array}$ \\
\hline weak & PRM. *loe2. & wide open & PRM. *mboo. \\
\hline
\end{tabular}




\begin{tabular}{|c|c|c|c|}
\hline widow & PRM. *balu2. & wire & $P R M$. \#kaba \\
\hline widower & PRM. *baluz. & with & PRM. *oo1. \\
\hline wife & PMeto. *fee1. & woman & PMeto. *fee 1 \\
\hline wig & PRM. *sadu. & & $P R M$. *ina. \\
\hline wild & $\begin{array}{l}\text { PnRote. *liiis; } \\
\text { PRM. *fui }\end{array}$ & $\begin{array}{l}\text { wood } \\
\text { wood, yellow }\end{array}$ & $\begin{array}{l}\text { PRM. *kaiu. } \\
\text { Rote. \#adulara }\end{array}$ \\
\hline $\begin{array}{l}\text { win } \\
\text { wind }\end{array}$ & $\begin{array}{l}\text { PRM. *isa2. } \\
\text { PRM. *anin. }\end{array}$ & worm & $\begin{array}{l}\text { PRM. *ulə; } \\
\text { Rote. *kalati. }\end{array}$ \\
\hline $\begin{array}{l}\text { wind (v.) } \\
\text { wind, east }\end{array}$ & $\begin{array}{l}n R M . \text { *laba; } \\
C E R . \text { *timu1. }\end{array}$ & wrap & $\begin{array}{l}n R M . \text { *fulə; } \\
P R M . \text { *lusə. }\end{array}$ \\
\hline windino & $P w R M$. *mbulə. & wrap up & $\begin{array}{l}C E R . \text { *balus. } \\
\text { Rote *ule }\end{array}$ \\
\hline window & $n R M$. \#dzinela & wrinkly & Rote. *kurus. \\
\hline wing & $\begin{array}{l}C E R . \text { *dila1; } \\
P R M . \text { *lida. }\end{array}$ & $\begin{array}{l}\text { write } \\
\text { wrong }\end{array}$ & $\begin{array}{l}\text { PRM. *tui1. } \\
\text { PRM. *sala. }\end{array}$ \\
\hline winnow & Rote. *tahi. & & \\
\hline wipe & $\begin{array}{l}\text { PRM. * rose; } \\
\text { Rote. *kose. }\end{array}$ & & \\
\hline
\end{tabular}

\section{$\mathbf{Y}-\mathbf{y}$}

\begin{tabular}{|c|c|c|c|}
\hline yam & PRM. *ufi. & \multicolumn{2}{|c|}{ yesterday, day before } \\
\hline yarn, spread out & $P w R M$. *mbuat. & & PRM. * esak. \\
\hline & $P R M$. *taun. & yet & PRM. *bei. \\
\hline yellow & PRM. *modo. & you (pl.) & PRM. *ke(m)i. \\
\hline yesterday & $\begin{array}{l}P R M . \text { *afi; } \\
\text { PRM. *beni. }\end{array}$ & $\begin{array}{l}\text { you (sg.) } \\
\text { young } \\
\text { youngest }\end{array}$ & $\begin{array}{l}P R M . \text { *koo2. } \\
P R M . \text { * yura. } \\
P R M . \text { *muri. }\end{array}$ \\
\hline
\end{tabular}

\section{$\mathbf{Z}-\mathbf{Z}$}

Ziziphus mauritiana Rote. *koo1.

Ziziphus oenoplia $\quad P R M . *$ nasi.

$1-2-3$

$\begin{array}{llll}\text { 1PL.EXCL } & \text { PRM. *ka(m)i. } & \text { 2PL } & \text { PRM. *ke(m)i. } \\ \text { 1PL.INCL } & \text { PRM. *kita; } & \text { 2SG } & \text { PRM. *kooz. } \\ & \text { Rote. *ata2. } & \text { 3PL } & \text { PRM. *sira. } \\ \text { 1SG } & \text { PRM. *au. } & \text { 3SG } & \text { PnRote. *ndia. }\end{array}$


6

\section{Proto-Malayo-Polynesian - Proto-Rote-Meto}


A - a

\begin{tabular}{llll}
\hline *adaduq & *daru. 'long'. & *anu & *nuu2. 'possession'. \\
*aku & *au. 'I, 1SG'. & *añam & *ane. 'braid'. \\
*ala[q/p] & *ala. 'fetch'. & *asu & *asu. 'dog'. \\
*alutən & *lutə. 'firebrand'. & *ata & *ata2. 'we (incl.), \\
*ama & *ama. 'father'. & & 1PL.INCL'. \\
*anaduq & *naru. 'long'. & *atas & *ata1. 'above'. \\
*anak & *anak. 'child'. & &
\end{tabular}

B - b

\begin{tabular}{|c|c|c|c|}
\hline *babaq & *bafa1. 'valley'. & $* *$ bata & *fata. 'female'. \\
\hline *babuy & *bafi. 'pig'. & *batu & *batu. 'stone'. \\
\hline *bahaq & *faa. 'current'. & *ba(w)baw & *bafo. 'top'. \\
\hline *bahi & *fee1. 'woman'. & *bayawak & *baiafa. 'monitor \\
\hline *bahuq & •boo2. 'smell'. & & lizard'. \\
\hline *bajaq & *fada. 'say'. & *bayu & *mbau2. 'stab'. \\
\hline *bakaq & $\begin{array}{l}\text { *faka1. 'split'; } \\
\text { *faka2. 'split'; } \\
\text { *paha. 'split'. }\end{array}$ & $\begin{array}{l}\text { *belas } \\
\text { *bəkaq } \\
\text { *bəkəlaj }\end{array}$ & $\begin{array}{l}\text { *belas. 'machete'. } \\
\text { *fekə. 'separate'. } \\
\text { *bela. 'spread out'; }\end{array}$ \\
\hline *baki & *ba?i. 'grandfather'. & & *fela2. 'spread out'. \\
\hline *balabaw & *lafo. 'rat'. & *boli & ‘beli. 'buy price'. \\
\hline *balik & $\begin{array}{l}\text { *baliz. 'turn back } \\
\text { around'; } \\
\text { *bali. 'mix'. }\end{array}$ & *bəluk & $\begin{array}{l}\text { *felu. 'bend'; } \\
\text { *fenu. 'bend'; } \\
\text { *helu. 'bend'. }\end{array}$ \\
\hline *baliw1 & *fali. 'help with'. & *bəntas & *beta. 'cut \\
\hline *baliw2 & *bali1. 'go back'. & & vegetation'. \\
\hline *balu & ‘baluz. 'widow'. & *bəntəy & *betə. 'tense'. \\
\hline *balun & $\begin{array}{l}\text { *balus. 'cover up'; } \\
\text { *mbalu. 'cover'. }\end{array}$ & *bəntuk & $\begin{array}{l}\text { *betu. 'bent'; } \\
\text { *fedu. 'bent'. }\end{array}$ \\
\hline *baluq & *balu1. 'mourn'. & *bəøkuq & *beku. 'bend'. \\
\hline *bañən & $\begin{array}{l}\text { *kesu/fani. } \\
\text { 'sneeze'. }\end{array}$ & $\begin{array}{l}\text { *bəqbəq } \\
\text { *bəRas }\end{array}$ & $\begin{array}{l}\text { *fefa. 'mouth'. } \\
\text { *mneas. 'husked }\end{array}$ \\
\hline *baykudu & $\begin{array}{l}\text { "bayakudu. } \\
\text { 'Morinda citrifolia'. }\end{array}$ & *bəRay & $\begin{array}{l}\text { rice'. } \\
\text { *fee2. 'give'. }\end{array}$ \\
\hline *baqbaq & *bafaz. 'mouth'. & *bəRəqat & *berat. 'heavy'. \\
\hline *baqəRu & $\begin{array}{l}\text { *beu1. 'new'; } \\
\text { *feu. 'in-law'. }\end{array}$ & *bəRyaw & $\begin{array}{l}\text { *ka|benu. 'fly'; } \\
\text { *mbena. 'fly'. }\end{array}$ \\
\hline *baqi & *bei. 'grandmother'. & *bəR ji & *beni. 'day after \\
\hline *baRaq & *baa. 'lungs'. & & tomorrow, day \\
\hline *baRat & *baat. 'across'. & & before yesterday'. \\
\hline *baRəq & *mbae. 'swell'. & *bəRsay & *sefe. 'paddle'. \\
\hline *baRu & $\begin{array}{l}\text { *bau. 'Hibiscus } \\
\text { tiliaceus'. }\end{array}$ & *bəRus & $\begin{array}{l}\text { *beu2. 'Garuga } \\
\text { floribuna'. }\end{array}$ \\
\hline *basəq & *fase. 'wash'. & *bəriq & *fei. 'open'. \\
\hline
\end{tabular}




\begin{tabular}{|c|c|c|c|}
\hline *botaw & *feto. 'sister'. & *bukij & *fuiz. 'wild'. \\
\hline *batəy & *beto. 'millet'. & *buku & *buku. 'node'. \\
\hline \multirow[t]{3}{*}{ *botuy } & *petun. 'giant & *bukuq & *mbuku2. 'bent'. \\
\hline & bamboo, & *bulan & *bulan. 'moon'. \\
\hline & Dendrocalamus'. & *bula $[\mathrm{n} / \mathrm{R}]$ & *fula. 'white'. \\
\hline *bibiR & *bife. 'lip'. & *bulat & *bula. 'open the \\
\hline *bilak & *mbila. 'blaze (v.)'. & & eyes'. \\
\hline *binəhiq & "bini. 'seed'. & *buliq & *fuli. 'cowrie shell'. \\
\hline *bintiq & *fitiz. 'kick'. & *buliR & *mbule. 'grain head \\
\hline *binaq & *bina. 'kind shell'. & & cob'. \\
\hline *biqak & *bia. 'split'. & *bulu & *bulu. 'hair'. \\
\hline *biRaq & *fia. 'taro'. & *buni & *funi1. 'hide'. \\
\hline *bisul & *bisu. 'ulcer'. & *buntər & *bunda. 'fat \\
\hline \multirow[t]{3}{*}{ *bitbit } & *bibi. 'pinch'; & & bellied'. \\
\hline & *biti. 'pinch'; & *bunut & *mbunut. 'coconut \\
\hline & *fitiz. 'carry'. & & husk'. \\
\hline \multirow[t]{2}{*}{ *bitiəs } & *biti1. 'calf (of & *buna & *buna. 'flower'. \\
\hline & leg)'. & *buqaya & *foez. 'crocodile'. \\
\hline \multirow[t]{2}{*}{ *bitik } & *bitiz. 'jerk up'; & *buqəni & *buni. 'ringworm'. \\
\hline & *fiti1. 'jerk'. & *buqi & *fui1. 'pour'. \\
\hline *bituqən & *fanduun. 'star'. & *buRiq & *fuiz. 'wash'. \\
\hline \multirow{3}{*}{ *buaq } & *bua. 'fruit'; & *buRit & *buit. 'backside'. \\
\hline & *mbuah. 'areca & *buRnay & *mbune. \\
\hline & palm nut'. & & 'Antidesma bunius'. \\
\hline *buay & *fue. 'legume'. & *buRuk & *mburuk. 'rotten'. \\
\hline *bubu & `bufu. 'fish trap'. & *burun & *funu. \\
\hline \multirow[t]{2}{*}{ *bubun } & *fufu1. 'fontanelle'; & & 'betel-pepper'. \\
\hline & $\begin{array}{l}\text { *fumbu. } \\
\text { 'fontanelle'. }\end{array}$ & *busuR & $\begin{array}{l}\text { *busu. 'cotton } \\
\text { bow'. }\end{array}$ \\
\hline *buhat & *fua1. 'carry'. & *butu & *futu. 'bundle'; \\
\hline *buhək & *buə. 'hair'. & & *mbutu2. 'bundle \\
\hline *bujəq & *fudzo. 'foam'. & & up'. \\
\hline *buka & *fuka. 'dig'; & *buu & *buu. 'blow'; \\
\hline *bul & $\begin{array}{l}\text { *huka. 'open'. } \\
\text { *bubu. 'bubble'. }\end{array}$ & & *fuu. ‘blow'. \\
\hline *bukbuk3 & *fufu2. 'weevil'. & & \\
\hline
\end{tabular}

D - d

$\begin{array}{llll}\text { *dahun } & \text { *doo. 'leaf'. } & \text { *dapuR } & \text { *raho. 'hearth'. } \\ \text { *daki } & \text { *daki. 'dirt'. } & \text { *daqan } & \text { *mba|raa. 'old'. } \\ \text { *dakih } & \text { *ndake1. 'climb'. } & \text { *daqih } & \text { *dei. 'forehead'. } \\ \text { *daləm } & \text { *dalə. 'in'. } & * \text { daRaq } & \text { *daa. 'blood'. } \\ \text { *damaR } & \text { *dama. 'resin'. } & * \text { daRəq } & \text { *dae. 'ground'. } \\ \text { *danaw } & \text { *dano. 'lake'. } & * \text { dəyəR } & \text { *rena. 'hear'. } \\ \text { *dayday } & \text { *dada. 'warm (v.)'. } & \text { *dəpa } & \text { *reha. 'fathom (n.)'. }\end{array}$




$$
\begin{aligned}
& \text { *dəpdəp } \\
& \text { *dəpdəp } \\
& \text { *dikit } \\
& \text { *dilap } \\
& \text { *dindin } \\
& \text { *dinin } \\
& \text { *diRi } \\
& \text { *diRus }
\end{aligned}
$$

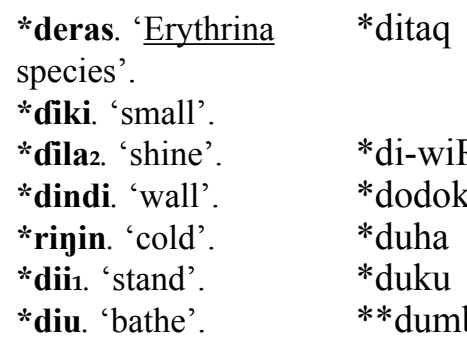

*dete. 'blackboard tree: Alstonia scholaris'.

*diii. 'left (side)'.

*dodo. 'kill'.

*dua. 'two'.

*ruku. 'bent'.

*dupi. 'wall'.

*dui1. 'bone'.

*duiz. 'dugong'.

\section{Ә-}

$\begin{array}{ll}\text { *əkit } & \text { *eki. 'shout out'. } \\ \text { *ənəm } & \text { *nee1. 'six'. } \\ \text { *əpat } & \text { *haa2. 'four'. } \\ \text { *əsa } & \text { *esa. 'one'. }\end{array}$

\section{$\mathbf{F}-\mathbf{f}$}

**fəran *feraz. 'landslide'.

\section{$\mathbf{G}-\mathbf{g}$}

$*_{\text {gatəl }}$

*gəmgəm *katə. 'itchy'.

*kame. 'knead';

*ke?e. 'knead';

*keme. 'knead';

*kumu2. 'fist';

*ygumu. 'fist,

squeeze'.

$$
\begin{aligned}
& \text { *gərgər } \\
& \text { *gatəl } \\
& \text { *giRin } \\
& \text { *gumi(s) } \\
& \text { *guru(q) }
\end{aligned}
$$

*ngengo. 'shake'.

"ygete. 'pinch off'.

*kiri1. 'bell'.

*ygomi. 'beard'.

*yguru. 'drone'.

\section{H - h}

*habaRat

*hadiRi

*hanin

*hapəjəs

*hapojis

*hapin

*hapun

*hapuy

*haRəzan *faat. 'rainy season'.

*dii2. 'post'.

*anin. 'wind'.

*meras. 'sick'.

*hedis. 'sick'.

*nahe. 'mat'.

*ahu. 'dew'.

*ahi2. 'fire'.

*eda. 'ladder'. *hasaq

*hawak

*hosi

*hikan

*hikət

*hipaR

*huaji

*hutək *saha1. 'whetstone'.

*ao. 'body'.

*sisi. 'meat'.

*ika. 'fish'.

*ikə. 'snare'.

*iha. 'sister-in-law'.

*wadi. 'younger

sibling'.

*kuto. 'brains'. 


\section{I - i}

\begin{tabular}{llll}
\hline *ia & *ia. 'this'. & *ina & *ina. 'mother'. \\
*ijuy $_{\text {*ikuR }}$ & *idu. 'kiss'. & *inum & *inu. 'drink'. \\
*iko. 'tail'. & $*_{\text {isi? }}$ & *isi. 'contents flesh'.
\end{tabular}

K - k

\begin{tabular}{|c|c|c|c|}
\hline *kabut & *habu. 'cloud'. & *kawanan & *kona. 'right'. \\
\hline *kaən & *ha. 'eat'. & *ka-wiRi & *kii. 'left'. \\
\hline **ka-felu & *?|fenu. 'candlenut'. & *kawit & *kai2. 'hook'. \\
\hline *kahiw & *kaiu. 'tree'. & *keRa & *kea. 'turtle'. \\
\hline *kahu & $\begin{array}{l}\text { *koo2. 'you (sg.), } \\
\text { 2SG'. }\end{array}$ & $\begin{array}{l}\text { *kəmiq } \\
\text { *kətuq }\end{array}$ & $\begin{array}{l}\text { *mii. 'urine'. } \\
\text { "ketu. 'cut off'. }\end{array}$ \\
\hline *kaka & $\begin{array}{l}\text { *kaka. 'elder } \\
\text { sibling'. }\end{array}$ & $\begin{array}{l}\text { *kilik } \\
* \text { kiluq }\end{array}$ & $\begin{array}{l}\text { *kili. 'tickle'. } \\
\text { *kilu. 'crooked'. }\end{array}$ \\
\hline *kakay & *hae|?. 'foot'. & $* *$ kilusa & *kalusa. \\
\hline *kali & *kali. 'dig'. & & 'fingernail'. \\
\hline *kalimatək & *kerumatu. 'leech'. & *kima & ‘kima. 'clam'. \\
\hline *kali-wati & $\begin{array}{l}\text { *kalati. 'worm'. } \\
\text { *kambe. 'saliva'. }\end{array}$ & *kiskis & $\begin{array}{l}\text { *sakiki. 'brush } \\
\text { teeth'. }\end{array}$ \\
\hline $\begin{array}{l}* \text { kambu } \\
*_{k}{ }_{k m i}\end{array}$ & *kambu. 'belly'. & *kita1 & *kita. 'we (incl.), \\
\hline *kamiri & $\begin{array}{l}\text { (exa(m)l. 'we } 1 \text { 1PL.EXCL'. } \\
\text { "kamiri. } \\
\text { 'candlenut'. }\end{array}$ & $\begin{array}{l}* \text { kita2 } \\
* * \text { klaRa } \\
* \text { koti }\end{array}$ & $\begin{array}{l}\text { *ita. 'see'. } \\
\text { *klaha. 'coals'. } \\
\text { *koti. 'cut'. }\end{array}$ \\
\hline *kampil & "kapir. 'basket'. & *kudən & •kurə. 'pot'. \\
\hline *kamuyu & *ke(m)i. 'you (pl.)'. & *kuhkuh & *kuku. 'finger'. \\
\hline *kanasay & $\begin{array}{l}\text { *nase. 'Blue-spot } \\
\text { mullet'. }\end{array}$ & $\begin{array}{l}* \text { kuluR } \\
* * \text { kumu }\end{array}$ & $\begin{array}{l}\text { *kunu1. 'breadfruit'. } \\
\text { "kumu1. 'wild }\end{array}$ \\
\hline *kapit & $\begin{array}{l}\text { *hapi. 'pinch'; } \\
\text { *ka6i. 'pinch'. }\end{array}$ & *kunij & $\begin{array}{l}\text { pigeon'. } \\
\text { *kuni. 'turmeric'. }\end{array}$ \\
\hline $\begin{array}{l}* \text { kaRat } \\
\text { *kaRus }\end{array}$ & $\begin{array}{l}\text { *kaa1. 'bite'. } \\
\text { *kao. 'scrape'. }\end{array}$ & $* \operatorname{kur}(\mathrm{u})$ & $\begin{array}{l}\text { *kuru1. 'call } \\
\text { chickens'. }\end{array}$ \\
\hline$* *$ karas & "kara. 'chest'. & *kurut & ‘kuru2. 'wrinkly'. \\
\hline $\begin{array}{l}\text { *karut } \\
\text { *kati }\end{array}$ & $\begin{array}{l}\text { *karu. 'scratch'. } \\
\text { *kati. 'call'. }\end{array}$ & *kutu & "kutu. 'head louse'. \\
\hline
\end{tabular}

\section{L - 1}

$\begin{array}{llll}\text { **lakateRu } & \text { *teu. 'pigeon'. } & \text { *lanit } & \text { *lani. 'heaven'. } \\ \text { *lakaw } & \text { *lako. 'go'. } & \text { *lanu } & \text { *lanu. } \\ \text { *laki } & \text { *la?i. 'king'. } & & \text { 'uncomfortable'. } \\ \text { *laluy } & \text { *lalu. 'rooster'. } & \text { *lapaR } & \text { *laha. 'hungry'. } \\ \text { *lamut } & \text { *lamu. 'seaweed'. } & \text { *laqia } & \text { *laia. 'ginger'. }\end{array}$




\begin{tabular}{|c|c|c|}
\hline *laRiw & *lai. 'run'. & *liaR \\
\hline *lasəR & *lasə. 'scrotum'. & $* *$ libun \\
\hline *-lat & $\begin{array}{l}\text { *laas. 'open the } \\
\text { eyes'. }\end{array}$ & *libut \\
\hline *latuq & *latu2. 'seaweed'. & *likud \\
\hline *lawaq & $\begin{array}{l}\text { *bo/lau. 'spider'; } \\
\text { \#k|naba|?. 'spider'. }\end{array}$ & $\begin{array}{l}* \operatorname{lilin} \\
* \operatorname{lima}\end{array}$ \\
\hline *layap & *laa4. 'fly (v.)'. & $* \operatorname{lin}$ \\
\hline *layaR & *laas. 'sail'. & *liqəR \\
\hline *lemba & *lemba. 'carry'. & $*$ luab \\
\hline **lemur & *lemuk. 'dolphin'. & *luay \\
\hline$*$ lesu & *lesu2. 'come out'. & *luhəq \\
\hline *letay1 & *lete1. 'mountain'. & \\
\hline *letay 2 & *lete2. 'bridge'. & *lukut \\
\hline *ləkən & *leke. 'coil'. & *lulun \\
\hline *ləku? & *leku. 'winding'. & *lumbu \\
\hline *ləyah & *lena. 'sesame'. & *luqaR \\
\hline *ləpaw & *lopo. 'shelter'. & \\
\hline *losun & *nesu. 'mortar'. & **lutuR \\
\hline *liay & *lea. 'cave'. & \\
\hline
\end{tabular}

*lii3. 'shy'.

*lifu. 'billabong'.

*libu. 'surround';

*rimbu. 'surround'.

*likuz. 'back'.

*lili. 'wax'.

*lima1. 'five'.

*lii1. 'sound'.

*liii. 'neck'.

*lua1. 'boil'.

*lua|t. 'cave'.

*luuz. 'tears

(crying)'.

*luku2. 'bent'.

*Iulun. 'roll up'.

*lombu. 'moss'.

*luaz. 'outside

(adj.)'.

*lutu2. 'pile up'.

$\mathbf{M}-\mathbf{m}$

\begin{tabular}{|c|c|c|c|}
\hline *ma-buhək & *mafu. 'drunk'. & $*_{\mathrm{ma}}(\mathrm{R}) \mathrm{i}$ & *mai. 'come'. \\
\hline *ma-əsa & *mesa. 'alone'. & *maRuqanay & *mane. 'male'; \\
\hline$*_{\text {mah }}$ & *ma. 'and'. & & *mone. 'male'. \\
\hline *ma-hataq & *mata2. 'raw'. & *maRus & *maus. 'tame'. \\
\hline *ma-həmis & *mamis. 'sweet'. & $*$ mata & *mata1. 'eye'. \\
\hline *ma-həyaq & *mae2. 'shy'. & *matay & *mate. 'die'. \\
\hline *ma-hipi & *mehi. 'dream'. & *ma-udəhi & *mone|?. 'outside'; \\
\hline$*_{m a-i R a q}$ & *mea. 'red'. & & *muri. 'last'. \\
\hline *maja & *mada. 'dry up'. & *maya & *maa. 'tongue'. \\
\hline *malip & *maliz. 'laugh'. & **maya & *mae1. 'itchy tuber' \\
\hline *mamaq & *mama. 'chew'. & **mbasaR & *mbasa. 'slap'. \\
\hline *mansər & *?|mauka|? & $* *$ mbio & *pio. 'garlic'. \\
\hline & 'cuscus'. & **meon & *meo1. 'cat'. \\
\hline *manuk & *manu. 'chicken'. & **məџi $(\mathrm{R})$ & *meni. 'fragrant'. \\
\hline **ma-neta & *neta. 'choke'. & *miñak & *mina. 'delicious'. \\
\hline *ma-putiq & *muti. 'white'. & $* *_{\text {mozo }}$ & *modo. 'yellow'. \\
\hline *ma-qasin & *masi. 'salty'. & *mukən & *mukə. 'dove'. \\
\hline *ma-qasu & *masu. 'smoke'. & *mulmul & *mumu1. 'suck'. \\
\hline *ma-qəti & *meti. 'low tide up'. & *muntay & *munde. 'citrus \\
\hline *ma-qitəm & *metam. 'black'. & & (fruit)'. \\
\hline *ma-quban & *mofa. 'grey hair'. & *muRmuR & *mumu2. 'gargle'. \\
\hline *ma-qudip & *mori. 'live'. & $* *$ musa & *muse1. 'seed'. \\
\hline *ma-Rayu & *maygu2. 'dry'. & *musay & *musez. 'civet'. \\
\hline
\end{tabular}




\begin{tabular}{|c|c|c|c|}
\hline$*_{\text {-na }}$ & *naa1. 'that'. & **nepi & *nehi. 'mat on'. \\
\hline *nabuq & *nafu. 'anchor (v.)'. & *nipay & *nihe. 'ant'. \\
\hline *nakaw & *nako. 'steal'. & *nipis & *nihis. 'thin'. \\
\hline *namaw & *namo2. 'coast'. & *niuR & *noh. 'coconut'. \\
\hline *nanaq & *nana. 'pus'. & *nuəs & *nuus. 'squid'. \\
\hline *nayuy & "naye. 'swim'. & *nuka & *nu?a. 'scabies'. \\
\hline **napi & *hani2. 'shell'. & *nunuk & *nunuh. 'banyan'. \\
\hline *naRa & $\begin{array}{l}\text { *naas. 'Pterocarpus } \\
\text { indica'. }\end{array}$ & **nuyan & $\begin{array}{l}\text { "nuya. 'Cordia } \\
\text { species'. }\end{array}$ \\
\hline$*$ nasu & *nasu. 'cook'. & *nusa & *nusa. 'island'. \\
\hline *natuq & *natu. 'ovary'. & & \\
\hline
\end{tabular}

*ñaRa

*naaz. 'brother (of *ñikñik

*nini. 'mosquito'. woman)'.

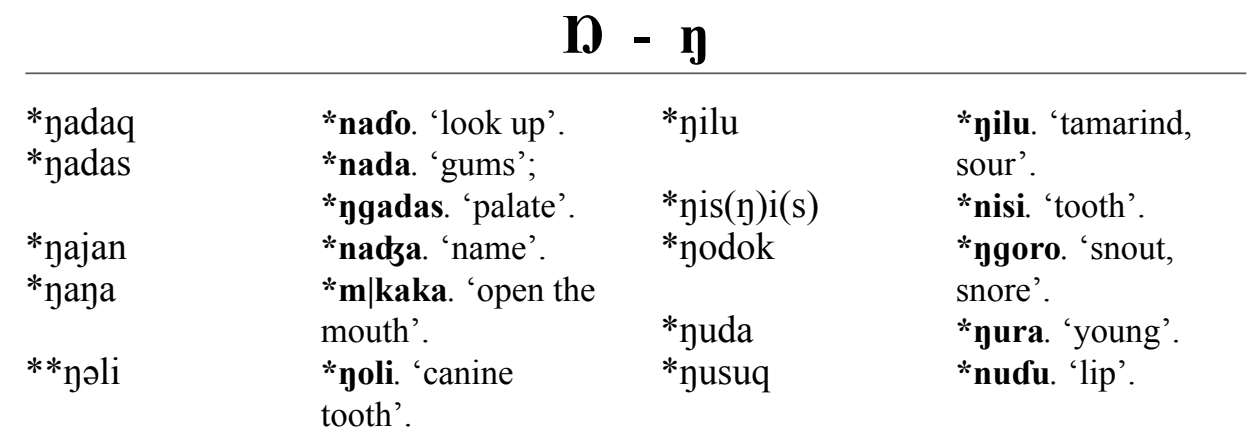

\section{O - o}

**oRo *oo1. 'with'.

\begin{tabular}{llll}
\multicolumn{4}{c}{$\mathbf{P}-\mathbf{p}$} \\
\hline *pagut & *heygu. 'eat'. & *panin & *hani. 'bait (v.)'. \\
**pai & *hai1. 'bucket'. & $* *$ panti & *fandi. 'cut'. \\
*pajay & *hade. 'paddy rice'. & **panga & *hanga. 'span span'. \\
*panahik & *hene. 'climb'. & *paydan & *hendam. 'wild \\
*panas & *hanas. 'hot'. & & pandanus'. \\
*panaw & *hano. 'spots'. & $* *$ pao & *hao. 'feed'. \\
*pandak & *mbada. 'short'; & *papaq & *6eba. 'palm leaf \\
& *pande. 'short'. & & stem'.
\end{tabular}




*paRih
*parud
*pasaqan
**penuk
*pəluk
*pənuq
*pəRəq
**pəsu
*pian
*pija

*hai2. 'stingray'. *piliq

*paru. 'grate'. *pitu

*saha2. "carry on the **pue

shoulders'.

*puləs

*henuh. 'beads'. *punti

*holu1. 'hug'. $\quad$ *pu(y)kaq

*henu. 'full'. *puqun

*hee2. 'press'. *pusəj

*hesu. 'fart'.

*putput

*hia. 'want'.

*puyuq

*hiri. 'choose'.

*hitu. 'seven'.

*hu?e. 'eucalyptus'.

*ule. 'wring'.

*hundi. 'banana'.

*huyga. 'chop'.

*huu. 'tree trunk'.

*husə. 'navel'.

*pupu. 'blow'.

*hida. 'how

*mbui. 'quail'.

much?';

*hida. 'few'.

\section{$\mathbf{Q}-\mathbf{q}$}

\begin{tabular}{|c|c|c|c|}
\hline *qabatəd & *bate. 'edible grub'. & *qatuan & *tuas. 'lord'. \\
\hline$*$ qabu & *afu. 'ash'. & *qaué & *auee. 'oh!'. \\
\hline *qahəlu & *halu. 'pestle'. & *qauR & *ooz. 'bamboo'. \\
\hline *qajəy & *kade. 'charcoal'. & *qayam & *aem. 'tame'. \\
\hline *qaləjaw & *ledo. 'sun'. & *qənəท & *nee2. 'quiet'. \\
\hline * qa-lima & *lima2. 'hand'. & *qənuR & *eno. 'way'. \\
\hline *qalu-hipan & *liha. 'centipede'. & *qota & *taa2. 'unhusked \\
\hline *qalunan & *lunu. 'cushion'. & & rice'. \\
\hline * qambawan & *mbao. 'mango'. & *qoti & *həndi. 'finish'. \\
\hline * qanahaw & *naa4. 'fibres'. & *qibaw & *kibo. 'shellfish'. \\
\hline *qanilaw & *lino. 'Grewia & *qihu & *iu. 'shark'. \\
\hline & slutaris'. & *qila & *ila. 'mole (skin)'. \\
\hline *qanitu & *nitu. 'spirit'. & *qizuR & *midu. 'saliva out'; \\
\hline *qapəju & *hedu. 'gall & & *ninu. 'spit out'. \\
\hline & bladder'. & $*[\mathrm{q} / \mathrm{k}] \mathrm{uma \eta}$ & *sayguma. 'hermit \\
\hline *qapuR & *aho. 'mineral & & crab'. \\
\hline & lime’. & *quay & *ue. 'rattan'. \\
\hline *qaqay & *ei. 'foot'. & *qubi & *ufi. 'tuber'. \\
\hline *qaRta & *atas. 'slave'. & *qudip & *hori|s. 'living'. \\
\hline$*$ qaRta $+*$ qudip & *hatahori. 'man'. & *quhənap & *unə. 'scale'. \\
\hline *qaRuhu & $\begin{array}{l}\text { *kai/ou. 'Casuarina } \\
\text { species'. }\end{array}$ & $\begin{array}{l}* \text { quləj } \\
*_{\text {qulin }}\end{array}$ & $\begin{array}{l}\text { *ulb. 'worm'. } \\
\text { *uli. 'rudder'. }\end{array}$ \\
\hline *qasawa & *sao1. 'marry'. & $*$ qulu & *hulu. 'first in \\
\hline *qasu & *aso. 'scoop'. & & front'; \\
\hline *qatay & *ate. 'liver'. & & *ulu. 'front, head \\
\hline *qatəluR & *təlo. 'egg'. & & hair'. \\
\hline *qatiməla & *mela. 'flea'. & *qusiR & *usi1. 'pursue'. \\
\hline *qatimun & *timu2. 'cucumber'. & *qutan & *utan. 'vegetables'. \\
\hline *qatip & *ati|s. 'breast beam & *qutin & *uti. 'penis'. \\
\hline & of loom’. & *quzan & ‘udan. 'rain'. \\
\hline
\end{tabular}




\section{$\mathbf{R}$ - $\mathbf{R}$}

\begin{tabular}{llll}
\hline *Rabiqi & *afi. 'yesterday'. & *Ribu & *rifu|n. 'thousand'. \\
*Rakit & *ra?i. 'bind'. & *Rumaq & *uma. 'house'. \\
*Raya & *nae. 'big'. & *Rusuk & *kai/usu. 'ribs'. \\
*(R)ədəm & *endən. 'soak'. & &
\end{tabular}

\section{$\mathbf{r}-\mathbf{r}$}

$\begin{array}{ll}\text { **rafu } & \text { *nafu|2. 'body hair'. } \\ \text { *rakup } & \text { *ra?u. 'scoop'. } \\ \text { *riba } & \text { *ifa. 'lap'. }\end{array}$

\section{$\mathbf{S}-\mathbf{S}$}

*sai
*sakay
**sakuray
*salaq
**salili
*salin
**sa(m)bura[t/s]
*saya
*sa-na-puluq
*sa-na-Ratus
*sayəlaR
*sau
**sauR
*sawa
*səbuh
**sədu(t)
*səksək
*səlaR
**səru
*si-ia

*see2. 'who?'. *si-ida

*sake. 'go up'. *siku

*kura. 'scorpion'. *sijaR

*sala. 'wrong'. *sinkab

*salili. 'armpit'.

*sali. 'pour'. *siwa

*sambudas. 'spurt'. * *suaR

*sana|?. 'branch of

fruit'. *suat

*sanahulu. 'ten'. *sudu

*natu|n. 'hundred'.

*seya. 'fry'.

*sauz. 'comb'.

*soo. 'sew'.

*saoz. 'snake'. *sukəd

*sufu. 'cool (v.)'. *sukun

*seru. 'weaving *sulə[n/d]

sword of loom'. *sumayəd

*sesə. 'cram'. *supu

*sela. 'coarse'. *suqun

*soru. 'meet'.

*ndia. 'she, he, it, *susu 3SG'. *sira. 'they, 3PL'.

"siku. 'elbow'.

*sina. 'light (n.)'.

‘sika. 'open';

*singa. 'open'.

*sio. 'nine'.

*suaz. 'digging

stick'.

*suhat. 'comb'.

*soro. 'spoon';

"suru. 'spoon'.

*sura. 'horn';

*sure. 'sword, caltrop'.

‘suaz. 'rafter'.

*suku. 'breadfruit'.

*sulə. 'plug'.

*sumanə. 'spirit'.

*suhu. 'edge'.

*suu2. 'carry on head'.

'susu2. 'breast'. *tabtab

*tabuni

*tabuqan *tata. 'split'.

*funi2. 'afterbirth'.

*katefuan. 'wasp'. *taduku

*takut

*taləs *taruku. 'chiton'.

*taku. 'fear'.

*tales. 'taro'. 


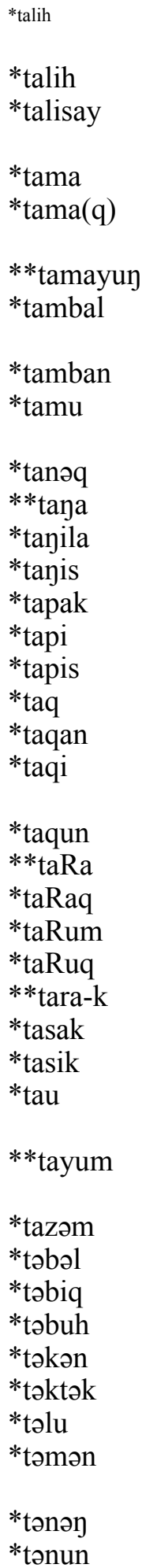

*tali. 'rope'.

*lise. 'Terminalia catappa'.

*tama1. 'enter'.

*tama2. 'fit together'.

*tamae. 'bedbug'.

*tamba2. 'patch, join'.

*temba. 'sardine'.

*tamu. 'close mouth'.

*tane1. 'mud'.

*tanaz. 'crisp'.

*ygela1. 'earwax'.

*tani. 'complain'.

*taa1. 'track'.

*tahi. 'winnow'.

*tais. 'cloth'.

*taa3. 'no'.

*taa4. 'endure'.

*tai. 'belly';

*tai2. 'faeces'.

*taun. 'year'.

*taha. 'answer'.

*tara. 'adze'.

*taum. 'indigo'.

*tao. 'put, do'.

*tana|?. 'thorn'.

*tasa. 'ripe'.

*tasi. 'sea'.

*toPo. 'man, male';

*tou. 'man, person'.

*k|teom. 'sea

urchin'.

*tande. 'sharp'.

*nembə. 'hard'.

*tebi. 'break up'.

*tefu. 'sugarcane'.

*tekə. 'staff'.

*teke. 'gecko'.

*telu. 'three'.

*temə.

'accustomed'.

*tenaz. 'calm'.

*tenu. 'weave'.
*təךəR

*təRas

**təRə

*tərin

*tikəd

*tila

*timid

*timuR

*tindaw

*tindəs

**tinaR

*tiqaw

*tiRəm

**tiRi

*tiRis

*titis

*tuak

*tuba

*tuduq

*tuduy

*tuhud

*tui

**tuir

*tukil

*tuktuk

*tuluy

*tumah

*tumbuk

*tu(m)buq

*tuna

*tunu

*tuqah

*tuquR

*tuzuq *tene. 'Ceriops, mangrove'.

*teas. 'heartwood'.

*tee. 'spear'.

*teri. 'giant

bamboo,

Dendrocalamus'.

*tinga. 'heel'.

*tila. 'vagina'.

*timi. 'chin'.

*timu1. 'east wind'.

*tino. 'peer';

*tiro. 'peer'.

*tede. 'press'.

*tina. 'field'.

*ti(?)o. 'goatfish,

Mullidae'.

*tiam. 'oyster'.

*tii. 'sea urchin'.

*tiri. 'drip'.

*titi. 'drip'.

*tua2. 'lontar palm'.

*tufa. 'Derris

elliptica'.

*turu. 'overflow'.

*turis. 'pigeon pea'.

*tuu1. 'knee'.

*tuiz.

'Dolichandrone

spathacea'.

*tuin. 'follow'.

*tuke. 'bamboo

container'.

*toko. 'beat';

*tutu. 'beat'.

*tuli. 'stop by'.

*tuma. 'clothes

louse'.

*tufu. 'punch'.

*numbu. 'grow';

*tumbu. 'heap'.

*tuna. 'freshwater

eel'.

*tunuz. 'roast'.

*tua1. 'big'.

*tuu2. 'dry'.

*tudu. 'point'. 


\section{$\mathbf{U}-\mathbf{u}$}

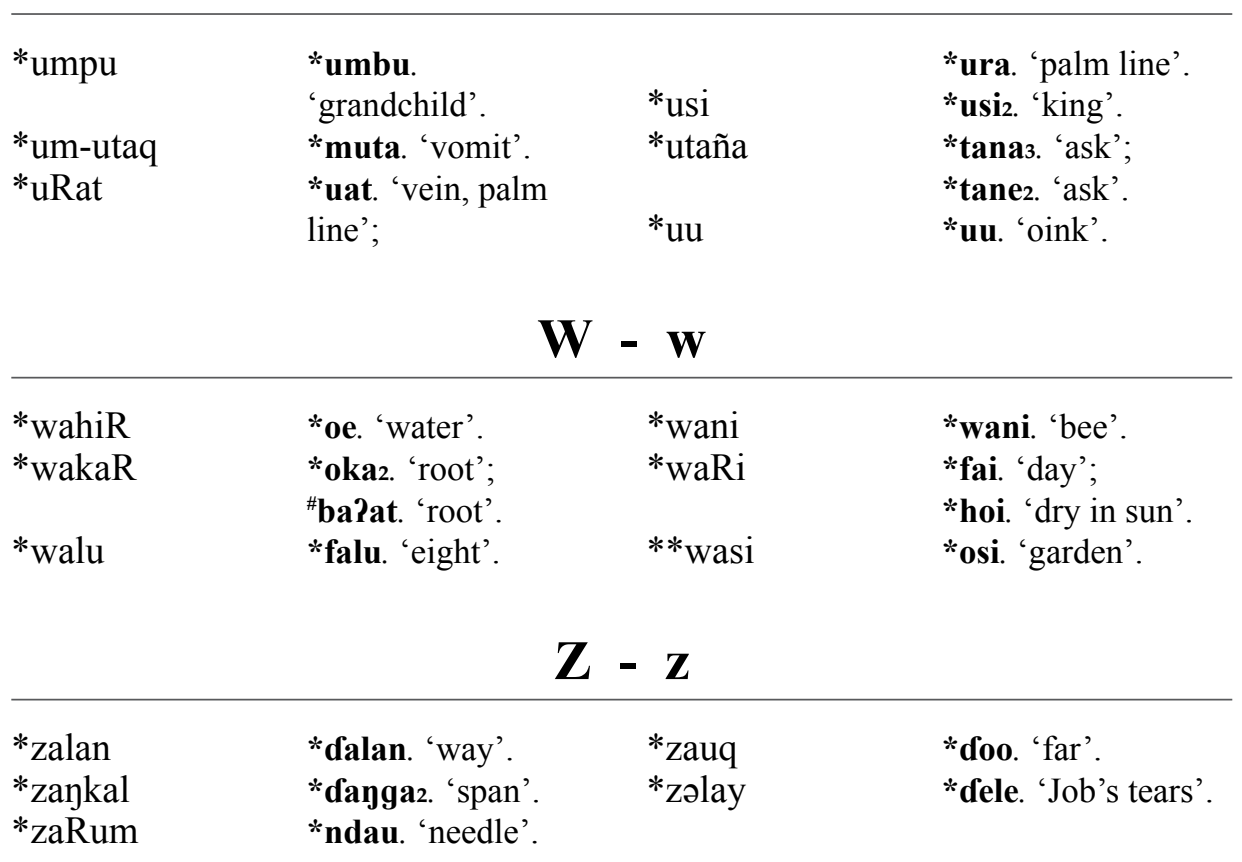

Total number of entries: 558 



\section{References}

Abas, Husen. 1995. Bugis wordlist. In Comparative Austronesian dictionary: An introduction to Austronesian studies, ed. Darrell T. Tryon, Parts 2-4. Berlin and New York: Mouton de Gruyter.

Adriani, Nicolaus. 1928. Bare'e-Nederlandsch woordenboek: met Nederlandsch-Bare'e register. Leiden: Brill.

Ali, Slamet Ryadi. 1995. Sasak wordlist. In Comparative Austronesian dictionary: An introduction to Austronesian studies, ed. Darrell T. Tryon, Parts 2-4. Berlin and New York: Mouton de Gruyter.

Alpher, Barry, and David Nash. 1999. Lexical replacement and cognate equilibrium in Australia. Australian Journal of Linguistics 19:5-56. doi.org/10.1080/072686099 08599573

Aoki, Eriko, and Satoshi Nakagawa. 1993. Endenese-English dictionary. Osaka: Osaka International University.

Arka, I Wayan, Fransiscus Seda, Antonius Gelang, Yohanes Nani, and Ivan Ture. 2007. Kamus Rongga-Indonesia dengan daftar pelacak kata Indonesia-Rongga. Jakarta: Penerbit Universitas Atma Jaya.

Arndt, Paul. 1961. Wörterbuch der Ngadhasprache. Posieux, Fribourg: Studia Instituti Anthropos.

Baird, Louise. 2002. A grammar of Kéo: An Austronesian language of East Nusantara. Doctoral Thesis, The Australian National University.

Balle, Misriani, and Stuart Cameron. 2014. Helong dictionary. Unpublished Toolbox file.

Bawa, I Wayan, and Adrian Clynes. 1995. Balinese wordlist. In Comparative Austronesian dictionary: An introduction to Austronesian studies, ed. Darrell T. Tryon, Parts 2-4. Berlin and New York: Mouton de Gruyter.

Blevins, Juliette, and Andrew Garrett. 1998. The origins of consonant-vowel metathesis. Language 74:508-556. doi.org/10.1353/lan.1998.0012

Blust, Robert. 1993. Central and Central-Eastern Malayo-Polynesian. Oceanic Linguistics 32:241-293. doi.org/10.2307/3623195 
Blust, Robert. 1999. Notes on Pazeh phonology and morphology. Oceanic Linguistics 38:321-365. doi.org/10.1353/ol.1999.0002

Blust, Robert. 2009a. The Austronesian languages. Canberra: Pacific Linguistics.

Blust, Robert. 2009b. The position of the languages of eastern Indonesia: A reply to Donohue and Grimes. Oceanic Linguistics 48:36-77. doi.org/10.1353/ol.0.0034

Blust, Robert, and Stephen Trussel. ongoing. The Austronesian comparative dictionary. www.trussel2.com/ACD/

Calon, L. F. 1891. Woordenlijstje bij het dialekt van Sikka (Midden-Flores). Tijdschrift voor Indische Taal-, Land-en Volkenkunde 34:283-363.

Cense, A. A. in collaboration with Abdoerrahim. 1979. Makassaars-Nederlands woordenbooek met Nederlands-Makassaars register. 's-Gravenhage: Martinus Nijhoff.

Chafe, Wallace. 1994. Discourse, consciousness, and time: The flow and displacement of conscious experience in speaking and writing. Chicago and London: University of Chicago Press.

Chlenov, Mikhail A., and Svetlana F. Chlenova. 2008. The Damar Batumerah (West Damar language) of south-eastern Indonesia. In Language and text in the Austronesian world: Studies in honor of Ülo Sirk, ed. Yury A. Lander and Alexander K. Ogloblin, 141-162. Munich: Lincom.

Chlenova, Svetlana F. 2002. Daweloor, a Southwest Moluccan language. Малайскоиндонезийские исследования [Malay-indonesian studies] 15:145-175. Special English language edition of Малайско-индонезийские исследования ed. В. В. Parnickel.

Christensen, John. in progress. Kisar dictionary. Unpublished Toolbox file.

Christensen, John, and Sylvia Christensen. 1992. Kisar phonology. In Phonological studies in four languages of Maluku, ed. Donald A. Burquest and Wyn D. Laidig, 33-65. Dallas: Summer Institute of Linguistics and the University of Texas at Arlington.

Clark, Ross. 2011. Birds. In The lexicon of Proto Oceanic: The culture and environment of ancestral Oceanic society, ed. Malcolm Ross, Andrew Pawley, and Meredith Osmond, volume 4: Animals, 271-370. Canberra: Pacific Linguistics.

Codrington, Robert H., and Jim Palmer. 1896. A dictionary of the language of Mota, Sugarloaf Island, Banks Islands. London: Society for Promoting Christian Knowledge.

Coolsma, Sierk. 1913. Soendaneesch-Hollandsch woordenboek. Leiden: A. W. Sijthoff.

Cristo Rei, João Maria, and Mark Donohue. 2012. Galolen dictionary. MS. 
Culhane, Kirsten. 2018. Consonant insertions: A synchronic and diachronic account of Amfo'an. Honours Thesis, The Australian National University. hdl.handle. net/1885/160794

Culhane, Kirsten, Laurence Jumetan, and Yedida Ora. 2018. Amfo'an dictionary. Unpublished Toolbox file.

Cunningham, Clark E. 1964. Order in the Atoni house. Bijdragen tot de Taal-, Land- en Volkenkunde 120:34-68. doi.org/10.1163/22134379-90002996

Daigle, Benjamin T. 2015. A grammar sketch of Batuley: an Austronesian language of Aru, eastern Indonesia. Master's Thesis, Leiden University.

da Silva, Eng. Guilherme. 2012. Disionáriu Wekais-Tetun. Timor-Leste: Secretaria de Estado da Cultura.

Dawson, Virginia. 2014. Manatuto survey (VD1). Digital collection managed by PARADISEC. catalog.paradisec.org.au/collections/VD1

de Josselin de Jong, J. P. B. 1947. Studies in Indonesian culture II: The community of Erai (Wetar) (texts and notes). Amsterdam: Noord-Hollandsche Uitgevers-Maatschappij.

de Josselin de Jong, J. P. B. 1987. Wetan fieldnotes: Some eastern Indonesian texts with linguistic notes and a vocabulary. Dordrecht-Holland: Foris Publications.

Dempwolff, Otto. 1938. Vergleichende Lautlehre des austronesischen Wortschatzes, volume 3: Austronesisches Wörterverzeichnis of Zeitschrift für Eingeborenensprachen. Berlin: Dietrich Reimer. Supplement 17. doi.org/10.1017/s0041977 $\mathrm{x} 00135360$

Djawanai, Stephanus. 1995. Ngada wordlist. In Comparative Austronesian dictionary: An introduction to Austronesian studies, ed. Darrell T. Tryon, Parts 2-4. Berlin and New York: Mouton de Gruyter.

Donohue, Mark. 2003. Daftar Kata Bata Lu'a [Palu'e wordlist]. MS.

Donohue, Mark, and Charles E. Grimes. 2008. Yet more on the position of the languages of eastern Indonesia and East Timor. Oceanic Linguistics 47:114-158. doi.org/10.1353/ ol.0.0008

Drabbe, Peter. 1932. Woordenboek der Fordaatsche taal, volume 61 of Verhandelingen van het Koninklijk Bataviaasch Genootschap van Kunsten en Wetenschappen. Bandoeng: Nix.

Eberhard, David M., Gary F. Simons, and Charles D. Fennig. 2020. Ethnologue: Languages of the world (23rd ed.). Dallas, Texas: SIL International.

Edwards, Owen. 2016. Parallel sound correspondences in Uab Meto. Oceanic Linguistics 55:52-86. doi.org/10.1353/ol.2016.0008 
Edwards, Owen. 2017. Epenthetic and contrastive glottal stops in Amarasi. Oceanic Linguistics 56:415-434. doi.org/10.1353/ol.2017.0020

Edwards, Owen. 2018a. Parallel histories in Rote-Meto. Oceanic Linguistics 57:359409. doi.org/10.1353/ol.2018.0016

Edwards, Owen. 2018b. Preliminary report on Funai Helong. NUSA: Linguistic studies of languages in and around Indonesia 65:1-27. doi.org/10.15026/92897

Edwards, Owen. 2018c. Top-down historical phonology of Rote-Meto. Journal of the Southeast Asian Linguistics Society 11:63-90. hdl.handle.net/10524/52421

Edwards, Owen. 2019. Reintroducing Welaun. Oceanic Linguistics 58:31-58. hdl.handle. net/1887/79038. doi.org/10.1353/ol.2019.0002

Edwards, Owen. 2020. Metathesis and unmetathesis in Amarasi. Berlin: Language Science Press. doi.org/10.5281/zenodo.3700413

Elias, Alexander. 2018. Lio and the Central Flores languages. Master's Thesis, Leiden University.

Fanggidaej, J. 1892. Rottineesche spraakkunst. Bijdragen tot de Taal-, Land- en Volkenkunde 41:554-571. doi.org/10.1163/22134379-90000187

Farjon, Aljos. 2017. A handbook of the world's conifers, volume 1. (2nd ed.) LeidenBoston: Brill.

Fogaça, Helem Andressa de Oliveira. 2017. O ecossistema fundamental da língua Mambae : aspectos endoecológicos e exoecológicos de uma língua austronésia de Timor-Leste. Doctoral dissertation, Universidade de Brasília. repositorio.unb.br/ handle/10482/31396

Forth, Gregory L. 2016. Why the porcupine is not a bird: Explorations in the folk zoology of an eastern Indonesian people. Toronto: University of Toronto Press.

Fox, James J. 1988. The historical consequences of changing patterns of livelihood in Timor. In Contemporary issues in development, ed. Deborah Wade-Marshall and Peter Loveday, 259-279. Darwin: The Australian National University North Australia Research Unit.

Fox, James J. 1991. The heritage of traditional agriculture in eastern Indonesia: Lexical evidence and the indications of rituals from the outer arc of the Lesser Sundas. Bulletin of the Indo-Pacific Prehistory Association 10:248-262.

Fox, James J. 2016a. Master poets, ritual masters: The art of oral composition among the Rotenese of eastern Indonesia. Canberra: ANU Press. doi.org/10.22459/mprm. 04.2016

Fox, James J. 2016b. Termanu ritual language dictionary. MS. 
Fox, James J., and Charles E. Grimes. 1995. Roti. In Comparative Austronesian dictionary: An introduction to Austronesian studies, ed. Darrell T. Tryon, Part 1, 611-622. Berlin: Mouton de Gruyter.

Friberg, Timothy. 1995. Konjo wordlist. In Comparative Austronesian dictionary: An introduction to Austronesian studies, ed. Darrell T. Tryon, Parts 2-4. Berlin and New York: Mouton de Gruyter.

Fricke, Hanna. 2014. Topics in the grammar of Hewa: A variety of Sika in Eastern Indonesia. Munich: Lincom Europa.

Fricke, Hanna. 2015. Field notes on Lamaholot-Kalikasa. In Lexirumah 3.0.0, ed. Gereon A. Kaiping, Owen Edwards, and Marian Klamer. Leiden: Leiden University Centre for Linguistics. lexirumah.model-ling.eu/lexirumah/

Fricke, Hanna. 2019. Traces of language contact: the Flores-Lembata languages in eastern Indonesia. Doctoral dissertation, Leiden University. hdl.handle.net/1887/80399

Geurtjens, Hendrik. 1921. Woordenlijst der keieesche taal, volume 63 of Verhandelingen van het Koninklijk Bataviaasch Genootschap van Kunsten en Wetenschappen. Weltevreden: Albrecht.

Grimes, Charles E. 1991. The Buru language of eastern Indonesia. Doctoral dissertation, The Australian National University. openresearch-repository.anu.edu.au/handle/1885/ 10925

Grimes, Charles E. 2010. Hawu and Dhao in eastern indonesia: Revisiting their relationship. In East Nusantara: Typological and areal analysis, ed. Michael Ewing and Marian Klamer, 251-280. Canberra: Pacific Linguistics.

Grimes, Charles E., Bernadus Lado, Thomas Ly, and Simon Tari. 2008. Kamus Lii Hawu (Sabu) online dictionary. Kupang: UBB-GMIT. ubb.or.id/download/kamus-lii-hawulexpro/

Grimes, Charles E., Ayub Ranoh, and Helena Aplugi. 2008. Kamus Lii Dhao (Ndao) online dictionary. Kupang: UBB-GMIT. ubb.or.id/download/kamus-lii-dhao-lexpro/

Grimes, Charles E., Evelyn Cheng, Enna Adelaide Hayer-Pah, Jonathan Pandie, Neng Mulosing, and Johnny M. Banamtuan. 2014a. Tii dictionary. Unpublished Toolbox file.

Grimes, Charles E., Carlos Marçal, and Paolino Fereira. 2014b. Introductory dictionary of Mambae (Same): Mambae-English, English-Mambae, Mambae-IndonesiaTetun Dili, Indonesia-Mambae, Tetun Dili-Mambae. Darwin: Australian Society for Indigenous Languages.

Grimes, Charles E., and Barbara Dix Grimes. 2020. Encyclopedic dictionary of the Buru language: With English-Buru finderlist. (3rd ed.). Kupang: Unit Bahasa \& Budaya (UBB). ubb.or.id/download/kamus-buru-inggris/ 
Grimes, Charles E., Yanri Suana, Yedida Ora, and Kirsten Culhane, compilers. 2021. Introductory dictionary of Amfo'an: With English-Amfo'an finderlist. Kupang: Unit Bahasa \& Budaya (UBB). ubb.or.id/bahasa/kamus/

Hammarström, Harald, Robert Forkel, Martin Haspelmath, and Sebastian Bank. 2020. Glottolog 4.3. Jena: Max Planck Institute for the Science of Human History. URL glottolog.org

Hendriks, H. 1897. Het Burusch van Masarete. The Hague: Martinus Nijhoff.

Heston, Tyler. 2015. The segmental and suprasegmental phonology of Fataluku. Doctoral dissertation, University of Hawai'i at Mānoa.

Heston, Tyler M. 2017. A first reconstruction of vowels in Proto-Timor-Alor-Pantar. Oceanic Linguistics 56:73-89. doi.org/10.1353/ol.2017.0003

Heyne, K. 1950. De nuttige planten van Indonesië. (3rd ed.).'s-Gravenhage and Bandung: NV Uitgeverij W van Hoeve. 2 volumes.

Himmelmann, Nikolaus P. 2001. Sourcebook on Tomini-Tolitoli languages: General information and wordlists. Canberra: Pacific Linguistics.

Himmelmann, Nikolaus P., John Bowden, Maurício C. A. Belo, Alex Hajek, John Tilman, and Alex Freitas. 2006. Waima'a lexical database. In DoBeS Waima'a documentation, ed. Belo Maurício C. A., John Bowden, John Hajek, Nikolaus P. Himmelmann, and Alexandre V. Tilman. DoBeS Archive MPI Nijmegen.

Hinton, Bryan. 1991. Aspects of Tugun phonology and syntax. Master's Thesis, University of Texas at Arlington.

Hinton, Bryan. 2000. The languages of Wetar: recent survey results and word lists, with notes on Tugun grammar. In Spices from the east: Papers in languages of eastern Indonesia, ed. Charles E. Grimes, 105-117. Canberra: Pacific Linguistics.

Hoogervorst, Tom. 2016. Problematic protoforms: Some 'hidden' Indic loans in Western Malayo-Polynesian languages. Oceanic Linguistics 55:561-587. doi.org/10.1353/ol. 2016.0025

Hoola van Nooten, Berthe, and P. Depannemaeker. 1880. Fleurs fruits et feuillages choisis de l'île de Java. Bruxelles: C. Muquardt. doi.org/10.5962/bhl.title.466

Ismail, Mansyur, Muhidin Azis, M. Saleh Yakub, M. Taufik H., and M. Kasim Usman. 1985. Kamus Bima-Indonesia. Jakarta: Pusat Pembinaan dan Pengembangan Bahasa.

Jacob, June, and Charles Grimes. 2011. Aspect and directionality in Kupang Malay serial verb constructions: Calquing on the grammars of substrate languages. In Creoles, their substrates, and language typology, ed. Claire Lefebvre, 337-366. Amsterdam and Philadelphia: John Benjamins. doi.org/10.1075/tsl.95.20jac

Jonker, J. C. G. 1893. Bimaneesch-Hollandsch woordenboek. Batavia: Landsdrukkerij. 
Jonker, J. C. G. 1906. Over de eind-medeklinkers in het Rottineesch en Timoreesch. Bijdragen tot de Taal-, Land- en Volkenkunde van Nederlandsch-Indië 59:263-343. doi.org/10.1163/22134379-90001964

Jonker, J. C. G. 1908. Rottineesch-Hollandsch woordenboek. Leiden: E. J. Brill.

Jonker, J. C. G. 1913. Bijdragen tot de kennis der Rottineesche tongvallen. Bijdragen tot de Taal-, Land- en Volkenkunde van Nederlandsch-Indië 68:521-622. doi. org/10.1163/22134379-90001774

Jonker, J. C. G. 1915. Rottineesche spraakkunst. Leiden: E. J. Brill.

Kähler, Hans. 1959. Vergleichendes Wörterverzeichnis der Sichule-Sprache auf der Insel Simalur an der Westkuste von Sumatra. Berlin: Dietrich Reimer Verlag.

Keraf, Gregor. 1978. Morfologi dialek Lamalera. Doctoral dissertation, Universitas Indonesia.

Kersten, J. 1984. Bahasa Bali. Ende: Nusa Indah.

Klamer, Marian. 2002. Timor-Leste survey word lists. In Lexirumah 3.0.0, ed. Gereon A. Kaiping, Owen Edwards, and Marian Klamer. Leiden: Leiden University Centre for Linguistics. lexirumah.model-ling.eu/lexirumah/

Klamer, Marian. 2011. A short grammar of Alorese (Austronesian). Munich: Lincom Europa.

Klamer, Marian. 2015a. Field notes on Hewa. In Lexirumah 3.0.0, ed. Gereon A. Kaiping, Owen Edwards, and Marian Klamer. Leiden: Leiden University Centre for Linguistics. lexirumah.model-ling.eu/lexirumah/

Klamer, Marian. 2015b. Field notes on Lamaholot-Lewoingu. In Lexirumah 3.0.0, ed. Gereon A. Kaiping, Owen Edwards, and Marian Klamer. Leiden: Leiden University Centre for Linguistics. lexirumah.model-ling.eu/lexirumah/

Kratochvíl, František. 2007. A grammar of Abui: A Papuan language of Alor. Doctoral dissertation, University of Leiden.

Lambrecht, Knud. 1994. Information structure and sentence form: Topic, focus, and the mental representations of discourse referents. Cambridge: Cambridge University Press. doi.org/10.1017/cbo9780511620607

Lekede’e Study Group. 2006. Disionáriu lia Tokodede - Tetun - Ingles. Likisá: Timor Loro Sa'e - Nippon Culture Center.

Lynch, John. 2001. Too much to swallow: On terms meaning 'swallow' in Oceanic languages. Oceanic Linguistics 40:336-341. doi.org/10.2307/3623445

Lynch, John, Malcolm Ross, and Terry Crowley. 2002. Proto Oceanic. In The Oceanic languages, ed. John Lynch, Malcolm Ross, and Terry Crowley, 54-91. Richmond: Curzon. doi.org/10.4324/9780203820384 
Mahdi, Waruno. 1994. Some Austronesian maverick protoforms with culture historical implications II. Oceanic Linguistics 30:431-490. doi.org/10.2307/3623137

Manafe, D. P. 1889. Akan bahasa Rotti. Bijdragen tot de Taal-, Land- en Volkenkunde van Nederlandsch-Indië 28:633-648. doi.org/10.1163/22134379-90000273

Masse, H. Abd. 2013. Kamus bahasa Bugis-Indonesia. Indonesia: CV. Gemilang Utama.

Mathes, B. F. 1859. Makassaarsch-hollandsch woordenboek. Amsterdam: Het Nederlandsch Bijbelgenootschap te Amsterdam.

Mathes, B. F. 1874. Boegineesch-Hollandsch woordenboek. 's-Gravenhage: M. Nijhoff.

McDonnell, Bradley. 2009. A preliminary description of Ende phonology. Journal of the Southeast Asian Linguistics Society 2:195-226.

Mead, David. 1998. Proto-Bungku-Tolaki: Reconstruction of its phonology and aspects of its morphosyntax. Doctoral dissertation, Rice University.

Meijer Drees, Ebertus. 1950. Daftar nama2 pohon dan perdu, Pulau Timor = lijst van boom- en struiknamen van het eiland Timor = list of tree and shrub names from Timor. Number 1 in Serie vegetatie-onderzoek = Seri pemeriksaan tumbuh-tumbuhan $=$ Ecological series. Bogor: Balai Penjelidikan Kehutanan.

Middelkoop, Pieter. 1950. Proeve van een Timorese grammatica. Bijdragen tot de Taal-, Land- en Volkenkunde 106:375-517. doi.org/10.1163/22134379-90002474

Middelkoop, Pieter. 1972. Nederlands-Timorees woordenboek. MS.

Mills, Roger. 1991. Tanimbar-Kei: An eastern Indonesian subgroup. In Currents in Pacific linguistics: Papers on Austronesian languages and ethnolinguistics in honour of George W. Grace, ed. Robert Blust, 241-263. Canberra: Pacific Linguistics. C-117.

Mills, Roger. 2010. Three common misconceptions about Proto-Lettic. In Studia anthropologica: сборник статей в честь М.А. Членова, ed. Svetlana F. Chlenova and Artem Fedorchuk, 284-296. Moscow-Jerusalem: Gesharim.

Moro, Francesca. 2016. Field notes on Alorese-Pandai. In Lexirumah 3.0.0, ed. Gereon A. Kaiping, Owen Edwards, and Marian Klamer. Leiden: Leiden University Centre for Linguistics. lexirumah.model-ling.eu/lexirumah/

Morris, Cliff. 1984. Tetun-English dictionary. Canberra: Pacific Linguistics.

Nako, Yustin Marince, Paulus Nako, Misriani Balle, and Johnny M. Banamtuan. 2014. Rikou dictionary. Unpublished Toolbox file.

Nishiyama, Kunio, and Herman Kelen. 2007. A grammar of Lamaholot, Eastern Indonesia: The morphology and syntax of the Lewoingu dialect. Munich: Lincom.

Onvlee, Louis. 1984. Kamberaas (Oost-Soembaas)-Nederlands woordenboek. Dordrecht: Foris. In collaboration with OE. H. Kapita and P. J. Luijendijk. 
Osmond, Meredith. 1998. Horticultural practices. In The lexicon of Proto Oceanic: The culture and environment of ancestral Oceanic society, ed. Malcolm Ross, Andrew Pawley, and Meredith Osmond, volume 1: Material culture, 115-142. Canberra: Pacific Linguistics.

Osmond, Meredith. 2011a. Fish. In The lexicon of Proto Oceanic: The culture and environment of ancestral Oceanic society, ed. Malcolm Ross, Andrew Pawley, and Meredith Osmond, volume 4: Animals, 25-136. Canberra: Pacific Linguistics.

Osmond, Meredith. 2011b. Insects and other creepy-crawlies. In The lexicon of Proto Oceanic: The culture and environment of ancestral Oceanic society, ed. Malcolm Ross, Andrew Pawley, and Meredith Osmond, volume 4: Animals, 371-420. Canberra: Pacific Linguistics.

Osmond, Meredith, Andrew Pawley, and Malcolm Ross. 2003. The landscape. In The lexicon of Proto Oceanic: The culture and environment of ancestral Oceanic society, ed. Malcolm Ross, Andrew Pawley, and Meredith Osmond, volume 2: The physical environment, 35-89. Canberra: Pacific Linguistics.

Osmond, Meredith, and Malcolm Ross. 2016a. The human body. In The lexicon of Proto Oceanic: The culture and environment of ancestral Oceanic society, ed. Malcolm Ross, Andrew Pawley, and Meredith Osmond, volume 5: People: body and mind, 75-208. Canberra: Asia-Pacific Linguistics.

Osmond, Meredith, and Malcolm Ross. 2016b. People: gender, age cohorts and marital status. In The lexicon of Proto Oceanic: the culture and environment of ancestral Oceanic society, ed. Malcolm Ross, Andrew Pawley, and Meredith Osmond, volume 5: People: body and mind, 37-74. Canberra: Asia-Pacific Linguistics.

Pareira, M. Mandalangi, and E. Douglas Lewis. 1998. Kamus sara Sikka Bahasa Indonesia. Ende, Indonesia: Nusa Indah.

Pawley, Andrew. 2011. Aquatic invertebrates. In The lexicon of Proto Oceanic: the culture and environment of ancestral Oceanic society, ed. Malcolm Ross, Andrew Pawley, and Meredith Osmond, volume 4: Animals, 161-216. Canberra: Pacific Linguistics.

Penn, David Trelly. 2006. Introducing Dadu'a: Uma língua de Timor-Leste. Honours Thesis, University of New England.

Rinnooy, N. 1886. Maleisch-Kissersch woordenlijst. Tijdschrift voor Indische Taal-, Land-en Volkenkunde 31:149-213.

Ross, Malcolm. 2003. Properties of inanimate objects. In The lexicon of Proto Oceanic: The culture and environment of ancestral Oceanic society, ed. Malcolm Ross, Andrew Pawley, and Meredith Osmond, volume 2: The physical environment, 3589. Canberra: Pacific Linguistics. 
Ross, Malcolm, and Meredith Osmond. 2016a. Bodily conditions and activities. In The lexicon of Proto Oceanic: The culture and environment of ancestral Oceanic society, ed. Malcolm Ross, Andrew Pawley, and Meredith Osmond, volume 5 People: body and mind, 209-333. Canberra: Asia-Pacific Linguistics.

Ross, Malcolm, and Meredith Osmond. 2016b. Cognition. In The lexicon of Proto Oceanic: The culture and environment of ancestral Oceanic society, ed. Malcolm Ross, Andrew Pawley, and Meredith Osmond, volume 5 People: body and mind, 535-566. Canberra: Asia-Pacific Linguistics.

Saad, George. 2015. Takalelang Abui word list. In Lexirumah 3.0.0, ed. Gereon A. Kaiping, Owen Edwards, and Marian Klamer. Leiden: Leiden University Centre for Linguistics. lexirumah.model-ling.eu/lexirumah/

Samely, Ursula. 1991. Kedang (Eastern Indonesia), some aspects of its grammar. Hamburg: Helmut Buske.

Schapper, Antoinette. 2009. Bunaq: A Papuan language of central Timor. Doctoral dissertation, The Australian National University.

Schapper, Antoinette. 2010. Field notes and dictionary on Kamang-Atoitaa. In Lexirumah 3.0.0, ed. Gereon A. Kaiping, Owen Edwards, and Marian Klamer. Leiden: Leiden University Centre for Linguistics. lexirumah.model-ling.eu/lexirumah/

Schapper, Antoinette. 2011. Phalanger facts: Notes on Blust's marsupial reconstructions. Oceanic Linguistics 50:258-272. doi.org/10.1353/ol.2011.0004

Schapper, Antoinette. 2017. Stress and gemination in Alor-Pantar languages: Revising Heston (2016). Oceanic Linguistics 56:257-266. doi.org/10.1353/ol.2017.0011

Schapper, Antoinette. 2020. Historical and linguistic perspectives on fortified settlements in southeastern Wallacea: Far eastern Timor in the context of southern Maluku. In Forts and fortification in Wallacea: Archaeological and ethnohistoric investigations, ed. Sue O'Connor and Andrew McWilliam, 221-246. Canberra: ANU Press. doi.org/10.22459/ta53.2020.10

Schapper, Antoinette, and Emilie Wellfelt. 2018. Reconstructing contact between Alor and Timor: Evidence from language and beyond. NUSA: Linguistic studies of languages in and around Indonesia 64:95-116.

Schulte Nordholt, H. G. 1971. The political system of the Atoni of Timor. The Hague: Martinus Nijhoff. Translation of Het Politieke Systeem van de Atoni van Timor (1966).

Smits, Leo, and C. L. Voorhoeve. 1992. The J.C. Anceaux collection of wordlists of Irian Jaya languages A: Austronesian languages (part I). Leiden/Jakarta: DSALCUL/IRIS.

Steven, Lee Anthony. 1991. The phonology of Roma, an Austronesian language of eastern Indonesia. Master's Thesis, University of Texas at Arlington.

Stresemann, Erwin. 1927. Die Lauterscheinungen in den ambonischen Sprachen, volume 10 of Zeitschrift für Eingeborenen-Sprachen. Berlin: Reimer. 
Taber, Mark. 1993. Toward a better understanding of the indigenous languages of southwestern Maluku. Oceanic Linguistics 32:389-441. doi.org/10.2307/3623199

Tamelan, Thersia. 2017. Dela dictionary. Unpublished Toolbox file.

Tamelan, Thersia. 2021. A grammar of Dela: An Austronesian language of Rote, eastern Indonesia. Doctoral dissertation, The Australian National University. hdl.handle. net $/ 1885 / 250953$

Therik, Tom. 2004. Wehali: The female land. Traditions of a Timorese ritual centre. Canberra: Pandanus Books.

Tregear, Edwards. 1891. The Maori-Polynesian comparative dictionary. Wellington: Lyon and Blair.

Unit Bahasa and Budaya. 2016. Manetualain Dede'a-kokolan: Hehelu-bartaa Beuk no Tutui Makasososak. Kupang: Unit Bahasa and Budaya in cooperation with Wycliffe Bible Translators.

van den Berg, René. 1991. Muna historical phonology. NUSA: Linguistic studies of languages in and around Indonesia 33:1-28.

van Ekris, A. 1864. Woordenlijst van eenige dialecten der landtaal op de Ambonsche eilanden. Mededeelingen vanwege het Nederlandsch Zendelinggenootschap 8:61-108, 301-336.

van Ekris, A. 1865. Woordenlijst van eenige dialecten der landtaal op de Ambonsche eilanden. Mededeelingen vanwege het Nederlandsch Zendelinggenootschap 9:109136.

van Engelenhoven, Aone. 2004. Leti, a language of Southwest Maluku. Leiden: KITLV Press.

van Hoëvell, G. W. W. C. 1877. Iets over de vijf voornaamste dialekten der Ambonsche landtaal (Bahasa Tanah). Bijdragen tot de Taal-, Land- en Volkenkunde van Nederlandsch-Indië 25:1-136. doi.org/10.1163/22134379-90000614

van Klinken, Catharina. 1995. Tetun Fehan dictionary. Unpublished Toolbox file.

van Klinken, Catharina L. 1999. A grammar of the Fehan dialect of Tetun: An Austronesian language of West Timor. Canberra: Pacific Linguistics.

Verheijen, Jilis A. J. 1967. Kamus Manggarai: I Manggarai-Indonesia. 's-Gravenhage: Martinus Nijhoff.

Verheijen, Jilis A. J. 1982. Komodo: het eiland, het volk en de taal. The Hague: Martinus Nijhoff. doi.org/10.1163/9789004287273

Verheijen, Jilis A. J. 1984. Plant names in Austronesian linguistics. NUSA: Linguistic Studies of Indonesian and Other Languages of Indonesia. 
Visser, Eline. 2019. Field notes on Uruangnirin. In Lexirumah 3.0.0, ed. Gereon A. Kaiping, Owen Edwards, and Marian Klamer. Leiden: Leiden University Centre for Linguistics. lexirumah.model-ling.eu/lexirumah/

Williams-van Klinken, Catharina, and Rob Williams. 2015. Mapping the mother tongue in Timor-Leste: Who spoke what where in 2010?. Dili: Dili Institute of Technology. www.tetundit.tl/Publications/Timor-Leste languages 2010.pdf

Wolff, John U. 2010. Proto-Austronesian phonology with glossary. Ithaca, NY: Cornell Southeast Asia Program Publications.

Zacharias, Albert, Adika Getroida Balukh, Misriani Balle, and Johnny M. Banamtuan. 2014. Lole dictionary. Unpublished Toolbox file.

Zorc, R. David. 1995. A glossary of Austronesian reconstructions. In Comparative Austronesian dictionary: An introduction to Austronesian studies, ed. Darrell T. Tryon, Part 1, 1106-1197. New York: de Gruyter. doi.org/10.1515/9783110884012 .2 .1105 